\title{
MTX FINAL REPORT
}

\author{
January 1994
}

E. B. Hooper, Scientific Editor

\author{
S. L. Allen \\ M. D. Brown \\ J. A. Byers \\ T. A. Casper \\ B. I. Cohen \\ R. H. Cohen \\ M. E. Fenstermacher \\ J. H. Foote \\ K. Hoshino \\ C. J. Lasnier \\ P. Lopez \\ M. A. Makowski \\ M. M. Marinak \\ W. H. Meyer \\ J. M. Moller \\ W. N. Nevins \\ K Oasa \\ T. Oda \\ K. Odajima \\ T. Ogawa \\ T. Ohgo \\ B. W. Rice \\ T. D. Rognlien \\ G. R. Smith \\ B. W. Stallard \\ K. I. Thomassen \\ R. D. Wood
}




\section{TABLE OF CONTENTS}

Executive Summary

The Microwave Tokamak Experiment (MTX)

Introduction (E B. Hooper)

Overview (E. B. Hooper)

Collaboration with JAERI (E. B. Hooper and K. Oasa)

Background

ECRH in Tokamaks (E. B. Hooper)

Nonlinear absorption of intense microwave puses

Descriplion of the ORPAT code (T. D. Rogntien)

Elextron distributions and X-ray spectra for MTX SEL Heating (T. D. Rogrien)

Nonlinear absorption: Scaling with plasma and FEL parameters (T. D. Rognlien)

Description of the experiment

Experimental goals and initial results

Quasi-Optical Transmission Line (B. W. Stallard)

Microwave absorption and heating experiments using the freeelectron laser

Initial experiments to measure the absorption of short, intense microwave pulses from the FEL (E. B. Hooper)

Free-Electron Laser Experiments in the Microwave Tokamak Experiment (S. L. Allen et al.)

Comparison of Propagation and Absorption of FEL-Generated Microwaves with Theory (M. E. Fenstermacher)

Nonlinear Absorption of High Power FEL-Generated

Microwaves at ECRH Frequencies in the MTX Tokamak (S.L.

Allen et al.)

LAPPS Results (J. H. Foote et al.)

Thomson scattering results (J. H. Foote)

ECRH experiments using a $140 \mathrm{GHz}$ Gyrotron

Overview (E. B. Hooper)

Results of ECRH experiments using a $140 \mathrm{GHz}$ gyrotron (E. B. Hooper, S. L. Allen, T. A. Casper, M. E. Fenstermacher, J. H. Foote, C J. Lasnier, M. J. Malowski, K. Oasa, T. Ogawa, B. W. Rice, B. W. Stallard)

Other Experiments

Poloidal Field Experiments (B. W. Rice and E. B. Hooper) Particle Transport (M. M. Marinak) 
8

8.1

8.2

8.2.A

8.2.B

8.3

8.3.A Development to Improve ETA-II: Feedback Timing

Comperation (C. J. Lasanier)

8.3.B Development to Improve ETA-II : Burst Mode Operation (C. J. Lavier)

8.3.C FEL Operation-Burst Mode (C. J. Lasnier)

Appendices

A.1 Documents pertaining to the collaboration

A.1.1 Formal agreement between the US and Japan

A.1.2 Steering Committee reports

A.2 Diagnostics: Microwaves

A.2.1 Microwave beam detectors (B. W. Stallard)

A.2.2 Pulsed Power Calorimeter (K. Onsa)

A.2.3 Microwave Calorimeter (S. Meassick and M. E. Fenstermacher)

A2.4 LAPPS (J. H. Foote et al.)

A.3 Diagnostics: Plasma

A.3.1 Thomson scattering (J. H. Foote)

A.3.2 Neutrons (T. Ogawa)

A.3.3 JAERI Soft $X$-ray (K. Osa)

A.3.4 ECE Dingnostics (C. J. Lasnier)

A.3.5 Diamagretic Loop (M. M. Marinak)

A.4 MTX Data Acquisition System (T. A. Casper)

A.4.1 MTX Data Acquisition and Analysis Computer Network (D. N. Butner, et al.)

A.4.2 Integrated, Multivendor Distributed Data-Acquisition System (D. N. Butner, et al.)

A.4.3 Diagnostic Data Management on MTX (D. N, Butner, et al.)

A.4.4 Maestro (J. M. Moller)

A.5 List of publications and reports 


\section{Executive Summary}

\subsection{Summary}

The Microwave Tokamak Experiment (MTX) was carried out to evaluate the application of high power, pulsed microwaves to electron cyclotron resonance heating (ECRH) of a tokamak. The experiment received significant dingnostics and physics support from the Japan Atomic Energy Research Institute (JAERD).

The microwaves were generated by the free-electron laser (FEL) consisting of the ETA-II electron accelerator and the IMP wiggler. Microwaves generated at $140 \mathrm{GHz}$ were injected into a high magnetic field tokamak moved to LLNL from the Massachusetts Institute of Technology where it had been operated as Alcator C.

This report documents much of the work on MTX in detail so that the information will be available as needed for future Magnetic Fusion Energy experiments.

Experimental highlights include:

- Generation of $140 \mathrm{GHz}$ microwave pulses at powers up to $2 \mathrm{GW}$ and pulse duration of 20-30 ns.

- Experimental demonstration of the feasibility of producing high average power by generating bursts of microwave pulses.

- High efficiency propagation of the microwave power to the tolamak using a quasi-optical transmission line.

- Injection of the microwaves into the tokamak and subsequent measurement of the efficiency of heating. This efficiency was in good agreement with theoretical predictions including nonlinear saturation effects; extrapolation to reactor heating and current drive predicts high efficiency.

- Measurement of the effects of the heating, including the generation of hot electrons. 
In addition to the ECRH experiments using the intense pulses, experiments were conducted using long pulses ( $<100 \mathrm{~ms}$ ) of $140 \mathrm{GHz}$ generated by a gyrotron.

- The heating at plasma density greater than $10^{20} \mathrm{~m}^{-3}$ was demonstrated to be in good agreement with the predictions, providing a basis for interpreting and understanding the high power, pulsed experiments.

- Electron temperature increases of greater than $2 \mathrm{keV}$ were observed on the magnetic axis.

- Significant effects were observed on sawteeth and MHD activity.

- Hollow electron temperature and current density profiles could be converted into peaked, sawtoothing profiles following on-axis ECRH.

- ECRH was observed to permit recovery from minor disruptions resulting from plasma cooling by high-Z impurities.

Three PhD theses resulted from experiments on MTX:

B. R. Rice, "Poloidal Magnetic Field Profile Measurements on the Microwave Tokamak Experiment Using Far-Infrared Polarimetry," awarded by the University of California at Davis, September, 1992.

M. M. Marinak, "Behavior of the Particle Transport Coefficients Near the Density Limit in MTX," awarded by the University of California at Berkeley, April, 1993.

Patricia Lopez, "Microwave Reflectometry: Observing Density Layer Motion in the Microwave Tokamak Experiment," awarded by the University of Michigan, 1993. 


\section{The Microwave Tokamak Experiment (MTX)}

\subsection{Introduction (E. B. Hooper)}

The MTX experiment was proposed in 19861 to apply high frequency microwaves generated by a free-electron laser (FEL) to electron cyclotron resonance heating (ECRH) in a high field, high density tokamak. As the absorption of microwaves at the electron cyclotron resonance requires high frequencies, the opportunity of applying a free-electron laser has appeal as the device is not limited to frequencies in the microwave or long millimeter wavelength regions, in contrast to many other sources. In addition, the FEL is inherently a high power source of microwaves, which would permit single units of $10 \mathrm{MW}$ or more, optimum for reactors. Finally, it was recognized early in the study of the application of the FEL based on the induction linear accelerator, that the nonlinear effects associated with the intense pulses of microwaves ${ }^{2}$ naturally generated would offer several unique opportunities to apply ECRH to current drive, MHD control, and other plasma effects. 1 It was consequently decided to adapt the induction accelerator based FEL to heating and controlling the tokamak, and to conduct experiments on the associated physics.

To this end, the Alcator C tokamak was moved from the Massachusetts Institute of Technology (MIT) to the Lawrence Livermore National Laboratory where it was installed in Building 431 and operated from March, 1989, until the conclusion of the experiment in October, 1992. The FEL, based on the ETA-II accelerator and IMP wiggler was brought into operation by the LLNL Electron Beam Group and power injected into the tokamak during an experimental run in the Fall, 1989. Following an upgrade by the MTX group, a second experimental run was made lasting from the Winter, 1992 through the end of the experiment.

Significant contributions to the ECRH experiments were made by the Japan Atomic Energy Research Institute (JAERD). Their effort included diagnostics and microwave antenna components for the microwaves, diagnostics to help determine the characteristics of the plasma hot electrons generated by the intense microwave pulses from the FEL, and scientific support induding scientists at LLNL.

The FEL generated microwaves at $140 \mathrm{GHz}$ in intense pulses with power up to $2 \mathrm{GW}$ and pulse duration of 20-30 ns. Theory predicted that these pulses would be intense enough that nonlinearities in the interaction with the plasma electrons would saturate the absorption. The saturation was, in fact, measured in good agreement with prediction. Theory also predicted that the absorption would increase if the spectrum of wave numbers parallel to the 
magnetic field were increased over that incident on the plasma in the nominal configuration. This was also observed in good agreement with theory. Hot electrons, predicted for the heating process were also observed.

It is important to emphasize that the nonlinear mechanism extrapolates to high, localized absorption in reactors, so that the saturation mechanism is not a limiting effect. Indeed, microwave injection predominately along one direction of magnetic field is predicted to be absorbed by "bucket lift" more strongly than linear theory predicts, and could be used to drive current more effectively than possible in the linear regime.

The FEL was also operated in burst mode, with more than 30 pulses generated in a $2 \mathrm{kHz}$ burst. This mode provides the possibility of high average power for heating, current drive, or control of MHD activity and disruptions. The burst mode pulses were not, however, injected into the toknmak.

In addition to the absorption measurements using the intense pulses of microwaves from the FEL, experiments were performed using a $140 \mathrm{GHz}$ gyrotron with up to $200 \mathrm{~kW}, 0.1$ second pulses absorbed in the plasma. The absorption of this power occurred in the linear regime, and so they provided a benchmark for the high-power puises from the FEL. In addition, the pulses demonstrated strong electron heating on the tokamak axis; the use of ECRH to convert hollow current profiles, occasionally observed during the initial plasma operation, into well peaked profiles; and the prevention of disruptions resulting from high- $Z$ impurity cooling.

Plasma current profiles were measured using Faraday rotation on the far infrared interferometer. A new technique increased sensitivity and time response beyond that achieved in other experiments. In addition to measurements during ECRH with the gyrotron, this sensitivity permitted the first observation of a very interesting poloidal field pulse during the sawtooth collapse. The on-axis 90 before the sawteeth collapse was typically 0.8 , and it remained below unity despite the reconnection process.

Other work on the tokamak included the establishing of a disruption data base, determination of the time evolution of distuptions, and use of gas pulses to determine the particle transport coefficients near the density limit.

1. K. I. Thomassen, et al., "Free-Electron Laser Experiments in Alcator C," LLL-PROP-00202, July 1986.

2. W. M. Nevins, T. D. Rognlien, and B. I. Cohen, Phys. Rev. Letters $\mathbf{5 9 , 6 0}$ (1987). 


\section{Overview (E. B. Hooper)}

The basic components of the Microwave Tokamak experiment are shown in Fig. 2.1-1. The tokamak is the Alcator C device, moved to LLNL from MIT because the electron cyclotron frequency at its high magnetic field matched the high frequencies available from the free-electron laser. The tokamak was installed in the high bay at the east end of Building 431, together with the required power supplies, neutron shielding, etc. A comprehensive list of references to publications describing the engineering as well as physics results can be found in Appendix A.5 at the end of this report.

The ETA accelerator was operated in the adjacent building extension. The experimental test accelerator was being upgraded to ETA-II; following later improvements it provided the necessary electron beam to drive the free-electron laser: I $>2 \mathrm{kA}, V>6 \mathrm{MeV}$, and pulse length $>50 \mathrm{~ns}$. The electron beam was transported to the Intense Microwave Prototype (MMP) wiggler.

In initial experiments microwaves were generated in an extended interaction oscillator (ETO) oscillator and approximately $50 \mathrm{~W}$ were injected into the wiggler, resulting in microwave pulses with powers of 100-400 MW. Up to $200 \mathrm{MW}$ were transmitted to the tokamak using a quasi optical transmission line. Experiments to measure the microwave absorption by the tokamak plasma were conducted at this initial power. The measurements were consistent with linear theory as expected.1

Following improvements to ETA-II, a second series of experiments were conducted using as an oscillator a microwave beam from a gyrotron mounted on the second floor above the wiggler. Approximately $10 \mathrm{~kW}$ were injected into the wiggler, resulting in output pulse power as high as $2 \mathrm{GW}$. Pulses injected into the tokamak at powers up to $1.5 \mathrm{GW}$ were used in a second set of absorption experiments, which found absorption reduced below the linear theory and in good agreement with the nonlinear predictions. ${ }^{2}$ The results, described in detail in Chapter 5, demonstrated the enhanced transmission through the plasma due to nonlinear effects. It is important to emphasize that for a reactor-grade plasma the absorption is close to $100 \%$, even with the nonlinear effects. Furthermore, as demonstrated in the experiment, the nonlinearities provide the opportunity for enhanced confinement. This could be used to provide directionality in absorption, and thus current drive, by appropriately tailoring the spectrum of wave numbers along the magnetic field.

Extensive diagnostics were utilized, both by LLNL and by the collaborators from the Japan Atomic Energy Research Institute (JAERD). Diagnostics included standard tokamak diagnostics, an advanced far infrared interferometer/ polarimeter, and a set dedicated to determining the effects of the microwave injection. The transmission of microwaves through the plasma, and thus the absorption for comparison with theory, was measured using a calorimeter mounted on the small major radius of the tokamak opposite the injection port and by a microwave horn mounted in the center of the calorimeter. The calorimeter is described in detail in Appendix A.2.3. Hot electrons, predicted by 
the theory, were observed by Thomson scattering, electron-cyciotron emission (ECE), and soft xrays. One of the ECE systems (heterodyne receiver) and the fast $(1 \mu \mathrm{s})$ soft $x$-ray system were constructed and operated by JAERI.

Three PhD theses were written from work on MTX: Measurements of the current density profile were made using the far infrared polarimeter, generating new information on tokamak current physics. ${ }^{3}$. The particle confinement near the density limit was measured using a gas-pulse technique, yielding transport coefficients which agreed well with nonlinear predictions based on nonlinear turbulence.4 In the third thesis, density layer motion in MTX was observed.5

1. S. L Allen et al., Plasma Physics and Controlled Nuclear Fusion Research 1990 (IAEA, Vienna, 1991), Vol 1, pp 783-787.

2 S. L. Allen et al., Plasma Physics and Controlled Nuclear Fusion Research 1992 (IAEA, Vienna, 1993), to be published.

3. B. W. Rice, "Pololdal Magnetic Field Profile Measurements on the Microwave Tokamak Experiment Using Far-Infrared Polarimetry," UCRL-LR-1I1863 (1993).

4. M. M. Marinak, "Behavior of the Particle Transport Coefficients Near the Density Limit in MTX," UCRL-LR-113984 (1993).

5. Patrici Lopez, "Microwave Reflectometry: Observing Density Layer Motion in the Microwave Tokamak Experiment," Ph.D. dissertation, Department of Nuclear Engineering, University of Michigan, 1993. 
Fig. 2.1-1

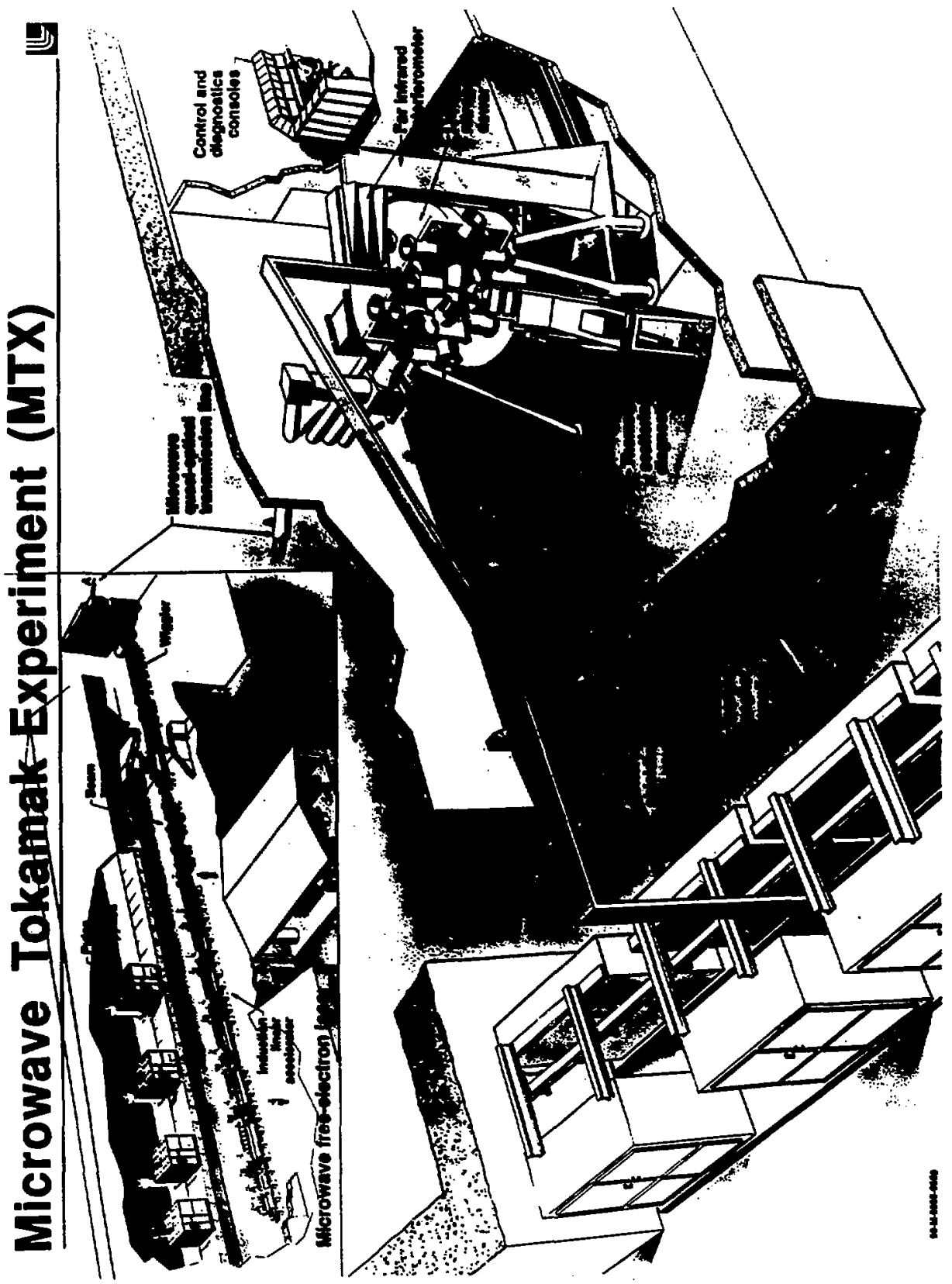




\subsection{Collaboration with JAERI}

The MTX experiement was supported by the Japan Atomic Energy Research Institute (JAERI) through a collaboration established by Annex 6 of the USJAPAN Collaboration on Magnetic Fusion Energy. The Annex, included as Appendix A.1.1, provided the formal basis for the joint effort.

The collaboration was-very fruitful providing essential support to the experiment in scientific, diagnostic, and microwave technology areas.

Oversight of the activities within the collaboration was provided by a steering committee consisting of representatives of JAERI Headquarters, JAERI scientists, DOE, and LLNL. Summaries of the Steering Committee meetings are attached as Appendix A.1.2.

The collaboration included both equipment and diagnostics for use in the experiement and scientists participating in the experiment. 


\subsection{ECRH in Tokamaks (E. B. Hooper)}

Electron cyclotron resonance heating (ECRH) has been extensively applied to tokamaks.1.2 Applications include: central heating both as a source of plasma energy and as a means of affecting sawteeth; localized heating utilizing the resonance with the magnetic field; control of MHD activity, for example by: heating in the close vicinity of the $m=2$ surface to reduce (or enhance) the mode amplitude and thus, indirectij; the likelihood of disruption; and current drive using the directional effects of the Duppler shift with the associated shift in the resonant location to enhance absorption in one direction.

- T-10 achieved $T_{e}=9 \mathrm{keV}$ using central electron heating with $2.4 \mathrm{MW}$ of ECRH power. ${ }^{3}$ They concluded that heat transport is not described by purely local piarameters, and that the effectiveness of off-axis heating is enhanced by weak oll-axis ECRH. However, experiments in PDX concluded that the transport model used in ohmic heating was valid for ECRH on axis. ${ }^{4,5}$

- DIID achieved H-mode operation when power was absorbed near the axis; power noar the edge did not generate the $H$-mode.5,7 JFT-2N found ECRH to generally more effective than neutral beams in establishing the $\mathrm{H}$ mode. ${ }^{8}$

- T-10, TFR, PDX, CLEO and DIID lengthened the sawtooth period or. stabilized sawteeth by heating near the $q=1$ surface $9,10,11,12,13,14,15$

- T-10 and TFR stabilized or modified the $m=2$ made by heating near the $q=2$ surface; if done early in the MHD activity, minor disnuptions were averted. ${ }^{11,16}$

- JFT-2M avoided disruptions at $q_{\Omega}=3$ by ECRH within a narrow radial region near the $q=2$ surface. ${ }^{17}$

- Current drive was demonstrated in CLEO, WT-2, and WT-3.18, 19, 20 In WT-2 and WT-3, ECRH power sustained a low current when the ohmic drive was turned off.

\section{References}

1. R. Prater in Applications of RF Waves to Tokamak Plasmas (Proc. Course and Workshop Varenna 1985), Vol. 1, Monotypia Franchi, Perugia, 354 (1985).

2. A. C. Riviere in Applications of Radiofrequency Power to Plasmas (Proc. 7th Top. Conf. Kissimmee, FL, 1987), AIP Conference Proceedings 159, 1 (1987). 
3. V. Alikaev et al., "Power Deposition Profile Effect on ECH Efficiency on T10", European Physical Society Meeting, Madrid, June 1987.

4. A. Cavallo, H Hsuan, D. Boyd, B. Grek, et al., Nucl. Fusion 25, 335 (1985).

5. A. H. Kritz, H. Hsuan, A. Cavallo, D. McCune, et al., Proc. 6th Topical Conf. RF Plasma Heating , AIP Conference Proceedings No. 129, 235 (1985).

6. J. Lohr et al., Phys. Rev. Letters 60, 2630 (1988).

7. B. W. Stallard et al., Nucl. Fusion 30, 2235 (1990).

8. K. Hoshino et al., Nucl. Fusion 28, 301 (1988).

9. R. M. J. Sillen et al., Nucl. Fusion 26, 303 (1986).

10. W. J. Goedheer and E. Westerhof, Nucl. Fusion 28, 565 (1988).

11. TFR Group, Proc. 13th European Plasma Phys. Soc., Schleersee, Vol II, 209 (1986).

12. H. Hsuan, R. Polman, D. Boyd, A. Cavallo, et al., Plasma Phys./Control Fusion 26, 265 (1984).

13. H. Hsuan, K. Bol, N. Bowen, D. Boyd, et al., Proc. 4th Intern. Symp. Heating in Toroidal Plasmas, Vol II, 809 (1984).

14. A. C. Riviere et al., in Heating in Toroidal Plasmas, Rome, 1984, Vol II, 795.

15. R. T. Snider et al., Phys. Fluids B 1, 404 (1989).

16. V. V. Alikasv and M. M. Stepanenko, JETP Letters 40, 1122 (1984).

17. K. Hoshino et al., Phys. Rev. Letters 69, 2208 (1992).

18. D. C. Rcbinson et al., Proc. 11th Intern. Conf. Plasma Phys. and Controlled Fusion Research, Kyoto, Japan, 1986, Vol 1, p. 575.

19. A. Ando et al, Phys. Rev. Letters 56, 2180 (1986).

20. H. Tanaka et al., Phys. Rev. Letters 60, 1033 (1988). 


\subsection{Nonlinear absorption of intense microwave pulses.}

The theory of absorption and its effects on the plasma electrons has been extensively developed elsewhere1,23 (c.f. Ref. 3 for further references) and thus will not be repeated here. Physically, the heating by the intense pulses is strong enough that the mass shift of the electrons is the dominant factor in determining the absorption. The consequent change in the cyclotron frequency causes an electron initially heated in phase with the microwaves to slip in phase relative to the wave and to feed its energy back into the electromagnetic field. The process repents, so the electron mass oscillates repetitively, and the electron is effectively trapped in a nonlinear ("bucket") resonance. This resonance is essentially the reverse of the nonlinear trapping which transfers energy from an electron beam to an electromagnetic wave in a free-electron laser.

In the simplest case, absorption of microwaves traveling across the magnetic field, the nonlinear effects limit the microwave absorption, reducing the opacity. This effect is not strong enough to limit the heating of a reactor-grade plasma, for which the absorption is close to $100 \%$. The bucket . trapping, however, offers possibilities of increasing absorption and of enhancing the directionality of current drive. For example, suppose the wave-vector, $k_{/ /}$along the magnetic field varies slowly enough in space that the electrons respond adiabatically as they travel along the magnetic field. The energy of the Doppler-shifted resonance will increase or decrease, depending on whether $k_{/ /}$increases or decreases along the field. From the "bucket" analogy, the central mass of the nonlinear resonance will change, carrying the trapped electrons along with it. Thus, the energy may be decreased or, of more interest, increased until the wave energy is absorbed or the electrons leave the region of heating. The result is that heating can be even stronger than linear theory predicts; the directionality of the effect can drive current in the plasma.

The experiments described later in this report demonstrated both the nonlinear saturation of the intense pulses and the effect of bucket lifting due to varying $k_{/ /}$. The results are in good quantitative agreement with heating predictions; current drive experiments were not attempted. Also, electron heating is predicted to generate a nonMaxwellian distribution which is observed. These two results provide strong support for the theory.

\subsubsection{Description of the ORPAT code (T. D. Rognlien)}

Here I give a brief description of the ORPAT code that has been used to calculate the nonlinear absorption of the FEL pulse in MTX. In aritition, the 
electron distribution functions calculated by the code have been used to compare with the heated distribution obtained from the Thomson scattering diagnostic and the $x$-ray signal emitted from the hot electrons.

ORPAT follows an ensemble of test electrons along a magnetic field line using the relativistic guiding-center equations of motion. In the absence of an If EM field, the magnetic mument and energy of the particles are conserved. Coulomb collisions off background distributions of electrons and ions can be included, and in this mode of operation, the code is sometimes referred to as MCPAT. Collisions do change the magnetic moment and energy of individual particles. Because the FEL pulse for MTX is only 20-50 ns, we usually omit the collisions as unimportant. It is possible to specify both electrostatic potential and magnetic field profiles, although the electrostatic potential is usually taken to be zero. Note that ORPAT is not a self-consistent simulation code, although potentials and background scattering distributions can be obtained by iterative procedures not discussed here. The magnetic field profile is taken to model a simple tokamak equilibrium, so passing particies and trapped particles are accounted for. Because the FEL heating in MTX is typically near the axis and the FEL pulse is on for a short time, trapping. which results in multi-pass heating for some electrons is not very important.

When an if EM field is present, ORPAT includes terms in the relativistic equations of motion for the magnetic moment, energy, and gyrophase angle arising from the wave. $A$ decomposition of the wave into cyclotron harmonics is used, and typically only one harmonic (the resonant one) is used. This is valid as long as the deviation of the electron from its zero-order gyromotion can be treated as a linear perturbation. Even though the if fields in MTX are strong, the approximate is valid for much larger fields. The ORPAT description of the wave-particle interaction has been checked extensively with leap-frog particle advance that makes no assumptions as to the size of th.e perturbation. However, the leap-frog scheme must operate with a time-step of $\omega_{c} \Delta t * 1$, whereas the ORPAT scheme is more efficient with $\omega_{c} \Delta t * 1$. Toroidal profiles of the electric field and parallel index of refraction $\left(N_{k}=k_{1} \in \omega\right)$ are obtained from the MTH code and fit with cubic splines. The effects of the rf magnetic field are included using Faraday's law $(\partial B / \partial t=-\nabla \times E)$; the resulting terms in the equations of motion are proportional to $N_{H}$.

In doing MTX calculations, two basic simulation modes are used: (1), net power absorption for comparison with the MTX calorimeter and (2), electron distributions including toroidal streaming for comparison with Thomson scattering and $x$-ray diagnostics.

\section{(1) Absorption Calculations}

For the absorption calculations, a short section near the FEL beam is usually simulated. An ensemble of Maxwellian electrons is injected from 
each side of the section and followed as they stream through the if beam. The energy change of each electron is recorded and deposited on a spatial grid in the toroidal direction, resulting in an energy absorption rate for the given density called $\dot{W}$ in Watts $/ \mathrm{cm}^{3}$. Integrating along the $B$-field line in the nearly toroidal direction is included to remove one of the dimensions. $A$ series of these calculations can be done for field lines at different vertical positions, taking into account the change in the amplitude of the rf beam to integrate in the second dimension. Typically, the effects of different vertical field lines is small, and one can get a good estimate of the absorption by using the vertical beam width as the vertical dimension of the beam power profile.

The final direction to integrate the power absorption is radially. Again, this can be done by using different magnetic field profiles, the primary effect being the 1/R increase in the magnitude of $B$ as the beam propagates toward the inner wall. The cyclotron resonance for $N_{1}=0$ is $\omega=\omega_{c} / \gamma$, where $\gamma$ is the relativistic mass shift. Consequently, as one moves to larger $B$, the number of electrons available to resonant with the wave decreases, and the absorption falls. For MTX, the absorption layer is roughly $1 \mathrm{~cm}$ thick. We have used two models; one assumes the if beam is not attenuated as the calculation is shifted to small radii, and the second attenuates the beam based on the power absorption found on the last radial surface (nearly a slab). Note that we cannot simple attenuate the if power exponentially with the absorption coefficient, as that applies to the linear absorption regime. The final absorption results we presented in the Phys. Rev. Letter paper used the attenuation model. The attenuation is based on the fact that the power propagation normal to the beam is the Poynting flux

$$
P=\frac{\in E_{n}^{2}(2, y)}{2} c
$$

where $\epsilon$ is the total permittivity of the plasma. Thus, if the calculated absorption at one radius is $\dot{W}$ and the Poynting flux there is $P_{\text {in }}$ then the Poynting flux at an adjoining radius separated by $\Delta R$ is

$$
P_{e}=P_{\text {in }}-\dot{W} \Delta R
$$

The $N_{1}$ of the beam is assumed to be unaffected by the attenuation.

\section{(2) Electron Distributions and Time Dependence}

The second mode of operation is to assemble the electrons into a distribution function to understand the heating mechanism in detail and to compare with Thomson scattering and $x$-ray diagnostics. The electron distributions are formed as contour plots of $f\left(\mathrm{YO}_{1} \mathrm{\gamma O}_{1}\right)$ at a given spatial location. An area-weighted, bi-linear interpolation scheme is used to transfer the particle data to a $30 \times 302-D$ velocity grid. This data is also integrated over $\gamma v_{1} \gamma \gamma_{\perp}$ or pitch angle to give emphasis to different aspects of the data. The 
Thomson scatter diagnostic views one velocity component normal to the magnetic field. Thus, if $h_{2}\left(r^{2} v^{2}\right)$ is the result of integrating $f$ over the parallel velocity, then the Thomson scattering will measure the 1-D velocity distribution

$$
h_{1}\left(\gamma \sigma_{x}\right)=\int_{0}^{\infty} h_{2} d y=-\int_{\gamma^{2}}^{\infty}\left(u-\gamma^{2} v_{x}^{2}\right)^{1 / 2} \frac{\partial h_{2}}{\partial u} d u
$$

where $u=\gamma^{2} v_{1}^{2}$. For typical MTX parameters, one may set $\gamma=1$ in the above expressions, although the mass is still retained in the equations of motion.

To determine the x-ray signal that the distribution $f_{\varepsilon}$ will emit, we integrate over pitch angle to obtain $f_{c}$. The $x$-ray spectrum is then calculated as

$$
F_{v}\left(\varepsilon_{v}\right) \propto \int_{e_{v}}^{\infty} f_{d}(\varepsilon) G\left(\varepsilon, \varepsilon_{v}\right) d \varepsilon
$$

where $F_{v}$ is the $x$-ray spectrum from bremsstrahlung at a photon energy of $\varepsilon_{v}$ for an electron energy distribution $f_{\varepsilon}$. The function $G$ can be obtained from Jackson, 5 and is given approximately by

$$
G\left(\varepsilon, \varepsilon_{v}\right)=\gamma\left(\gamma^{2}-1\right)^{1 / 2} \ln \left[\frac{\varepsilon^{1 / 2}+\left(\varepsilon-\varepsilon_{v}\right)^{1 / 2}}{\varepsilon_{v}^{1 / 2}}\right]^{2}
$$

where $\gamma=1+\varepsilon / m c^{2}$ is the relativistic mass factor. For MTX, it is probably sufficient to use tile nonrelativistic expansion for $G$, but ORPAT uses the full form given above.

Finally, a word about time dependence. ORPAT can follow the whole ensemble of electrons for a series of different time intervals, during which time input to the code can be changed, and diagnostics can be view for only a specified time interval. This is important because the FEL beam is only on for about $20 \mathrm{~ns}$; thus, only electrons within about $50 \mathrm{~cm}$ of the port will be heated. The Thomson scattering diagnostic at $134 \mathrm{~cm}$ from the FEL port records its data about 100 ns after the initiation of the FEL pulse, so transit time effects can be important. In our comparison with the Thomson scatter data, we use this same delay to get as accurate a model as possible. The $x$-ray data is taken 1 us after the FEL pulse, and we likewise model this time delay which results in essential toroidal symmetry of the electron distribution. 
3.2.2 Electron distributions and X-ray spectra for MTX FEL Heating (T. D. Rognlien)

I have used ORPAT to performed a set of calculations for FEL heating in MTX to assess the expected X-ray spectrum for the two basic modes of operation: a straight FEL port with no insert and a tapered FEL port with a corrugated insert. The primary difference between these configurations is in the RF beam profile at the center of the plasma as shown in Fig. 3.2-1. The profiles for the no insert case are cubic-spline fits to output from the MTH wave propagation code plus a contribution from the toroidal curvature; for the case with an insert, a Gaussian electric field profile and a linear $N_{\| 1}$ profile are used as these are good fits to the MTH code plus the curvature effect. The most important difference between the two cases is the larger $N_{\|}$variation for the insert case which results in a large contribution to the heating from

bucket lift. ${ }^{6}$ The detailed structure of $N_{\|}$seen in case (a) is of little importance as nearly the same results can be obtained with a linear $N_{\|}$approximation.

The parameters chosen for the comparison are those corresponding to MTX shots 16296-16309. These are the shots with an insert for which Foote obtained the Thomson scattering data showing an energetic electron tail. The on-axis parameters are

$$
\begin{gathered}
n_{e}=6.5 \times 10^{13} \mathrm{~cm}^{-3} \\
T_{e}=1.55 \mathrm{keV} \\
f_{f f}=140 \mathrm{GHz} \\
P_{f f}=1 \mathrm{GW}(0-\text { mode }) \\
E_{f f}(z, y)=E_{f f}(z) \exp \left[-\left(y / y_{e}\right)^{2}\right]
\end{gathered}
$$

where $z$ is in the toroidal direction, and $y$ is in the vertical direction. For both cases, I use $y_{e}=2.83 \mathrm{~cm}$ obtained from the MTH code and adjust $E_{\text {rf }}(0)$ to give 1 GW; for no insert, $E_{r f}(0)=2.82 \times 10^{5} \mathrm{~V} / \mathrm{cm}$ while with the insert, $E_{r f}(0)=$ $2.37 \times 10^{5} \mathrm{~V} / \mathrm{cm}$. Because the experiment used the ordinary mode, $E_{r f}$ is parallel to the magnetic field. I consider three different magnetic field strengths to simulate on-axis heating: $B=5.00 \mathrm{~T}, 5.05 \mathrm{~T}$, and $5.10 \mathrm{~T}$. More detailed calculations have shown that most of the power is absorbed in this region and including the gradient in the magnetic field for heating above or below the aris has little effect.

The simulation is performed by initializing an ensemble of electrons with a Maxwellian velocity distribution and uniform spatial distribution along the entire flux-tube length of $2 \pi R=402 \mathrm{~cm}$; typically 15,000 electrons are used. ORPAT follows the relativistic equations of motion for the electrons as they pass through the beam, retaining only the fundamental cyclotron harmonic interaction.4 The FEL beam is then turned on at $t=0$ and switched off at $t=20$ ns. The electrons then continue to stream toroidally and collisionally scatter off the Maxwellian background at $1.55 \mathrm{keV}$ and the ions. However, in the 
time period of $1 \mu \mathrm{s}$, which is when the $x$-ray measurements are taken, the collision time for a thermal electron is about $10 \mu \mathrm{s}$ and for a $5 \mathrm{keV}$ electron, it is $50 \mu \mathrm{s}$. Thus, the collisions have negligible effect on the high-energy electrons. Also, at $1 \mu \mathrm{s}$, the toroidal streaming has mixed the electrons sufficiently that there is no spatial dependense remaining for the distribution function, i.e., viewing the xrays near the FEL port or on the opposite side of the machine would yield the same result.

The electron distributions at $t=1 \mu$ for the case of no insert are shown in Fig. 3.2-2 for the three magnetic field strengths that would correspond to radial positions of $R-R_{0}=0 .,-0.64,-1.28 \mathrm{~cm}$. The FEL beam profile is not attenuated to account for absorption in these calculations. Other calculations predict that about $50 \%$ of the FEL beam would be absorbed for this case. The contour levels in Fig. 3.2-2 are spaced logarithmically with (a) being close to the initial Maxwellian. For this $B=5.00 \mathrm{~T}$ case, the $\omega=\omega_{c} / \gamma$ resonance occurs for zero energy where $\gamma=1$, and there is not much Doppler shift from $N_{\mathrm{fi}}$ little net heating results. For the $B=5.10 \mathrm{~T}$, the cyclotron resonance occurs for 10 $\mathrm{keV}$ electrons, and more nonlinear bifurcation heating is observed.

The corresponding $x$-ray spectrum is calculated from the distribution function. Here I average over pitch angle, and consider the distribution to be a function of energy. The spectrum is then given by

$$
F_{v}\left(\varepsilon_{v}\right)=\int_{\varepsilon_{v}}^{\infty} f_{d}(\varepsilon) G\left(\varepsilon, \varepsilon_{v}\right) d \varepsilon
$$

where $F_{v}$ is the $x$-ray spectrum from bremsstrahlung at a photon energy of $\varepsilon_{v}$ for an electron energy distribution $f(E)$. The function $G$ can be obtained from Jackson,, 5 and is given approximately by

$$
G\left(\varepsilon, \varepsilon_{v}\right)=\gamma\left(\gamma^{2}-1\right)^{1 / 2} \ln \left[\frac{\varepsilon^{1 / 2}+\left(\varepsilon-\varepsilon_{v}\right)^{1 / 2}}{\varepsilon_{v}^{1 / 2}}\right]^{2}
$$

where $\gamma=1+\varepsilon / m c^{2}$ is the relativistic mass factor. For MTX, it is probably sufficient to use the nonrelativistic expansion for $G$, but ORPAT uses the full form given above.

The results for the "no insert" case are shown in Fig. 3.2-3 for the distributions in Fig. 3.2-2. For $B=5.00 \mathrm{~T}$, the straight line for $f(E)$ shows that the distribution is close to the initial Maxwellian. The x-ray photon distribution, $F_{v}\left(\varepsilon_{v}\right)$ is also nearly Maxwellian in shape. At higher magnetic fields, more heating occurs, and the photon distribution shows a more pronounced tail than the electron particle distribution.

Turning to the case with the insert, the electron distributions are shown in Fig. 3.2-4. Considerably more heating occurs here because of the larger change in $N_{\|}$across the beam leading to substantial bucket-lift heating. The 
corresponding electron and photon distributions versus energy are shown in Fig. 3.2-5; note that the energy scale is a factor of two larger than in Fig. 3.2-3. Again, the $E_{\gamma f}$ amplitude is not attenuated at larger magnetic fields here, but absorption calculations find that about $85 \%$ of the beam would be absorbed for this case. The high-energy electron and, especially photon, distributions are strongly enhanced over the "no insert" case.

In order to quantify the enhancement of the x-ray spectrum, I compared the $x$-ray signal at a given energy with that from a Maxwellian at $1.55 \mathrm{keV}$; the results are shown in Fig. 3.2-6. At energies of $10 \mathrm{keV}$ and greater, the results with the insert show at least an order of magnitude more enhancement than the "no insert" case. At $5 \mathrm{keV}$, the case with the insert shows a factor of 2-3 greater enhancement than the "no insert" case.

To summarize, un increase in the hot electron production and wave absorption is obtained when the FEL port insert is used owing to the larger $N_{\|}$ variation across the beam in the toroidal direction and the concomitant increased heating from the bucket-lift effect. The x-ray photon emission at moderate to high energies $(5-40 \mathrm{keV})$ is enhanced over the electron distributions at those energies. At $5 \mathrm{keV}$, the $x$-ray signal with the insert would be 2-3 times greater than that with no insert; for 10-20 keV, the $x$-ray signal with the insert should be at least 10 times greater than without the insert. The effect of collisions for the $1 \mu$ interval before the $x$-ray signals are recorded has been included, but is not important. The effect of toroidal streaming of electrons has also been included. The streaming results in a toroidally symmetric electron distribution after $1 \mu s$. Thus, the same $x$-ray signal would be obtained whether one was viewing near the FEL port or on the opposite side of the torus.

\subsubsection{Nonlinear absorption: Scaling with plasma and FEL parameters (T. D. Rogrilien)}

Here we present some results on how the nonlinear FEL absorption in MTX scales with various parameters, namely, density, power, and electron temperature. As mentioned earlier, we perform calculations here at the vertical midplane, i.e., $y=0$, assuming that the vertically integrated transmission over the FEL beam is the same, except for effects of refraction. Other calculations have been done to verify that this procedure produces nearly the same result as doing calculations at a series of vertical locations. The attenuated-beam model described previously is used for these absorption calculations.

The effects of plasma refraction shown in Fig. 3.2-7 have been taken from the calculations of Fenstermacher using the TORCH ray-tracing code. ${ }^{7}$ Refraction from the beam port to the magnetic axis and the additional 
refraction from the magnetic axis to the wall or calorimeter are shown. Data points represent calculations for a range of measured density profiles characteristic of MTX operation. The second order polynomial fits (dashed curves) were used for the FEL absorption simulations. With this refraction effect, the parallel electric field on axis, Erf, is modified for the absorption calculations, and the power at the calorimeter is further reduced by refraction from the axis to the wall.

To clarify what is meant by central transmission, we define three different power transmission factors $T_{1}, T_{2}$ and $T_{3}$ based on the local transmission at the inner wall, $T(z, y)$ :

$$
\begin{gathered}
T_{1}=T(0,0) \\
T_{2}=\int T(z, 0) d z \\
T_{3}=\iint T(z, y) d z d y
\end{gathered}
$$

Here $z$ and $y$ are in the toroidal and vertical directions, respectively. Because we assume there is no $y$ variation to the transmission factor here, $T_{2} / T_{3}=$ refraction factor from the magnetic axis to the wall; thus, $T_{2}<T_{3}$.

We first consider results with the tapered insert in the FEL port that produces the Gaussian beam shape in $z$ shown in Fig. 3.2-1(b). The Erf, variation in the vertical direction is taken to be Gaussian with a scale length of $y_{e}=2.83 \mathrm{~cm}$. The transmission factors $T_{1}, T_{2}$ and $T_{3}$ versus various parameters are given in Figs. 3.2-8 to 3.2-11.

Note that the central transmission, $T_{1}$, is always the largest, arising because the absorption is largest at the edges of the beam. This effect could arise because the nonlinearity at the edge of the beam is weaker, but we believe the major contributor is the nature of the nonlinear "bucket-lift" absorption. Thus, as the beam propagates through the absorption region near the magnetic axis, the shape becomes non-Gaussian and more peaked at $z=0$. Also, there is a significant reduction of the transmission with $T_{e}$ as seen in Fig. 3.2-11.

Finally, a wider range of on-axis densities was considered for both the cases with the port insert and those without the insert. The profile of $E_{r f}$, and Nin used for the case without the insert are again shown in Fig. 3.2.1(a). The results of the toroidally averaged transmission at $y=0, T_{2}$, are shown in Fig. 3.2-12 for two power levels: $1.0 \mathrm{GW}$ and $0.75 \mathrm{GW}$. Here we have set $T_{e}=1$ $\mathrm{keV}$. We think $T_{2}$ is the transmission factor that most closely models the signal on the calorimeter. There are a number of experimental shots in this power and density range, and Fig. 3.2-12 forms the final theoretical data set that is used to compare with the MTX data.

\section{References}

1. W. M. Nevins, T. D. Rognlien, and B. I. Cohen, Phys. Rev. Letters 59, 60 (1987). 
2. R. H. Cohen and T. D. Rognlien, Phys. Fluids B 3, 3406 (1991).

3. B. I. Cohen, R. H. Cohen, W. M. Cohen, and T. D. Rognlien, Rev. Mod Phys. 63, 949 (1991).

4. T. D. Rognlien, Phys. Fluids 26, 1545 (1983).

5. J. D. Jackson, Classical Electrodynamics, (Wiley, New York 1975) Second Ed., Chapter 15.

6. R. H. Cohen and T. D. Rognlien, Phys. Fluids B 3, 3406 (1991).

7. G. R. Smith, M. E. Fenstermacher, and E. B. Hooper, Nucl. Fusion 30, 2505 (1990). 
Fig. 3.2-1
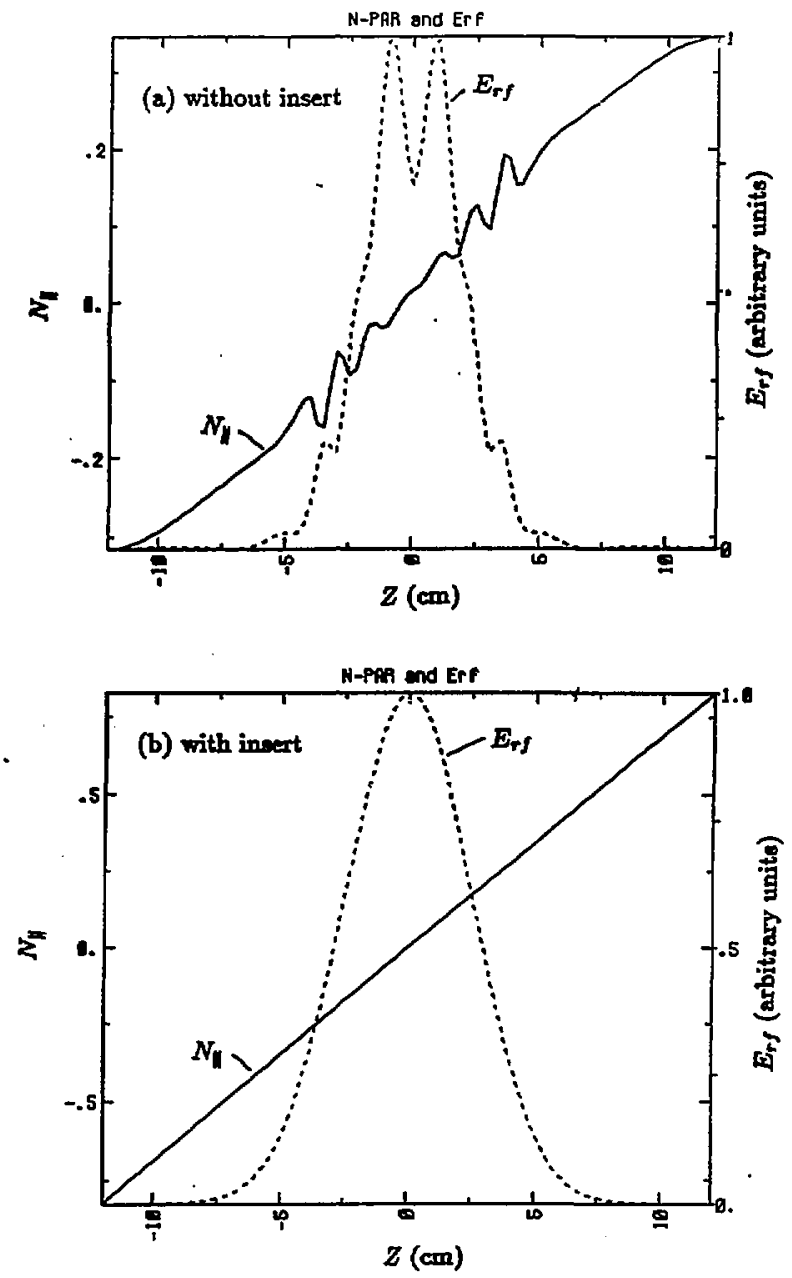

Fig. 1. Toroidal profiles of parallel index of refaction, $N_{\|} \equiv k_{\|} c / \omega$, and ordinary mode parallel electric field, $E_{r f}$ for the two case of (a), no FEL port insert, and (b), with the tapered FEL port insert. Profiles are fits to the MTH code output. 

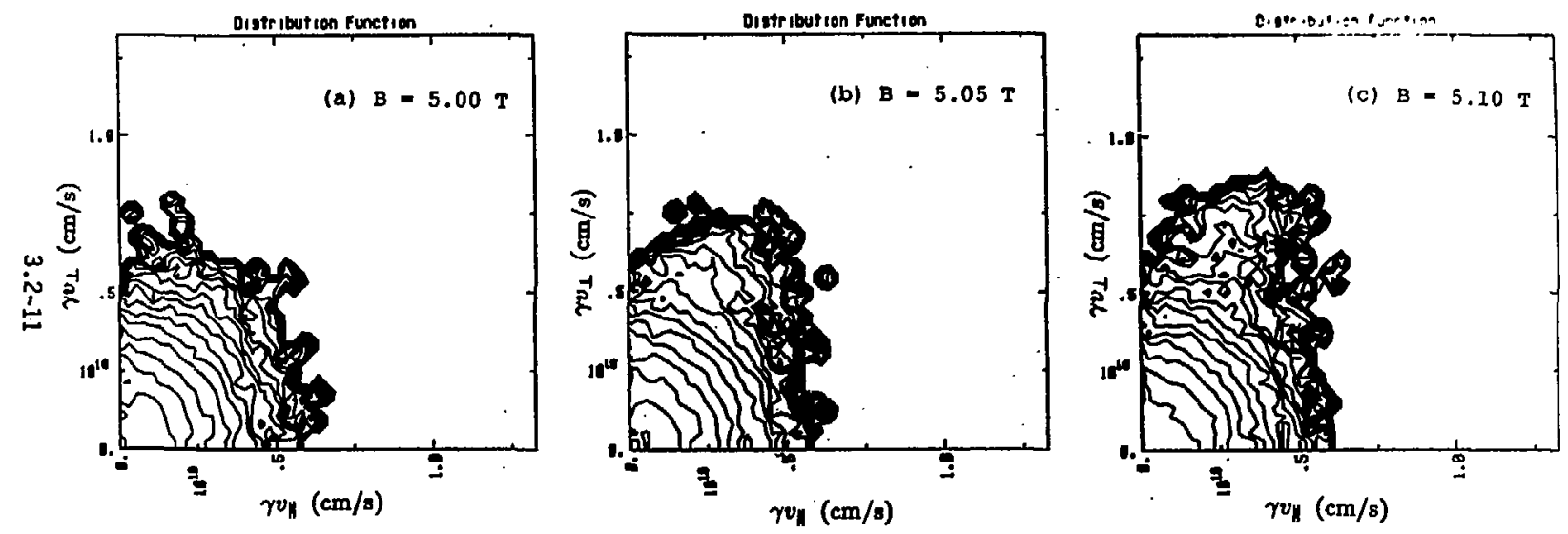

: Fig. 2. Logarithmic contours of the electron distribution functions for the case without the FEL port insert for thrice magnetic field strengths. 


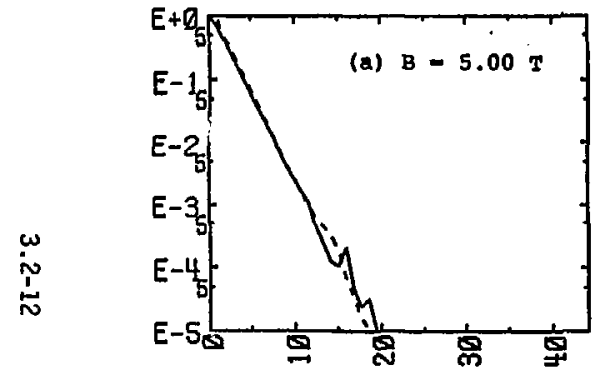

$\varepsilon, \varepsilon_{\text {v }} \mathrm{keV}$

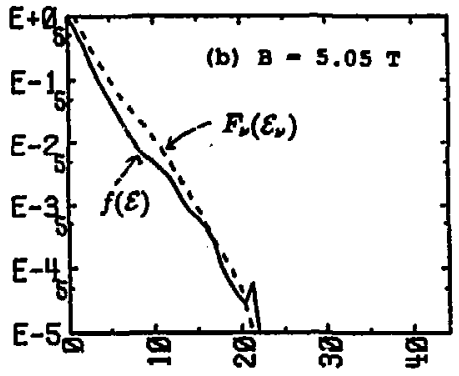

$\varepsilon, \varepsilon_{,} \mathrm{keV}$

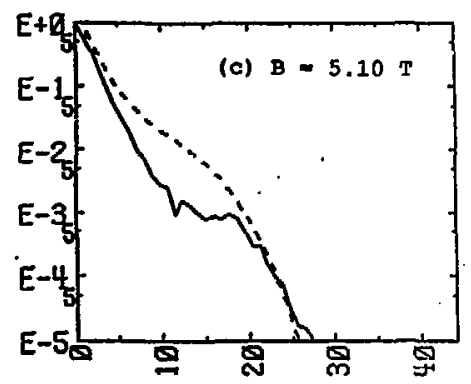

$\varepsilon, \varepsilon_{\nu} \mathrm{keV}$

Fig. 3. Electron energy distribution corresponding to the three cases in Fig. 2 having no insert and the associated $x$-ray photon apectrum, $F$. 

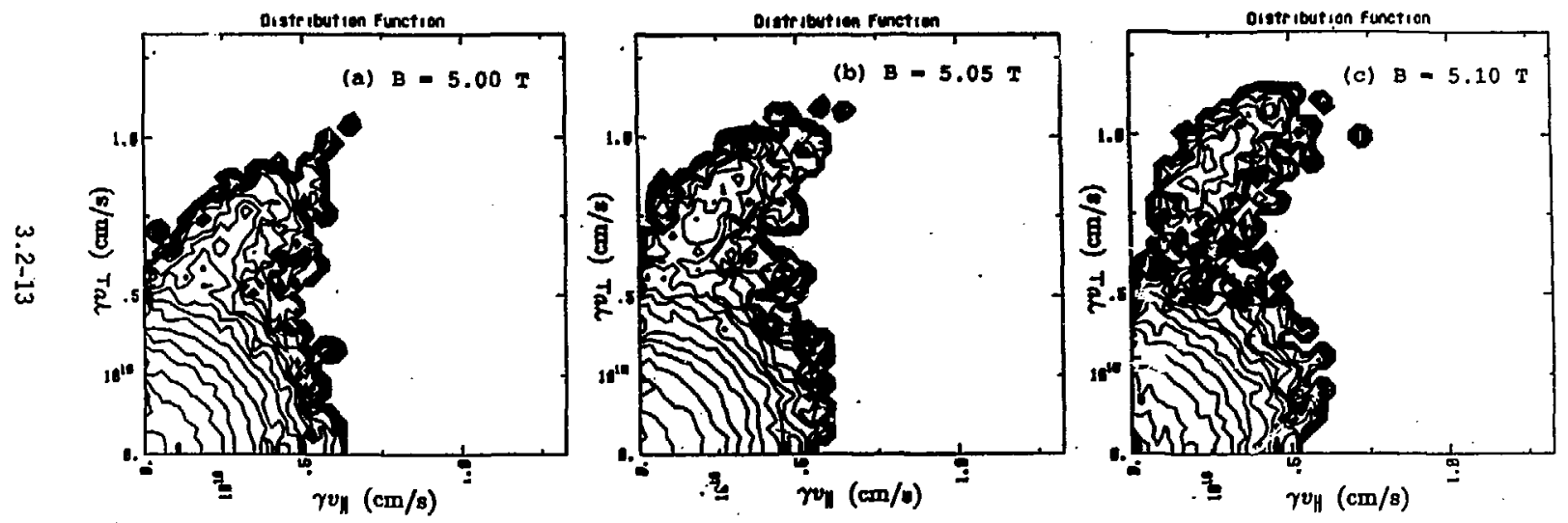

Fig. 4. Logarithmic contours of the electron distribution function 3 for the case including the . FEL port insert for three magnetic field atrengths. 

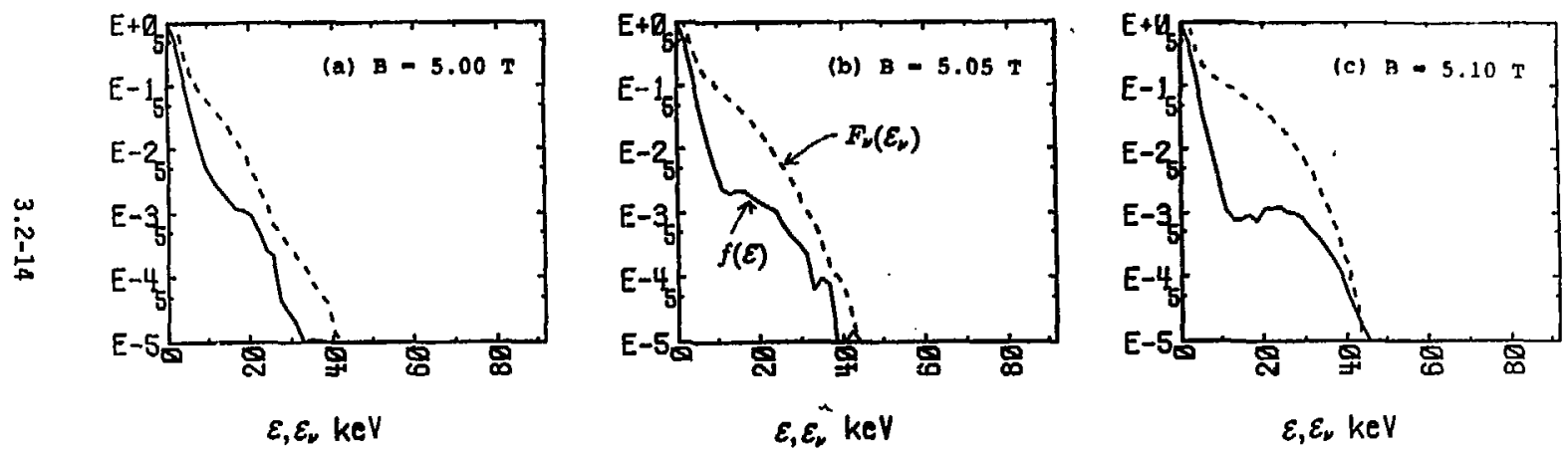

Fig. 5. Electron energy distribution corresponding to the three cases in Fig. 4 including the insert and the associated x-ray photon epectrum, $F_{p}$. Note that the energy scale is twice that in Fig. 3 
Fig. 3.2-6

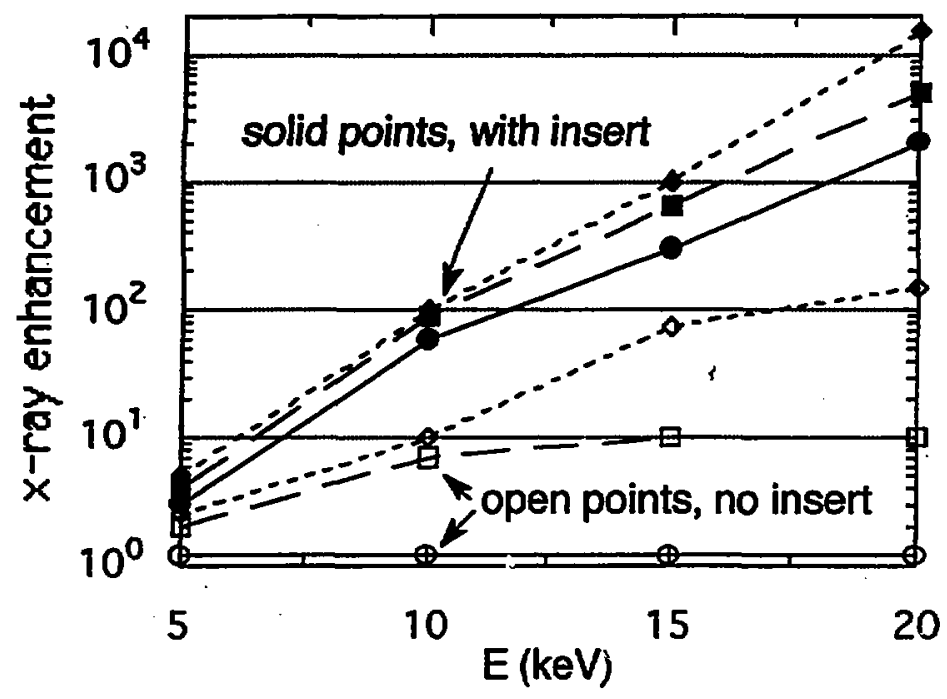

Fig. 6. Enhancement of the $x$-ray signal relative to that from a $1.55 \mathrm{keV}$ Maxwellian for the case with no insert (open points) and the case including the FEL port insert (solid points). Three magnetic field strengths are shown for each case: circle, $5.00 \mathrm{~T}$; square, $5.05 \mathrm{~T}$; and diamond, 5.10 T. 
Fig. 3.2-7

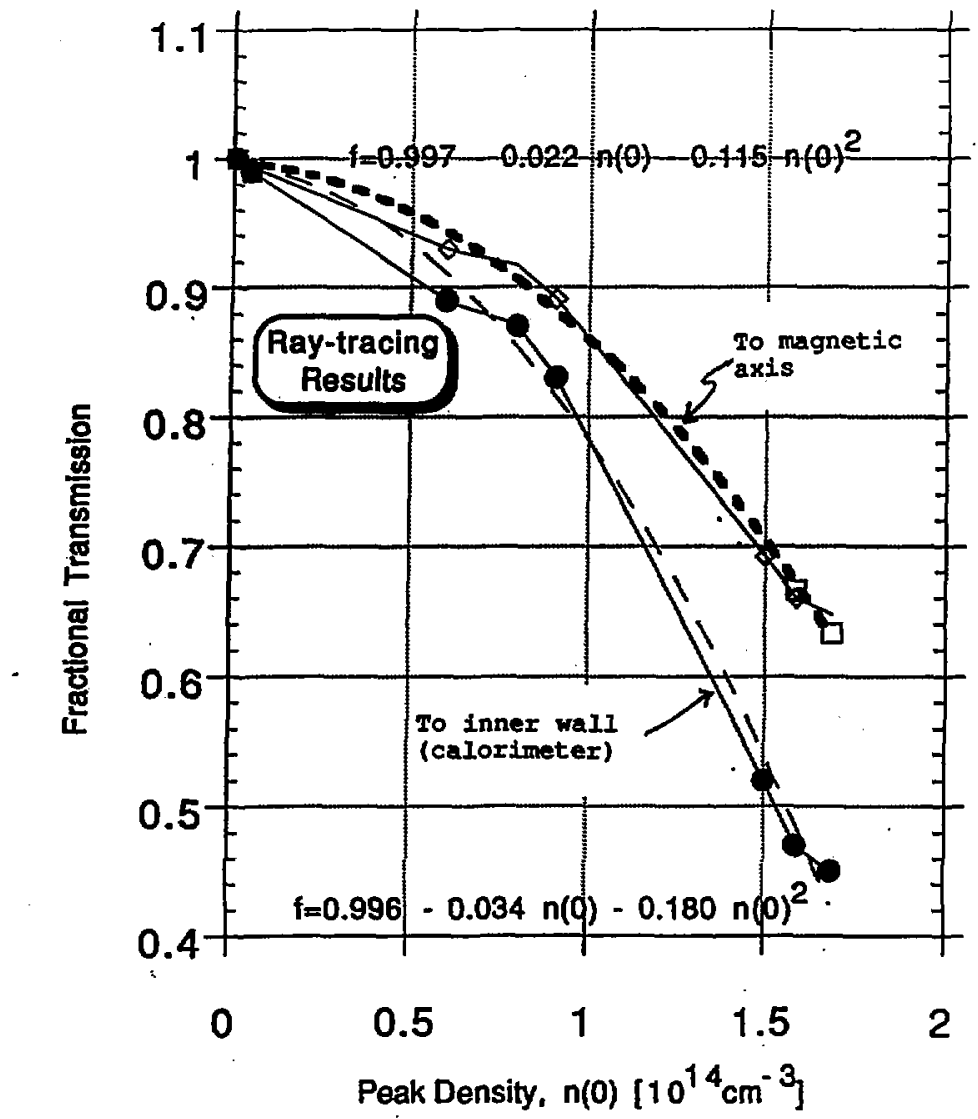

Fig. 1. Fractional transmission of the FEL beam at the magnetic axis and at the inncr wall (calorimeter) including refraction only from the TORCH code. 
Fig. $3.2-8$

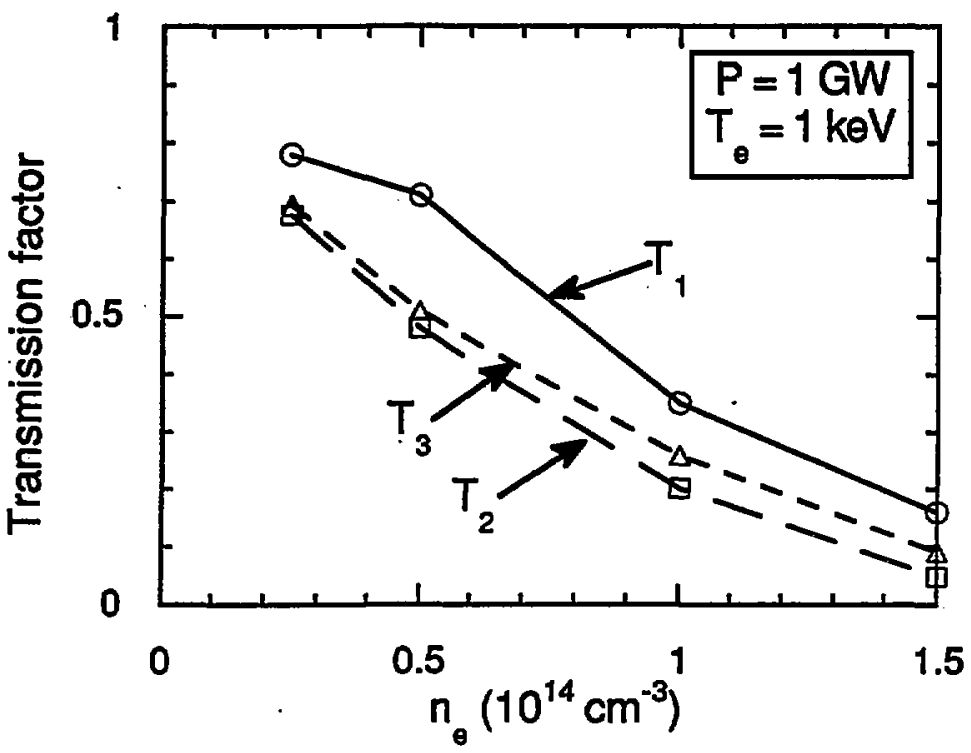

Fig. 2. Transmission factors versus electron density for the case of the tapered insert in the FEL port at a FEL power of $1 \mathrm{GW}$. Includes nonlinear absorption from ORPAT and refraction and has $T_{e}=1.0 \mathrm{keV}$. The transmission factors are: $T_{1}$, central; $T_{2}$, toroidal average; $\boldsymbol{T}_{3}$, toroidal plus vertical average. 
Fig. 3.2-9

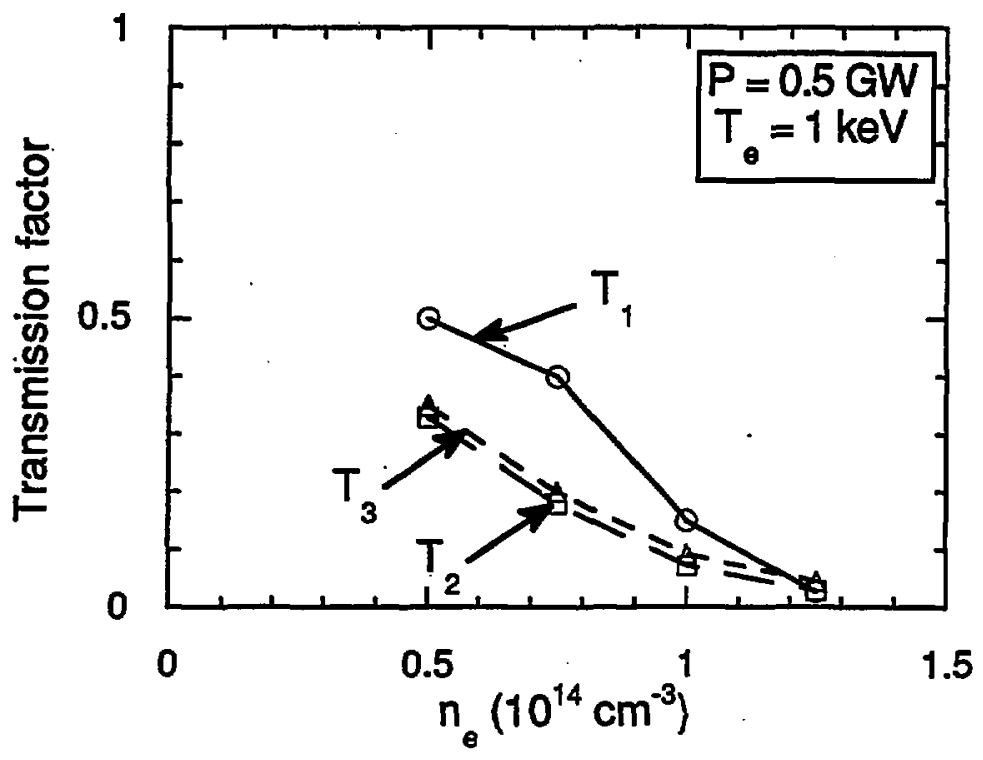

Fig. 3. Transmission factors versus electron density as in Fig. 2, but with FEL power of 0.5 GW. 
Fig. $3 \cdot 2-10$

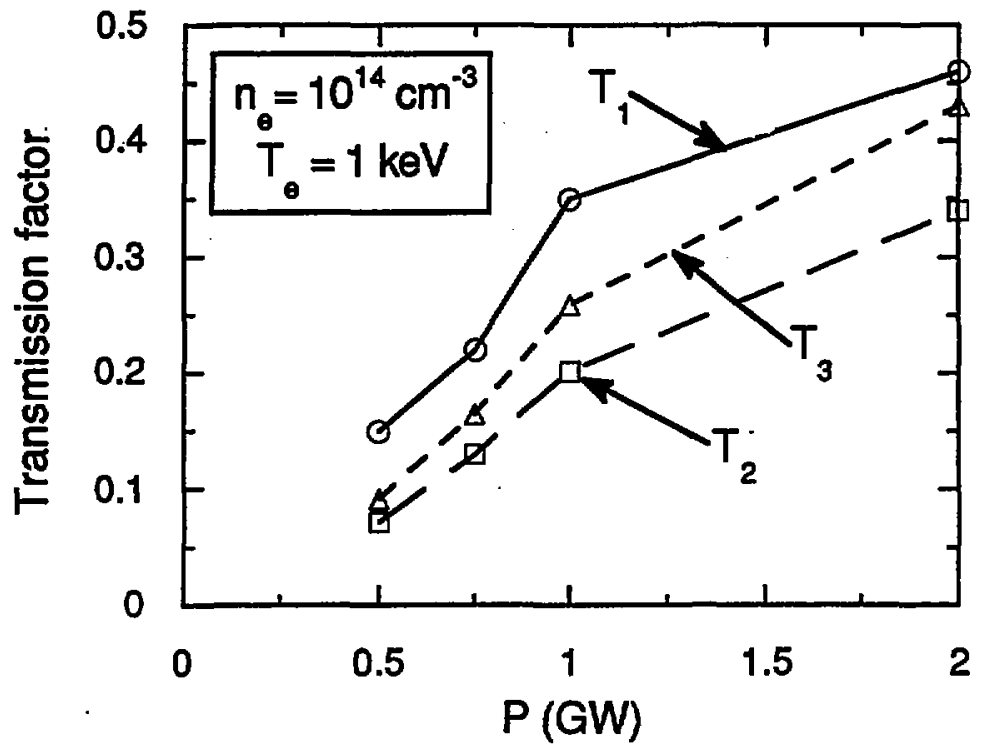

Fig. 4. Transmission factors versus FEL power for tapered insert case in Fig. 2 with $n_{e}=$ $1 \times 10^{14} \mathrm{~cm}^{-3}$. 
Fig. $3.2-11$

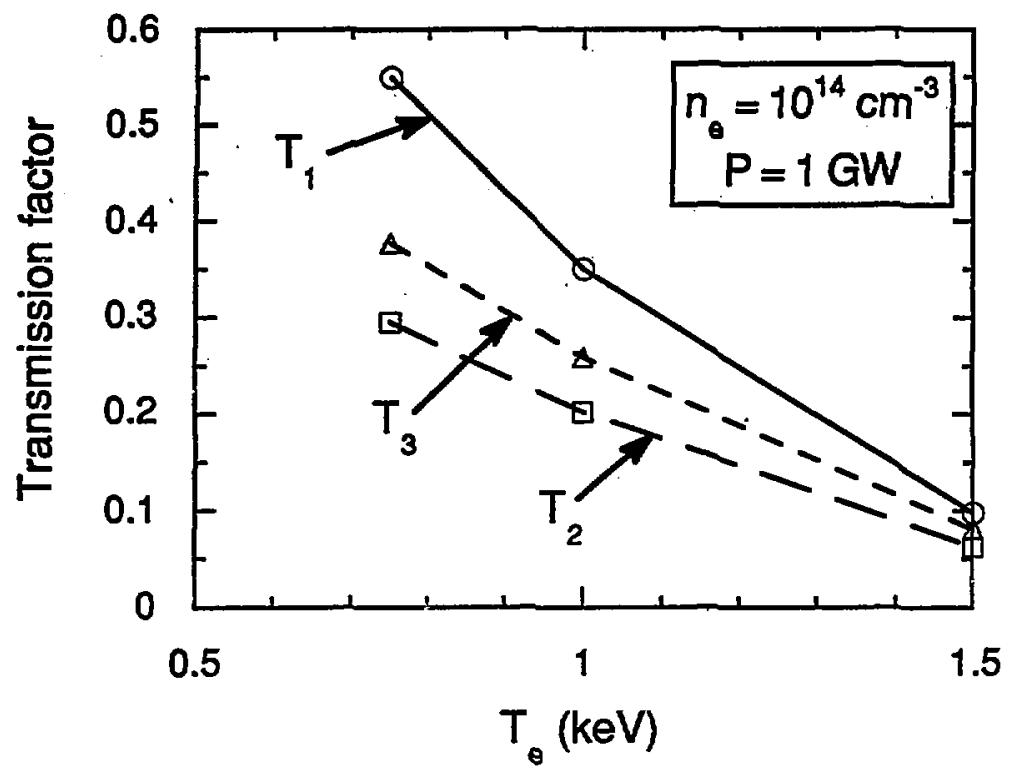

Fig. 5. Transmission factors versus $T_{e}$ for the parameters of Fig. 2 with $n_{e}=1 \times 10^{14} \mathrm{~cm}^{-3}$. 
Fig. 3.2-12

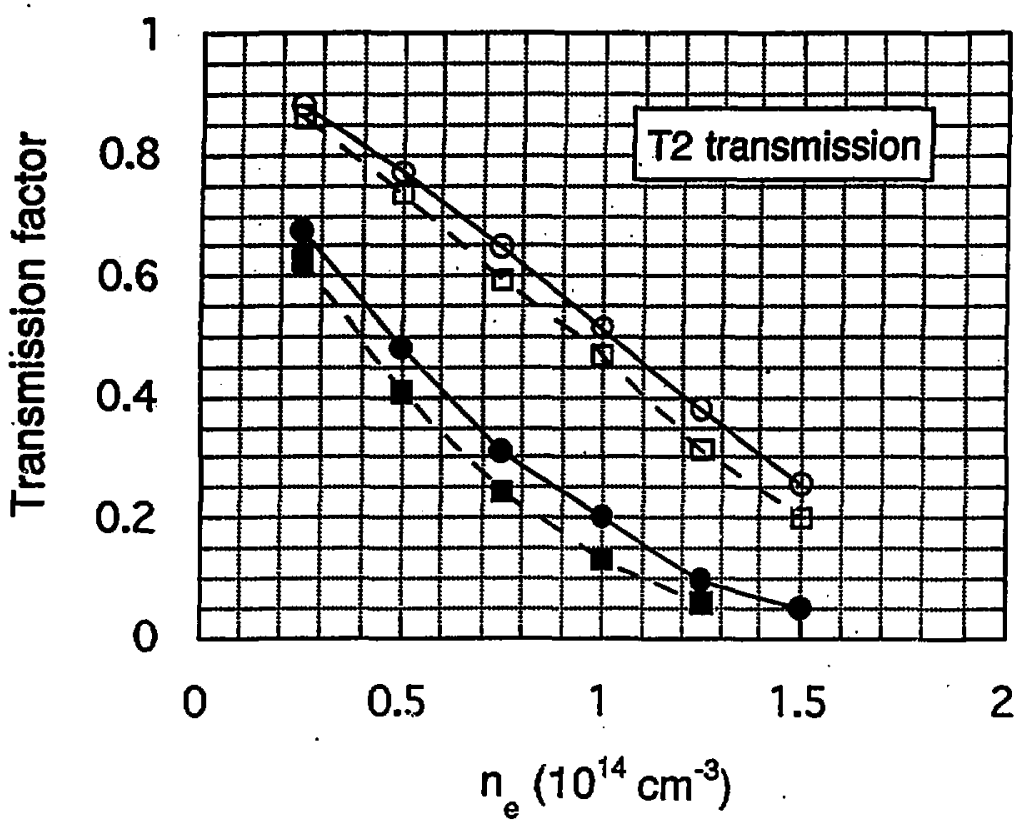

Fig. 6. Toroidally averaged transmission, $T_{2}$, versus density for the case with the tapered insert (solid points) and without the insert (open points) at two power levels: circles, $0.75 \mathrm{GW}$; and squares, $1.0 \mathrm{GW}$. Here $T_{e}=1.0 \mathrm{keV}$. 


\section{DESCRIPTION OF THE EXPERIMENT}

\subsection{Experimental goals and initial results}

The following 10 pages describe the experimental components and goals. Some initial results are included, especially a brief description of the initial FEL experiments; $d$. Section 5.1 for details. The papers referred to on the following 10 pages were presentations at the 1991 Annual Meeting of the Division of Plasma Physics of the American Physical Society held in Tampa, Florida, during November 4 through 8. The reference is: Bulletin of the American Physical Society 36, 2312-2314 (October 1991).

Details of the ECRH experiments using the gyrotron are given in Section 6. The final FEL, experiments, conducted at powers up to $2 \mathrm{GW}$, are described in Section 5.2 .

The following section (4.2) describes the microwave quasioptical system used to transmit power from the FEL or gyrotron to the tokamak. 


\section{THE MICROWAVE TOKAMAK EXPERIMENT}

The experiment consists of:

- MTX Tokamak - Alcator C moved from MIT

$$
\begin{aligned}
& R=0.64 \mathrm{~m} \\
& a=0.165 \mathrm{~m} \\
& B_{\max }=13 \mathrm{~T}
\end{aligned}
$$

- Pellet Injector (4 barrel) to generate peaked density profiles for confinement experiments

- Extensive diagnostlcs, especially directed to electron physics

- Free-electron laser

ETA-II accelerator

Wiggler: ELF for Inltial experiments at $140 \mathrm{GHz}$

IMP for future experiments at $140 \mathrm{GHz}$ (and $250 \mathrm{GHz}$ )

- Gyrotron - $140 \mathrm{GHz}$ at $0.4 \mathrm{MW} \mathrm{cw}, 1 \mathrm{MW} \geq 10 \mathrm{~ms}$ 


\section{MTX EXPERIMENTAL GOALS}

Physics experiments are directed towards microwave Interactlons with tokamak plasmas

- Microwave transport from the source to the tokamak using a windowless, quasloptical system

- Microwave absorptlon measurements and comparison with theory

- Plasma heating experiments, especlally with peaked density profiles

- Energy transport and confinement during ECRH

- Current drive using microwaves

- Disruption characterization and control

- Sawtooth suppression 


\section{MTX FOUR SHOT PELLET INJECTOR IS USED FOR FUELING STUDIES}

- Three Different Size Pellets are Avallable-a Single Pellet was Injected on This Shot.

- ECH Heating Experiments with Peaked Density Profiles are Planned

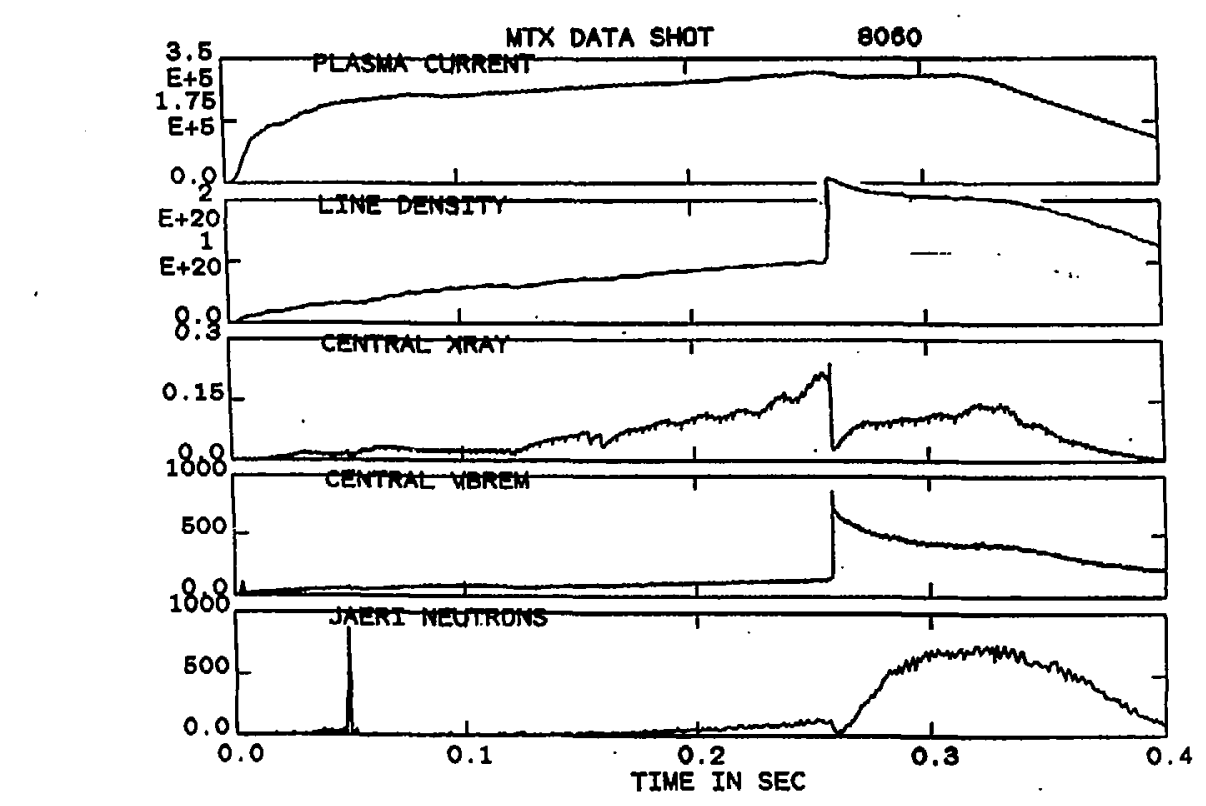




\section{QUASI-OPTICAL TRANSMISSION OF MICROWAVES}

- Quasi-optical (QO) transmission systems are usually choosen for reactor designs

- High power carrying capaclty as the electrlc fleld on the wall is small, minlmizing breakdown

- Low loss in Gausslan-mode propagation ( $\approx 0.1 \%$ loss/mirror reflection)

- No problems with mode-conversion, unlike highly overmoded waveguldes

- Fewer parts than multiple wavegulde systems

- The MTX quasi-optical transmission line is the first mm-wave, high power QO system used In fusion research

o Same line used for FEL and gyrotron systems

- The transmission line Is windowless - for the FEL, there is no window between the accelerator cathode and the tokamak plasma 


\section{$140 \mathrm{GHz}$ ECRH Experiments in MTX at $\mathrm{B}=5 \mathrm{~T}$}

Our experlments use O-mode Injected using a quasl-optlcal system

- InItial FEL experiments measured single-pulse absorptlin

- $\mathbf{P} \leq \mathbf{2 0 0} \mathrm{MW}$

- $\tau \leq 10 \mathrm{~ns}$

- Absorption consistant with predictions of llnear theory

- Gyrotron experiments reported here measured heating and proflle control

- $P \leq 200 \mathrm{~kW}$

- $\tau \leq 60 \mathrm{~ms}$

- Slgnificant heating and proflle effects observed

- See Poster $2 S$ 20, B. Stallard, "Performance or Gyrotron coupling . . ."

- Multl-pulse FEL experiments are planned for Spring, 1992

- $P=1-4 \mathrm{GW}$

- $\tau_{\text {pulse }}=30-40 \mathrm{~ns}$

- 50 pulses at $5 \mathrm{kHz}$; Average power $\geq 0.5 \mathrm{MW}$ 


\section{$140 \mathrm{GHz}$ Gyrotron Experiments}

- Purposes:

- Provide data base to compare with intense-pulse experiments using the FEL

- Evaluate ECRH at densitles $>1020 \mathrm{~m}^{-3}$

- Examine To proflle effects from ECRH, Including effects on current profiles, MHD, otc.

- General conclusions:

- Strong central heating observed, nato $\leq 1020 \mathrm{keV} / \mathrm{m}^{3}$

- Electron temperature increases on axis $>2 \mathrm{keV}$ observed at low density

- No density pumpout observed

- Power usually deposited withln $r=5 \mathrm{~cm}$, a typlcal sawtooth radlus (See Poster 2511, M. Fenstermacher, "Measurements of single pass absorption of ECH in MTX")

- Significant effects observed on sawteeth and MHD activity

- Hollow electron temperature and current density proflles converted into peaked, sawtoothing proflles

- ECRH occasionally caused recovery from minor disruptlons 


\section{Single-pulse FEL experiments}

- Single-pulse FEL experiments were conducted in 1989 and reported at the APS meetling

- Purposes:

- Demonatrate high-power FEL technology at $140 \mathrm{GHz}$

- Measure microwave propagation and absorption at high power

- Peak powers of $\mathbf{4 0 0} \mathrm{kW}$ were obtalned.

- It was concluded that the FEL needed to be modified for future FEL experiments

- The modiflcations are almost complete. (See Poster 25 10, S. Allen, "Initial operation of the ETA-II 60 cell accelerator and IMP wiggler"

- Power transmitted through the plasma was compared with ray tracing and absorption calculations. (See Poster 2S11, M. Fenstermacher, "Measurements of single pass absorption of ECH in MTX" 


\section{CENTRAL HEATING BY GYROTRON}

- Maxímum heating power $=200 \mathrm{~kW}$ at $140 \mathrm{GHz}$

- Pulse length = 5 to $50 \mathrm{~ms}$

- Less than maximum heating is due to short pulselength, heating durlng Inltla! current ramp, or poor conflnement during minor disruptions

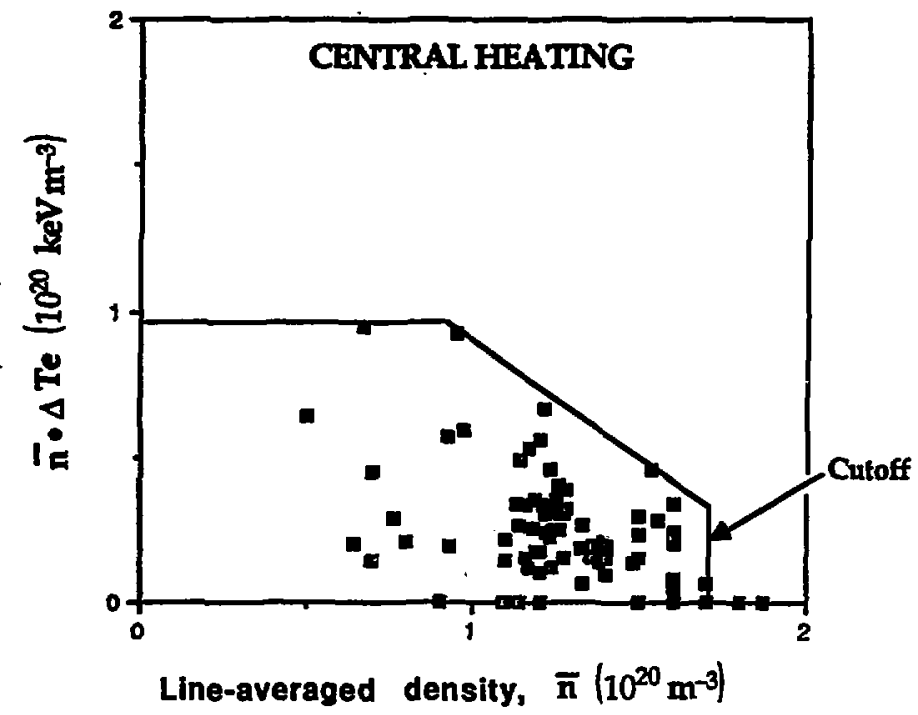




\section{FEL plasma transmission measurements for central resonance in MTX}

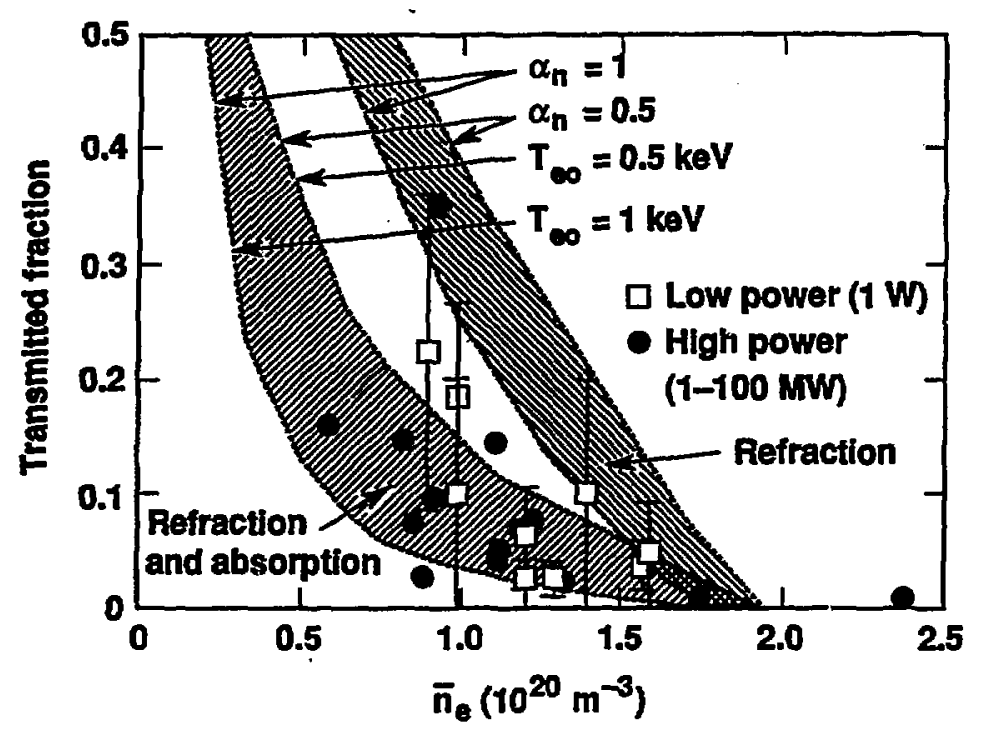

$$
\text { Plasma profiles } \begin{aligned}
n & =n_{0}\left(1-\frac{r^{2}}{a^{2}}\right)^{\alpha_{n}} \\
-T_{6} & =T_{60}\left(1-\frac{r^{2}}{a^{2}}\right)^{\alpha_{n}}
\end{aligned}
$$




\title{
LOW POWER TRANSMISSION IN MTX
}

\author{
$140 \mathrm{GHz}$ and $5 \mathrm{~T}$
}

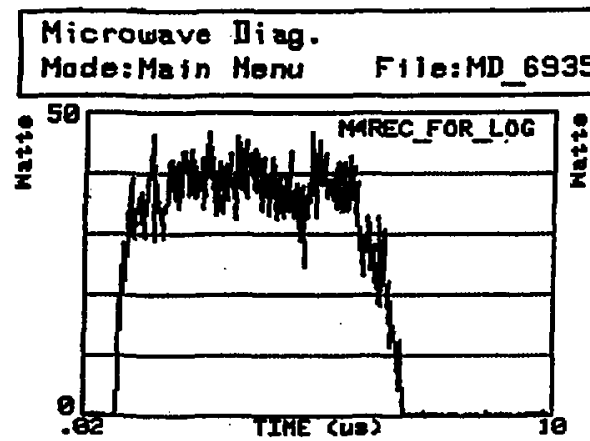

$40 \mathrm{~W}$ Incldent Signal

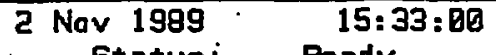

Status:

Ready

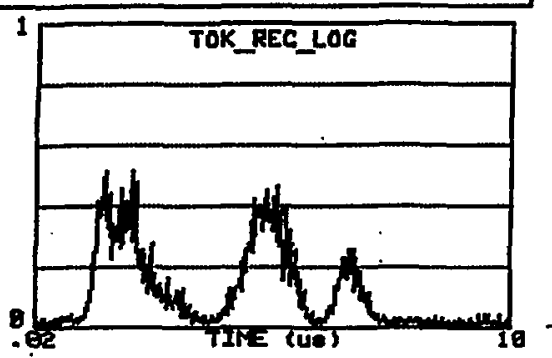

Transmitted Signal

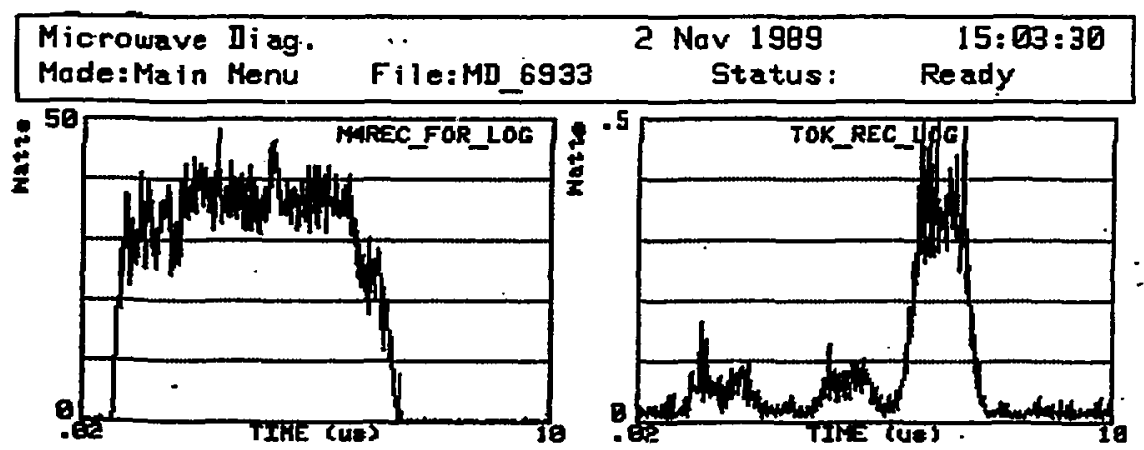




\section{CONCLUSIONS}

Physics of the Initial FEL experiments in MTX has been analyzed.

-. Diffraction effects - Huygens Integral code (MTH); transmission of waveguide modes in the duct.

-- Plasma refraction - Ray tracing using the cold plasma approximation.

-- Plasma absorption - Single pass absorption from linear optical depth.

Physics of non-linear FEL experiments in MTX has also been examined.

-- Nonlinear absorption - Relativistic orbit - calculation with "bucket-lift" 


\subsection{Quasi-optical transmission system (B. W. Stallard)}

The MTX quasi-optical (Q.O.) transmission system consists of aluminum reflective optics mounted within a $50 \mathrm{~cm}$ diameter vacuum envelope, with a total transmission distance $>30 \mathrm{~m}$ from the if source to the tokamak. No windows were used between the if source output and the tokamak. This transmission system was used for both gyrotron and FEL heating of the MTX plasma. Fig. 4.2-1 shows a drawing of the system where both heating configurations are indicated.

The optics, both planar and focusing, were designed for optimized coupling of the rf source (gyrotron or FEL) into a Gaussian-like mode for efficlent transmission to the tokumak. The optics were designed by use of the MTH (Microwave Transmission Huygen's) code1, which includes diffractive effests. This code predicted transmission effidencles of $\approx 98 \%$ for a Gaussian beam mode.

\subsubsection{Gyrotron heating}

Fig. 4.2-1 shows the transmission system configured for direct gyrotron heating of the plasma. We operated the gyrotron at 350 to $400 \mathrm{~kW}$ power for pulses up to $100 \mathrm{~ms}$. The gyrotron produces power in 3.5 in waveguide nominally in the $\mathrm{TE}_{15,2}$ waveguide mode. This mode is unsuitable for efficient long distance transmission and for beam-like illumination of the plasma. Consequently, a Vlasov type mode converter ${ }^{2}$ was employed to convert the if power into a Gaussian-like mode for transmission. Fig. 4.2-2 shows the JAERIdesigned Vlasov converter used for MTX. The converter consisted of a helically cut waveguide launcher (2.28 in diameter) and two doubly curved optics ( $V_{1}$ and V2), which produced linear, horizontal output polarization. Geometric optics was used for design of the converter. Rays, defined by the caustic surface for the TE15,2 mode, were launched from the cut waveguide and propagated to mirrors $V_{1}$ and $V_{2}$. The mirror curvatures were designed to transform the waveguide aperature profile, defined by geometric optics, into a Gaussian amplitude profile

Four mirrors, $\mathrm{G}_{2}$ and $\mathrm{M}_{2}$ to $\mathrm{M}_{4}$, comprised the transmission system, where $M_{2}$ and M4 were focusing optics. The transmission optics do not all lie in the plane of the drawing in Fig. 4.2-1, but make two bend angles of about $39 \mathrm{deg}$. at optics $\mathrm{M}_{2}$ and $\mathrm{M}_{4}$, for a total bend angle of $78 \mathrm{deg}$. For launch of the $\mathrm{O}$-mode at MTX (horizontal polarization), a twist polarizer was required for optic $\mathrm{M}_{2}$ to rotate the polarization $78 \mathrm{deg}$. The twist polarizer consisted of a $44 \mathrm{~cm}$ diameter planar mirror containing rectangular-shaped, parallel groves with depth $h / \lambda=0.267$. There were two periods per wavelength, and teeth and groove widths were equal. ${ }^{3}$ Within measurement accuracy, low power measurements showed $=100 \%$ polarization efficiency.

Starting from an initial He-Ne laser alignment, we aligned the optics using movable if probes to scan the beam profile at each mirror. Each mirror was 
adjusted in sequence to center the beam on the next "downstream" mirror. Horizontal and vertical profiles of the output beam were measured in the diagnostic box near the MTX input port and are shown in Fig. 4.2-3 along with Gaussian profiles. Departures of the measured points from a Gaussian profile are believed to arise from imperfect alignments, from side-Jobe power produced by the mode converter, and also from non-TE15,2 mode content of the gyrotron output. Because there is very little dissipation in the transmission system, sidelobe power can propagate to MTX and produce interference with the main beam.

The input port to MTX is a narrow duct of $4 \mathrm{~cm}$ width, $30 \mathrm{~cm}$ height, and 22 $\mathrm{cm}$ length, located $242 \mathrm{~cm}$ from the last optic (M4). Strong focusing on the final optic and careful allignment were required to launch the beam into the duct. For accurate alignment into the duct, we used short pulses (few ms) of the gyrotron and an IR camern to view the beam on abeorbing macor ceramic. Fig. 4.2.4 shows the narrow duct and the $2 \mathrm{~cm}$ diameter image of a misaligned beam. By adjusting M4 to obtain a second image on the other side of the duct, the correct aligned setting for M4 was calculated. Vertical centering was obtained by using the segmented calorimeter (section A.2.3) on the inside wall.

The transmitted power to MTX was about $200 \mathrm{~kW}$, as measured on the JAERI calorimeter. From this measurement the overall gyrotron-to-MTX efficiency is $\eta$ $-55 \%$. Most of the low efficiency $(=0.8 \times 0.8=0.64)$ resulted from two factors: 1$)$ non- $\mathrm{TE}_{15,2}$ modes from the gyrotron and 2) diffraction losses at the first Vlasov optic $V_{1}$. Using a k-spectrometer 4 we measured a power content of $\approx 0.8$ for the $T_{15,2}$ mode from the gyrotron. Large diffraction losses (power spillage) on $V_{1}$ resulted from the geometric optics approximations used for the design. StrattonChu diffraction calculations made subsequent to fabrication of the converter predicted losses $\approx 0.2 .5$ Most of the remaining small losses can be attributed to waveguide dissipation and small edge diffraction losses at the spiral-cut waveguide launcher of the converter.

\subsubsection{FEL heating}

For FEL heating of MTX plasmas the Q.O. system was reconfigured as shown in Fig. 4.2-5. For this operation the FEL was operated as an amplifier and the gyrotron was used as a driver at the FEL input. In the driver mode the gyrotron output power was first transmitted through evacuated 3.5 in overmoded, smooth-wall waveguide to a Vlasov mode converter. Two planar-mirror miter bends were used in the transmission system and several stainless steel lossy sections were used as Q-dampers for undesired modes. Since the miter bend mirrors were not phase-corrected for the TE15,2 mode, mode conversion into other modes was expected. High efficiency for driver coupling was not a requirement because only low drive power was required for FEL operation. At the waveguide output a linear down-taper to 2.28 in coupled the power to the Vlasov converter.

We designed the Vlasov antenna to generate a Gaussian-like beam for propagation in $50 \mathrm{~cm}$ pipe to the linac beam line. Two optics, $S_{1}$ and $S_{2}$, relayed 
the beam to a side coupler at the wiggler input. The side coupler contained a port with widely spaced parallel conductors to carry e-beam return current at the nominal wall position in the absence of the port. This port admitted the driver beam onto a flat mirror so that the microwave driver beam and the e-beam were co-linear at the wiggler input. The e-beam passed through a $4 \mathrm{~cm}$ hole in this optic, and mirror $S_{2}$ was curved to focus the driver if beam into the FEL waveguide. For the side coupler the computed diffraction coupling losses from a Gaussian beam to the TE11 mode in the $3.25 \mathrm{~cm}$ wiggler waveguide were $30 \%$.

The overall efficiency of conversion from gyrotron power to beam power at the FEL input, while adequate for FEL operation, was very low. We measured about $7 \mathrm{~kW}$ at the input for an estimated gyrotron output power of about 300 kW. While not carefully optimized, we attribute the low efficiency to excessive mode conversion at the miter bends and the attending power spillage at the Vlasov converter and possible system optics misalignment. Fig. 4.2-6 shows the measured $\mathrm{E}$ and $\mathrm{H}$-plane power profiles near the wiggler input, showing that interference effects resulting from multiple modes or misalignments play a role.

For transmission of the FEL output to MTX, six optics are used: J0, I1, and $M_{1}$ to M4 The focusing on mirrors J0 and $\mathrm{M}_{2}$ was designed to match the $\mathrm{TE}_{11}$ mode FEL output into the Q.O. system. In Fig. 4.2-7 we compare the TE11 mode with the measured beam profile in the E-plane at the FEL output. The beam output power was $1.6 \mathrm{GW}$ for an optimally tapered wiggler magnetic field. Also shown in Fig. 4.2-7 is the transmitted beam at MTX, again for the E-plane, and the data is compared with the Gaussian-like design profile. For the TE11 mode the design tranmission efficiency to MTX is $89 \%$, although calorimeter measurements at the FEL output are unavailable to confirm the transmission efficiency. Small interference effects are visable in the profile, but we conclude that the transmission system performs as designed.

4.2.2.1 Control of $k_{\|}$gradient. In order to study the nonlinear heating physics, beams with two different geometries were injected into MTX. At the resonance the beam spot sizes were similar but the toroidal gradients in $k_{\|}$were made different by a large factor. The two beam geometries are shown in Fig. 4.2-8. For the first experiments a circular beam was injected into the smooth wall input duct ( $4 \mathrm{~cm}$ width, $30 \mathrm{~cm}$ height, and $22 \mathrm{~cm}$ length). For this beam the 1/e power diameter in vacuum at the plasma resonance $t$ as $3.6 \mathrm{~cm}$. The $k_{\|}$spectrum of the beam was $\left\langle k_{\|}\right\rangle=0$ and the variation of $k_{\|}$across the beam was $\Delta k_{\|} / k=0.1$.

To test the predictions of the theory for enhanced absorption we used a beam with a large gradient in the toroidal $k \|$. For a second series of experiments the transported beam was injected into a corrugated vertical side wall duct, tapered in the horizontal (toroidal) direction (the side wall normal to the horizontal electric field for the O-mode). .A parabollic taper was employed for the design. 6 The Gaussian-shaped input beam coupled to the HE11-like mode in rectargular waveguide and the length of the 3.4:1 down taper was set to reconstruct the HE11 mode at the output. ${ }^{7}$ The beam size at the resonance in vacuum was 
elliptical and elongated in the horizontal direction with dimensions $4.8 \times 3.6 \mathrm{~cm}$. This beam had a smooth variation of $k_{\|}$across the beam $\Delta k_{\|} / k=0.35$.

4.2.2.2 Power calibrations. Single point measurements of the FEL beam were made using fundamental mode waveguide horns on movable probes located at both the FEL output and the MTX input and at fixed locations at the centers of several mirro: optics. These waveguides were connected to calibrated $140 \mathrm{GHz}$ heterodyne receivers to measure the beam pulse shape and the absolute power (to several dB accuracy). The most accurate measurements of If power were done using the IAERI-provided calorimeter at the tokamak input diagnostic box to measure the total FEL beam pulse energy Using the measured pulse shape from an $r f$ probe, the peak power was determined.

\section{References}

1. B. Stallard, J. Byers, B. Hooper, et al., Eighth Top. Conf. on Radio-Freq. Power in Plasmas, Irvine, CA, p. 84 (1989); also T. Samec, Bull. Amer. Phys. Soc. 32, 1872 (1987)

2. S.N. Vlasov, LI. Zagryadskaya, and M.I. Petelin, Radio Engr. and Electronic Phys. 20, 10 (1975).

3. J.L. Doane, (groove depth, private communication); also F.M. Smits, EC-7, Hefei, China (1989).

4. W. Kasparek and G.A. Müller, Int. J. Electronics 64, 5 (1988).

5. M. Blank, J.A. Casey, R.J. Kreischer, et al., Int. J. Electronics 72, 1093 (1992).

6. J.L. Doane, Int. J. of Infrared and Millimeter Waves 5 , 737 (1984).

7. J.L. Doane, Int. J. of Infrared and Millimeter Waves 8, 13 (1987). 


\section{IMP-MTX MICROWAVE SYSTEM}

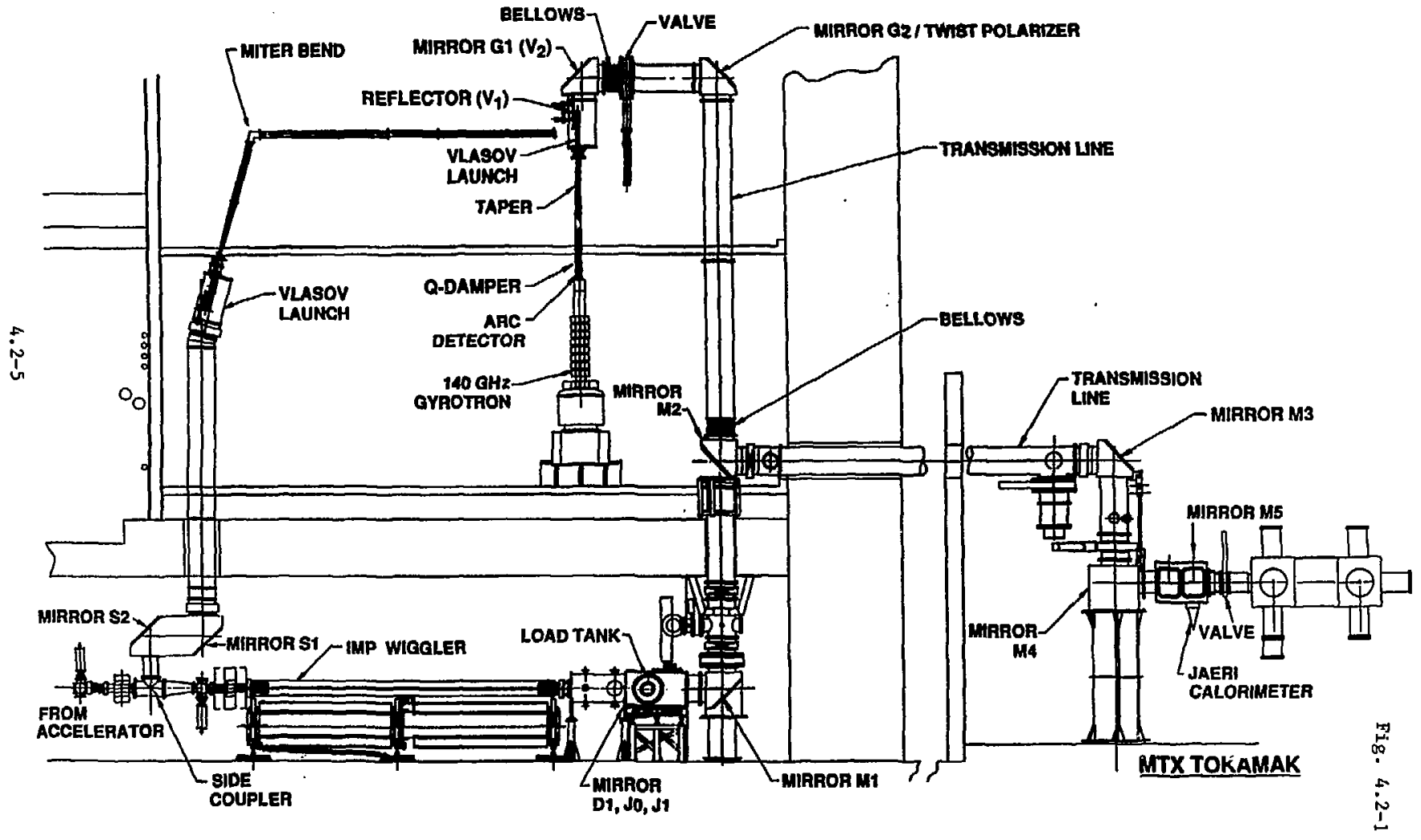




\section{Improved (JAERI) Vlasov reflector for MTX}
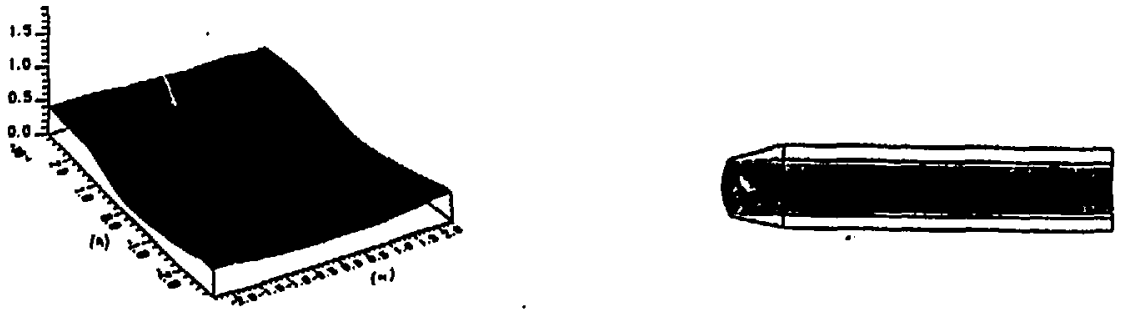

i
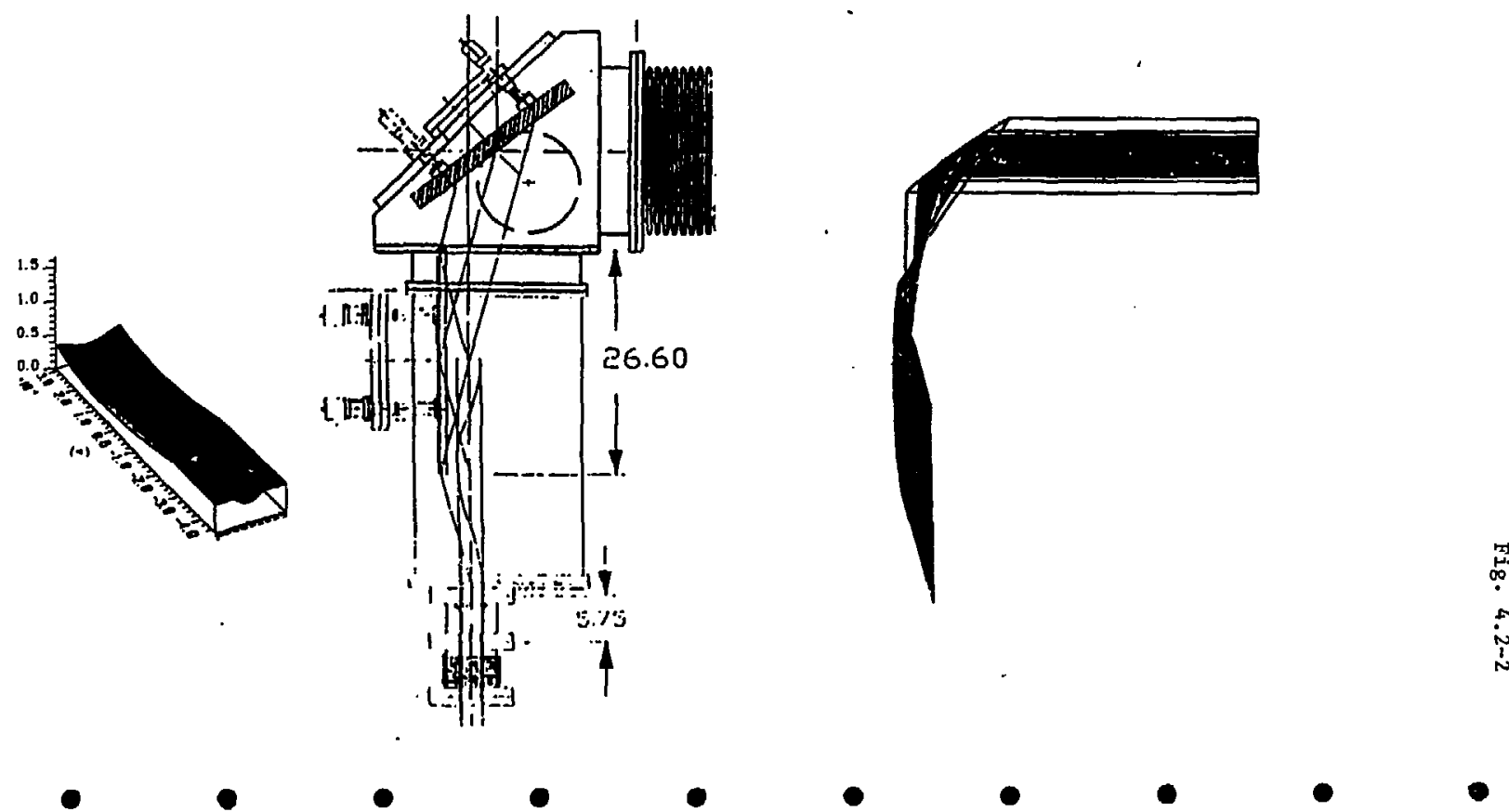


\section{Q.O. Output Beam Profiles - $400 \mathrm{~kW}$ Gyrotron Power, 200 kW Output Power

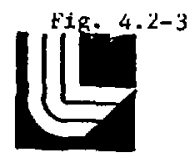

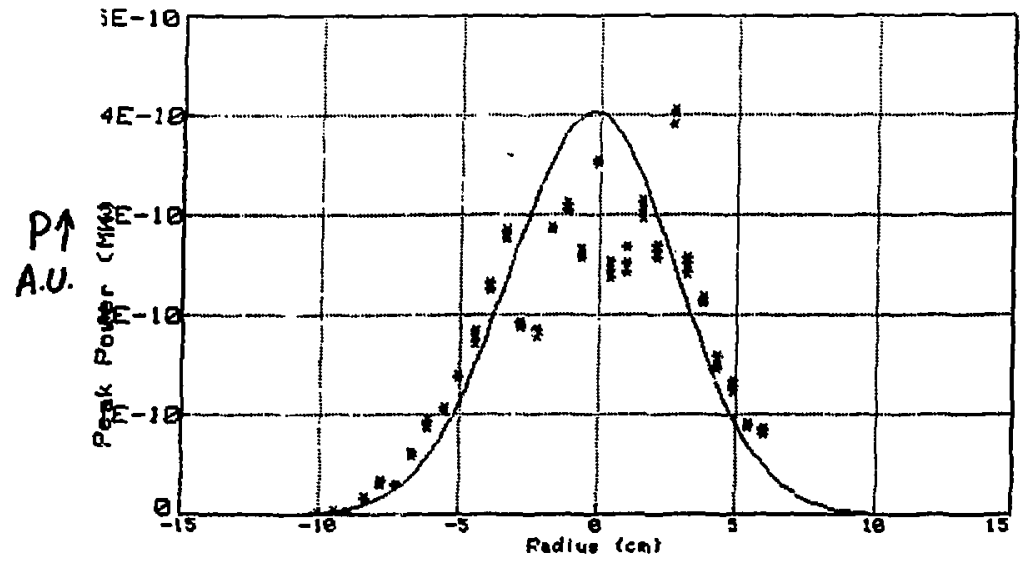

$117 \mathrm{~cm}$ to $M \pi x$ port

Horizontal

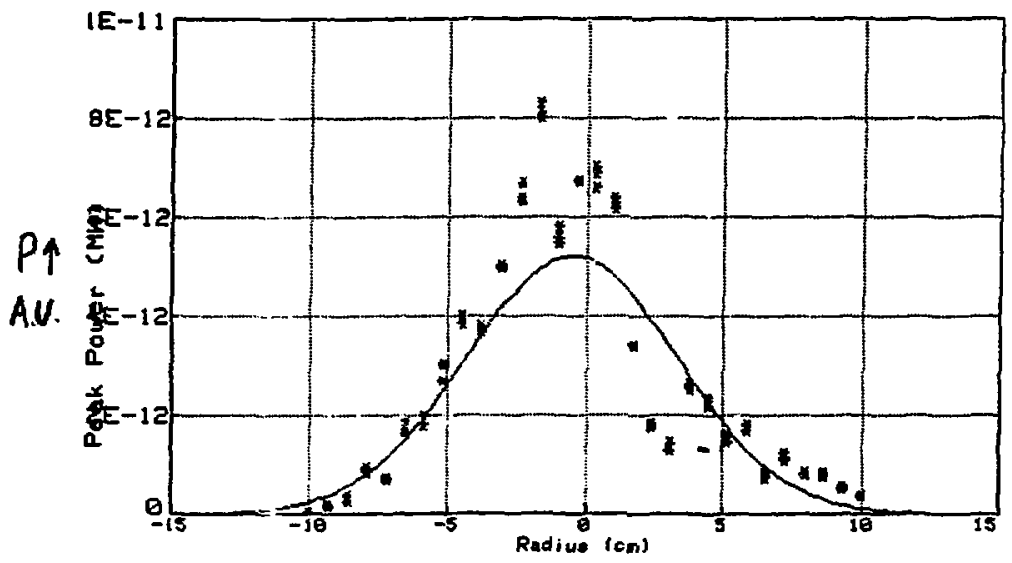

$142 \mathrm{~cm}$ to $M T X$ Port

Verticai 


\section{Beam at MTX-IR Image on MACOR LL}

For this data the beam is offset from the input slot; for the final alignment the beam is centered into the MTX slot by adjustment of the final optic M4

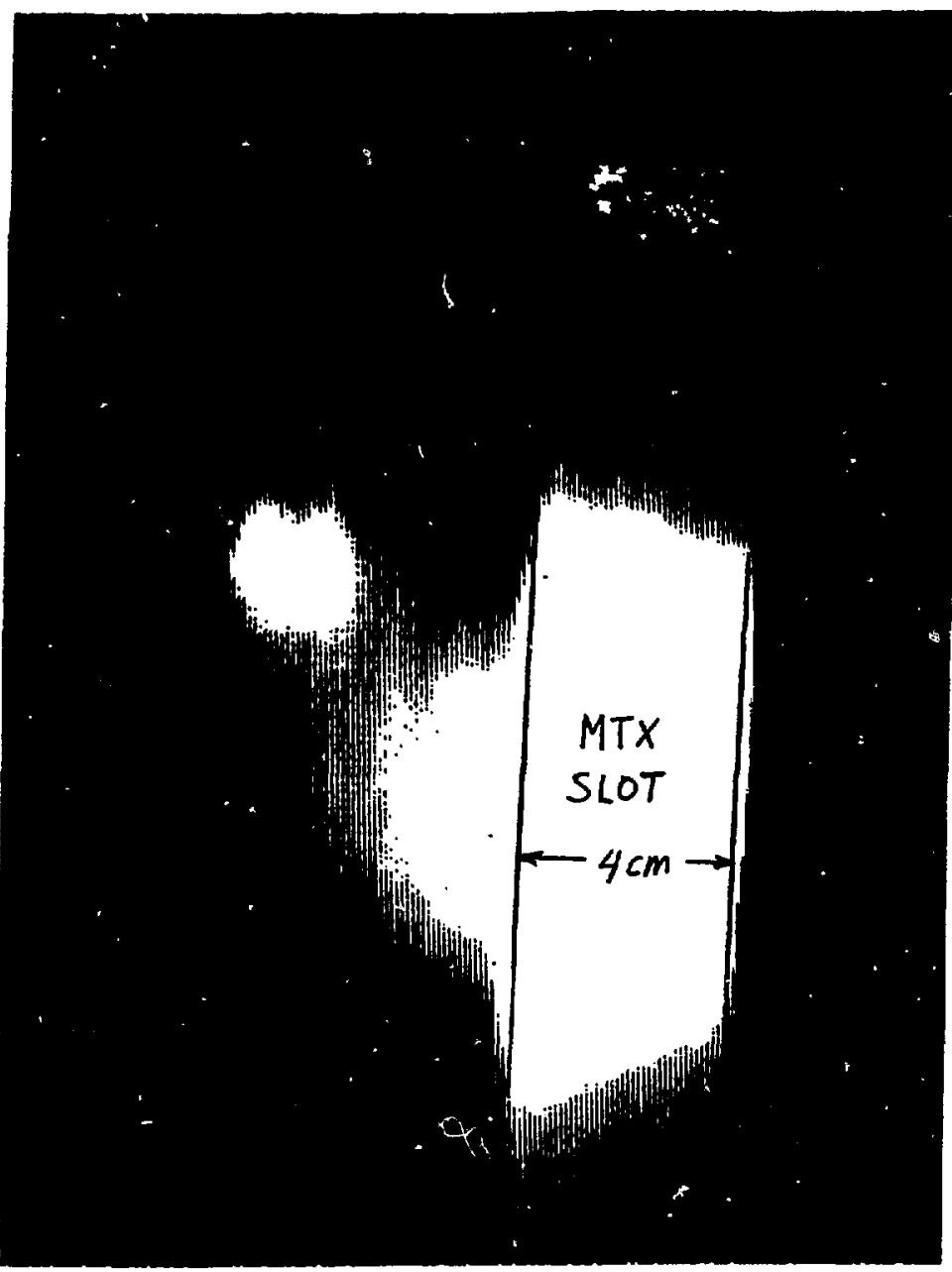




\section{IMP-MTX Microwave System}

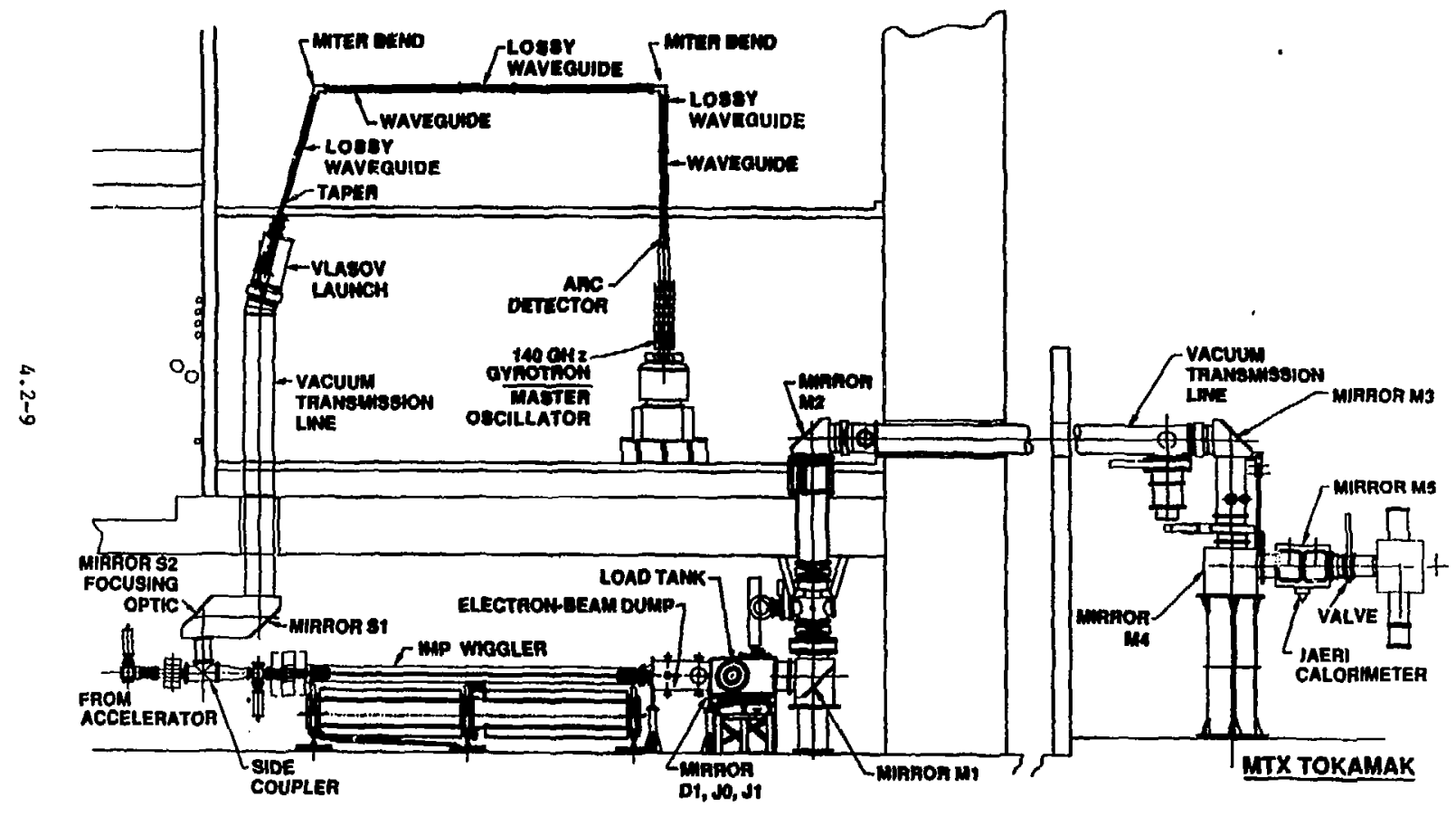




\section{IMP FEL Input Drive Power}

- $140 \mathrm{GHz}$ gyrotron with Vlasav mode converter used as input source - total drive power $=7$ KW at IMP input.

- Low drive power results from poor mode content at the Vlasov input (two miter bends without phase correction), Vlasov losses, and misalignmonts.
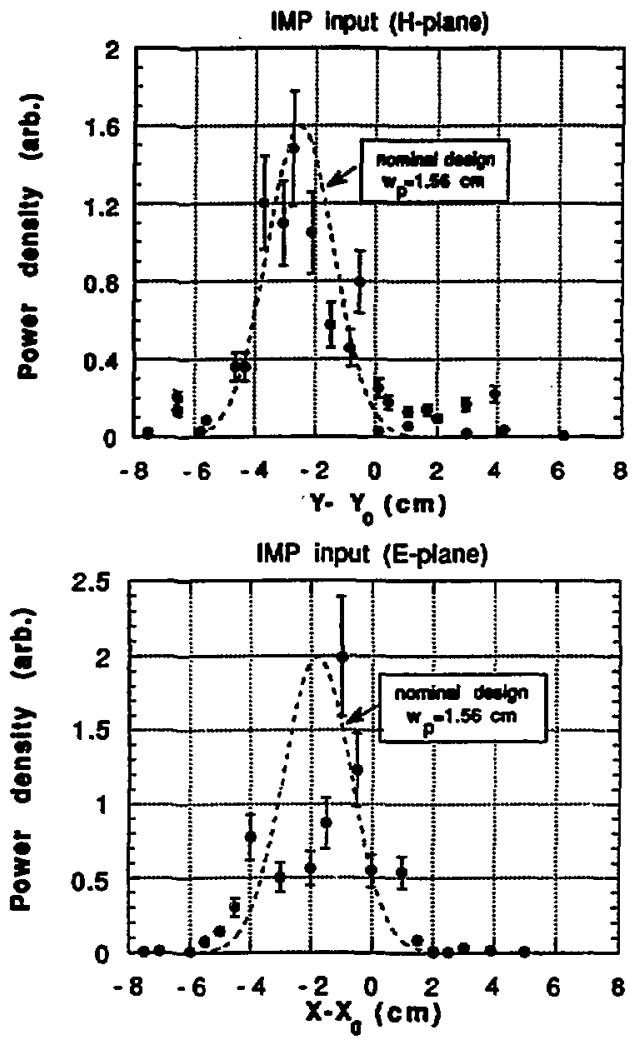


\subsection{GW Tapered FEL Operation}

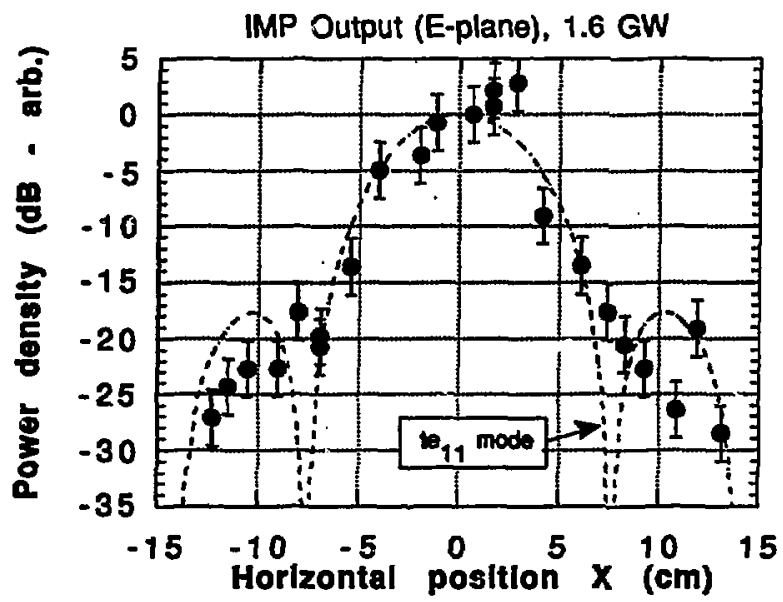

FEL Beam at MTX

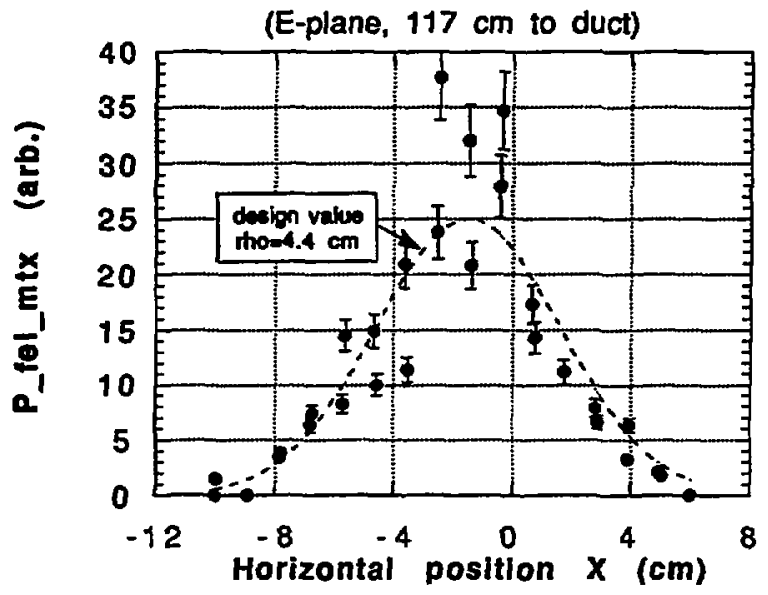




\section{Toroidal Profiles of FEL Beams}

\section{Injected into MTX}

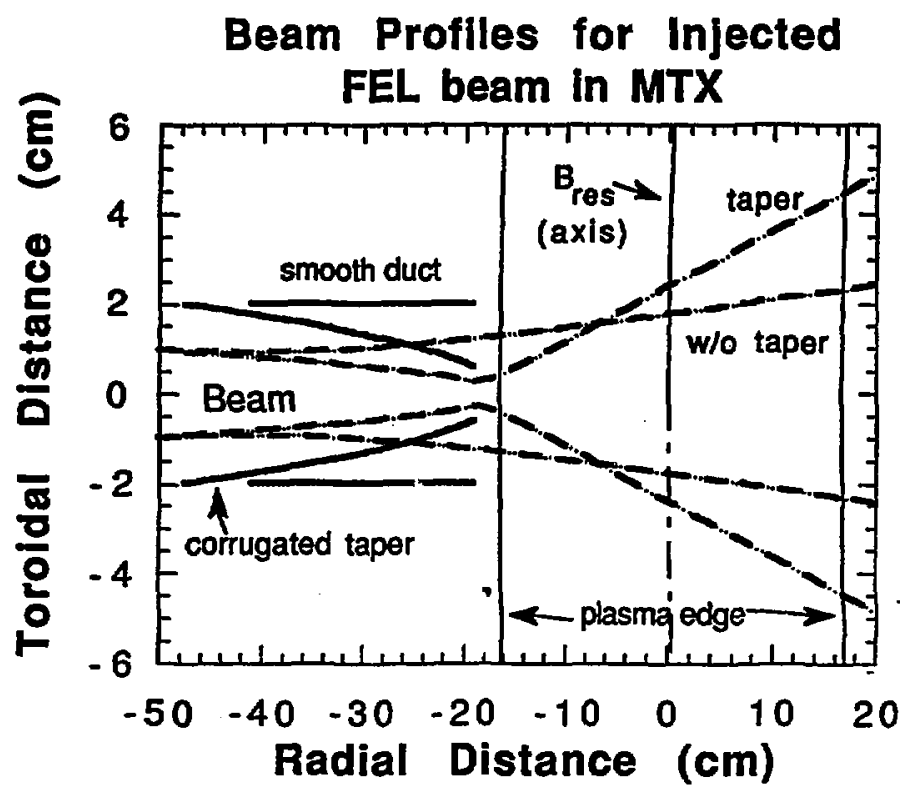




\section{Microwave absorption and heating experiments using the free-electron laser}

5.1 Initial experiments to measure the absorption of short, intense microwave pulses from the FEL (E. B. Hooper)

The initial experimental operation measuring intense microwave pulse absorption in MTX were performed in the summer-fall of 1989. Because of limitations to the accelerator operation (c.. Sec. 8.1), the peak power was limited to $<200 \mathrm{MW}$ (with $400 \mathrm{GW}$ generated but not injected) and pulse lengths of $<10$ ns. Despite these limitations, the absorption was measured. As expected from theory for these powers, the data were full consistent with predictions for linear absorption, although the scatter was large enough to prevent highly accurate comparisons. The scatter was speculated to be due (in part) to scattering from fluctuations from fluctuations in the edge plasma of MTX; c.f. Smith et al for the theory of this process, including application to MTX.1

The experimental measurements of the absorption are summarized in Allen et al., attached as Sec 5.1.1. Detailed discussion of the data and the analysis techriques is found in Fenstermacher, ${ }^{3}$ attached as Sec. 5.1.2.

1. G. R. Smith, D. R. Cook, A. N. Kaufman, A. H. Kritz, and S. W. McDonald, submitted to Phys. Fluids.

2. S. L. Allen et al., Plasma Phys. and Control. Fusion Res. 1990 (IAEA, Vienna, 1992), Vol. 1, pp. 783-87.

3. M. E. Fenstermacher, "Comparison of Propagation and Absorption of FEL-Generated Microwaves with Theory," LLNL Report UCRL-ID-103808, May, 1990. 
5.1.1 Free-Electron Laser Experiments in the Microwave Tokamak Experiment (S. L. Allen et al.)

S. L. Allen et al., Plasma Phys. and Control. Fusion Res. 1990 (IAEA, Vienna, 1991), Vol. 1, pp. 783-87. 


\title{
FREE-ELECTRON LASER EXPERIMENTS IN THE MICROWAVE TOKAMAK EXPERIMENT*
}

\author{
S.L Allen, M.D. Brown, J.A. Byers, T.A. Casper, B.L Cohen, \\ R.H. Cohen, D.R. Cook(b), J.C. Cummings(b), M.E. Fenstermacher, \\ J.H. Foote, E.B. Hooper, R.A. Jong, A.B. Langdon, B.F. Lasinski, \\ CJ. Lusnier, M.A. Makowski(c), Y. Matsuda, W.H. Meyer, \\ J.M. Moller, W.E. Nexsen, K.Oasa(d), T. Oprwa(d), B.W. Rice, T.D. \\ Rogaliea, G.R. Simith, B.W. Stallmd, K.I Thomassen, A.L. Throop, \\ W.C. Turner, and R.D. Wood
}

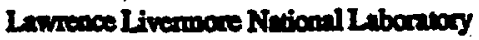 Livermore, CA 94551, USA}

\begin{abstract}
Microwave pulses have been injected from a free eloctron-laser (FEL) into the Microwave Tolamak Experiment (MIX) at up to $0.2 \mathrm{GW}$ at $140 \mathrm{GHz}$ in shart puises (10-ns duration) with O-mode polarization. The power transmitted through the plasma was measured in a first experimental study of high power pulse propagation in the plasma; no nonlinear effects were found at this power level. Calcularions indicate that nonlinear effects may be found at the higher power densities expected in future experiments.

\section{INTRODUCTION}

The primary goal of MTX [?] is to study electron-cyclatron resonance heating and control of a high-density wokmak ( $A$ lcitor $C$ ) by microwaves from an FEl or a gyrotron. Here we report the first measurements of high peakedpower micrownve absorpion in the plasma. Microwaves were generated by the FEL, and their transmission and absorption in MTX were experimentally evaluated. In agreement with theory, nonlinear effects [2] were not found at these power levels $(0.2 \mathrm{GW})$ but are predicted at higher levels.
\end{abstract}




\section{FREE-ELECTRON LASER OPERATIONS: MICROWAVE GENERATION AND TRANSMISSION TO MTX}

For the microwave transmission and absorption experiments, the EL.F-II wiggler ( $9.8 \mathrm{~cm}$ wiggler wavelength and $4 \mathrm{~m}$ total length) was used [3]. With nontapered operation of the wiggler, peak power levels up to $0.2 \mathrm{GW}$ and pulse lengths up to $10 \mathrm{~ns}$ were achieved at $140 \mathrm{GH}$. Figure 1 hhows an experimental gain curve for the wiggler, obtained by varying the wiggler length (number of energized magnets). At the aviliable drive power and electron berm curxent, the Fill interaction just reached saturation. Operation with the wigeler tupered achioved $0.4 \mathrm{OW}$, but this power level was not ued during tolamak experimencs.

The FEL microwave pulve we injected into the windowless, quasioptical transport system [4] for transmission to the tokemak. This system consists of an evacuated, stainiess steel pipe of $50 \mathrm{~cm}$ diamever, six aluminum mirras tranemit the bear $33.3 \mathrm{~m}$. Mearurements outride the tolamak part demonstrated that the transmitted mierowave beam envelope was well approximated by a Ganssian cuve, in good agreement with theory.

\section{ABSORPTION PHYSICS EXPERIMENTS}

FEL produced microwave pulses were injected into the solamak for a variety of plasma parameters. Local measurement of the transmiuled fraction of injected power was made using a microwave ham located jus: below the midplane on the inside wall of the tolkamak. At toroidal magnetic fielis sufficiently high (6.5 T) that there was no electron-cyclotron resonance in the plasma, the tranemitued power showed the expected drop to negligible levels as the peak density approached cutoff. Ray-tracing calculations [S] based on measured density profiles qualitatively reproduced the observed falloff in transmitted power ve density, indicating thut refraction was strong enough to be the dominant mechanism for the measured result.

Experiments were also performed with a central magnetic field of $5.0 \mathrm{~T}$ so that the cyclotron resonance passed through the plasma center. Figure 2 shows the dut from these experiments compared to ray-tracing calculations of refraction effects and of the additional effects of linear absorption. Analysis indicates that the FEl power levels were below that at which nonlinear absorption [2], Brillowin backscatter instabilities [6], and pondermotive selffocusing $[7,8]$ are important. 
The transmitted power fraction data from the FEl experiments are shown by solid circles. For comparison, the upper hatched region shows the calculated effect of refinction alone. This region spans the range of plasma density profiles $\left(\alpha_{n}=0.5-1.0\right.$, where $\left.n(r)=n(0)\left[1-(r / a)^{2}\right] \alpha_{n}\right)$ measured in the experiments. The lower hatched region shows the combined effects of refraction and linear absorption. This bund spans the range of density profiles and electron temperatures (0.5-1.0 keV) observed. Within the variation, there is no evidence tha non-linear sanumion of the absorption plays a rote in these experiments, in agreement with theory [2].

Data from 5.0-T experiments at low power (1 W) and lons pulselength $(1-2 \mu)$ are shown ss aquares in Fic. 2 . The bers on the din points represent the RMS variation is the emplitude of the microwave hom signal during the pulse. These variations also occumed in the high power experiments. Ray tracing calculations with edpo-density floctuntions predicted scatbering of 10$15 \%$ of the power through angles of about $20^{\circ}$, insufficient to explain the observed signal variations. The timescale of the variations is also much shorter than for edse turbulence periods. Multiple spatill modes (generated by wall inveruction to the narow MTX socess doct) together with time varying, moltipath transmission of power through the plasma (due to edge turbulence) could produce a npidly varying interference pattem on the talamak inside wall. This might account for the variations in the observed signals. Experiments are planned to quantify these effects.

\section{INTENSE WAVE ISSUES}

\subsection{Ponderomotive self-focusing}

Ponderomotive self-focusing calculations are relevant for future highpower microwave propagation experiments in MTX. Numerical solutions of a scaled, paraxial self-focusing equation for ordinary modes perpendiculariy incident to an applied magnetic field show qualiative differences from theories in which the laser beam remains Gaussian: self-focusing cocurs in a shotrer distance and the beam does not remain Gaussian [8]. In the limit that ion inertia is neglected, calculations for $T_{e}=T_{i}=1 \mathrm{keV}, 3-\mathrm{cm}$ beam radius, $\omega_{p e} 2 / \omega^{2}=$ $0.5, \mathrm{f}=140 \mathrm{GHz}$, and $v_{o} / v_{e}=0.24\left(0.1 \mathrm{GW} / \mathrm{cm}^{2}\right.$ at $1 \mathrm{keV}$ core temperature) show that self-focusing would occur near the magnetic axis in 3-5 ns. The numerical studies further.indicate that the self-focusing time and distance 
increase approximately as ( $\left.v_{o} / v_{e}\right)^{-1}$ for powers above threshold for selffocusing in agreement with a self-similar analysis [7]. Thus, self-focusing is less severe for higher plasma temperetures and larger bean cross sections, which will make FEL heating applications in CTT less susceptible to selffocusing than in MTX.

Our numerical calculations overestimate the degree of self-focusing because they do nox include several effects that significantly delay or prevent the focusine ion ineria [7], scatuting by edge fluctuations that incresse the effective beum divergence, and aborption. Analytical and mumerical calculations including ion inertia [7] and adtitional mumerical calculations including absorpoion, plasma profiles, end beam divergence with ion.inertia onitued (8] have sbown that these effocts greatly reduce the amount of selffocusing expected in MTX. These calculations also indicave how self-rocusing can be avoided; es. increaring the bean divergence in the plasma.

\subsection{Microinstability}

Microinstability of the Fetheared eloctrons conld rapialy relax the distribution function, thus affecting nonlinear physics. We have developed a new computer code that solves the electromagnetic linear dispersion for a relativistic piasma with en arbitrary fistribution [9]; and we have analyzed distributions predicted by a particle-orbit code for whistler, upper-bybrid losscone (UHLC) and cyclowon-maser instabilities in an infinite homogeneous geometry. For a representative distribution we chose the one generated by injecting into an MTX plasma (at $5 \mathrm{~T}$ ) 2 GW of power at $140 \mathrm{HHz}$ in a beam with cross section of $6 \times 8 \mathrm{~cm}$. At a typical density given by $\omega_{p} / \omega_{c e}=0.6$, the whistler mode has a maximum growth rate of $y / 0_{c e}=7 \times 10^{-3}$, the UHLC mode has $y / 0_{c e}=5.6 \times 10^{-3}$, and the cyclotron-maser mode is stable. The UHLC made is unstuble for a range of wave numbers $0.344 / \omega_{c e}<1.5$ and $12<k_{1} c / w_{c e} 22$. The cyclotron-maser mode becomes unstable at a lower density, $\omega_{p} d \omega_{c e}=0.3$. The maximum growth rates for the whistler and UHLC modes increase monotonically with density in ranges of interest. Thus, high frequency linear microinstabilities should be observable in the openating regime of MTX and could enhance the relaxation of the heated distribution. 


\section{REFERENCES}

- This work was performed under the auspices of the U. S. Deparment of Energy by the Lawrence Livermore National Laboratory under contract W. 7405-ENG-48. (1) Lawrence Berkeley Laboratory. (b)Present address:

Princeton University, Princeton, NJ. (c)TRW, Redondo Beach, CA. (d)Japan Aromic Energy Research Institute.

(1) THOMASSEN, K. I, et al, LLNL Repoct LLLPROP-00202, (1986).

[2] NEVINS, W.M., ROGNLIEN, T.D., COHEN, B.I, Phys. Rev. Lett. 59 (1987) 60.

[3] ORZZECHOWSKI, T. Jn et Il, Phys. Rev, Lett 57 (1986) 2172; THROOP, A, et il, Nucl. Instrum. Meth. A272 (1988) 15.

[4] STALLARD, B.W., et al, Proc. 16th Symp. Fusion Techn, London (1990), to be publisked.

[5] SMTH, GR, FENSTERMACHER, M.E, HOOPER, E.B., LLNL Repart UCRL-JC-103455 (June 1990), submitued to Nucl. Fusion.

(6] PORKOLAB, M, and COEEN, B. I, NucL Fusion 28 (1988) 239.

[7] LONTANo, M., SERGEeV, A.M., CARDINALI, A., Phys. Fluids B 1 (1989) 301.

[8] COHEN, B.L, LASINSK, B.F., LANGDON, A.B., CUMMINGS, J.C. LLNL Report UCRL-JC-103524 (June 1990), submitted to Phys. Fluids.

[9] MATSUDA, Y., SMITH, G,R., LLNL Report UCRL-JC-104559 (July 1990), submitted to J. Comp. Phys.

\section{FIGURE CAPTIONS}

FG. 1. Measured Gain curve for nontapered FEL operation at $139.9 \mathrm{GHz}$ The electron beam parnmevers were $6 \mathrm{MeV}, 2 \mathrm{kA}$, and $50 \mathrm{~ns}$ pulse length. The input drive power was $20 \mathrm{~W}$ from an extended interaction oscillator (ETO) source, injected into the wiggler wheguide co-linear with the electron beam.

FIG. 2. Measured plasma transmission for FEL and EIO power for resonance at plasma center. See text for discussion. 


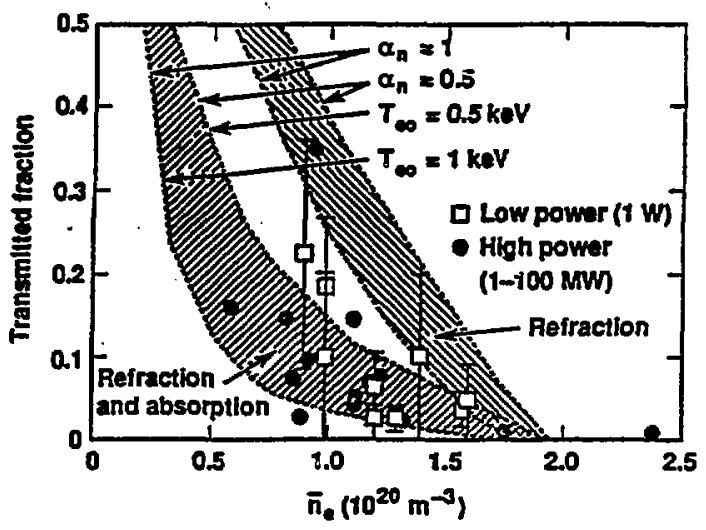

Fig. 1

6 


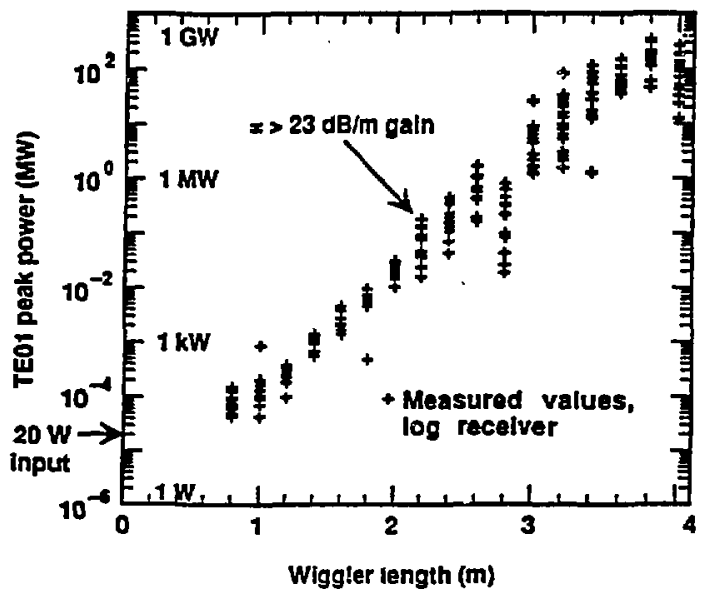

Fig. 2 
5.1.2 Comparison of Propization and Absorption of FEL-Generated Microwaves with Theory (M. E. Fenstermacher)

M. E. Fenstermacher, LLNL Report UCRL-ID-103808, May, 1990. 


\title{
COMPARISON OF PROPAGATION AND ABSORPTION OF FEL-GENERATED MICROWAVES WITH THEORY
}

\author{
DOE MILESTONE REPORT \\ MICROWAVE TOKAMAK EXPERIMENT \\ March 1990
}

M. E. Fenstermacher

April 26, 1990 


\section{Introduction}

The ECH propagation and absorption experiments performed in MTX during November, 1989 have been analyzed in detail. These experiments used a microwave horn mounted behind the microwave calorimeter on the tokamak inside wall to measure the $\mathrm{ECH}$ power transmitted through the plasma from the injection port. The transmitted power fraction was obtained by normalizing this measurement with the incident power measured by a horn in the last mirror (M4) of the micowave transmission line. This configuration in shown in Fig. 1. Analysis of shots without plasma showed that the powers measured by the two horns were linearly dependent (Fig. 2).

The experiments were done at 5.0 and $6.5 \mathrm{~T}$ magnetic field. At the higher field the ECH resonance for $140 \mathrm{GHz}$ microwaves is outside the plasma on the outboard side of the tokamak so that absorption does not affect the beam as it transits the plasma. Measurements at this field allow an independent investigation of plasma refraction effects. Measurements at $5.0 \mathrm{~T}$ show the effects of both refraction and absorption near the center of the plasma. For all of the data obtained to date, the FEL power level delivered to the tokamak, $P_{F E L}$, has been in the range $0.05<\mathrm{P}_{\mathrm{FEL}}<0.25 \mathrm{GW}$ so that linear absorption theory should be applicable (non-linear absorption effects begin to be important for PFEL $>0.5 \mathrm{GW}$ [1]). The data at both magnetic field levels show the expected decrease in the fraction of the power transmitted through the plasma as density increases, with negligible transmission for densities above the theoretical cutoff density.

The remainder of this report is organized as follows. The next section presents the analysis of the data from the FEL experiments. A broad comparison of the data with ray tracing results using fixed density and temperature profiles is described. Following this, detailed analyses of individual shots are given in which density profiles inferred from FIR interferometer measurements and electron temperature profiles from ECE measurements are used in 
the ray tracing and absorption calculations. The next section discusses possible causes for the scatter in the experimental data and the differences between the measurements and calculational predictions. The final section is a summary of the results.

II. Data Analysis

A. Refraction experiments at 6.5 Tesla.

The data from the transmitted power experiments using the FEL in MTX are shown in Figs. 3 and 4. Transmitted power fraction as a function of line averaged density at $6.5 \mathrm{~T}$ magnetic field on axis is shown in Fig. 3. These experiments test the effects of refraction in the plasma, so the electron density and density profiles are importent. The theoretical predictions from the ray tracing calculations [2] using a nearly parabolic radial density profile are shown by the solid line. This density profile is typical of some MTX operation prior to these experiments. The data confirms the expected decrease in transmitted power and the cutoff as the density increases. However, for densities below the cutoff the measurement of the transmitted power fraction is typically lower than the theoretical prediction for the density profile used.

Closer examination of the density profiles for several of the shots in Fig. 3 showed that the assumed profile in the ray tracing calculation was broader than measured experimentally. Data from the 10-channel FIR interferometer were inverted, assuming insideoutside symmetry, for the shots where good data were available [3] and these profiles were used in ray tracing calculations on a shot-byshot basis. The transmitted power fraction from these calculations is shown in each case as an offset from the original theory line. This offset is a measure of the scatter in the data which might be attributable to shot-by-shot variability in the density profiles. The FIR inteiferometer data typically show higher line averaged density on the inside of the plasma compared with the outside and this 
asymmetry varies both during the shot and from shot-to-shot. At present, however, the ray tracing analysis is only available for symmetric profiles. In all but one of the cases the agreement between theory and experiment improved with this detailed analysis. Reasons for the remaining differences will be discussed in Section III below.

B. Refraction plus absorption experiments at 5.0 Tesla

Data on the fraction of the power transmitted through the plasma as anction of line averaged electron density for MTX experiments at 5.0 $\mathrm{T}$ are shown in Fig. 4. In this case the ECH resonance is on the plasma axis so that the effects of both refraction in the plasma and absorption at the resonance are important. As in the experiments at $6.5 \mathrm{~T}$, the refraction depends on the density and density profile. For these experiments in the linear absorption regime ( $P_{F E L}<0.25 \mathrm{GW}$ ) the transmitted power fraction through the resonance region is exp(- $\tau$ ) with the linear (low intensity) opacity for the ordinary mode $[4,5]$ given by

$$
\tau=\pi^{2}\left(T_{d} / \mathrm{mc}^{2}\right)\left(\mathrm{R} / \lambda_{0}\right) \delta(1-\delta)^{1 / 2}\left(1+0.05 \delta^{2}\right)
$$

where $R$ and $T_{e}$ are the plasma major radius and the electron temperature, respectively. Here the vacuum wavelength is $\lambda_{0}=\mathrm{c} / \mathrm{f}$, the microwave frequency $f=140 \mathrm{GHz}$ and $\delta=\omega_{p}{ }^{2} / \Omega^{2}$ with $\omega_{p}$ and $\Omega$ denoting the electron plasma and cyclotron frequencies, respectively. All spatially dependent quantities were evaluated for each ray at the point where that ray crosses the cyclotron resonance. Absorption effects, therefore, depend on both the density and temperature at the resonance surface. This surface spans the plasma vertically at a major radius $R=64 \mathrm{~cm}$ so that absorption for the beam as a whole depends on the density and temperature profiles as well. The prediction from the ray tracing calculation using the same parabolic density profile as in Fig. 3 and the square of a parabolic temperature profile is shown by the solid line. For reference the dashed line 
shows the prediction for refraction alone without absorption. The peak temperature for these calculations was $1 \mathrm{keV}$. Again, the data exhibits the appropriate decrease and cutoff with increasing density, but in this case for densities below cutoff the data shows higher transmitted power fraction than predicted.

The same analysis [3] of the FIR interferometer data used in the refraction experiments was applied to several of these shots at $5.0 \mathrm{~T}$ to give the experimental density profile. In addition, analysis of the ECE data was done to obtain the electron temperature profile. For most of the shots this data was normalized to the absolutely calibrated Thompson scattering diagnostic measurement of the peak temperature on axis. Ray tracing results using these density and temperature profiles with the measured peak temperature are shown as offsets from the theoretical line in Fig. 4 . As in the refraction experiments, the agreement between theory and experiment improves with the detailed analysis but substantial differences still remain.

The ratio of the theory prediction to the measured transmitted power fraction for all of the data that have been analyzed in detail, from both the refraction and the refraction plus absorption experiments, is shown in Fig. 5. Scenarios which could produce this scatter in the data are discussed below.

\section{Discussion}

The data presented above are for experiments with power measurements at essentially a single point on the inside wall of the tokamak normalized to similar measurements at essentially a single point on the input optics in the transmission line. With such a point measurement, a variety of small effects, not considered in the modeling, can produce large variations in the transmission measurements. The scenario we believe is affecting the measurements in MTX involves either density fluctuations at the plasma edge (or varying asymmetries in the density profile), mode structure in the microwave beam, or a combination of these effects. 
This is summarized in Table 1 . There is strong evidence that the scatter is not due to the high-power, short-pulse nature of the FEL power interacting with the plasma. Data supporting this view are described below.

There are two different effects that can produce mode structure in the microwave beam used in these experiments. First, full three-dimensional calculations of the wave fields [6] for a pure $T_{01}$ mode entering the transmission line from the FEL show that when that beam propagates through the tall, narrow MTX port, the horizontal field pattern at the duct exit has large electric fields at the duct side walls. These modes interfere as they propagate to the far wall of the tokamak, even in the absence of plasma, producing an interference pattern there with spatial scale length comparable with the dimensions of the microwave horn. Notw that this might explain part of the scatter in the data of Fig. 2.

Other wave calculations have been done [7] to predict the mode structure exiting the FEL when, as in all the MTX experiments to date, the wiggler is not tapered. This work shows that substantial $T E_{21}$ and $T M_{21}$ components can be amplified by the untapered wiggler so that the beam entering the transmission line will contain structure other that a pure $\mathrm{TE}_{01}$ mode. This makes the field pattern reaching the inside wall of the tokamak more complicated than for the duct propagation calculations described above. In addition, this mixture of modes is subject to shot-to-shot variation when conditions in the FEL change, possibly contributing to the scatter in the transmission data.

In addition to containing mode structure as described above, the beam is scattered by density fluctuations at the plasma edge. The edge plasma fluctuations have yet to be characterized in detail for these experiments. However, evidence for these fluctuations was seen during refraction (6.5 $\mathrm{T}$ ) and refraction plus absorption (5.0 T) experiments using the power from the EIO oscillator (1-2 W) sent through the wiggler and transmission line to MTX. Data from these experiments are shown in Figs. 6 and 7. Note that in this case preferential amplification of the $T_{21}$ and $T M_{21}$ modes does not play a role since the accelerator electron beam is not used. Output from 
the wiggler waveguide does include a mixture of modes but the beam pattern should be reproducible from shot-to-shot. The data implies that this is the case; both microwave horns measure a nearly constant EIO pulse with a flattop time of about 1 microsecond when no plasma is present in the tokamak. The bars on the plasma data in these figures represent the RMS fluctuations on the signal seen by the tokamak hom normalized to the flattop signal seen at M4 during the 1 microsecond pulse. Two representative EIO pulse measurements at the tokamak horn are shown in Fig. 8 . Fluctuations greater than $50 \%$ of the peak amplitude were seen on many of the EIO plasma shots.

The data using the EIO beam implies that density variations of some sort on a sub-microsecond time scale are present in the plasma and that the mode pattern produced by propagating the EIO beam through the narrow MTX duct is affected before the power is detected by the tokamak horn. Only small variations in the effective index of refraction for power in different parts of the beam cross section are required to significantly change refraction and thus the location of features in the interference pattern on the tokamak inside wall. Also, relatively small changes in density along the ray trajectory can produce large changes in wave phases and thereby significantly affect the interference pattern. Since the tokamak horn is comparable in size with the structure in the interference pattern, large variations in the horn measurements could result. Since this variation is seen in the low power (1-2 W) EIO experiments, there is every reason to assert that it affects the FEL measurements as well. Note that this picture of a changing interference pattern does not require that there be gross plasma fluctuations at near megahertz frequencies. Only sufficient changes in the effective index of refraction (possibly at much lower frequency) to change the position of fringes in the interference pattern on these fast time scales are required to explain the EIO data. 
IV. Summary

Microwave transmission experiments in the MTX tokamak using low power pulses (1-2 W, 1 microsecond) from the EIO oscillator and high power pulses ( $0.25 \mathrm{GW}, 10 \mathrm{~ns}$ ) from the FEL clearly show the expected decrease in transmitted power fraction with increasing density. Negligible transmission was observed above the cutoff density for $140 \mathrm{GHz}$ ordinary mode waves as predicted from theory. These results were observed both in high field (6.5 T) experiments where only refraction played a role and in the resonance experiments $(5.0 \mathrm{~T})$ in which refraction and absorption occurred. Similar transmission results were found at both power levels indicating that no "burn-through" occurred in the high power experiments. Detailed computations with a ray tracing code, using the experimentally measured density and temperature profiles, qualitatively agree with the data as a function of plasma density. Quantitative agreement (within better than a factor of 2) is hampered by scatter in the data. A plausible scenario for producing the scatter in both the low and high power data involves a combination of density variations in the plasma and multiple modes in the microwave beam that interfere in the plasma for these experiments. The measurements made here with a microwave horn on the tokamak inside wall are sensitive to temporal and spatial variations of such an interference pattern. Such a variation might be caused by edge density fluctuations or density changes in the plasma. 
1. T.D. Rognlien, private communication (Feb. 1989) and, J.A. Byers, R.H. Cohen, M.E. Fenstermacher, et. al., Preparation for Propagation and Absorption Experiments in MTX, in Proc. of 8th Top. Conf. on Radio Frequency Power in Plasmas, Irvine, CA, 1989.

2. G.R. Smith, M.E. Fenstermacher, and E.B. Hooper, Modelling of Electron Cyclotron Power Profiles on a Calorimeter in the Microwave Tokamak Experiment, Lawrence Livermore National Laboratory report UCRL-(to be determined), 1990 (to be submitted to Nucl. Fusion). A draft of this report is attached as an appendix.

3. T.A. Casper, private communication (March 1990), and T.A. Casper et. al., Bull. Am. Phys. Soc 34, 1956 (1989).

4. M. Bornatici, R. Cano, O. Debarbieri, and F. Engelmann, Nucl. Fusion 23, 1153 (1983).

5. See National Technical Information Service Document No. DE88016919 (Lawrence Livermore National Laboratory report No. LLLPROP-00202 by K.I. Thomassen, Scientific Editor, 1986).

6. J.A. Byers, private communication (LLNL memo, Dec. 12, 1988).

7. J.A. Byers, private communication (LLNL memo, Oct. 3, 1989), and J.A. Byers and R.H. Cohen, A Microwave FEL Code Using Waveguide Modes, Lawrence Livermore National Laboratory report UCRL-97348, (1987). 
Acknowledgements

This work benefitted from the support of the entire MTX Experimental Team. Special thanks to E. B. Hooper, T. A. Casper, C. J. Lasnier and S. I Allen for valuable assistance and critical comments. 
Figure Captions

Figure 1. Geometry for the FEL and EIO experiments in MTX showing diagnostic horns in the M4 mirror and on the tokamak inside wall.

Figure 2. Power measured on the tokamak horn as a function of power measured on the M4 horn, without plasma in the tokamak. Power values in $\mathrm{MW}$ are estimates using signals from sensitive receivers ( $\mathrm{mW}$ ) with approximately $110 \mathrm{db}$ of attenuation in the waveguide.

Figure 3. Transmitted power fraction as a function of line average plasma density $\left(10^{20} \mathrm{~m}^{-3}\right)$ for FEL input to MTX at $6.5 \mathrm{~T}$ magnetic field. The theoretical prediction of the effect of refraction for parabolic density profiles is shown by the solid line. The cutoff density for this profile corresponds to a line averaged density of $1.85 \times 10^{20} \mathrm{~m}^{-3}$. Variations in the theoretical prediction due to the experimentally measured density profiles are shown as offsets.

Figure 4. Transmitted power fraction as a function of line average plasma density $\left(10^{20} \mathrm{~m}^{-3}\right)$ for FEL input to MTX at $5.0 \mathrm{~T}$ magnetic field. The theoretical prediction of the effect of refraction for parabolic density profiles is shown by the dashed line. The prediction including refraction plus absorption, assuming a peak electron temperature of $1 \mathrm{keV}$, is shown by the solid line. Variations in the theoretical prediction due to the experimentally measured density and temperature profiles are shown as offsets.

Figure 5. Transmitted power fraction measured in the FEL experiments normalized to theoretical predictions for shots that have been analyzed with the experimentally measured density and temperature profiles. The data is scattered within a factor of 3 from the theory (horizontal lines). 
Figure 6. Transmitted power fraction as a function of line average plasma density for EIO input to MTX at $6.5 \mathrm{~T}$ magnetic field. The theoretical prediction of the effect of refraction for parabolic density profiles is shown by the solid line. The bars represent the RMS variation of the signals on the tokamak horn during the 1 microsecond pulse.

Figure 7. Transmitted power fraction as a function of line average plasma density for EIO input to MTX at $5.0 \mathrm{~T}$ magnetic field. The theoretical prediction of the effect of refraction for parabolic density profiles is shown by the dashed line. The prediction including refraction plus absorption, assuming a peak electron tempe: ature of $1 \mathrm{keV}$, is shown by the solid line. The bars represent the RMS variation in the signals on the tokamak horn durirg the 1 microsecond pulse.

Figure 8. Signals from the M4 and tokamak horns as functions of time for two typical shots with EIO input into an MTX slasma at 5.0 T. Large variations in the transmitted power on a sub-microsecond time scale are shown. 
TABLE 1. Possible Causes of Scatter in the Transmitted Power Data.

\author{
Effect \\ Spatial structure \\ in the beam power \\ profile entering \\ MTX plasma. \\ Cause \\ Narrow MTX Access \\ duct.
}

Untapered wiggler
Beam propagation that is not well modeled by ray tracing calculations

\section{Result}

interference

in the plasma

due to non-zero

edge fields at

the duct exit.

Mixture of $\mathrm{TE}_{0 \mathrm{i}}$

$\mathrm{TE}_{21}$ and $\mathrm{TM}_{21}$

modes at FEL

output transmitted

to MTX plasma

Temporal and spatial mixing of model ray trajectories for various parts of the bean profile (ie. a time varying interference pattern in the plasma).
Remarks

Reflection of power missing transmission line mirrors further complicates the beam power profile.

On the time scale of the FEL, the turbulent density in the edge plasma is stationary

\author{
microwing of \\ plasma density \\ variations which \\ have yet to be char- \\ acterized by fluctu- \\ ation diagnostics.
}




\section{Microwave Transmission Diagnostics}

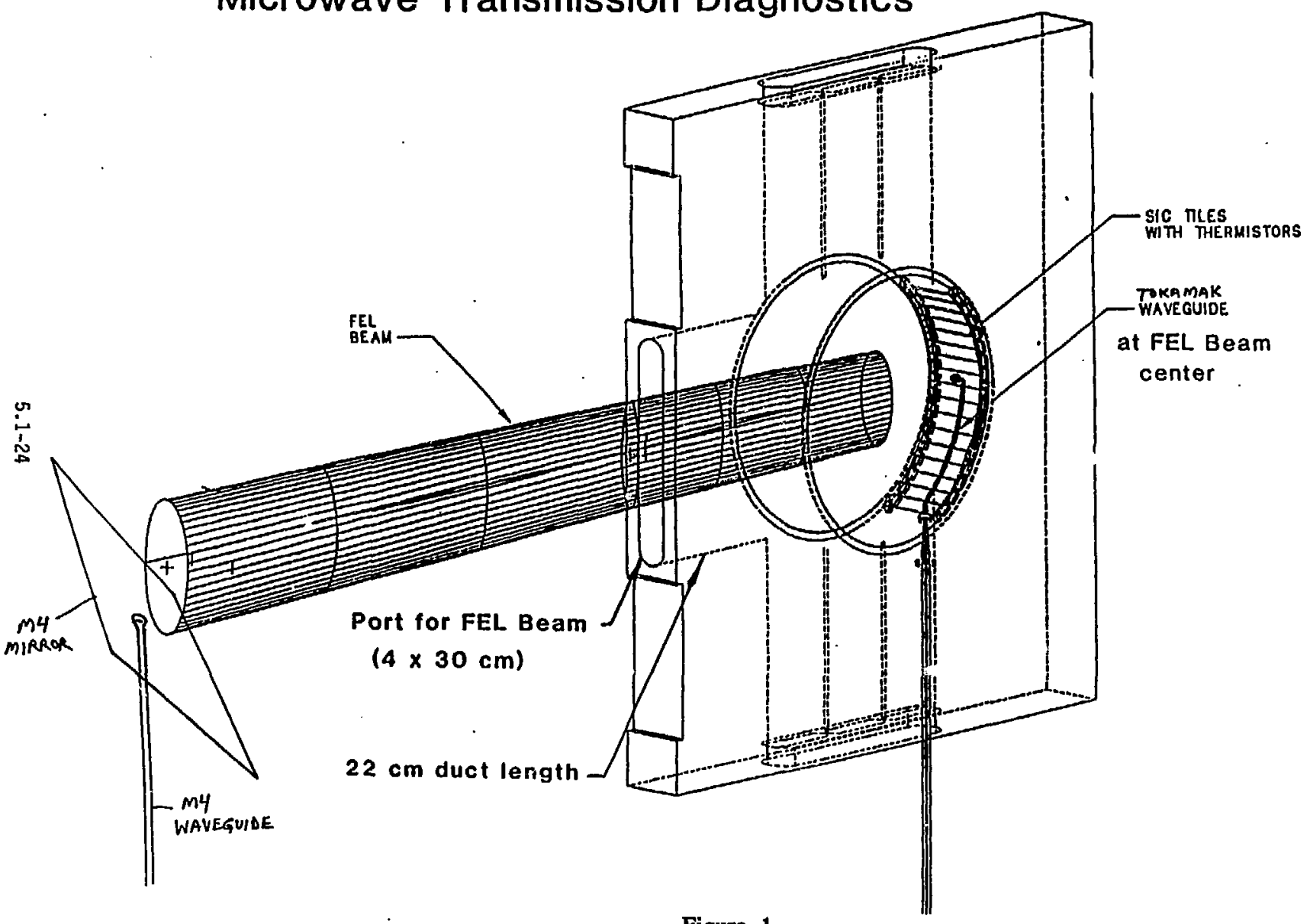

Figure 1. 
CALIBRATION - NO PLASMA

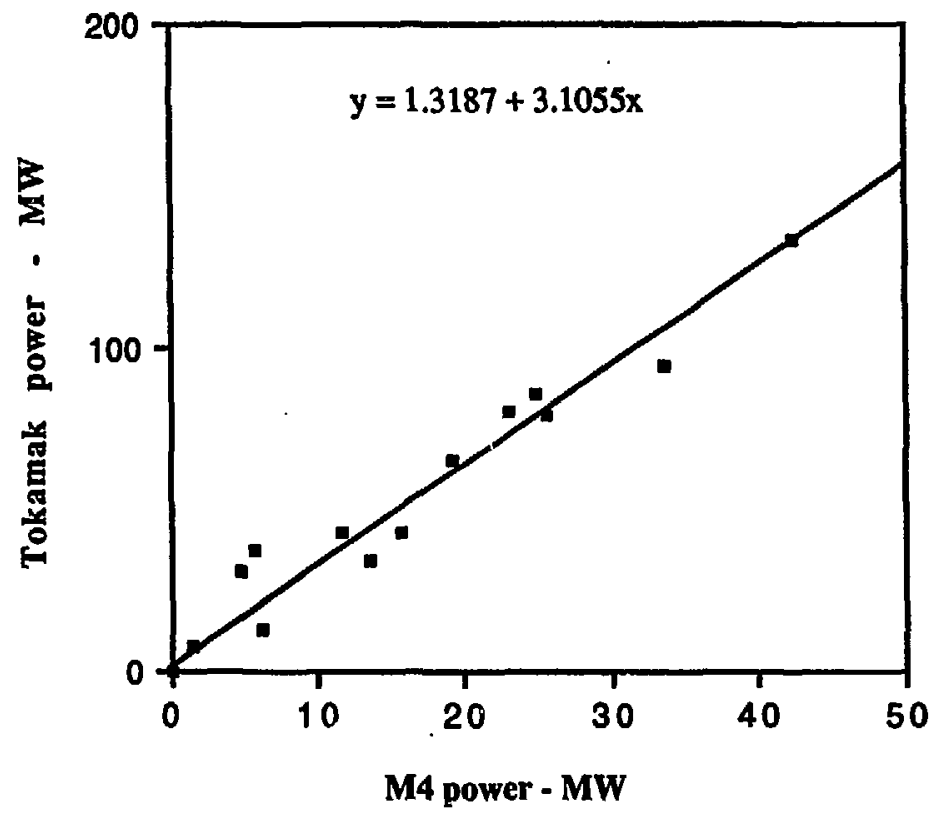

Figure 2. 

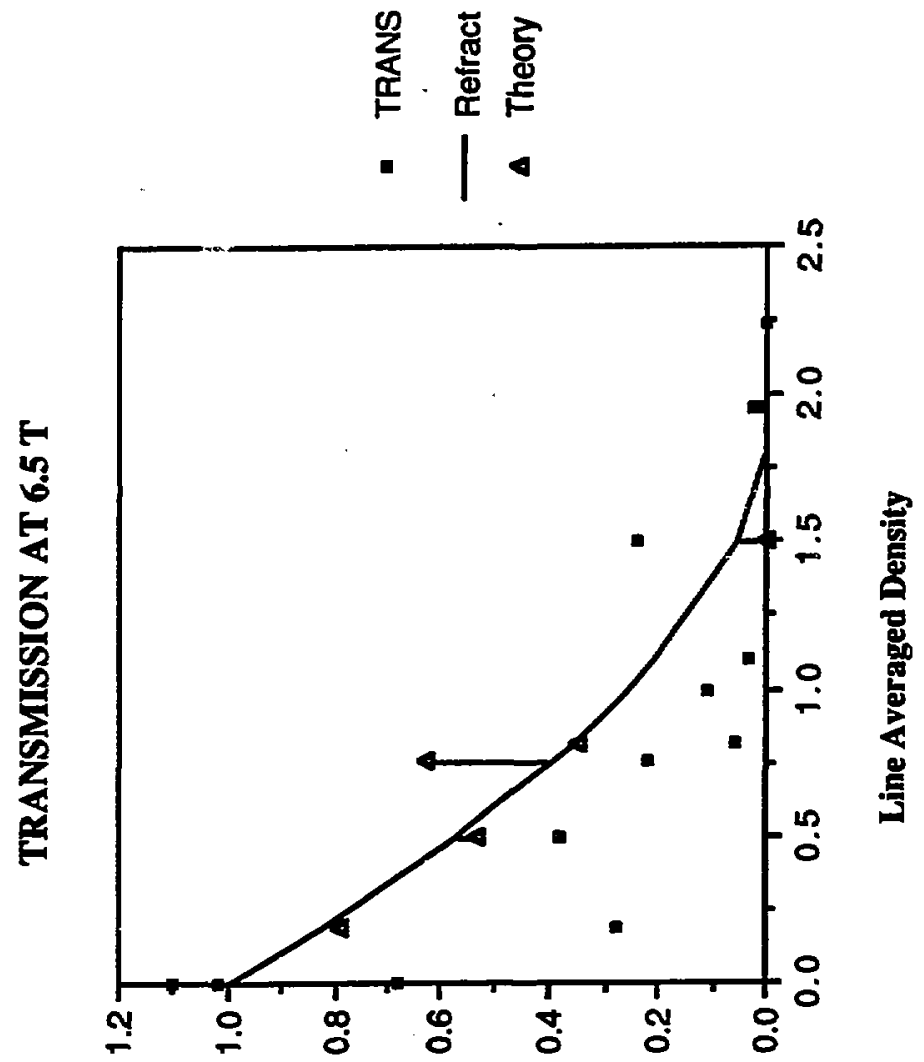

禹 nogfed JaAod pap1!usuedJ 
TRANSMISSION AT $5 \mathrm{~T}$

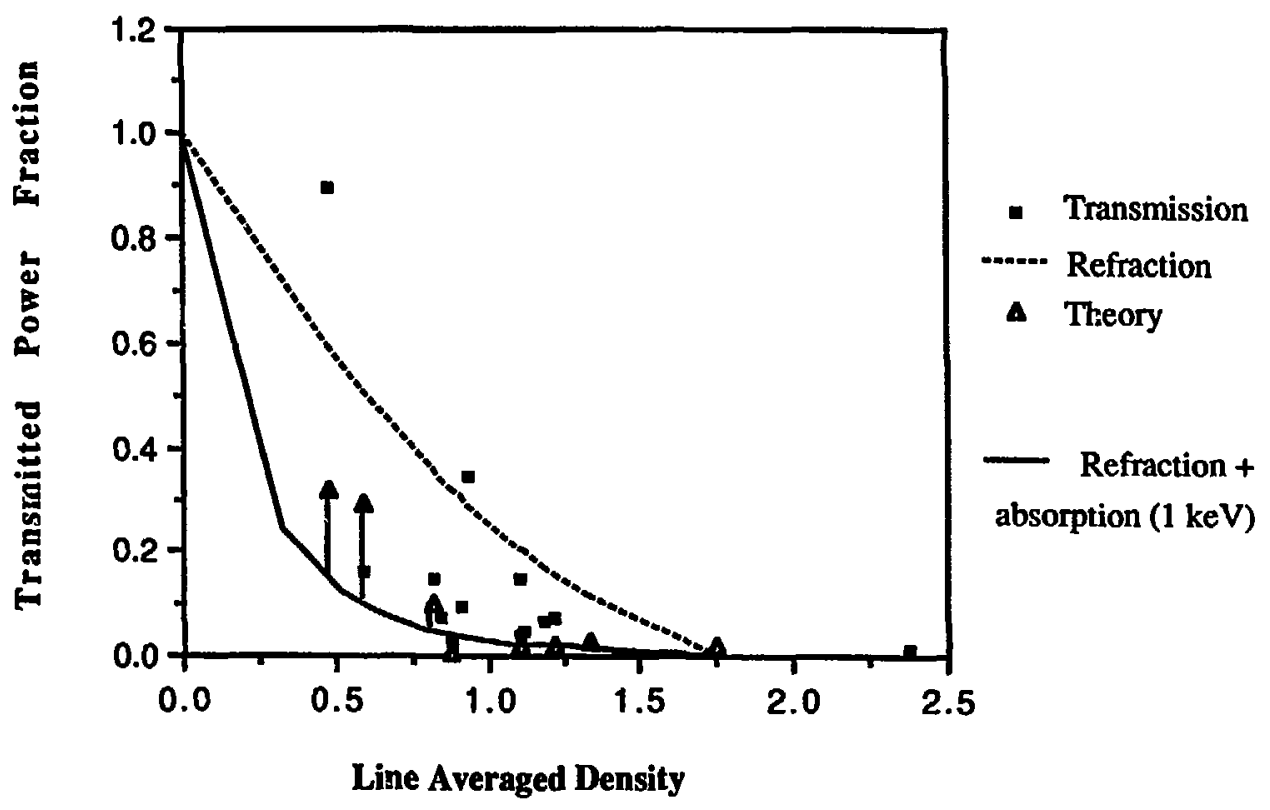

Figure 4. 
Transmission Data Normalized to Theory

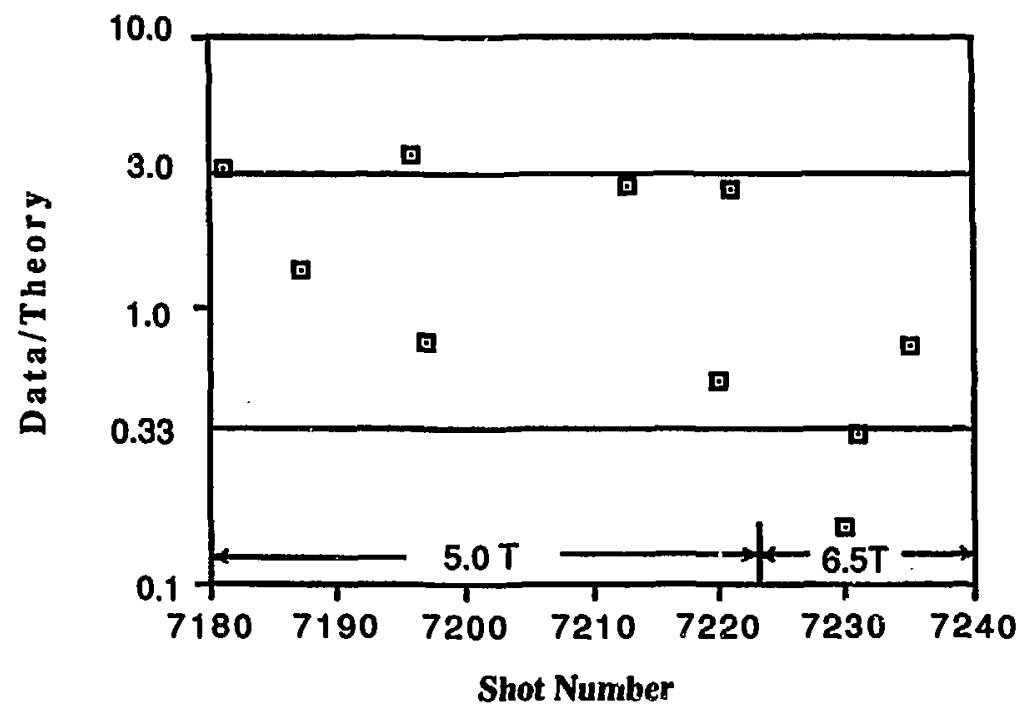

Figure 5. 
6.5 T - LOW POWER (EIO) TRANSMISSION

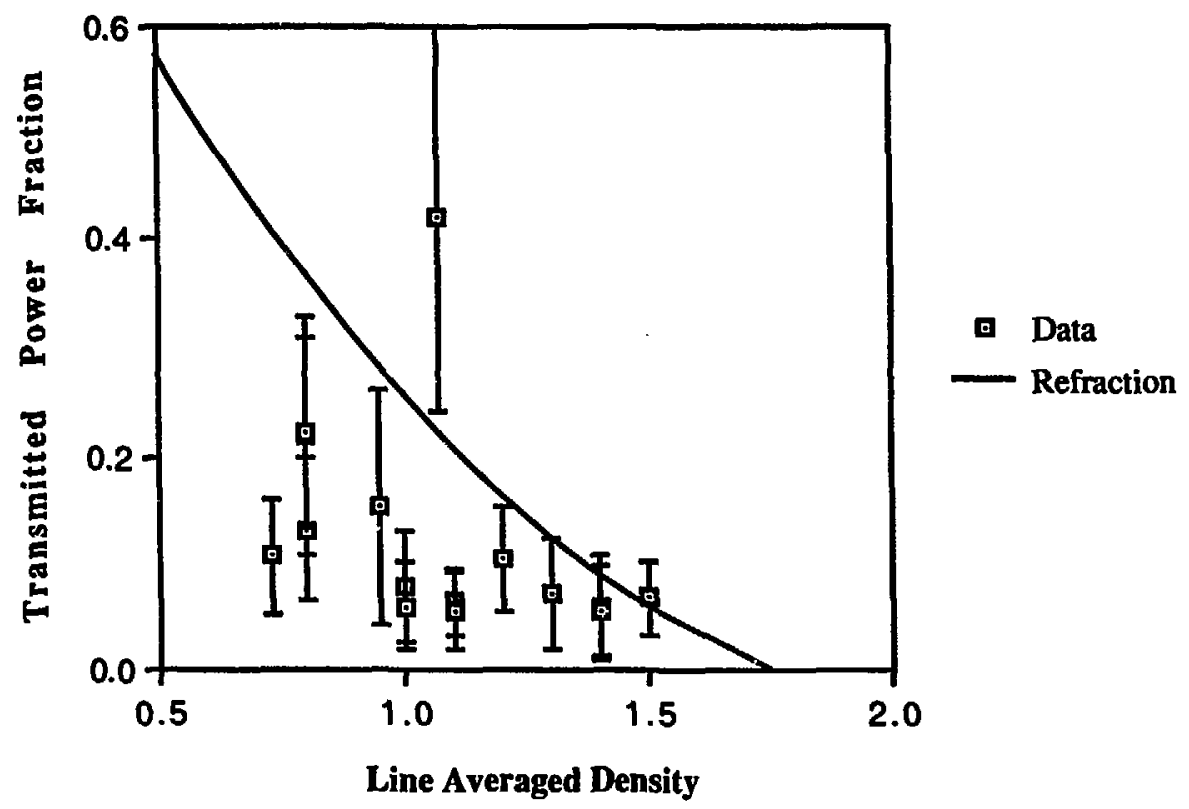

Figure 6. 


\section{S T - LOW POWER (EIO) TRANSMISSION}

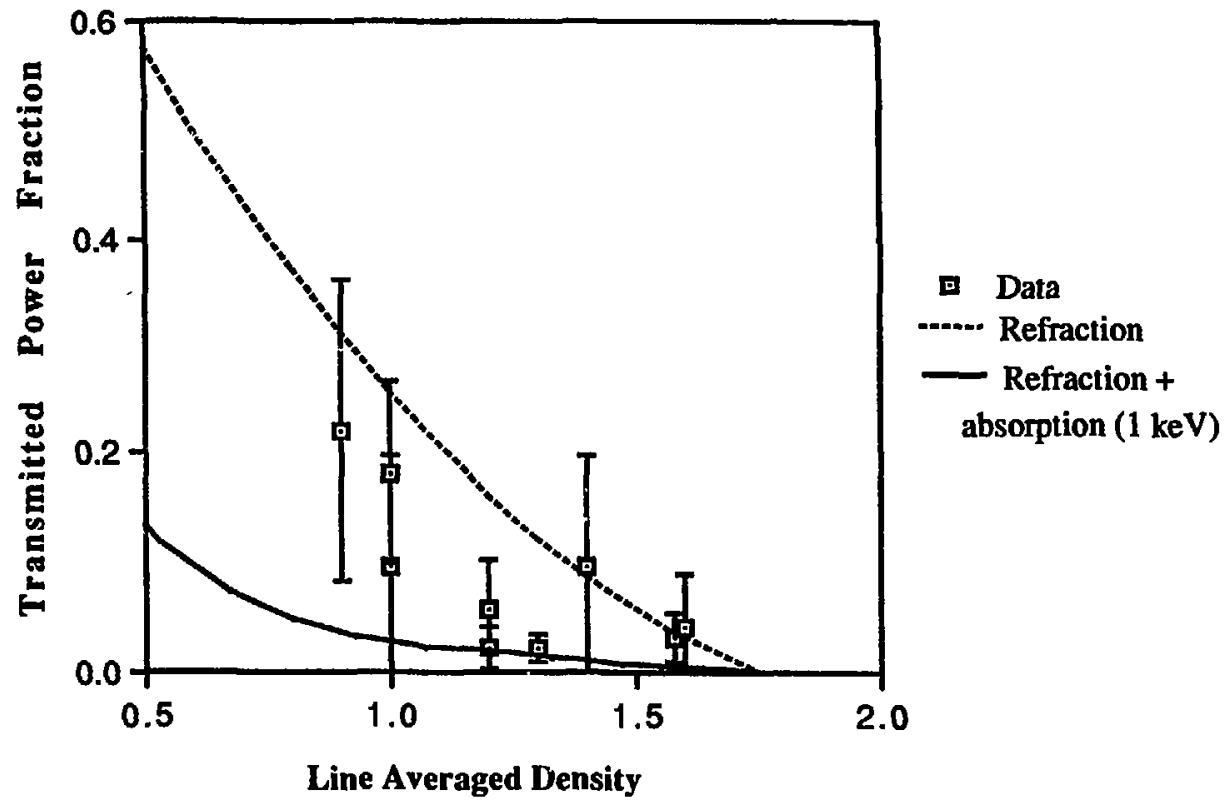

Figure 7. 


\title{
LOW POWER TRANSMISSION IN MTX
}

\author{
$140 \mathrm{GHz}$ and $5 \mathrm{~T}$
}
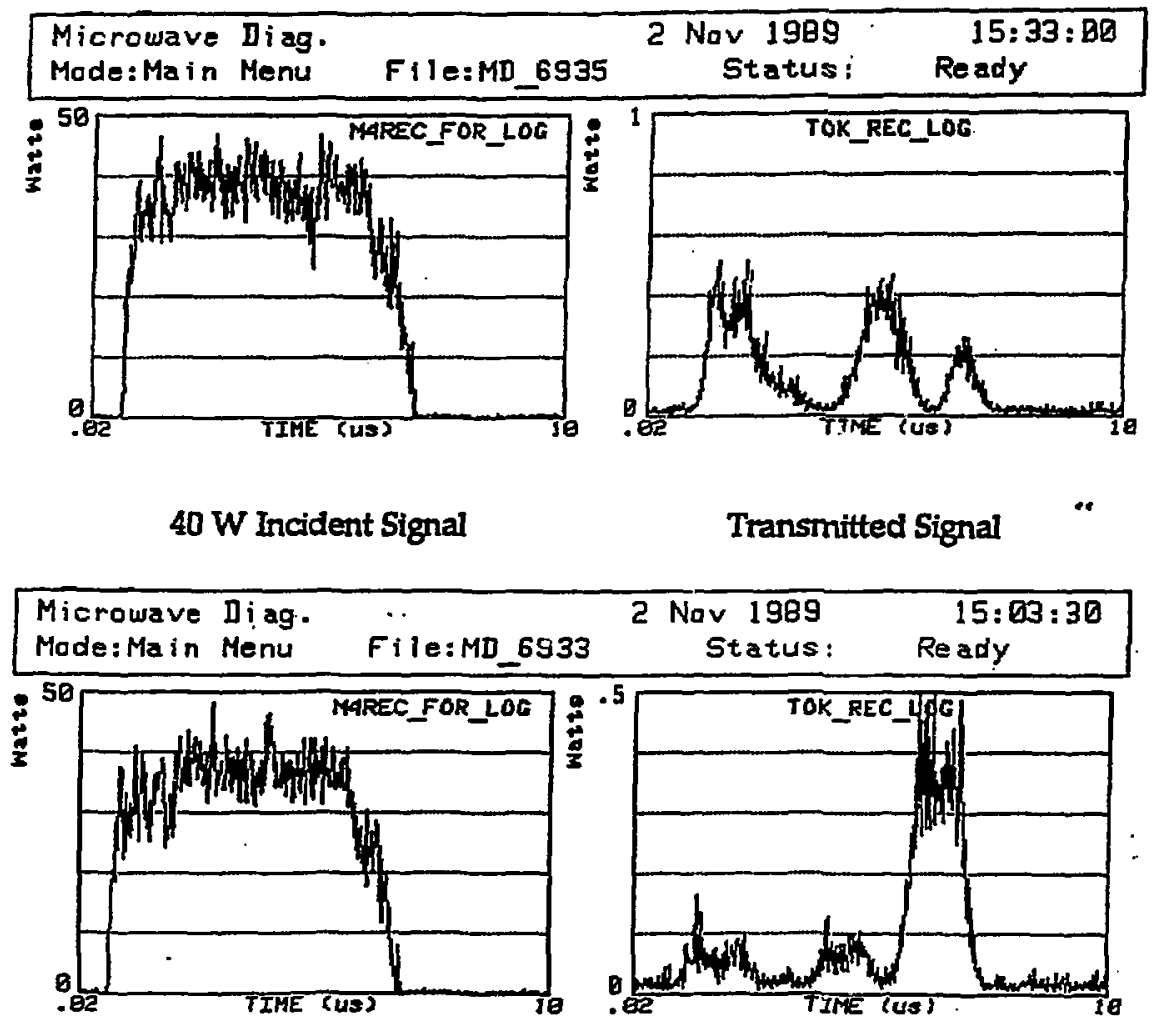

Figure 8.

5.1-31 


\subsection{Nonlinear Absorption of High Power FEL-Generated Microwaves at ECRH Frequencies in the MTX Tokamak (S.L. Allen, M.D. Brown, J.A. Byers, T.A. Casper, B.I. Cohen, R.H. Cohen, M.E. Fenstermacher, J.H. Foote, E.B. Hooper, C.J. Lasnier, P. Lopez, M.A. Makowski, M.M. Marinak, W.H. Meyer, J.M. Moller, W.M. Nevins, B.W. Rice, T.D. Rognlien, G.R. Smith, B.W. Stallard, E.T. Scharlemann, K.I. Thomassen, R.D. Wood, K. Hoshino, K. Oasa, T. Oda, K. Odajima, T. Ogawa, T. Ohgo)}

\section{(Submitted to Physical Review Letters.)}

We report the first measurements of single-pass propagation of intense $(>$ 1-GW, 20-ns) microwaves through a high-density $\left(n_{e}=0.5-2.4 \times 10^{20} \mathrm{~m}^{-3}\right)$ tokamak plasma at $140 \mathrm{GHz}$, the fundamental electron cyclotron resonance frequency. Compared with low-power pulses, these FEL-generated intense pulses were not as strongly absorbed by the plasma, in agreement with predictions of nonlinear theory. Enhanced absorption of intense pulses was achieved by increasing the gradient in the parallel index of refraction $\left(N_{\mid 1}\right)$ of the microwave beam.

The decrease of the Coulomb collision frequency with increasing temperature places a fundamental limitation on ohmic heating in toroidally confined plasmas. Consequently, a major thrust of controlled thermonuclear fusion research has been the development of auxiliary heating techniques for both ions and electrons. In many cases, these same techniques can be used to drive plasma current and provide local control of plasma profiles. The purpose of the Microwave Tokamak Experiment (MTX) is to study one particular type of electron heating in tokamaks: Electron Cyclotron Resonance Heating (ECRH). The major advantage of ECRH is that very localized electron heating is achieved, as absorption occurs where the wave frequency equals the local electron cyclotron frequency $f_{c e}=28 \mathrm{GHz} / \mathrm{T}(140 \mathrm{GHz}$ at $5 \mathrm{~T}$ for MTX. In contrast to lower-frequency heating methods, ECRH can be accomplished with a launching structure that is well separated from the plasma, thereby eliminating plasma-antenna interactions.

On the MTX experiment, we measured for the first time the interaction of very intense (> 1-GW, 20-ns) microwave pulses with a high-density $\left(n_{e}=0.5-\right.$ $\left.2.4 \times 10^{20} \mathrm{~m}^{-3}\right)$ plasma confinad in a high-toroidal-field tokamak $\left(B_{\mathrm{T}}=5 \mathrm{~T}\right)$. These pulses were generated by a free electron laser (FEI.) ${ }^{1}$ and represent the highest peak power generated to date at 140 GHz. The use of an FEL for ECRH has several technological advantages, including the potential of frequency sweeping $(-10 \%)$ to vary the heating zone ${ }^{2}$ and the ability to generate high frequency microwaves for heating at high density. ECRH with an FEL also makes possible several novel heating and current drive schemes. ${ }^{3}$ 
For comparison, we have also performed more conventional ECRH experiments with moderate-power, long-pulse $(200-\mathrm{kW}, 100-\mathrm{ms})$ microwaves generated by a gyrotron. These results, discussed in detail elsewhere ${ }^{4}$, are similar to those obtained on other tokamaks, such as DIII-D $D^{5}$ and T-106. In general, these experiments show efficient heating and are in agreement with thoroughly developed linear theories of $\mathrm{ECRH}^{7}$

The ECRH theory has been extended to the nonlinear (high-microwave electric field) regime ${ }^{8}$ relevant to the FEL experiments. A simple phase space picture of the nonlinear effect is, that electrons are strongly heated by the intense microwave beam and become nonlinearly trapped near the cyclotron resonance. While trapped, they exhibit adiabatic motion with a concomitant reduction in opacity compared to the linear theory. However, the nonlinear opacity increases with electron temperature and plasma size 8,9 , so that estimates for high-temperature reactor-grade plasmas indicate good absorption of these intense pulses. The nonlinear theory also predicts the generation of energetic electrons (5-25 keV in MTX). Theoretical estimates of the magnitude of other mechanisms that could degrade the absorption, such as parametric effects ${ }^{9}$, indicate that these are not large in MTX. In all, theory predicts that the MTX FEL experiments should show significant, observable differences in absorption from linear theory, and heating by intense pulises can be an attractive option for future tokamaks.

The MTX experiment consists of the microwave source (FEL or gyrotron), a quasi-optical microwave transport system, the MTX tokamak, and the microwave and plasma diagnostics. The FEL operates as an amplifier and is an improved version of the system developed by the Beam Research Group at LLNL. 2 It consists of an electron beam accelerator, beam transport section, master oscillator, and a tunable steady-state wiggler. The upgraded ETA-II linear induction accelerator ${ }^{1}$ generates the $2-3 \sim \mathrm{kA}, 6-\mathrm{MeV}$ beam for the FEL. Previous FEL results at $140 \sim \mathrm{GHz}^{10,11}$ with ETA-II were limited to peak powers of 100-150 MW and short pulses of $10 \mathrm{~ns}$ by poor electron beam quality. Magnetic field errors in the accelerator and energy sweep of the beam due to variations in the power supplies ( $\triangle E / E$ of up to 10\%) resulted in a helical ("corkscrew") beam motion $(1 \mathrm{~cm})$. Computer models 12,13 indicated that a reduction of the corkscrew by nearly a factor of ten was required for generation of multi-GW microwave pulses. Several improvements were implemented in ETA-II, including a new magnetic alignment technique, computerized tuning of error-correction coils ${ }^{1,14}$, and an improved pulsedpower supply system. The corkscrew amplitude was reduced by an order of magnitude to $\pm 1.5 \mathrm{~mm}$, and the energy was regulated to $1 \%$ for over $30 \mathrm{~ns}$. The beam brightness was not measured for these experiments, but previous experiments with a $3-\mathrm{MeV}$ version of ETA-II indicated values 15 of $4 \times 10^{8} \mathrm{~A} \mathrm{~m}^{-}$ $2 \mathrm{r}^{-2}$, exceeding the requirements of the computer models by a factor of 2-4.

The electron beam is transported to the wiggler by a series of magnetic lenses. The master oscillator power (5-10 kW) is coupled quasi-optically at the input of the wiggler; the electron beam passes through a hole in the input 
optic. The Intense Microwave Prototype (IMP) wiggler is a new steady-state tunable design ${ }^{16}$. The measured magnetic field errors were less than $0.2 \%$ RMS over the length of the wiggler as required by the computer models. Beam shape was verified along the wiggler with a moveable optical target; betatron (sausage-like) motion was small when the wiggler was properly tuned.

Tapered IMP operation (tailoring of the magnetic field to maintain FEL resonance as the microwaves gain energy from the electron beam) resulted in a peak microwave power at the exit of the FEL in the range of 1-2 GW. Spatial mode pattern measurements were consistent with a predo.ninantly $\mathrm{TE}_{1,1}$ mode. While results from computation models indicated that peak powers of nearly a factor of two greater were achievable, further optimization was not performed due to time constraints. Multiple-pulse bursts of microwaves were also generated after the conclusion of MTX experiments. ${ }^{17}$

The microwave beam was transported to MTX by a windowless quasioptical transport system. ${ }^{16}$ Horizontally polarized microwaves (ordinary mode) were launched into the plasma from the low-field side of the tokamak (outside). A precision calorimeter measured the input power to MTX. Peak powers up to 1.4 GW were observed, consistent with theoretical estimates (89\%) of the transmission of the transport system. Measurements of the vacuum FEL-generated electric field (LAPPS, Sec. 5.3) were aiso consistent with this power level.

The transmission of the microwaves was measured as a function of position in the MTX tokamak with a 48-channel electronic calorimeter mounted on the inside wall of the machine. We have examined transmission values obtained for both the core $( \pm 3 \mathrm{~cm})$ and the whole beam. For core measurements, we used the ratio of the temperature rise with and without plasma. The whole beam analysis used the difference in the temperature rise from plasma shots with and without the FEL. Both calculations included appropriate normalizations to the output power of the FEL measured both at the entrance to MTX and by the MTX calorimeter (without plasma).

Figure 1 presents representative plasma data from a discharge used for FEL experiments. The plasma current is $200 \mathrm{kA}$, the electron density is 5 $\times 10^{19} \mathrm{~mm}^{-3}$, and the central electron temperature is $\sim 1.2 \mathrm{keV}$. The data fiom the $x$-ray diagnostic located at the FEL port are also shown; the FEL pulse was injected at approximately $200 \sim \mathrm{ms}$. The $x$-ray sampling rate is increased during the time of the FEL pulse; the expanded data for three spatial chords are shown. Note the very localized nature of the $x$-ray response: only the detector viewing the central chord shows heated electrons.

Figure 2 shows the core $( \pm 3 \mathrm{~cm})$ transmission data $T_{F E L}$ from calorimetry as a function of peak electron density (from inverted interferometer linedensity profiles) for FEL input powers in the range of 0.8-1.4 GW (r : d circles). For comparison, data from low-power $T_{\text {?ow }} \sim 3 \mathrm{~kW}$, i.e., master oscillator orly with no FEL gain) are shown (green diamonds). Note that $T_{F E L}$ 
$>T_{\text {low }}$ for all densities, demonstrating the nonlinear behavior. Theoretical predictions for both nonlinear (red) and linear (green) theory are included.

The linear theoretical calculation is the product of transmission times refraction; the latter is calculated from a ray-tracing code ${ }^{18}$ using electron density profiles measured with a 15-chord far-infrared interferometer. There is good agreement between the linear theory $T_{\text {lin }}$ theory (green line) and the $T_{\text {low }}$ (green diamonds) data. Previous experiments $\mathrm{j}$ terformed at $\sim \mathbf{2 0 0} \mathrm{kW}$ with a gyrotron on MTX showed similar agreement ${ }^{4}$. Also, earlier measurements with the FEL 10, 11 (at 100-150-MW) were consistent with linear theory, as predicted.

In the nonlinear theoretical calculations for the FEL case, a refractionmodified on-axis electric field was used in the orbit-following code ${ }^{19}$; the transmission was then further reduced by refraction from the magnetic axis to the wall. Curves for $1 \mathrm{GW}$ (dashed red) and $0.75 \mathrm{GW}$ (solid red) are shown; the latter curve is approximately the average power obtained from the time history of the FEL pulse.

Note there is good agreement between the nonlinear theory curve $T_{F E L}$ thsory $\left(0.75 \mathrm{GW}\right.$, red solid) and the data $T_{F E L}$ (red circles), indicating that the theory accurately models the data. We have attempted to ascertain power and electron temperature scalings from these calorimeter data; we also have time-resolved transmission measurements from an on-axis microwave hom. However, due to the scatter in these data, we could not derive any significant scalings with input power (0.7-1.4 GW) or electron temperature $(0.8-1.4$ $\mathrm{keV}$. The scatter in the $T_{F E L}$ data is not well understood, but may be due to variations in undetermined plasma effects (e.g., scattering from edge plasma fluctuations).

We have also analyzed the calorimeter data by integrating the signals from the whole detector. These bulk transmission data were compared with theoretical calculations of transmission (i.e., no refraction). Within the estimated error in fitting and integrating the 2-D temperature profiles on the calorimeter, these data show the same increased transmission relative to linear data as seen in the analysis of the beam core (Fig. 2), and a similar agreement with the theoretical prediction for the nonlinear transmission.

The nonlinear theory also predicts that the opacity increases with the gradient in $N_{1 .}{ }^{20}$ To test this theory, we installed a corrugated-wall, tapered insert in the entrar.e port to the MTX tokamak. By tapering the beam to small toroidal dimension at the output of the taper, diffraction spreads the beam in the toroidal direction. The on-axis beam size is similar in the two cases, but the $N_{\mid l}$ (toroidal) spread is increased from $\Delta N_{\|}=0.1$ without the insert to 0.35 with the insert. The beam shape with the insert was verified by low-power side-lab experiments.

The theoretical predictions for the transmission with the insert

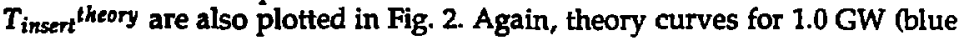


dashed) and 0.75 (blue solid) are presented. Note that the theoretical transmission is reduced for the case with the insert: $T_{\text {insert }}$ theory (blue) $<\$$ $T_{F E L}$ yheory (red). Furthermore, the insert case approaches the linear prediction $T_{\text {lin }}$ theory (the nonlinear opacity can even exceed the linear opacity).

The core transmission with the insert $T_{F E L}$ insert was also measured with the calorimeter and the data are presented in Fig. 2 (blue circles). Again, there is good agreement between the theory (blue lines) and the data (blue circles); these data are also approaching the low-power data values (green diamonds) and the linear theory (green line). We observed the same trends in the total calorimeter data.

During FEL experiments, we have observed energetic electrons with soft $x$-ray, electron cyclotron emission (ECE), and Thomson scattering diagnostics. Decay times of soft $x-$ says and of ECE were typically $\sim 20 \mu$, corresponding to an energy of $-5 \mathrm{keV}$ if the energy loss is purely collisional. The change in the electron distribution function due to the FEL pulse was measured by the Thomson scattering diagnostic located $120^{\circ}$ toroidally away from the FEL port; these data are presented in Fig. 3. The photon count as a function of wavelength for data with (solid circles) and without (open diamonds) the FEL are shown; six shots have been averaged to increase the signal-to-noise ratio. The dashed curve shows the expected signal for a Maxwellian electron distribution at the measured plasma electron temperature (1.5 $\mathrm{keV})$ corrected for relativistic effects; note the FEL data deviate from this cur..... high energies signifying the presence of energetic electrons. The oroit-following code $^{19}$ was used to calculate the effect of the FEL pulse on the electron distribution function, taking into account the relative locations of the FEL port and the Thomson scattering diagnostic and the time of the measurement. The nonrelativistic Thomson scattering spectrum was calculated from the distribution function, and a two-temperature relativistic correction 21 was then applied to the model spectrum for comparison with the data. Figure 3 shows good agreement between the theoretical molel and the data, showing that the influence on the electron distribution function is as expected. A high-energy cutoff at $\sim 25 \mathrm{keV}$ predicted by theory is above the energy range of the Thomson measurements, but preliminary analysis of the $x$-ray data suggests a cutc $f$ in the range of $10 \mathrm{keV}$.

A limited set of experiments was performed to measure backscattered radiation that might provide information on the presence of the most worrisome parametric instabilities. The reflected microwave signals measured by a horn in the MTX port were below the receiver sensitivity, resulting in a ratio to the incident signai on the order of $10^{-5}$. The receiver frequency was scanned $1.3 \mathrm{GHz}$ downward from the FEL frequency with similar ratios, so we have no evidence of tackscattered radiation. Brillouin backscatteririg is expected to be small because of the 20-ns FEL pulse length.

In summary, transmission measurements in the MTX produced several key results: (1) the transmission for the high-power FEL data is significantly greater than that measured in low-power experiments, including the gyrotron experiments discussed elsewhere; (2) the transnission is reduced (the 
absorption is increased) when the gradient in the parallel index of refraction of the beam is increased; (3) the on-axis FEL transmission decreases with increasing electron density; and (4) hot electrons are produced. All of these observations are in approximate quantitative agreement with theoretical models of the nonlinear ECRH interaction.

We would like to acknowledge the help from the Alcator Group at Massachusetts Institute of Technology (MTX was previously the Alcator-C tokamak). The FEL group at LLNL provided valuable technology development. The team from JAERI and the MTX engineers and technicians were an integral part of the overall experiment. This work was performed under the auspices of the U.S. Department of Energy by the Lawrence Livermore National Laboratory under contract W-7405-ENG-48.

Fig. 1. The plasma current, electron density, and central $T_{e}$ from a representative discharge used for FEL absorption experiments. The FEL is injected at 200 ms; the expanded $x$-ray data shows a spatially localized response.

Fig. 2. FEL high-power core transmission data (red circles) are greater than low-power data (green diamonds), demonstrating nonlinear absorption. Theoretical predictions of the FEL transmission without the insert (red: 1 GW, dashed; $0.75 \mathrm{GW}$, solid) compare well with the FEL data. An insert increases the $N_{11}$ gradient of the microwave beam, thereby increasing the experimentally measured absorption (blue circles), in agreement witi: nonlinear theory (blue: $1 \mathrm{GW}$, dashed; $0.75 \mathrm{GW}$, solid). The insert data and theory approach the linear theory (green line).

Fig. 3. Thomson scattering data with (solid circles) and without (open diamonds) the FEL; note the presence of high-energy electrons in the FEL case near 7-8 keV. The Maxwellian distribution (dashed line) and an orbitfollowing code prediction for the FEL case (solid line) have both been corrected for relativistic effects.

1. S.L. Allen and E.T. Scharlemann, to be published in Proc. of the 9th Int. Conf. on High Power Particle Beams, Washington, DC, 1992.

2. T.J. Orzechowski et al., Nucl. Instrum. Methods in Phys. Res. A250, 144 (1986).

3. R.H. Cohen, in Nonlinear Phenomena in :'lasov Plasmas (Editions de Physique, Orsay, France, 1989), p 335.

4. M.E. Fenstermacher et al., in Proc. RF Power in Plasmas, AIP Proc. 244, 20 (1991).

5. B.W. Stallard et ai, Nucl. Fusion 30, 2235 (1990).

6. V.V. Alikaev et al., in Plasma Physics and Cont. Fus. Res. 1986 (Proc. 11th Int. Conf., Kyoto, 1986) vol. 1, (IAEA, Vienna, 1987) p 111.

7. M. Bornatici et al., Nucl. Fus. 23, 1153 (1983).

8. W.M. Nevins, T.D. Rognlien, B.I. Cohen, Phys. Rev. Lett. 59, 60 (1987). 
9. B.I. Cohen et al., Rev. Mord. Phys. 63, 949-990 (1991).

10 S. L. Allen et al., in Plasma Physies and Cont. Fus.Res. 1991 (Proc. 13th Int. Conf., Washington, 1990) vol. 1, (IAEA, Vienna, 1991) p 783.

11. B.W. Stallard et al., in Pruc. 16th Symp. on Fus. Tech. vol. 2, (London, 1990) p 1050.

12. E.T. Scharlemann, Laser Handbook, vol. 6, (Nörth-Holland, New York, 1990) p 291.

13. R.A. Jong et al., Nucl. Instrum. Meth. in Phys. Res. A285, 379 (1989).

14. Y.I. Chen, Nudl. Instrum. Meth. in Phys. Res. A292, 455 (1990).

15. A. Paui et al., Proc. 1991 IEEE Part. Accel. Conf. (San Francisco, 1991) p. 3106.

16. B. Felker and S.W. Ferguson, Proc. of the 14th IEEE-NPSS Fus. Eng. Symp. (San Diego, 1991) p. 132.

17. C.J. Lasnier et al. to be published in the Prac. 1993 IEEE Part. Accel. Conf., Washington, DC.

18. G.R. Smith, M.E. Fenstermacher, and E.B. Hooper, Nucl. Fusion 30, 2505 (1990).

19. T.D. Rognlien, Phys. Fluids 26, 1545 (1983).

20. R.H. Cohen and T.D. Rognlien, Phys. Fluids B 3, 3406 (1991).

21. J. Sheffield, Plasma Scattering of Electromagnetic Radiation (Academic, New York, 1975) p. 200. See also T. Matoba et al., Jpn. J. Appl. Phys. 18, 1127 (1979). 


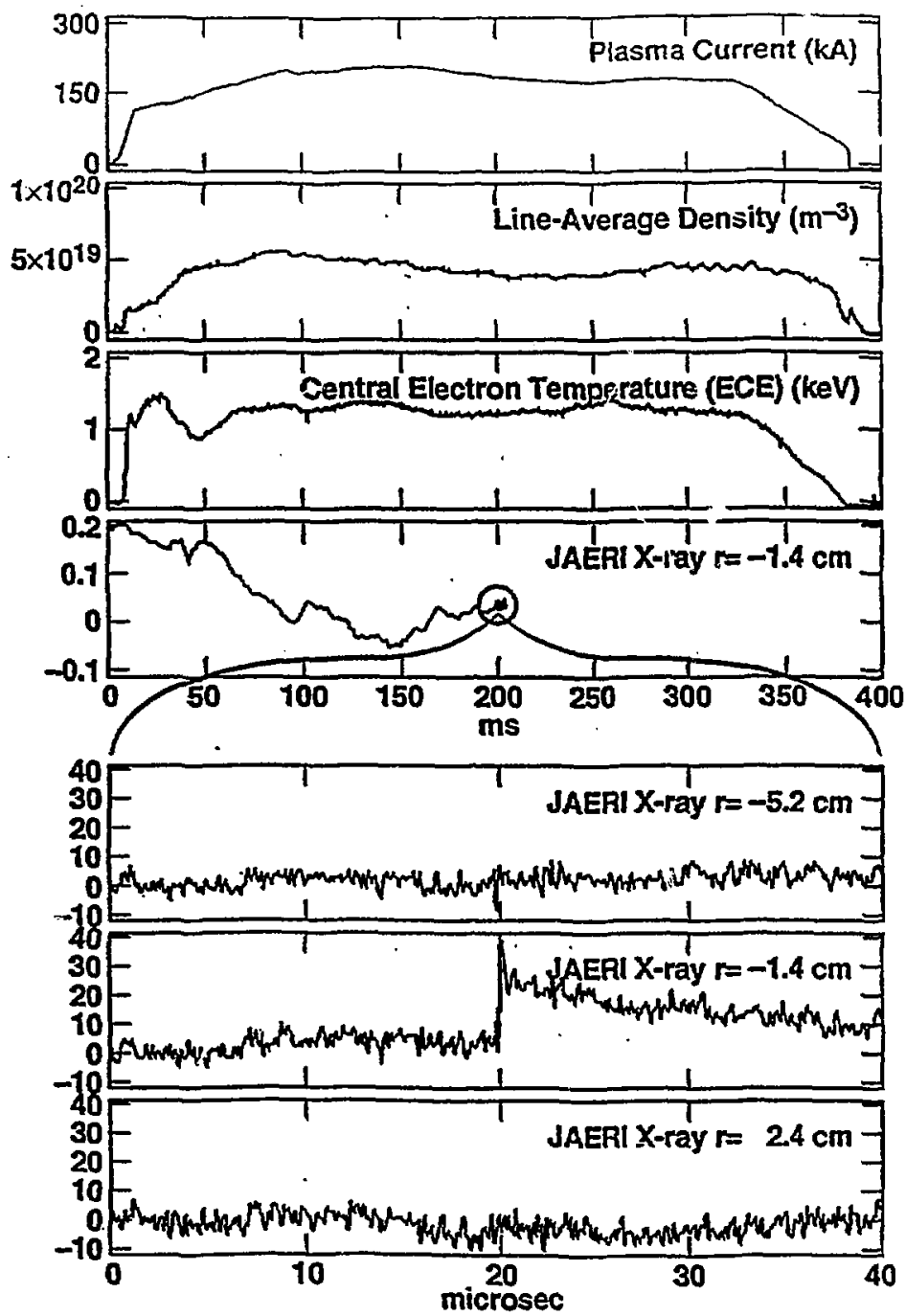


Fig. 2

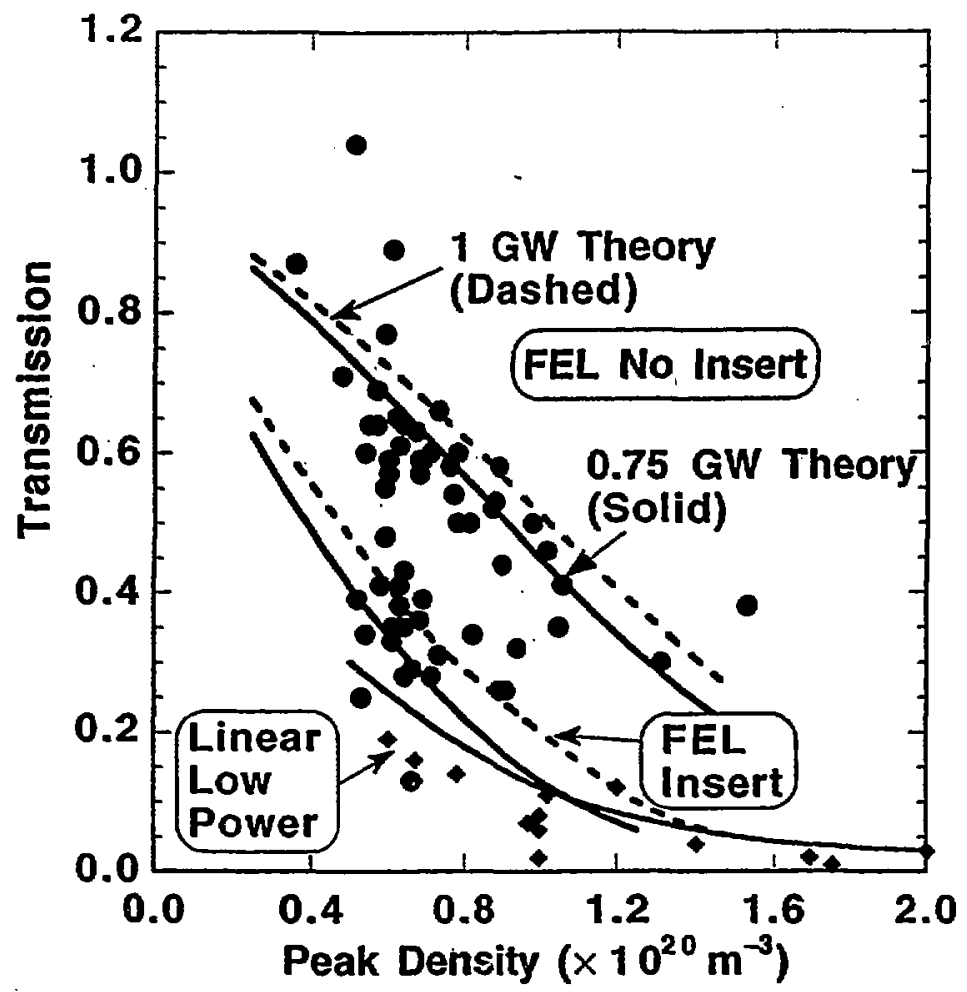


Fig. 3

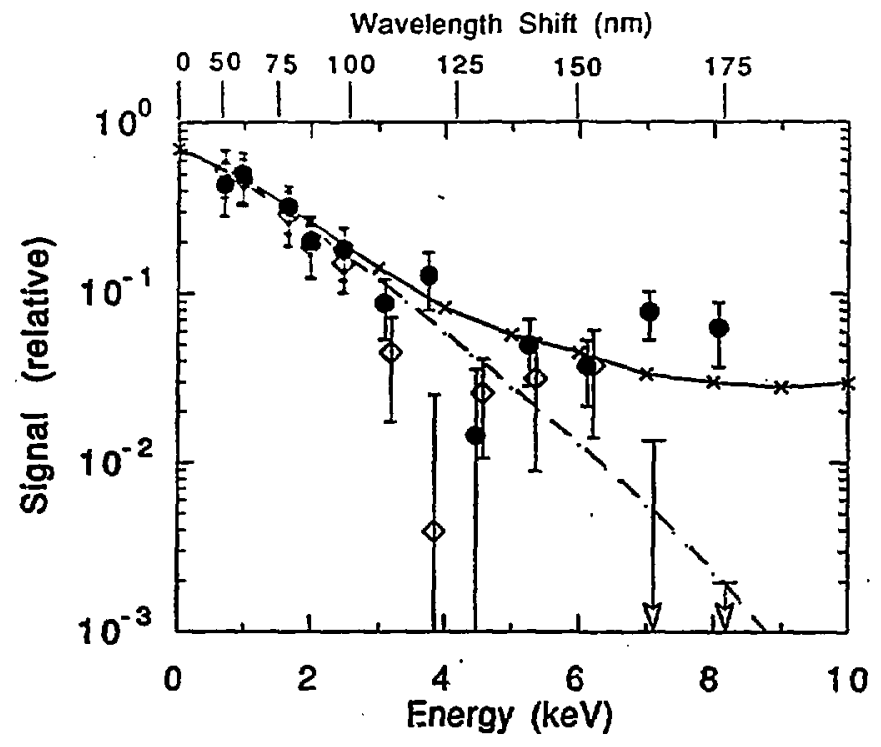




\subsection{LAPPS results (J. H. Foote, T. Oda, K. Odajima, T. Ogawa, T. Ohgo)}

\subsubsection{Introduction.}

The LAPPS (Laser-Aided Particle Probe Spectroscopy) diagnostic system was designed and constructed to measure the electric field of the FEL when injected into the MTX plasma chamber. We discuss in Appendix A.2.4 the LAPPS technique, equipment, and the cascade-fluorescence, allowedtransition measurements that checked out this diagnostic system.

In this section, we present the LAPPS measurements of intense electric fields using forbidden transitions occurring because of the Stark effect. We first discuss the calibration of our FEL electric-field measurements using the motional ( $v \times B$ ) Stark effect. We then present the method of LAPPS measurement and subsequent raw-data reduction for determining the FEL electric field at the center of the MTX toroidal vacuum chamber. Finally, we combine the calibration and FEL results to obtain an estimate of the FEL electric field in vacuum.

This measurement was made in vacuum using the triplet metastable helium-atom state, $2^{3} \mathrm{~S}$, which was produced in the deuterium neutralizer. We had also planned to use the singlet metatstable state, $2^{1} \mathrm{~S}$, which can be produced in the plasma, to measure the FEL electric field in the plasma. But, because of time constraints, the LAPPS equipment was removed from MTX before a concentrated attempt could be made.

\subsection{2 v×B electric-field measurements.}

We calibrated the FEL electric-field measurements by rsing the motional Stark effect and measuring the electric field produced when the $45-\mathrm{keV}$ neutral-helium beam perpendicularly crossed the 6.5-T MTX magnetic field. The calculated electric field is

$$
E(\mathrm{kV} / \mathrm{cm})=\frac{v_{l}(\mathrm{~cm} / \mathrm{s}) \times B(T)}{10^{7}}=\frac{1.48 \times 10^{2} \times 6.5}{10^{7}}=96(\mathrm{kV} / \mathrm{cm}) .
$$

We made this type of measurement during MTX magnetic-field pulses, without plasma or FEL present.

To measure the constant $\mathrm{V} \times \mathrm{B}$ electric field, we observed a spectroscopic line resulting from a forbidden-excitation transition arising from the Stark effect created by the electric field. The energy-level and transition diagram pertaining to this measurement is shown in Fig. 5.3-1. Because of the Stark effect, the forbidden transition $2^{3} S$ to $3^{3} \mathrm{D}$ could occur. We observed the light emitted when the $3^{3} \mathrm{D}$ state decayed to the $2^{3} \mathrm{P}$ level by spontaneous emission.

To make these measurements, we used the same optical viewing-system arrangement as for the cascade-fluorescence measurements described in 
Appendix A.2.4 (see Figs. A.2.4-2 and A.2.4-3 there). All optical components were the same, including the dichroic mirror, the interference filters and the photomultiplier tubes (PMTs).

Examples of the signals obtained are shown in Fig. 5.3-2. Here, the signal of interest is from PMT2 rather than PMT1 (which recorded the cascadefluorescence signal) because the signal is near the wavelength of $587.6 \mathrm{~nm}$ rather than $706.5 \mathrm{~nm}$. The interference filter before PMT2 was centered near $584.9 \mathrm{~nm}$ rather than at $587.6 \mathrm{~nm}$ to allow for the approximately $2.4-\mathrm{nm}$ Doppler shift toward shorter wavelengths of the emitted light because of the forward motion of the excited helium atoms.

In Figs. 5.3-3 and 5.3-4, we show the vxB electric-field data for laserwavelength scans obtained on two different MTX operation days. Both are included to show the consistency of the results after wavelength-scale calibration, even though the wavelength scans were made differently. We plot the measured PMT2 signal (which recorded the $587.6-\mathrm{nm}$ transition) vs the wavelength of the laser beam. As in the similar plot in Fig. A.2.4-5, for the cascade-fluorescence measusements, we calibrated the wavelength scale using the $388.865-\mathrm{nm}$ wavelength in air corresponding to the $2^{3} \mathrm{~S}$ to $3^{3} \mathrm{P}$ transition.

Data points plotted in Fig. 5.3-3 are single-shot values, while those in Fig. 5.3-4 are averages over two or three values. We do not show the statistical error bars for the data points near the peak in Fig. 5.3-4 because it would be too crowded on the plot.

The maximum heights of the fitting curves in these two figures are almost the same - about $1200 \mathrm{mV}$-ns. We use this value in the calibration for the FEL electric-field results discussed later.

At the left in each of these two figures is a tall vertical line that shows the calculated laser-wavelength position for the PMT2 signal arising frorn the $\nabla \times B$ electric field. To obtain the position of this vertical line, we start at $388.865 \mathrm{~nm}$ and first shift $7.958 \mathrm{~nm}$ to shorter wavelengths. This allows for the energy difference between the $2^{3} \mathrm{~S}$ to $3^{3} \mathrm{P}$ and the $2^{3} \mathrm{~S}$ to $3^{3} \mathrm{D}$ transitions. We next adjust another $0.234 \mathrm{~nm}$ toward shorter wavelengths to account for the Doppler shift created because the angle between the laser and neutralhelium beams was not $90^{\circ}$ (see Appendix A.2.4).

The final adjustment is $0.088 \mathrm{~nm}$ toward longer wavelengths because this Zeeman-shift correction appears to be needed to obtain agreement with the data. (We did not realize this until after the LAPPS equipment was removed from MTX, so we could not check for the other Zeeman peaks.) Figure 5.3-5 shows the calculated Stark-Zeeman pattern for 6.5-T MTX magnetic field, 96$\mathrm{kV} / \mathrm{cm}$ electric field, and for our measured polarization of the laser beam.

We assume we were measuring the high $\Delta \lambda / \Delta \lambda_{L}=2$ peak.

There is good agreement in both Figs. 5.3-3 and 5.3-4 between the calculated wavelength of the $\mathrm{V} \times \mathrm{B}$ peak and the measured peak. The 
differences are only $0.01-0.02 \mathrm{~nm}$, to be compared with the shift of $8.10 \mathrm{~nm}$ from the 388.865-nm position.

In adjusting the wavelength-dial readings in Fig. 5.3-4 for the dial calibration, we made an extra adjustment not needed in Fig. 5.3-3 because of the measured $0.07-\mathrm{nm}$ shift in dial reading when we changed the direction of wavelength scan. We estimate that this correction is reasonably accurate but could be off by 0.01-0.02 $\mathrm{nm}$. This uncertainty could explain the difference between our calculated and measured positions of the PMT2 signal peak. This correction was not needed for the data of Fig. 5.3-3 because we continued to move in the same direction, toward shorter wavelengths, as that in the daily dial calibration at $388.865 \mathrm{~nm}$.

If we shift the measured peak in Fig. 5.3-4 to line up. with the calculated position, then the indicated $0.044-\mathrm{nm}$ position would approximately line up with the left-most data point. The possible upturn of the data there could suggest other Zeeman peaks at shorter wavelengths (as shown in Fig. 5.3-5). It is not clear why there is no evidence for a longer-wavelength peak in Fig. 5.34 or for other peaks in Fig. 5.3-3.

The widths of the measured peaks in Figs. 5.3-3 and 5.3-4 are similar to that obtained for the cascade-fluorescence peak in Fig. A.2.4-5. Thus, the widths of these peaks can also be explained by the range of Doppler shifts possible because of the spread of angles between the laser and neutral-helium beams.

\subsubsection{FEL electric-field measurements in vacuum.}

To determine the FEL electric field, we measured the same spectroscopic line as for the $\nabla \times B$ electric-field investigations. The energy-level and transition diagram pertaining to this measurement is shown in Fig. 5.3-6. It is similar to the diagram for the vXB case (Fig. 5.3-1) except for the energy increment of the FEL photon, represented in Fig. 5.3-6 as $\omega_{\mathrm{FEl}}$. The viewing system was again the same.

Examples of the signals obtained are shown in Fig. 5.3-7. The FELmonitor signal was derived from a microwave horn in the JAERI Box near the edge of the FEL beam. It intercepted a small portion of the beam power. We recorded the linear output of the microwave receiver that processed the signal. There is now a signal from PMT1 as well as PMT2, unlike the vxB measurements where any PMT1 signal was small. The origin of the PMT1 signal and much of the PMT2 signal is a large wide-wavelength-band light burst that occurred when the FEL pulse reached the MTX plasma chamber. Any PMT2 signal derived from the $2^{3} S$ to $3^{3} \mathrm{D}$ forbidden transition arising from the FEL electric field is in addition to the FEL light-burst signal.

To extract the part of the PMT2 signal that was due to the FEL electric field, we used the PMTI signal as a monitor. The wavelength bands 
measured by the two PMTs were close enough together to encourage us to assume a constant shot-to-shot ratio of the full PMT1 signal to the part of the PMT2 signal that was due to the FEL light burst.

In applying this procedure, we recorded "background" shots in which the laser beam was fired $200 \mathrm{~ns}$ earlier than noimal, well before the FEL. Then, there could be no Stark-effect signal and all the PMT2 signal should be from the FEL light burst. For these conditions, we adjusted the recorded PMT1 signal to match the PMT2 signal in wave form as closely as possible. We shifted the PMT1 signal in time; adjusted its peak height, and varied its time variation to maximize the match. Because both signals arose from the FEL light burst, it was reasonable to align in time their rises. (The differences in recorded times are caused by differences in cable lengths, electronic time constants, and times of flight.) By determining the factor necessary to match the heights in a background shot, we hoped to measure any additional PMT2 signal in the "data" shots. And, the rational for adjusting the PMT1 time variation is that this detector appeared to have a faster response time than did the PMT2. Figure 5.3-8(a) shows an example of the PMT1 and PMT2 signals for a background shot after these adjustments were made.

The three adjustment parameters averaged over several background shots could then be applied to data shots in which the laser and FEL pulses reached the center of the plasma chamber at the same time. We had arranged the electronics and cabling so that when this synchronization occurred, the laser-monitor and FEL-monitor pulses would also be aligned on the recordeddata plots. Tests during our experimentation confirmed that this was true. Because of time jitter in either or both the laser and FEL pulses, the alignment was not always perfect. Thus, we normalized our result for each shot to the overlay in time of these two signals.

Figure 5.3-8(b) shows a data shot, for which we w-uld expect to observe a signal derived from the forbidden transition arising from the FEL electric field. The average fitting parameters obtained from the background shots are used. A difference in area under the signal peaks is observed; much of this difference is assumed to be the desired forbidden-transition signal.

We began the area integration for this and other shots at a level about $10 \%$ of the peak value along the signal rise. The period of integration [the darkened area in Fig. 5.3-8(b)] was the amount of laser-FEL overlap mentioned above plus $20 \mathrm{~ns}$. The latter increment was based on the $3^{3} \mathrm{D}$ to $2^{3} \mathrm{P}$ decay time and an estimate of the detector-electronics response time.

The residual (darkened) areas for a series of 11 constant-condition data shots with high-power FEL pulses injected into the MTX vacuum chamber are plotted in Fig. 5.3-9 (the circles). We have normalized the residual area of each data shot to the amount of laser-FEL overlap as described above. The $X^{\prime}$ ' represent 6 background shots. For these shots, we have plotted the darkened area obtained [as in Fig. 5.3-8(a)] when we adjusted the PMT1 signal to the PMT2 signal using the three adjustment parameters averaged over all six background shots. The two shots from Fig. 5.3-8 are included in Fig. 5.3-9. 
The averages of the background and data sets of shots are shown at the right in Fig. 5.2-9, with their standard-deviation error bars.

The diamond in Fig. 5.3-9 shows the result ( $415 \pm 362 \mathrm{mV}$-ns) after the background level has been subtracted. Although the scatter of the plotted values is larger than desired, leading to a sizable uncertainty in the result, the diamond is at least a standard deviation away from zero in the positive direction. The peak FEL power estimated from the JAERI calorimeter for this group of shots was in the range $0.99-1.39 \mathrm{GW}$ (averaged over the 140-GHz FEL cycle).

A second set of background and data shots, analyzed in a similar fashion, also gave a positive result, agreeing with the result in Fig. 5.3-9 to well within the error bars. Experimental conditions were best for the set of shots shown here, so we use this set to estimate the FEL electric field.

For these FEL data, we were set at the same wavelength as the data point for the $v \times B$ measurements in Fig. 5.3-4 at $1054 \mathrm{mV}$-ns, the point just to the right of the fitted peak. For the FEL measurements, there was no MTX magnetic field and thus no Zeeman effect. But, we needed to adjust the laser wavelength to allow for the energy of the FEL photon (as depicted in Fig. 5.36). The calculated change necessary is $+0.068 \mathrm{~nm}$, which is different by 0.020 $\mathrm{nm}$ from the adjustment for the 0.088-nm Zeeman peak. This would suggest that, for the FEL measurements, we were actually located on the wavelength scale relative to the peak two data-point positions to the right of where we were on the $\nabla \times B$ peak (again referring to Fig. 5.3-4). That would put us at the position of the data point plotted at $458 \mathrm{mV}$-ns, about 0.4 of the peak value. The LAPPS equipment was removed from MTX before we were able to redo the FEL measurements and check this tentative conclusion.

\subsubsection{Estimation of the FEL electric field in vacaum.}

We now combine the FEL data just summarized with the $\mathrm{v} \times \mathrm{B}$ results discussed earlier to obtain an estimate of the FEL electric field in vacuum at the center of the MTX plasma chamber.

First, we consider the $\mathbf{v} \times B$ electric-field measurement. The number, $I_{v B}$ $\left(\mathrm{cm}^{-3} / \mathrm{shot}\right)$, of laser-induced-fluorescence photons at wavelength $587.6 \mathrm{~nm}$ due to the forbidden absorption is given by

$$
I_{s}=\int C_{s}^{n} E_{w}^{2} I_{L}(t) N_{2 s} d t \text {. }
$$

Here, $C_{s}^{\text {dl }} E_{w}^{2}$ denotes the forbidden absorption coefficient ( $C_{s}^{\text {d }}$ is a constant, and $E_{\mathrm{vB}}$ is the electric-field strength, which is constant in time), $\mathrm{I}_{\mathrm{L}}(t)$ is the laser intensity, and $\mathrm{N}_{2 S}$ is the triplet metastable atom density. We write

$$
I_{t}(t)=G_{t} g(t) \text {, }
$$

where $g(t)$ is the waveform of the laser-monitor signal and $G_{L}$ is a constant. 
It is also true that the time-integral of the total possible signal, $S_{L}^{\text {w }}(t)$, representing the laser-induced-fluorescence photons at wavelength $587.6 \mathrm{~nm}$, should be proportional to $\mathrm{I}_{\mathrm{vB}}$, that is

$$
I_{v t}=S_{v B} \int S_{L}^{v B}(t) d t,
$$

where $S_{\mathrm{VB}}$ is the proportionality constant.

Second, we consider the FEL electric-field measurement. The corresponding photon number is given by

$$
I_{F z L}=\int C_{s}^{F L} E_{F E L}^{2}(t) I_{i}(t) N_{2 s} d s,
$$

where $E_{k z}^{2}(t)$ should be proportional to the FEL-monitor waveform. The other parameters have meanings similar to those in Eq. (5.3.1). As above, the time integral of the 587.6-nm signal can be expressed as

$$
I_{F x}=S_{F s t} \int S_{L}^{F r L}(t) d t \text {. }
$$

We can now write the ratio $I_{\mathrm{VB}} / \mathrm{I}_{\mathrm{FEL}}$ in two ways. Dividing Eq. (5.3.3) by Eq. (5.3.5), we can write

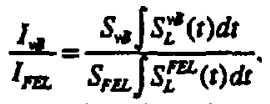

Then, dividing Eq. (5.3.1) by (5.3.4), assuming that $\mathrm{N}_{2 S}$ is the same in both cases, and canceling the constant $G_{L}$, we can write

$$
\frac{I_{v 1}}{I_{r x}}=\frac{\int C_{s}^{N} E_{w d}^{2} g(t) d t}{\int C_{s}^{F z} E_{F E L}^{2}(t) g(t) d t} \text {. }
$$

We now insert numerical values. The constants $S_{\mathrm{vB}}$ and $\mathrm{S}_{\mathrm{FEL}}$ are inversely proporional to the sensitivity of the photomultiplier tube used. Because different high voltages were used on PMT2 for the two sets of measurements, the gains were different by a factor of nine. Thus, we set $S_{F E L}$ $=9 S_{\mathrm{vB}}$.

We also set $C_{s}^{\text {rz }}=\frac{C_{s}^{*}}{2}$. The two $C_{s}$ constants are equal when all possible transition channels are available. But, one of the two possible channels for the FEL measurement was not available because of the laser-wavelength setting. This second channel corresponds in Fig. 5.3-6 to the horizontal dashed line being a distance of $\omega_{\mathrm{FEL}}$ above the $3^{3} \mathrm{D}$ level. Thus, $C_{s}^{F E}$ is only half its maximum possible value.

When Eqs. (5.3.6) and (5.3.7) are combined, and we insert the numerical values just discussed, we obtain

Rearranging Eq. (5.3.8), we can write

$$
\frac{\int S_{L}^{n z}(t) d t}{9 \int S_{L}^{F 2}(t) d t}=\frac{2 \int E_{w d s}^{2} g(t) d t}{\int E_{F E L}^{2}(t) g(t) d t} .
$$

$$
\int E_{r \varepsilon L}^{2}(t) g(t) d t=\int E_{v E}^{2} g(t) d t \times 9 \times 2 \times \frac{\int S_{L}^{F L}(t) d t}{\int S_{L}^{\nu d}(t) d t} .
$$


By moving the electric-field parameters outside the integrals and using time-average values ( $E_{r b}^{2}$ is a constant), we can write

$$
\left\langle E_{F z L}^{2}\right\rangle \int g(t) d t=E_{v B}^{2} \int g(t) d t \times 9 \times 2 \times \frac{\int S_{L}^{F E z}(t) d t}{\int S_{L}^{v d}(t) d t} .
$$

The laser-monitor signals were fairly constant for all the $\mathbf{v} \times \mathbf{B}$ and FEL data we are using here, so the $\int g(t) d t$ factors on each side of the equation cancel. Equation (5.3.10) then becomes -

$$
\left\langle E_{r z z}^{2}\right\rangle=E_{v s}^{2} \times 9 \times 2 \times \frac{\int S_{L}^{F t z}(t) d t}{\int S_{L}^{w}(t) d t} .
$$

For the numerical values of the integrals, we use

$$
\int S_{i}^{f x t}(t) d t=415 \pm 362 \mathrm{mV} \cdot \mathrm{ns}
$$

and

$$
\int S_{L}^{*}(t) d t=1200 \mathrm{mV}-\mathrm{ns} \times 5 \text {. }
$$

The first number (415 $\pm 362 \mathrm{mV}$-ns) comes from the anaiysis of Shots 16090 16108 in Fig. 5.3-9. The second number ( $1200 \mathrm{mV}-\mathrm{ns})$ comes from the peaks in Figs. 5.3-3 and 5.3-4, as discussed earlier. The factor of 5 multiplying the 1200 $m$ m -ns value is calculated from Fig. 5.3-5 showing the Stark-Zeeman pattern for our $\nabla \times B$ measurements. This factor represents the inverse of the ratio of the +0.088-nm peak, which we assume we measured, to the sum of all the peaks (i.e., the total possible signal). We have added together here the contributions from both the $S$ and $P$ polarizations because our optical components were insensitive to the direction of polarization.

Putting these numbers into Eq. (5.3-11), and dropping the standarddeviation uncertainty in Eq. (5.3.12) until later, we have

$$
\left\langle E_{F x}^{2}\right\rangle=E_{w s}^{2} \times 9 \times 2 \times \frac{415}{1200 \times 5}=1.245 E_{v B}^{2} \text {. }
$$

To obtain the square of the peak electric field, we multiply by a factor of $\approx 1.4$ [see Fig. 5.3-10(a)] and also by another factor of 2 [see Fig. 5.3-10(b)]. These are adjustments needed to obtain the peak in time of the electric field. Applying these two time adjustments to Eq. (5.3.14), we obtain

$$
\begin{aligned}
& \hat{E}_{F Z}^{2}=1.245 E_{v i d}^{2} \times 1.4 \times 2=3.49 E_{v i}^{2} \\
\text { or } & \hat{E}_{F Z}=1.87 E_{v s} .
\end{aligned}
$$

Any adjustment needed because our measurement is an average over the electric-field peak in space as well as time is expected to be not more than $10 \%$. The full dimensions of the laser at the plasma are approximately $1.5 \mathrm{~cm}$ wide and $1.7 \mathrm{~cm}$ high. When these full widths are compared with the estimated horizontal and vertical 1/e halfwidths of the electric field of the FEL beam (see below), and good alignment of the two beams is assumed, we find that we are measuring an average spatial electric field near the peak value. Any adjustment here is small compared with the other uncertainties in the measurement, so no additional adjustment has been made. 
Using $E_{v d}=96 \mathrm{kV} / \mathrm{cm}$ (for 45-keV helium energy and $6.5 \mathrm{~T}$ magnetic field) in Eq, (5.3.15), we obtain

$$
\hat{E}_{F E}=180 \mathrm{kV} / \mathrm{cm} \text {. }
$$

To relate the peak electric field to the FEL rms power, we use the equation

where

$$
P_{\text {mu }}=\frac{\hat{E}_{f a}^{2}}{2 \eta_{0}} \times \frac{\pi W_{H} W_{V}}{2},
$$

$W_{H}$ and $W_{V}$ are the horizontal and vertical 1/e halfwidths of the FELbeam electric field at the plasma center in cm (estimated at 3.6 and 2.6 $\mathrm{cm}$, respectively, for the tapered entrance waveguide),

$\eta_{0}=377$,

$\hat{E}_{\mathrm{Fr}}$ is in $\mathrm{V} / \mathrm{cm}$,

and $P_{r m s}$ is the FEL, root-mean-square power in watts.

Using this equation, we obtain $0.63 \pm 0.54 \mathrm{GW}$ for the rms power corresponding to a peak electric field of $180 \mathrm{kV} / \mathrm{cm}$. (We have now included the standard-deviation uncertainty from Eq. (5.3.12). This value is somewhat lower but comparable to the values of $0.99-1.39 \mathrm{GW}$ for the average maximum measured rms power of our analyzed shots \#16090-16108 (Fig. 5.39) estimated from the JAERI-calorimeter power measurements. (The possible correction discussed in the last paragraph of Sec. 5.3.3 would increase the value of peak power measured by LAPPS, adjusting the result to be in even better agreement with the calorimeter measurements.)

These results show that the LAPPS equipment functioned well and made the type of measurement for which it was designed. Although our measurements with LAPPS on MTX were severely restricted because of the limited experimental time available to obtain data, LAPPS has proven to be an effective diagnostic for determining high electric fields (including those of an FEL). 


\section{FIGURE CAPTIONS}

Fig. 5.3-1. The energy-level and transition diagram pertaining to the LAPPS $\mathbf{v} \times \mathbf{B}$ electric-field measurements.

Fig. 5.3-2. Examples of the data signals obtained during the $\mathbf{v} \times \mathbf{B}$ electric-field measurements.

Fig. 5.3-3. The $v \times B$ electric-field data for ore wavelength scan. The calculated position of the $587.6-\mathrm{nm}$ peak is at the left tall vertical line.

Fig. 5.3-4. Further $v \times B$ electric-field data, from a second and more extensive wavelength scan. Again, the calculated position of the $587.6-\mathrm{nm}$ peak is at the left tall vertical line.

Fig. 5.3-5. Relative intensity of the components of the Stark-Zeeman pattern for $B=6.5 \mathrm{~T}$ and $\mathrm{E}=96 \mathrm{kV} / \mathrm{cm}$, where $\Delta \lambda_{L}=0.044 \mathrm{~nm}$. The measured ratio of $4.8 / 1$ for the vertical/horizontal intensity components of laser polarization at the MTX vacuum chamber is assumed.

Fig. 5.3-6. The energy-level and iransition diagram pertaining to the LAPPS FEL elerth ield measurements.

Fig. 5.3-7. Examples of the data signals obtained during the FEL electric-field measurements.

Fig. 5.3-8. (a) An example of adjusting the PMT1 signal to fit the PMT2 signal for a background shot. (b) An example of a data shot, where we have used fitting parameters averaged over several background shots to adjust PNiT1.

Fig. 5.3-9. Reduced data for a series of FEL electric-field measurements in vacuum. The averages of the 6 background and 11 data sets of shots are shown at the right, with their standard-deviation error bars. The diamond shows the final averaged result after the background level has been subtracted.

Fig. 5.3-10. (a) Time average of the FoL power over the time interval of the LAPPS measurement (using the FEL-monitor signal), and the estimated adjustment to the peak of the pulse. (b) The 140-GHz electric-field sine waveform and its square (proportional to the power). The rms time average and peak of $E^{2}$ are shown, related by a factor of two. 
Fig. 5.3-1

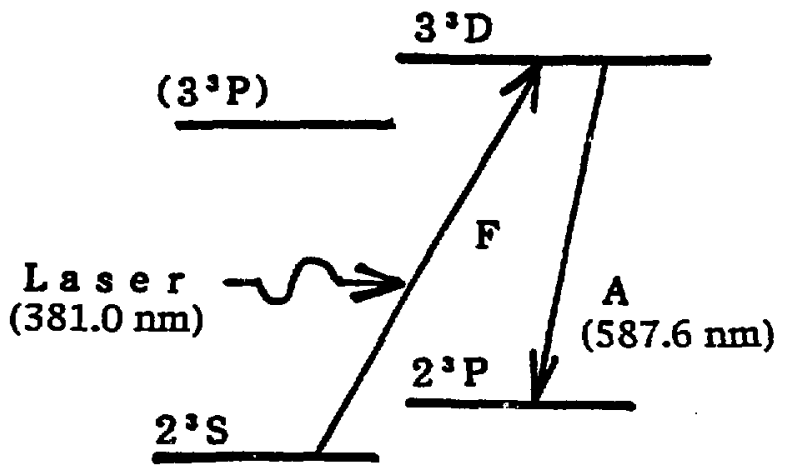



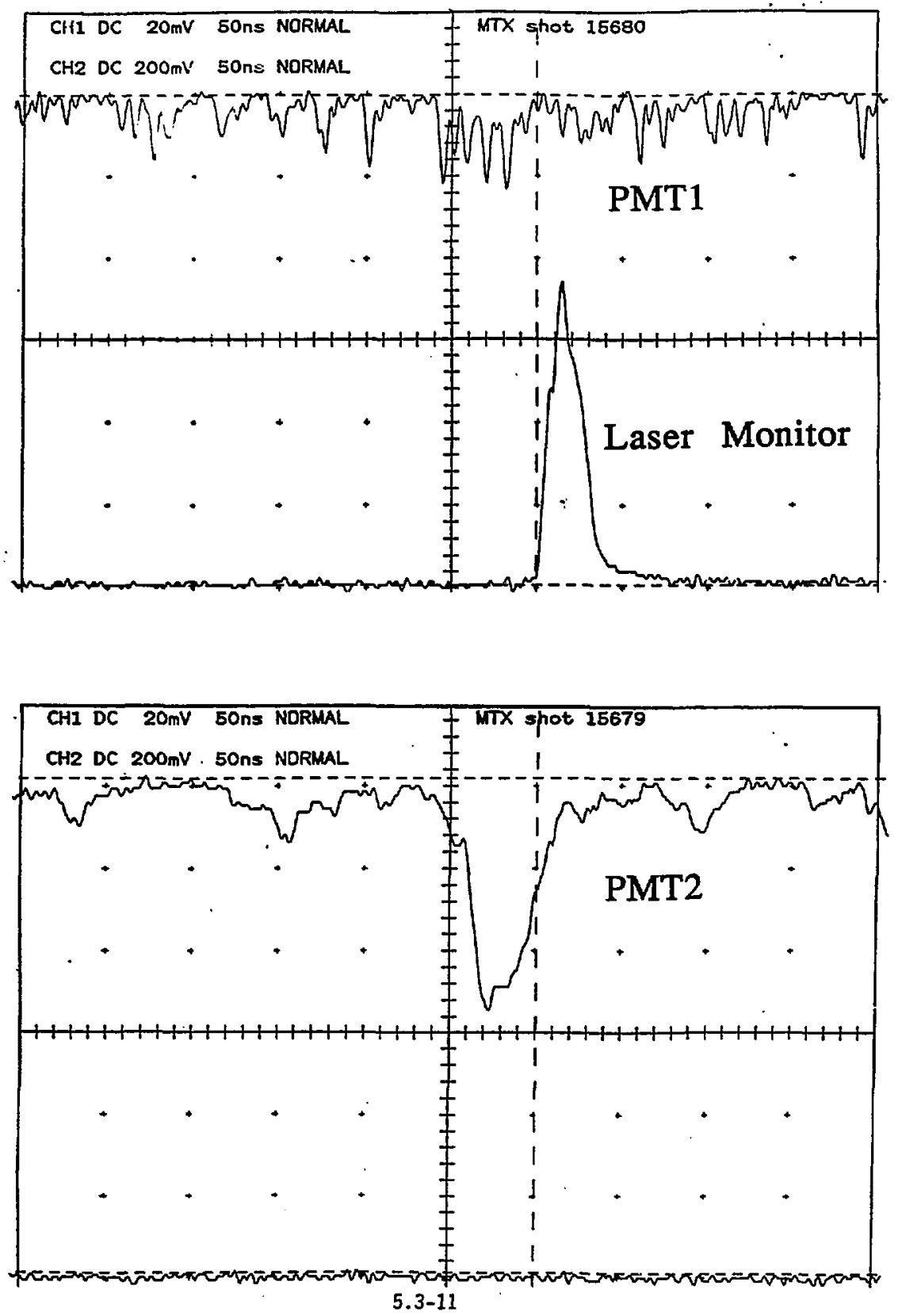


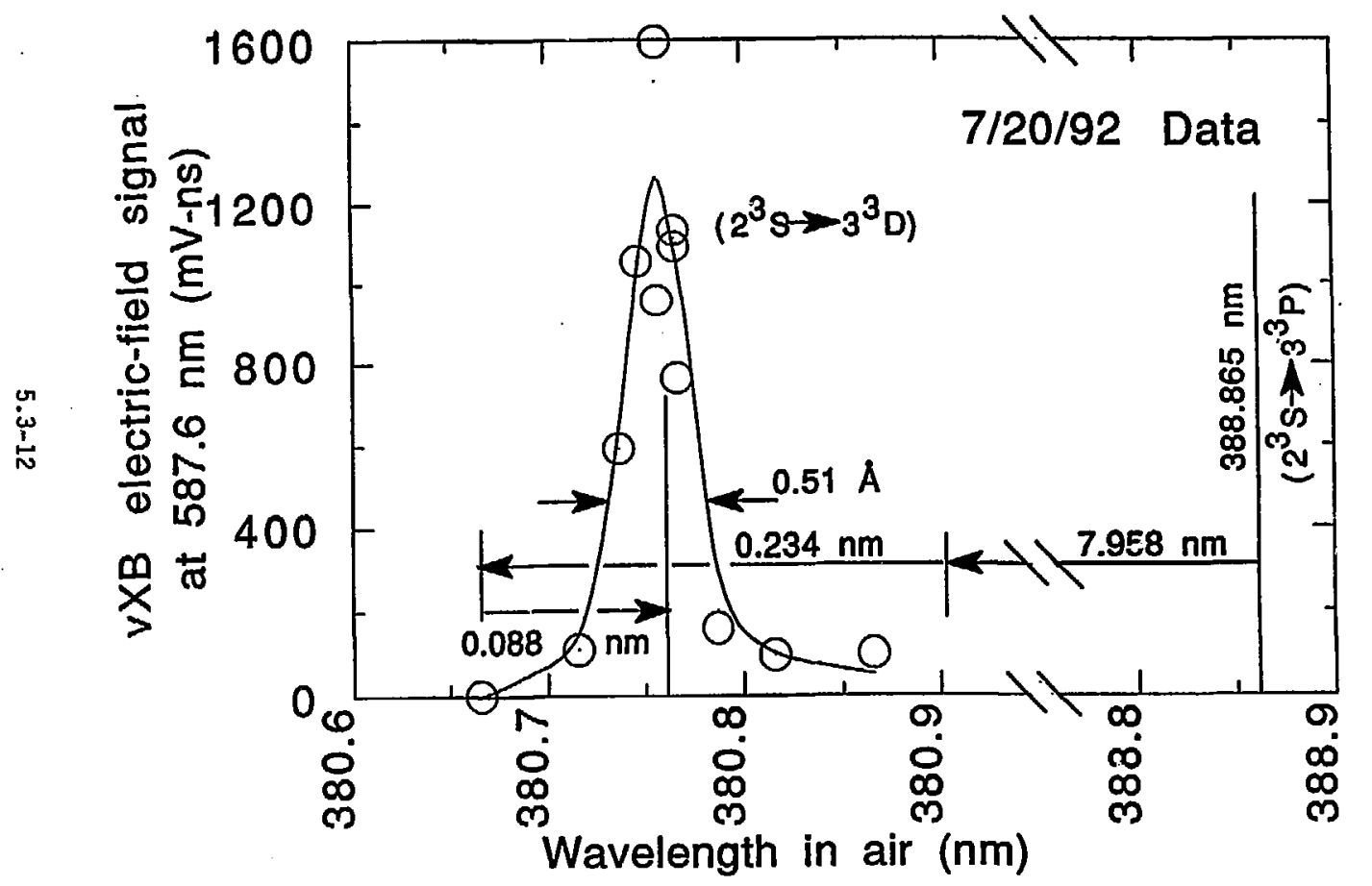

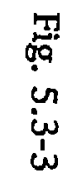


vXB electric-field signal at $587.6 \mathrm{~nm}$ (mV-ns)

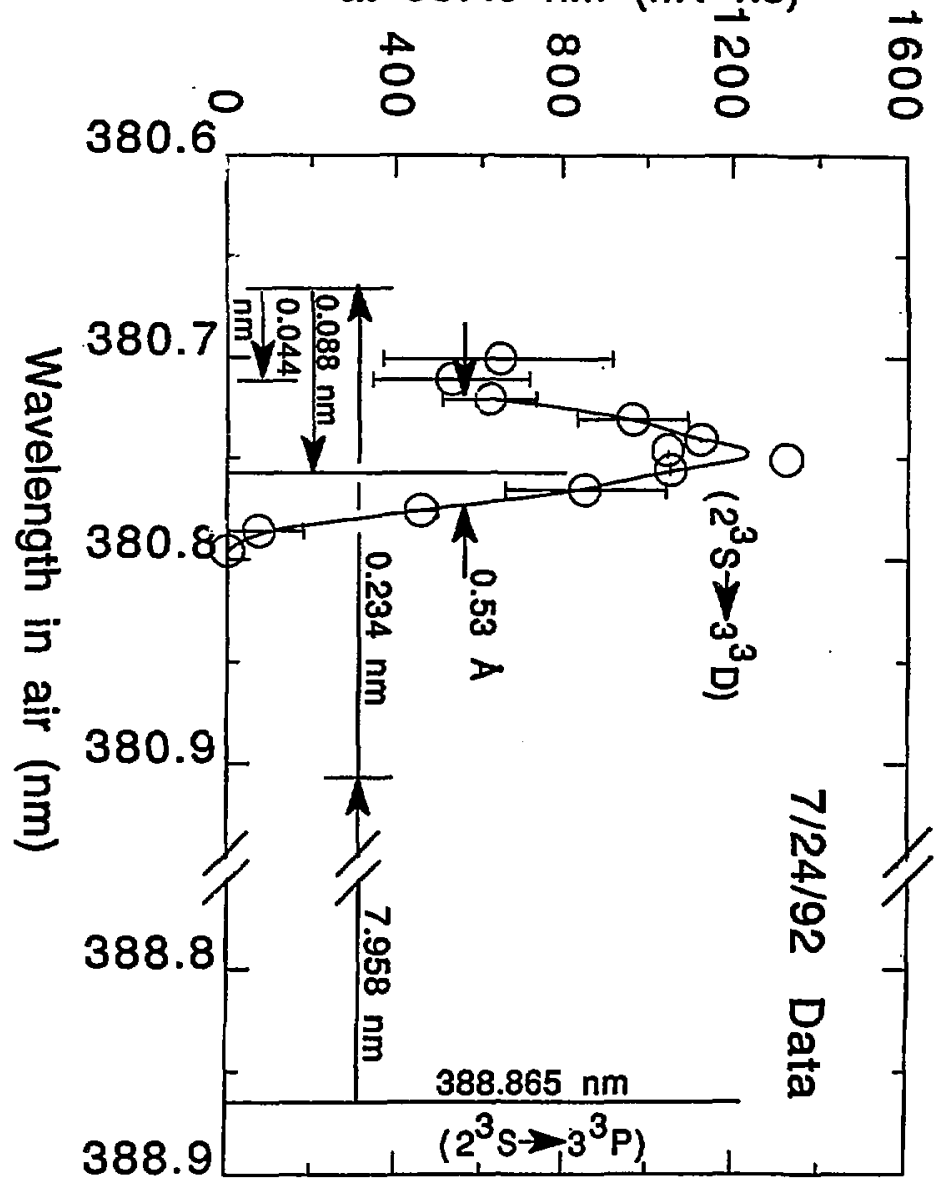


Fig. 5.3-5

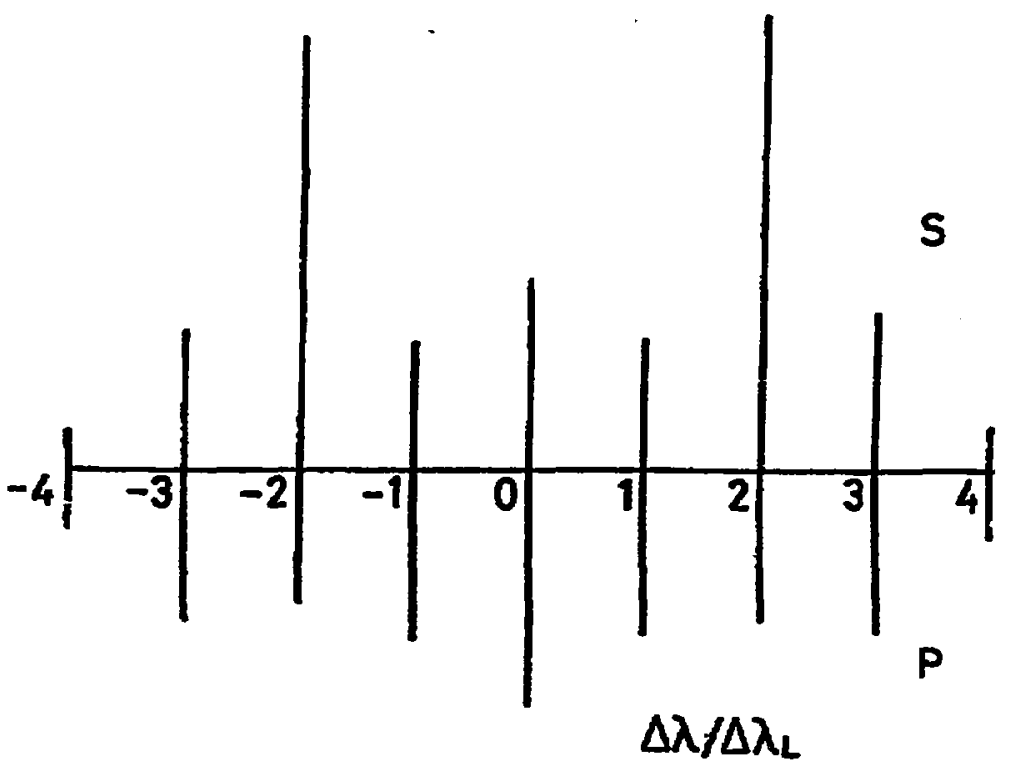


Fig. 5.3-6

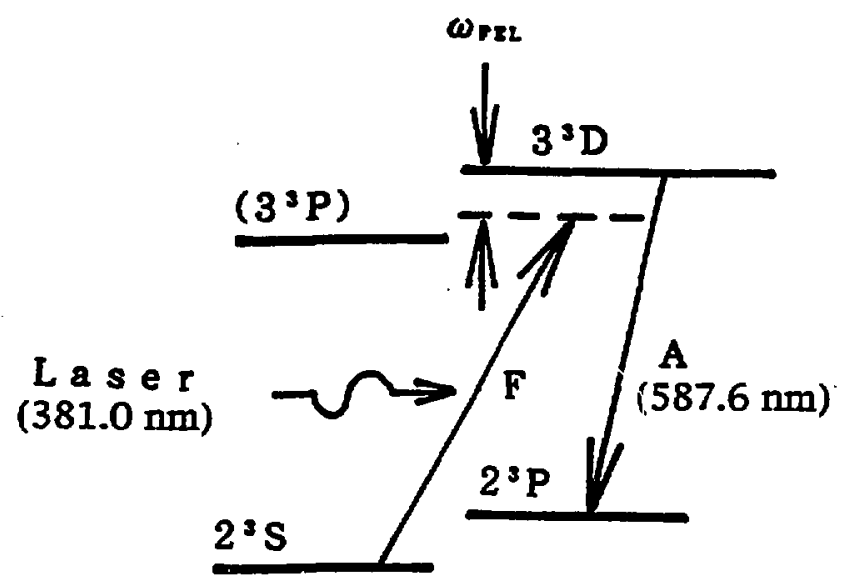


$9 \tau-\varepsilon \cdot g$
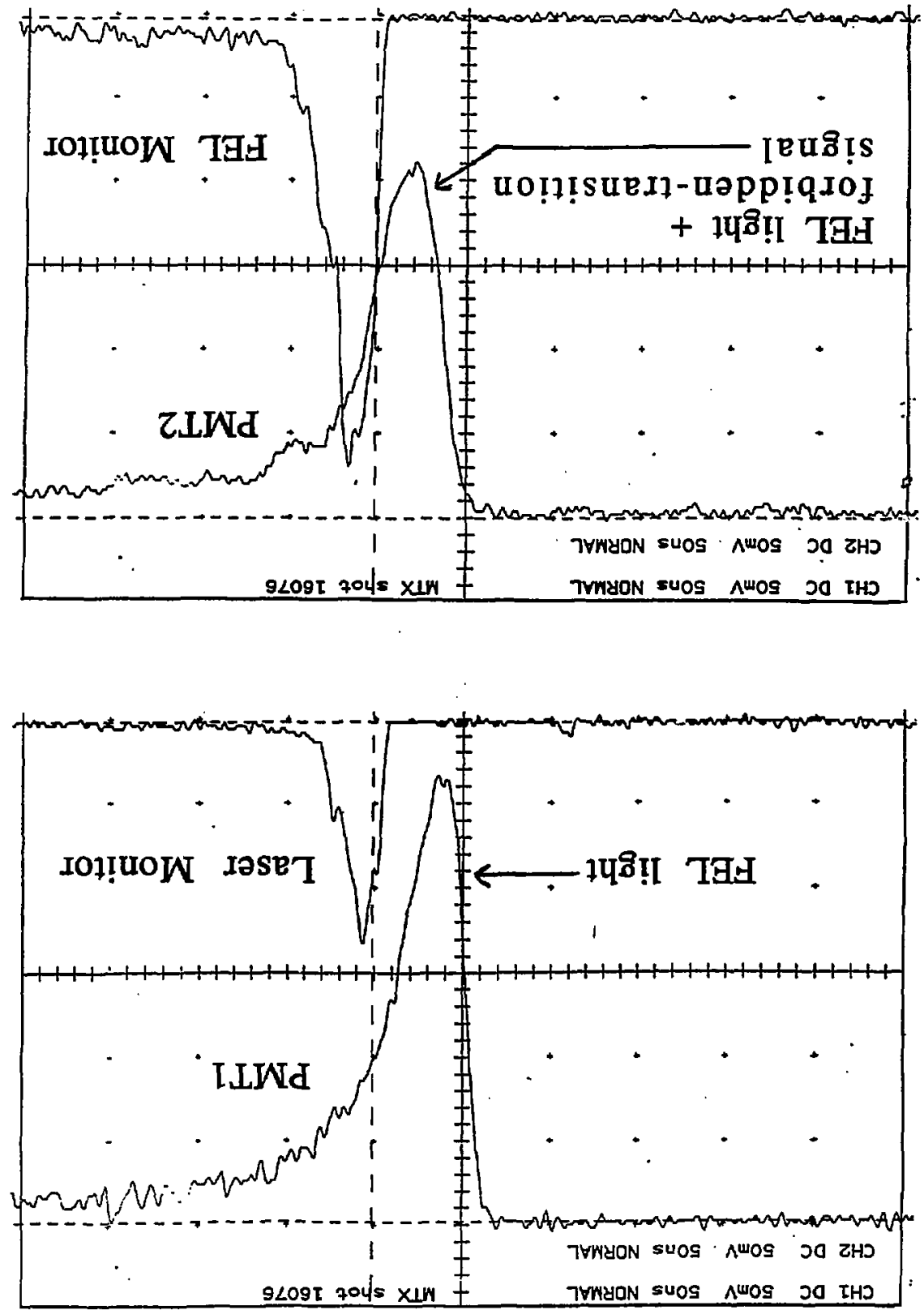
Fig. 5.3-8
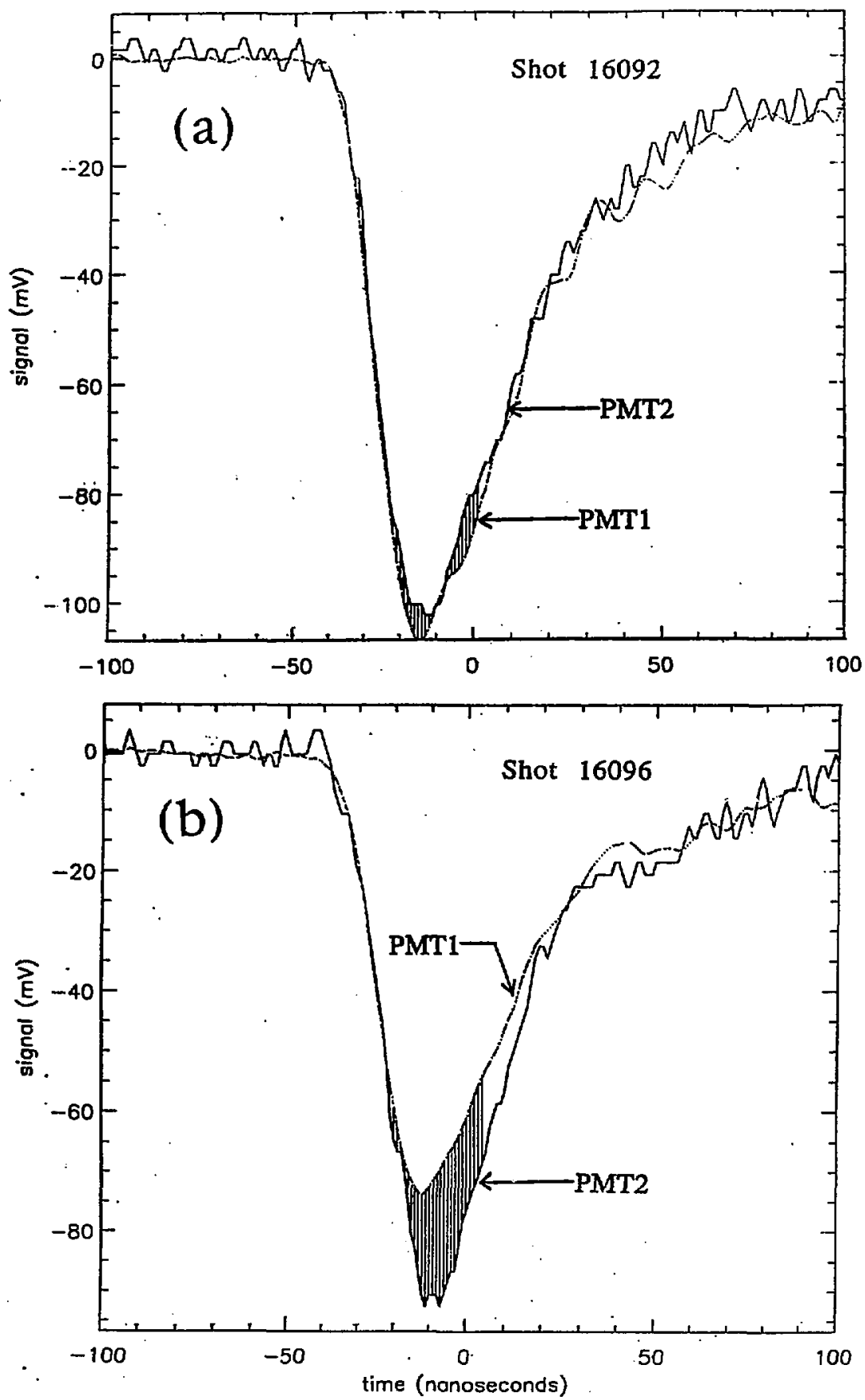
Fig. 5.3-9

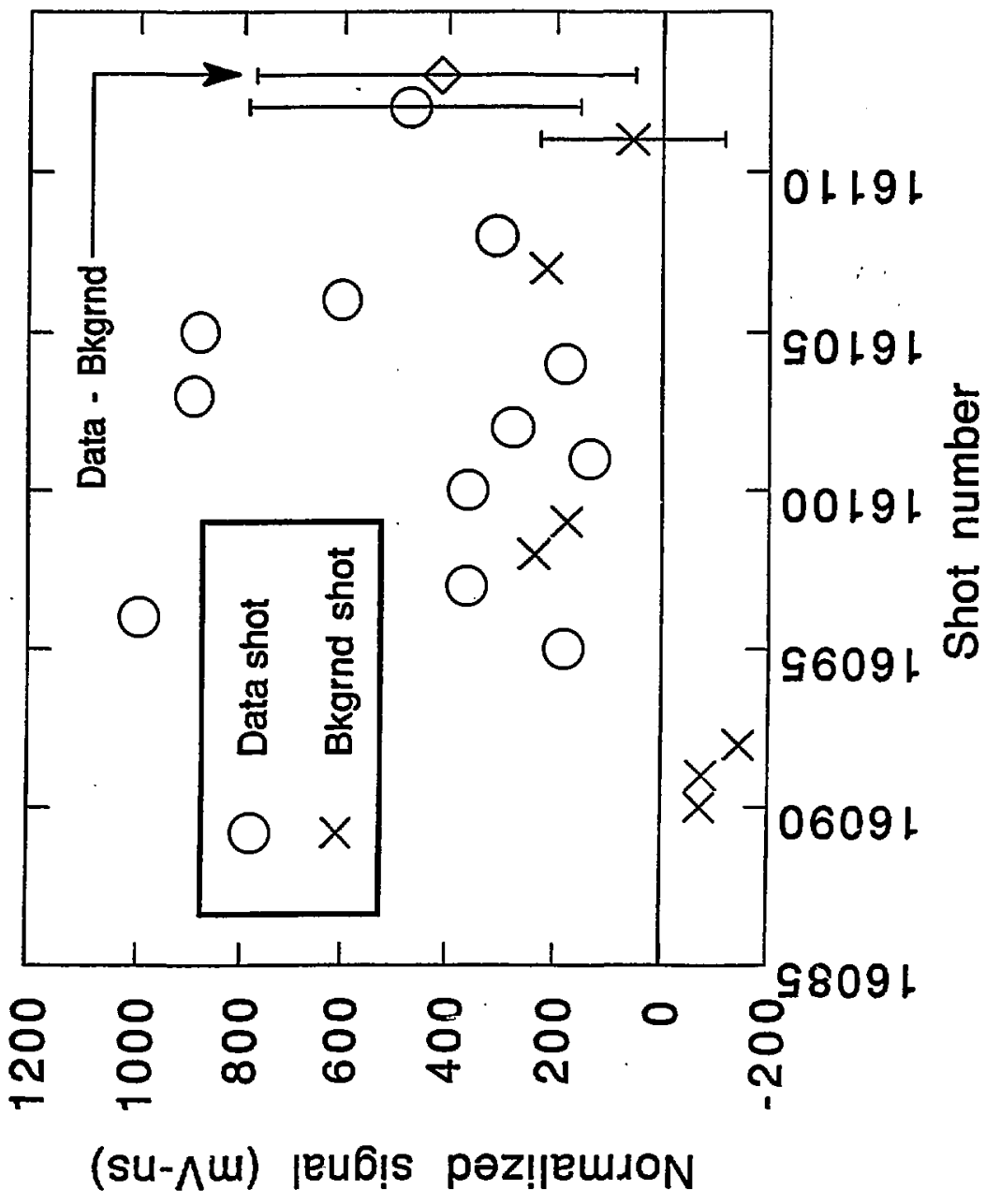


(a)

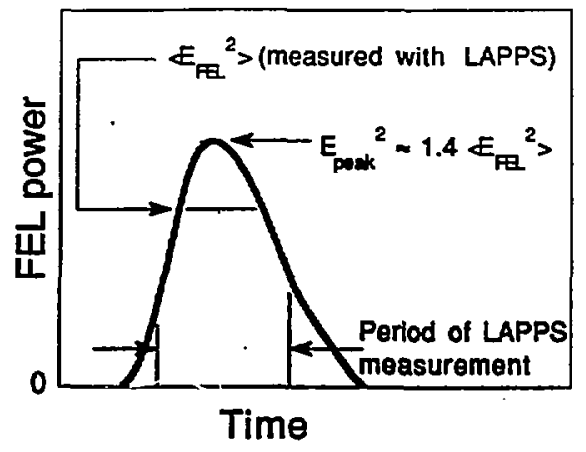

(b)
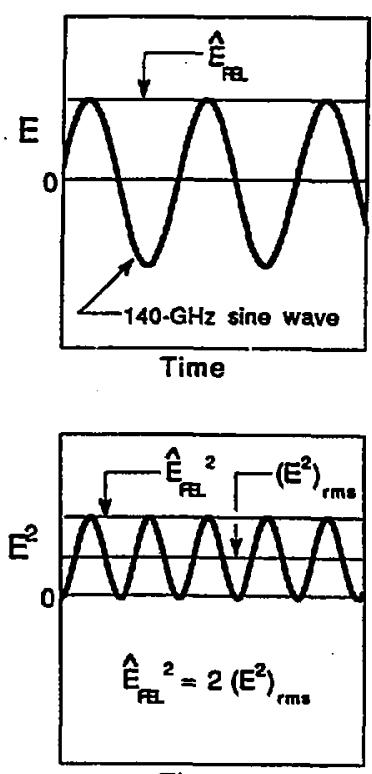

Time 


\subsection{Thomson scattering results (J. H. Foote)}

\subsubsection{Introduction}

A high-energy group of plasma electrons heated by the FEL is expected to show up as a large-wavelength-shift tail in the Thomson scattering data. (See Appendix A.3.1 for a description of this diagnostic, referred to as TSS.) We present here a set of TSS data obtained during constant MTX plasma and FEL sonditions. Plasma electron temperature and peak density were approximately $1.5 \mathrm{keV}$ and $0.65 \times 10^{14} \mathrm{~cm}^{-3}$ (at $5.0 \mathrm{~T}$ magnetic field). The FEL peak power and total energy of each pulse were about $1.0 \mathrm{GW}$ and $20 \mathrm{~J}$, respectively (at $140 \mathrm{GHz}$ ). The tapered insert at the FEL entrance was in place.

The plasma location at which the TSS measurements were made was on the plasma centerline one-third the total distance around the torus from where the FEL beam crossed the plasma and heated it. The distance between these locations along the plasma centerline was $134 \mathrm{~cm}$. We timed the firing of the TSS laser so that the peak of the 40-ns (FWHM) light burst would reach the plasma about $85 \mathrm{~ns}$ after the FEL power peak reached the plasma. This delay was to allow time for the electrons heated by the FEL to drift around to the TSS measurement region.

\subsection{Data with and without FEL heating}

Figure 5.4-1(a) shows standard MTX TSS plasma data for a single shot (the open squares toward the right, with $T_{e}=1.55 \mathrm{keV}$ ) and also large-wavelengthshift data for an average of six plasma shots with injected FEL beam (the filled circles toward the left). This plot is similar to our customary MTX TSS plot (see Fig. A.3.1-2 in Appendix A.3.1) except that the vertical scale is semilog rather than linear and the wavelength-shift region has been extended to shorter wavelength.

We acquired the two sets of plasma data at two rotation settings of the spectrometer diffraction grating, to extend the measured wavelength span. Plasma conditions were similar for all the data. For better comparison, the two sets overlap along the wavelength scale and are plotted using the same scaling factor. The solid curve is a least-squares fit to the open squares by a Gaussian with a relativistic correction. We have extended this solid fitting line to the region of larger wavelength shift, for easier comparison with the FEL data.

Figure 5.4-1(b) shows a plot similar to Fig. 5.4-1(a), with the same set of open-square data, but now the open diamonds are an average of six plasma shots acquired with experimental arrangements in which any FEL heating could not have been observed by the TSS. For these no-FEL shots, we either 
kept closed the valve in the FEL beam line just before MTX or we fired the TSS laser before the FEL beam reached the plasma. The last two points at the left, indicated by the arrows, are no-FEL data points that lie below the plot. Their top horizontal bars show the limit of the one-standard-deviation error bars.

We checked to see if the TSS viewing system was detecting any FELderived light other than actual Thomson-scattered light. This was accomplished by leaving closed for several shots the valve that permitted the TSS laser pulse to enter the plasma region. No other changes were made, and the plasma and FEL were at normal levels. The resulting data suggest that our customary method of subtracting plasma and laser background light took into account any small amount of additional FEL background light.

Error bars shown in these plots include estimated statistical standard deviations. They are obtained from the counting rates of the various types of signals in the different wavelength channels and from statistical averaging procedures when six shots are combined. In addition, for the largewavelength-shift data (the filled circles and open diamonds), we have included an estimate of the systematic errors.

In Fig. 5.4-1(c), we combine the results of the above two plots in a way to emphasize the difference between the sets of data in which we would and would not expect to see the effects of FEL electron heating. Each data value of the six-shot averages of Figs. 5.4-1(a) and 5.4-1(b) has had the calculated fit value (the solid curve) at the same wavelength shift subtracted from it and the result divided by that fit value, to show the relative displacement from the predicted fit (which represents no FEL heating). Each standard-deviation error has been divided by the corresponding fit value. The filled circles are from the Fig. 5.4-1(a) FEL data averages and the open diamonds are from the Fig. 5.4-1(b) no-FEL averages. The diamonds average out to approximately zero when normalizing their displacements to their standard deviations, while the circles appear to show a statistically significant positive FEL heating effect.

\subsection{Comparison with theory}

We replot the data of Fig. 5.4-1(a) and (b) in Fig. 5.4-2, using a linear electron-energy scale to emphasize the longer wavelength-shift region. We also show the nonlinear wavelength-shift scale at the top of the plot, now increasing in magnitude to the right. The solid fitting curve of Fig. 5.4-1 (shown as dashed here) would be a straight line in Fig. 5.4-2 if it were not for the relativistic correction factor (see below). The data symbols have the same meaning as in the earlier figure. For clarity, we have omitted in Fig. 5.4-2 the open-square data points of Fig. 5.4-1 used to obtain the fitting curve. The last 
two points at the lower right, indicated by the arrows, are no-FEL diamond data points that lie below the plot. As in Fig. 5.4-1(b), their top horizontal bars show the limits of the one-standard-deviation error bars.

The X's in Fig. 5.4-2, connected by straight-line segments, are the results of computer-modeling calculations of FEL electron heating using an orbitfollowing code (see Sec. 3.2.1).1 The input parameters correspond to the MTX plasma and FEL experimental conditions given in Sec. 5.4.1 In the calculation, electrons are heated by the FEL at the toroidal position at which the FEL passes through the plasma. The electron energy distribution is then calculated at a later time corresponding to the TSS sampling time and at a toroidal axial position corresponding to the TSS measurement location. From this distribution, the Thomson-scattering spectrum can be calculated.

To compare the calculated values (the $X ' s$ ) with the experimental data, a "blue shifr" relativistic correction has been applied to the results of the modeling. This correction arises principally from the forward bias in the radiation pattern of a relaivistic electron, so that approaching electrons scatter more light toward the observer than those that are receding. To apply the correction to the calculated non-relativistic Thomson-scattering spectrum, a function that is the sum of two Maxwellians was fitted to the calculated results, with $T_{e}=1.55 \mathrm{keV}$ corresponding to the core plasma and $T_{e}=10.4 \mathrm{keV}$ corresponding to the FEL-heated portion of the plasma. (The choice of 10.4 $\mathrm{keV}$ is explained below.) The relativistic correction appropriate to the FELheated componeni could then be applied to it separately. The effect of the correction was to increase the calculated values at the highest energy for which we have data by as much as $56 \%$. Although the relativistic correction we used was accurate to second order ${ }^{2}$ in $v / c$, the difference compared with the correction using terms only up to the first power ${ }^{3}$ of $v / c$ was only a few percent.

Figure 5.4-3 shows the modeling results before the relativistic correction (the triangles), the two Maxwellians used in the fitting of these results (the dashed straight lines) and their sum (the solid line). The corrected mo iing data are shown by the X's (which are also the X's of Fig. 5.4-2). The range of the energy scale in Fig. 5.4-3 is greater than in Fig. 5.4-2. The electron temperature $\left(T_{e}=10.4 \mathrm{keV}\right)$ used for the higher-energy Maxwellian part of the fit to the modeling results was chosen for consistency. The low-energy part had been determined from a standard MTX-TSS shot taken near the time of the FEL data and under similar plasma conditions. When the low-energy part is subtracted from the uncorrected distribution (the triangles), the computed mean energy of the remaining part (representing the FEL-heated electrons) is $5.2 \mathrm{keV}$. Because the modeling results are for a 1-D distribution, to be able to compare with the TSS measurernents, we can write

$$
\bar{W}=\frac{1}{2} m \bar{v}^{2}=\frac{1}{2} k T \text {. }
$$


Including $\mathrm{k}$ in $\mathrm{T}_{\mathrm{e}}$, we obtain the $\mathrm{T}_{\mathrm{e}}$ value usec above:

$$
T_{.}=2 \bar{W}=2 \times 5.2 \mathrm{keV}=10.4 \mathrm{keV} \text {. }
$$

The relativistic correction in the energy region where we have data is only slightly sensitive to the values of $T_{e}$ and zero-energy intercept chosen for the higher-energy Maxwellian fit to the modeling results (ove: the range 10 to 40 $\mathrm{keV})$.

In summary, the relativistically corrected calculated results in Fig. 5.4-2 show a variation with energy similar to that of the TSS experimental results and are of the same magnitude. This variation at high energy is quite different from the dashed curve, which was experimentally obtained at lower energy for no FEL heatin and extended to th higher-energy regime. Thus, it appears that the TSS measurements show Fï electron heating and support the results of the modeling calculations. 


\section{FIGURE CAPTIONS}

\section{Fig. 5.4-1.}

(a) Standard MTX TSS plasma data (the open squares, Shot 16316, with $T_{e}=1.55 \pm 0.29 \mathrm{keV}$ ) and also large-wavelength-shift data for an average of six plasma shots with injected FEL beam (the filled circles toward the left). The solid curve is a least-squares fit to the open squares by a Gaussian with a relativistic correction.

(b) Similar to (a), with the same set of open squares, but now the diamonds are an average of six plasma shots acquired with experimental arrangements in which FEL electron heating could not have been observed by the TSS.

(c) Data compared with and normalized to the Gaussian fit. The filled circles and open diamonds correspond to the data represented by the same symbols in (a) and (b) above.

Fig. 5.4-2. The data of Fig. 5.4-1 replotted vs electron energy. The wavelength-shift scale is shown at the top, now increasing in magnitude to the right. The dashed curve is the solid curve of Fig. 5.4-1, and the X's are computer-modeling results of FEL electron heating (see text for details). Some of the diamonds are slightly offset to the right in energy for clarity. The last two points at the lower right, indicated by the arrows, are no-FEL diamond data points that lie below the plot. Their top horizontal bars show the limits of the one-standard-deviation error bars.

Fig. 5.4-3. The FEL-heating modeling results for the Thomson-scattering distribution (the triangles), the two Maxwellians used in the fitting of these results (the dashed straight lines) and their sum (the solid line), and the relativistically corrected modeling data (the $X ' s$, which are also the X's of Fig. 5.4-2). See text for details. 
i. T. D. Rognlien, Phys . Nuids 26, 1545 (1983).

2. T. Matoba, T. Itagaki, T. Yamauchi, and A. Funahashi, Japanese Journal of Applied Physics 18, 1127 (1979).

3. J. Sheffield, Plesma Scattering of Electromagnetic Radiation (Academic, New York, 1975), p. 200. 
Fig. 5.4-1.

(a)

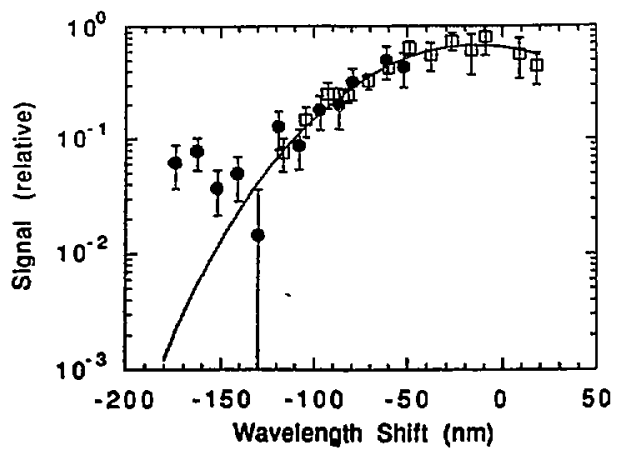

(b)

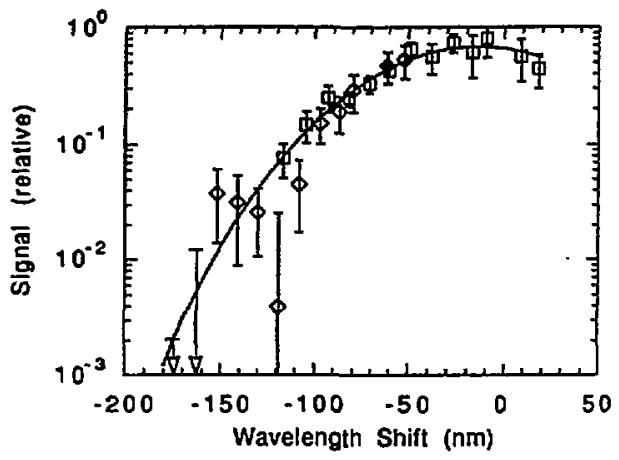

(c)

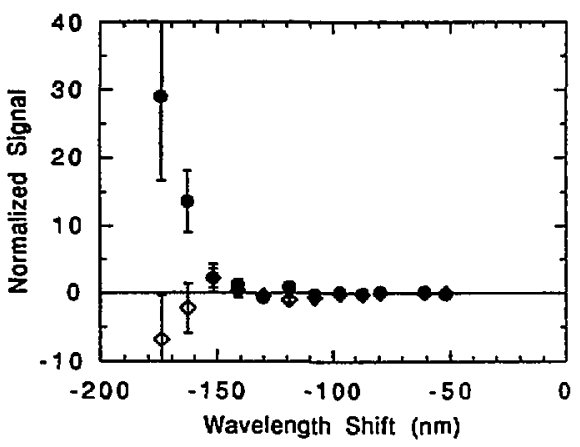


Fig. 5.4-2.

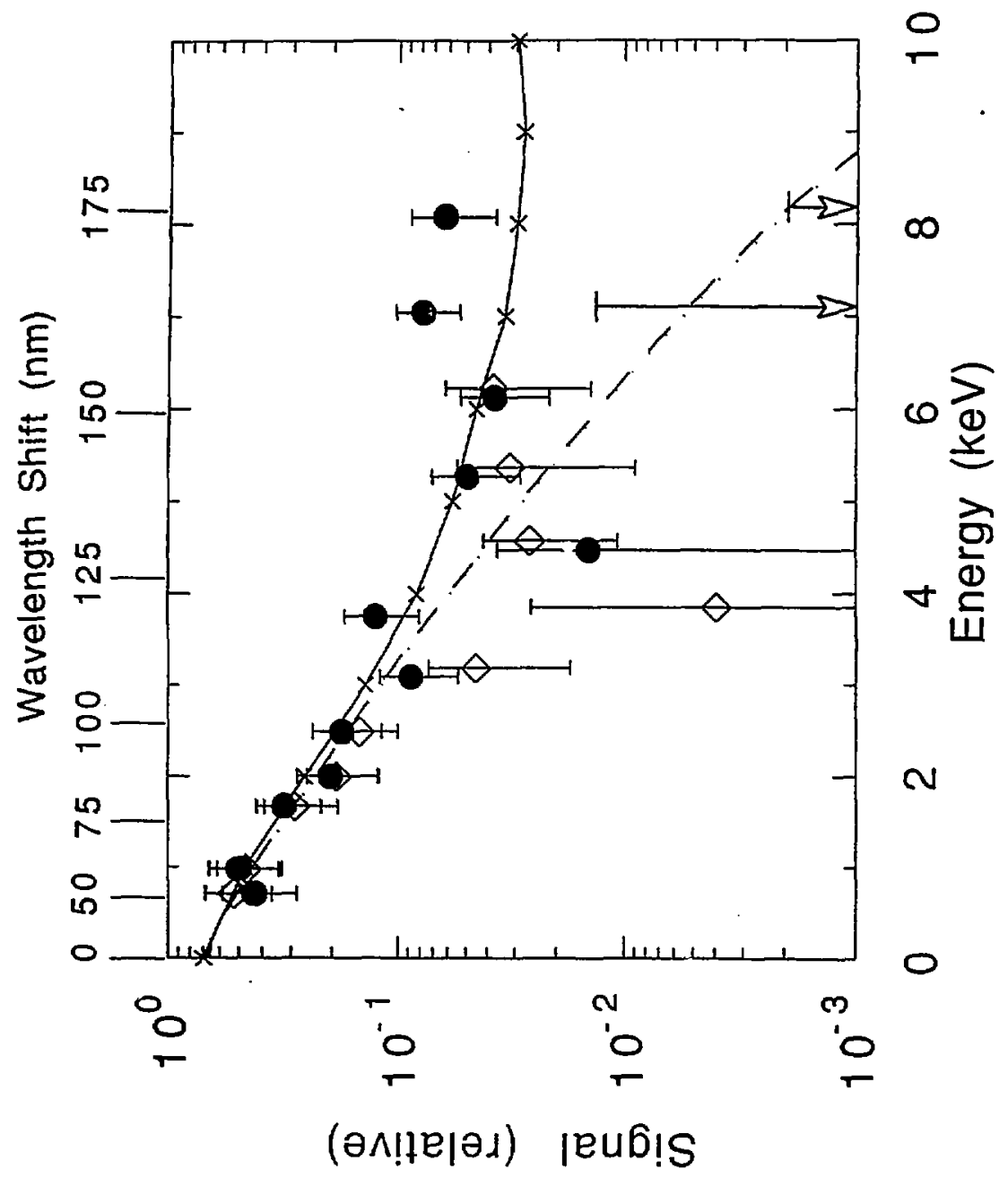


$6-6 \div 9$

Signal (relative)

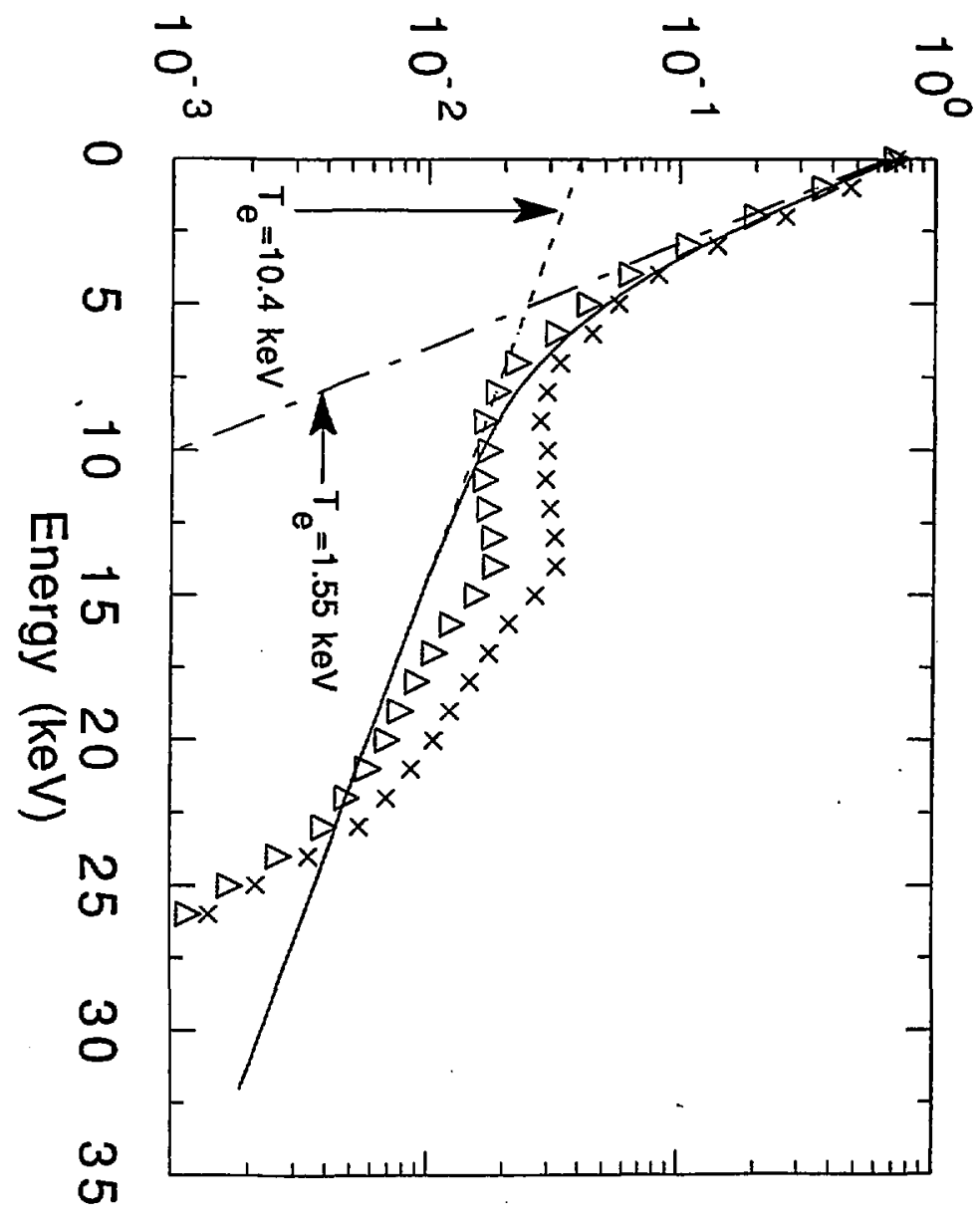




\section{ECRH experiments using a $140 \mathrm{GHz}$ Gyrotron}

\subsection{Overview (E. B. Hooper)}

A series of experiments were conducted in the summer-fall 1991 using the $140 \mathrm{GHz}$ gyrotron to heat plasma. The purposes of these experiments included:

- Establishing an ECRH data base for comparison with high-peak power experiments using a FEL.

- Demonstrating heating by ECRH at densities in excess of $10^{20} \mathrm{~m}^{-3}$.

- Exploring the physics of ECRH in a high density plasma:

The experiments were conducted at powers up to $200 \mathrm{~kW}$ and pulse lengths up to $60 \mathrm{~ms}$, with the resonance on or near the magnetic axis of the tokamak. The conclusions can be summarized as:

- Strong central heating was observed, with $n \Delta T_{e} \leq 10^{20} \mathrm{keV} / \mathrm{m}^{3}$.

- Electron temperature increases (on axis) were observed up to $2 \mathrm{keV}$.

- No density pumpout was observed.

- Power could be depasited within $r=5 \mathrm{~cm}, \leq$ than a typical sawtooth radius.

- Significant effects were observed on the sawteeth and on MHD activity.

- Hollow electron temperature and current density profiles could be converted into peaked, sawtoothing profiles.

- ECRH occasionally caused recovery from minor disruptions.

The results of the experiments were presented in a poster at the 1991 American Physical Society meeting. ${ }^{1}$ Selected figures from that presentation are included below, together with review of the results and references to other publications on particular items.

1. E. B. Hooper et al., Bull. Am. Phys. Soc 36, 2312 (1991). 
6.1 Results of ECRH experiments using a $140 \mathrm{GHz}$ gyrotron' (E. B. Hooper, S. L. Allen, T. A. Casper, M. E. Fenstermacher, J. H. Foote, C. J. Lasnier, M. J. Malowski, K. Oasa, T. Ogawa, B. W. Rice, B. W. Stallard)

Energy increase and confinement; effects on sawteeth. The increase in stored energy due to central heating by the gyrotron is plotted versus line-averaged density in Fig. 6.1-1. As expected, no heating was observed for the central density above cutoff. Strong refraction ${ }^{2}$ causes the rays to miss the axial region, absorption is weak off axis as temperatures are lower, and power which renches the rear wall is largely absorbed by the calorimeter ${ }^{3}$ so that multipass absorption is weak. Scatter in the data arises from short microwave pulse lengths or poor energy confinement due to minor disruptions in the tokamak. At $200 \mathrm{~kW}$ incident on the plasma, the maximum increase in temperature corresponded to $n \Delta T_{e}=1 \times 10^{20} \mathrm{~m}^{-3}$.

An example of on-axis heating is shown in Fig. 6.1-2, with more details in Figs. 6.1-3-6.1-6. Note that:

- The on-axis heating increases the amplitude and frequency of sawteeth.

- The electron temperature is greater following the heating than before, suggesting that the plasma had generally not reached full equilibrium before the ECRH was applied.

- The sawtooth amplitude and frequency during and following the heating, as does the inversion radius (c.f. Fig. 6.1-4).

- The temperature increase rate during heating and decrease rate following heating are at a rate of $1 / 3$ to $2 / 3$ the neoAlcator time; c.f. Fig. 6.1-5. This result is consistent with the energy increase measured by the diamagnetic loopt, although there is sufficient noise in the signal to make accurate conclusions difficult.

Sawtooth postcursors. During on-axis ECRH a "postcursor" ( $\mathrm{m}=1$ mode) is observed ${ }^{5}$ on the sawteeth, as shown in Figs. 6.1-7-6.1-10. The observation implies that the reconnection is incomplete during ECRH. In the absence of ECRH no postcursors were observe, consistent with complete reconnection. Precursors were observed with and without the additional heating.

Recovery from a minor disruption. Heating on axis immediately following a minor disruption was observed to cause the plasma to recover into a well- 
behaved, sawtoothing discharge, as seen in Figs. 6.1-11-6.1-18. (Note that recovery was not observed on most shots.) The timing may be important; note from Fig. 6.1-14 that the $T_{e}$ profile at $\mathrm{r} \geq 10 \mathrm{~cm}$ had not been significantly affected at the time of the microwave puise.

Strong MHD activity was observed on the magnetic loops immediately proceeding the minor disruption, as seen in Fig. 6.1-12 and detailed in Figs. 6.1-15-6.1-18. The mode was identified as $\mathrm{m}=2$ (Fig. 6.1-18).

The likely mechanism for the disruption has been identified ${ }^{6}$ as cooling due to the radiation from molybdenum from the limiters, with the ECRH heating the electrons above the "radiation barrier" so that a stable discharge is re-established. Cf. Sec 7.1 for details.

Figures 6.1-19-6.1-21 show a discharge which did not recover to a stable equilibrium. The large amplitude variations following the ECRH are not sawteeth, although the high frequency mode seen, for example on the electron temperature (Fig. 6.1-19) and magnetic loops (6:1-20) is an $m=1$ mode. This mode was very clear on the fast $x$-ray measurements. ${ }^{7}$

Filling hollow current profiles. Occasionally, on the startup of low-density discharges the current and electron temperature were hollow, apparently due to the radiation for molybdenum. ECRH converted these into well-behaved, sawtonthing discharges, apparently also due to overcoming the radiation barrier.6 Data illustrating such a discharge is shown in Figs. 6.1-22-6.1-26. The hollow current profile is shown in Sec 7.1, along with further analysis of the effect.

1. E. B. Hooper et al., Bull. Am. Phys. Soc. 36, 2312 (1991).

2. G. R. Smith, M. E. Fenstermacher, and E. B. Hooper, Nucl. Fusion 30, 2505 (1990).

3. See Section A.2.3, this report.

4. M. M. Marinak, Bull. Am. Phys. Soc 36, 2313 (1991). See also Sec A.3.5, this report.

5. C J. Lasnier, M. Fenstermacher, and K Oasa, Bull. Am. Phys. Soc 36, 2313 (1991):

6. B. W. Rice and E. B. Hooper, submitted to Nucl. Fusion. See also, Sec. 7.1, this report.

7. K. Oasa, T. Ogawa, K. Odajima, M. Shiho, and H. Maeda, Bull. Am. Phys. Soc. 36, 2S13 (1991). 
Fig. 6.1-1

\section{CENTRAL HEATING BY GYROTRON}

- Maximum heating power $=200 \mathrm{~kW}$ at $140 \mathrm{GHz}$

- Pulse length $=5$ to $50 \mathrm{~ms}$

- Less than maximum heating is due to short pulselength, heating during initial current ramp, or poor confinement during minor disruptions

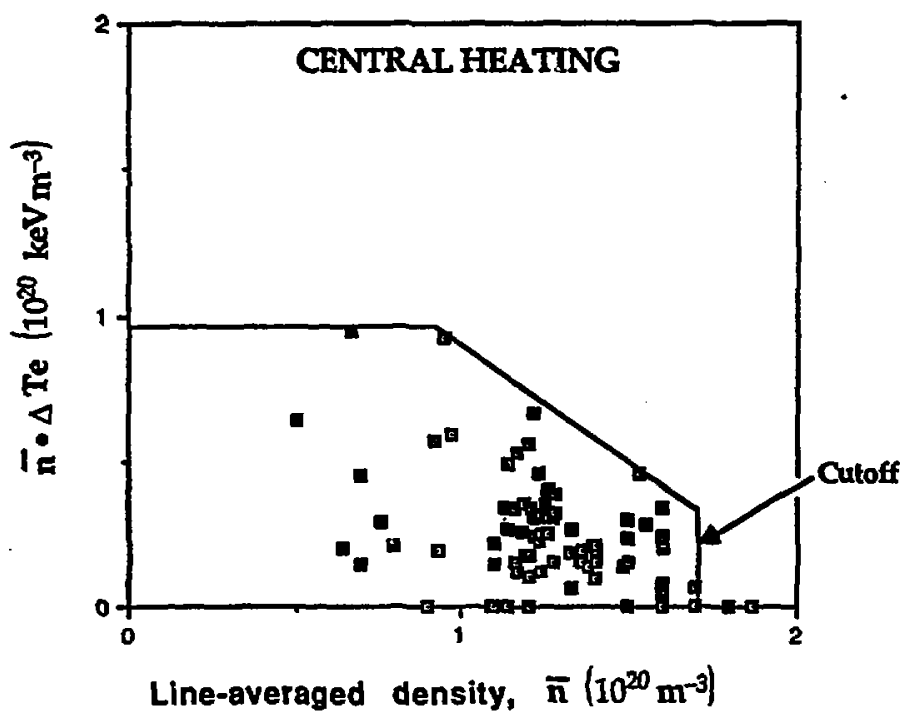


Fig. 6.1-2

SHOT $=12002$

Central temperature

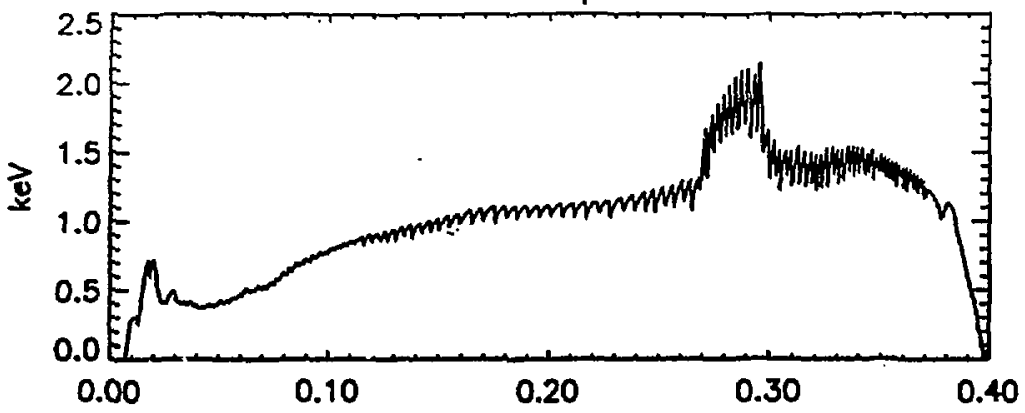

JAERI soft xroys

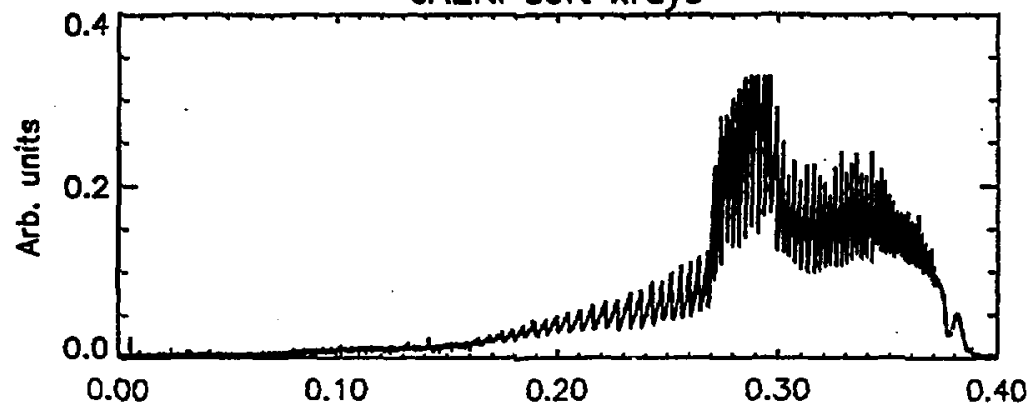

Microwave power

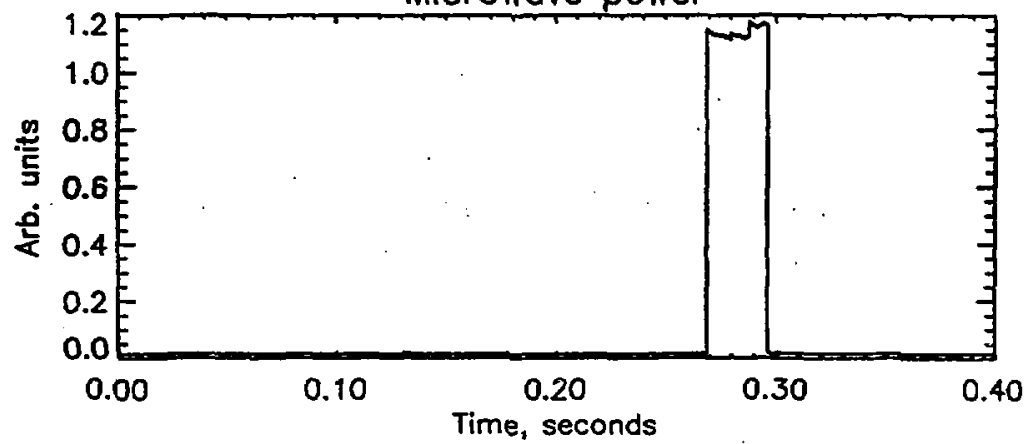


Fig. $6.1-3$

SHOT $=12002$

Toroidal current
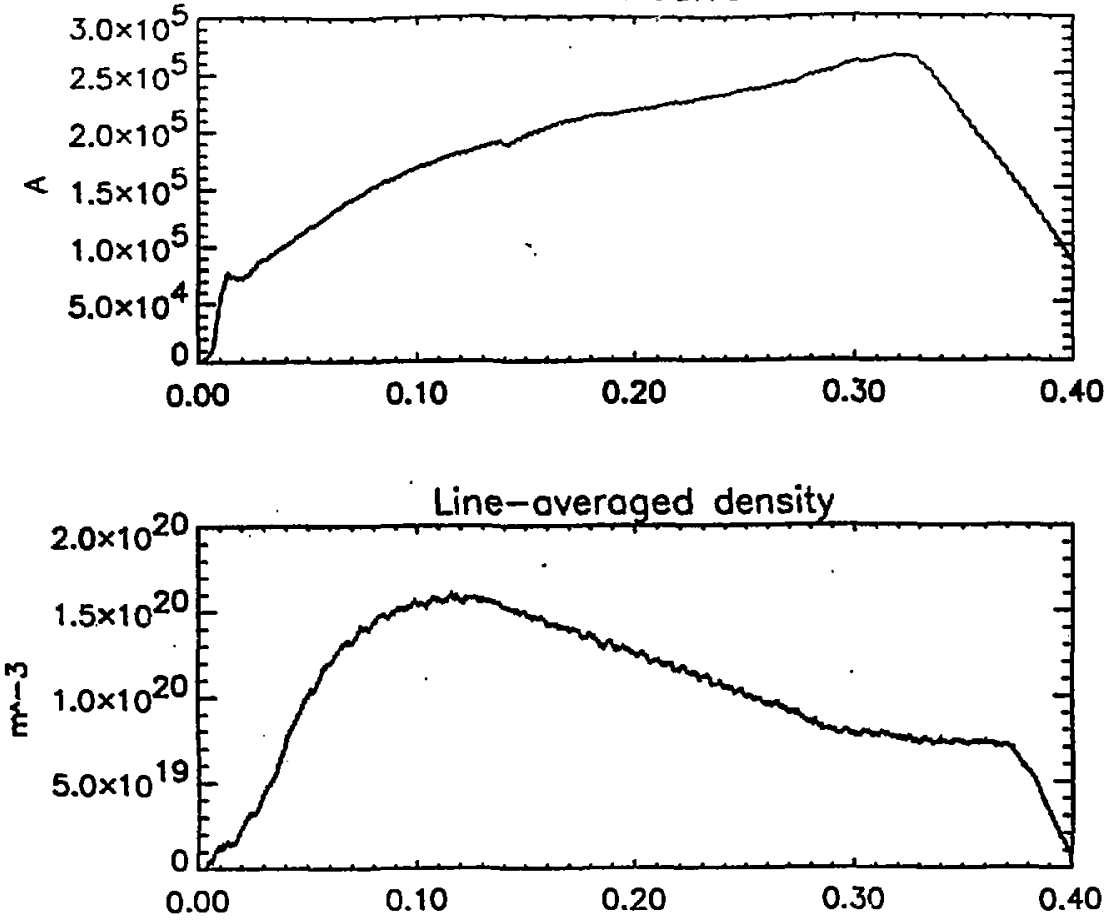

MHD activity

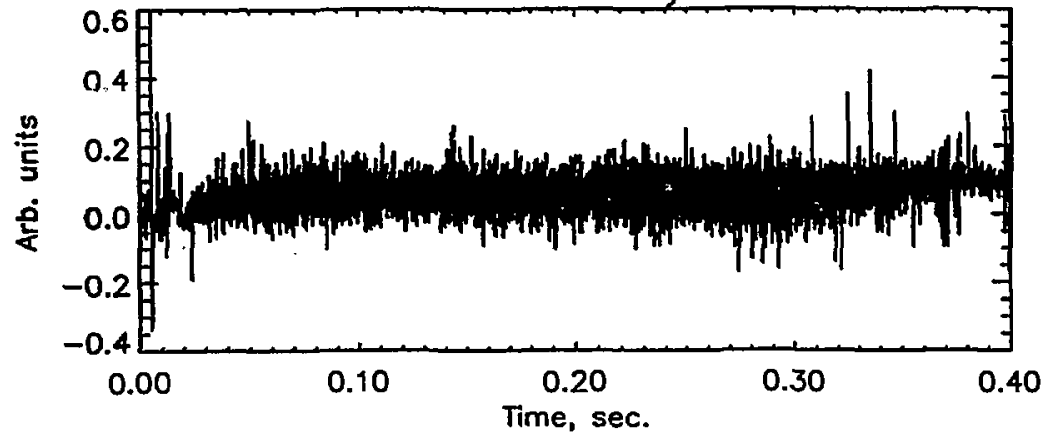


Fig. 6.1-4

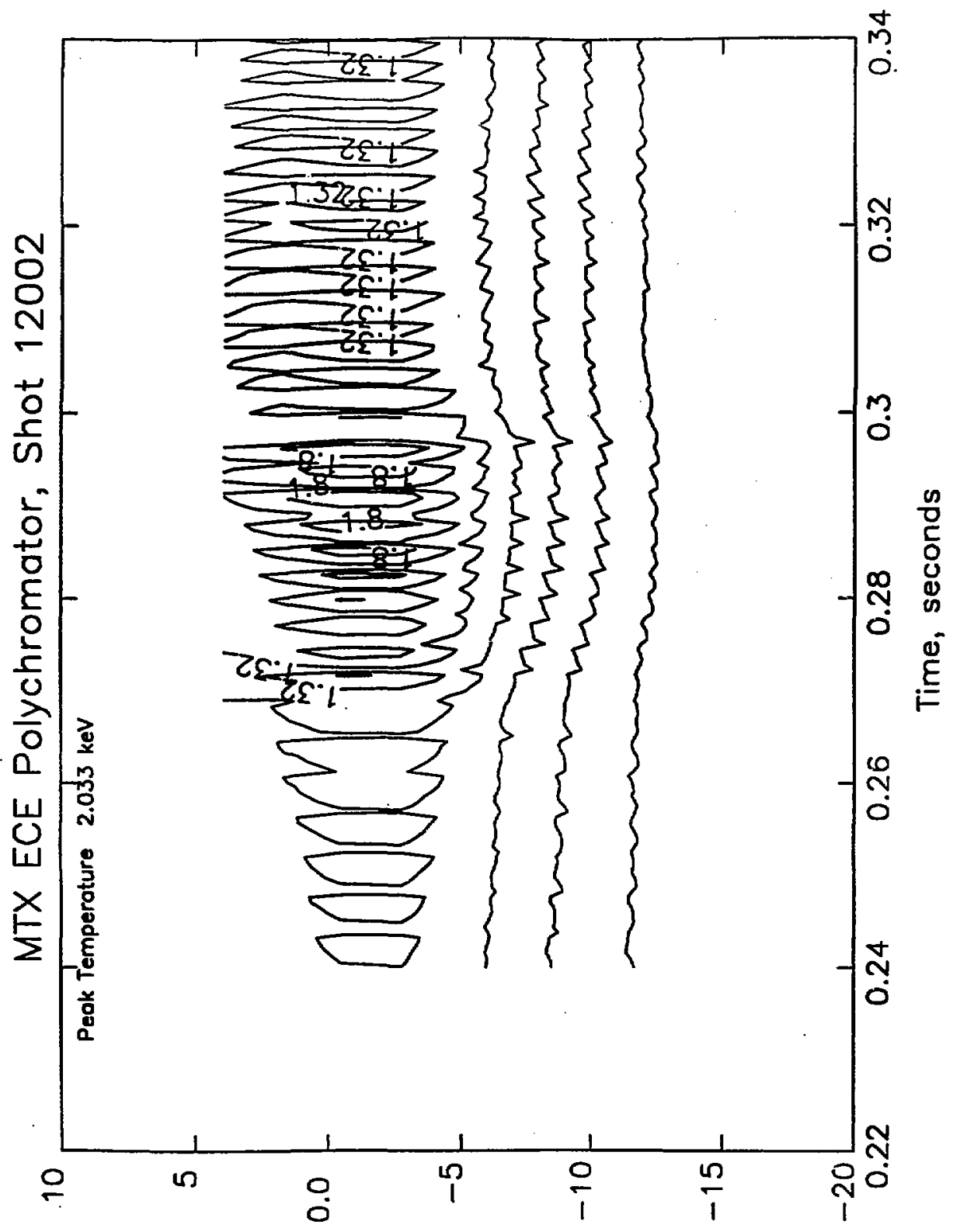




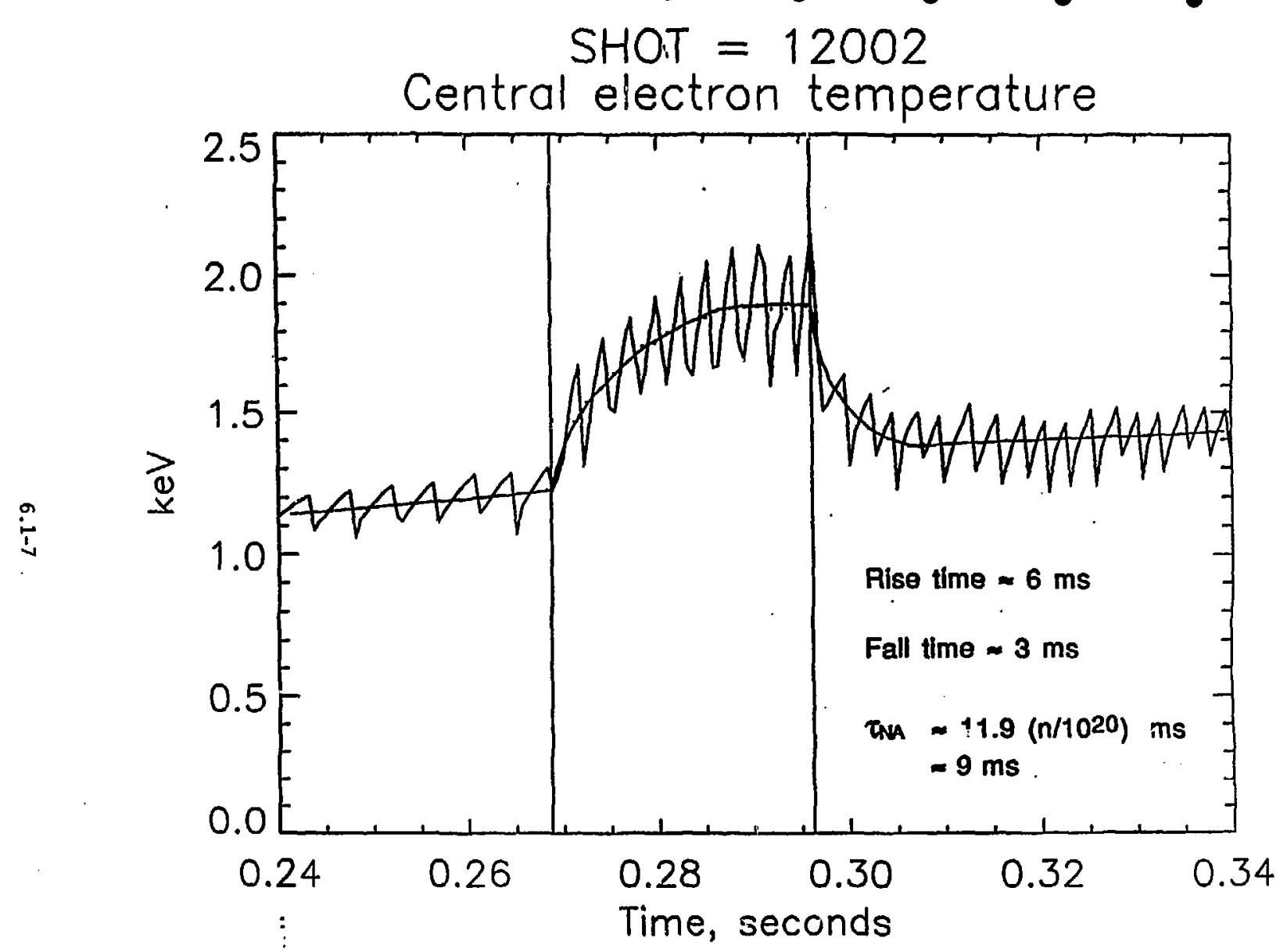


PLASMA THERMAL ENERGY SHOT 12002

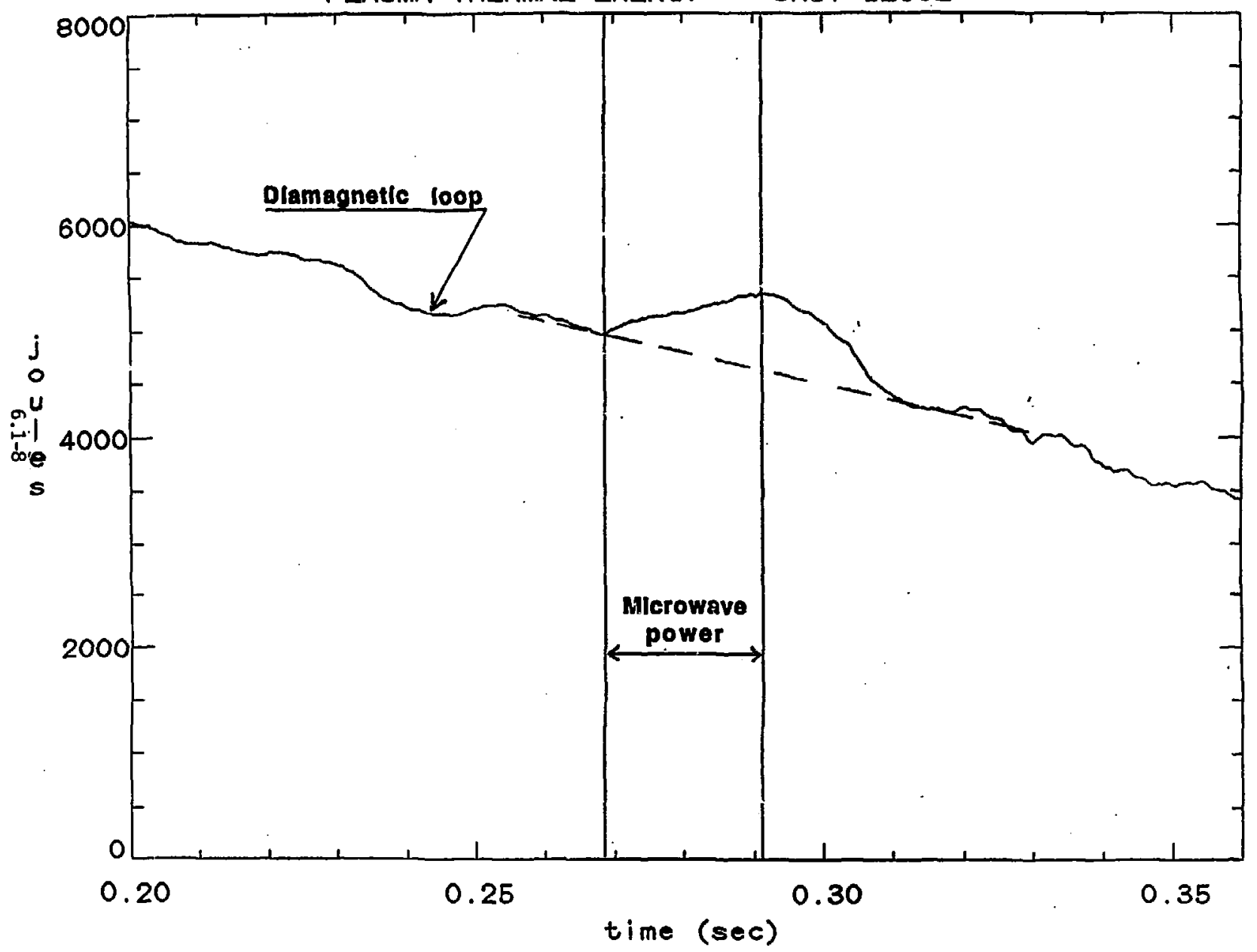

$n$
0
0
0
0
0 
Fig. 6.1-7

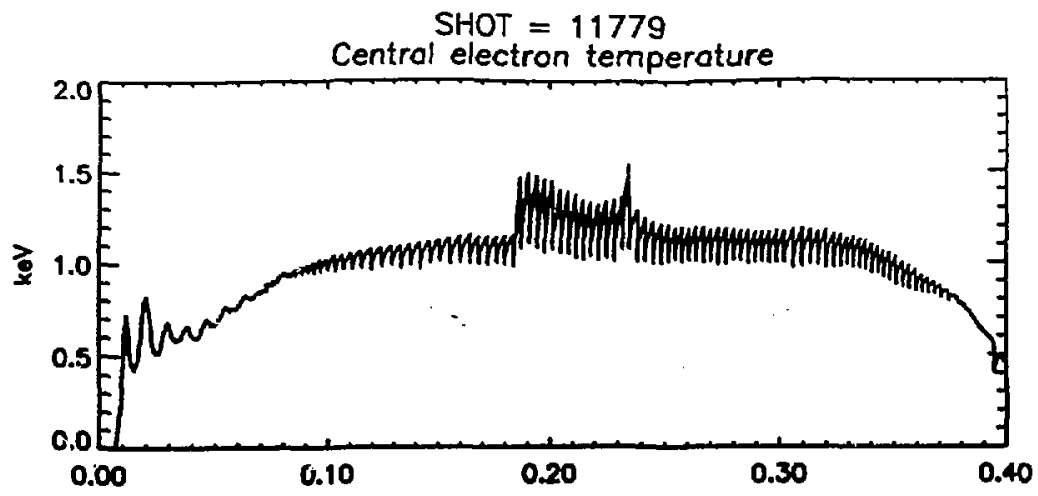

JAERI soft xrays

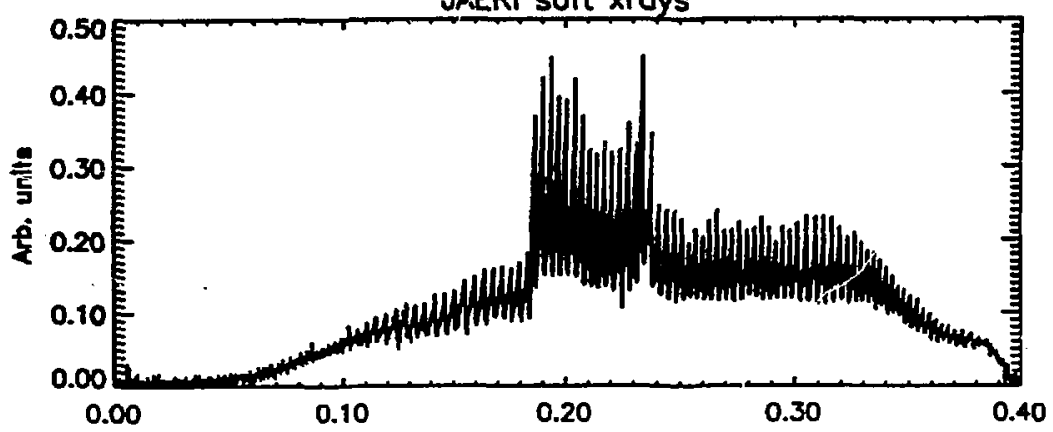

Microwave power

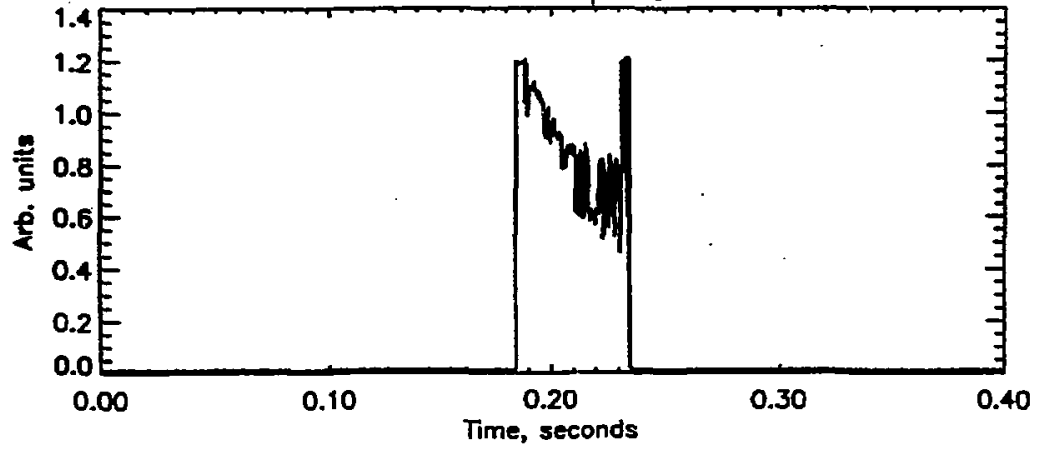

6.1-9 
Fig. $6.1-8$

$$
\text { SHOT }=11779
$$
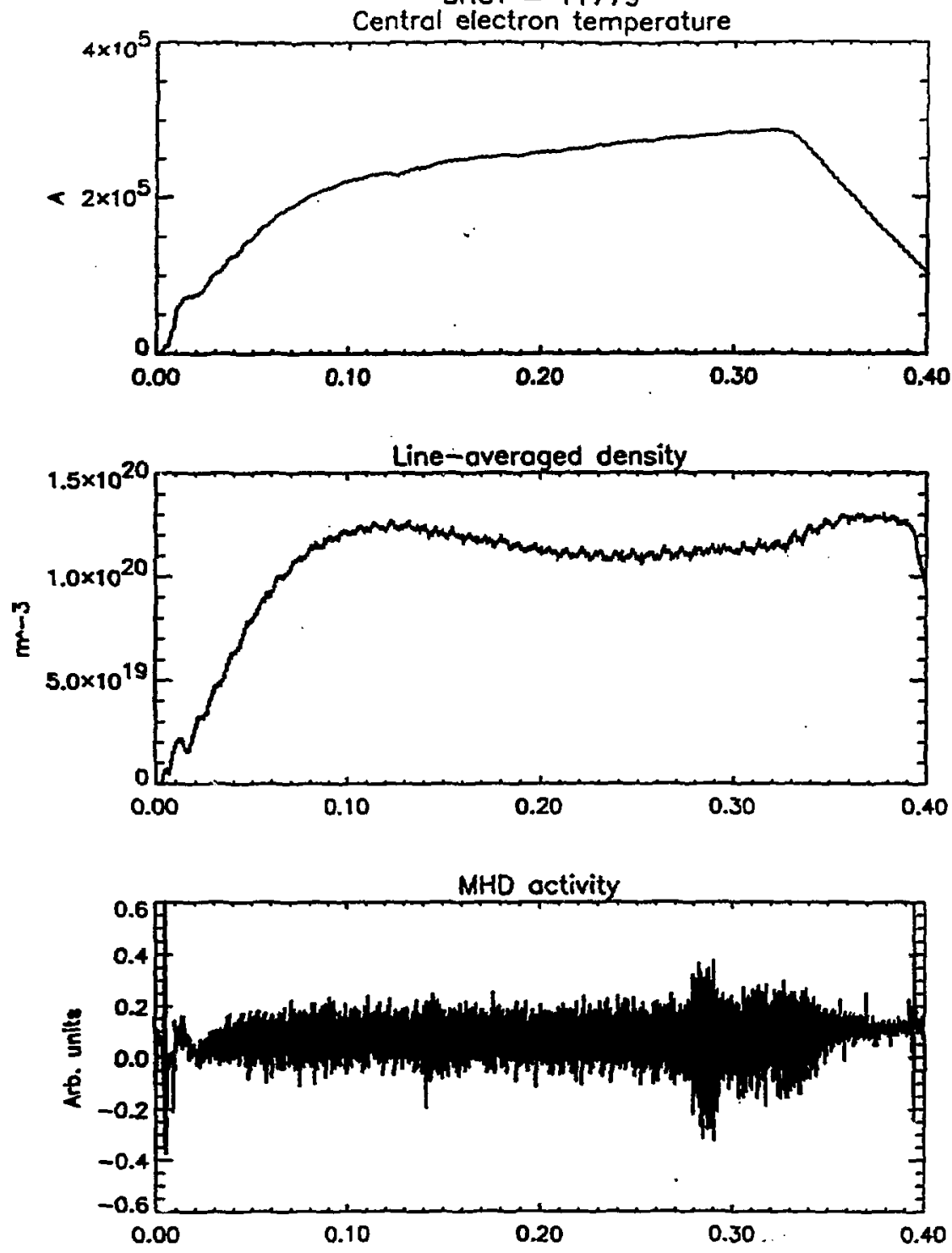
naMPM 


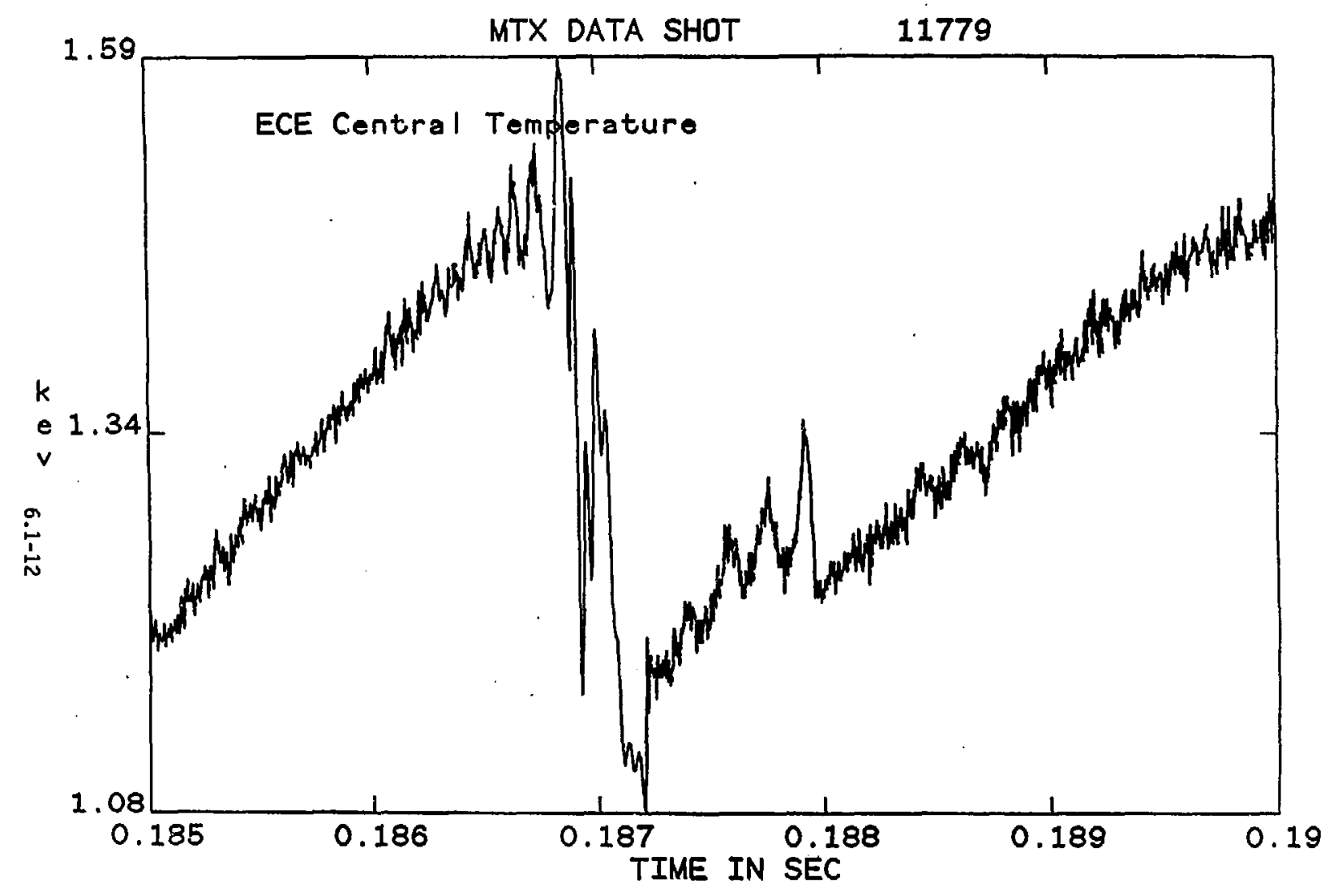

苛 
Fig. 6.1-11
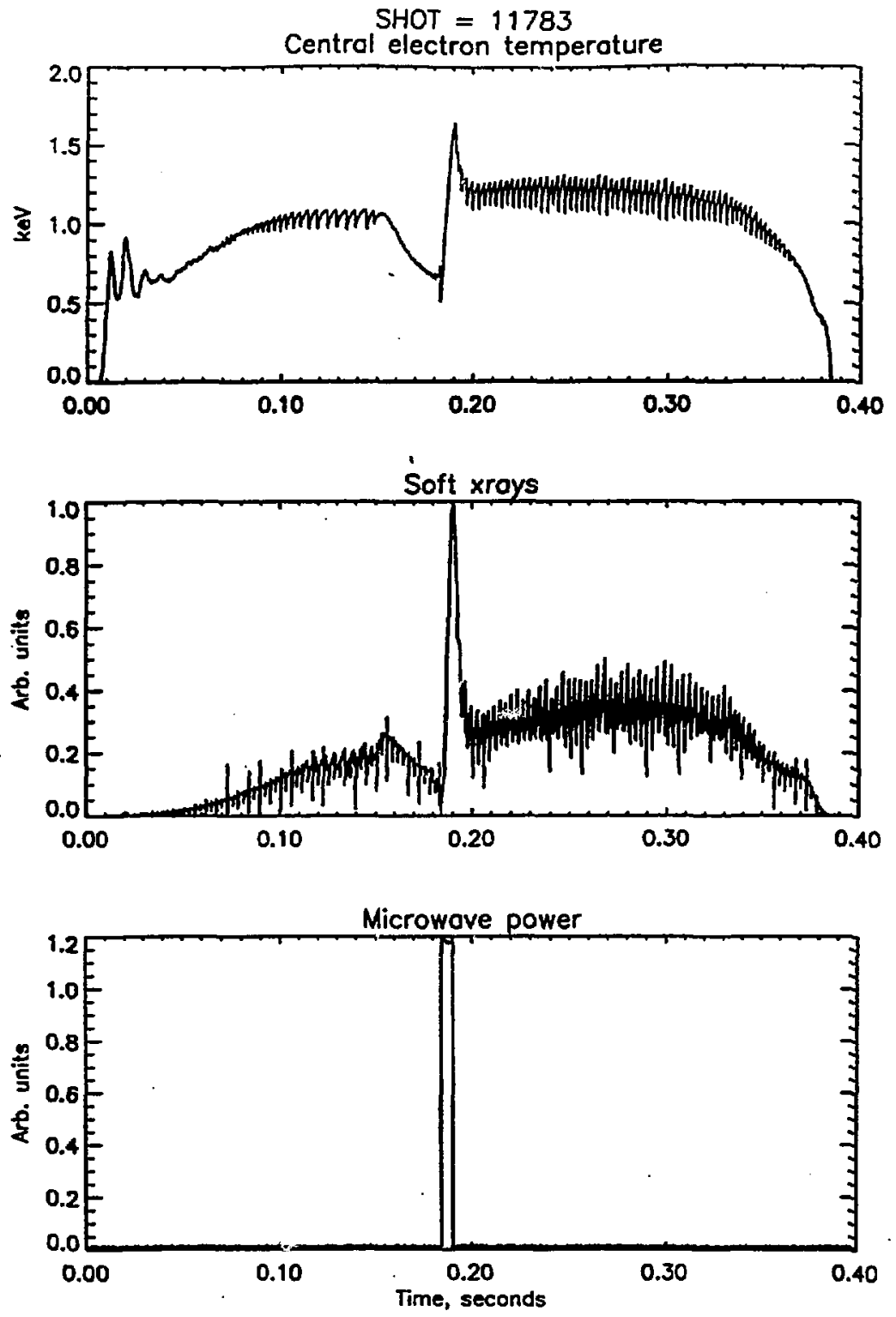
Fig. 6.1-12
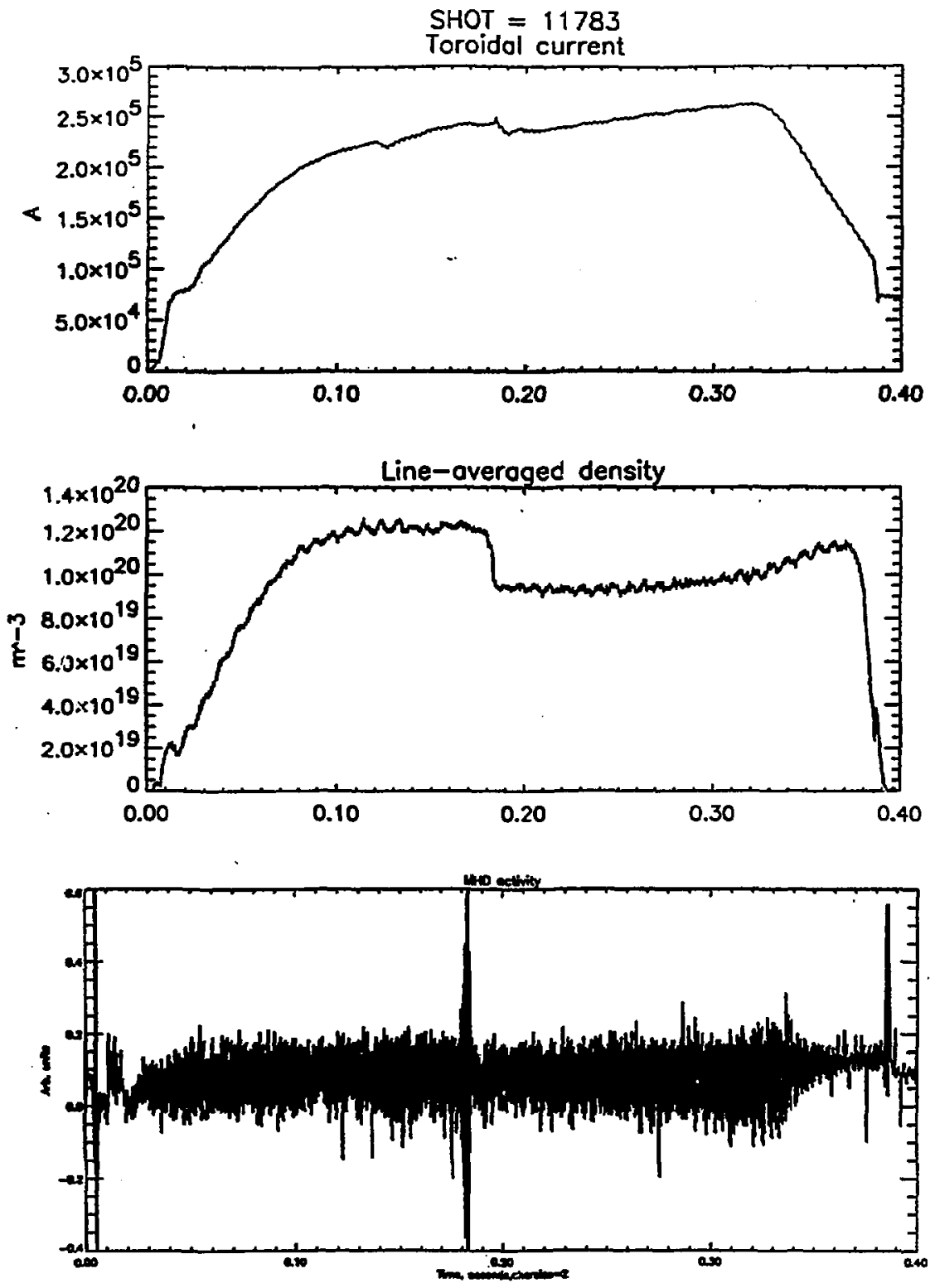
Fig. $6.1-13$

SHOT $=11783$

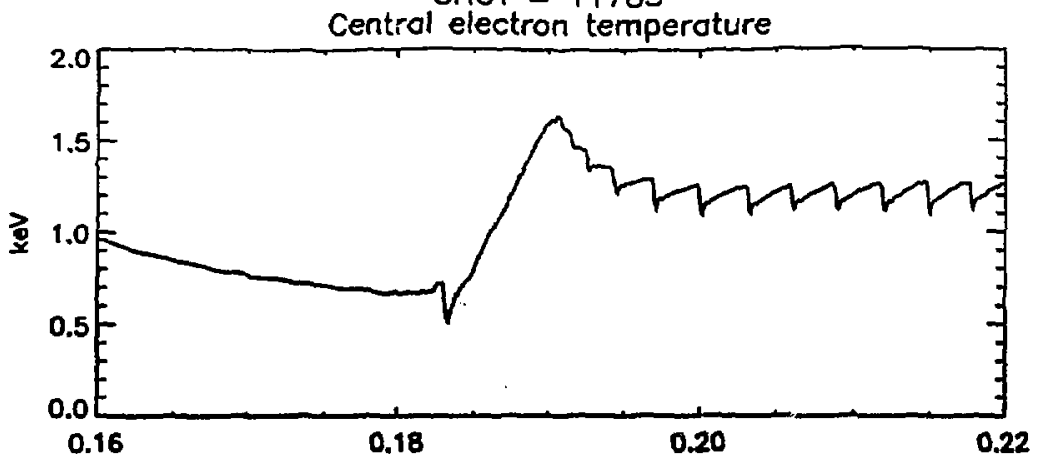

Soft xroys

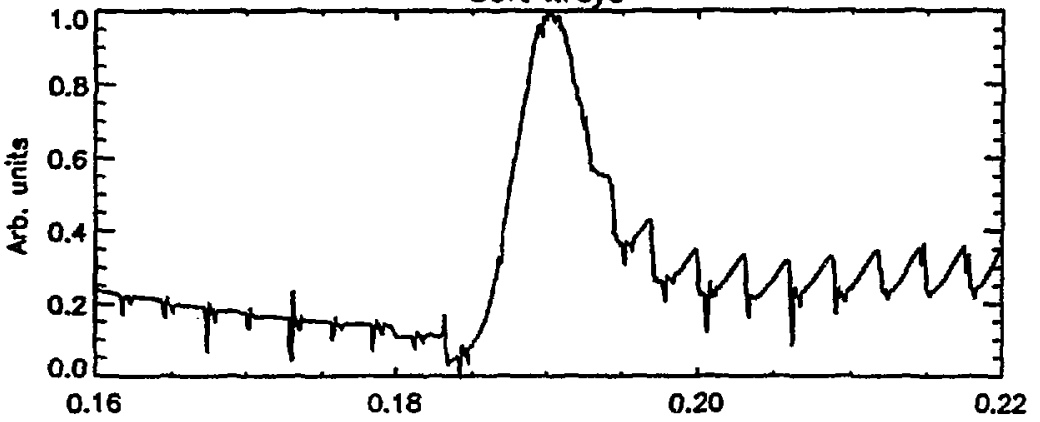

Microwave power

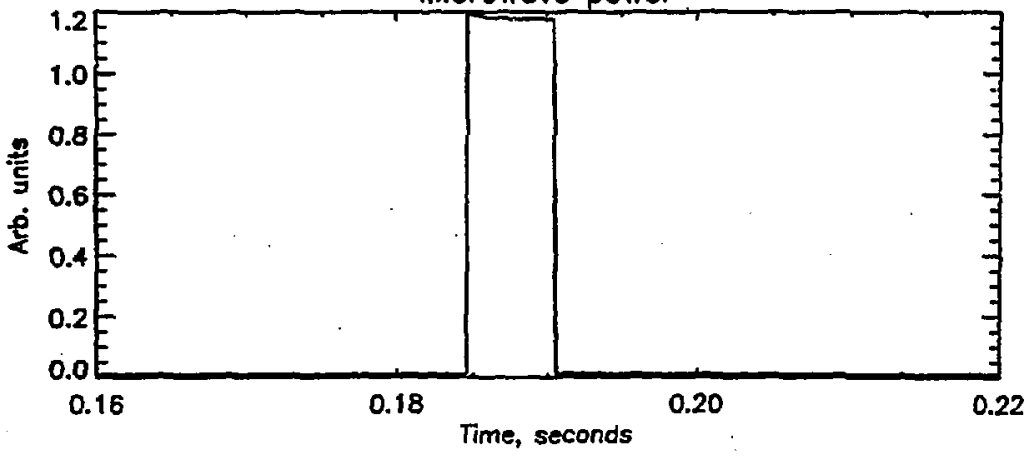


Fig. $6.1-15$
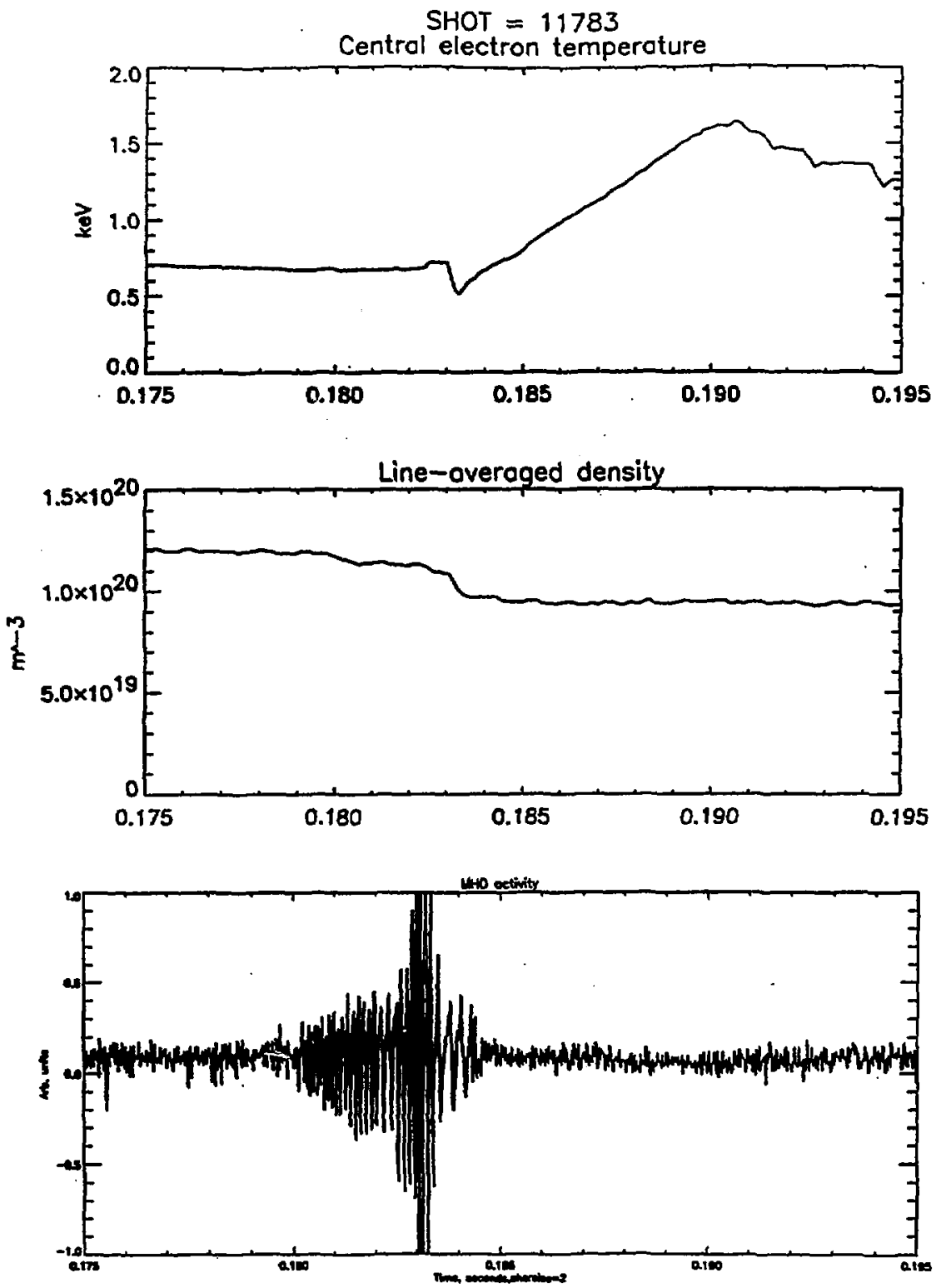
MHO activity and Central electron temperature. Stert of ECRH is indicated.

年

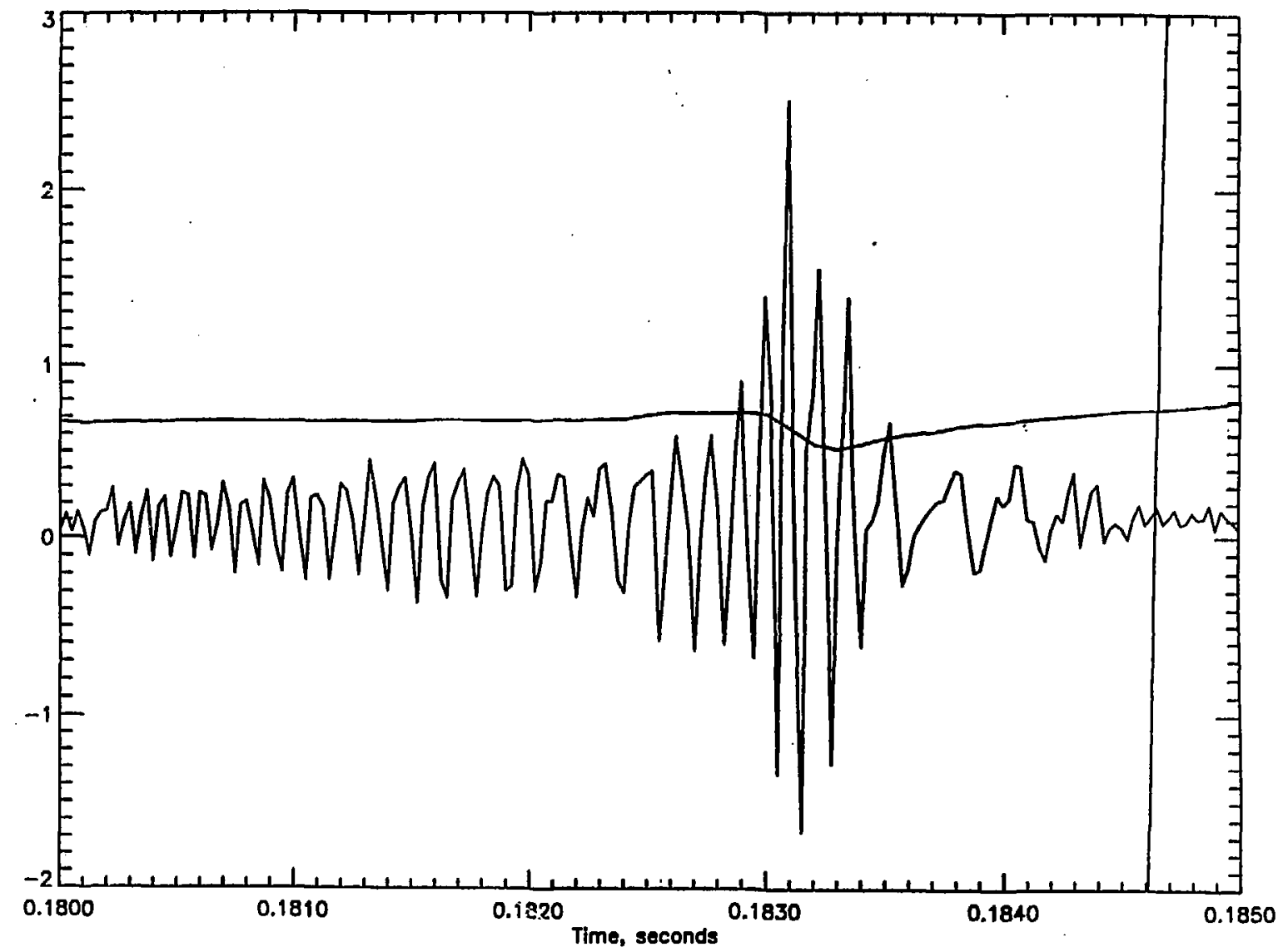




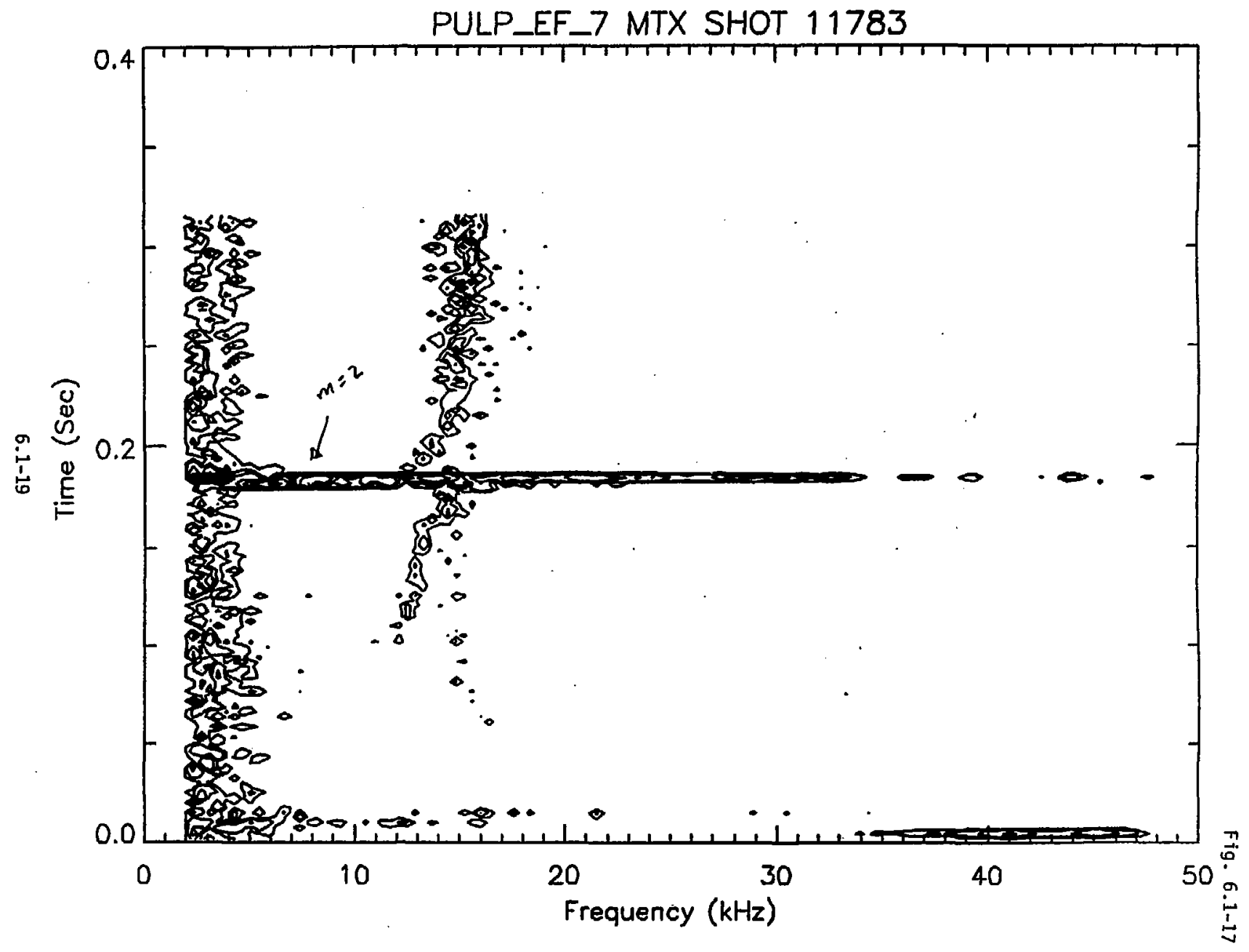




\section{Mode amp iltuce vs po ollca}

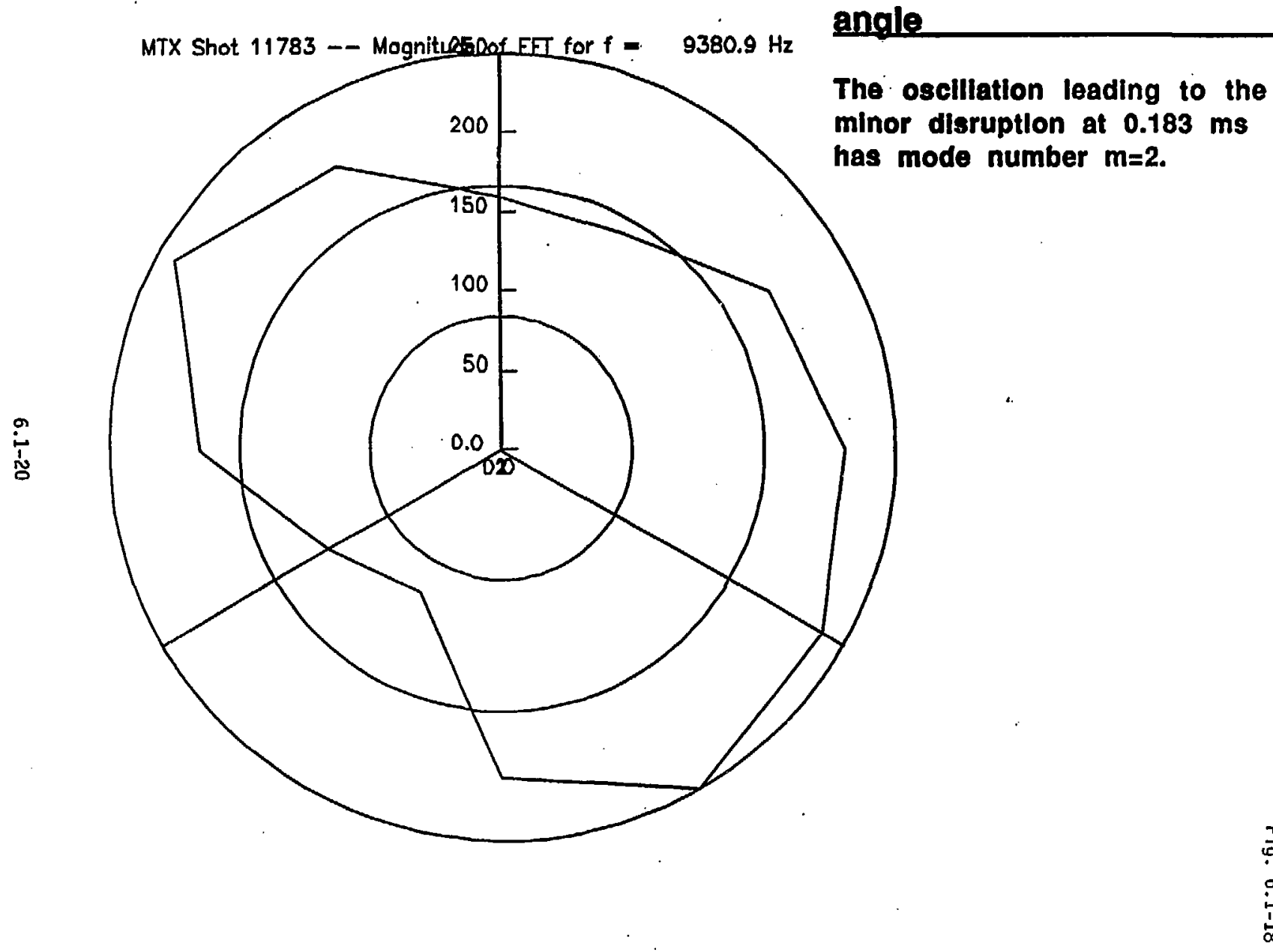


Fig. 6.1-19
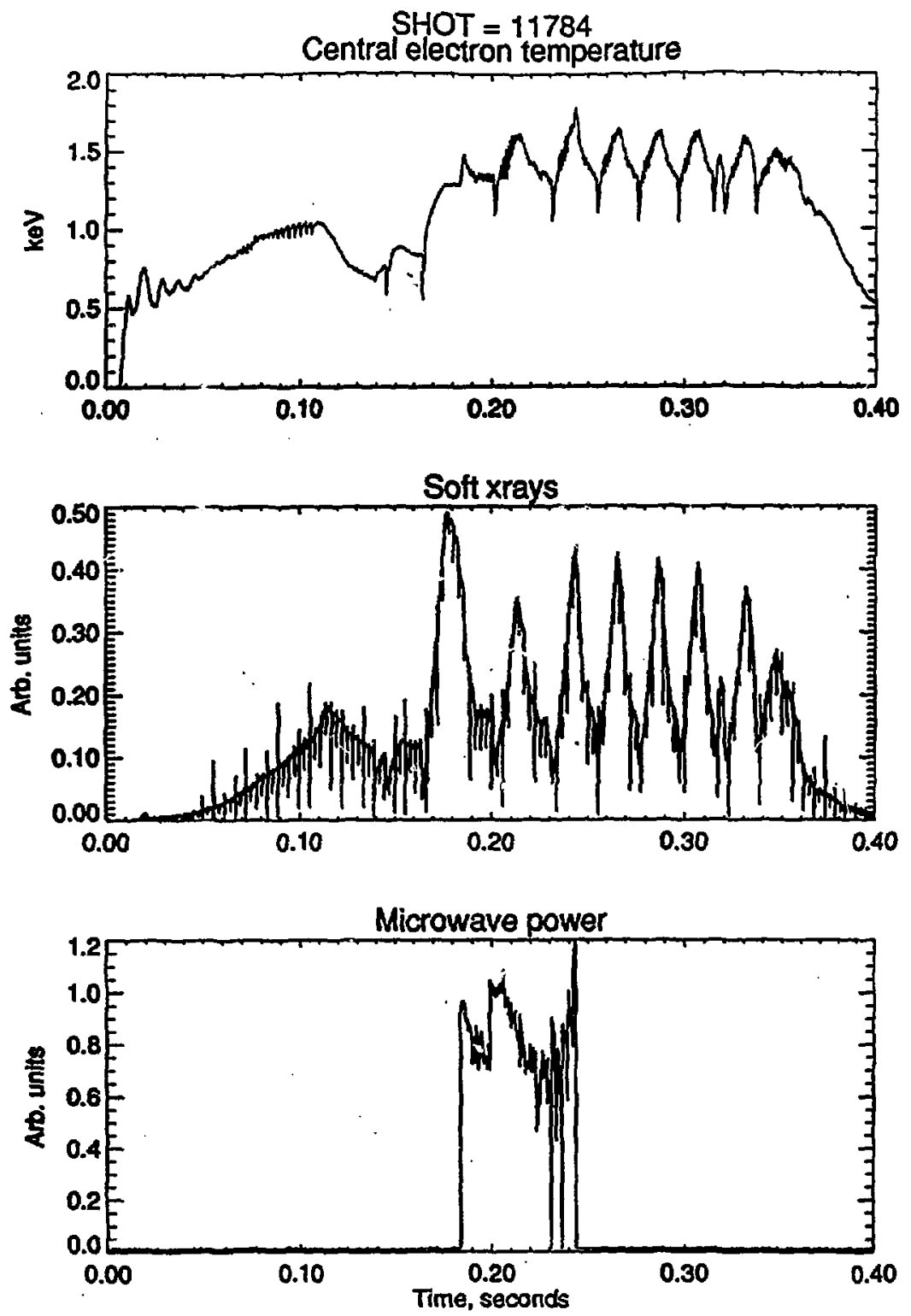

6.1-21 
Fig. $6.1-20$

SHOT $=11784$

Toroidal current

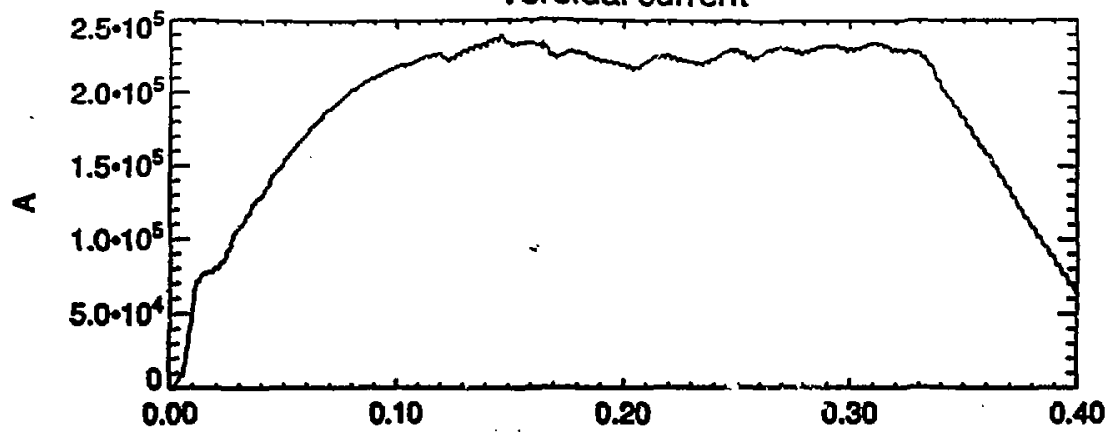

Line-averaged density
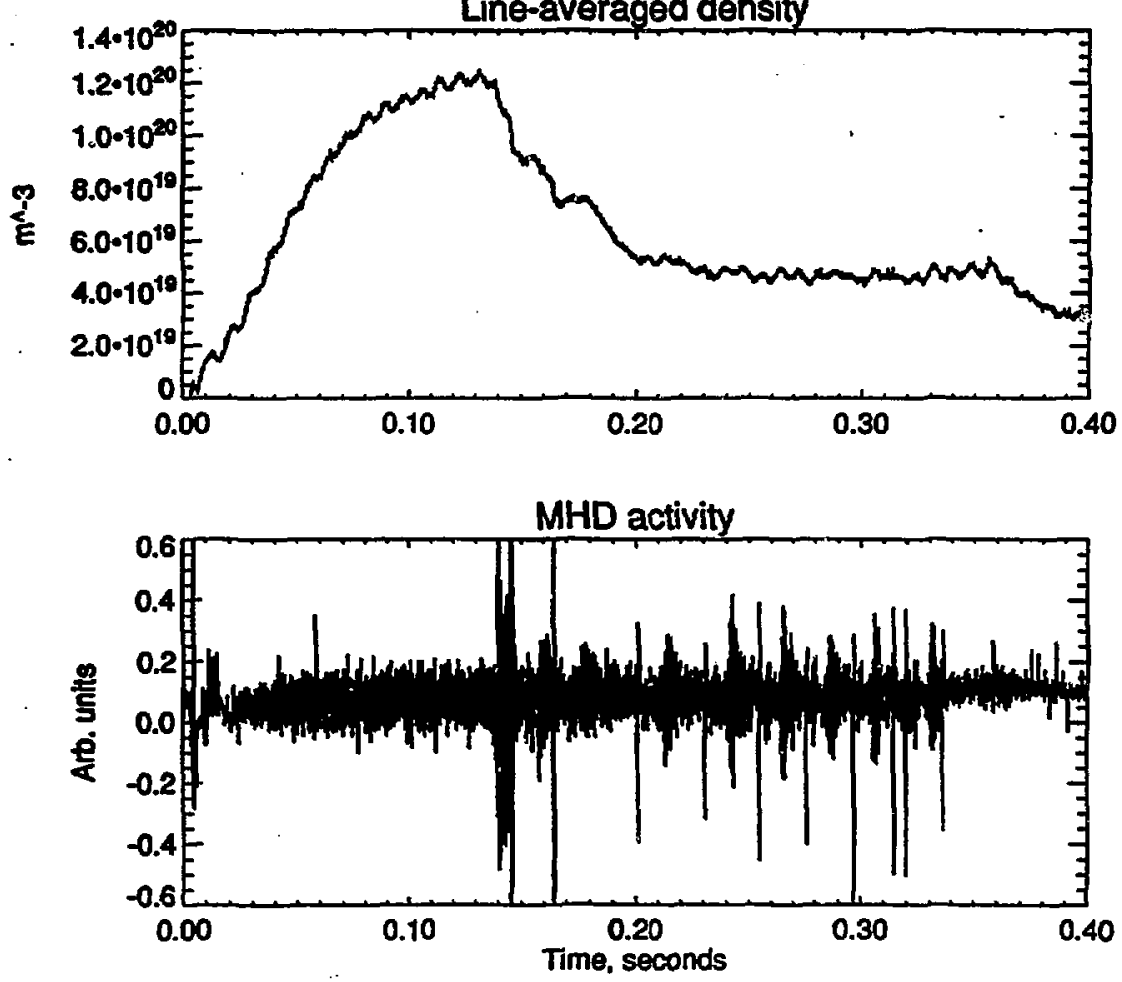
Fig. 6.1-21

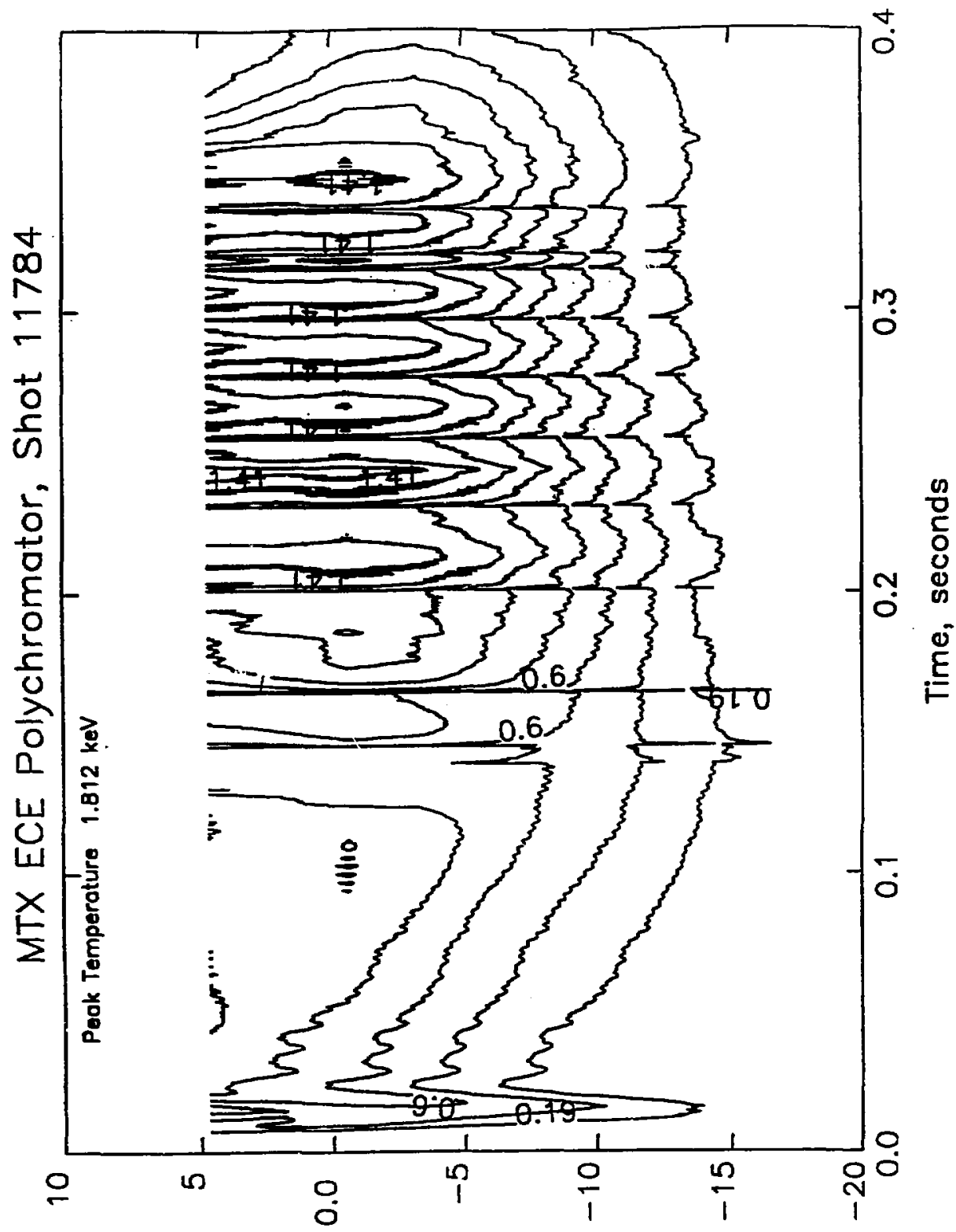

$$
\text { wo 'sn!poy sou!w }
$$


Fig. 6.1-22

SHOT $=12252$

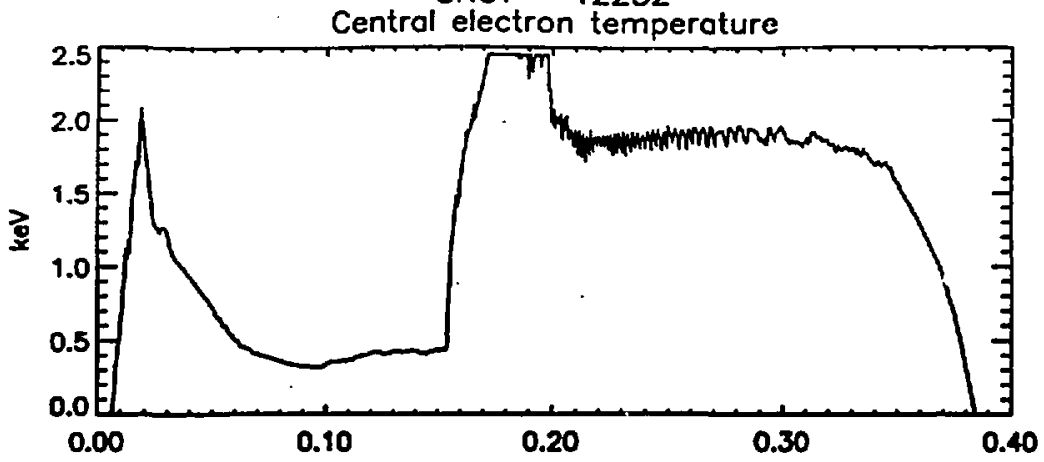

Soft xroys

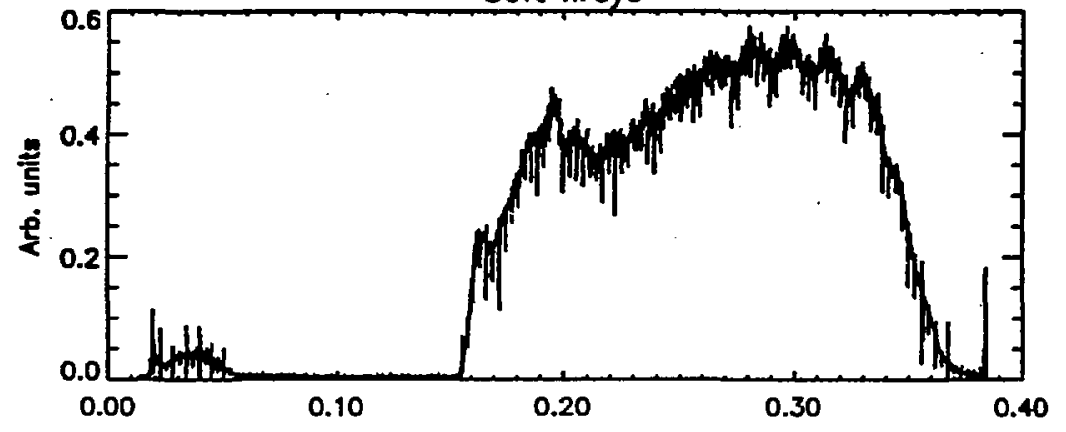

Microwave power

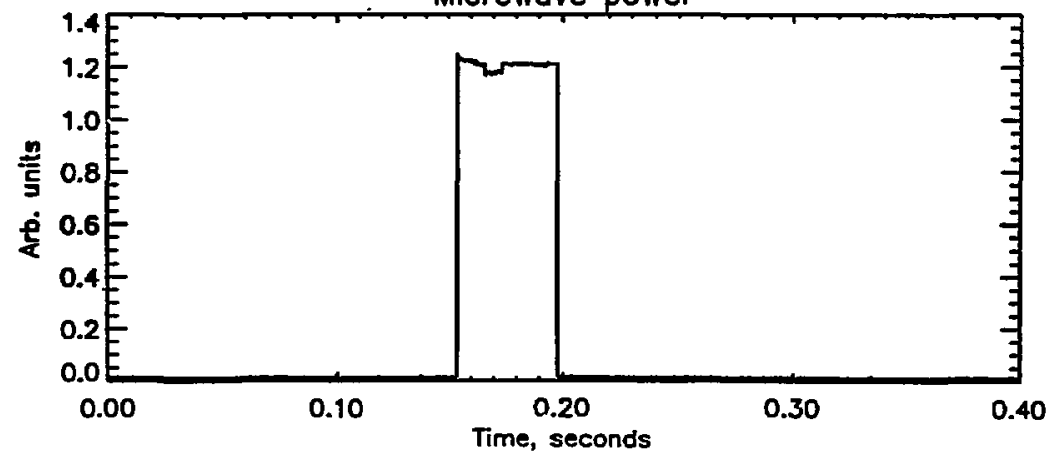


Fig. 6.1-23
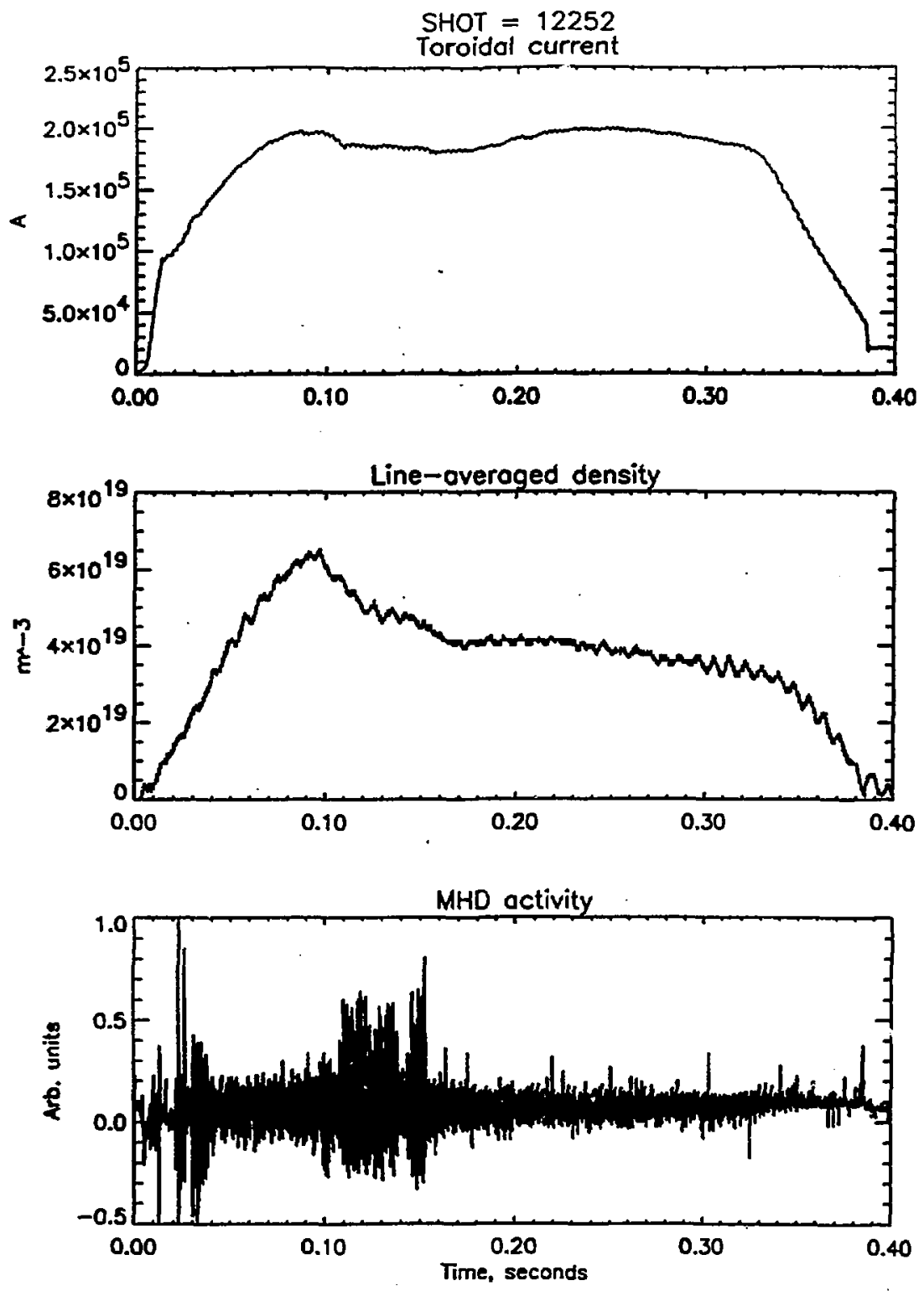

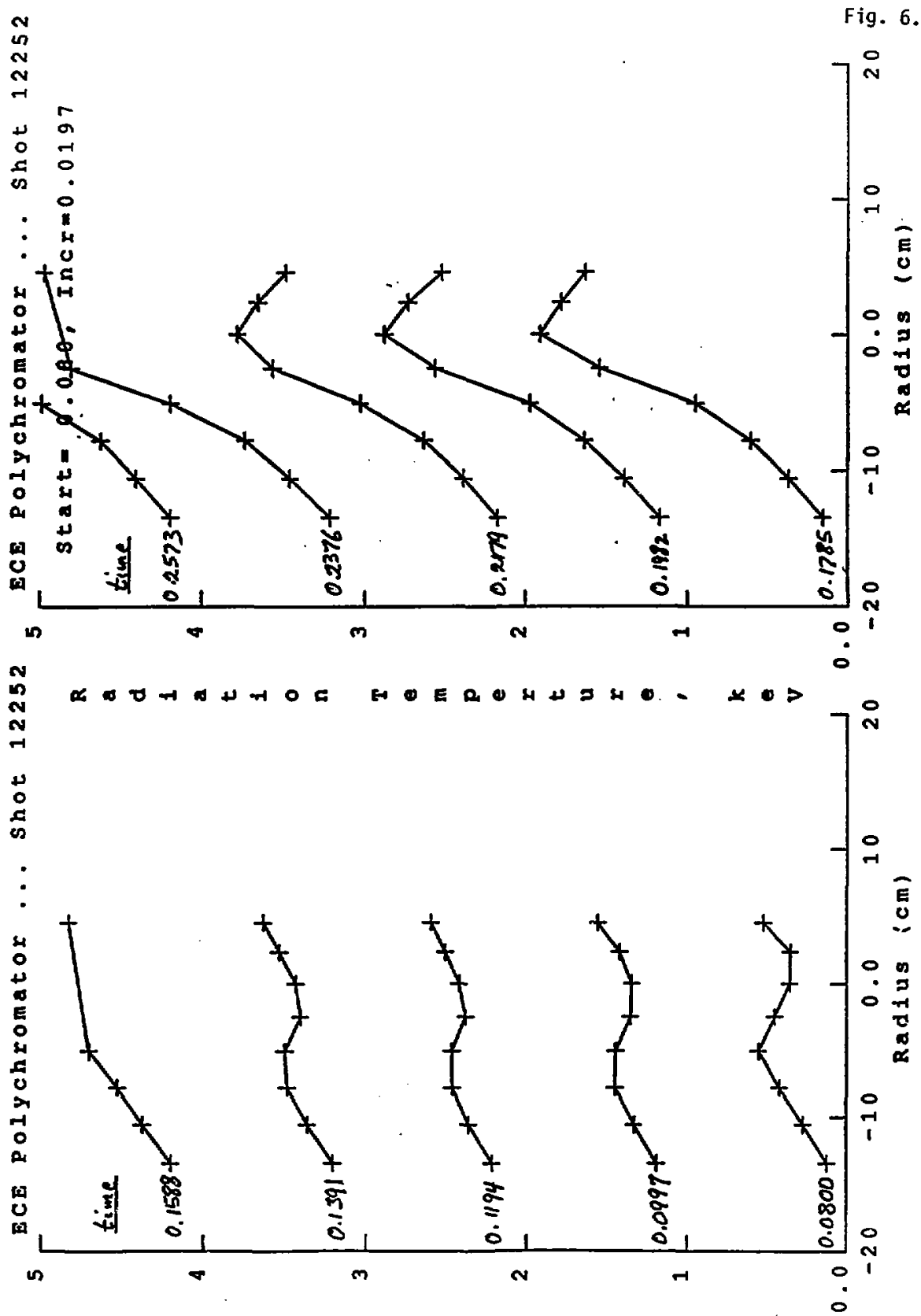

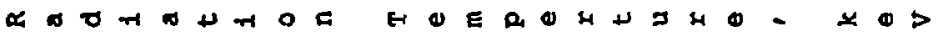




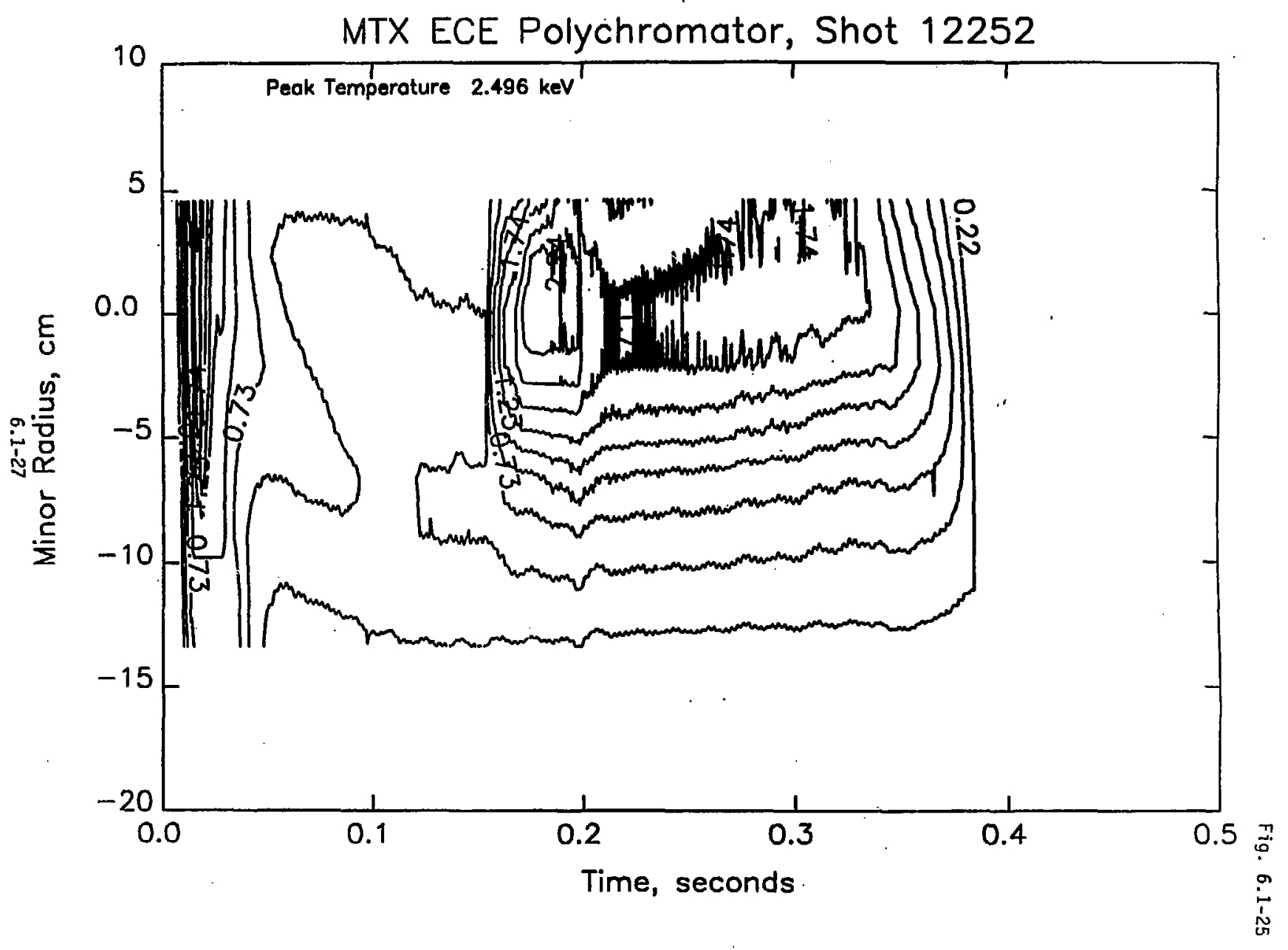




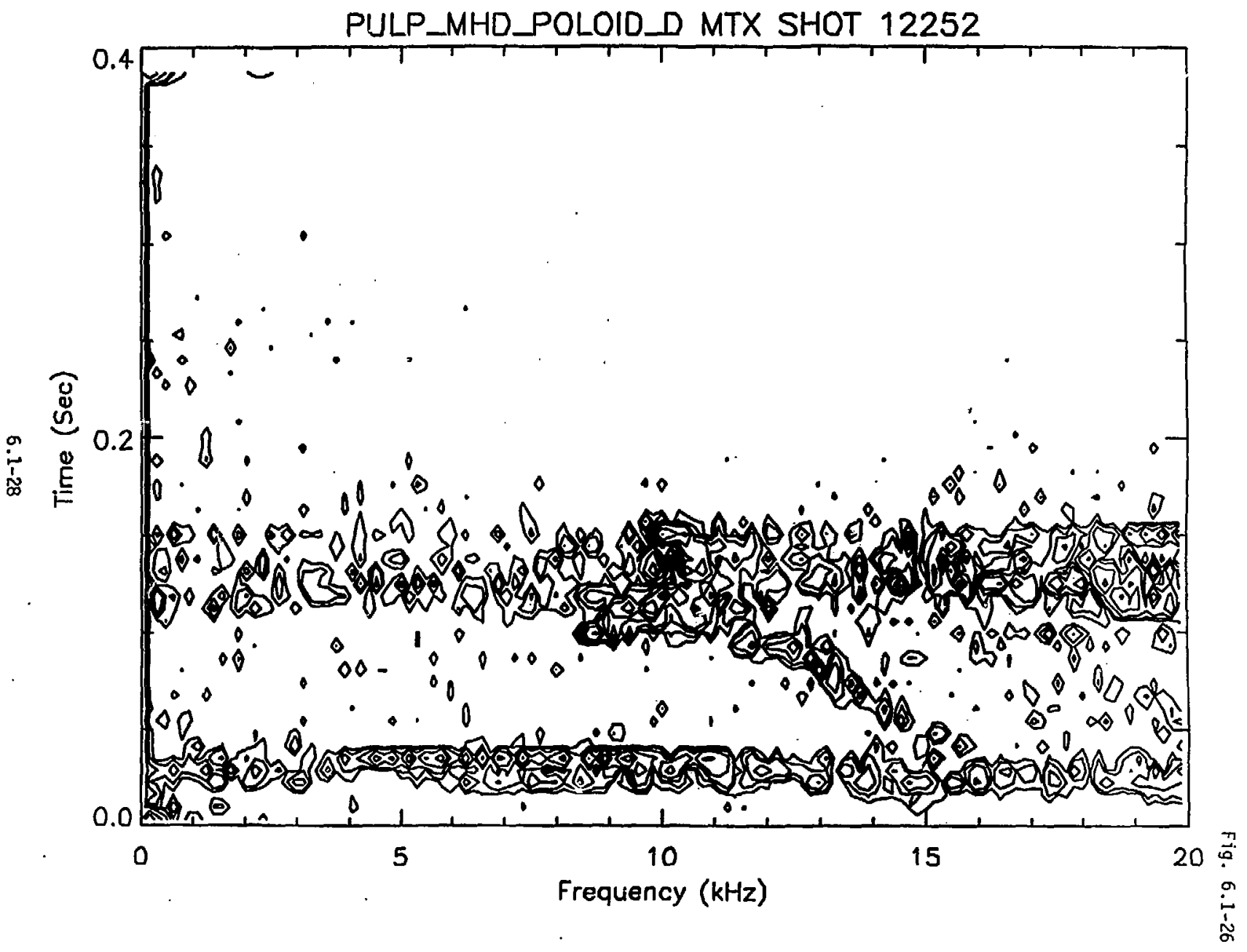




\section{Other experiments}

7.1 Poloidal Field Measurements During Sawteeth and Disruptions on MTX (B. W. Rice and E. B. Hooper)

B. W. Rice and E. B. Hooper, "Poloidal Field Measurements During Sawteeth and Disruptions on MTX," submitted to Nucl. Fusion. 


\title{
Poloidal Field Measurements During Sawteeth and Disruptions on MTX
}

\author{
B. W. Rice and E.B. Hooper \\ Lantence Livermore National Laboratory
}

\begin{abstract}
A fifteen chord far-infrared polarimeter has been used to measure internal poloidal field profiles in the Microwave Tulamak Experiment (MTX) during sawteeth, disruptions, and electron cyclotron heating (ECH). We preeent a brief overview of the instrument and tochniques used to analyze the profile data, followed by experimental revults. Polarimeter current density $(J)$ measurements are shown to be in good agreement with the expected resistivity profile based on $T_{e}$ measurements. Measurements of safety factor ( $q$ ) profiles during sawtoothing ohmic dischargets are presented ind are compared with various sartooth models. We find $q_{0} \sim 0.7-0.85$ during sawteeth and $q_{0}>1$ without swwteeth. The change in $q_{0}$ during the sawtooth ramp is small at $6 q_{0} \sim 0.01$. Finally, data are presented for ohmic discharges with higher core impurity radiation levels which produces hollow $T_{e}$ and $J$ profiles, and often leads to discuptions. This type of disruption has been stabilized on MTX by repeaking the $J$ profile through electron cyclotron heating in the core.
\end{abstract}




\section{Introduction}

Measurement of the poloidal field, $B_{p}$ in a tokamak, along with the related current density, $J$, and safety factor, $q \sim r B_{T} / R B_{p}$, is critical for a complete understanding of the magnetohydrodynamic (MHD) stability properties of the plasma. The sawtooth instability [1] and maior disruptions are two examples of MHD phenomena which have been observed on essentially all tokamaks for many years now, yet remain poorly understood. In addition to MHD effects, recent current-ramp experiments have shown that the shape of the current density profile also affects the transport properties of the plasme. Thus, poloidal Geld measurements will be especially important in next-generation tolamaks, where non-inductive current drive techniques will be used for steady-state current drive and to modify the $J$ profile to control MHD instabilities and improve plasma confinement.

In this paper, we describe poloidal field measurements obtained from the operation of a 15 channel far-infrared (FIR) polarimeter on the Microwave Tokamak Experiment (MTX). We present measurements of the $q$ profile during sertooth activity, as well as the profile evolution prior to major disruptions. The stabilization of distuptions with Electron Cyclotron Heating (ECH) is also demonstrated.

Although it is generally agreed that sawteeth are caused by a rapidly growing $n=m=1$ internal kink that results when $q_{0} \leq 1_{1}$ the details of the trigger mechenism and rapid crash are not well understood. The original sawtooth model developed by Kadomtsev [2] is based on the resistive reconnsction of magnetic surfaces. Here the growth of the $m=1$ island is terminated by a rapid reconnection of magnetic flux inside the $q=1$ surface, resulting in the oboerved $T_{e}(0)$ crash and a return of $q_{0} \geq 1$. Early measurements of sawtooth characteristies on smaller circular crass-section machines gave reasonable agreement with this model; however recent measurements $i \mathrm{~L}$ larger tokamaks show many discrepancies, including faster crash times, successor oscillations, and indications of $q_{0}<1$ after the crasin. This has prompted the development of numerous other sawtooth models based on turbulence [3], stochasticity $[4,5,6,7,8]$, quasi-interchange [9], and double-layer reconnection [10].

Clearly, detailed measurements of the $q$ profile during sawteetb, and the change in $q$ at the crash, would help distinguish between the various thearetical models. Models based 
on full magnetic reconnection or quasi-interchange require $q_{0} \sim 1$ after the crash, while other models such as the stochastic or double-layer reconnection allow $q_{0}<1$. Recent Faraday rotetion measurements on TEXTOR $[11,12]$, JET [13], and initial results from MTX [14] show an on-axis safety factor of $90 \sim 0.7-0.8 \pm 0.1$ in contradiction with the complete magnetiv reconnection and quasi-interchange models. Motional Stark Effect (MSE) measurements made on DIII-D [15] and TFTR [16] also show $q_{0} \sim 0.7-0.8 \pm 0.1$, although recent multichannel MSE results from DIII-D indicate $g_{0}$ closer to one [17]. The perturbation to qo during the sawtooth ramp was obtained in some of these measurements and was found to be small at $\delta g_{0}<0.1$ as expected considering the long current diffusion times.

Like the sawtooth instability, major disruptions are another type of MHD phenomena not well understood, mainly because they occur over a broad range of operating regimes and often display different charscteristics. The empirical Hugill diagram [18] is used to help identify the disruption-free operating regime in tokamaks. There are two boundaries in this plot, a low $q$ boundary at $q=2$, and a density limit represented by the parameter $\bar{n} R_{0} / B_{T}$. The ares inside these limits represents the usual disruption-free operating space. Several experimental studies have shown that the $m=2$ mode plays a role in the disruptions that occur near these boundaries.

In addition to the disruption regimes on the Hugill plot, disruptions can also occur occasionally throughout the remaining normally stable regime. For example, locked-modes have been observed on many machines [19] and can lead to disruptions, especially at low density. Another disruption mechonism is thermal instability due to high impurity radiation from the plasma core $[20,21]$. On MTX, high-Z impurities such as molybdenum (the limiter material) sometimes collect in the core. If the line radiated power exceeds the ohmic input power, the central temperature begins to collapse leading to hollow $T_{e}$ and $J$ profiles. Since the $q$ profile is no longer monotonic in this case, the overlap of islands on closely-spaced rational surfaces can lead to a disruption.

Our polarimetry measurements focus on this last type of disruption; since the hollowing of the $J$ profile prior to the disruption occurs on a relatively slow time scale (compared with the disruption itself), reliable measurements can be made during the collapse. Also we prestint cases where central ECH has been applied to repeak the $T_{e}$ and $J$ profiles, thus 
suppressing the disruption.

The paper is organized as follows. The experimental apparatus is described briefly in Section 2. Readers interested in details of the instrument design are referred elsewhere [14, 22]. An overview of the density and Faraday rotation profile inversion methods is outlined in Section 3, with details given in Appendix A. The experimental results for sawtoothing discharges are presented in Soction 4, while disruptions and ECH are covered in Section 5.

All experimental measurements were made on MTX (originally the Alcator-C machine) at toroidal fields of $5 \mathrm{~T}$ to $6.5 \mathrm{~T}$ with a major radius of $64 \mathrm{~cm}$ and a minor radius of $16.5 \mathrm{~cm}$. The plasma was operated with a circular molybdenum limiter and $\mathrm{D}_{2}$ gas fueling. The only form of auxiliary heating used in this work is $200 \mathrm{~kW}$ ECH (gyrotron) at $140 \mathrm{GHz}$.

\section{Experimental Apparatus}

Polarimetry makes use of the Faraday rotation effect in far-infrared (FIR) beams propegating through a magnetired plasma to determine the internal poloidal field. The general relations describing the evolution of the state of polarization were developed by Segre and De Marco $[23,24]$. For the plasma parameters on MTX $\left(B_{T}=5-8 \mathrm{~T}, I_{p}=300-400 \mathrm{kA}\right.$, and $n_{e}=1-3 \times 10^{20} \mathrm{~m}^{-3}$ ), the Faraday rotation angle for an FIR beam of wavelength $\lambda$ propagating the in poloidel plane reduces to the simple expression (MKS units),

$$
\Psi(x)=2.62 \times 10^{-1 s} \lambda^{2} \int B_{x}(x, z) n(x, z) d z
$$

where $B_{x}$ is the vertical component of the poloidal field parallel to the FIR beam, $n$ is the plasme density, and $x=R-R_{0}$. If we now pass several parallel chords through the plasme, or equivalently send a contimuous beam imeged onto an array of detectors, the Faraday rotation profile can be determined. Knowing the density $n(x, z)$ independently from interferometry, the above equation can be inverted to give the poloidal feld profile.

Unfortunately, this bas traditionally been a difficult measurement because the rotation angle is small and many measurement chords are required to accurately invert the profile. The first polarimeter system to operate routinely, with enough chords (9) to obtain the poloidal field profile, was constructed by Soltwisch on TEXTOR [25, 26]. A similar 6 chord system was installed on JET. 
On MTX, we have installed a 15 chord inteferometer/polarimeter system with a minimum chord spacing of $1.5 \mathrm{~cm}$ and a polarimeter frequency response up to $\sim 1.8 \mathrm{kHz}$, providing the best spatial and time resolution obtained to date. The viewing geometry is shown in Fig. 1. Because of blockage in the ports, the 15 chords are not spaced evenly across the plasme, but are grouped into three sections as shown. The Faraday rotation angle is determined using a new technique based on phase measurements of a rotating polarization ellipse [14, 22]. The main advantages of this technique are simplicity, low cost, and, most importantly, a reduction in the errors due to signal amplitude variations caused by refraction. An FIR wavelength of $185 \mathrm{~mm}$ is used giving the best tradeoff between rotation angle and refraction. At this wavelength, typical Faraday rotation angles on MTX are 5-15 . The instrument resolution during a beckground magnet shot is $\pm 0.18^{\circ}$ at a bandwidth of $1.5 \mathrm{kHz}$.

\section{Profile Inversion Technique}

Determination of the density and poloidal field profiles requires simultaneous inversion of the line-integrated interferometer and polarimeter date. Although the well-kown Abel inversion technique can be used in a pure cylindrical geometry, it is not well-suited to handle asymmetries due to the toroidal effects (oftset Bux. surfaces), which are quite significant for the polarimetry date. Other complicating effects that should be incorporated in the inversion algorithm include FIR beam refrection, plasma position offsets, and density variations on flux surfaces near the edge. Non-circular flux surfaces are not generally encountered on MTX and are not considered here. There are also some higher-order corrections to Eq. 1 that become important at large toroidal and poloidal fields (or longer $\lambda$ ) as discussed by Segre [24]; these are small in the present operating regime on MTX and will be neglected.

For results presented in this paper, we have developed two inversion techniques that satisfy the requirements stated above. They are discussed briefly here, with full details given in Appendix A.

The first is a spline-fit/matrix-inversion technique along the lines suggested by Soltwisch [25] and Park [27]. A flux surface geometry is set up, consisting of circular flux surfaces offset by the Shafranov shift function $\Delta(r)$ (see Fig. 16 in Appendix A). Although $\Delta(r)$ depends 
on the profiles of pressure and $B_{p}$ which gre not yet known, an initial estimate for $\Delta(r)$ is made and subsequently improved through an iterative approach. The flux surfaces are divided into a number of equally-spaced rings so that line-integrals can be represented by a matrix miltiplication, i.e. the value at the ring midpoint times the path length through the ring. The resulting matrix equations are then inverted to yield the desired density or poloidal field. This method is yuite general, allowing any profile shape to be obtained. It does, however, require a smooth spline fit of the measured chord dats onto the constructed grid.

A second technique used here is a tittiug method, where the desired quantities $n(r)$ and $J(r)$ (or $g(r)$ ) are represented by functions with free parameters. Given the flux surface geometry, these functions can be line-integrated and compared with measurements. The free parameters are then adjusted to give a least-squares fit to the measured data. The advantage of this method is that no spline fit is required and the smoothness of the function can be controlled by limiting the number of free parameters. The disadvantage is that with a limited number of free parameters, it is often difficult to fit the wide variety uf profile shapes encountered.

Since the final calculated profiles can vary depending on the inversion method used, we have developed a flexible analysis cods which allows different inversion techniques to be used on the same data for comparison. Likewise, effects such as density variations on a fux surface or FIR beam refraction can be turned on or off to judge the effect on the final results. In this way, the uncertainties associated vith the inversion methods can be assessed.

\section{Sawtoothing Discharge Results}

A typical profile analysis using the spline-fit inversion tochnique is shown in Fig. 2 for a single time point during the current flattop. This is a standard sawtoothing ohmic discharge with $B_{T}=5 \mathrm{~T}$ and $I_{p}=330 \mathrm{kA}$. The measured data are represented by the points, while the solid lines give the fit and the resulting inverted profiles. The weighting for each Faraday rotation point is the inverse of the error bars shown (some error bars are smaller. then the circles). The enror bars on most channels are given by the standard deviation $\sigma$, whis:. is : 
determined from the baseline noise level. Channels near the plasraa edge, or the slot walls, are given somewhat larger error bars to account for larger fluctuations and measurement uncertainty. The value of $q$ on axis for this timeslice is 0.82 , which is typical of most obmic discharges on MTX where $q_{0}$ falls in the range of $0.7-0.9$. As will be discussed in more detail later, the uncertainty in $q_{0}$ is estimated to be \pm 0.08 or abcut $\pm 10 \%$.

As a general test of the validity of the polarimeter data, the current density profile can be sompared with the conductivity profile during the $I_{p}$ flattop where the electric field has completely diffused in. In. the cylindrical approximation, $J(r)=V_{\mathrm{kop} o} \sigma(r) /\left(2 \pi R_{0}\right)$ where $V_{\text {loop }}$ is the plasma lnopp voltage. The classical Spitzer [28] cotductivity $\sigma$ is given by

$$
1 / \sigma=1.65 \times 10^{-9} \frac{\operatorname{in} \Lambda Z_{a} N(Z)}{T_{e}^{3 / 2}} \Omega \cdot m \quad\left(T_{c} \text { in } \mathrm{keV}\right)
$$

where $\ln \Lambda$ is the coulomb $\operatorname{logarithm}(\ln \Lambda \approx 15$ on $M T X)$ and $N(Z)$ is a function dependeat on the impurity charge state $Z_{\text {ar given approximately by }}$

$$
N(Z)=0.58+\frac{0.91}{1.08+Z_{\text {off }}}
$$

Using $T_{\boldsymbol{f}}$ from the ECE Michelson interferometer and $Z_{\text {eff }}$ from visible bremsstrablung measurements (we assume $Z_{\text {ef }}$ to be constant across the plasma), this expression for $J$ is compared $\$$ ith the measured $J$ profile in Fig. 3 , where good agreement is observed. The effect of sawteeth is smoothed out by averaging over several cycles. The neoclassical correction to $\sigma$ has been calculated using the ONETWO [29] transport code. However, MTX plasma parameters are in the plateau collisional regime where the trapped particle fraction is small, typically reaching a maximum of 0.2 at $r / a \cdot 0.5$. Given the uncertainties in the $J, T_{e}$ and $Z_{a}$ measurements (all about $\pm 10 \%$ ) and the complicating effects of sawteeth, we cannot clearly distinguish small neoclassical corrections to the Spitzer conductivity.

In Fig. 4, the time history of the polarimeter $q_{0}$ is plotted along with the plasma current, density, and electron temperature. In this plot, $q_{0}(t)$ is smoothed to less than the sawtooth frequency. This diacharge was chosen for illustration because a current ramp is initiated from 0.2-0.25 sec causing a transient increase in $\ell_{i}$ and an eventual rise in $q_{0}$, leading to the termination of sawteeth. As expected, we see that $q_{0}$ drops below one when the sawteeth oscillations begin, reaching an equilibrium value of $\sim 0.8$, and rises above one is the 
sawteeth stop during the current ramp. Despite the existing uncertainty in the theoretical understanding of sawteeth, it is generally agreed that a necessary condition for sawteeth is $q_{0} \leq 1$. Thus the good correlation between the time history of $q_{0}$ and the presence of sawteeth belps alleviate concerns that some systematic error may be affecting the measurement. This has beea checked for many obmic discharges, and to within an uncertainty of $\delta q_{0} \sim \pm 0.08$, $q_{0}$ always drops below unity et the start of sawtooth activity. No discharges with $q_{0}<1$ without sawteeth have been observed on MTX.

The location of the sawtooth inversion radius, $r_{\text {war }}$, has been obtained using both polychromator measurements of $T_{e}$ and interferometer measurements of $p_{e}$ (the $n_{e}$ inversion radius is correctly determined from the inverted $n_{e}$ data not the line-integrated date). In Fig. 5 , this date is plotted against the polarimeter $q=1$ radius $T_{q 1}$. The data. was taken from 9 different discharges at times between $0.1-0.3 \mathrm{sec}$. The uncertainty in $r_{q 1}$ and $r_{\text {iny }}$ is estimated at $\pm 0.5 \mathrm{~cm}$. Although $r_{q}$ and $r_{\text {iar }}$ egree quite well in the range whrre $r_{q 1}<4$ cm (typically earlier in the discharge), these is poorer agreement for larger $r_{q 1}$ where $r_{\text {bov }}$ is about $70-80 \%$ of $r_{q 1}$. Given the measurement uncertainties, it is difficult to judge the significance of the difference between $r_{11}$ and $r_{\text {bry. }}$. Sawtooth models developed to date predict the sawtooth inversion radius to be located at the $q=1$ radius. It is possible that the $q=1$ radius is changing during the saptooth crash on a time scale too fast to detect. However, we rote that recent polarimetry measurements of the $q=1$ radius on TEXTOR [30] show that $r_{i e n}$ is $\sim 15 \%$ smalles than $r_{q 1}$ similar to our results.

\subsection{Analysis of Systematic Error in $q_{0}$ Measurement}

Since the questior: of whether $q_{0}$ is equal to or significantly less than one is 80 critical in the understanding of sawteeth, and since conflicting measurements of $q_{0}$ have been reported, we provide bere a stildy of the systematic uncertainty in $q_{0}$ resulting from Faraday rotation measurements. The uncertainty in $g_{0}$ due to signal noise is only \pm 0.04 at the maximum bandwidth of $1.8 \mathrm{kHz}$ (see next section), and this can be reduced further by filtering or smoothing. A larger systematic uncertainty is associated with the inversion methods.

It is useful to diverge for a momant to consider how the Faraday rotation profile is related to $q_{0}$ and what leads to uncertainty in $q_{0}$. The slope of the Faraday rotation profile near the 
magnetic axis is approximately proportional to $1 / q_{0}$. This is shown by writing the Faraday rotation angle in the cylindrical approximation and substituting $B_{\theta}=\tau B_{T} / q R_{0}$, giving

$$
\Psi(x)=\frac{c_{p} B_{T} x}{R_{0}} \int \frac{\pi}{q} d z
$$

where $I=R-R_{0}$ and $c_{p}$ is a constant. To illustrate scalings, assume a density profile $n=n_{0}\left(1-(r / c)^{2}\right)$ and a $q$ profile $q=q_{0}\left[1+\left(q_{a} / q_{0}-1\right)(r / a)^{\alpha}\right]$, where $q_{a}$ is the edige safety factor. For small $x$, the Faradey rotation integral is written approcimately as

$$
\Psi \approx \frac{c_{p} B_{T} x n_{0}}{R_{0} g_{0}} 2 a \int_{0}^{*} \frac{1-(z / a)^{2}}{1+\left(q_{a} / q_{0}-1\right)(z / a)^{\alpha}} d z / a \text {. }
$$

The slope at the origin is

$$
\left.\frac{d \Psi}{d x}\right|_{x=0} \approx \frac{s B_{T} n_{0} a}{n_{0} q_{0}} f(\alpha),
$$

where $f(\alpha)$ represents the above integral function. Since the integral is heavily weighted toward small $z$, the value of $f(\alpha)$ is not very dependent on the $q$ profile parameter $\alpha$, varying by less than $20 \%$ over the range $3<q_{a} / q_{a}<5$ and $2<\alpha<4$. Considering $f(\alpha)$ roughly constant, we have the result

$$
s_{0} \propto n_{0} /\left.\frac{d \Psi}{d x}\right|_{x=0} .
$$

Thus, uncertainties in $q_{0}$ are most directly related to uncertainties in $n_{0}$ and the slope of the Faraday rotation profile through the origin. With six chords located near the plasma center, we expect $n_{0}$ and $d \Psi / d x$ to be quite accurately determined.

The dependence of $q_{0}$ on the inversion method has been studied by inverting the same data with different techniques. For example, the data shown in Fig. 2 has been inverted using the parametrized fitting function method, representing $J$ as the product of a 3-term (even terms only) Hermite polynomial with a Gaussian. In this case the fit was slightly better with $x^{2}=0.78$ (versus 1.0 for Fig. 2), and $q_{0}$ was 0.77 , a change of 0.05 from the spline fit. Similarly, the data was also fit using a parametrized form for $q$ which forces $q_{0}=1$ unside the sawtooth inversion radivs. In this case, $x^{2}$ increased to 2.5 and the fit through the central chords was noticeably worse. For most sawtoothing discharges, we find that forcing $g_{0}=1$ results in a fit which is outside of reasonable error bars on the central chords.

Another source of error is uncertainty in the density profile, and no specifically, as seen in Eq. 7. One problem which has been investigated extensively is small offsets or asymmetries 
in the density profile and the resulting effect on inverting the polarimetry data. In Fig. 2, note that the fitted line density profile is shifted by about $0.5 \mathrm{~cm}$ towards the inside relative to the measured data. This type of offset is frequently observed. The geometry of the flux surfaces is determined from the polarimeter data analysis and the $\Delta(r)$ calculation, so if $n$ is assumed to be constant on a flux surface, then there is no freedom to shift the density profile to obtain a better fit. The offset cannot be due to alignment problems between the two instruments since the polarimeter and interferometer share the same chords.

Instead ve believe there are two contributing factors to the offset: 1) slightly non-circular flux surfaces due to the nonuniform equilibrium vertical field and; 2) small density variations on the edge flux surfaces. Poloidal density asymmetries behind the limiter were observed previously on Alcator-C [40].

Our main interest here is whether such density asymmetries significantly affect the inferred velue of $g_{0}$. Using the methods described in Appendix A, the date from Fig. 2 is inverting assuming a first-order density correction which varies as a $\delta n(r) \cos \theta$. The result is shown in Fig. 6, where a better fit to the density data is obtained. Fortunately, the Faraday rotation fit is only slightly affected by including this correction, with $q_{0}$ femaining almost unchanged at 0.81. The function $\delta n(r)$ is shown on the lower left; it is small at the center and larger toward the edge, which agrees with the belief that poloidal asymmetries should be confined to the edge. The maximum perturbation is about $8 \%$ of the peak density.

In summary, by applying the various inversion methods described above to many different discharges, we estimate the uncertainty in $q_{0}$ due to systematic error to be $\leq \pm 0.08$ or about $\pm 10 \%$.

\subsection{Fast Time Resolution $q_{0}$ Measurements}

In addition to the equilibrium value of $q_{0}$, it is of great interest to know the detailed time evolution of $q_{0}$ during the sawtooth cycle. The time history of $q_{0}$ at the maximum polarimeter frequency response of $\sim 1.8 \mathrm{kHz}$ (5 kHz sampling rate) is shown in Fig. 7 .

First, note the relatively large randomly polarized spikes in $q_{0}$ which are correlated with the sawtooth irash. It was initially believed that this was a real poloidal field effect since the density change is relatively small and regular. However, we have shown that the spikes are a 
suotle instrumental effect due to refraction perturbations associated with the rapid density crash. The Faraday rotation measurement technique used here relies on the phase measurement of a rapidly rotating polarization ellipse (see Ref. [14]). In general, this technique has the advantage of being insensitive to waveform amplitude changes caused by refraction. In this case, though, the refraction-induced signal amplitude change triggered by the sawtooth crash is very rapid ( 20ps), occuring in-less than one cycle of the polarimeter waveform

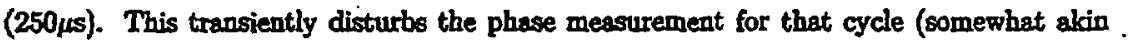
to the pre blem of interferometry phase jumps during pellet injection). The random spike polarity is caused by the random phase of the sastooth crash relative to the polarimeter waveform.

The data is valid immediately following the spike, however, where $q_{0}(t)$ remains essentially constant during the sawtooth ramp. Neglecting the spikes, the standard deviation in $q_{0}$ due to noise is \pm 0.04 at this bandwidth. By summing many savtooth cycles together, the noise is reduced and a very slight drop in $q_{0}$ is observed during the wtooth ramp of $\delta q_{0} \sim 0.01$. The expected change in $q_{0}$ due to magnetic diffusion is given roughly by [9]

$$
\delta q_{0} \sim \frac{T_{e}}{T_{r}} \frac{\delta T_{e}}{T_{e}}
$$

where $\tau_{r}=\mu_{0} \sigma_{0} \tau_{Q 1}^{2} / 4$ is a characteristic resistive diffusion time and $\tau_{\text {, is }}$ is the sawtooth period With $T_{s}=4.5 \mathrm{~ms}, T_{r}=30 \mathrm{~ms}$, and $\delta T_{e} / T_{e}=0.08$, we have $\delta q_{0} \sim 0.01$ in agreement with observations.

The conclusion is that $q_{0}$ remains well below one throughout the sawtooth cycle. This appears to be in contradiction with the Kadomtsev and quasi-interchange models which require $g_{0} \approx 1$ after the crash. The stochastic and double-layer reconnection models allow for $q_{0}<1$ throughout the cycle. There are several variations of the stochastic model, but genesally it is postulated that stochasticity arising from the overlap of second-order islands in a region surrounding the $q=1$ surface leads to the sawtooth crash and prevents full reconnection from occurring. After the crash, a zero-shear pedestal at the $q=1$ surface could stabilize the $m=n=1$ mode, even with $q_{0}<1[5,6]$. The evaciation of pressure from the core may also play a role in stabilizing the mode after the crash [7]. In the doublelayer reconnection model [10], the conservation conditions for magnetic flux and plasma 
volume are considered for two reconnecting layers rather than one. In this case, it is shown that full magnetic reconnection can take place with $q_{0}<1$.

We note that there are no indications of $m=1$ postcursor oscillations on MTX, so assuming $q_{c}<1$, then some mechanism- such as a zero-shear pedestal, or pressure effectsmust exist to suppress the $m=1$ mode after the sawtooth crash.

\subsection{Non-Sautoothing Discharges}

During clear, hirh current $\left(q_{e} \sim 2.5-4\right)$ ohmic discharges on MTX, sawtooth oscillations are usually present. But if the density of heavy-metal impurities is too high, large radiated posver associated with the impurities cools the plasma core resulting in $q_{0}>1$. In this section, we examine this behavior by analyzing two neighboring discharges, one with sawteeth (22625) and one without sawteeth (12624), as shown in Fig. 8. In discharge 12625, the sawtisth instability begins at $80 \mathrm{~ms}$ in good agreement with the time when $q_{0}=1$. Discharge 12624 shows $T_{e}(0)$ about $25 \%$ less than 12625 and no sawteeth, consistent with $q_{0}>1$ throughout the discharge.

The most striking difference in 12624 is the increased radiated power from the core $\left(P_{\text {rod }}\right)$ which keeps the central $T_{e}$ lower and $q_{0}$ higher. Measurements of $Z_{\mathrm{ed}}$ for the two shots gives a similar value of $\sim 1.2 \pm 15 \%$. Therefore, the increased radiated power must be due to line radiation from heavy impurities. EUV spectrometer measurements indicate that molybdenum, the limiter material, is the dominant impurity.

A comparison of $T_{e}, n$, and Faraday rotation profiles for the two shots at $175 \mathrm{~ms}$ is presented in Fig. 9. The "bole" in the $T_{e}$ profile due to the increased radiated power is clearly visible for shot 12624. The profile is not simply hollow, but has an unusual asymmetry. The density appears to be reasonably symmetric but more sharply peaked in shot 12624 . The Faraday rotation data for 12624 is shifted to inside slightly and also shows a larger asymmetry than the sawtoothing discharge.

The asymmetry in the Faraday rotation data is seen more readily when attempting to invert the profile as shown in Fig. 10. Some asymmetry is expected in the Faraday rotation profile due to toroidal effects as seen from Eq. 18, where the degree of asymmetry depends on the $\Delta(r)$ profile. In Fig. 10, however, the asymmetry is too large to fit using the standard 
$\Delta(r)$ calculation.

The asymmetries observed here are puzzling, but occur quite repraducibly during nonsawtoothing discharges with high impurity radiation. Generally, the thermal conductivity along field lines is so large that flux surfaces can be considered constant $T_{e}$ surfaces. The asymmetry in $T_{e}$ then implies either a large asymmetric power sink-which could be due to an asymmetric distribution of heavy impurities-or a distortion of the magnetic flux surfaces awry from the standard assumption of offset circles. A combination of these two effects is also a possibility.

We have explored different flex surface geometries by allowing the $\Delta(r)$ profile to very in the inveraion algorithm. Indeed, better fits to the date can be obtained with this method, but there is no physical basis for the resulting $\Delta(r)$ profiles (i.e., they do not satisfy Eq. 14). Fortunately, the value of $g_{0}$ is not affected significantly by these changes in $\Delta(r)$, varying by less than $10 \%$ and always remaining well above one.

The reason for the increased impurity level in discharges like 12624 is not clear, but probably results from subtle differences in the breakdown and early current-ramp phose of the discharge. We note in Fig. 8 that $T_{e}(0)$ evolves quite differently during the first $50 \mathrm{~ms}$. This type of behavior has been reported previously in tokamaks with metal limiters such as PLT using tungsten limiters $[20,21]$ and on Alcator-C with molybdenum limiters [31]. The problem was especially prevalent at low densities where reduced gas puffing at the edge led to bigher edge temperatures, resulting in the generation of more limiter impurities.

\section{Disruption Measurements}

If the impurity level in the plasma core continues to increase, the radiated power can exceed the input ohmic power leading to a thermal instability followed by a distuption. An example is shown in Fig. 11. The disruption occurs at $0.283 \mathrm{sec}$ and is preceded by a drop in $T_{e}(0)$, which raises $q_{0}$ above one and stops the sawtooth activity. It is clear from the bolometer trace that the power radiating from the core is increasing rapidly causing the temperature collapse.

Polarimeter profiles are shown in Fig. 12 at $200 \mathrm{~ms}$ and $279 \mathrm{~ms}$. The profiles at $0.20 \mathrm{sec}$ 
are representative of the sawtoothing portion of the discharge, and are typical of most ohmic discharges with a Gaussian $J$ profile. The only unusual feature at this time is the relatively poor fit to the inside three points (compare with Fig. 2). Low level $m=2 \mathrm{MHD}$ activity is observed on magnetic loops at this time, consistent with tearing mode stability analysis for the peaked $J$ profile. During the temperature collapse at $0.279 \mathrm{sec}$, the $J$ profile has become hollow, following the $T_{e}$ profile. The safety factor is above one and there are no sawteeth at this time. In addition to being hollow, the $T_{t}$ profile shows an asymmetry similar to the non-sawtoothing discharge discussed in the last section.

Considering the long global current diffusion time of $\sim 200 \mathrm{~ms}$, it is reasonable to ask if the $J$ profile can become hollow during time $20-30 \mathrm{~ms}$ prior to the disruption due to resistive diffusion alone, or if some anomalous diffusion process is required to explain the hollow $J$ profile. Defining a "local" resisitive diffusion time as $\tau_{\tau}=0.5 a_{t}^{2} \sigma_{0} \mu_{0}$, where $a_{t}$ is some characteristic core radius and $\sigma_{a}$ is the on-axis conductivity, and using $T_{e} \sim 700 \mathrm{ev}$ and $a_{t} \sim 4 \mathrm{~cm}$, gives $\tau_{\mathrm{r}} \sim 18 \mathrm{~ms}$, indicating that $J$ can become hollow on this time scale. This result has also been confirmed using a numerical cylindrical difusion model.

The initial minor disruption cocurs at $283 \mathrm{~ms}$ followed by a major disruption about $10 \mathrm{~ms}$ later. Since only low-level MHD ectivity is observed during the time 250-280 ms, it is clear that only radiation, not MHD fluctuations, is responsible for the hollow $T_{e}$ and $J$ profiles. The minor disruption is triggered by a rapid MHD event, however, and is most likely due to the unstable hollow current distribution. Analysis of the ECE polychromator data immediately following this event shows that rapid heat transport in the core bas temporarily flattened the $T_{e}$ profile. Hollow current density profiles are known to be quite unstable [33], particularly to double tearing modes which result when the nonmonotonic $q$ profile allows more than one rational surface of the same mode number to be present in the plasma. As the q profile evolves, the islands on these closely spaced rationel surfaces can overlap and reconnect leading to a distuption [32]. It has long bsen thought that this mechanism leads to the enhanced current diffusion observed on many tokamalss during the initial current ramp when skin currents are present. Given the evolution of the $T_{e}$ profile from hollow to flat following the initial disruption, it is plausible that che reconnection associated with the double tearing mode is the dominant mechanism. Although a mode number could not be 
identified during this short MHD burst, judging from the $q$ profile in Fig. 12, the $m / n=3 / 2$ or $2 / 1$ modes are likely candidates. We also note that $q_{a}$ is quite low in the range of 2.5-3, and near the $q_{a}=2$ limit. which helps explain why the discharge could not sustain after the initial disruption.

More insight into the $T$, collapse can be gained by considering the electron power balance on axis

$$
\frac{3}{2} \frac{\partial}{\partial t}\left(n_{0} T_{\infty}\right)=\frac{3}{r} \frac{\partial}{\partial r} r\left(n \chi_{e} \frac{\partial T_{e}}{\partial r}+\frac{3}{2} T_{e} D \frac{\partial n}{\partial r}\right)_{0}-\frac{3}{2} \frac{n_{0}\left(T_{e 0}-T_{i 0}\right)}{T_{e l}}-P_{r}+P_{\mathrm{OH}} .
$$

where $P_{\mathrm{OH}}$ is the obmic power density and $P_{\mathrm{r}}$ is the radiated power density. During normal operation with peaked profiles, the source power $P_{\mathrm{OH}}$ is belanced by the loss terms on the right side of the equation. With fat or hollow $T_{e}$ profiles, however, the thermal diffusivity term and possibly the electron-ion exchange term become source terms on axis. Thus, changes in $\chi_{e}$ alone cannot generate hollow $T_{e}$ profiles. The increase in radiated power $P_{r}$ must be the dominant loss mechanism here. Bremsstrablung measurements indicate $Z_{\text {eff }}$ remains relatively constant at $\sim 1.2-1.5$, so the bolometer rediation power increase must be due to predominantly molybdenum line radiation. The peak ohmic power density prior to the collapse obtained from $\mathrm{J} \cdot \mathrm{E}$ is $\sim 7.5 \mathrm{~W} / \mathrm{cm}^{3}$. Figure 11 shows that by $0.25 \mathrm{sec}$ the radiated power density is indeed approaching this ohmic power density.

The line radiation power for molybdenum is expressed as

$$
P_{r}=n_{e} n_{z} f\left(T_{e}\right)
$$

where $n_{x}$ is the impurity density. The function $f\left(T_{e}\right)$ varies strongly with temperature and is calculated for molybdenum in coronal equilibrium by Jensen, et al., in Ref. [34]. Some values of $f\left(T_{e}\right)$ relevant to MTX are given in Table 1 . The molybdenum inpurity density at the beginning of the $T_{e}$ collapse can then be estimated using $n_{x}=P_{T} /\left(n_{e} 2 \times 10^{-25}\right)$, with $P_{\tau}$ in units of W/cm $\mathrm{cm}^{3}$. The bolometer radiated power at $0.25 \mathrm{sec}$ is $\sim 5 \mathrm{~W} / \mathrm{cm}^{3}$ and $n_{0}=2.5 \times 10^{14} \mathrm{~cm}^{-3}$, which gives a molybdenum density of $n_{z} \sim 10^{11} \mathrm{~cm}^{-3}$. This is slightly higher than to molybdenum densities measured previously on Alcator-C [31] for comparable plasma densities. 


\begin{tabular}{|ll|}
\hline$T_{c}(\mathrm{keV})$ & $\mathrm{f}\left(\mathrm{W}-\mathrm{cm}^{3}\right)$ \\
\hline 0.5 & $2 \times 10^{-25}$ \\
1.0 & $2 \times 10^{-25}$ \\
1.5 & $9 \times 10^{-26}$ \\
2.0 & $6 \times 10^{-25}$ \\
3.0 & $5 \times 10^{-26}$ \\
\hline
\end{tabular}

Table 1: Radiated power function $f\left(T_{e}\right)$ for molybdenum in coronal equilibrium, after Jensen, et al. [34].

\subsection{ECH Stabilization of Disruptions}

We have found that the radiative disruption described above can be stabilized through ECH heating as shown in Fig. 13. In this discharge, $T_{e}(0)$ begins to drop at $\sim 0.15$ sec. Although polarimetry data was not available on this shot, the characteristics of the collapse are identical to discharge 12540 analyzed above, so we expect the $J$ profile to become hollow in as similar manner. The initial disruption begins at $183 \mathrm{~ms}$, as evidenced by the drop in deasity, the notch in $I_{p}$, and the burst of MHD activity. A rapidly growing $m=2$ mode is observed beginning 3 ms prior to the disruption. Again the disruption probably results from the double tearing mode and the overlap of islands on adjacent $q=2$ surfaces. The overlap of the $q=2$ and $q=1.5$ ( $3 / 2$ mode) surfaces is also a possibility. A shott $5 \mathrm{~ms} 150 \mathrm{~kW}$ ECH pulse is applied $1 \mathrm{~ms}$ after the initial disruption. The toroidal field is tuned to $5 \mathrm{~T}$ placing the electron cyclotron resonance on axis at $140 \mathrm{GHz}$. ECH produces a rapid increase in $T_{e}(0)$, restoring sawtooth activity and allowing the discharge to continue normally. The discharge is stabilized because the rapidly peaked $T_{e}$ profile also peaks the $J$ profile, suppressing MHD modes associated with the hollow profiles.

The increase in $T_{e}(0)$ is accompanied by a drop in bolometer radiation as seen in Fig. 13 (the bolometer time response is only $10 \mathrm{~ms}$ ). Although a complete time history of molybdenum density $n_{z}$ is not available, it is reasonable to assume that $n_{z}$ remains roughly constant over the timescale of the $T_{e}$ collapse and repeaking ( $\sim 50 \mathrm{~ms}$ ). From Table $\mathrm{I}_{\mathrm{t}}$ the impurity radiated power drops by a factor of 2.3 as the temperature increases from 0.7 to $1.6 \mathrm{keV}$, indicating that the drop in bolometer radiation may be attributed to the change in $T_{e}(0)$ alone. It is interesting that after the ECH pulse, the temperature and bolometer radiation levels are 
not much different from earlier in the discharge, yet the $T_{e}$ collapse does not return after the ECH is turned off. Comparing the times $0.15 \mathrm{sec}$ and $0.24 \mathrm{sec}, T_{e}$ goes from $1.05 \mathrm{keV}$ to 1.2 $\mathrm{keV}$ while $P_{\mathrm{r}}(0)$ decreases from $2.5 \mathrm{~W} / \mathrm{cm}^{3}$ to $2.0 \mathrm{~W} / \mathrm{cm}^{2}$. So $P_{O H}=J E \propto T_{e}^{3 / 2} E$ increases by $22 \%$ while $P_{r}(0)$ decreases by $20 \%$. We conclude that this slightly more favorable power balance in the core prevents the thermal instability from recurring.

A second example of an ECH discharge that demonstrates significant change in the current density profile is shown in Fig. 14. In this discharge, high-impurity levels early in the discharge koep $T_{e}(0)$ very low almost from the start. The total current is about $100 \mathrm{kA}$ lower than usual. Strong bursts of MHD activity are observed during the time $100-155 \mathrm{~ms}$. The poloidal mode number is mainly $m=3$, consistent with the measured $q_{0} \sim 2.5-3$. Also, the confinement is poor as evidenced by the dropping density.

Because of the low density, the $200 \mathrm{~kW}$ on-axis ECH pulse initiated at $155 \mathrm{~ms}$ has a tremendous heating effect, increasing $T_{2}(0)$ to over $2.5 \mathrm{keV}$ (polychromator detector saturates). The temperature profile rapidly peaks, MHD activity stops within $3 \mathrm{~ms}$, and sawtooth activity returns. The central radiated bolometer power, which was as high as $3 \mathrm{~W} / \mathrm{cm}^{3}$ before the ECH pulse, drops to $1.2 \mathrm{~W} / \mathrm{cm}^{3}$ after the $\mathrm{ECH}$ pulse.

The time evolution of the polarimeter $J$ profile is shown in a surface plot in Fig. 15. For comparison, two time slices overlaying $J$ and $T_{e}^{1.5}$ before and after the ECH puise are also shown; the $T_{e}^{1.5}$ curve is scaled to match the polarimeter $J$. The uncertainty in $J$ is somewhat larger here than in previous discharges because the low density and current in this discharge gives a very small Faraday rotation angle. Nevertheless, there is a clear evolution of the $J$ and $T_{e}$ profiles from hollow to peaked following the ECH pulse. Again we would like to know if the rapid peaking of the current density following the ECH pulse is due to classical diffusion or some other anomalous process. Because of the high resistivity caused by the initial low temperature of $350 \mathrm{eV}$, the local diffusion time is small at $\tau_{\mathrm{r}} \sim 6 \mathrm{~ms}$ using a core radius of $a_{t}=4 \mathrm{~cm}$. The first $7 \mathrm{~ms}$ following the $\mathrm{ECH}$ pulse have been simulated with a simple cylindrical diffusion code us'ag measured temperature profiles: Snitzer resistivity, and $I_{p}$ for the boundary condition. Results show that the $J$ profile evolves from hollow to flat (or slightly peaked) in only $4 \mathrm{~ms}$, in qualitative agreement with polarimeter $J$ measurements and consistent with the suppression of MHD fuctuations within a few milliseconds after 
$T_{e}(0)$ begins to rise. The $J$ profile does not reach its fully diffused equilibrium state until about $50 \mathrm{~ms}$ later.

As with discharge 11783, the stabilization of the strong MHD activity is attributed to a rapid peaking of the $J$ profile. Unlike discharge 11783 , there is a large $T_{e}$ difference here when comparing before the ECH pulse at $0.14 \mathrm{sec}$ with after the pulse at $0.24 \mathrm{sec}$. Using Eq. 10 and Table 1, the molybdenum impurity density is $n_{z}=2.3 \times 10^{11} \mathrm{~cm}^{-3}$ at $0.14 \mathrm{sec}$, and remains roughly constant through the remainder of the discharge. So the drop in power radiated from the core, and the suppression of MHD activity, is mainly due to the large on-axis $T_{e}$ change induced through ECH.

\section{Conclusion}

Polarimeter measurements of poloidal field profiles have been used to investigate a variety of phenomena on MTX including sawteeth, disruptions, and electron cyclotron heating. Measurements show $0.7<q_{0}<0.9$ during sawteeth, with the change in $q_{0}$ during the sawtooth ramp being small at $\delta q_{0} \sim 0.01$ consistent with classical current diffusion. The results are in disagreement with the Kadomtsev reconnection and quasi-interchange models, but may be compatible with the stochastic or double-layer reconnection models. Measurements of the $J$ profile during the collapse of $T_{\varepsilon}$ in the core due to high- $Z$ impurity radiation shows the development of a hollow $J$ profile and nonmonotonic $q$ profile, which often leads to disruptions. The effectiveness of ECH heating in the core to suppress disruption was demonstrated. Peaking of the $T_{a}$ and $J$ profiles through ECH has the beneficial effect of reducing impurity line radiation from the core, as well as eliminating MHD modes associated with nonmonotonic $q$ profiles. 


\section{Appendix A}

\section{A Profile Inversion Methods}

The determination of density and poloidal field profiles requires simultaneous inversion of the line-integrated interferometer and pollarimeter signals

$$
\begin{aligned}
& \Phi(x)=c_{n} \int_{0}^{\infty} n(x, z) d z \\
& \Psi(x)=c_{s} \int_{0}^{n_{n}} B_{x}(x, z) n(x, z) d z,
\end{aligned}
$$

where $c_{n}$ and $c_{p}$ are constants, and $z$ is the vertical dimension parallel to the probe beam. As mentioned in the text, we have developed two methods for inverting these profi is: a spline fit/matrix inversion method and a parametrized fitting technique.

\section{A.1 Flux Surface Geometry}

Before discussing the actual inversion techniques, it is necessary to set up the flux surface . geometry on which the inversion will take place. In the large aspect ratic,$\left.R_{0} / a\right)$ expansion, the poloidal flux function $(\psi)$ in a circular cross section machine is give $\lrcorner$ by $[35,36]$

$$
\psi=\psi_{0}-\Delta(r) \frac{\partial \psi_{0}}{\partial r} \cos \theta,
$$

where $\theta$ is the poloidal angle. This ssiution describes concentric circular flux surfaces offset from the major radius of the vesel $R_{0}$ by the Shafranov shift $\Delta\left(r^{\prime}\right.$ given by

$$
\Delta(r)=\frac{1}{R_{0}} \int_{r}^{a}\left[\beta_{p}(r)+\frac{\ell_{(}(r)}{2}\right] r d r,
$$

where the poloidal beta $\beta_{p}(r)$ and internal inductance $h_{i}(r)$ are defined as

$$
\begin{aligned}
& \beta_{p}=-\frac{2 \mu_{0}}{B_{\partial_{0}}^{2}}\left(p(r)-\frac{2}{r^{2}} \int_{0} p r d r\right) \\
& \frac{\ell_{i}}{2}=\frac{2 \mu_{0}}{B_{b_{0}}^{2}} \frac{1}{r^{2}} \int_{0}^{r} \frac{B_{0_{0}}^{2}}{2 \mu_{0}} r d r .
\end{aligned}
$$

The inversion geometry is set up as shown in Fig. 16, using an initial guess for $\Delta(r)$. After the pressure and poloidal field profiles are determined, $\Delta(r)$ is recalculated and the 
inversion process is repeated. Note that $\mathrm{r}$ now represents the radius of a given flux surface and not the radius from the origin. The goal is to express Eqs. 11 and 12 in terms of flux surface quantities only. Equation 11 is unchanged except that now $n(r)$ is the density on a flux surface of radius $r$.

For Eq. 12, we follow the method used by Soltwisch $[25,26]$ and write $B_{x}$ in terms of the flux function $\psi$ as

$$
B_{x}=\frac{1}{R} \frac{\partial \psi}{\partial R}=\frac{1}{R_{0}+x} \frac{\partial \psi}{\partial r} \frac{\partial r}{\partial x} .
$$

Given $r^{2}=z^{2}+(x-\Delta(r))^{2}$, we can rewrite Eq. 12 as

$$
\Psi(x)=\frac{2 c_{p}}{R_{0}+x} \int_{0}^{x_{0}} n(r) \frac{\partial \psi}{\partial r}\left[\frac{x-\Delta}{r+\frac{\partial \Delta}{\partial r}(x-\Delta)}\right] d z .
$$

Expressing density is in units of $10^{20} \mathrm{~m}^{-3}$, and everything else in MKS units, the constant is given by $c_{p}=51.08$ for a wavelength of $\lambda=184.3 \mu \mathrm{m}$. All quantities in this equation are functions of the flux surface radius $r$ only.

- The toroidal current density can also be written in terms of flux surface quantitiea using

$$
\begin{aligned}
\mu_{0} J_{\phi} & =(\nabla \times B)_{\phi}=\frac{\partial B_{R}}{\partial z}-\frac{\partial B_{x}}{\partial R} \\
& =-\frac{\partial}{\partial z}\left(\frac{1}{R} \frac{\partial \psi}{\partial r} \frac{\partial r}{\partial z}\right)-\frac{\partial}{\partial R}\left(\frac{1}{R} \frac{\partial \psi}{\partial r} \frac{\partial r}{\partial x}\right),
\end{aligned}
$$

which leads to

$$
J_{\phi}=\frac{\text { Dnst }}{\left(R_{0}+x\right)} \frac{r^{2}}{w^{2}}\left[\frac{\partial u}{\partial r}+\frac{u}{r}\left(2-\frac{r}{w}\left[1-v^{2}+\frac{\partial v}{\partial r}(x-\Delta)\right]-\frac{w(x-\Delta)}{r\left(R_{0}+x\right)}\right)\right],
$$

where $u=\frac{\partial \psi}{\partial r}, v=\frac{\partial \Delta}{\partial r}$, and $w=r+v(x-\Delta)$. The safety factor $q$ is defined as

$$
q(r)=d \phi / d \psi=\frac{\partial \phi}{\partial r} / \frac{\partial \psi}{\partial r},
$$

where $\phi$ is the toroidal flux: In terms of flux surface quantities we have

$$
q(r)=\frac{R_{0} B_{\alpha_{0}}}{u}\left[v-\frac{\left(R_{0}+\Delta(r)\right) v-r}{\sqrt{\left(R_{0}+\Delta(r)\right)^{2}-r^{2}}}\right] .
$$

Note that in the limit $R_{0} \rightarrow \infty$ and $\Delta(r) \rightarrow 0$, the above relations return the. expected cylindrical plasma form. 


\section{A.2 Path Length Matrix}

Regardless of the inversion technique used, it is useful to define a path length matrix that is consistent with the flux surface geometry shown in Fig. 16. The poloidal cross section is dividea into a number (usually $m=50$ ) of equally-spaced radial ring zones of radius $\tau_{j}$ and offset $\Delta_{j}$. A set of $2 m$ integration chords located at $x_{i}$ are positioned at the intersection of each ring midpoint and the $x$ axis. A path length matrix $I$ is then constructed according to

$$
\begin{aligned}
L_{i j} & =2\left[\left(r_{j}^{2}-\left(x_{i}-\Delta_{j}\right)^{2}\right)^{1 / 2}-\left(r_{j-1}^{2}-\left(x_{i}-\Delta_{j-1}\right)^{2}\right)^{1 / 2}\right], \quad \text { for } r_{j}>x_{i} \\
& =2\left[\left(r_{j}^{2}-\left(x_{i}-\Delta_{j}\right)^{2}\right)^{1 / 2}\right], \quad \text { at midplane } \\
& =0, \quad \text { for } r_{j}<x_{i} .
\end{aligned}
$$

For quantities assumed to be constant on a flux surface, such as density, the vector of line integrals can be represented as the sum

$$
\Phi_{i}=c_{n} \sum_{j=1}^{m} L_{i j} n_{j}
$$

where $r_{j}$ is the density at the center of each ring. This reduces to the simple matrix equation

$$
\Phi / c_{n}=\mathbf{L} \cdot \mathbf{n} .
$$

This equation can now be used to perform the line irtegrals needed for the functional fitting technique or can be inverted directly to salve for $r\left(r_{j}\right)$ when using the spline fitting technique.

Similarly, for the polarimeter system, referring to Eq. 18, a new matrix $G$ is defined as

$$
G_{i j}=c_{p} L_{i j} n_{j} \frac{1}{R_{0}+x_{i}}\left[\frac{x_{i}-\Delta_{j}}{r_{j}+\frac{\partial \Delta_{j}}{\hat{\sigma}_{T}}\left(x_{i}-\Delta_{j}\right)}\right] .
$$

This matrix contains the path length information in $L$, the density profile in $n$, and the toroidal asymmetry effects in the term in brackets. The Faraday rotation profile is then given by the matrix equation

$$
\Psi=\mathbf{G} \cdot \mathbf{L}
$$

where $u_{j}=\left(\frac{\partial p}{\partial r}\right)_{j}$. The assumption here is that all quantities in $\mathbf{G}$ are known, so the only unknown in this equation is $u$. 
The above equations can easily be extended to include the possibility of small variations in density on a flux surface. This does not violate the requirement that pressure must be constant on a flux surface, although usually the parallel thermal conductivity is so large that temperature and density are each constant on a flux surface. An obvious violation of this is the MARFE [37] phenomenon where a region of low $T_{e}$ and high $n_{e}$ plasma is localized near the edge at a certain poloidal angle. Although the information on poloidal density variations is limited with a single view system, if we allow a small perturbation on each flux surface given by $\delta n_{j}$, and assume some type of poloidal distribution for this perturbed density, for example $\cos \theta$ (linear with $x$ ), the line-integrated density can be written as

$$
\Phi_{i}=c_{n} \sum_{j=1}^{m} L_{i j}\left(n_{j}+\frac{\left(\Sigma_{i}-\delta_{j}\right)}{r_{j}} \delta n_{j}\right) .
$$

Likewise, for the Faraday rotation the above term in parenthesis can be substituted into Eq. 27 for $n_{j}$.

\section{A.3 Refraction Corrections}

Refraction of the EIR beam due to density gradients in the plasma will change both the path length (phase shift) and the position of the probe beam relative to the geometry set up in Fig. 16. These effects are more severe on MTX than most machines because the small minor radius coupled with high density lead to large density gradients. For small refraction angles $(\alpha<.01 \mathrm{rad})$, the refraction angle for a chord located a distance $x$ from the midplane is given by

$$
\alpha(x)=-\frac{x}{r_{x}} \int_{x}^{a} \frac{\partial r}{\partial r} \frac{d r}{\sqrt{r^{2}-x^{2}}}
$$

where $n_{c}$ is the cutoff density $n_{c}=c^{2} \varepsilon_{0} \pi 4 \pi^{2} /\left(\lambda^{2} \mathrm{e}^{2}\right)$, which is equal to $326 \times 10^{20} \mathrm{~m}^{-3}$ at $\lambda=184.3 \mu \mathrm{m}$.

The MTX interferometer/polarimeter is somewhat unique in that the illumination beam is a continuous sheet (except for port rib. blockage) incident on an array of detectors. This means that cach detector is not vieming a fixed chord position $x$, but rather the measured chord can move in the plasma depending on the refraction angle as illustrated in Fig. 17. The path BA represents the no-plasma ray path, while CD represents the refracted ray path. 
The chord displacement from its nominal no-plasma position is $d=Z \sin \alpha$. In addition, the refracted chord path $A C$ is longer than the nominal chord path $A B$ by the amount

$$
\delta_{\text {path }}=Z-\frac{Z}{\cos \alpha} \approx Z\left(\frac{\alpha^{2}}{2}\right) \text {. }
$$

For a typical plasme with a parabolic density profile with $n_{0}=2.5 \times 10^{20} \mathrm{~m}^{-3}$, the maximum refraction angle is about $0.007 \mathrm{rad}\left(0.4^{\circ}\right)$ giving $d=1.2 \mathrm{~cm}$ and $\delta_{\text {pach }}=45 \mu \mathrm{m}$. Note that the chord offiset toward higher density (shorter path length for $\mu<1$ ) is partially cancelled by the increased path length $\delta_{\text {pack }}$.

Both the chord offset correction and the path length change are incorporated into the line density data before inversion. Since the $n(r)$ profile is needed to calculate the corrections, but it is not yet known, we adopt an iterative approsch. An initial guess for $n(r)$ is used to calculate initial refraction corrections. The profile is then inverted to get a second estimate for $n(r)$ and the process is repeated. Since refraction corrections are small, this iterative technique converges quickly.

The final chord offsets, calculated from $n(r)$ profiles, are also used to correct the polarimeter chord positions. The additional phase shift $\delta_{\text {path }}$ is not relerant for the polarimeter however.

\section{A.4 Spline Fit Inversion Technique}

Returning to Eqs. 25 and 26 , we that if $\Phi$ and $\Psi$ are known at the grid points $x_{i}$ then the desired profiles $n(r)$ and $u(r)$ are obtained by simply inverting the respective matrix equations. In general, the preferrod method for solving a set of linear equations is by singular value decomposition (SVD) [38]. However, since $L$ is essentially a triangular matrix, there is a more direct method as pointed out by Park [27]. Starting at the plasma edge, where there is only one path length element on each side of the plasme, the density in the outermost ring is written immediately as

$$
n_{m}=\frac{1}{2 c_{n}}\left(\frac{\Phi_{1}}{L_{1, m}}+\frac{\Phi_{2 m}}{L_{2 m, m}}\right)
$$

This density ring is then subtracted off $\mathrm{CI} \Phi_{2}$ and $\Phi_{2 m-1}$ and the above step is repeated to get $n_{m-1}$, and so on. This is equivalent to performing Gaussian elimination on a triangular 
matrix. Asymmetries about the midplane are effectively averaged out by adding the inner and outer chord data and dividing by two.

After the dessity profile is found, the matrix $G$ is constructed and Eq. 26 is inverted to obtain $u(r)$ exactly as described above.

The key to this method is the spline interpolation of the measured data at chord locations $x_{k}$ onto the aric of chord positions $x_{i}$ shown in Fig. 16. An ordinary cubic spline applied to the raw dats will pass tiarough every data point regardless of noise or calibration error, which is aot desirable suce small fluctuations in the spline fit will result in large fluctuations in the inverted density or poloidal feld profile. Inotead, a smoothing spline routine is used [39], which attempts $t 0$ minimize the square of the second derivative of the fitting function while maintaining a fit to the data with $\chi^{2}$ less than a user specified input parameter 8 . By adjusting $s$, one can control the smoothness of the spline fit.

The spline fits to the density and Faraday rotation data are also required to satisfy certain boundary conditions. For density, past Langmuir probe [40] measurements have shown that $n$ just outside the limiter can range from 1 to $10 \%$ of the peak density, with a 1/e scrape-off length on the order of $0.4 \mathrm{~cm}$. For the purpose of spline fitting, we extend the radial ring zones out to $\sim 18 \mathrm{~cm}$ and set the line-integrated density there equal to a constant of $\sim 1-2 \%$ of the peak line-integrated density. The spline fit is then forced to go through these points on both the inside and outside of the profile. For the Faraday rotation data, the edge poloidal field calculated from the total plasma current is used along with the edge density to produce a. boundary condition for the spline fit. In addition, the spline fit and inverted poloidal feld profile are forced to be smooth through the origin, which is numerically unstable.

The plasma position is always considered a free parameter in thsse inversions and is usually adjusted to give a best fit to the Farsday rotation data rather than interferometer data. Due to the steep zero crossing exhibited by the typical Faraday rotation profile, very accurate measurement of the plasma offset is possible.

Once the functions $u=\frac{\partial \partial}{\partial r}$ and $n(r)$ are determined, the temperature profile is obtained from ECE diagnostics, $\ell_{i}$ and $\beta_{p \alpha}$ are calculated from Fq.'s 16 and 15, and the Shafranov shift $\Delta(r)$ is calculated from Eq. 14. A routine-ion temperature profile measurement is not available in MTX, so for the purpose of calculating $\beta_{\mathrm{p}}$ it is assumed that $T_{i}=0.8 T_{\mathrm{e}}$. With 
this new estimate of $\Delta(r)$, a new flux surface geometry is constructed, and the density and poloidal field inversions are repeated. Generally, the function $\Delta(r)$ does not vary significantly after the 2nd iteration, so a total of two, or at most three, iterations are required.

With the final estimate of $u(r)$ in hand, the desired quantities $J(R, z)$ and $q(R, z)$ are obtained from Eq. 20 and 22 respectively.

The spline fit tschnique can be useful for generating error bars on the inverted profile by applying the method repeatedly to the same data with random errors added in. When the measured line densities were perturbed by random errors with a standard deviation of $1 \%$, which is typical of noise and calibration uncertainty, the resulting density error at $|x|>3 \mathrm{~cm}$ is relatively amall at about $\pm 1.5 \%$, while the error on exis is somewhat larger at $\sim \pm 3.5 \%$. The smoothing parameter was set to $8=15$ for this case. Error analysis for polerimetry is discussed in the main text.

\section{A.5 Non-linear Least Squares Fitting}

Although the spline fitting inversion technique described above is most gensral, a parametrized fitting method has been developed to check the spline fit inversion and to handle data which has larger noise or uncertainty. Here a parametrized form is chosen for the zero-order (cylindrical) current density $J_{0}(r)=f\left(\beta_{1}, \beta_{2}, \ldots\right)$. The density profle can also be parametrized if desired.

A useful form for $J_{0}$ (we write $J_{0}$ to avoid coufusion with the final current density $J(R, z)$ calculated from Eq. 20) is the product of a Gaussian with the even Hermite polynomials,

$$
J_{0}(r)=C e^{-15\left(r / a_{i}\right)^{2}} \sum_{i=0}^{n} \beta_{i} H_{2 i}
$$

where $a_{i}$ is a variable profile width (the factor 1.5 is included in the exponent so that $a_{t}$ is equivalent to the temperature profile width), and $\beta_{i}$ are the amplitudes of each polynomial term. The sonstant $C$ is chosen to give the correct total current. Note that the lowest order profile is a Gaussian, while higher-order terms allow profiles ranging from hollow to peaked. The function $u$ is then calculated from Maxwell's equation

$$
u(r)=\frac{R_{0} \mu_{0}}{r} \int_{0}^{r} J_{0}\left(r^{\prime}\right) r^{\prime} d r^{\prime}
$$


The reason that $J_{0}(r)$ is chosen as the fitting function rather than $u$ is just convenience since we expect $J_{0}(r)$ to have a Gaussian-like shape similar to the $T_{e}$ profile. Another useful form for $J_{0}(r)$ is a super Gaussian $e^{-1.5\left(r / a_{0}\right)^{s}}$.

Once the parametrized forms for $n(r)$ and $u^{\prime}(r)$ are given, the line-integrated profiles are calculated using Eqs. 25 and 27. The free parameters are then adjusted to give a best $\chi^{2}$ Git to the measured data. A non-linear least squares fitting routine based on the method of Marquardt [41] is utilized. The technique requires the calculation of both the line-integrated quantities from Eq. 27 and the partial derivatives of $\Psi$ with respect to each free parameter $\partial \Psi / \partial \beta_{1}, \partial \Psi / \partial \beta_{2} \ldots$... Since the vector $\Psi$ is calculated at the chord locations $x_{i}$, which are different from the measurement chord locations designated $x_{k}$, we must interpolate $\Psi$ onto the measurement chord locations. Defining the fitting function rotation angles as $\Psi\left(I_{k}\right)$ and the measured rotation angles as $\Psi_{k}$, the least squares fitting routine attempts to minimize the quantity

$$
x^{2}=\sum\left[\frac{1}{\sigma_{k}^{2}}\left(\Psi_{k}-\Psi\left(x_{k}\right)\right)^{2}\right]
$$

Since the Faraday rotation profile is essentially two integrations removed from the current density profile, lerge variations in $J_{0}(r)$ are greatly smoothed in the final Faraday rotation profile. This makes it difficult for the fitting routine to converge to a unique solution when more than three or four polynomial terms are used to represent $J_{0}(r)$.

\section{Acknowledgment}

"We would like to thank the entire MTX physics, engineering, and technician staff for their contributions to this work. We also thank our JAERI collaborators for providing soft $X$-Ray data. This work was supparted by Lawrence Livermore National Laboratory under U.S. DOE Contract No. W-7405-ENG-48.

\section{References}

[1] Van Goeler, S.L., Stodiek, W., Sauthoff, N., Phys. Rev. Lett. 33 (1974) 1201.

[2] Kadomtsev, B.B., Sov. J. Plasma Phys., 1 (1975) 389 .

(3) Dubois, M.A., Samain, A., Nucl. Fusion; 20 (1980) 1101. 
[4] Lichtenberg, A.J., Nucl. Fusion, 24 (1984) 1277.

[5] Lichtenberg, A.J., Itoh, K., Itoh, S.-I., Fukuyama, A., Nucl. Fusion 32 (1992) 495.

[6] Dubois, M.A., Hennequin,P., Nakach, R, Sabot, R, in Controlled Fusion and Plasma Physics (Proc. 19tb Eur. Conf., Innsbruck, 1992) Vol. 16C, Part II, European Physical Society (1992) 1409.

[7] Baty, H., Lucian1, J.F., Bussac, M.N., Nucl. Fusion 32 (1992) 1277.

[8] Holmes, J.A., Phys. Fluids B 3 (1991) 594.

[9] Wesson, J.A., Plasma Phys. Controll. Fusion 28 (1986) 243.

[10] Kolesnichenko, Y.I., Yakovenko,Y.V., Andersnn, D., Lisak, M., Wising, F., Phys. Rev. Lett. 68 (1992) 3881.

[11] Soltwisch, H., Rev. Sci. lostrum. 57 (1986) 1939.

[12] Soltwisch, H., Rev. Sci. Instrum. 59 (1988) 1599.

[13] O'Rourke, J., Plasma Phys. and Controlled Fusion, 33 (1991) 289.

[14] Rice, B.W., Rev. Sci. Instrum. 63 (1992) 5002.

[15] Wroblewski, D., Lao, L.L., Phys. Fluids B 3 (1991) 2877.

[16] Yamada, M., Nagayama, Y., Davis, W., Fredrickson, E., Janos, A., Levinton, F., Rev. Sci. Instrum. 63 (1992) 4623 .

[17] Wroblewski, D., Lao, L.L., Rev. Sci. Instrum. 63 (1992) 5140.

[18] Fielding, S.J., Hugill, J., McCracken, G.M., Paul, J.W.M., Prentice, P., Stott, P.E., Nucl. Fusion, 17 (1977) 1382.

[19] Snipes, J.A., Campbell, D.J., Haynes, P.S., et al., Nucl. Fusion, 28 (1988) 1085 .

[20] Hinnov, E., Bol, K. Dimock, D., et al, Nucl. Fusion, 18 (1978) 1305.

[21] Hawryluk, R.J., et al., et al., Princeton Pysics Laboratory Report PPL-1534, April 1979.

[22] Rice, B.W., Ph.D. Dissertation, University of California, Davis (1992)

[23] De Marco, F. Segre, S.E., Plasma Phys., 14 (1972) 245.

[24] Segre, S.E., Plasma Phys., 20 (1978) 295.

[25] Soltwisch, H., Rev. Sci. Instrum 57 (1986) 1939.

[26] Soltwisch, H., Institut fur Plasmaphysik, Julich, Report Jul-1638 (1980).

[27] Park, H.K., Plasma Fhys. and Controlled Fusion, 31 (1989) 2035. 
[28] Spitzer, L. Jr., Physics of Fully Ionized Gases, John Wiley \& Sons Inc., New York (1962).

[29] Pfeiffer, W., Davidson, R.H., Miller, R.L., Waltz, R.E., General Atomic Report GAAl6178 (1980). ONETWO code runs performed by M. Marinak.

[30] Soltwisch, H., Fuchs, G., Koslowski, H.R., Schluter, J., Waidmann, G., in Controlled Fusion and Plasma Physics (Proc. 18th Eur. Conf., Berlin, 1991) Vol. 15C, Part II, European Physical Society (1991) 17.

[31] Pickrell, M.M., Ph.D. Dissertation, MIT, Cambridge (1982).

[32] Stix, T.H., Phys. Rev. Lett., 36 (1976) 521.

[33] Furth, H.P., Rutherford, P.H., Selberg, H. Phys. Fluids, 16 (1973) 1054.

[34] Jensen, R.V., Post, D.E., Grasberger, W.H., Tarter, C.B., Lokke, W.A., Nucl Fusion, 17 (1977) 1187.

[35] Mukhovatov, V.S., Shafranov, V.D., Nucl. Fusion, 11 (1971) 605.

[36] Wesson, J.,Tokamaks, Oxford University Press, New York (1987).

[37] Lipschultz, B., LaBambard,B., Marmar, E.S., et al., Nucl. Fusion, 24 (1984) 977.

[38] Press, W.H., Flannery, B.P., Teukolsky, S.A., Vetterling, W.T "smerical Recipes in Pescal, Cambridge University Press, New York (1989).

[39] Cline, A. K., Fitpack software package, Pleasant Valley Software. (1987).

[40] LaBambard, B., Lipschultz, B., Nucl. Fusion, 27 (1987) 81.

[41] Bevington, P.R., Data Reduction and Error Analysis for the Physical Sciences, McGrawHill, New York (1969). 


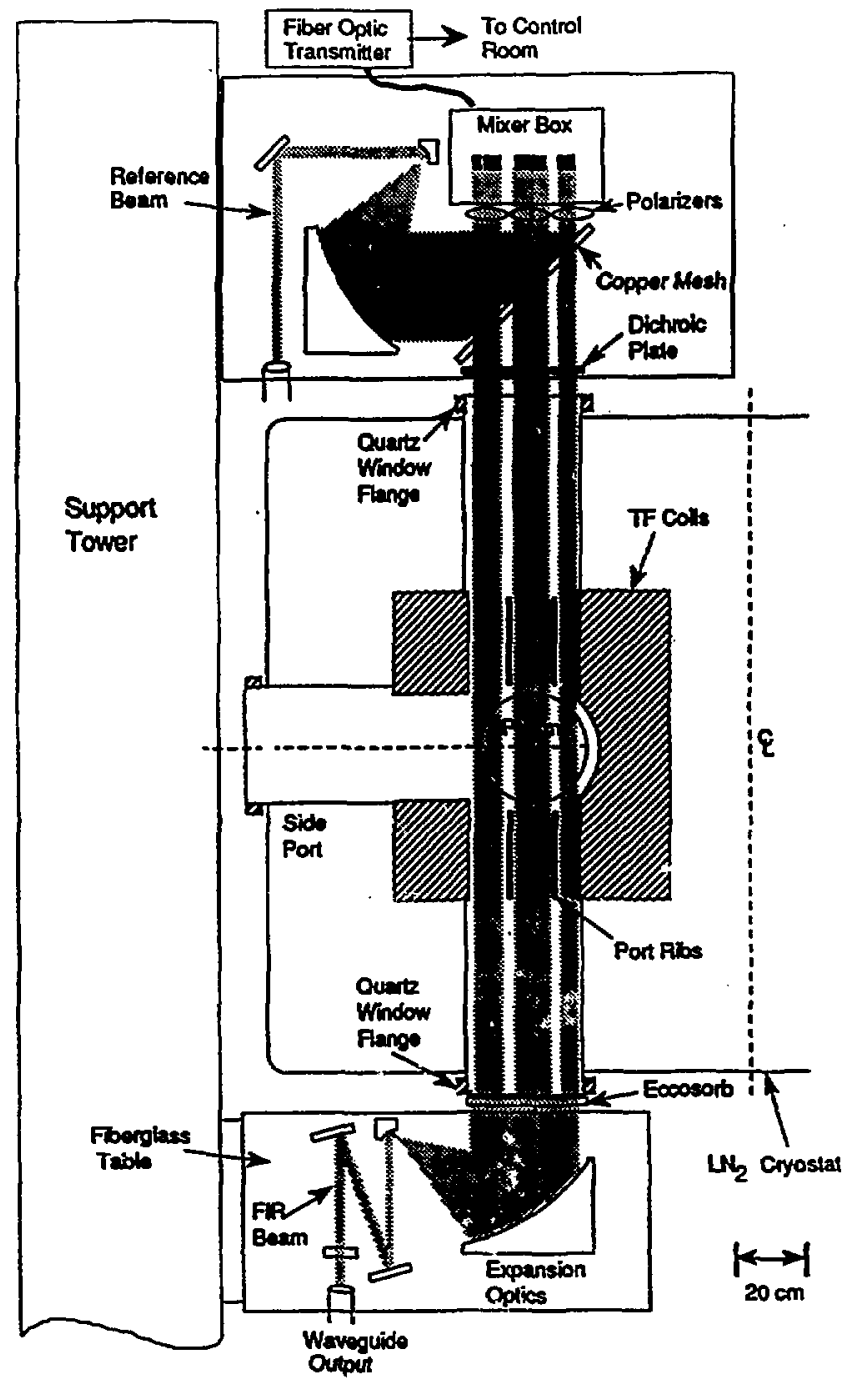

Figure 1: Overview of the MTX interferometer/polarimeter diagnostic. 

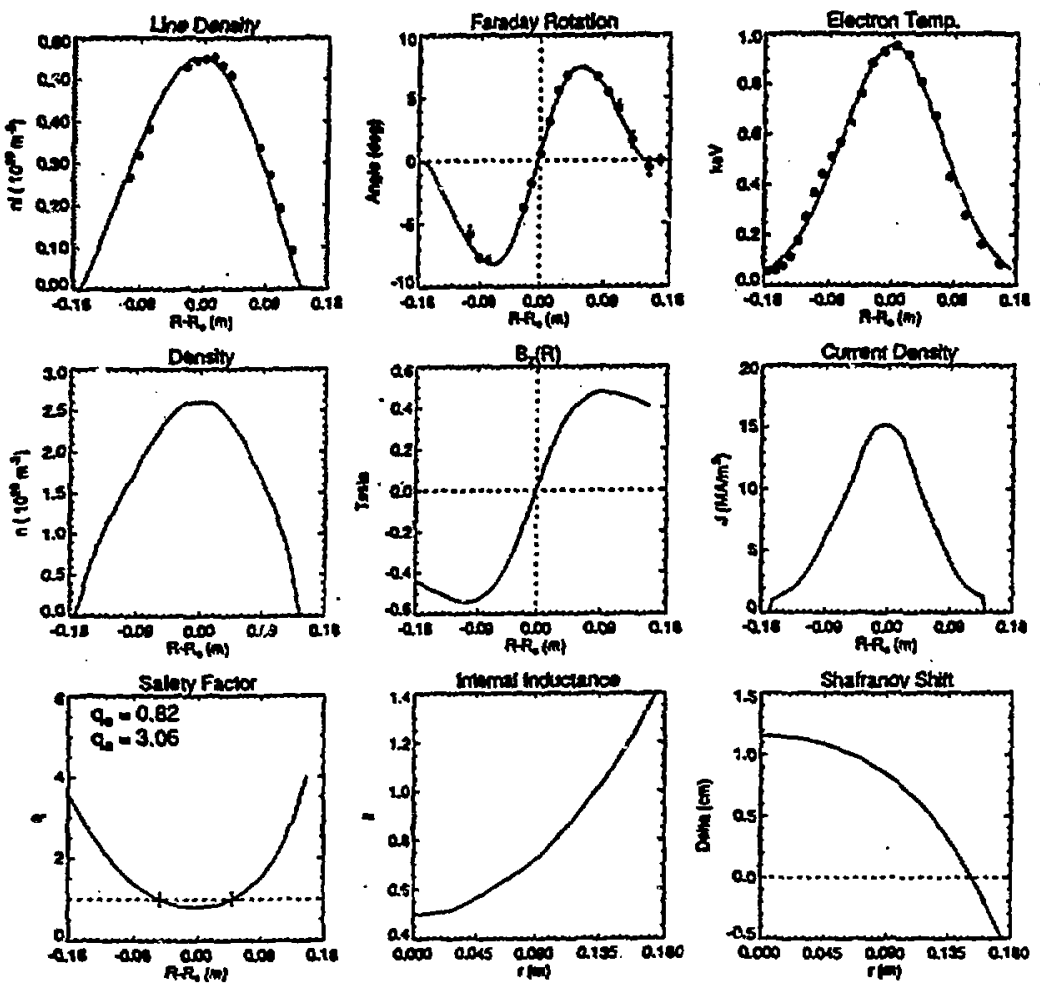

Figure 2: Profile analysis for ohmic discharge 12599 at $t=0.185 \mathrm{sec}$ using the spline-fit inversion technique. : 


\section{Current Density Profiles}

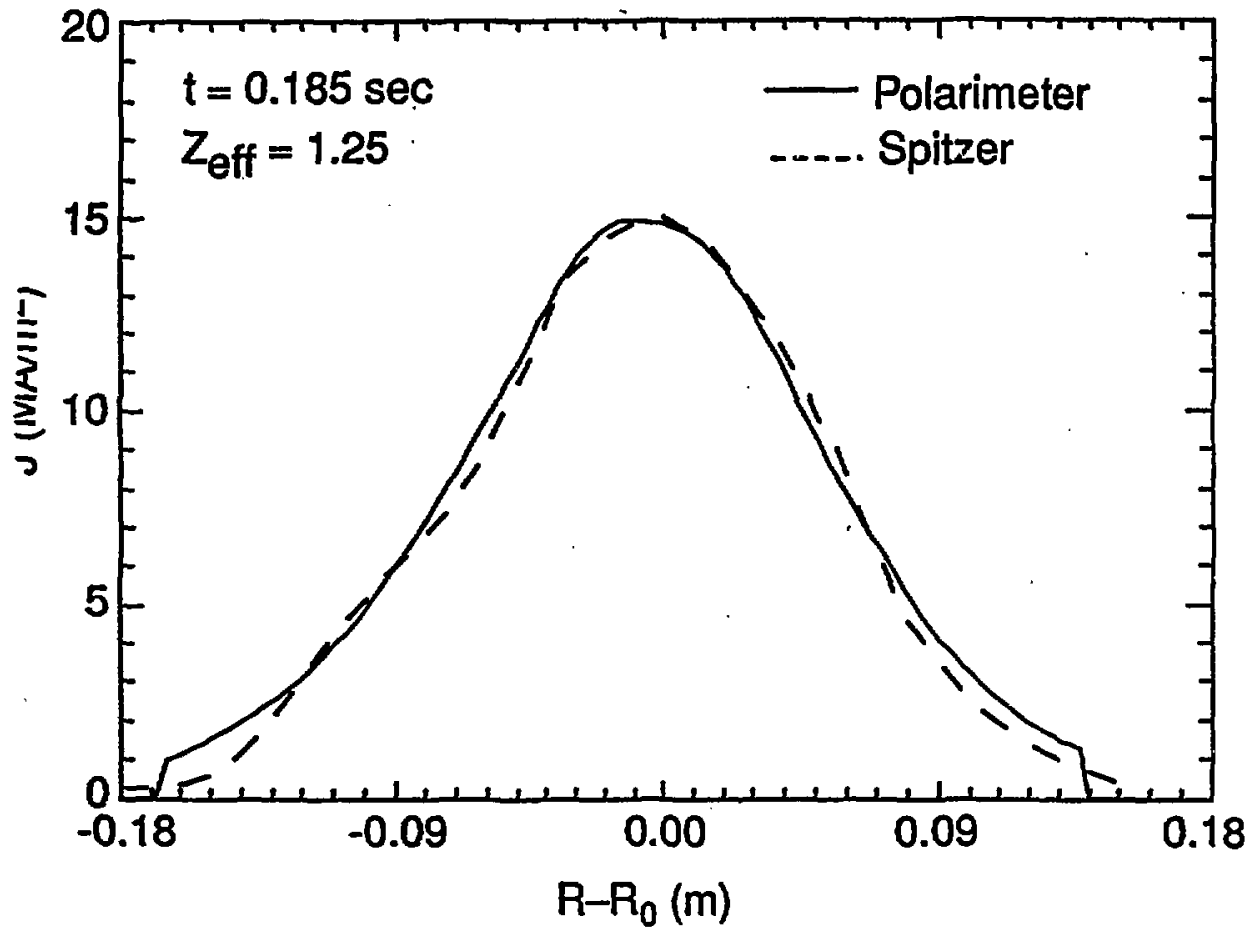

Figure 3: Comparison of the measured polarimeter $J$ profile with the classical Spitzer prediction. 


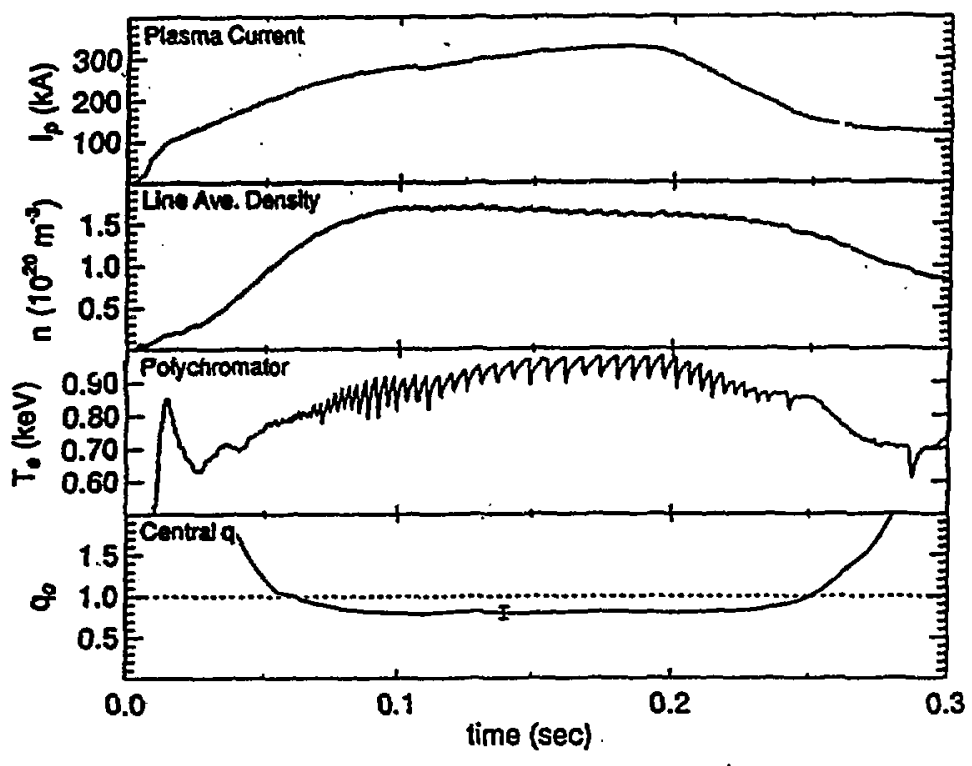

Figure 4: Polarimeter measurements of $q_{0}(t)$ shows $q_{0}<1$ during sawteeth as expected. A current ramp initiated at $0.2 \mathrm{sec}$ leads to an eventual rise in $g_{0}$ and the suppression of sawtooth activity. 


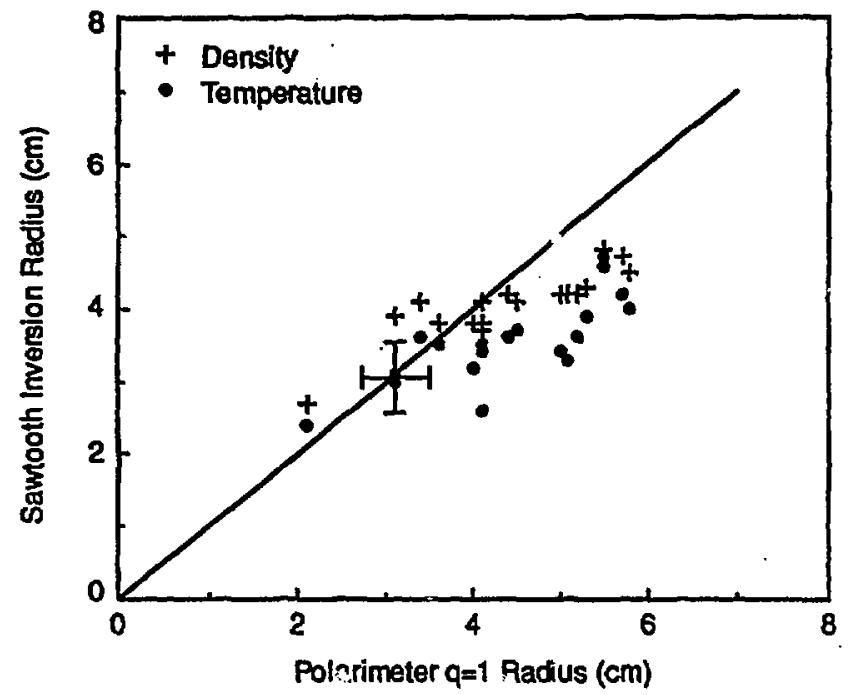

Figure 5: Tempersture, and density sawtooth inversion radius versus the $q=1$ radius obtained from polarimetry. 

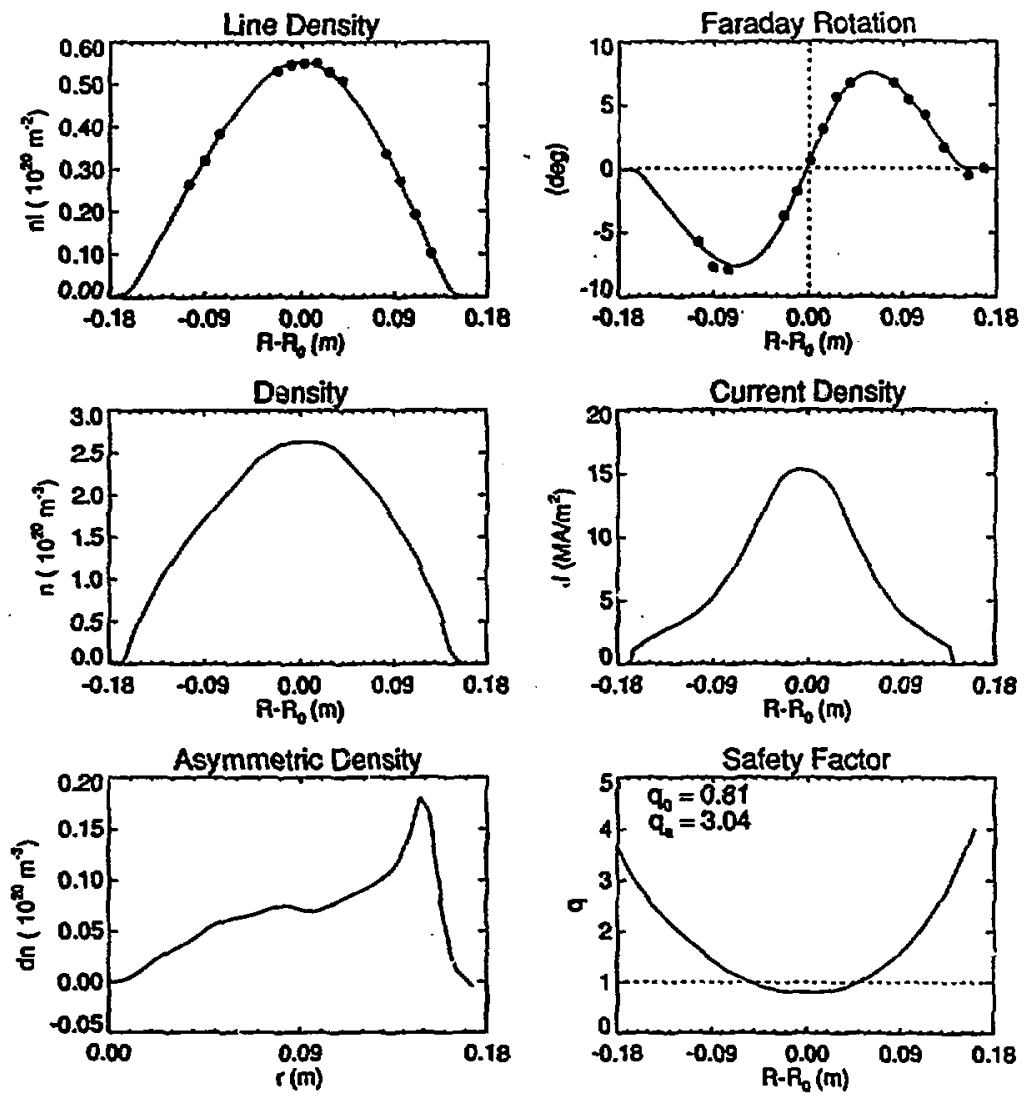

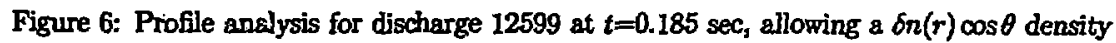
variation on a flux surface. The value of $q_{0}$ remains unchanged. 


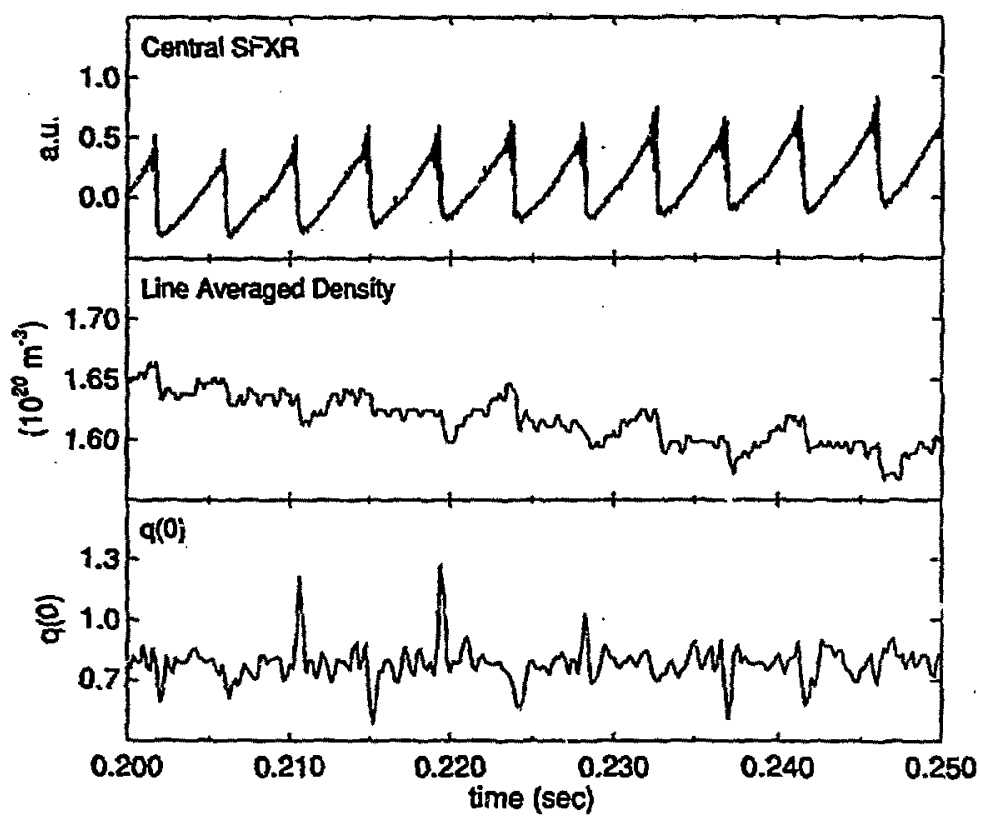

Figure 7: High time resolution $q_{0}$ measurements duing sawtooth activity. The spikes in $q_{0}$ correlated with the sawtooth crash are instruy ental effects related to the rapid change in refraction as the density profile fattens. 


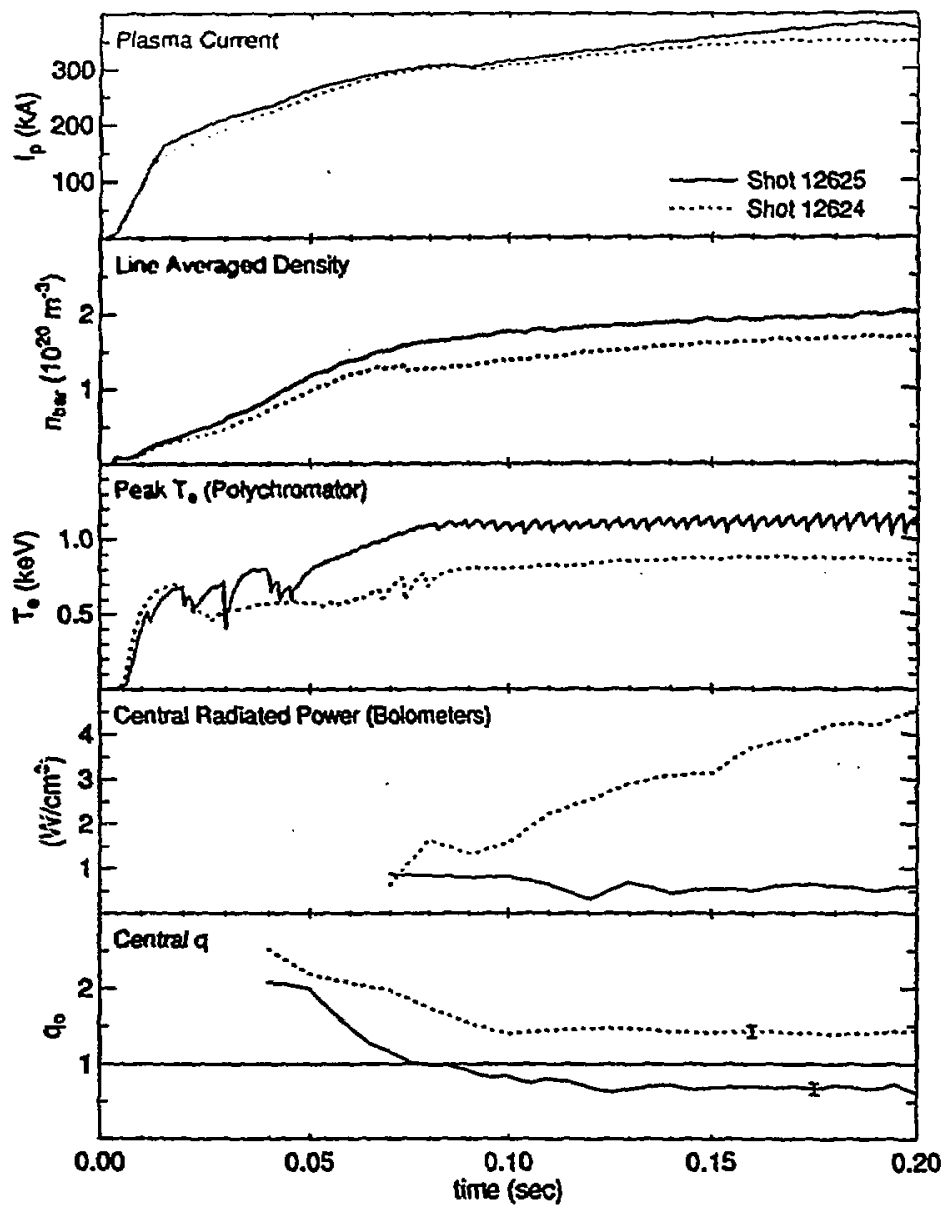

Figure 8: Time history comparison of non-sawtoothing discharge 12624 (dashed) and sawtoothing discharge 12625 (solid). The increased radiated power in 12624 reduces $T_{e}(0)$ keeping $q_{0}>1$. 

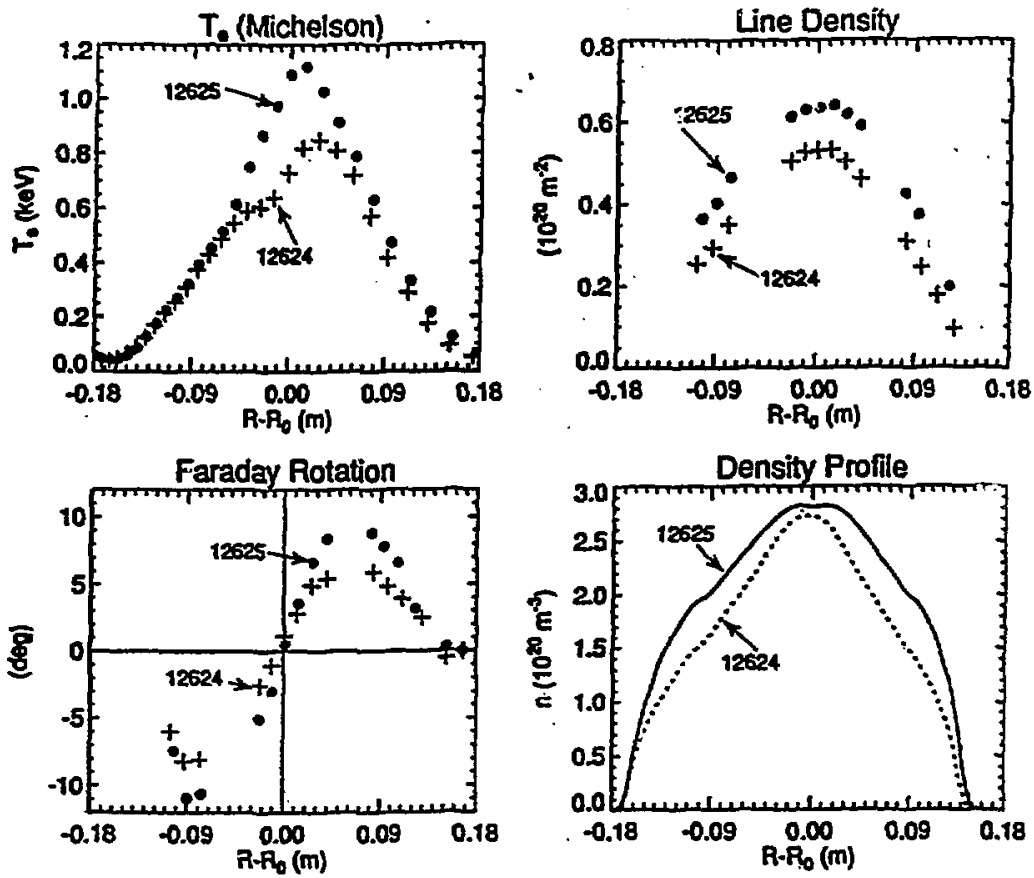

Figure 9: Comparison of profiles for non-sawtoothing discharge 12624 and sawtoothing discharge 12625 at $175 \mathrm{~ms}$. 

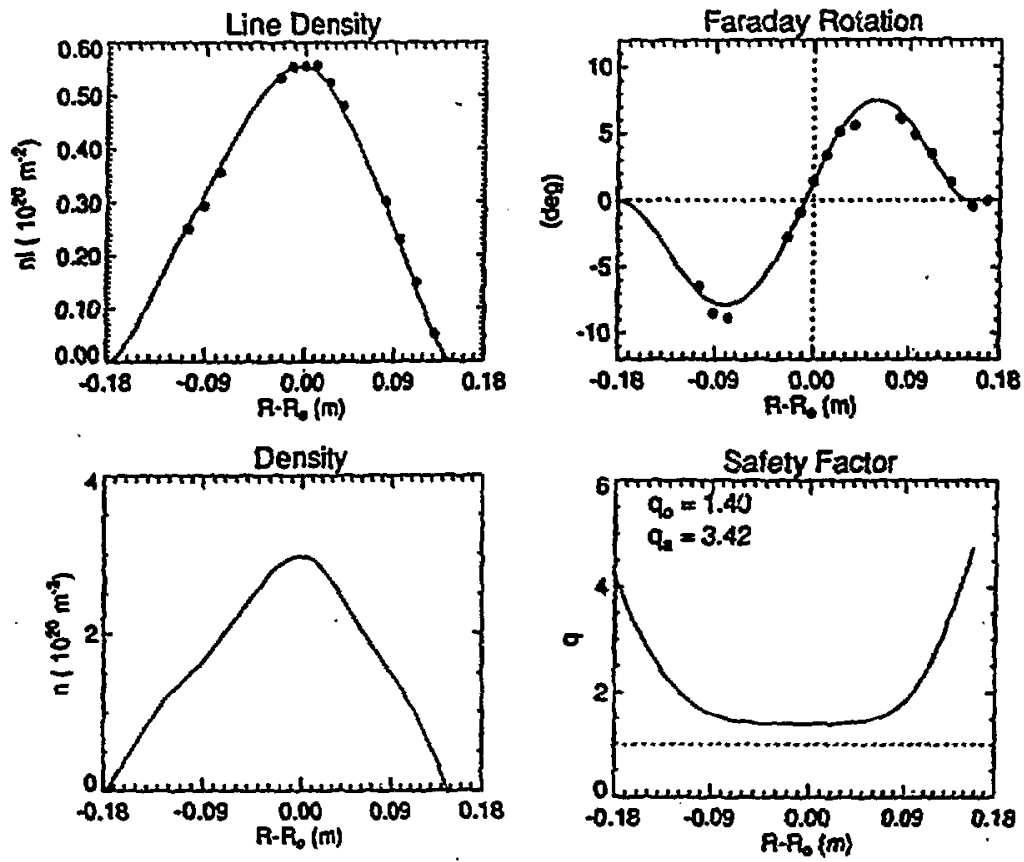

Figure 10: Profile inversion for discharge 12624 at $0.2 \mathrm{sec}$ shows $q_{0}>1$ and a sizable asymametry in the Faraday rotation data. 


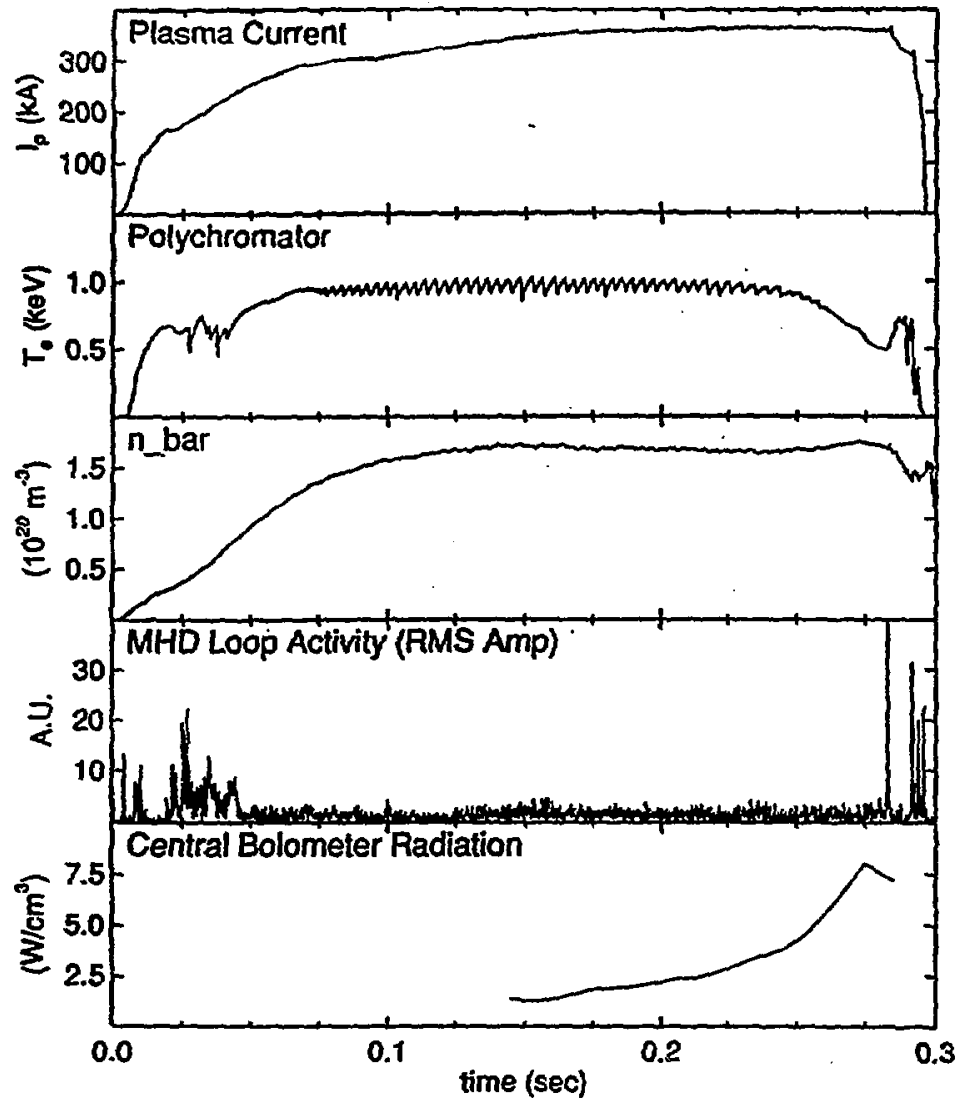

Figure 11: Overview of disruption discharge 12540. Increasing core radiation causes central $T_{e}$ to collapse beginning at $0.25 \mathrm{sec}$, resulting in a disruption at $0.29 \mathrm{sec}$. 
a)
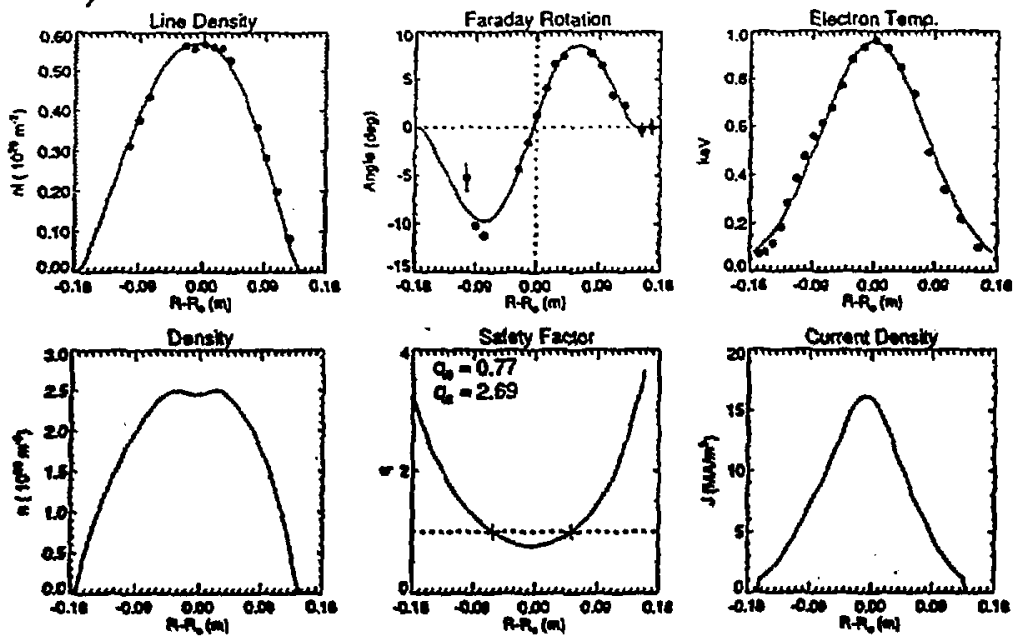

b)
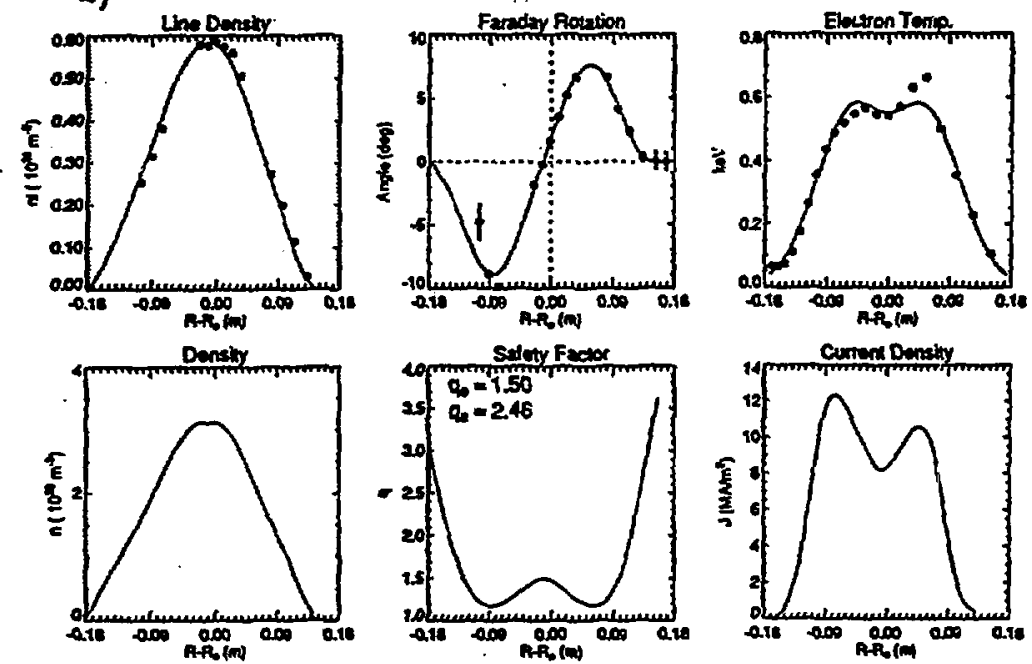

Figure 12: Profile data for discharge $12540 ;$ a) prior to the $T_{c}$ collapse (200 ms) and b) during the $T_{e}$ collapse just prior to the distuption $(279 \mathrm{~ms})$. Development of hollow $T_{e}$ and $J$ profiles is evident. 


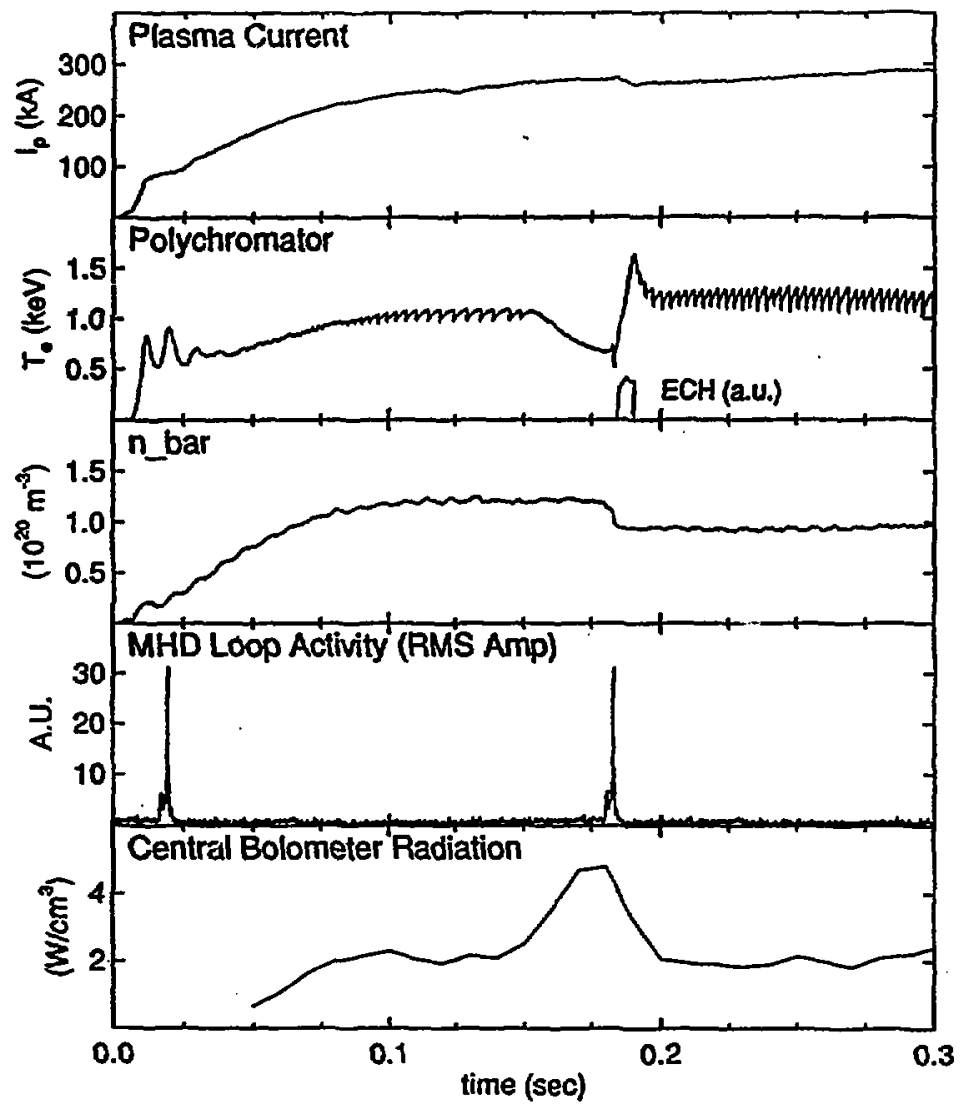

Figure 13: A disruption due to the radiative collapse of $T_{e}$ is evoided through application of an ECH pulse at $180 \mathrm{~ms}$ (discharge 11783). 


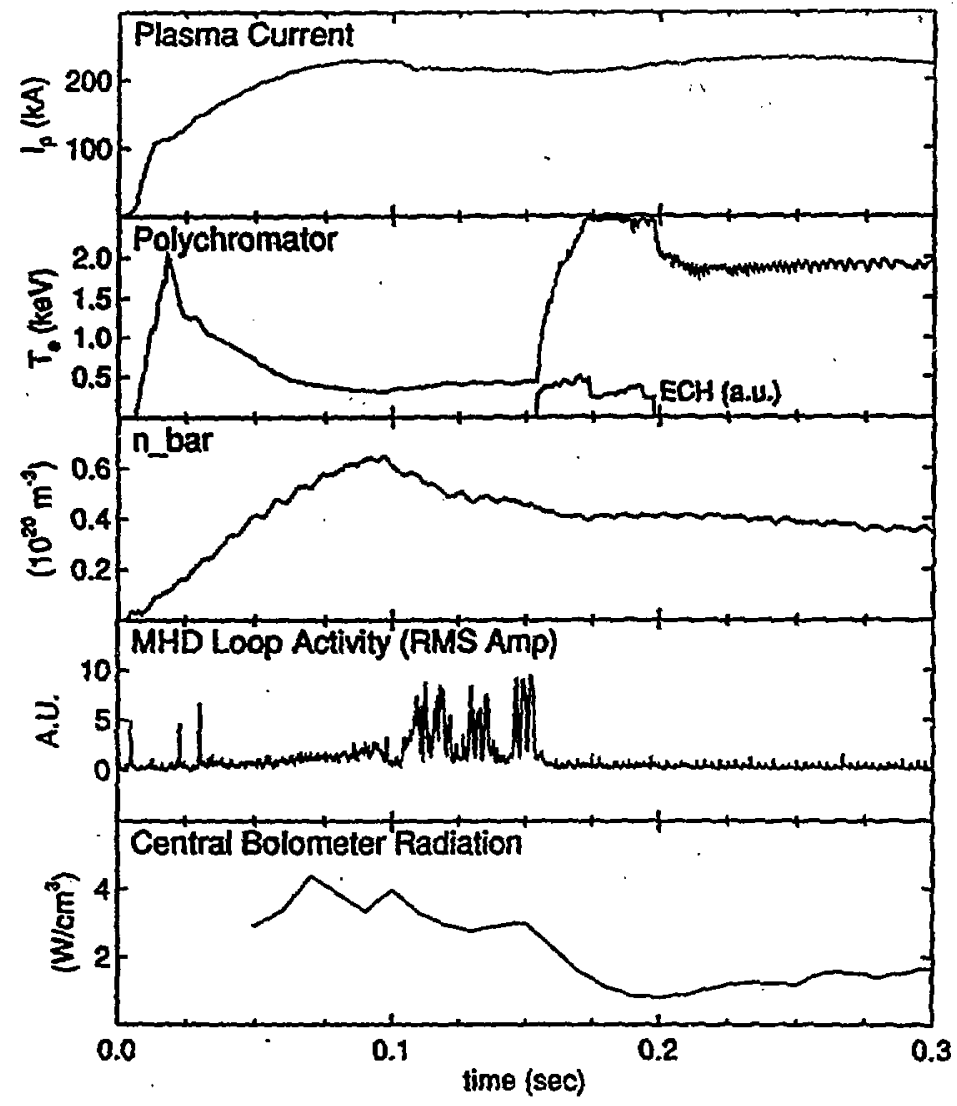

Figure 14: Strong MHD activity ( $m=3$ ) is suppressed by peaking the $T_{e}$ and $J$ profiles with central ECH in discharge 12252. 


\section{Current Density Profiles, Shot 12252}
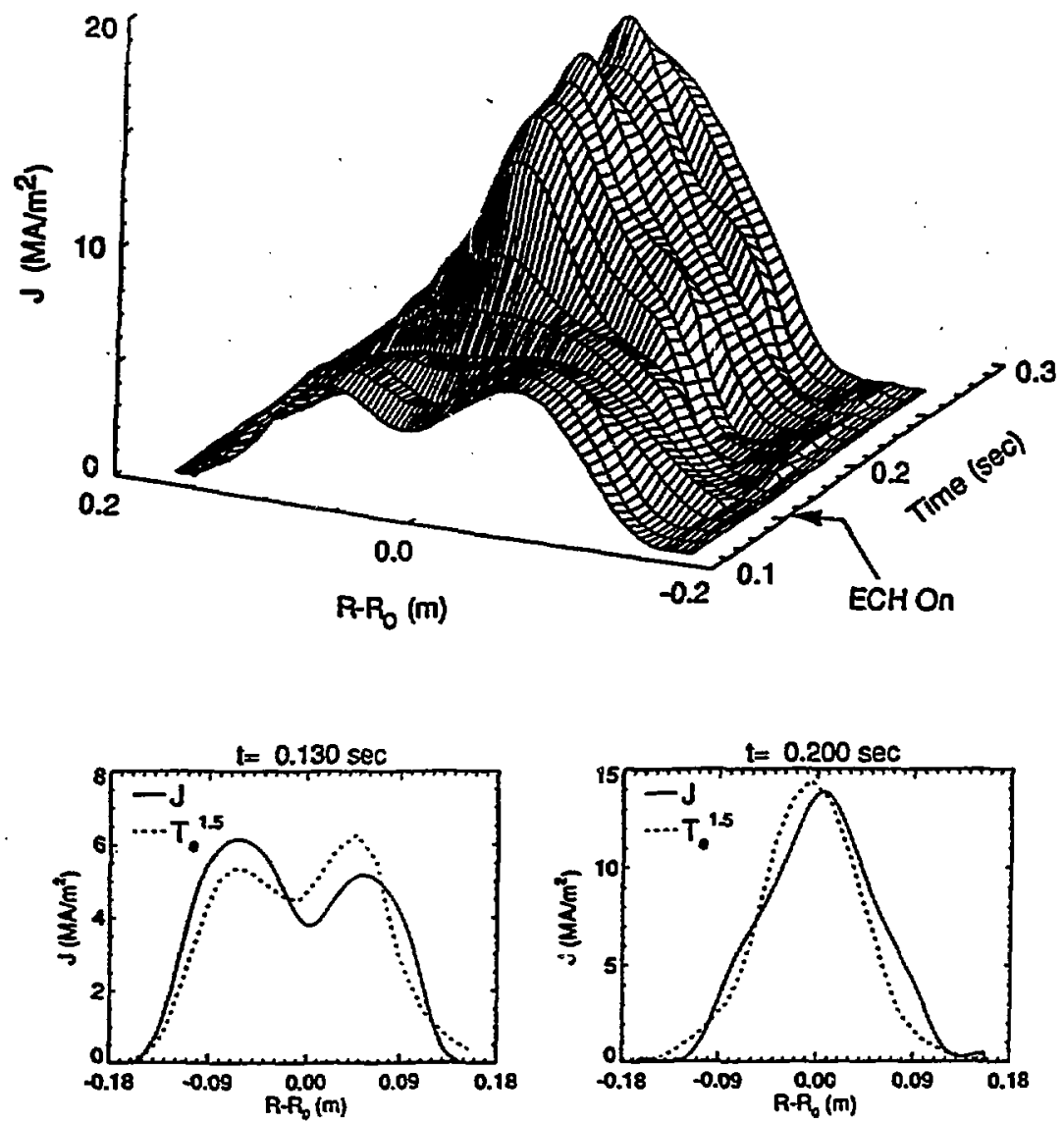

Figure 15: Current density profile evolution during ECH. Comparison with $T_{e}^{1.5}$ profiles before and after the ECH pulse show good agreement. 


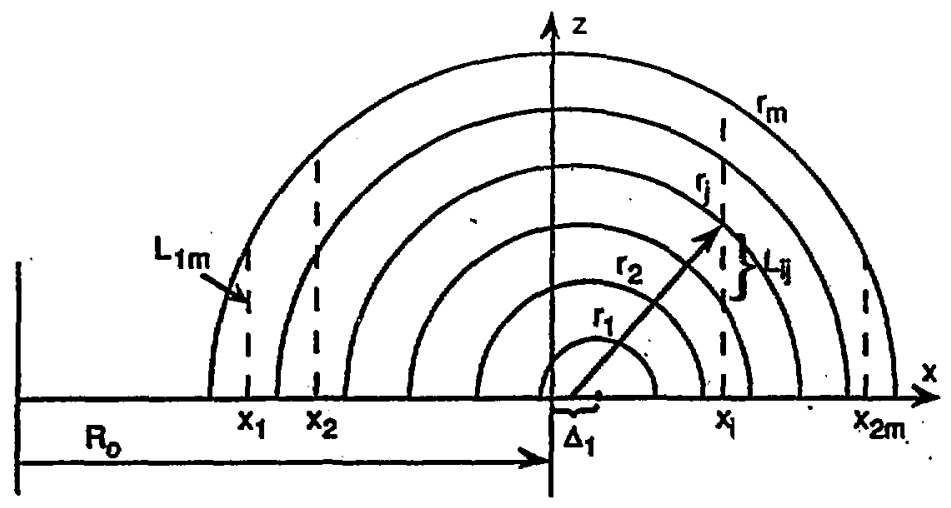

Figure 16: Toroidal geometry used for profile inversion.

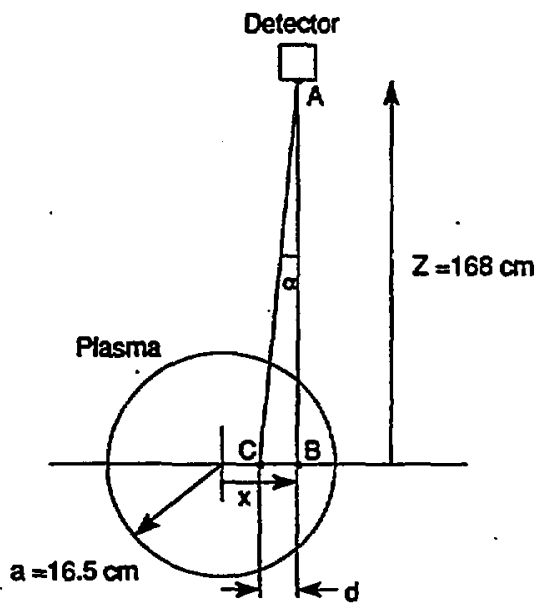

Figure 17: Geometry for refraction correction. 


\subsection{Particle Transport (M. M. Marinak)}

In order to achieve ignitioli in future magnetic fusion plasma experiments, stable plasmas must be prociuced with central densities near $(2-5) \times 10^{20} \mathrm{~m}^{-3}$. On ITER the average densities for these ignited plasmas are expected to be near or beyond the limits according to the empirical Greenwald scaling. Tokamak plasmas at the density limit often terminate by disruptions that would adversely impact the reliability of operations in this machine. Thus it is important to increase our understanding of the mechanisms responsible for this limit.

The density limit disruption is thought to result from cooling of the edge plasma, which causes the current profile to contract, destabilizing the $m / n=2 / 1$ mode 1 . A deterioration in confinement, scrape-off.layer collapse, and radiation have been advanced as mechanisms responsible for the cooling of the edge plasma. On several machines, including Alcator $\mathrm{C}^{2}, \mathrm{~T}-10^{3}, \mathrm{DITE} 4$, Tuman- $3^{5}$ and TEXT 6 an increase in transport is evident near the density limit. Experiments on Alcator $C$ revealed a dramatic decrease in the density decay time following pellet injection near this Greenwald limit. This decrease was symptomatic of a deterioration in particle confinement in the plasma core.

The increase in particle transport and its relation to plasma conditions were examined in a series of gas modulation experiments on MTX. Detailed measurements with a 15-chord FIR allowed radial profiles of the perturbed particle transport coefficients to be resolved. The particle transport coefficients in the core of the deuterium plasma were studied as the density limit was approached during a density scan at constant plasma current and toroidal field $\left(B_{t}=6.5 \mathrm{~T}, \mathrm{q}_{\mathrm{a}}=4.8, \mathrm{R}=64 \mathrm{~cm}, \mathrm{a}=16.5\right.$ $\left.\mathrm{cm}, 1 \leq Z_{\text {eff }} \leq 1.2\right)$. Near the density limit a dramatic increase occurred in both the perturbed diffusion and convection coefficients in the relatively cool outer portion of the plasma. The coefficient profiles provide evidence that the mechanism responsible is confined to regions of relatively low temperatures and causes particle transport coefficients to increase through an inverse dependence on $\mathrm{T}_{e}$ and/or $\mathrm{L}_{\mathrm{Te}}$ characteristic of several resistive edge turbulence theories.

These gas-fueled, ohmically heated plasmas were produced at a relatively high value of $q_{a}$ to avoid disruptions. No substantial MHD activity was observed during the quasi-steady-state portion of any of the gas modulation discharges. Although both periodic and single gas puff modulations could be used at low densities, at higher densities ( $n_{e} \geq 2 \times 10^{20} \mathrm{~m}^{-3}$ ) only a single gas puff could be used due to increased recycling and slower plasma density response. These small single gas puffs created small changes in the local variables $\delta n / n \sim 0.1, \delta \nabla n / \nabla n \sim 1-.2, \delta T_{e} / T_{e} \sim .05-.10$, which corresponded to large fractional changes in the particle flux $\delta \Gamma / \Gamma>1$. The perturbed flux is represented as the sum of diffusive and inward convection terms $\tilde{\Gamma}=-D \partial \bar{n} / \partial r-V \tilde{n}$ For the Alcator C plasma the particle source calculated with the ONETWO transport code falls rapidly from the plasma edge and can be neglected in the region $\mathrm{r} / \mathrm{a} \leq 0.8$. The continuity equation for the perturbed density in the core reduces to 


$$
D(x) \frac{\partial \bar{n}(x, t)}{\partial x}+V(x) \bar{n}(x, t)=\frac{I}{x} \int_{0}^{x} \frac{\partial \bar{n}^{-}\left(x^{\prime}, t\right)}{\partial t} x^{\prime} d x^{\prime}
$$

The values of $\mathrm{D}$ and $\mathrm{V}$ are obtained experimentally by applying multidimensional lirear regression analysis at each radial position for many time slices during the density perturbation according to Eq. 1 , taking $\mathrm{n}$ and $\partial \mathrm{n} / \partial \mathrm{r}$ as independent variables. Shots were repeated a total of 8-10 times to obtain a good signal-tc-noise ratio. The terms D and $V$ represent first-order expansions of the expression for the flux in terms of plasma parameters according to linear perturbation theory. The expansion was obtained by Gentle ${ }^{7}$

$$
\begin{aligned}
& D=D_{\ldots+}+\frac{\partial D^{-}}{\partial \frac{\partial x}{\partial x}} \frac{\partial R_{e x}}{\partial x}
\end{aligned}
$$

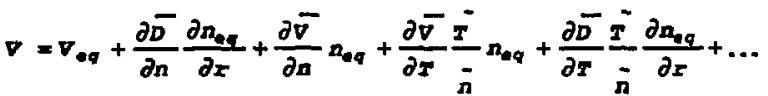

with significant contributions to the flux dependent upon $\nabla n$ grouped with $D$. The perturbed transport coefficients, which are distinctly different from the equilibrium coefficients, may be compared with predictions of transport theories through the above expansions. The effect of temperature perturbations is manifest in the perturbed convection velocity $\mathrm{V}$. 
Figure I shows the density profiles at six different line-averaged densities from the scan as a function of flux surface radius, which is normalized to the last closed flux surface. The density at the last closed flux surface is higher than that at the outside limiter. These are separated due to the slight inward offset of the plasmas $\sim 0.06 \mathrm{a}$. The discharge with $h_{e}=3.3 \times 10^{20} \mathrm{~m}^{-3}$ is at the Greenwald limit threshold $\tilde{n}_{e}=k \bar{j}$ and was the highest density at which transport coefficients were obtained. The highest density achieved at this current was $20 \%$ higher than the limit scaling of Greenwald, who pointed out that it was an approximate relation. Values of the $20 \%$ higher than the limit were also obtained with gas fueling at MIT.

Marfes anc present during the "plateau phase" of all the discharges with $\hbar_{e} \geq 2.2 \mathrm{X}$ $10^{20} \mathrm{~m}^{-3}, I_{\mathrm{p}}=285 \mathrm{kA}$, consistent with the scaling reported previously ${ }^{8}$. In the high-
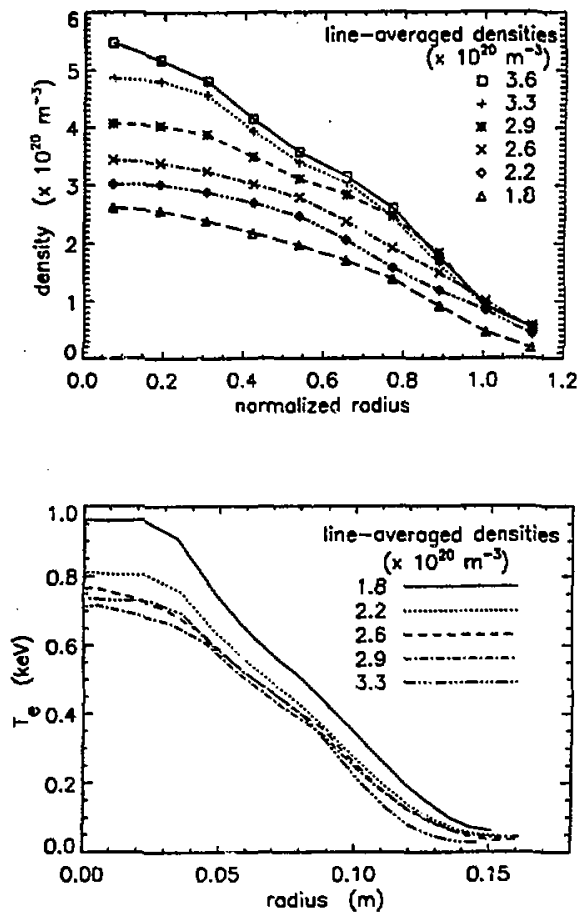

Fig. 1(a) Density profiles for $285 \mathrm{kA}$ plasmas (b) Black body temperature profiles. Outside of $r / a \sim 0.8$ the emission is not optically black. 
density discharges the scrape-off layer (SOL) density saturates, becoming independent of $h_{e}$. Measurements on Alcator C with DENSEPACK, a poloidal array of Langmuir probes, indicated that the poloidal average of the boundary density is relatively insensitive to $\bar{n}_{e}$ in the high-density regime where Marfes are present, consistent with profiles of this scan. DENSEPACK measurements showed strong poloidal asymmetries in the plasma density at minor radii za that persist for all values of $\bar{n}_{e} / I_{p}$. The normalized density asymmetry increases only slightly at high values of $\bar{n}_{e} / l_{p}$. Normalized magnitudes and poloidal locations of the extrema of the density asymmetries are insensitive to the presence of a Marfe. In fact, the Marfe was detected as a relatively weak poloidal density perturbation.

Edge asymmetries contribute to the modulation signal only to the extent the edge plasma density changes during the modulation. Fig. 2 shows the change in FIR chord signals following a single gas puff at various time intervals. The two outermost chords show very little change, indicating that the change in the density outside the last closed flux surface is small compared to changes occurring in the bulk plasma. Concurrently, Fig. 2 reveals no indication of significant asymmetries in the change in the chord signals following the puff.

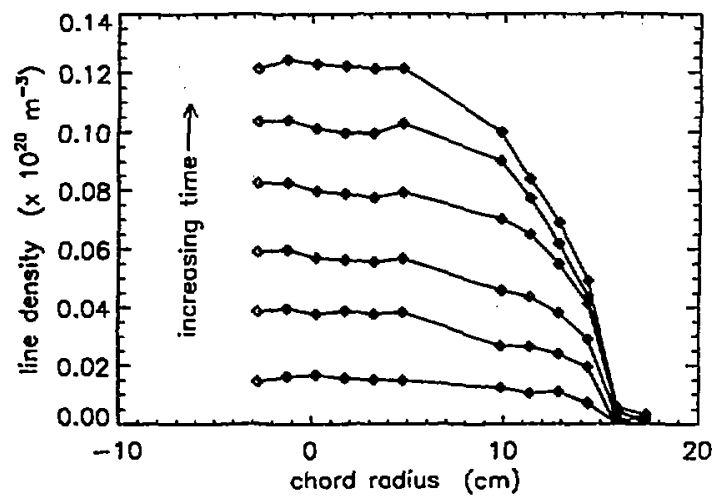

Fig. 2 Change in 12 FIR thord signais following a gas puff for $\overline{n_{\mathrm{e}}}=3.3 \times 10^{20} \mathrm{~m}^{-3}, \mathrm{I}_{\mathrm{p}}=285 \mathrm{kA}$. 
A scanning Michelson interferometer was used to obtain profiles of plasma electron temperature shown in Fig. 1. There is an obvious trend towards lower electron temperature as the density is increased.

The analysis described earlier was applied to data from these discharges to obtain the particle transport coefficients shown in Fig. 3. A dramatic increase in the transport coefficients is seen in the outer portion of the profiles at $\bar{n}_{e, 20}=3.3$, where the density is at the limit value predicted by the Greenwald scaling. The increase in both $D$ and $V$ is most pronounced at the outer edge of the region analyzed, but is apparent over a region that extends well into the plasma core. Near the plasma center the coefficient values are not increased over the values at lower densities. Density profiles maintain a similar
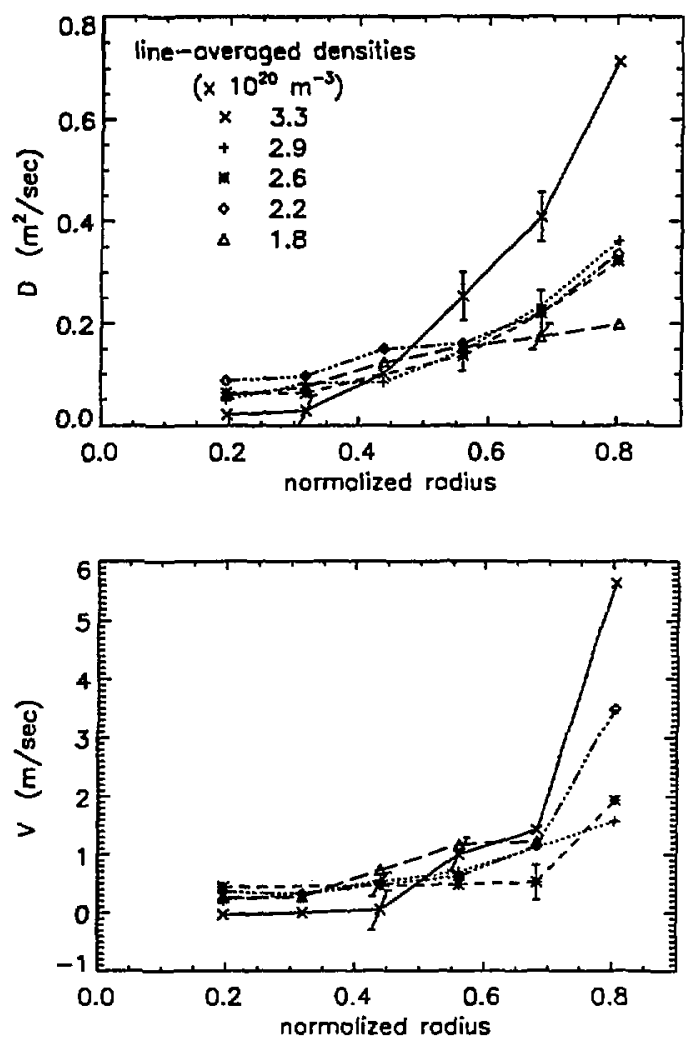

Fig. 3 Particle transport coefficient overlaid from discharges with $\hbar_{e, 20}=1.8,2.2,2.6,2.9,3.3$. 
shape near the density limit, congruous with the similar increase in the perturbed transport coefficients. Values of aV $/ \mathrm{D}$ for $0.4<\mathrm{r} / \mathrm{a}<0.8, \mathrm{I} p=285 \mathrm{kA}$ fell in the range (1-1/4) a/Ln, indicating the effect of dependences of particle transport coefficients on plasma parameters and off diagonal terms was significant. The rapid increase of the particle transport coefficients over a region extending into the core is qualitatively consistent with density limit behavior in the aforementioned pellet fueling experiments.

The temperature perturbations measured with a polychrometer are found to be highly correlated with density perturbations. When $\tilde{\mathrm{T}}_{\mathrm{e}} \nabla \tilde{\mathrm{T}}_{\mathrm{e}}$ are included explicitly as independent variables, the diffusion-coefficients obtained are essentially unchanged from values obtained from Eq. 1 in which temperature effects are considered implicitly. This is consistent with Eqs. 2 and 3, where the temperature effects are manifest in V. The error bars reflect uncertainties in the plasma position and geometry and the regression correlation?.

In earlier experiments at MIT Gomez found that the $\chi_{e}$ values determined from sawtooth heat pulse propagation scaled proportional to 1 / he. Although they show little variation the values of $D$ for densities $1.8<h_{e, 20}<2.9$ are typically smaller than the $\chi$ e values determined by Gomez by a factor of (2-5).

At line densities $h_{e}>2 \times 10^{20} \mathrm{~m}^{-3}$ the global energy confinement time $\left(\tau_{e}\right)$ saturates due to anomalous ion thermal conduction. Values of $\tau_{e}$ determined from the diamagnetic loop, which are close to the values from ONETWO calculations, are nearly independent of $\mathrm{h}_{\mathrm{e}}$ in the saturated ohmic confinement regime (he $>2 \times 10^{20} \mathrm{~m}^{-3}$ ). The profiles of $D(r)$ exhibit this same behavior over most of this regime. There is, however, no substantial deterioration of the global energy confinement time near the density limit, even though the particle transport coefficients increase rapidly. If the anomalous $\chi \mathrm{i}$, eq profile increased in a manner similar to $D$, a measurable deterioration in global energy confinement (20-30\%) would have occurred. Furthermore, in the pellet injection experiments on Alcator $\mathrm{C}$ no deterioration in energy confinement was reported at the density limit except that directly associated with the convected flux of particles. This lange increase in $D$, unaccompanied by id decrease in $\tau_{e}$, is difficult to explain in the framework of ion temperature gradient driven turbulence $\left(n_{i}\right)$ theory, which predicts $\chi_{1}$ *D. Also, the $\eta_{\mathrm{i}}$ mode is weak at low temperature ${ }^{10}$.

The outer third of these high-density, high-q MTX plasmas, where the increases in particle transport coefficients are most pronounced, have relatively low $T_{e}(-250 \mathrm{eV})$ and satisfy the criteria used to derive the reduced Braginskii equations ${ }^{11}$, similar to the equations of resistive MHD. Models of resistive fluid turbulence have been advanced to explain transport specifically in the relatively cool edge plasma regions. These models typically have particle diffusion coefficients scaling with inverse dependences on $T_{e}$ and/or LTe. Examples are the scalings of particle diffusivity given with the theories of resistivity-gradient-driven turbulence ${ }^{12}$.

$$
D_{\text {REDI }}=\left(\frac{E_{0} I_{x}}{B_{x} I_{\eta}}\left(I+\eta_{2}\right)\right)^{4 / 3}\left(\chi_{x} k_{11}^{\prime 2}\right)^{-1 / 3}
$$




$$
-x^{-5 / 6} n^{1 / 3} q^{2} B_{x}{ }^{-4 / 3}\left(I_{\eta}^{-1}+I_{z}^{-1}\right)^{4 / 3} \bar{s}^{-2} E_{0}{ }^{1 / 3} x^{2 / 3} R^{14 / 3}
$$

and resistive ballooning turbulence ${ }^{13}$

$$
\begin{aligned}
& D_{x I}=(2 \pi q)^{2} p_{e}^{2} v_{e x}\left(R / I_{h}\right) \\
& =I^{-1 / 2} n g^{2} B_{x}{ }^{-2} R I_{w^{2}}{ }^{-1}
\end{aligned}
$$

Here $x_{e}=-x_{0} / \frac{\partial x_{c}}{\partial x}=\frac{3}{2} I_{n}, \chi_{z}=V_{i}^{2} / Z^{2} v_{i i} k^{\prime} \mid I=k_{y} / L_{s}, \eta_{z}=L_{\eta} / L_{z}$ and the shear length $L_{5}=\mathrm{Rq} / \mathrm{s}$. The toroidal electric and magnetic fields are $E_{0}$ and $B_{2}$ respectively. The RGDT theory was originally an outgrowth of the rippling mode. Detailed calculations of the linear theory, including impurity gradlent and diamagnetic effects, show that the rippling mode is stabilized at rather v temperatures 14 . Kwon, Diamond and Hahm ${ }^{15}$ state that in the nonlinear regime the mode dynamics are vastly different from those of the linear theory and that the mode is nonlinearly robust. They also argue that since the edge plasma is rarely quiescent "the picture of a linearly unstable mode developing from a quiescent plasma at equilibrium is not necessarily appropriate". Estimates obtained from the theory ${ }^{16}$ indicate that the turbulent enhancement due to radiation drive, which would reduce the sensitivity to the loop voltage, is insignificant for the plasma where $0.6<r / a<0.8, \mathrm{Te}-150 \mathrm{eV}$. Thus the scaling of Eq. 4 is the most appropriate version for this region. The scaling for DRGDT is evaluated using data from shots at the five densities with $\eta_{\mathrm{z}}=0$. Results in Fig. 4 show a trend in the profiles of $D$ similar to that in the experimentally determined values. The plasma current, $Z_{\text {eff }}$ and $q$ are constant across this scan. The largest changes occur in the density and temperature. The substantial increase calculated at $\bar{n}_{e, 20}=3.3$ is due primarily to decreases in $\mathrm{T}_{\mathrm{e}}, \mathrm{L}_{\mathrm{Te}}$ and to a lesser extent to an increase in the loop voltage. Through its inverse temperature dependence Eq. 5 also gives diffusion coefficients generally increasing toward the limit, but the variation is weaker and bears less resemblance to the measured coefficient variation. 
Results given here suggest that the mechanism responsible for the increased particle transport near the density limit in Alcator $C$ is localized to regions of relatively low temperatures. A resemblance to the RGDT scaling suggests that the particle transport coefficients were increased there through a similar inverse dependence on $\mathrm{T}_{\mathrm{e}}$ and $\mathrm{L}_{\mathrm{Te}}$. ONETWO analysis indicates that a fractional increase in the particle flux similar in magnitude to that observed in D would not cause a significant change in the global energy confinement time. But in a region where the convected energy flux is a substantial fraction of the overall energy flux, such as might occur at the plasma periphery, a rise in the convected flux would also lead to a significant increase in the local thermal transport.

Plasmas in the T-10 tokamak also show a deterioration in confinement near the density limit. The evolution of certain parameters of these $T-10$ plasmas has been simulated reasonably well with an adjustable model that combines several forms for the transport coefficient profiles. It includes two key features also consonant with MTX plasma behavior: an inverse temperature dependence in the edge transport coefficients and an SOL density that saturates with regard to the particle flux at fixed power. A reduced form of the RGDT scaling was used $n^{1 / 3} q^{2} / T^{5 / 6}$ and found to be the most appropriate to model the edge region ${ }^{17}$.

In the gas-fuelled Alcator C discharges the particle source is localized to the scrapeoff layer and very edge of the bulk plasma. The rapid increase in particle transport

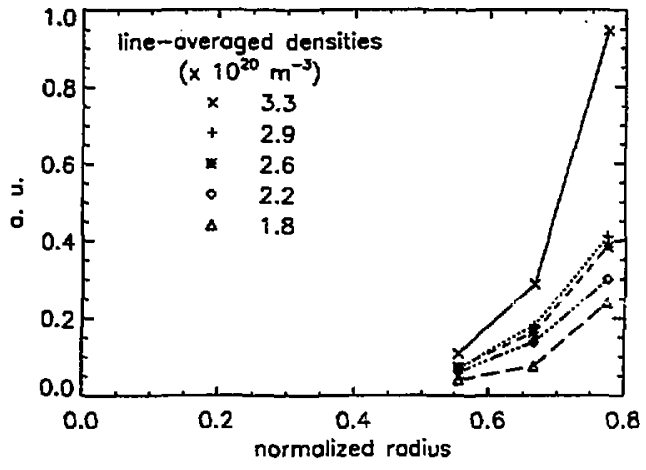

Fig. 4 Scaling of resistivity-gradient-driven turbulence diffusion coefficients for plasmas of the scan.

coefficients in the outer plasma near $\bar{n}_{e}=x \bar{j}$, combined with the saturation in the scrapeoff layer density make the plasma density profile in the region where significant particle source exists insensitive to further increases in this source. This causes the entire density profile to saturate resulting in the density limit in Alcator C. 
In large tokamaks the density limit appears to be controlled by processes that occur at the plasma edge. Recent pellet injection experiments with neutral beams on TFTR revealed no deterioration in the core particle confinement for average densities as large as $\mathbf{2 . 2}$ times the Greenwald limit value ${ }^{18}$. The central densities and toroidal field of these circular plasmas were similar to those of the MTX plasma. Although many differences exist between them, such as size and detailed profile shapes, the most obvious difference is in the temperature profiles. The regicns with low $\mathrm{Te}(\leq 250 \mathrm{eV})$ exist in these TFTR discharges only in the SOL and sometimes at the limiter. An instability that exists only in relatively low $T_{e}$ plasma, such as a resistive instability, would be absent in the core of this plasma.

In summary, both the perturbed particle diffusion and convection coefficients are seen to increase near the Greenwald density limit in Alcator $C$. The increases occur in regions with relatively low temperatures. A resemblance to the scaling given by a theory of RGDT suggests that the coefficients were enhanced there through a similar type of inverse dependence on $\mathrm{T}_{e}$ and $\mathrm{L}_{\mathrm{T}}$.

1. K. Borrass, Y. L. Igitkhanov, N. A. Uckan, Report of the ITER Experts' Meeting on Density Limits, ITER-TN-PH-9-6, Garching, September 1989.

2. M. Greenwald, J. L. Terry, S. M. Wolfe et al., Nuclear Fusion 28, 2199 (1988).

3. V. V. Alikaev et al., Plasma Physics and Controlled Nuclear Fusion Research (Proc. 13th Int. Conf., Washington, 1990), Vol. I, IAEA, Vienna (1991) 163.

4. J. Allen, G. E. Austin, K. B. Axon, et al., Plasma Physics and Controlled Nuclear Fusion Research 1986, (Proc. 11th. Int. Conf., Kyoto, 1986), Vol. 1, IAEA, Vienna (1987) 227.

5. T. Yu. Akatova, et al., Plasma Physies and Controlled Nuclear Fusion Research (Proc. 13th Int. Conf., Washington, 1990), Vol. I, IAEA, Vienna (1991) 509.

6. D. L. Brower, C. X. Yu, et al,, Phys. Rev. Lett., 67, 200 (1991).

7. K. W. Gentle, Phys. Fluids 31, 1105 (1988).

8. B. LaBombard, Ph.D. Thesis, Poloidal Asymmetries in the Limiter Shadow Plasma of the Alcator C Tokamak, MIT (1986).

9. M. M. Marinak, Ph.D. Thesis, Behavior of the Particle Transport Coefficients Near the Density Limit in Alcator C, Department of Nuclear Engineering, University of California at Berkeley (1993).

10. P. H. Diamond, J. F. Drake et al., Comments Plasma Phys. Controlled Fusion 13(6), 327 (1990).

11. J. F. Drake, T. M. Antonsen, Phys. Fluids 27(4), 898 (1984).

12. T. S. Hahm, P. H. Diamond, P. W. Terry, L. Garcia, B. A. Carreras, Phys. Fluids 30, 1452 (1987).

13. D. R. MCCarthy et al., Phys. Fluids 4, 1846 (1992).

14. W. M. Tang, F. Romanelli, S. Briguglio, Phys. Fluids 31(10), 2951 (1988).

15. O. J. Kwon, P. H. Diamond, T. S. Hahm, Phys. Fluids B1, 2172 (1989), also D. R. Thayer and P. H. Diamond, private communications.

16. D. R. Thayer, P. H. Diamond, Phys. Rev. Lett. 65, 2784 (1990), D. R. Thayer, P. H. Diamond, Phys. Fluids 30, 3724 (1987).

17. Yu. N. Dnestrovskij, Yu. V. Esipchuk, K. A. Razumova, P. N. Yushmanov, Plasme Physics and Controlled Nuclear Fusion Research (Proc. Thirteenth Int. Conf., Washington, 1990), Vol. I, LAEA, Vienna, 393 (1991). 
18. M. G. Bell et al., Nuclear Fusion 32, 1585 (1992). 


\section{FEL development}

\subsection{FEL status during initial microwave experiments (A. L. Throop, et al.)}

Details of the free-electron laser facility status during the initial microwave experiments on MTX can be found in a Beam Research Report. ${ }^{1}$ The ELF-II (Electron Laser Facility) FEL (Free Electron Laser) experiment used the ETA-II (Experimental Test Accelerator) Induction-Linac accelerator facility. This experiment was the first phase of the development program to investigate the use of high-power pulsed microwave FEL for plasma heating.

The experiments covered the period of May 17 through November 14, 1989. An experimental log summary and breakout of activities is given in Appendices A and B. The experiments included the following major activities:

- Operation of the ETA-II accelerator using the "D1" diode injector geornetry to verify the capability of producing and accelerating a $3 \mathrm{kA}$ beam, as required for subsequent plasma heating experiments.

- Transport and matching of the electron beam from the accelerator into the wiggler. These experiments were performed using a $1.8 \mathrm{kA}$ beam from the injector to improve accelerator reliability and performance during initial FEL operation.

- Operation of the FEL with an untapered wiggler for initial start-up and characterization of the FEL. Incremental and saturated amplifier gain were measured, along with detuning curves and spontaneous noise measurements, and compared with simulation codes.

- Low-power operation of the FEL for joint experiments with MTX. These included measurements of microwave transport, diagnostic tests, and linear-absorption experiments on MTX.

- Initial tapering experiments of the FEL. A quick evaluation of tapering the wiggler under lower-current operation was made.

Due to non-technical constraints, we were unable to continue with the remaining experiments that involved raising the beam current about $500 \mathrm{~A}$ to achieve FEL operation at the gigawatt peak-power level, followed by nonlinear absorption and heating experiments on MTX.

The report provides an internal reference summary of the results of the FEL portion of the FEL/MTX experiments. Sufficient background 
information (e.g., cailibration accuracy) is included to put the results in context and to document procedure's and issues for future reference. The operational performance of the FEL subsystems (accelerator, beamline, wiggler, and microwave diagnostics) is sunmmarized to serve as a reference for the next phase of experiments. Outstanding issues that remained to be addressed were identified.

The major results of the ELF-II experiments are summarized as follows:

- The D1 injector was capable of providing a $3 \mathrm{kA}$ of beam using the existing MAG-1D design, in good agreement with design codes.

- The ETA-II accelerator routinely provided a $6 \mathrm{MeV}, 1700 \mathrm{~A}$ beam with a brightness of $6 \times 10^{8} \mathrm{~A} /(\mathrm{m}-\mathrm{r})^{2}$, as measured by a two-slit emittance diagnostic. Operation at $3 \mathrm{kA}$ was limited by breakdown in the accelerator cells due to mismatch. Aside from small loss at the injector, the beam was transported through the accelerator without measurable current or charge loss (3-5\% accuracy).

- Energy sweep and corkscrew beam motion in the present accelerator significantly limited FEL performance, resulting in short pulse width and reduced output power. The sources of these problems have been identified and modifications are in progress to correct them for the next FEL experiments.

- The ELF beamline transported the beam to the wiggler with about $8 \%$ current and 50\% charge loss, with the dominant singular loss where the beam is matched into the beamline.

- The tuned-receivers used for microwave power measurements functioned well, although the unplanned short FEL pulse-width (5-10 ns) limited their use for quantitative measurements. The microwave transport also functioned well, although alignment procedures and reproducibility of a movable mirror required some improvement to insure measuring consistent power levels at the various detectors.

- Saturated peak-power levels up to $210 \mathrm{MW}$ at $140 \mathrm{GHz}$ were measured for an untapered wiggler, with pulse widths of $8 \mathrm{~ns}$. Incremental and total amplifier gains of $23 \mathrm{~dB} / \mathrm{m}$ and $70 \mathrm{~dB}$, respectively, were measured. The measured gain curve showed good agreement with simulation results when beam offset is included. This was obtained with $1600 \mathrm{~A}$ of beam current, somewhat below the planned current level, and without any phase-space conditioning of the beam.

- In a preliminary tapering experiment at the $1600 \mathrm{~A}$ current level, a significant increase in power over untapered operation was measured using an unoptimized linear taper. The peak-power is estimated in the 
range of $300-500 \mathrm{MW}$, in reasonable agreement with simulation predictions. The extremely short pulse width (1.5 ns), however, made quantitative analysis difficult and confirmational experiments important.

- While not detailed in the report, joint plasma-absorption experiments with the MTX facility using the FEL were rouainely accomplished. The ability to reliably transport the FEL microwave pulses over long distances and to absorb and diagnose them in a tokamak plasma using a unique array of pulsed microwave diagnostics was demonstrated.

- While the FEL and tokamak experiments were made at the 100-200 MW power level, the results and modeling indicates nothing that would have prohibited the generation of gigawatt peak-power levels at this present phase, using only the modest increase in beam current that was planned. The power-distribution and alignment modifications that were implemented in ETA-II allowed these levels to be achieved in the next phase of experiments.

The experiments represented the combined efforts of individuals responsible for the accelerator, FEL, microwave, and tokamak subsystems. In the report, the authors summarized the results in reasonable detail for future reference. Background information (calibrations, etc.) that might not be documented elsewhere were also included for reference. Section. 2 describes each subsystem and summarizes its operational performance. Results of FEL measurements are described in Section 3.

1. A. L. Throop et al., "Summary of Single-Pulse Induction-Linac FEL Experiments at $140 \mathrm{GHz}$ Using the ETA-II/ELF-II Facility - Run of Summer/Fall, 1989," Beam Research Memo RM 90-22-12/10/90. 
8.2 Generation of High Power $140 \mathrm{GHz}$ Microwaves with an FEL for the MTX Experiment* (S.L. Allen, C.J. Lasnier, B. Felker, M. Fenstermacher, S.W. Ferguson, S. Fields, E.B. Hooper, S. Hulsey, M. Makowski, J. Moller, W. Meyer, D. Petersen, E.T. Scharlemann, B. Stallard, R. Wood)

\subsubsection{Introduction}

We have used the improved ETA-II linear induction accelerator (ETA-III) and the IMP steady-state wiggler to generate high power (1-2 GW) microwaves at $140 \mathrm{GHz}$. The FEL was used in an amplifier configuration with a gyrotron driver. Improved control of energy sweep and computerized magnetic alignment in ETA-III resulted in small beam corkscrew motion (<1.5 mm) at $6 \mathrm{MeV}, 2.5 \mathrm{kA}$. Reduction of wiggler errors $(<0.2 \%)$, improved electron beam matching, and tapered wiggler operation resulted in peak microwave power (single-pulse) of up to $2 \mathrm{GW}$. These pulses were transported to the MTX tokamak for microwave absorption expel.ments. In addition, the FEL was run in a burst mode, generating 50-pulse bursts of microwaves; these results are discussed elsewhere.1

Generation of high power microwaves with an FEL is advantageous for fusion experiments for several reasons: 1) the output is a simple TE01 mode which couples directly to the plasma, 2) there is no output window which could fail at high power, and 3) frequency sweeping is possible by varying a low power source. The FEL for the MTX experiment is made up of four major parts: the ETA-II linear induction accelerator, the electron beamline, the MMP wiggler, and a quasi-optical microwave transport system connected to the MTX tokamak. FEL performance in 1989 was limited to short (5-10 ns) $0.2 \mathrm{GW}$ pulses at $140 \mathrm{GHz}$ because of a substantial corkscrew motion ( 1 $\mathrm{cm}$ ) of the beam and the nonrepioducibility of the electron beam pulse (making empirical wiggler tapering difficult). The corkscrew motion is caused by the energy sweep of the beam during the pulse, coupled with misalignments of the solenoidal transport system of the accelerator. ${ }^{2}$

The 2-D FEL simulation code FRED ${ }^{3}$ was used to estimate the beam parameters required for high-power FEL operation. At a beam energy of 7.35 $\mathrm{MeV}, 2 \mathrm{kA}$ of beam current, and $50 \mathrm{~W}$ of drive power, calculated output power is approximately $5 \mathrm{GW}$ [4].4 This estimate requires a $\pm 0.1 \mathrm{~cm}$ beam. displacement, a $\pm 1 \%$ energy sweep, and a $0.1 \%$ error in the wiggler magnetic fields. The sensitivity of the cutput power to these quantities is shown in Table 1, along with achieved parameters.

Several hardware and operational improvements were first tested on a 20 -cell version of the whole ( 60 cell) ETA-II system, resulting in reduction of the corkscrew motion and improved energy regulation.2, 5,6 These improvements were then implemented on a 60-cell version called ETA-III, 
Table 1

FRED Predictions of FEL Output Power

\begin{tabular}{|c|c|c|c|}
\hline Purameter & Variation & $80 u t$ & Achieved \\
\hline Beam Energy & 7.5 to $6 \mathrm{MeV}$ & 50 & $\mathrm{MeV}^{6.3}$ \\
\hline Bean Current & 2.5 to $2 \mathrm{kA}$ & 60 & $2.5 \mathrm{kA}$ \\
\hline & 2.0 to $2 \mathrm{kA}$ & 17 & \\
\hline Brightness & $\begin{array}{r}100.2 \times 10^{8} \\
A \mathrm{~m}^{-2} \mathrm{r}^{-2}\end{array}$ & 60 & $>10^{8}$ \\
\hline Beam Motion & & & $\begin{array}{l} \pm 1 \mathrm{~mm} \\
(40 \mathrm{~ns})\end{array}$ \\
\hline Encery Swoed & $\pm 1 \%$ to $\pm 2 \%$ & 80 & $\pm 1 \%$ (35 ns) \\
\hline Wirler Error & & & $0.2 \%$ RMS \\
\hline
\end{tabular}

and a steady-state wiggler was installed. Note from Table 1 that the required electron bexm parameters have been achieved. Tapered FEL operation resulted in the generation of single pulse microwaves up to $2 \mathrm{GW}$ which were transported to the MTX tokamak with a quasi-optical microwave transport system. Further optimization of the output power was not performed because of extremely limited experimental time. Electron Cyclotron Resonance Heating (ECRH) experiments on MTX with the FEL demonstrated nonlinear absorption of the high power microwaves, in agreement with theory? Extrapolation of this theory shows good absorption for reactor conditions, indicating that FELs are useful for future fusion machines.

\subsubsection{Description of the FEL}

8.2.2.1. The ETA-II 60-Cell Accelerator. The ETA-II accelerator consists of an electron beam injector and 60 accelerator cells. The injector consists of a thermionic, osmium-coated (12.7-cm-diameter) dispenser cathode operated in a diode configuration to generate about $2500 \mathrm{~A}$ at $1 \mathrm{MeV}$ in the space-charge limited regime. Several improvements tested on the 20-call prototype were implemented on the 60-cell version of ETA-II for these experiments: 1) a multicable pulse distribution system ${ }^{8}, 2$ ) arc and overvoltage protection, 3) feedback control of pulse timing, 4) an improved cell design to minimize arcing and the Beam Break-Up Instability, 5) in-situ magnetic alignment, and 6) a computerized tuning algorithm to minimize corkscrew directly. ${ }^{10}$ The computer processing was substantially improved compared to the 20 cell case so that the whole accelerator could be tuned in about 1 day. Improvements (1) and (3) resulted in much better energy regulation, as shown in Table 1, $\Delta \mathrm{T} / \mathrm{T}$ of $\pm 1 \%$ for $35 \mathrm{~ns}$ was achieved. The feedback system enabled pulse-to-pulse corrections in the timing of each magnetic compressor (MAG-1D) to be maintained within $\sim 1-2 \mathrm{~ns}$; daily variations of over $20 \mathrm{~ns}$ 
were continuously corrected. Energy flatness was also improved by optimization of the operating point of the MAG-1D; a slightly rising waveform was used on the first 20 cells to compensate for a falling waveform from the injector. The corkscrew amplitude was found to depend weakly on the operating point ( $\pm 10 \mathrm{kV}$ from $90 \mathrm{kV}$ per cell gap).

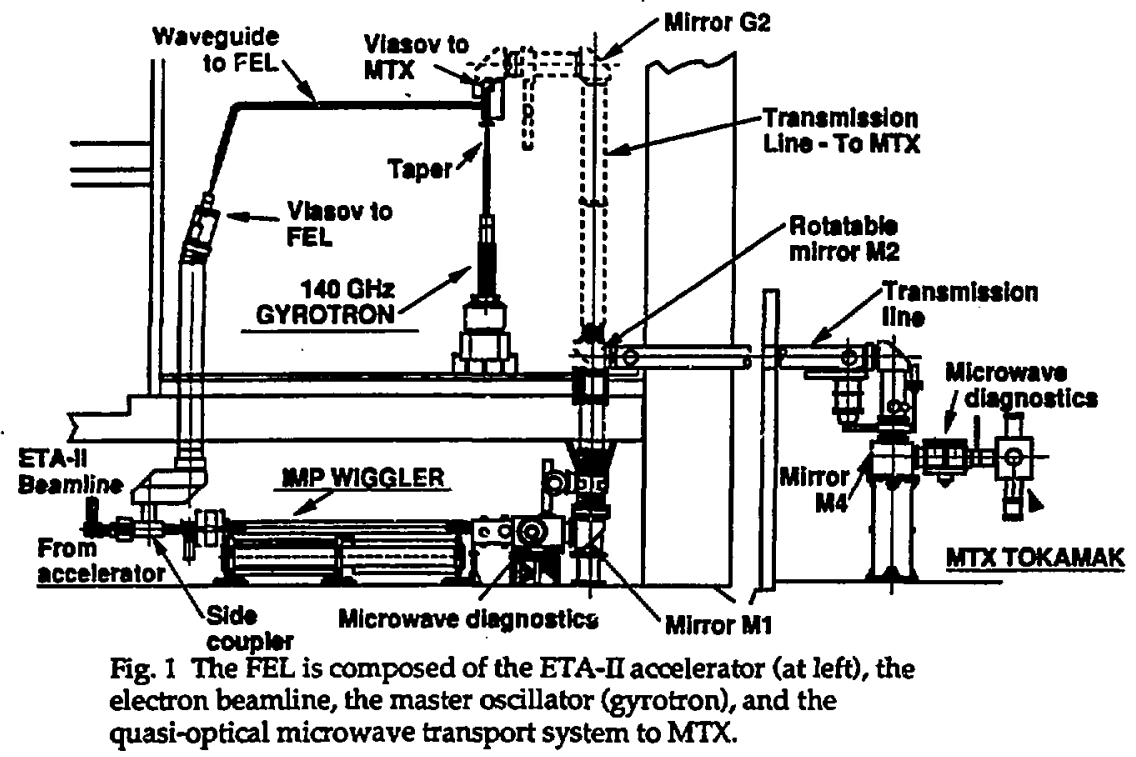

Reduction of magnetic errors with (5) and (6) resulted in transverse beam motion nearly comparable to that obtained for the 20-cell experiment ${ }^{5}$, i.e., \pm 1 $\mathrm{mm}$ for $40 \mathrm{~ns}$. A Stretched Wire Alignment Technique (SWAT) was used for alignment before operation. During electron beam operation, the MAESTRO10 computerized control system minimized the corkscrew directly. The $\mathrm{x}$ and $\mathrm{y}$ components of the beam motion (referenced either to the beam or apparatus centroid) were measured with current return monitors and processed into a corkscrew amplitude $A$ by the MAESTRO sysiem. The MAESTRO system also controls the 60 pairs of power supplies for the sin and cos correction coils in each cell block used to minimize A. The computer system has been optimized so that $A$ is calculated and the currents are changed between pulses at $1 \mathrm{~Hz}$. MAESTRO can automatically process all 60 cells in a few hours.

8.2.2.2 The IMP Wiggler and Microwave System. Shown in Fig. 1 is the FEL system; a series of magnetic lenses are used to transport and match the 
electron beam into the input of the wiggler. The $110 \mathrm{GHz}$ gyrotron is the master oscillator (MO); a mirror in the sidecoupler focuses the microwave beam into the wiggler; a 4-cm diameter central hole is provided to pass the electron beam. The IMP wiggler ${ }^{11}$ is a tuneable $5.5 \mathrm{~m}$ hybrid (both permanent and electromagnets) steady-state wiggler with a $10 \mathrm{~cm}$ period. The field is adjustable from 2.7-5.5 kG in the first half, and 0.6-4.1 kG in the second half of the wiggler. After careful construction, hall probe measurements indicated that the RMS wiggler errors were reduced to $0.1 \%$ for elettromagnets alone, and $0.2 \%$ for the whole wiggler. We have verified the electron beam matching in the wiggler with a moveable optical target; no strong evidence of beam steering or abnormal growth in beam size was observed.

\subsubsection{Experimental Results: FEL Output Power at 140 GHz}

The FEL system was operated at $1 \mathrm{~Hz}$, and scans of the wiggler magnetic field w:ire used to determine the IMP operating point. The viggler was then tapered to optimize the output power, again using the MAESTRO system for control. We started with a theosetically-derived taper, and then empirically optimized the output power. The output power waveform was measured with both a calibrated diode and a receiver. A more precise calibration of the time-integral of this signal was provided by a precision calorimeter. An example of a pulse is shown in Fig. 2; we measured peak powers up to about 2 GW.

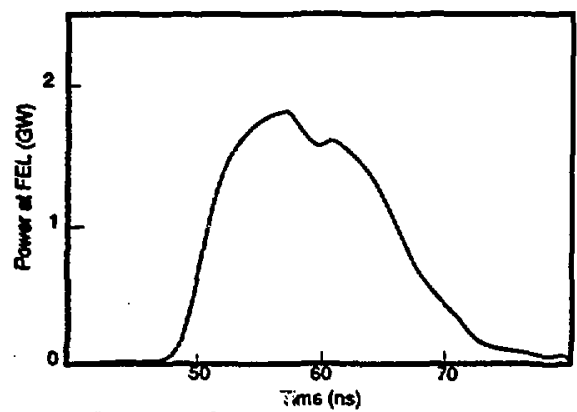

Fig. 2 A sample trace showing the output power versus time measured at the output of the FEL.

At this point, even though the theoretical models indicated that powers of 2-3 times greater were possibie, we concentrated on MTX piasma experiments rather than FEL optimization. (In addition, one IMP coil was damaged, limiting the tuning range and therefore the usable tapers). The FEL output power was transmitted to the MTX tokamak with a quasi-optical transmission system consisting of several mirrors. This system is very efficient, transmitting nearly $90 \%$ of the power. FEL operation in the single- 
pulse mode was fairly reproducible; routine operation for several days with only minor tuning was achieved. An example of the peak power measured at MTX for one experimental day is shown in Fig. 3 (the FEL runs at $1 \mathrm{~Hz}$, MTX shots are 5 minutes apart.)

\subsection{Discussion}

Improved energy regulation and reduced corkscrew motion of the electron beam from the ETA-II accelerator have made possible high power FEL operation. Peak powers of $-2 \mathrm{GW}$ at $140 \mathrm{GHz}$ has been generated in single pulses. Further optimization of the FEL power was not possible due to limited experimental time. These high-power pulses were used in microwave transmission experiments in the MTX tokamak; nonlinear ECRH behavior was observed, in semi-quantitative agreement. with theory. Burst mode operation of the FEL was also demonstrated, and is described in Ref. 1.

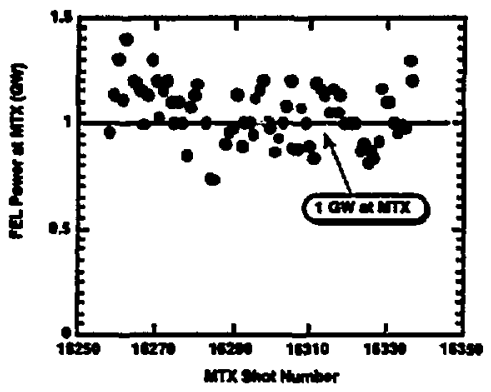

Fig. 3. The peak microwave power (measured at MTX) varies from 0.75-1.4 GW during a day of experiments

We would like to acknowledge the fundamental accelerator and FEL work done by the Beam Research Group at LLNL that made these FEL experiments on the MTX tokamak possible.

- S.L. Allen et al., Proc. 1993 Part. Accel. Conf. (Washington, 1993).

1. C J. Lasnier et al., "Burst Mode FEL with ETA-III Induction Linac," Proc. 1993 Part. Accel. Conf. (Washington, 1993); also, Sec. 8.3

2. W.C. Turner, "Control of Energy Sweep and Transverse Beam Motion in Induction Linacs," in Proc. 1991 Part. Accel. Conf. (San Francisco, 1991) 3094.

3. E.T. Scharlemann, Laser Handbook, Vol 6, (North Holland, New York, 1990) 291. 
4. R.A. Jong et al., Nuc: instrum. Meth. Phys. Res. A285 (1989) 379, also private communications April, 1992.

5. S.L. Allen et al., "Measurement of Reduced Corkscrew Motion on the ETA-II Linear Induction Accelerator," Proc. 1991 Part. Accel. Conf. (San Francisco, 1991) 3094.

6. W.E. Nexsen et al., "Reduction of Energy Sweep of the ETA-II Beam," Proc. 1991 Part. Accel. Conf..(San Francisco, 1991) 3103.

7. S. L. Allen et al., "Nonlinear Absorption of High Power FEL-Generated Microwaves at ECRH Frequencies in the MTX Tokamak," submitted to Phys. Rev. Lett.

8. S.E. Sampayan et al., "Energy Sweep Compensation of Induction Accelerators," in Proc. 1990 LINAC Conf. (Albuquerque, 1990).

9. L. Griffith and F. Deadrick; "Progress in ETA-II Magnetic Field Alignment Using Stretched Wire and Low Energy Electron Beam Techniques," in Proc. 1990 Linac Conference, Albuquerque, NM, September 10-14, 1990.

10. Darrel L. Lager et al., "Artificial Intelligence Techniques for Tuning Linear Accelerators," Proc. 1991 Part. Accel Conf. (San Francisco, 1991) 3082.

11. R. Schlueter and G. Deis, "Tuneability Enhanced Electromagnetic Wiggler," US Patent 5099175, March 24, 1992. 


\subsection{A Development to Improve ETA-II: Magnetic Alignment (C. J. Lasnier)}

A technique referred to as SWAT (Stretched Wire Alignment Technique) was used for correction of mechanical magnet positions, mechanical and electromagnetic correction of tilts for intercell focus magnets, and electromagnetic correction of tilts in cell magnets by steering coils. This was a refinement of a technique previously used to align the accelerator, which in turn was a variation on a technique used to correct wiggler magnets at other laboratories. ${ }^{1-3}$

A Beryllium-Copper wire was stretched down the axis of the accelerator while the ends were open. Micrometer-adjustable positioning fixtures were plnced at both ends, with one end having a pulley. The wire passed over the pulley, and a weight was hung from the end of the wire. Electrical connections were made to the ends of the wire, and current pulses were passed through the wire. (Fig. 8.2.A-1)

One focus magnet at a time was turned on, and if the wire did not pass down the magnetic axis, the transverse field would pluck the wire when the current pulse passed. The pulse traveled down the wire, and the displacement was detected near the end by two light emitting diode and photo-sensor pairs for, respectively, horizontal and vertical motion.

The signatures of offset or tilt errors in the magnet were distinct (Fig. 8.2.A1). The photo sensor signals were digitized by an oscilloscope and acquired in a Macintosh computer using LabView. The data was processed to separated the tilt and offset components by projecting the signal on orthogonal basis functions representing pure tilt and pure offset. The offset signal is an even function about its center, while the tilt is odd. The program then gave estimates of the displacement from the magnetic axis in thousandths of an inch, and the tilt correction coil current change needed in Amperes.

The catenary sag in the wire was calculated, and removed using the micrometer adjustments to position the wire at the mechanical center of the magnet.

The wire was moved by the micrometers at both ends until the offset signal was nulled out, and the trim (steering) coil currents were adjusted for minimum tilt signal. These alignment values were recorded for the horizontal and vertical adjustments of each magnet.

The coil currents were used at the option of the accelerator operations crew as the starting values for the steering coils, for corkscrew minimization. The displacement values for each of the magnets in a 10-cell block were plotted and an axis was selected to minimize the overall magnetic error. The cell block was moved mechanically to bring the selected axis in line with the mechanical axis of the accelerator. The SWAT procedure was repeated after this to check the alignment.

The next alignment step was to run the accelerater and interactively minimized the beam corkscrew by adjusting steering magnets. This was done either by the operator watching beam-bug signals on an oscilloscope display and 
turning knobs to adjust. magnet currents, or by running the MAESTRO tuning $V$ procedure.

The Tuning $\mathrm{V}$ consisted of a steering current scan executed by the computer while digitizing beam bug signals. The corkscrew was calculated by one of several algorithms, and plotted against steering magnet currents (Fig. 8.2.A-2). The minimum could be chosen by the computer and set as the new steering current, or the operator could choose a value after examining the plot. The MAESTRO system is discussed in more detail in section A.4.4.

1. F. J. Deadrick and L. V. Griffith, "Progress in ETA-II Magnetic Alignment Using Stretched Wire and Low Energy Electron Beam Techniques," 1990 Linac Conference, Albuquerque, NM, UCRL-103780.

2. R. W. Warren, "Limitations on the Use of the Pulsed-Wire Field Measuring Technique," Nucl. Instrum. Meth. Phys. Res. A272, 257 (1988).

3. D. J. Liska, L. B. Daulsberg, and G. Spalek, "Precision Alignment of Permanent-Magnet Drift Tubes," LA-UR 86-1718, LANL 1986. 

Magnetic Offsets and Tilts During ETA-II Construction

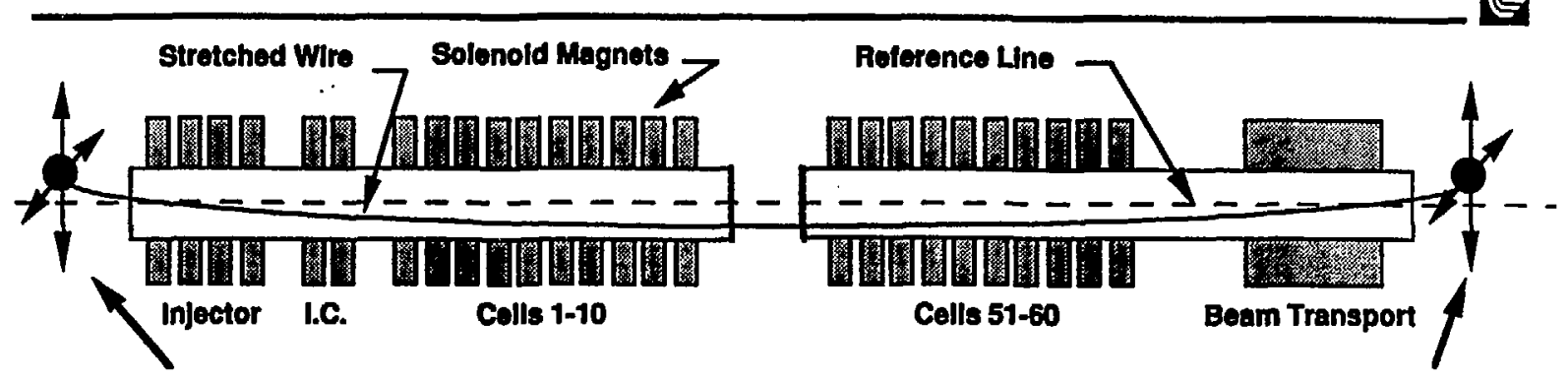

Current Pulse is Injected at this End

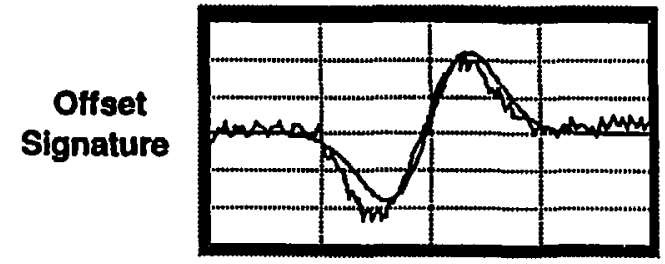

Computer Acquires Photodetector Signal at This End

Tilt Signature

- Computer Deconvolves Tilt and Offset and Indicates Required Correction (Coils are Done One at a Time)

-Offset (Mechanical Adjustment)

--Tilt (Trim Current)

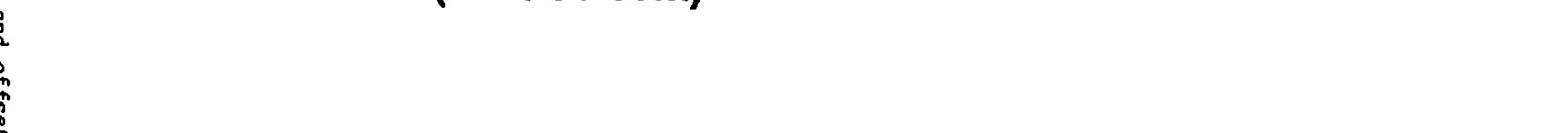




\section{MAESTRO system is used to tune the ETA-II accelerator $B$ fields}

- "Tuning V's" minimize corkscrew directly by scanning trim fields

- Automated procedure can tune 10 cells in about 10 minutes (compared to 1 day in previous 20 cell experiment last year)

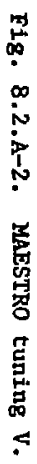

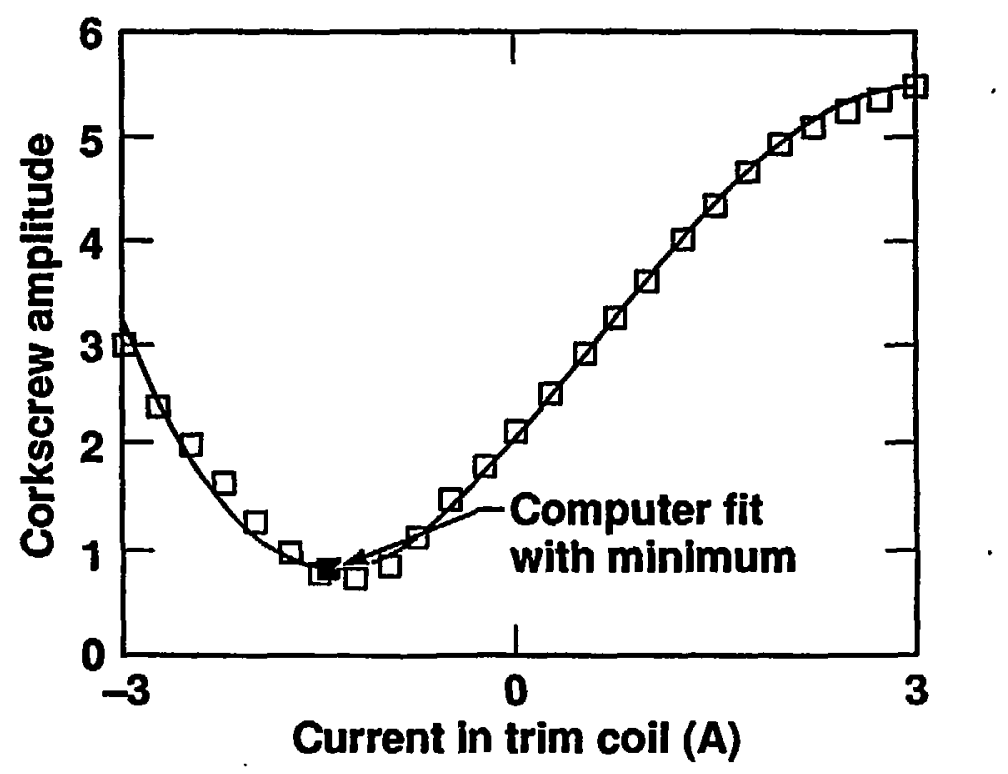




\subsection{B FEL Operation: Single Pulse (C. J. Lasnier)}

Accelerator. The focus tune of the accelerator for beam transport used as a starting point was calculated by Y.J. Chen. Small changes were made to optimize this empirically.

The accelerator was tuned to minimize corkscrew and energy sweep in the electron beam ( see 8.2.A Magnetic Alignment). The initial SWAT alignment was followed by interactive tuning of the beam by using the MAESTRO tuning V's (see Section A.4.4 MAESTRO) and manual beam operator adfustments. The corkscrew was reduced to less than $\mathrm{mm}$ at the end of the accelerator for $40 \mathrm{~ns}$ (Fig. 8.2.B-1). Energy sweep of $\pm 1 \%$ for $30 \mathrm{~ns}$ was achieved.

All ETA-II magnet settings were archived by the MAESTRO system, and could be downloaded to the ETA-II magnet control VAX, so that previous operational setups were easily repeatable.

The timing of the voltage pulses relative to the beam in the accelerator was found to be critical for high peak FEL power. The best results were from "front-loading" the beam, which meant the voltage pulse was not centered on the beam timing, but $4-6$ ns later.

Transport Section. The initial values of the focus magnets in the transport section were based on calculations by A. Paul. This was adjusted to maximize the beam transport through the slits. The transport section of the beam line is shown in Fig. 8.2.B-2.

During the process of steering the beam into the wiggler, several holes were melted in the sides of the wave guide. Each time, this took the wiggler vacuum to air. The holes were patched using sections of copper sheet held in place by Torr-Seal. This allowed continued operation the following day.

As a result of the holes in the wave guide, a protection scheme of 'diverting' the electron beam was arrived at. Transport magnets in the beam line were turned off for a period, then turned on to allow beam into the wiggler. This was repeated with a duty cycle of perhaps 20 seconds on $(0.5 \mathrm{~Hz}$ pulses) followed by $30-60$ seconds off. This allowed heat built up in the wave guide to dissipate before the next series of pulses. The MTX timing system sent a signal when a shot was in progress which told the ETA-II magnet control computer to 'un-divert' the beam during combined operations, so that FEL pulses could be fired into MTX plasma.

Wiggler. The small beam bug at the entrance to the wiggler was damaged early on by stray beam, and could not be used to steer into the wiggler. The initial steering into the wiggler was done using beam bugs further upstream, and the final steering was accomplished by maximizing the FEL microwaves.

The beam steering in the wiggler was checked by dragging a quartz foil down the wave guide. Initially the foil was attached to a copper "boat", but the copper was distorted by the beam heating. The next boat was machined of 
carbon, and endured satisfactorily. Due to the small spot size of the beam in the wiggler, the foil was good for only a few hours of operation before a hole was gradually burned in the center.

A gated video camera looking through a window and periscope assembly from the down-stream end of the wiggler wave guide captured images of the foil (Fig 8.2.B-3). The video data was analyzed to obtain the beam path data shown in Fig 8.2.B-4. this shows the 'wiggle' of the beam in the wave guide.

Several of the electromagnets in the wiggler were damaged by overheating or short circuits during the course of operation. Various wiring changes were made to reduce the effect of the bad magnets, and some magnets were run with low current limits.

FEL. Peak FEL power was up to $2 \mathrm{GW}$ out of the wiggler. The integrated power was measured at the JAERI calorimeter at MTX for a series of pulses. The same series of pulses was digitized from a diode detector connected to the J0 mirror near the wiggler output end. By comparing the ratio of the integral of the digitized diode signal with the energy integrated by the calorimeter, the diode calibration was obtained. The resulting calibration gave the diode signal in units of GW at MTX (JAERI Box). The transmission of FEL power from the wiggler to the JAERI Box at MTX was near 70\% ( see Section 4.2 Quasi-Optical Transmission Line).

Length scans and resonance scans were done with the wiggler. The resonance was uniformly found at 12-15\% lower field than predicted by the expression derived by E.T. Scharlemann,

$$
E=0.511 M e V\left(5 \sqrt{1+0.46 B^{2}}-1\right)
$$

(where $E$ is the kinetic energy of an electron in $\mathrm{MeV}$, and $B$ is the resonant wiggler field in $\mathrm{kG}$ ) in rough agreement with previous experiments. The length scans showed anomalously high gain for short wiggler lengths. The short wiggler was obtained by de-tuning sections of the wiggler. Code runs by Scharlemann indicate the maximum de-tuning possible for this wiggler still allowed some gain in the "non-resonant" sections, so that the "short" wiggler had a longer effective length. 


\section{Corkscrew and Energy Sweep are Greatly Reduced in ETA-II}

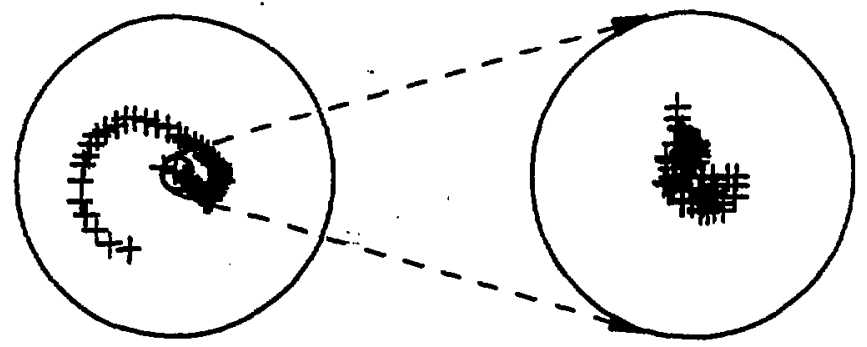

BEFORE $< \pm 2 \mathrm{~cm}$ $1.5 \mathrm{kA}, 6 \mathrm{Mev}, 40 \mathrm{~ns}$ Fib Do170FO1
AFTER $< \pm 0.2 \mathrm{~cm}$ $2.5 \mathrm{kA}, 6$ Mev $40 \mathrm{~ns}$ File D2007204

\section{Energy Sweep of Less Than $\pm 1 \%$ for Over $30 \mathrm{~ns}$}

\section{Mean Ener
6.1 MeV}

5
8
$\frac{1}{5}$
4

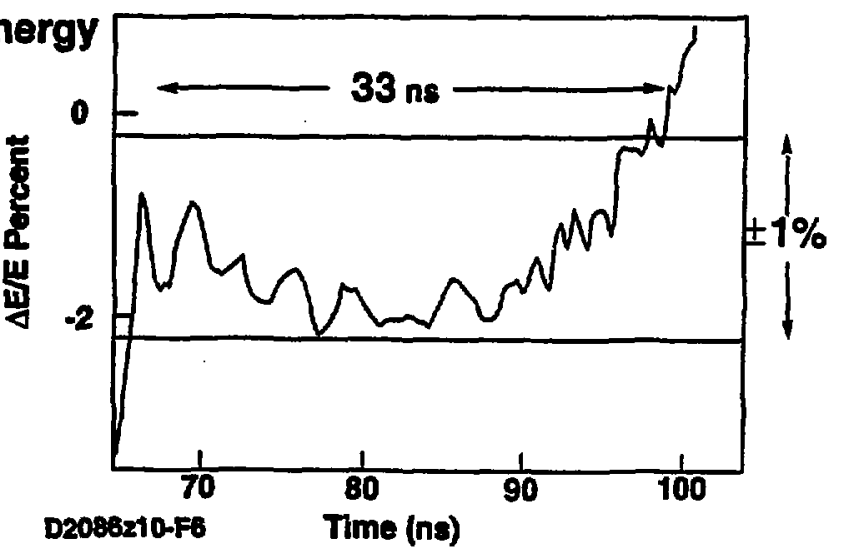

Fig. 8.2.B-1. Corkscrew after magnetic alignment of the beam was reduced to less than $1 \mathrm{~mm}$ at the end of the accelerator for $40 \mathrm{~ns}$. Energy sweep was held to $\langle+|-1$, for $30 \mathrm{~ns}$. 


\section{The Electron Beam Line ETA-II to IMP}

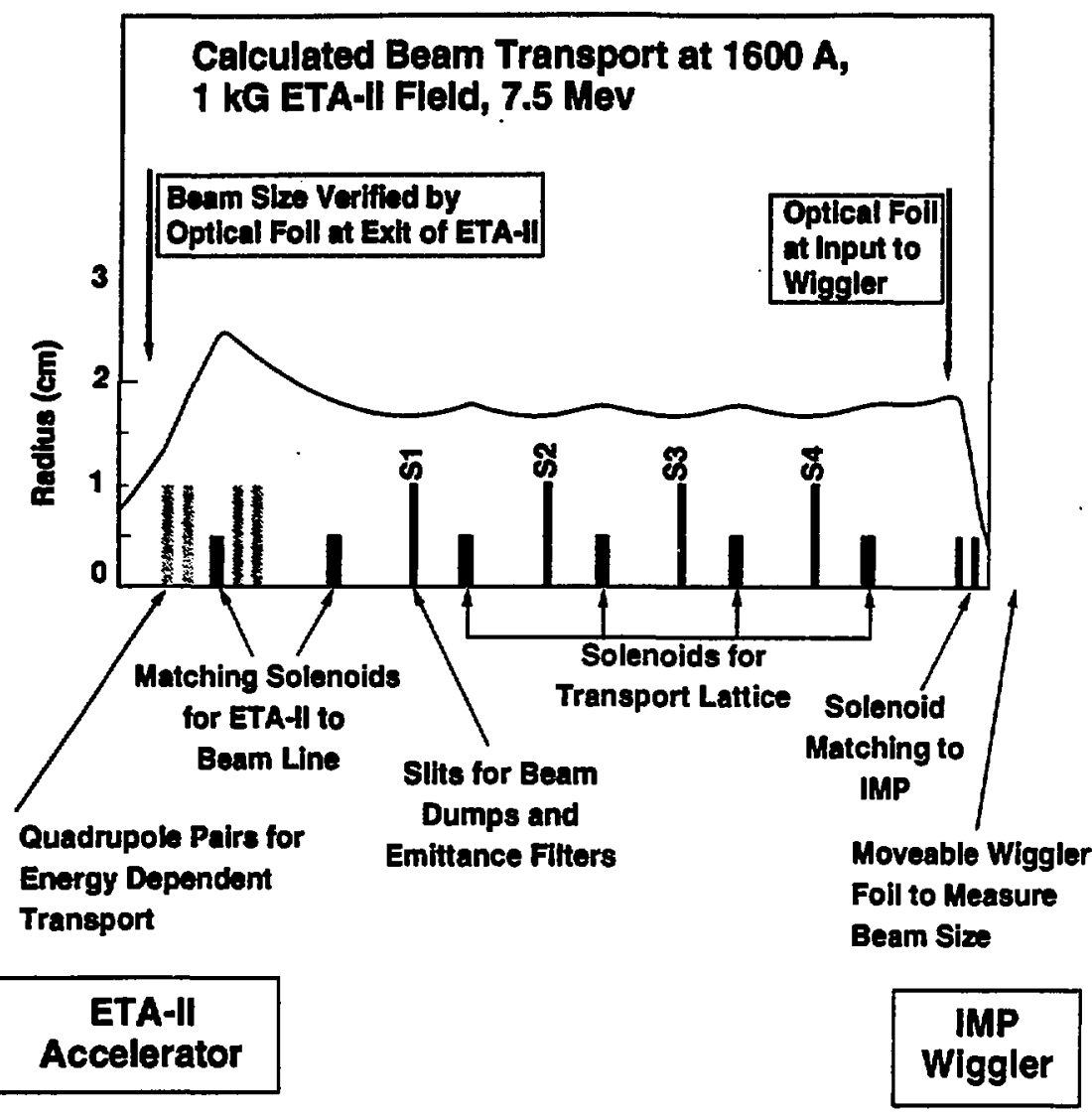

Fig. 8.2.B-2. Diagram of the transport section of the beamline. 


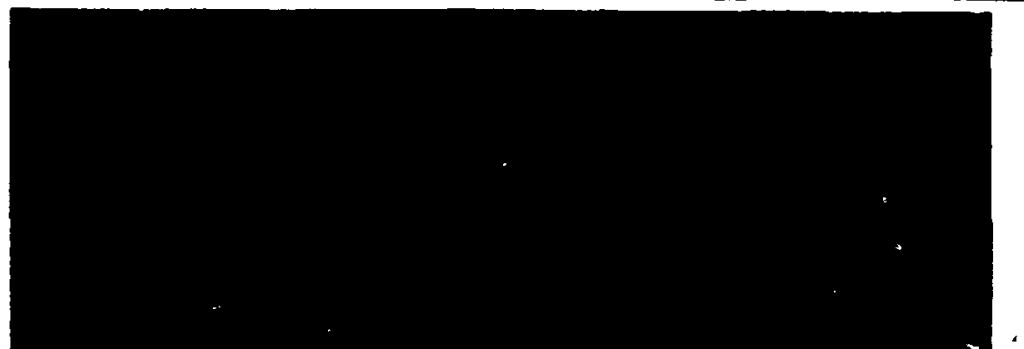




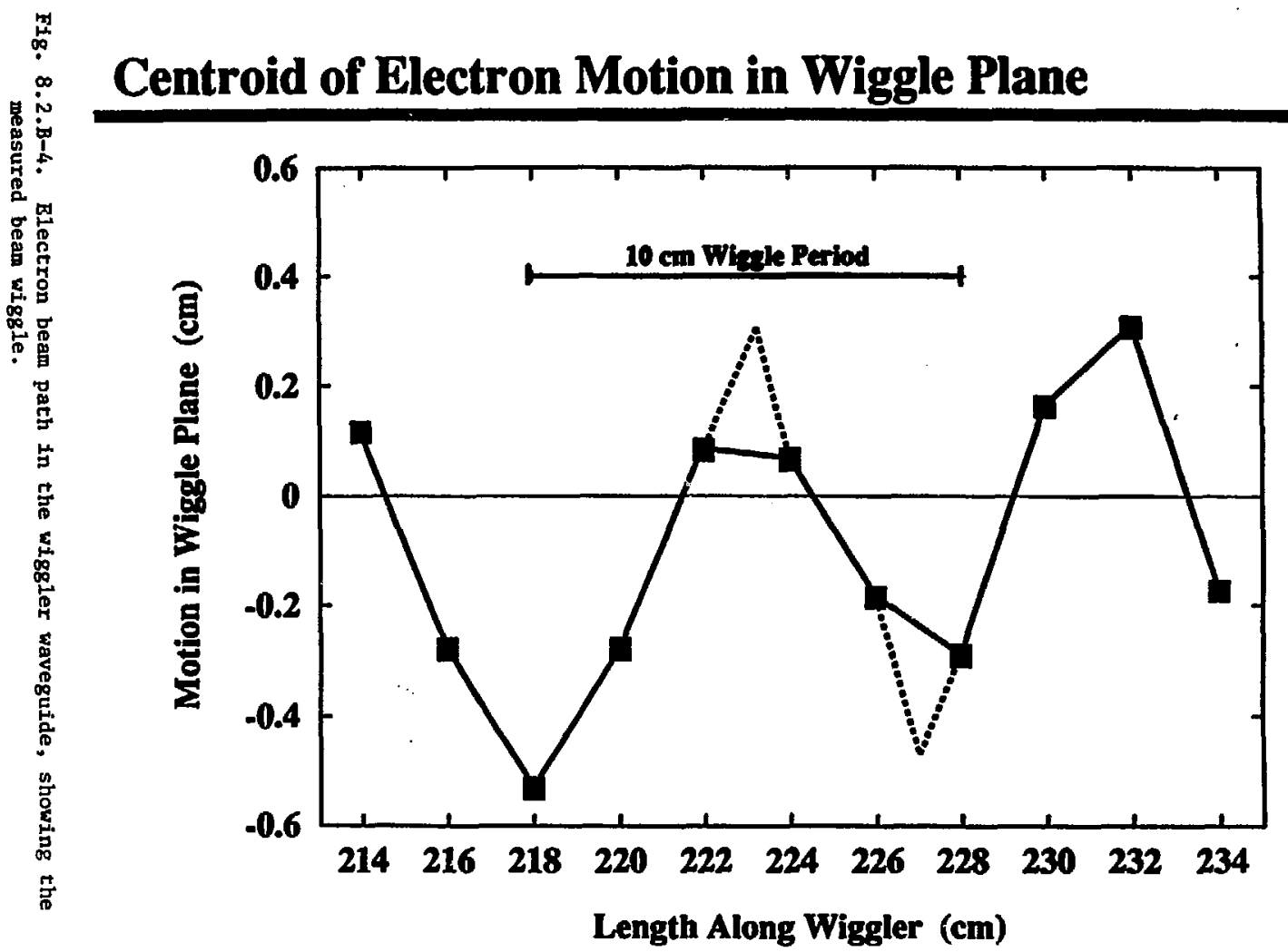

R2126FO6-FOH 
8.3 Burst Mode FEL with the ETA-III Induction Linac (C.J. Lasnier, S.L. Allen, B. Felker, M.E. Fenstermacher, S.W. Ferguson, S.D. Hulsey, E.B. Hooper, M.C. Jackson, M.A. Makowski, W.H. Meyer, J.M. Moller, D.E. Petersen, S.E. Sampayan, B.W.Stallard, W.F. Fields, K. Oasa)

\subsubsection{Introduction}

Pulses of $140 \mathrm{GHz}$ microwaves have been produced at a $2 \mathrm{kHz}$ rate using the ETA-III induction linac and IMP wiggler. The accelerator was run in bursts of up to 50 pulses at $6 \mathrm{MeV}$ and greater than $2 \mathrm{kA}$ peak current. $A$ feedback timing control system was used to synchronize acceleration voltage pulses with the electron beam, resulting in sufficient reduction of the corkscrew and energy sweep for efficient FEL operation. Peak microwave power for short bursts was in the range 0.5-1.1 GW, which is comparable to the single-pulse peak power of 0.75-2 GW. ${ }^{1}$ FEL bursts of more than 25 pulses were obtained.

Pulses of high-power microwaves were generated using the ETA-III (an upgrade of ETA-II), a linear induction accelerator at the Lawrence Livermore National Laboratory, and the IMP (Intense Microwave Prototype) wiggler. The microwaves were produced as part of the MTX fusion energy project. To progress from single pulse operation to bursts of pulses at multi-kilohertz rates, modifications were made in the pulsed-power systems, and a new timing compensation scheme was implemented for synchronizing the acceleration voltage pulses with the electron beam.

The timing system was especially critical. ${ }^{2}$ The electron beam provided most of the loading for the high-voltage pulse at the induction gaps. If the high voltage pulse did not arrive at the gap within $+/-10 \mathrm{nS}$ of the electron beam pulse, the unloaded voltage would have been too high, causing an arc and damaging insulators. To achieve the best acceleration of the beam, the high voltage pulse timing needed to be controlled to within $+/-2 \mathrm{nS}$ for each pulse of a burst. This was shown to be within the capability of the feedback timing compensation system. Note that it is not the standard deyiation of timing measurements which is critical in preventing arc damage, it is the largest excursion of timing from the target value.

ETA-III was run in $2 \mathrm{kHz}$ bursts of up to 50 pulses. The electron beam reached peak currents of over $2 \mathrm{kA}$ at $6 \mathrm{MeV}$. An example of a long burst is shown in Fig 3. For FEL operation, the beam used in IMP wiggler with $7 \mathrm{~kW}$ of master oscillator power at $140 \mathrm{GHz}$ generated by a gyrotron.

The FEL peak output power exceeded $1 \mathrm{GW}$ during many bursts. This is not as great as the highest power $(=2 \mathrm{GW})$ measured during single-pulse operation. ${ }^{1}$ Corkscrew and energy sweep of the beam during a burst remained within the acceptable range during short bursts, as evidenced by 
consistent FEL output (Fig 4). The experimental time to optimize operation was limited, so the longest recorded burst of microwaves contained slightly more than 25 pulses (Fig. 5).

\subsubsection{Description of the Experiment}

8.3.2.1 The ETA-III Accelerator The accelerator was configured in nearly the same way as for the previous 20-cell experiment,1,3,4 except that 60 accelerator cells were in place to provide $6 \mathrm{MeV}$ beam energy. The high voltage pulses to drive the ind:rion gaps were provided by four pulsed power systems, each consisting of a MAG-1D magnetic pulse compressor fed by a Pulsed Power Unit (PPU) (Fig 1). The PPU's were energized by DC power supplies typically run at 10-14 kV. A capacitor bank for each DC supply helped maintain voltage during bursts.

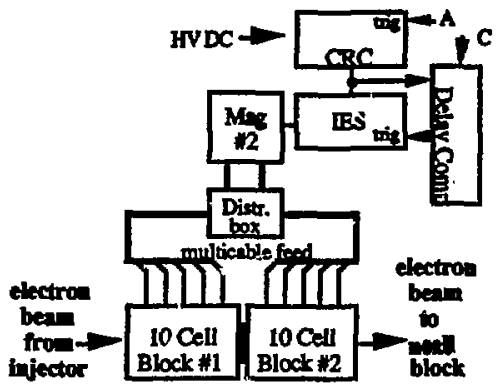

Fig. I A typical Pulsed Power system drove 20 cells.

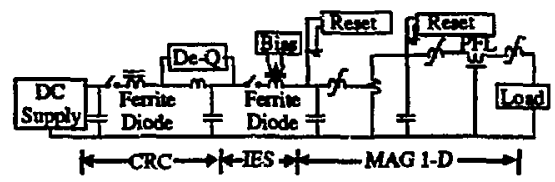

Fig. 2 Pulsed Power Schematic (from Ref. 3).

The first stage of each PPU was the Charge Resonant Command (CRC) circuit, which regulated the voltage. The second stage was the Intermediate Energy Storage (IES) which was triggered to fire the accumulated charge into the MAG-1D. 
Thyratron tubes were used as switches in the PPU's. The PPU's, the MAG-1D's, and the accelerator cells all contained ferrite material to act as magnetic switches. The ferrites were reset to the bottom of their hysteresis loops by currents in field windings.

The PPU typically generated a voltage pulse of $20-22 \mathrm{kV}$ peak value. This pulse was compressed by the MAG-1D units, which were able to step up the peak roltage to over $130 \mathrm{kV}$. The output pulse had a full width at half maximum of $70 \mathrm{nS}$.

The injector whs run by a dedicated PPU and MAG-1D unit, ordinarily at $100-120 \mathrm{kV}$ peak voltage. Three other PPU's each ran a MAG-1D, which each in turn drove 20 accelerator cells in parallel at $80-100 \mathrm{kV}$ peak. A distribution box split the output of each MAG-1D into 10 cables, each of which drove two cells. The cable length provided transit time isolation during the pulse, protecting cells from reflected voltage originating at cells attached to other cables.

Changes made to the pulsed power system for burst mode included an additional high voltage DC power supply and capacitor banks for the PPU's. Thyratron tubes were replaced with high repetition rate units, new ferrite diodes were installed in the PPUs, and new bias circuits were made for the IES reset. MAG-1D pre-compression reset inductors were installed, thyratron trigger chassis were modified; and the DC power supply for the trigger chassis was upgraded. The high voltage trigger distribution units were improved, and circulation pumps were added for the fluid in the cell resistors."

83.22 IMP Wiggler. The IMP wiggler 5 is a steady-state hybrid laced wiggler with a period of $10 \mathrm{~cm}$ and an active magnetic length of $545 \mathrm{~cm}$. This replaced the pulsed ELF wiggler to maintain resonance during long bursts. The wiggler field in the upstream resonance region, tunable from $2700 \mathrm{G}$ to $5500 \mathrm{G}$, was ondinarily near $3 \mathrm{kG}$. The downstream part had a linear range of $600-4100 \mathrm{G}$. Each electromagnet was cooled by separately instrumented and interlocked water lines. Independently computer-controlled power supplies were used to drive the electromagnets. A copper circular wave guide of $3.25 \mathrm{~cm}$ inner dimension was used between the wiggler magnet poles. The wiggler field errors were measured during assembly at $0.2 \%$ RMS.

The computer control of the wiggler allowed repeatable remotecontrolled tapering of the wiggler field without physically moving magnets. Different magnet configurations were stored and re-loaded easily. The compater was also helpful in performing scans of the wiggler field, raising and lowering whole sets of magnet poles together.

Because of the permanent magnets, when lower field was selected on any pole, the computer ran an hysteresis loop of the whole wiggler to repeatably arrive at the requested field.

8.3.2.3 Timing Compensation System. The feedback timing compensation system for the high-voltage pulses was expanded to handle four MAG-1D pulse compression units independently. The previous single-correction 
LabVIEW control system ${ }^{6}$ was modified by using a LeCroy 2228A Time-toDigital Converter to measure the firing time of each MAG-1D, from capacitive probe samples of the output. The computer adjusted the delay generator triggering each of the PPU's to cause firing at the target time.

For burst mode, a Kinetics List Sequencing Crate Controller was used to record the delay values for each of the $\mathbf{5 0}$ pulses of a burst. Timing corrections were written to a Digital-to-Analog converter. The analog voltages, one for each pulse of a burst, went to a delay compensation chassis for each PPU, which converted the voltage into a trigger delay.7

The timing system was able to time-align the pulses to within 2-4 nS of the target value when the jitter in the MAGs and PPU's was low (normal operation).

To achieve operation at normal voltage $(80 \mathrm{kV})$ on the accelerator gaps and 2300 Amps injector current (FEL parameters), the accelerator was started at low voltage and raised slowly. This was because the voltage pulses had to be precisely timed with the beam load to avoid over-voltage and gap arcs. Changing voltage caused large timing changes in the PPU's.

For burst mode at full current, it was necessary to start with 2- or 3-pulse bursts at low voltage and slowly raise voltage. The operators found that a sodium thiosulfate solution giving a resistance of $150 \mathrm{Ohms}$ in the cell load resistors prevented arcing and allowed easier startup than de-ionized water, and did not place an unreasonable load on the MAG-1D units. This was due to the resistors providing some load even if beam loading was poor. Ideal beam load was $\mathbf{4 0}$ Ohms.

\subsubsection{Experimental Results}

The injector was operated independently at up to $3 \mathrm{kHz}$, in bursts of 50 pulses. The entire accelerator was run at $2 \mathrm{kHz}$ because some trigger chassis required reduced inductance in ferrite diodes to run at higher rates This was not done due to time constraints. Repeatable operation of up to 50 pulse bursts of electron beam at over $2 \mathrm{kHz}$ and over $6 \mathrm{MeV}$ was achieved. A. 45pulse burst is shown in Fig. 3.

Short bursts of FEL pulses were repeatedly obtained with peak power near $1 \mathrm{GW}$ (Fig 4). For longer bursts, the microwave output was less repeatable but still produced many good pulses (Fig 5).

The reduced effectiveness of long bursts is at least partially attributed to the beam falling out of resonance in the wiggler, due to the pulsed power capacitor banks being drained during the burst. Power supplies connected to the banks were able to recharge capacitors between bursts, but not maintain voltage during a burst. For long bursts, the capacitor bank supplying the injector was drained so that less beam current was produced (Fig. 6). Due to reduced load on the induction gaps, this most likely increased the beam energy unless gap voltage dropped at the corresponding rate. 
In some cases, FEL microwave output power dropped off during a burst due to a mode shift in the gyrotron master oscillator, seen as a change in gyrotron microwave monitors.

The FEL microwave power was measured by a diode which detected a sample of the wiggler output power, by means of a small coupling hole in the center of the first mirror of an evacuated quasi-optical transport system. The diode was calibrated by calorimetry at the end the transport section. 8

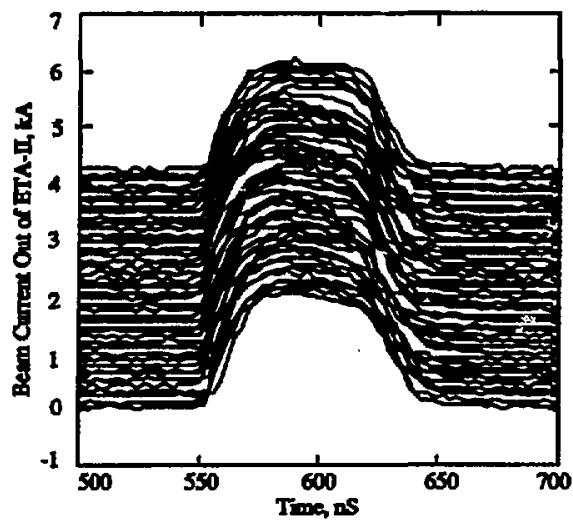

Fig. 3 Electron Beam Current vs. Time for a Burst of 45 Pulses of Electron Beam at $6 \mathrm{MeV}$.

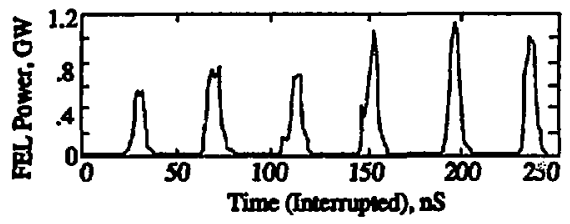

Fig. 4 Microwave Power vs. Time for a Short FEL Burst. The time axis is interrupted: the dead time between pulses recurring at a $2 \mathrm{kHz}$ rate has been removed. 


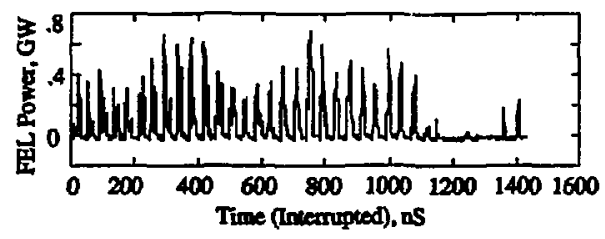

Fig. 5 Microwave Power vs. Time for a Long FEL Burst.

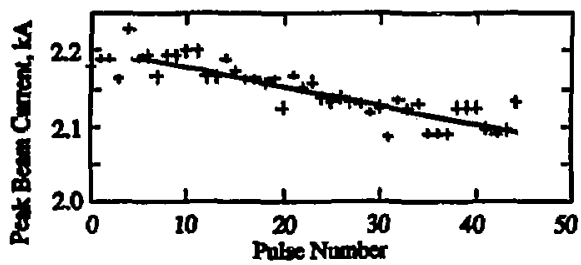

Fig. 6 Peak Current vs. Pulse Number in a 45-Pulse Burst Peak current falis off slightly during the burst as copacitor banks are drained. The line is a leastsquares fit to the points.

\subsubsection{Discustion}

Burst Mode Free Electron Laser operation using an induction linear accelerator has been demonstrated for the first time, and was used to produce bursts of high power $(-1 \mathrm{GW})$ pulsed microwaves. The accelerator hardware was upgraded for high repetition rate, and the timing compensation system was expandec' to control burst mode timing synchronization.

There is potential for improvement. Using a much larger power supply (5 MW compared to $695 \mathrm{~kW}$ total for ETA-III), the High Average Power Test Stand at LLNL was able to consistently demonstrate operation of a PPU and MAG-1D pulse compressor into a dummy load for 800-pulse bursts (a result of the work described in ref [4]). Burst length could also be extended by using larger capacitor banks.

1. S.L. Allen et al., "Generation of High Power $140 \mathrm{GHz}$ Microwaves with an FEL for the MTX Experiment," these proceedings.

2. S.E. Sampayan et al., "Performance Characteristics of an Induction Linac Magnetic Pulse Compression Modulator at Multi-Kilohertz Pulse Repetition Frequency," Proc. 1991 Part. Accel. Conf. (San Francisco, 1991) 3097.

3. S.L. Allen et al. "Measurement of Reduced Corkscrew Motion on the ETA-II Linear Induction Accelerator", Proceedings of the 1991 Part. Accel. Conf. (San Francisco, 1991) 3094. 
*Performed jointly under the auspices of USDOE by LLNL W-7405-ENG48 and DOD under SDIO/SDC MIPR No. W42-GBL-0-5-

7

4. W.C. Turner, "Control of Energy Sweep and Transverse Beam Motion in Induction Linacs," Proc. 1991 Part. Accel. Conf. (San Francisco, 1991) 581.

5. B. Felker and S.W. Ferguson, "The Intense Microwave Prototype (IMP) Free Electron Laser, 140 Gigahertz Microwave System for the Microwave Tokamak Experiment (MTX", Proc. 14th IEEE Symposium on Fusion Engineering (San Diego, 1991)

6. Previous Single-pulse timing system by F.E. Coffield

7. M.A. Newton and J.A. Watson, "Timing and Voltage Control for Magnetic Modulators on ETA-II," Proc. 7th IEEE Pulsed Power Conf. (Monterey, CA 1909).

8. B.W. Stallard et al., "ECH by FEL and Gyrotron Sources on the MTX Tokamak," in Proceedings of the 16th Symp. on Fus. Tech., Lordon, Sept. 1990. 


\subsection{A Development to Improve ETA-II: Feedback Timing Compensation (C. J. Lasnier)}

The feedback timing compensation was expanded to handle four MAG units independently. The previous single-correction Labview control system was adapted by using a CAMAC LeCroy 2228A Time-to-Digital Converter to measure the firing time of each MAG, using C-probe signals from the MAG output. The computer adjusted the delay generator triggering each of the PPU's to cause firing at the target time.

For burst mode, a Kinetics List Sequencing Crate Controller was used to record the delay values for each of the 50 pulses of a burst. Timing corrections were written to a CAMAC Digital-to-Aralog converter. The resulting sequence of voltages, one value for each pulse of a burst, was fed to a delay compensation chassis for each PPU. This chassis converted the voltage into a trigger delay.

The timing system was able to time-align the pulses to within 2-4 ns of the target value when the jitter in the MAGs and PPU's was low (normal operation).

For operation at high voltage $(80 \mathrm{kV})$ on the accelerator gaps and $2300 \mathrm{Amps}$ injector current (FEL parameters), it was necessary to start the accelerator pulsing at low voltage and slowly raise voltage. This was because the voltage pulses had to be precisely timed with the beam load to avoid over-voltaging and arcing the gaps. Changing voltage caused large timing changes in the PPU's.

For burst mode, it was usually necessary to start with 2-or 3-pulse bursts at low voltage and slowly raise voltage. however, it was found that a copper sulfate load solution giving a resistance of $150 \mathrm{Ohms}$ in the cell load resistors gave better insurance against arcing and easier startup than de-ionized water, and did not place an unreasonable load on the MAGs. 
8.3.B Development to Improve ETA-II : Burst Mode Operation (C. J. Lasnier)

Burst mode testing was initially done at HAPTS (High Average Power Test Stand), where techniques and circuits were developed for running a MAG ID into a dummy load for bursts of $5 \mathrm{kHz}$, up to $R C O$ pulses. Some of these developments were translated to ETA-II, in addition to modifications planned specifically for ETA-II.

- A third DC power supply ( $45 \mathrm{~kW}$ ) was added to run PPU 2 separately, in addition to the existing $50 \mathrm{~kW}$ supply for PPU1 and the $600 \mathrm{~kW}$ supply for PPU3 and PPU4.

- Capadtor banks with crowbar protection circuits were hooked into the $\mathbf{4 5}$ $\mathrm{kW}, 50 \mathrm{~kW}$, and $600 \mathrm{~kW}$ supplies which drive the Pulsed Power Units (PPUs).

- Thyratron tubes were replaced with high repetition rate thyratrons.

- New CRC ferrite diodes were installed.

- New bias filter circuits were installed for the IES reset.

- MAG-1D pre-compression reset inductors were installed.

- Thyratron trigger chassis were modified

- The DC power supply for trigger chassis was modified.

- A delay compensation chassis of the type developed by Jim Watson at HAPTS was built and installed in each PPU. This chassis had an input for cap bank voltage, as well as an input to receive a voltage signal from the feedback timing compensation system. (See section 8.3.A Feedback Timing Compensation). The summed signal determined a comparison voltage level. A voltage ramp in the compensition chassis was started by the IES (Intermediate Energy Storage) trigger from the timing system, and when the ramp reached the comparison voltage, the chassis issued an IES trigger to the PPU. This allowed the delay in firing the IES to be adjusted by approximately $\pm 500 \mathrm{~ns}$.

- The feedback timing system was extended to control each PPU independently, and do burst mode correction. (See sections 8.3.A and 8.3.C.)

- The high voltage trigger distribution chassis were modified after a number of resistor fires, and no further failures occurs in those units.

- During burst mode operation, two HYPO circulating pumps were hooked up to the accelerator cell resistors. One pump serviced the cells whose insulators were prone to electrical breakdown, with low-resistance copper sulfate solution. The other pump circulated higher-resistance solution to the resistors of the cells with good insulators. The fluid in the injector insulators was not circulated. The injector was run at various times with either HYPO or oil in its resistors. 


\subsection{FEL Operation- Burst Mode (C J. Lasnier)}

The accelerator was run in bursts of up to 50 pulses at $2 \mathrm{kHz}$ (Fig 8.3.C-1). The Injector was run at $5 \mathrm{kHz}$ with 50-pulse bursts on one occasion, without feedback timing compensation (Fig 8.3.C-2). Bursts of 50 pulses were shot into the wiggler, with FEL pulses out for up to 28 pulses of the burst (Fig 8.3.C-3). Many bursts contained pulses with peak power of order $1 \mathrm{GW}$ at MTX (Fig 8.3.C4).

The accelerator was run at $2 \mathrm{kHz}$ instead of $5 \mathrm{kHz}$ due to limitations in some of the PPU (Pulsed Power Unit) trigger chassis. Some of the ferrite cores had too many turns of wire, resulting in excessive inductance. This would have taken a few days to correct.

The maximum beam current into the wiggler was -2000 amps. The most current seen at the output of the wiggler was $-800 \mathrm{~A}$, but usually $<600 \mathrm{~A}$.

The burst mode feedback timing compensation system development continued during the period of burst mode operation. It reached a level where the timing of the pulses within the burst could be aligned to $\pm 2-3 \mathrm{~ns}$. 


\section{Pulse Electron Beam Burst Achieved on ETA-II at Full FEL Parameters}

\section{OMTX}

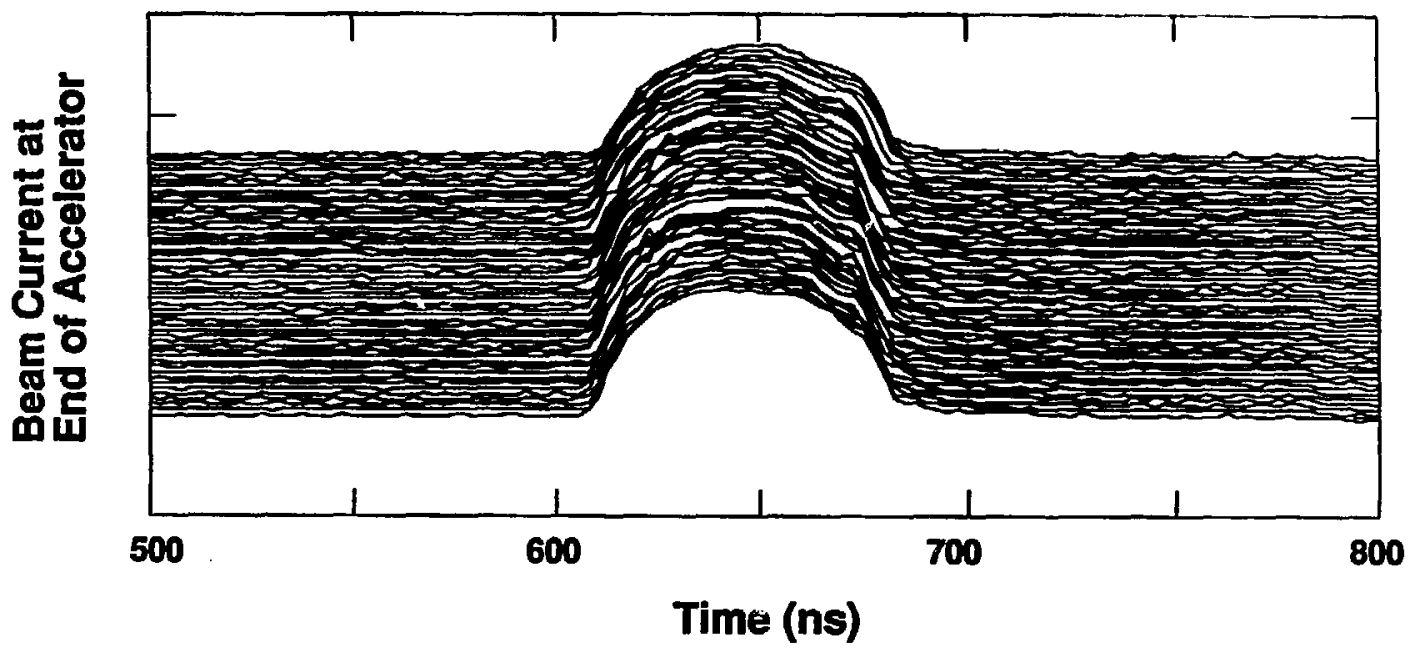

- ETA-II Accelerator Has Been Run in BURST MODE

-50 Pulse Burst at Full FEL Parameters--2 kHz, 2 kA, $6 \mathrm{MeV}$

-FEL Designed for Use with MTX ECRH Heating Experiments

Fiir D2252f02.dig 


$$
\text { 용 }
$$

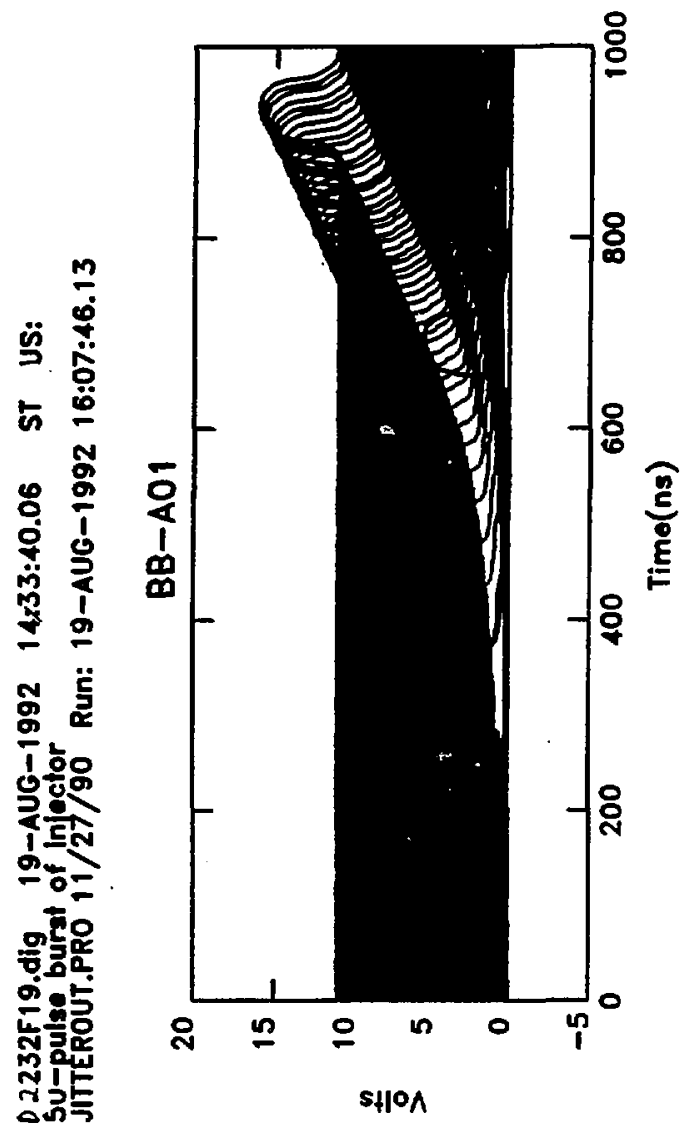

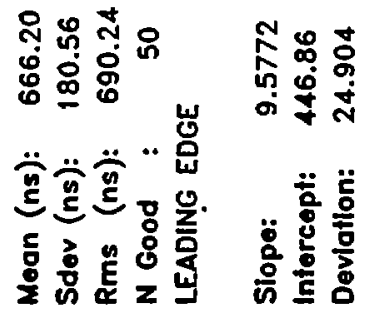

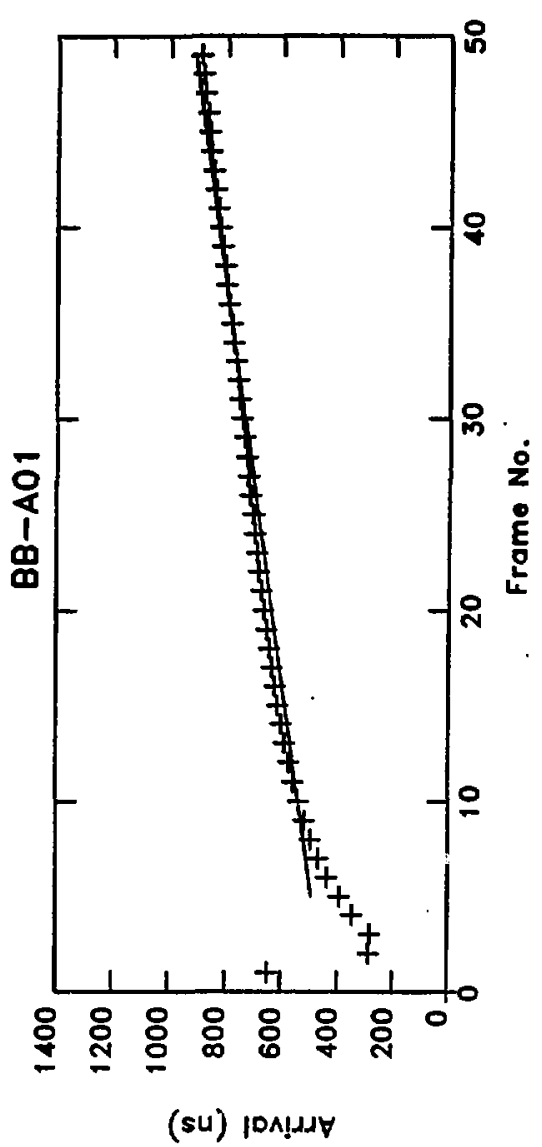

Fig. 8.3.C-2. The injector was run at $5 \mathrm{kHz}$ in 50-pulse bursts, without feedback timing compensation. 


\section{BURST OF MICROWAVE PULSES FOR MTX AT 2 KHZ}

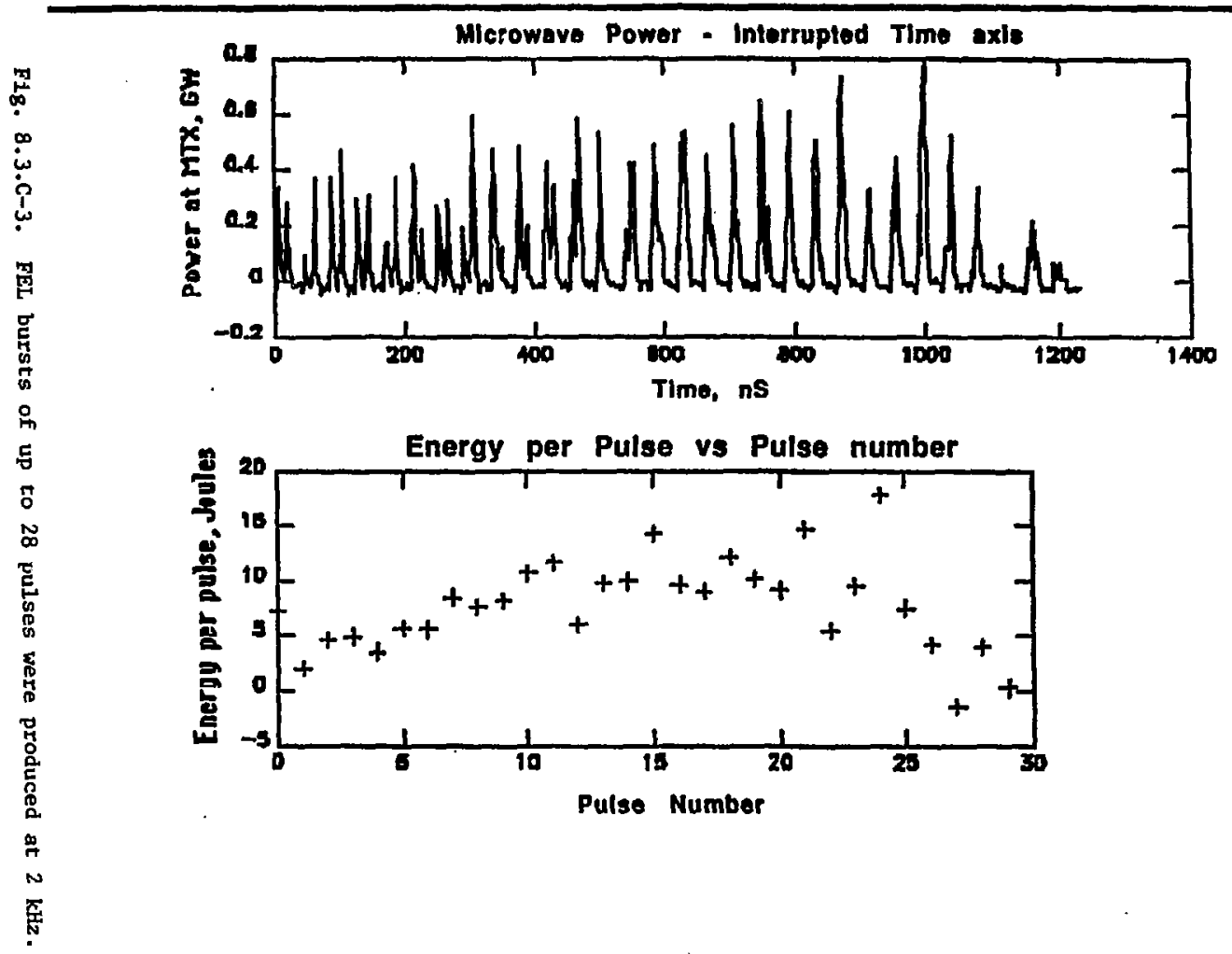




\section{BURST AT HIGH POWER}

OMTX
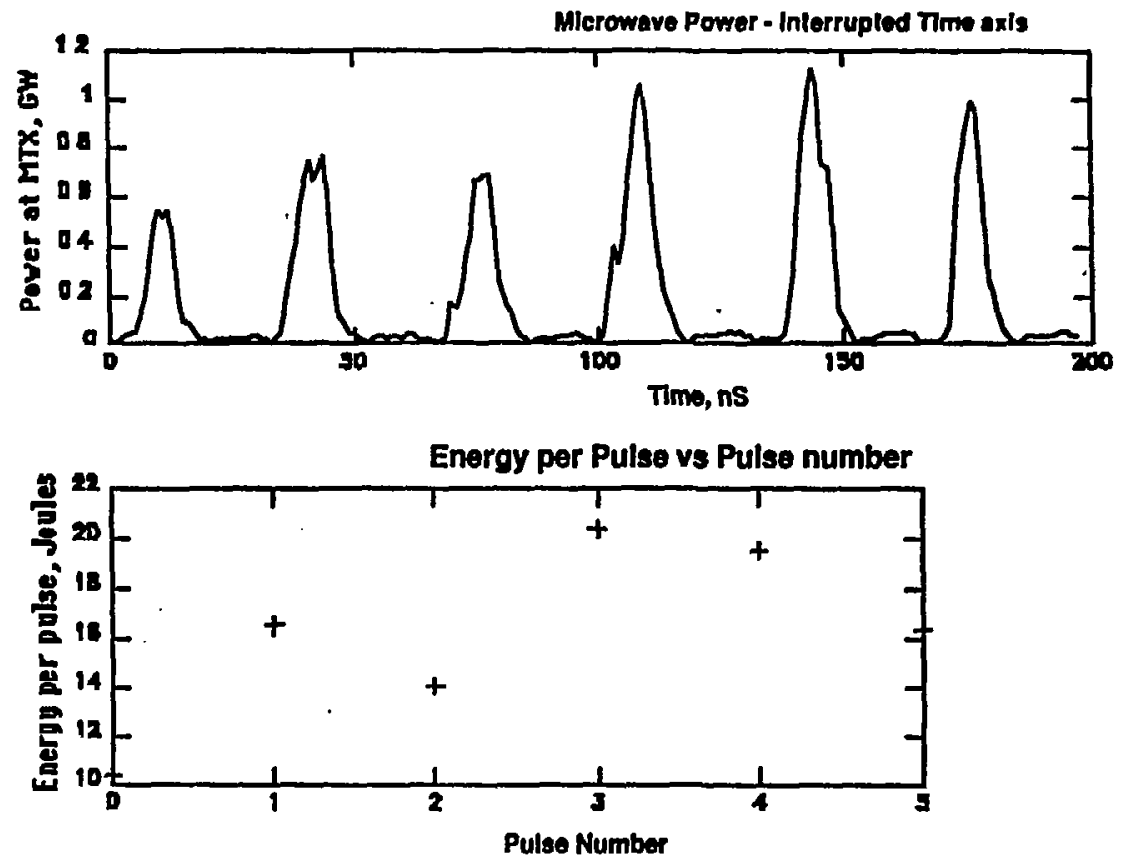


\section{A.1 Documents pertaining to the collaboration between DOE/LL̃NL and JAERI}

A.1.1 Formal agreement between the United States and Japan 
ANNEX VI TO THE IMPLEMENTING ARAANGEYENT

BETHEEN THE JAPAN ATOHIC ENERGY RESEARCH INSTITUTE

AHD THE UNITED STATES DEPARTMENT OF ENERCY

ON COOPERATION IN FUSION RESEARCH AND DEVELOPMENT

FOR THF DOE-JAERI COLLABORATIVE PFOGRAY IN THE

MICROHAVE TOKAMAK EXPERIMENI (MTX) PROJECT OF

FRES ELECTRON LASER (FEL) MICROWAVE HEATING AND CURRE!TT DRIVE

APPLIEATIONS TO MAGNETIC FUSION

1. INTRODUCIZW ANE TERHS OF REFERENCE

Whereas Article III.2 of the Implementing Arrangement between the United States Department of Energy (hereinarter referred to as ${ }^{\circ} D O E^{\prime \prime}$ ) and the Japan Atonic Energy Research Institute (hereinarter referred to as -JAERI $)$ on Cooperation in Nusion Research ind Development or November 8. 1983 (herelnarter referred to as "the 1aplenenting Arrangenent") provides for additional activities through written Annexes to the Inplesenting Arrangewent,

Whereas DOE and JAEAI wish to aake advances in fusion through the application of free lectron laser (FEL) alerowave power for heat ing and current drive in plasmas and to determine the sultability of the mlcrowave FEL for rusion appl lcations,

Whereas JAERI has extensire experience in tokawak diagnostles and operation on $J T-60$ and $J F T-2 M$, and DOE has a unique racility (hereinarter referred to as "MTX") consisting or a high-fielc tokazak and a weroweve transmission system to transport high power FEL atcrowaves to the tokamak.

DOE and JAERI (hereinafter referred to as "the parties") agree to a Collaborative Progran in accordance with the provisions of the Implesenting Arrangewent, as rollows:

\section{OBJECTIVES}

The objectlve of the collaborative Program, derined in article 3 below, are to:

(a) conduct and evaluate plasma heating and current drive experinents in the MTX Tokamak racility at Lawrence Liverwore National Laboratory (LLNL), using idcrowaves froa a high-average-power alcrowave FEL,

(b) accelerate the development of information from HTX through use of additlonal equipment and personnel support from JAERI, and

(c) conduct joint studies of the application of EEL wlorowave technology in rollow-on rusion tokamaks to determine the benerits for rusion applications. 


\section{COLLABORATIVE PROGRAM}

3.1 The Collaborative Program is a joint program of research on MTX and studies of further applications of FEL microwaves for fusion tokanaks. The Collaborative Progran shall use the HTX facility at LLNL, which is supported by DOE, and shal 1 be consisient with the DOE approved HIX Prosram Plan. The activities of this collaboration wlil Include:

(a) planning for JAERI participation in heating and current drive experinents in MTX and in measurements to characterize the aicrowave FEl Input to the tokamak,

(b) preparation for and participation in these experiments at LLM,

(c) Jolnt evaluation of the above experiaents, Inciuding their ieplications for future applications to fusion research and developent,

(d) Joint :tudies of fur ther applications of FEL alcrowares for rusion, and

(e) provision of personnel, equipment and information necessary to carry out the Col 1 aborative Progran.

3.2 The responsiblilties of each Party in the Collaborative Program are as follows:

3.2.1 DOE shall be responsible for:

(a) Installation of the ilcator-C tokawak at LLNL, coupling the tokanak to the ETA-II free electron laser fac1lity, and operations. on MTX.

(b) Supporting an operational tean at LLHL capable of operating and antaining wix.

(c) Providing access to conputer equipment capable or being used for collecting and analyzing diagnostics data from the experinents at LLWL.

(d) Is the host country for the Collaborative Program, providing offices, facilities and other support at LLNL for the risiting personnel from JAERI.

\subsubsection{JAERI shall be responsible for:}

(a) Providing scientific and other personnel, both at LLNL and at JAERI, to participate in the Col laborative Program.

(b) Developing and constructing, in each of the first three years or this Collaborative program, diagnostics or other equipment as will be speciried by the Steering Committee or Article 4 below. All equipment constructed or purchased for this Collaboration shall remain at LLNL throughout the duration of

$$
\text { A.1-3 }
$$


this Arnex.

(c) Providing offices, facilities and other support fur the visiting personnel from LLNL, when experirents, studies or tests for the Collaborative Program are bsing perforned at JAERI.

\section{MANAGEMENT}

4.1 The Partles agree to establish a Steering Coumittee.

4.2 The steering comittee shal l be responsible for anagement of the Collaborative Progran.

4.3 The Steering Comittee shall composed of four members, two exch to be assigned by DOE and JAERI. One of the DOE neabers shall be assigned fron LWN. The Steering Connittee shall have the functions as described in paragraph 4.5 below.

4.3.1 DOS and JAERI each shall Ident1fy person to serve as cochairean of the Stering Condtee. Orficlal cownulcations shall be channeled through co-chairuen or their designees.

4.3.2 DOE and JAERI shal I deslgnate an approprlate al ternate who shall serve if a member is unable to do so and each shell inform the other in writing of 11 such designations. DOE and JAERI shall each hare two rotes in the Steering Compittee and all declsions shall be by unaniolty. All meetings shall be chaired by the hosting country whe the weetings are held.

4.4 The Steering Condtee shall meet annually at LLNL unless an alternative location is mutualiy agreed upon, on a date mutualiy agreed upon. An exchange or letters may serve as a substitute for a meeting of the Steering Cowittee.

4.5 The functions or the Steering connittee shall include

(a) planning of the Collaborative Program,

(b) reviewing progress in preparation for, and during conduct or, the Collaborative Progran.

(c) evaluating the results of the experinents,

(d) reporting to the U.S.-Japan Coordinating Conittee on Fusion Energy through Contact Persons described in Article I of the Exchange or Letters between the Science and Technol08J Agency or Japan and DOE on January 25, 1983.

(e) reaching agreesent or, arrangement for the assignee(s); and

(f) discussing other watters necessary for conduct of the Collaborative Program. 


\section{FINANCE}

Except when otherwise mutually agreed in writing, each Party shall bear the costs or its own activitles in accordance with its responsibilities as described in this Annex. The activities to be conducted under this Annex shal 1 be subject to the avallability of appropriated funds in each country.

\section{INFORMATION AND PATENTS}

6.1 The Parties shall exchange information necessary to carry out the Collaborative Program. This information shall be non-proprietary and shall not include detajled canufacturing know-how. Information developed at linl outside of the HTX program but relevant to this Collaborative Prograa may be provided after normal revieu and release procedures have been followed.

6.2 Each Party shall provide proaptiy to the other Party all inforation arising frow the Collaborative Prograw.

6.3 The information provided under and arising from the Collabixative Progras any be made available to the public by elther Party through customary channels and in accordance with-the normal procedures of the parties, subject to the provisions of puragraph 6.5 below.

6.4 Inventions ande or conceived in the course of or under the Col laborative Program of this innex (hereinafter referred to as "arising inventions") shall be identifled and reported promptly by the Inventing Party to the other Party. Information regarding inventions on which patent protection is to be obtained shall not be published or publicly disclosed by. the parties until a patent application has been flied in elther country of the Parties, provided, however, that this restriction on publication or disclosure shall not extend beyond six months from the date of the Invention. It shall be the responsiblilty of the Inventing party to appropriately mark reports which disclose inventions that have not been appropriately protected by filing of a patent application.

6.5 Arising inventions shall be owned

(a) by JAERI in Japan subject to a royalty-sree, non-exclusire, . irrevocable 1 lcense to DOE, Its Covernent, and the nationals of Its country designated by $1 t$, and

(b) by DOE in the United States and the third countries subject to a royalty-free, non-exclusive, irrerocable license to JAERI, its Governsent, and the nationals of Its country designated by 1t.

6.6 The provisions of paragraph 6.6 shall apply outatis mutand is to the protection of utility adel and of design.

6.7 Each Party shall assume the responsiblilty to pay awards or compensation required to be peid to its own nationals according to its own laws. Each Party shall, without prejudice to any rights or

$$
\text { A. 1-5 }
$$


Inventors under its nationals own laws, take al I necessary steps to provide the cooperation from its inventors required to carry out the provisions of this Article.

\section{ASSIGNMENT OF PERSONNEL}

DOE and JAERI may assign to the other Party at least one scientific or technical person at any $t$ lwe, in accordance with irticle IX or the Implementing Arrangement, in order to carry out the coljaborative Program. Each such assignment of personnel shal 1 be the subject of a separate assignment agreeaent. -

8. LOAN OF COMPONENTS, EQUIPMENT, INSTRUMENTS AND MRTERIAL

Loan of components, equipient, instruments and material by JAERI under the Coldaborative Prograw shald be done in accordance ulth article $X$ of the Implementing Arrangement. For the duration of the Col 1 aborative Progran, components; equipwent, instrumenta and waterial provided by JAERI shall be considered to be property of the Government of Japan.

\section{INCORPORATION BY REFERENCE}

Article $V$, VII and VIII of the Implenenting Arrangewent shall apply to this Collaboirative Progran.

\section{DURATION AND TERMIMATION}

The Collaborative Progran shall enter into force upon signature of this Annex by DOE and JAERI and shald remain in rorce for a period of four years or until ternination of the Implewenting Arrengement, whichever occurs rirst. The col laborat I ve Progran any be renewed or amended by written agreement between DOE and JAERI. The Collaborative Prograw may be terninated at the discretion of either DOE or JAERI upon $s i x(6)$ nonths advance not-ice in writing by the side seeking to terminate the collaborative Program. Specific activities initiated hereunder but not completed at the termination of this Annex may be continued until their completion under the terms of this Annex.

Done at Tokyo . , this 19th day of Hay. 1988 in duplicate. in English and Japanese languages, both being equaliy authentic.

for THE UNITED STATES DEPARTHENT OF ENERCY
FOr THE JAPAN ATONIC ENERGY RESEARCH IHSTITUTE

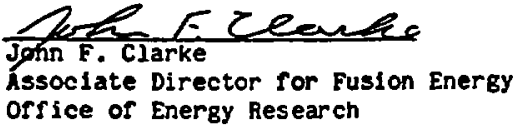

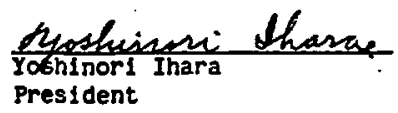

President 


\section{A.1.2 Steering Committee reports}


Summary of Annex VI Activity in 1988-1989

Collaboration between LLNL and JAERI under Annex VI to the US-Japan Inplementing Arrangement began In May 1988. Objectives of this Annex are to conduct and evaluate plasma heating and current drive experiments in MTX, using a bigh power microwave FEL, and to conduct joint studies of the application of FEL microwave technology in follow-on fusion tokamaks to determint the benefits for fusion applications.

Under this agreement JAERI is supplying equipment, primarily plasma and microwave diagnostics, for use on MTX. The equipment for JPY8s includes a section of the microwave transmision sgstem between the wiggler and MTX, namely thot portion between the last focusing mirror and the tokamak port. This section, loosiy called the "mlcrowave box", houses several microwave diagnostics to be bullt partly by LLAL and partly by JAERI. The section, welghing 2 tons, Is belag constructed by Toshlba Corp. and will be delivered to LLNL In March, 1989.

Another diagnostle to be delivered in March 1989 by JAERI is a set of neutron detectors, with assoclated data analysis equipment, for measuring lon temperature in the plasma. Dr. T. Ogawa 18 responsible for this equipment and will be coming to LLKL on an extended stay in March (1989) to operate the system and particlpate in the MTX experiments. Another long term visitor to ILNL, Dr. R. Ohasa, arrived la August 1988 for the the purpose of evaluating fast detectors for a sof X-ray bystem, and also to particlpate in MTX operations. We expect to expand this soft X-ray system to multiple spatial and energy channels in 1989.

In support of the Annex, there have been severel exchanges in 1988-89 under the US-Japan exchange program (see attachments). Exchange Ml, the Steering Committce meeting occurred in February; exchange M2 was split lato two parts, the first part was a visit to LLNL by Drs. 
Maeda and Odajima 1n August, 1988, to discuss details of the microwave box and the second part is a visit to LINL by Dr. Odajima for two weeks in March, 1989; and exchange M3 by Drs. Price, Trocessen, and Booper in December 1988 at Tokal to make preliminary freperations for the steering commitee meeting by proposiag turther equipment for MTX. Also, two long term exchanges. QL6J and MLI Involving Drs. Ohasa and Ogawa were begun. F 1 nally, exchange KL2 w111 send LLNL engineers to Japan In Karcb to vitness ecceptance tests of the microwave box. Other planned exchanges, M4-H7 are to be cancelled. The summary of personal exchenge in 1988-89 is glven In Attachment 1.

A steering committee has been establised, as called for in the Annex, to montor progress and approve additional JAERI equipment for MTX. Members from Japan are Dr. A. Kitsunezaxi (Mr. S. Satake, alternate) and Dr. H. Maeda, wh1le Dr. E. Oktay and Dr. R. Thomssen (Dr. E. B. Hooper, elternate) represent the US. In preparation for the first meeting et LLNL February 16-17, 1989, we Jointly agreed at the December 1988 meeting to propose to the MTX steering Committee three diagnostic systems for JAERI construction In JFY89. These are (a) microwave dignostics for the microwave box, (b) and expanded soft X-ray system as mentloned above, and (c) three channels of 2nd harmonic ECE heterodjne recelpers at separate frequencles around $280 \mathrm{GBz}$. We aloo ldentified three posible dlagnostics for the tbird year of the collaboration, Including microwave components, a scatering syotem besed on either FIR or $\mathrm{CO}_{2}$ lasers, and an if electic field diagnostic based on stark coupling to forbldden lines in He. These last three wilI be examed In more detall starting immediately, so that at the nexi two steering Committee meetings we can comment on these ptions.

At the first MTX steering Committee on February 16-17, 1989 at ILNI we voted to accept the above proposed list of diagnostic equipment to be provided by JAERI in JFY89. A personal exchange plan in the next year is given in Attachment 2. We also reviewed all activities of the coliaboration during this first year, and

$$
\text { A. } 1-9
$$


adopted action thems that will aid in the selection of diagnostic equipement for JFYgo. A report of the meeting was prepared ard signed by members of the steering Committee.

These diagnostics, which cost about $\$ I M$ In equipment each year (exclusive of JAERT pepple) are having a major benefletal lmpact of the MTX program, with its limited resources. Without this collaboration the HTX program would be delayed by having to construct these Items from its budget. In addition, the expertise and experlence of the JAERI staff from the JFT 2-H group will add significantly to the capability of the MTX staff.

Good working relations have been established and communication between ILNL and JAERI fo very good. Communtcation links vie computer haje been established and are working well. This good commulcation is the primary reason for cancelling the M4-M7 exchenges, since they were found to be unnecessary after onr working relations were established.

Tokamak operations have begun and microwave palses from the FEL w111 be sent to MTX next spring so that jolnt experimental operations are now beginning.
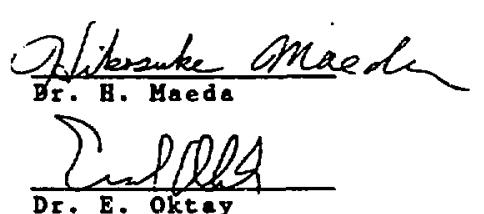

Dr. E. Okteg

February, 1989

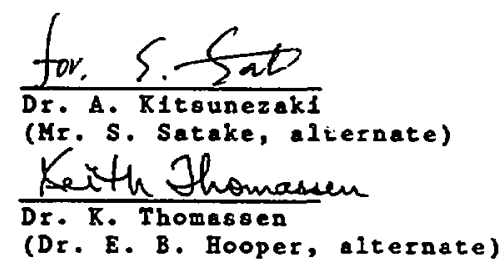




\section{JAPAN-OS FUSION COOPERATION PROGRAM}

April 1989 through March 1990.

iterachent, $2:$ :

NO TITLE TYPE FEEK PERSON RARTICIPANTS DATE

JABRI to LLNL

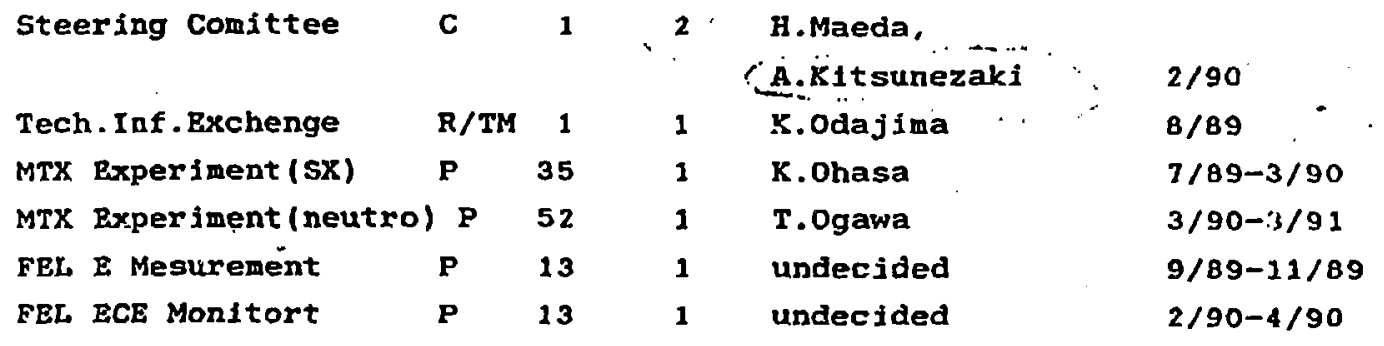

\section{TS to JAPAN}

$\begin{array}{lllllll}\text { Equip.Coodination } & R & 1 & 2 & \text { undecided } & \text { June-Oct./89 } \\ \text { Equip.Coodination } & R & 1 & 2 & \text { undecided } & \text { Oct./89 } \\ \text { Diagnostic Review } & R & 1 & 2 & \text { undecided } & \text { Jan.-March/90 }\end{array}$




\section{JAERI to LLNS}

ML1 MTX Collaboration

M2 MTX Collaborat Ion

$P \quad 52$
$R / T M \quad 2$

1

2 H.Maeda

K. Ddajima

$\stackrel{2}{\grave{2}}$

\section{M1 Steering Couittee}

C

M2 MTX Collaboration

QL61 MTK Callaboration

\section{US to JAPAN}

M3 MTX Collaboration

R/TM 1

2

K.Thomassen

B. Hooper

R.Price

ML2 MTX Collaboration

$\mathbf{P}$

4

2

C.Brooksby

B.Rice

M1 MTX Coilaboration

M5 MrX Collaboration

M6 MTX Collaboration

MI MTX Collaboration

R 1 1 2 cancèl

$R \quad 1 \quad 1-2$ cancel

R 1 1-2 cancel

R 1 1-2 cancel
$31 / 7 / 86-30 / 7 / 89$

$29 / 8-1 / 9 / 88$

$26-27 / 2 / 89^{\circ}$

27/3-6/4/89 plan

29/3/89-28/3/90 plan

$1-2 / 12 / 88$

7-14/3/89 plan 
Summary of Annex VI Activity in April 1989 - March 1990

Collaboration between LLNL and JAERI under Annex VI to the US-Japan Implementing Arrangement, begun in May 1988, continued throughout April 1989 March 1990. Objectives of this Annex are to conduct and evaluate plasma heating and current drive experiments in MTX, using a high power microwave FEL, and to conduct joint studies of the application of FEL microwave technology in follow-on fuston tokamaks to determine the benefits for fusion applications.

Under this agreement JAERI is supplying equipment, primarily plasma and microwave diagnostics, for use on MTX Diagnostics dellvered at the end of the previous period were installed and operated, new diagnostics were completed, and JAERI scientists participated in MTX experiments. The JAERI scientists were involved in microwave FEL expertments on MTX during the year, applying their diagnostics and interacting on a wide range of physics issues.

The diagnostics and exchanges have a major beneficial impact on the MTX program with its limited resources. Good working relations continued during the year, aided by exchanges in both directions and rapid communication vla computer link between personnel at both sites.

The attached tables summarize the technical accomplishments, individual exchanges for this year, and personnel exchange plan for the next year.
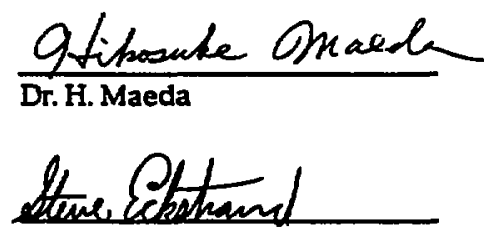

Dr. S. Eckstrand

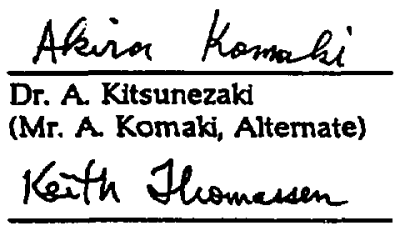

Dr. K Thomassen

(Dr. E. B. Hooper, Alternate)

February 16, 1990 
Teshnical Accomplishments

A. Installation and operation of JAERI diagnostics on MTX

ITEM

1. Microwave diagnostic Installed on MTX box

2. Soft $x$-ray system

3. Neutron system
STATUS

Installed on MTX

Installed on MTX

\section{COMMENT}

Diagnostics installed by LLNL were used in FEL microwave experiments

Measurements were made of plasma $x$-ray emission; results used for $x$-ray camera design

Operational; measurements made during deuterium operation of MTX

B. JAERI activity for preparation of MTX diagnostics in JFY89

ITEM

1. Dummy load system

2. $\mathrm{JO}$ and $\mathrm{M} 2$ mirrors

3. SX camera

4. ECE

5. Helium neutral beam ion source for LAPPS
STATUS

Design and fabrication completed

Design and fabrication completed

Design and fabrication completed

Design and fabrication completed

Design and fabrication completed
COMMENT

On schedule

On schedule

On schedule

On schedule

On schedule

\section{COMMENTS:}

1. Items 1 to 4 are scheduled to be shipped from JAERI to LLNL by the end of March 1990.

2. Item 5 is a part of LAPPS (Laser Assisted Particle Brobe Spectroscopy). LAPPS is scheduled to be completed in JFY90. 


\section{IAPAN-US FUSION COOPERATION PROGRAM}

\begin{tabular}{|c|c|c|c|c|c|c|}
\hline No & TITLE & TYPE & WEEK & PERSONS & PARTICIPANTS & DATE \\
\hline M8 & Steering committee & C & 1 & 2 & $\begin{array}{l}\text { H. Maeda } \\
\text { A. Komaki }\end{array}$ & $2 / 15-2 / 16 / 1990$ \\
\hline M9 & $\begin{array}{l}\text { Technical } \\
\text { information exchange }\end{array}$ & $R / T M$ & 1 & 1 & K. Odajlma & planned \\
\hline M10 & $\begin{array}{l}\text { Equipment coor- } \\
\text { dination meeting }\end{array}$ & R/TM & 1 & 2 & $\begin{array}{l}\text { K. Thomassen } \\
\text { E.B. Hooper }\end{array}$ & $11 / 29-30 / 1989$ \\
\hline M11 & $\begin{array}{l}\text { Equipment coor- } \\
\text { dination meeting }\end{array}$ & $\mathrm{R} / \mathrm{TM}$ & 1 & 2 & $\begin{array}{l}\text { J. Foote } \\
\text { D. Nilson }\end{array}$ & $1 / 29-2 / 2 / 1990$ \\
\hline M12 & $\begin{array}{l}\text { Plasma diagnostic } \\
\text { review }\end{array}$ & $\mathrm{R} / \mathrm{TM}$ & 1 & 2 & $\begin{array}{l}\text { S. Allen } \\
\text { T. Casper }\end{array}$ & $1 / 29-2 / 2 / 1990$ \\
\hline ML3 & $\begin{array}{l}\text { MTX Collaboration } \\
\text { (Neutron) }\end{array}$ & $\mathbf{P}$ & 32 & 1 & T. Ogawa & 4/1989-3/1990 \\
\hline ML4 & $\begin{array}{l}\text { MTX Collaboration } \\
\text { (X-ray) }\end{array}$ & $\mathbf{P}$ & 52 & 1 & K Oasa & $4 / 1989-3 / 1990$ \\
\hline ML5 & $\begin{array}{l}\text { MTX Collaboration (FEL } \\
\text { electric field measurement) }\end{array}$ & $\mathbf{P}$ & 13 & 1 & M. Shiho & $10 / 23-10 / 31 / 1990$ \\
\hline ML6 & $\begin{array}{l}\text { MTX Coliaboration } \\
\text { (FEL ECE monitor) }\end{array}$ & $\mathbf{P}$ & 13 & 1 & K. Hoshino & planned \\
\hline$\because 12$ & MTX Collaboration & L & & & & 4/1989-3/1990 \\
\hline
\end{tabular}


PROPOSED JFY 90 EXCHANGES FOR MTX ANNEX ACTIVITIES

\section{IAERITOILNL}

Participation in MTX Experiments

1. MTX Experiment 1 person

2 MTX Expaterient 1 person

3. MTX Experiment 1 person

4. MTX Experiment

1 person

Technical Information Exchange

1. Tech. Exchange

2. Tech. Exchange

MTX Steering Committee 2 people
1 year

4 wks

3 wiks

13 wks

$1991 / 3$

1 wk

1 wk

1 wk
$1951 / 3$

$1990 / 10$

$1991 / 2$

\section{ILNLTO IAERI}

1. MTX Plasma Diagnostic Review

2 people

1 wk

$1990 / 6$

2. MTX Technical Info. Exchange 2 people

3. MTX Coordination/ Planning

1 person

4. MTX Technical Info. Bxchange

2 people

1 wk

1 wk

$1990 / 8$

$1990 / 9$

2 wks 1991/2 
DOE-JAERI Collaborative Program in the

Microwave Tokamak Experiments (MTX)

Second Steering Committee Meeting

February 15-16, 1990

I. Membership

Th collaboration between LLNL and JAERI on MTX was established under Annex VI to the U.S.-Japan Implementating Arrangement on Fusion. The second meeting of the Steering Committee called for in the Annex took place at LLNL on February 15-16, 1990. Members from japan are Dr. H. Maeda and Dr. A Kitsunezaki (Mr. A Komaki, Alternate) Members from the U.S. are Dr. S. Eckstrand and Dr. K Thomassen (Dr. E B. Hooper, Alternate). In attendance were Dr. M. Shiho and Dr. E. B. Hooper.

\section{Adoption of Agenda}

The agenda (Attachment 1) was adopted by unanimous consent.

IIL Minutes from last Meeting

- The minutes from the first Steering Committee Meeting were read and distributed to the members.

IV. Status Report on MTX

LLNL reported on experimental activities (see Agenda for speakers) on MTX this year. Due te priority use of the ETA-II accelerator by the Beam Research program the single pulse FEL experiments were delayed several months, but began at the end of the summer. During the run period ending the 10th of November microwave pulses up to $150 \mathrm{MW}$ at $140 \mathrm{GHz}$ were generated and transmitted on the quasi-optical system to the tokamak Transmission measurements were carried out showing no evidence of nonlinear effects at this power level, as expected. Transmission at densities up to cutoff was observed to be in general agreement with refraction and linear absorption theory. Immediately following this run a quick attempt to taper the wiggler was made, resulting in $400 \mathrm{MW}$ pulses from the FEL that were deflected from the transmission line to a dummy load.

Beam energy regulation of about $1 \%$ was achieved for only $10 \mathrm{~ns}$ or so, resulting in shorter microwave pulses than desired. Changes in the feed cables to the accelerator are now underway to correct this, delaying the installation of the IMP wiggler. A combination of these technical problems, and budget reductions in both the fusion and beam research budgets have 
delayed the program (see Attachment 2), raising concerns that the objectives of this Annex will not be met in the 4 year period it covers. The current schedule for MTX in FY90 and FY91 is part of Attachment 2. This schedule presents no technical difficulties in meeting it, but the budget requirement may exceed the MTX budget for next year and further delay the schedule.

V. JAERI Activities for MTX

Activities by JAERI in constructing the diagnostics adopted by the Steering Committee last year were summarized by Dr. Maeda. These instruments (Attachment 3) will be delivered on schedule at the end of March, 1990. Conceptual design work was done on items proposed for construction next year to determine their cost and feasibility.

\section{Plans for Next Year}

Agreement was reached on arrangements for assignees and exchanges within the Collaborative Program for next year. JAERI and LLNL each proposed a Hist of exchanges (Attachment 4) that will be presented to our respective organizations. Many of these exchanges are necessary to coordinate the work at both sites on the equipment to be completed next year. There are a number of interface issues, and both LLNL and JAERI have technical responsibilites for various issues raised in the conceptual designs.

The committee was pleased that Dr. T Ogawa and Dr. K. Ohasa will remain at LLNL on assignment to participate in the MTX experiments.

\section{Actions Adopted by the Committee}

The Steering Committee adopted by unarimous vote a resolution that JAERI will provide the following diagnostics and equipment next year: (a) A Laser Assisted Particle Probe Spectroscopy (LAPPS) system for microwave field measurements in the plasma, (b) A multichannel reflectometer, and (c) 3 different Vlasov couplers, designed to convert whispering gallery modes in the FEL driver gyrotron on MTX to linearly polarized free space modes needed at the wiggier input.

The committee also agreed to prepare a summary of activities on the Collaborative Program this year, to be presented at the JFPCC in a few months. 
VIII. Future Activities

The next meeting of the Steering Committee is planned for February, 1991 at LLNL

Qtiknouke made

Dr. H. Maeda

Akiva Kamaki

Dr. A. Kitsunezaki

(Mr. A. Komakj for Dr. A. Kitsunezakj)
Keith Thomassen

Dr. K Thomassen

Ste Elathand

Dr. S. Eckstrand

A.1-19 


\author{
Agenda \\ MTX Steering Committee Meeting \\ Exchange M8 \\ February 15-16, 1990 \\ Bldg. 543, Room 2023 \\ LLNL
}

\title{
Thursday, February 15
}

9:00 a.m.-10:00 a.m.

Welcome, Preparation of Agenda

10:00 a.m.-12:00 p.m. MTX Tours, Summary of Progress

Lunch

130 p.m.-5:00 p.m.

MTX Steering Committee

LNL Technical Presentations

LAPPS \& Reflectometry Studies - Casper et al

Experimental Plans for FY90 - Hooper

Progress on 1 MW Gyrotron Installation - Stallard, et al

JAERI Presentations

Friday. February 16

9:00 a.m. - 12:00 p.m.

Decisions on JAERI Diagnostics for Next Year

Preparation of JFPCC Summary of MTX Annex

Activity for this Year

Preparation of M8 Summary

Lunch

Discussion of Future Exchanges

1:00 p.m. - 3:00 p.m. General Discussion

$3.00 \mathrm{pm}$.

Adjourn

Contact: Keith Thomassen, 2-9815 
- Schedule has been stretched out for several reasons - FEL and Fusion budgets, technical problems on ETA II, delays in starting single pulse experiments because of Beam Research priority use.

- Single pulses to $400 \mathrm{MW}$ have been achieved, and plasma transmission experiments at the $100 \mathrm{MW}$ level performed.

- The low field IMP (2.8 kG) wiggler is completed and the high field (4.6 kG) add-on coils are partly fabricated.

- IMP operations are now delayed into FY 91, and FEL funding to the Beam Research porgram is uncertain. ETA-II facility may require funding solely from MTX program.

- Objectives of MTX Annex may not be met in 4-year time frame of the Annex. 


\section{NEW MTX OPERATIONS PLAN}

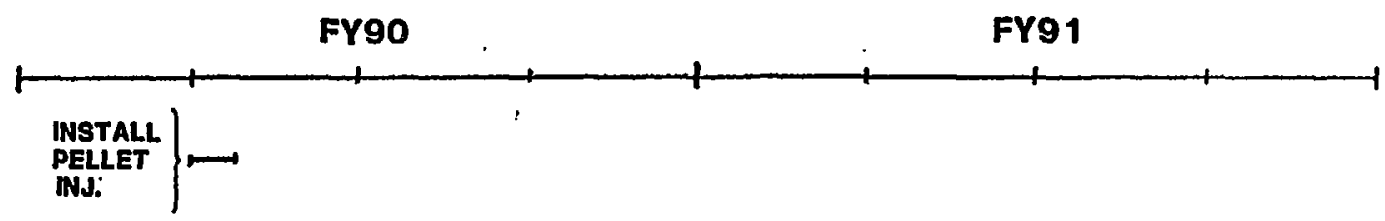

HARDWARE $\frac{\text { INSTALL 1/2-1 MW }}{140 \text { GHZ GYROTRON DRIVER }}$

$\frac{\text { IMP SYSTEM }}{\text { INSTALL (WIGQLER, TRANSMISSION; COUPLING) }}$

140 aHz

ELF-UNTAPERED

OPERATIONS

PÉLLET IMJ./NEW DIAG.

CHECKOUT

CW GYAOTRON - PELLET FUELNG

HEATING/TRANBPORT/FLUCTUATIONS

$\sim$ 1/2-TIME OPERATION

SHARED WITH BEAM PROGRAM

140 GHZ IMP - SINGLE PULSE

- BURST MODE 
JAERI ACTIVITY FOR PREPARATION OF MTX DIAGNOSTICS IN JFY89

ITEM

1. Dummy load system

2. J0 and $M 2$ mirrors

3. SX camera

4. ECE

5. Hellum neutral beam ion source for LAPPS
STATUS

Design and fabrication completed

Design and fabrication completed

Design and fabrication completed

Design and fabrication completed

Design and fabrication completed
COMMENT

On schedule

On schedule

On schedcule

On schedule

On schedule

\section{COMMENTS:}

1. Items 1 to 4 are scheduled to be shipped from JAERI to LLNL by the end of March 1990.

2. Item 5 is a part of LAPPS (Laser Assisted Particle Probe Spectroscopy). LAPPS is scheduled to be completed in JFY90. 
PROPOSED JFY 90 EXCHANGES FOR MTX ANNEX ACTIVITIES

\section{IAERITOLLNL}

Participation in MTX Experiments

1. MTX Experiment 1 person

2 MTX Experiment 1 person

3. MTX Experiment 1 person

4. MTX Experiment 1 person

1 year

4 whs

3 wiks

13 wks

$1991 / 3$

Technical Information Exchange

1. Tech. Exchange 2 people

2. Tech. Exchange 1 person

1 wk

1 wk

$1991 / 3$

MTX Stearing Committee 2 people

1 wk

$1990 / 10$

$1991 / 2$

\section{LLNLTOIAERI}

1. MTX Plasma Diagnostic Review

2. MTX Technical Info. Exchange

3. MTX Coordination/ Planning

4. MTX Technical Info. Exchange

2 people

2 people

1 person

2 people
1 wk

1 wk

1 wk

2 wks
$1990 / 6$

$1990 / 8$

$1990 / 9$

$1991 / 2$ 
DOE-JAERI Collaborative Program in the

Microwave Tokamak Experiment (MTX)

Addenda to the Minutes of the

Second Steering Committee Meeting

February 15-16, 1990

The following addenda to the indicated paragraphs have been agreed to by the Steering Committee Members.

\section{Plans for Next Year}

Subsequent to the Steering Committee meeting. LLNL prepared their Field Work Proposal for the U.S. Department of Energy. This proposal includes detalls of the work planned for the U.S. Fiscal Years 1991 and 1992. The attached schedules for MTX ETA/IMP Construction and Operations, extracted from the proposal, describe the planned work at LLNL Key dates include operation of the accelerator in a 20 cell mode by October, 1990, to test modifications designed to provide a constant energy electron beam, installation of the modified 60 cell accelerator and IMP wiggler by Apri., 1991, tests of this system in stingle pulse mode by July, 1991, and initial tests of the system in a burst mode by October, 1991. Extensive operation and physics experiments are planned for Fiscal Year 1992.

VII. Actions Adopted by the Committee

The committee discussed the likely schedule for MTX The Japanese side expressed concem at the slow pace and that the new schedule does not match the agreement. Both sides agreed that they would explore ways for effective action to speed up the achievements. As part of their action, LLNL is changing the management of the ETA accelerator and the IMP wiggler so as to bring it under the control of the MTX management.

April 11, 1990

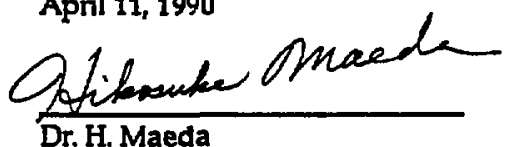

Dr. H. Maeda

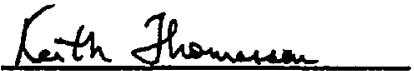

Dr. K. Thomassen
Akina Komaki

Dr. A. Kitsunezaki

(Mr. A. Komaki, alternate)

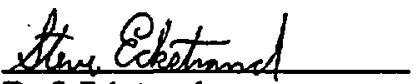

Dr. S. Eckstrand 


\section{MTX ETA/MP OPERATIONS SCHEDULE A FOR FY90, FY91, FY92}

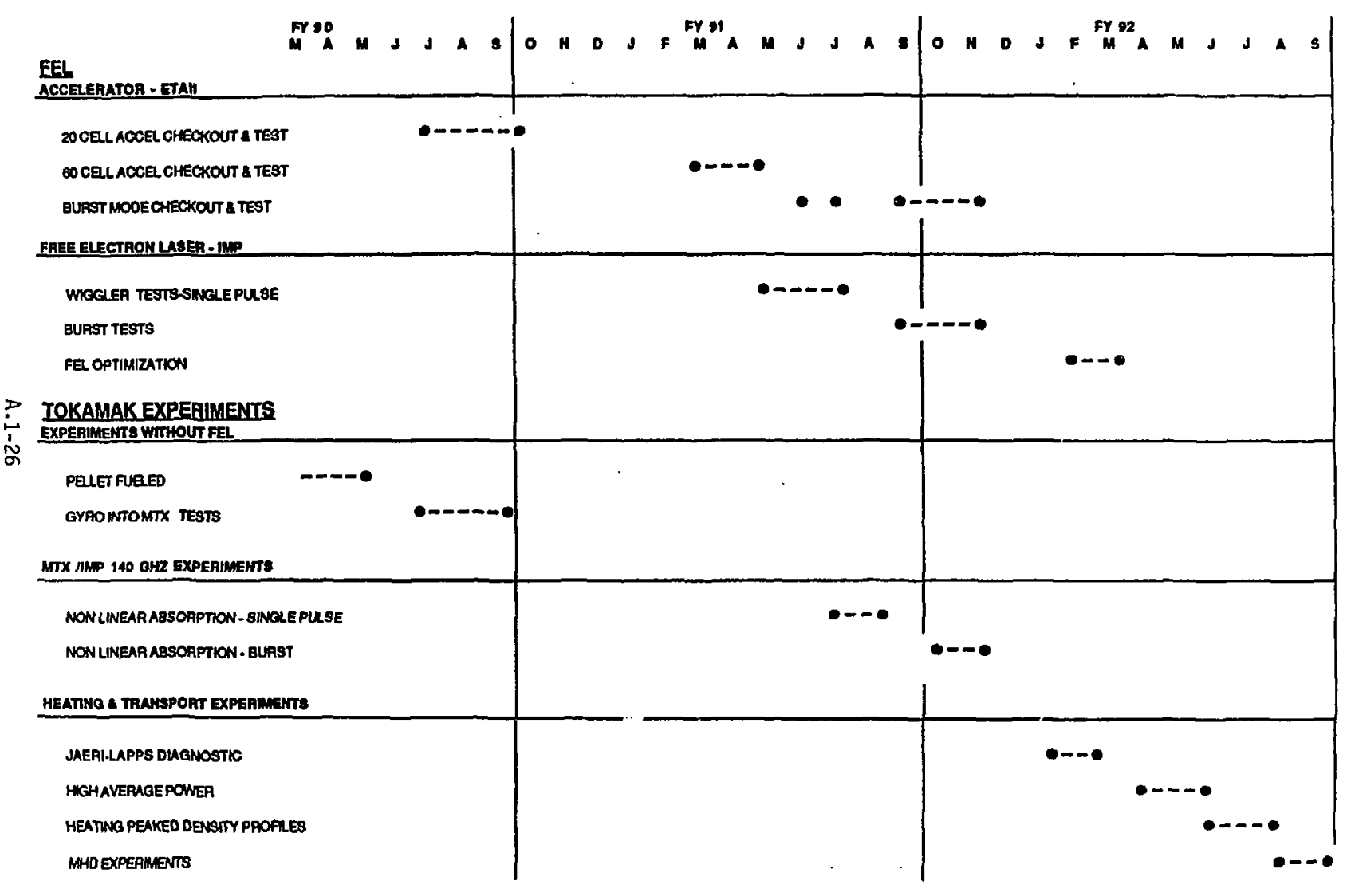

D. D. LANG 


\section{MTX ETA/MP CONSTRUCTION SCHEDULE A FOR FY90, FY91, FY92}

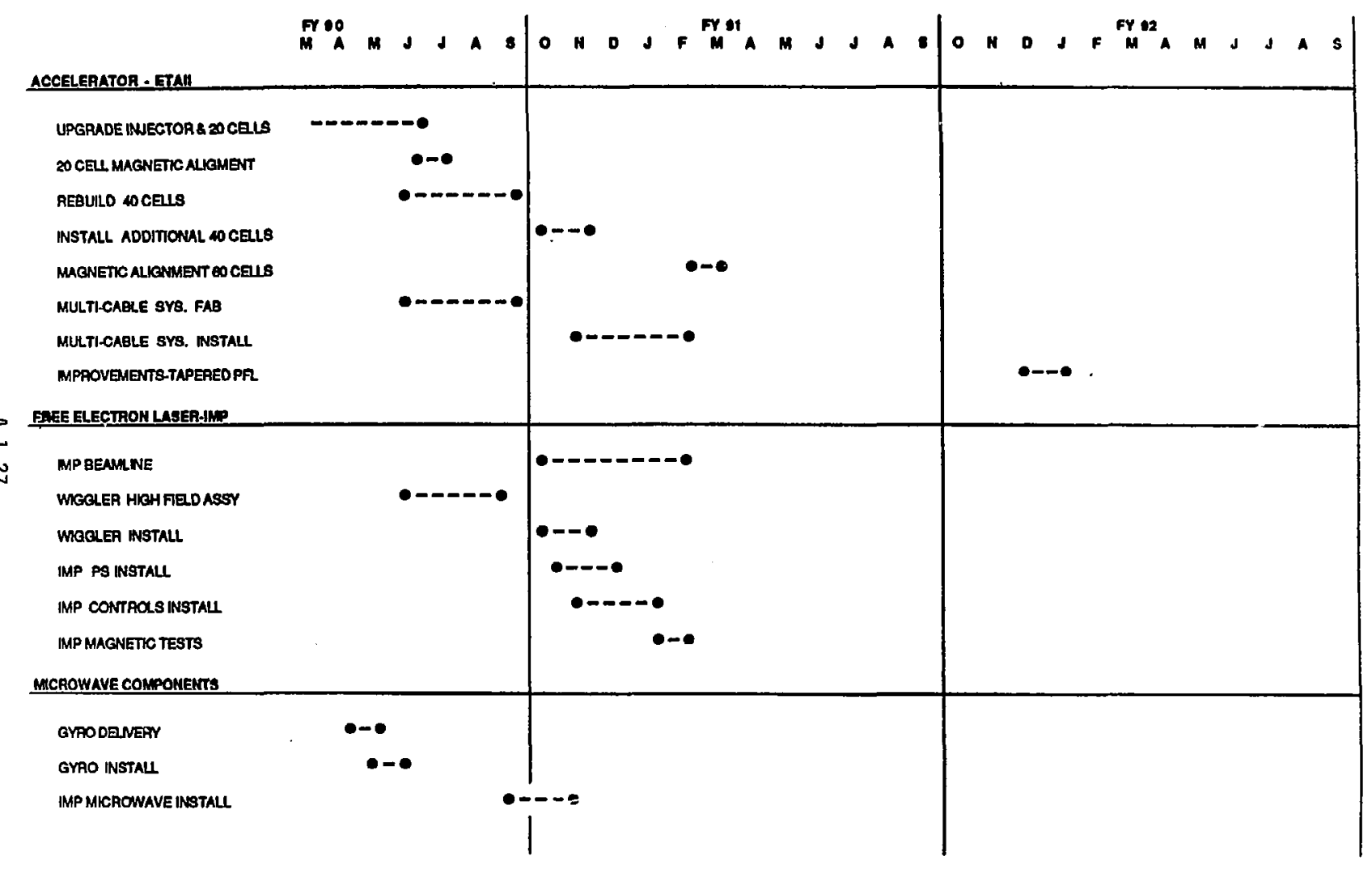


Summary of Annex V1 Activity in April 1990 - March 1991

DOE-JAERI Coliaborative Program in the

Microwave Tokamak Experiment (MTX)

Collaborations between LLNL and JAERI under Annex VI to the USJapan Implementing Arrangements, begun in May 1988, continued throughout April 1990-March 1991. Objectives of this Annex are to conduct and evaluate plasma heating and current drive experiments in MTX, using a high power microwave FEL, and to conduct joint studies of the application of FEL microwave technology in follow-on fusion tokamaks to determine the benefits for fusion applications.

Under this agreement, JAERJ is supplying equipment, primarily plasma and microwave diagnostics, for use on MTX. Diagnostics delivered at the end of the previous period were installed and operated, new diagnostics were completed, and JAERI scientists participated in MTX experiments. The JAERI scientists were involved in microwave FEL experiments on MTX during the year, applying their diagnostics and interacting on a wide range if physics issues.

The diagnostics and exchanges have a major beneficial impact on ihe MTX program with its limited resources. Good working relations continued during the year, aided by exchanges in both directions and rapid communication via computer link between personnel at both sites.

The attached discussion and tables summarize the technical accomplishments, individual exchanges for this year, and personn ${ }^{\prime}$. exchange plan for next year.

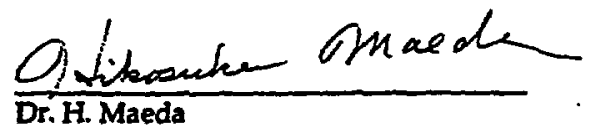

Dtancelsetrand

Dr. S. Eckstrand

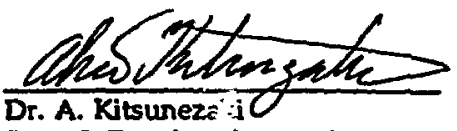

(Mr. H. Terada, Alternate)

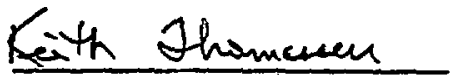

Dr. K. Thor assen

(Dr. E. B. Fioper, Alternate)

April 8, 1991 
Technical Accomplishments

During the period April, 1990 - March 1991, the major FEL experimental activity was experiments by the LLNL accelerator group to test improvements to the ETA-II induction linear accelerator. These improvements were required to correct problems with energy resolution, magnetic alignment, and pulse-to-pulse reproducibility identified in the FEL experiments on MTX during the previous year. The improvements were successful, and their implemention on the full accelerator started in late fall, 1990. In addition, final magnet installation on the isew wiggler, IMP, was started, and the wiggler will be moved into the accelerator pit later in the spring, 1991.

In parallel with the FEL effort, a Varian gyrotron $(140 \mathrm{GHz}, 400 \mathrm{~kW} \mathrm{cw})$ was installed. The gyrotron will provide the oscillator power for the FEL. In addition, tokamak plasma heating experiments will be compared with the pulsed, high peak power heating due to the FEL in order to compare the two different microwave interaction regimes.

Diagnostics were provided by JAERI on schedule. During the gyrotron operation, they made significant contributions. A calorimeter, which will be used with the FEL, was used to determine the efficiency of transport of microwave power from the gyrotron to the FEL using the MTX quasi-optical system. The measurements yielded an efficiency of $80 \%$, in good agreement with theory which predicted $20 \%$ loses at the Vlasov convertor. The convertor changes high order gyrotron modes into a Gaissian mode for coupling into the transmission line; a more efficient Vlasov convertor itom JAERI will be tested in Spring, 1991. In addition, measurements were made by the JAERI upgraded fast soft $x$-ray and neutron diagnosties during a brief tokamak experimental run using the gyrotron. The JAERI ECE heterodyne diagnostic was successfully tested in an exerimeitil run during June. The finai system was installed in September: the signal-to-noise ratio was low because of antenna and transmission line losses. Modifications are being icientified to correct the problem at LLNL in Aprii, 1991. A prototype reflectrometer was tested, and a high directivity optical coupler for the final installation of JAERI equipment was designed and tested. Several meeting were held with JAERI and LLNL personnel to finalize the design of the LAPPS diagnostic

The technical accomplishments of the exchange are summarized in the following tables. 
A. Installation and operation of JAERI diagnostics on MTX

ITEM STATUS COMMENTS

1. Microwave diagnostic Installed on MTX box

Used in gyrotron experiments; to be used in FEL experiments

2. Microwave calorimeter (Dummy load system)

3. Fast soft $x$-ray system (SX-camera)

4. Neutron system

5. ECE

6. J0 and M2 mirrors
Installed on MTX; attached to Micro wave diagnostic box

Upgraded system installed on MTX

Installed on MTX

Installed on MTX

At LLNL awaiting installation
Used to verify the transmission efficiency of the quasioptical system. Operational for FEL experiments.

Measured heating during gyrotron experiments. Operational for FEL experiments.

Integrated into MTX diagnostic system for routine operation. Operational for FEL experiments

Signal-to-noise improvements are being done.

To be used in the FEL microwave system.

B. JAERI activity for preparation of MTX diagnostics in JFY90

1. LAPPS

2. Vlasov convertor

3. Reflectrometer
Design and fabrication completed, including diagnostic neutral beam, laser, and optical system

Design and fabrication completed

Prototype constructed; testing and final corstruction underway
Under testing at JAERJ through the end of August, 1991. To be shipped to LLNL in Fall, 1991.

To be shipped to LLNL by the end of April, 1991, after cold tests at Stuttgart, Germany.

Prototype tests led to a final design of the optical system; components are on hand at LLNL for construction of the reflectrometer. 
IAPAN-US FUSION COOPERATION PROGRAM

\begin{tabular}{|c|c|c|c|c|c|c|}
\hline No. & TITLE & TYPE & WEEK & PERSONS & PARTICIPANTS & DATE \\
\hline M19 & MTX Coordination/Planning & TM & 1 & 1 & K. Thomassen & $2 / 18-22 / 91$ \\
\hline U12 & MTX Collaboration & $\mathbf{L}$ & 1 & & K. Thomassen & $4 / 90-3 / 91$ \\
\hline ML7 & MTX Experiment & $\mathbf{P}$ & 52 & 1 & K. Oasa & $4 / 90-3 / 91$ \\
\hline ML8 & MTX Experiments & $\mathbf{P}$ & 4 & 1 & K. Odajima & $7 / 90$ \\
\hline ML9 & MTX Experiments & $\mathbf{P}$ & $\begin{array}{l}\mathbf{3} \\
\therefore\end{array}$ & 1 & K. Hoshino & $3 / 91-4 / 91$ \\
\hline ML10 & MTX Experiment & $\mathbf{P}$ & 13 & 1 & K. Sakamoto & planned \\
\hline M15 & MTX Steering Conımittee & C & 1 & 2 & $\begin{array}{l}\text { H. Maeda } \\
\text { H. Terada }\end{array}$ & $3 / 91$ \\
\hline M16 & $\begin{array}{l}\text { Development of Spectroscopic } \\
\text { System for Electric Field } \\
\text { Measurement of High Power } \\
\text { Microwave in Tokamak }\end{array}$ & $\mathbf{P}$ & 2 & 1 & T.Oda & $10 / 90$ \\
\hline
\end{tabular}


PROPOSED JFY91 EXCHANGES FOR MTX ANNEX ACTIVITIES

\section{IAERI TO LLNL}

Participation in MTX Experiments

1. MTX Experiment

2. MTX Experiment

1 person 13 weeks

1 person 8 weeks

3. MTX Experiment

1 person 6 weeks

4. MTX Experiment

1 person 52 weeks

Technical Information Exchange
1. Technical Exchange
1 person 1 week
2. Technical Exchange
1 person 2 weeks
3. Techrical Exchange
1 person 2 weeks
4. Technical Exchange
1 person 2 weeks

MTX Steering Committee

2 people 1 week

LLNL to IAERI

1. MTX Plasma Diagnostic Review

2. MTX Technical Information

3. MTX Technical Information

4. Program Coordination

2 people 1 week

2 people 1 week

2 people 2 weeks

1 person 1 week 


\section{MTXIETAIIMP CONSTRUCTION SCHEDULE FOR FY91, FY92}

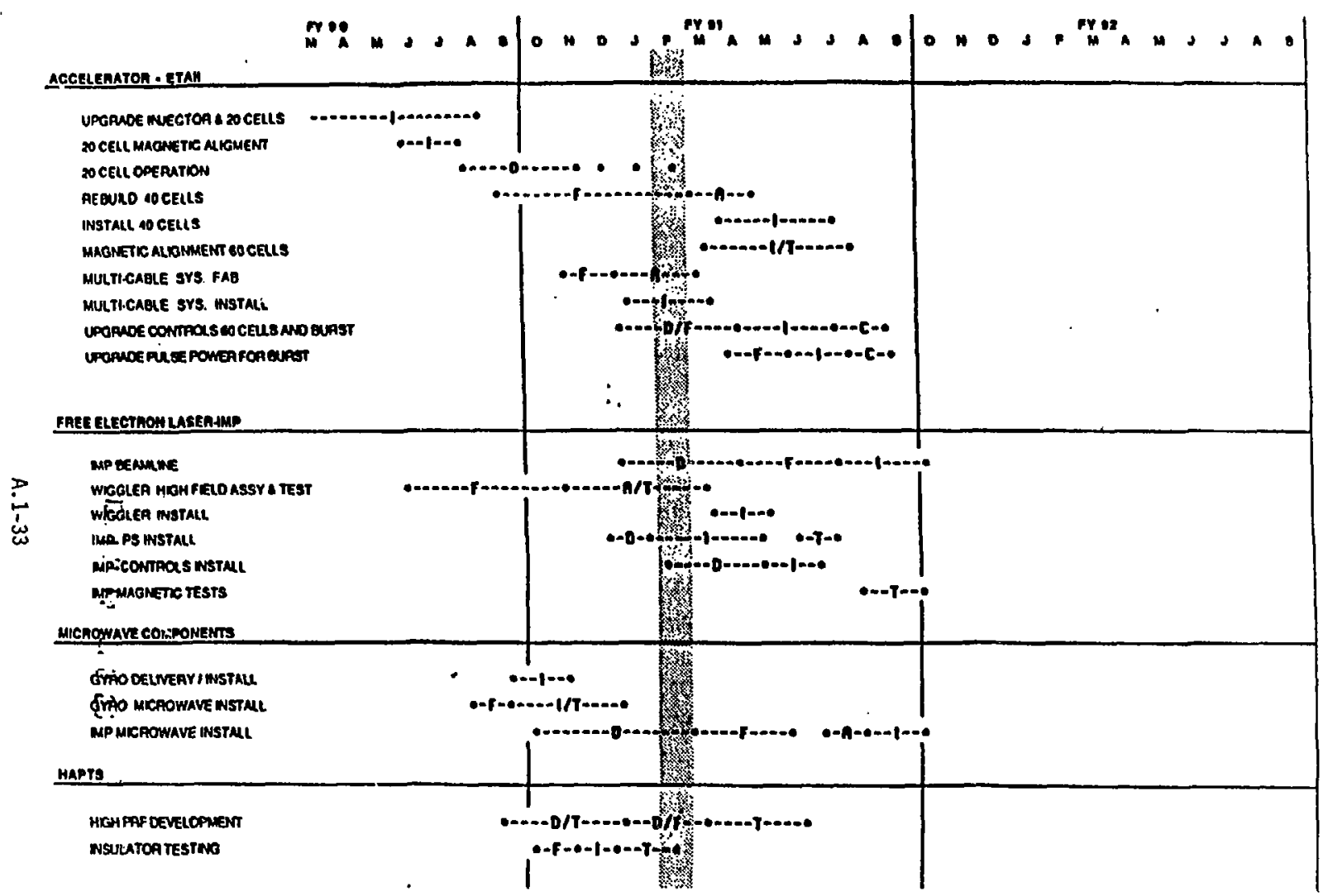

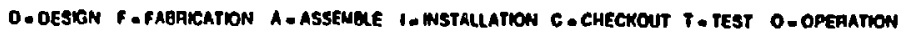




\section{MTX STEERING COMMITTEE \\ March 11, 1991}

\section{FINDINGS \& RECOMMENDATIONS}

The MTX Steering Committee reviewed the FEL construction plans for FY 91 and the MTXJFEL operations for FY 92 as presented by LLNL. It also heard the JAERI views on desired results from this collaboration. in summary, the JAERI team wants to see the feasibility of using H-wave FEL's for tusion established in the experiments next year. Specifically, their physies and technology goals were discussed. On the physics side, both absorption and transport physics results were desired. Non-linear absorption coefficients are to be determined for comparison with theory by measuring the macroscopic heating of the plasma. Global transport measurements are desired to determine whether there are any deleterious effects. A sub-set of the transport goals expressed by JAERI include (a) comparison of linear vs non-linear effects, (b) assessments of relaxation processes on the various fast and slow time scales, including any development of potentials to alter the ion motion, and (c) a search for multi-pulse effects. These will use the JAERI diagnostics provided under the collaboration.

On the technology side, some of their objectives have aiready been met by earlier FEL operations. These include control of the ouput mode (gaussian-like with linear polarization), transmission of high power pulses on a Quasi-Optical line, and transmission of high average power (gyrotron source) microwaves on the Q.O line. Remaining objectives are the transmission of high-average power FEL $\mu$-waves, and an investigatiof of the limits (by breakdown or any other phenomena) to power density for different background pressures in the tine and tokamak port. While the desire to show the tunability and higher frequency $(250 \mathrm{GHz}$ ) operation was expressed JAERI did not consider them necessary to meet the minimum objectives of the collaboration.

The LLNL budget request for FY $92(\$ 7.01 \mathrm{M})$ needed to meet the sciedules for construction (attacned) and allow time for burst mode operation was acknowledged by DOE and it was noted that attempts were being made to secure the funding. If this funding becomes available, the Steering Committee finds that there is a reasonable expectation that the minimum ohysics and technology goals as expressed above by JAEBL could be met. $\angle \mathrm{NL}$ and DOE agree that, if 
that funding is secured, a best-efforts attempt will be made to meet these goals during the existing Annex.

One of the objectives of the collaboration is to evaluate the results of the experiments and the applicability of FEL's for fusion. DOE suggested to JAERI that this evaluation and analysis could take place in the period immediately following the experiments, set now to conclude at the end of the current Annex. The Steering Committee strongly recommends an extension of the Annex for the purpose of these joint analysis activities. The DOE suggested a period of about six months for this work. The fruits of this collaboration will be in the form of the analyses and joint reports of the JAERI/LLNL team. This extension will allow the formal continuance of personnel exchanges between LLNL and JAERI, and for meetings as necessary to complete these reports.

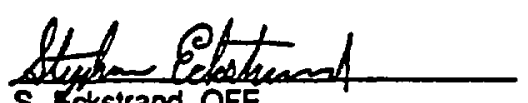

S. Eckstrand, OFE

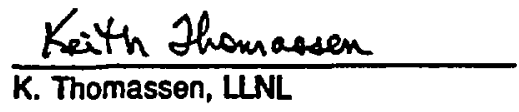

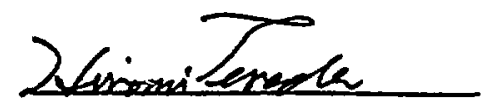

H. Torada, JAERI

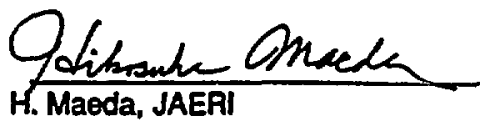

$$
\frac{9-12-91}{\text { Date }}
$$




\section{MTX/ETA/IMP CONSTRUCTION SCHEDULE FOR FY91, FY92}

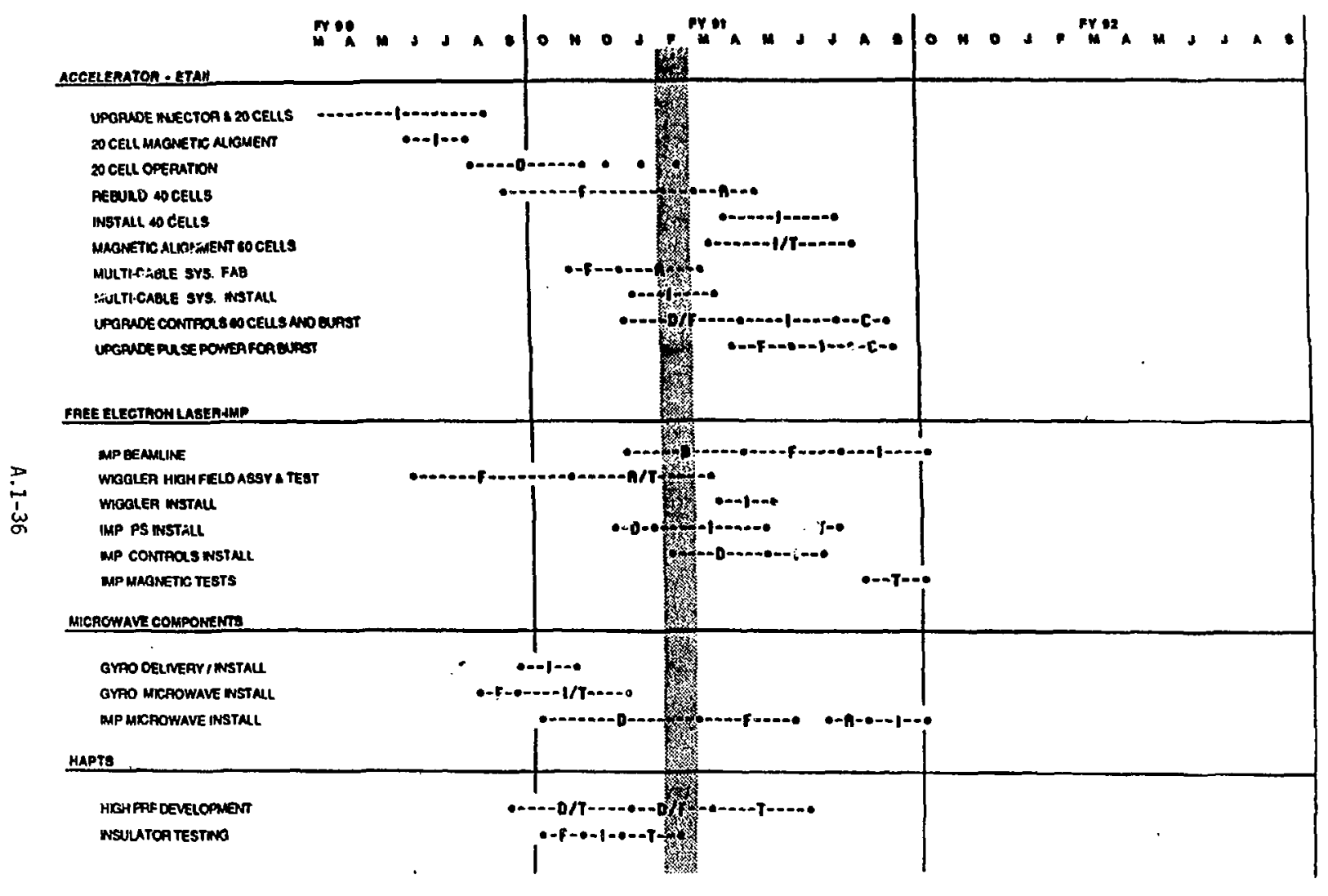

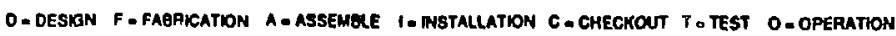


Surnmary of Annex VI Activity in April 1991 - March 1992

DOE-JAERI Collaborative Program in the

Microwave Tokamak Experiment (MTX)

Collaborations between LLNL and JAERI under Annex VI to the USJapan Implementing Arrangements, begun in May 1988, continued throughout April 1991-March 1992. Objectives of this Annex are to conduct and evaluate plasma heating and current drive experiments in MTX, using a high power microwave FEL, and to conduct joint studies of the application of FEL microwave technology in follow-on fusion tokamaks to determine the benefits for fusion applications.

Under this agreement, JAERI is supplying equipment, primarily plasma and microwave diagnostics, for use on MTX. Diagnostics delivered at the end of the previous period were installed and operated, new diagnostics were completed, and JAERI scientists participated in MTX experiments. The JAERI scientists were involved in microwave gyrotron experiments on MTX during the year, applying their diagnostics and interacting on a wide range of physics issues. During this period the ETA-II Accelerator was rebuilt.

The diagnostics and exchanges have a major beneficial impact on the MTX program with its limited resources. Good working relations continued during the year, aided by exchanges in both directions and rapid communication via computer link between personnel at both sites.

The attached discussion and tables summarize the technical accomplishments, individual exchanges for this year, and personnel exchange plan for next year.

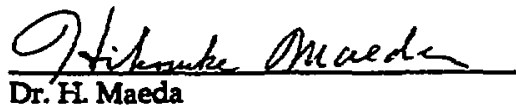

Dr. H. Maeda

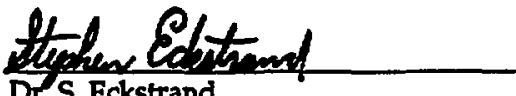

Dr.S. Eckstrand
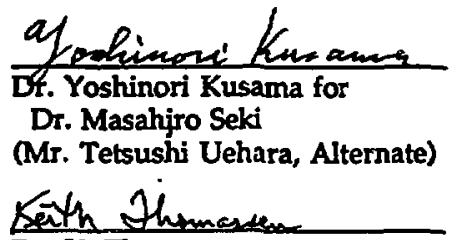

Dr. $K$ Thomassen

(Dr. E. B. Hooper, Alternate)

March 12, 1992 


\section{Technical Accomplishments}

During the period April, 1991 - March, 1992, experimental work was conducted on the MTX tokamak and on the FEL. Microwave power up to $200 \mathrm{~kW}$ was injected from the $140 \mathrm{GHz}$ gyrotron into the tokamak to provide a data base for comparison with future experiments on heating using the free-electron laser. Significant heating was documented for resonance on the magnetic axis at pulse lengths varying from a few ms to $100 \mathrm{~ms}$. These experiments were carried out for line densities of $5 \times 10^{19} \mathrm{~m}^{-3}$ to $3 \times 10^{20} \mathrm{~m}^{-3}$. In some discharges, on axis heating was observed to convert pathological behavior to well behaved, sawtoothing: In one case, microwaves were applied immediately following a minor disruption apparently due to locked modes, and caused recovery. At low densities, hollow current and electron temperature discharges could be made peaked by ECRH.

The ETA-II accelerator was rebuilt and brought into operation with timing control good enough to permit $5 \mathrm{kHz}$ burst operations. The rebuild included replacing the insulators in most of the accelerator gaps and a careful magnetic alignment of the assembled accelerator to minimize the cork screw motion of the beam. The MAG timing units were rebuilt to provide better timing reliability, and a timing feedback system installed to compensate for slow (ms) drifts of the units. Pulse-to-pulse timing stability of 1-2 ns was observed. The IMP wiggler was installed and careful magnetic measurements used to adjust the permanent magnets so that the fractional field error is less than $2 \times 10^{-3}$, required for efficient FEL operation. The microwave transmission line from the gyrotron to the wiggler was installed. Experimental work leading to full FEL operations is underway.

Burst mode operation of the pulsed-power units and MAGs, coupled to an acceleration column, was demonstrated at $5 \mathrm{kHz}$ on the HAPTS. The ETA-II equipment has been modified for burst mode and will be tested in th 3 near future.

The microwave equipment provided by JAERI was important to the success of the gyrotron experiments, and the diagnostios from JAERI generated significant data. The Vlasov reflector was used to convert the whispering-gallery mode output from the gyrotron into a Gaussian mode for propagation to the tokamak. The micrc wave calorimeter was used as the primary measurement of the microwave power reaching the tokamak. The microwave diagnostic box, which included the calorimeter, also was used to obtain profiles of the microwave beam. The fast-soft $x$-ray system yielded very high quality data of the $x$-ray emission during heating. Neutron measurements were used to search for ion heating during the ECRH. The heterodyne ECE and reflectrometer were tested and improvements installed for use during the FEL experiments.

The Laser Aided Particle Probe (LAPPS) diagnostic to measure the microwave electric field in the plasma was delivered to LLNL and has been installed on the tokamak. Alignment of the optios is underway by a team of Japanese and US scientists. 
Installation and operation of JAERI equipment and diagnostics on MTX

ITEM

1. Microwave diagnostic box

2. Microwave calorimeter (Dummy load system)

3. Fast soft "-ray system (SX-camera)

4. Neutron system

5. ECE

6. J0 and M2 mirrors

7. Vlasov convertor

8. Reflectrometer

9. LAPPS
STATUS

Installed on MTX

Installed on MTX; attached to Microwave diagnostic box

Installed on MTX Installed on MTX

Installed on MTX

Installed in the FEL microwave system

Installed in the grrotron system

Installed on MTX

Installed on MTX
COMMENTS

Used in gyrotron experiments; to be used in FEL experiments

Used to measure gyrotron power transmitted to the tokamak. Operational for FEL experiments.

Used in gyrotron experiments. Operational for FEL experiments.

Integrated into MTX diagnostic system for routine operation. Operational for FEL experiments

Signal-to-noise improvements have been made, but signal level still marginal. Operational for FEL experiments; improvement in receiver coupling planned.

To be used to transmit microwave power to the tokamak.

Used to convert microwave power from wlispering galley modes to low order modes

Prototype tested during gyrotron run. Single channel of final receiver built and ready for FEL operations.

Undergoing optical alignment and debug of neutral beam and laser systems 


\section{IAPAN-US FUSION EXCHANGES FOR IFY92}

\begin{tabular}{|c|c|c|c|c|c|c|}
\hline No. & THLE & TYPE & WEEK & BERSONS & PARTICIPANTS & DATE \\
\hline M17 & MTX Plasma Diagnostic Review & $\mathbf{T M}$ & 1 & 2 & & Cancelled \\
\hline M18 & $\begin{array}{l}\text { MTX Technical Information } \\
\text { Exchange }\end{array}$ & $\mathbf{T M}$ & 1 & 2 & Thomassen & Deferred \\
\hline M20 & $\begin{array}{l}\text { MTX Technical Information } \\
\text { Exchange }\end{array}$ & $\mathbf{T M}$ & 2 & 1 & Foove & $5 / 17-6 / 3 / 91$ \\
\hline M21 & Steering Committee Meeting & $\mathbf{C}$ & 1 & 2 & Maeda & $3 / 12-13 / 92$ \\
\hline M22 & Technical Meeting on LAPPS & TM & 1 & 1 & Odajima & $\begin{array}{l}\text { Conbined } \\
\text { with } M L-11\end{array}$ \\
\hline M23 & Vlasov U-Wave Installation & $\mathbf{P}$ & 2 & 1 & Sakamoto & $5 / 6-31 / 91$ \\
\hline M24 & FEL Discussions & $\mathbf{T M}$ & 2 & 1 & Shiho & $9 / 3-4 / 91$ \\
\hline M25 & Theory/Analysis of Results & $\mathbf{P}$ & 2 & 1 & Kishimoto & $9 / 3-4 / 91$ \\
\hline ML11 & Installation of LAPPS & $\mathbf{P}$ & 11 & $\begin{array}{l}1 \\
2\end{array}$ & $\begin{array}{l}\text { Odajima } \\
\text { Ogawa, Shibata }\end{array}$ & $\begin{array}{l}9 / 23 / 91-3 / 5 / 92 \\
3 / 24 / 92-3 / 31 / 92\end{array}$ \\
\hline MI.12 & ECE/Reflectometer & $\mathbf{P}$ & 8 & 1 & Hoshine & $3 / 29 / 92-3 / 31 / 92$ \\
\hline ML13 & LAPPS Experiment & $\mathbf{P}$ & 6 & 1 & Oda & $3 / 24 / 92-3 / 31 / 92$ \\
\hline ML14 & MTX/FEL Experiment & $\mathbf{P}$ & 52 & 1 & Oasa & $4 / 1 / 91-3 / 31 / 92$ \\
\hline
\end{tabular}




\section{IAPAN-US FUSION EXCHANGES FOR IFY93}

NO. TIIE TYPE WEEKS ERRSONS RARTICIPANTS

TO JAERI

MX-1 MTX Data Analysis

WR.

2

3.4

Hooper, Allen,Casper

M18 MTX Technical Information

$\mathbf{P}$

1

1

Thomassen

To LLNL

$\underset{⿱ 亠 乂}{2}$

Measurement by LAPPS and

Data Analysis

Neutron Diagnostics

Analysis of ECE Data and

Reflectrometry Data

LAPPS Experiment

MTX/FEL Experiment

$\mathbf{P}$

6

1

Oda

P 13

1

Ogawa

P

4

1

Hoshino

P $\quad 26$

1 Odajima

P

26

1

Oasa 


\section{MTX STEERING COMMITTEE \\ March 13, 1992}

\section{SUMMARY OF THE MEETING}

The MTX Steering Committee reviewed the FEL construction activities in FY 92 and the MTXJFEL operations since last year, as presented by LLNL. It also heard a summary of the JAERI activity on this collaboration. A summary of the activity on Annex VI during the year was prepared and signed by both parties.

This summary included details of activities on the exchanges last year, and the proposals for exchanges starting on April \%, 1992. These exchanges will occur both during the Annex, which both sides recognize will terminate on May 18, 1992, and after that date.

Those exchanges beyond the termination date are for the purposes of collaborating on activities initiated under the Annex, but not completed at the termination, according to article 10 of the Annex. These would include, specifically, completing work on the JAERI instruments (LAPPS, reflectrometry, ECE, and neutron detectors), gathering data from MTX experiments using these instruments, and analyzing and reporting this data. The LLNL/Tokai data link will be used to transmit data so that report writing can take place at both laboratories.

It was noted by DOE that activites on MTX would be termiriated during the U.S. FY92, the exact time yet to be determined. The Steering Cornittee recommends that experimental activities on MTX continue for a few months beyond the Annex termination date to increase the amount of data on FEL heating in MTX.

Diagnostics provided by JAERI during the 4-year period of the Annex often required LiNL labor or components for their completion. In those cases this results in partial ownership of a diagnostic by each party, with rrajority ownership sometimes in question. Although the parts supplied by each party belong to that party, it was agreed that if LLNL proposed to retain a particular diagnostic instrument. it would present a request to JAERI to transfer orynership to LLNL. The Steering Committee recommends that a final disposition of instruments be determined jointly by the parties on termination of the MTX experimental operations.

A JAERI scientist was injured in a nitrogen asphyxiation accident on February 13, 1992. His very good recovery was the result of prompt aution by LLNL emergency services personnel. As a result of this incident, an accident investigation team is preparing a 
report to be completed on March 30, 1992. JAERI expressed an appreciation for the prompt action and irformal information from LLNL and DOE since the accident. JAERI also expressed its interest in receiving full details of the event in a formal report from DOE as soon as possible. LLNL has reviewed its proceedures on MTX, and its training and supervision of visiting JAERI scientists who participate in experimental activities.

One of the objectives of the collaboration is to evaluate the results of the experiments and the applicability of FEL's for fusion. Last year DOE suggested to JAERI that this evaluation and analysis could take place in the period immediately following the experiments. The Steering Committee recommends that this assessment be included in the final reports of the Collaboration.

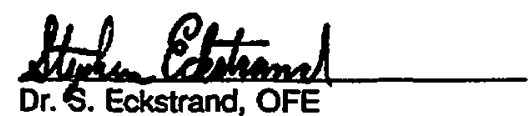

Keith Thomassen

Dr. K. Thomassen, LLNL

(Dr. E. B. Hooper, Alternate)

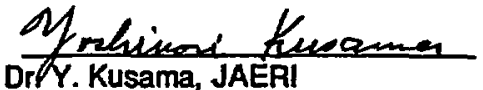
for Dr. M. Seki (Mr. T. Uehara, Alternate) Dr. H. Maeda, JAER!

$\frac{3-13-92}{\text { Date }}$ 


\section{A.2 Diagnostics: microwaves}

\section{A.2.1 Microwave beam cetectors (B. W. Stallard)}

The diagnostics used to measure the gyrotron and FEL beam in the Q.O. transmission system and in the MTX tokamak were the following:

- A manually scannable if probe consisted of a WR8 waveguide probe and diode detector. The probe was transportable and was moved from mirror to mirror for beam alignment and profile measurements.

- A remotely operated scanning probe, located $107 \mathrm{~cm}$ from the output of the FEL waveguide, was movable in the horizontal direction (E-plane) . The probe signal was detected by a calibrated $140 \mathrm{GHz}$ receiver. A block diagram of the receiver circuit is shown in Fig. A.2.1-1.

- Single-hole couplers (WRE waveguide), were located at the centers of mirror optics Jo and M4. Signals from these couplers were detected by a receiver.

- Two probes, remotely scannable in the horizontal (E-plane), located 117 $\mathrm{cm}$ from the port, and vertical (H-plane), positioned $142 \mathrm{~cm}$ from the port, were connected to If receivers to measure the beam profiles at MTX. For FEL injection into the tokamak the horizontal probe was inserted into the edge of the FEL beam to measure the pulse shape.

- A calcrimeter (JAERI diagnostic), located before the MTX port, measured the total pulse energy. With the pulse shape measured by the rf probes the peak If power was determined.

- An infrared (IR) camera viewing the MTX input port was lised to measure the size and position of the beam upon absorbing macor located on both sides of the MTX port. Using the IR camera the beam was aligned into MTX by adjustment of the final optic M4.

- A microwave horn connected to an rf receiver and located on the inside wall of the tokamak opposite the injection port measured FEL beam transmission.

- A segmented calorimeter measured the power profile of the transmitted FEL beam. 


\section{MTX Microwave Receiver Diagnostic}

$140 \mathrm{GHZ}$ RECEIVER (4 CHANNEL)

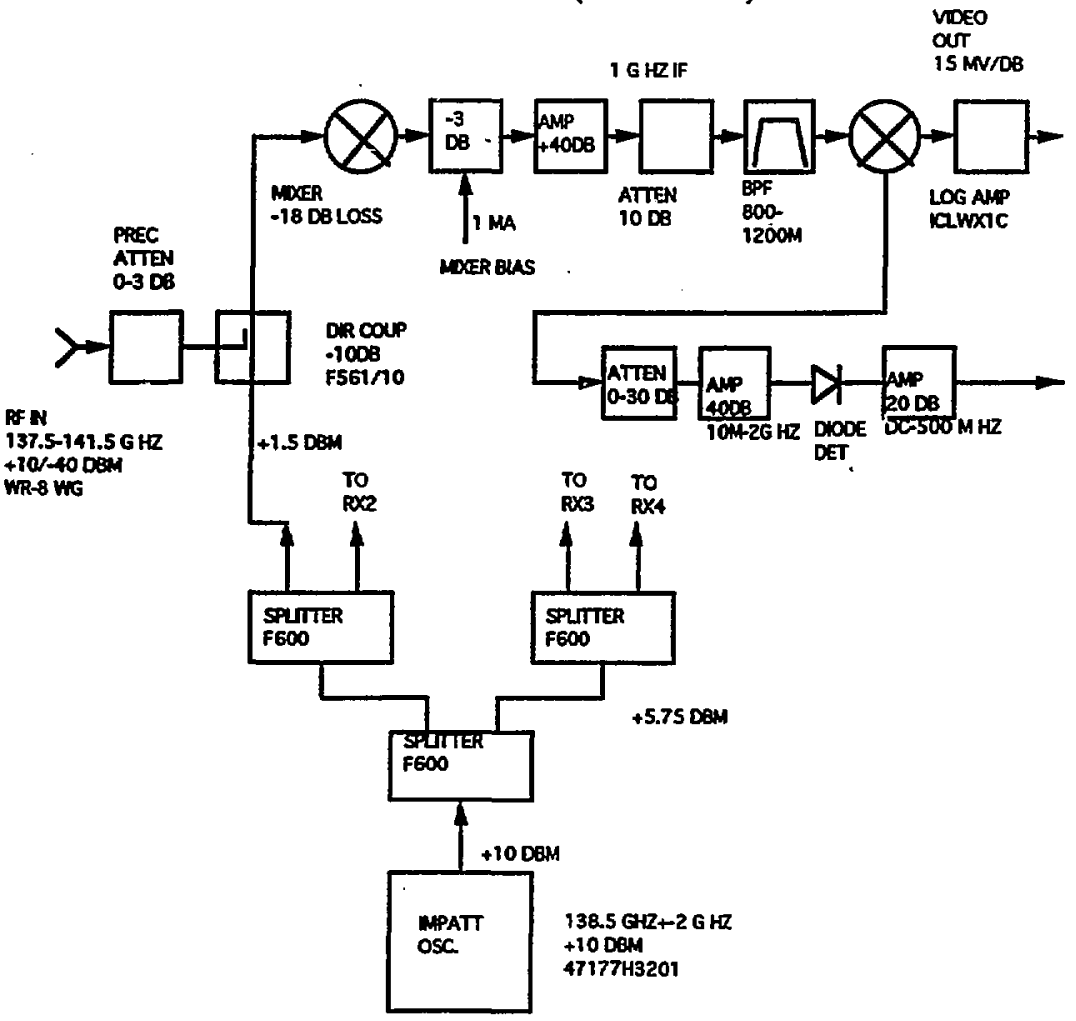




\section{A.2.2 PULSED POWER CALORIMETER (K OASa)}

Pulsed microwave power from the FEL was measured at the entrance port to MTX using a high-power calorimeter which was water-cooled between microwave pulses. The calorimeter, designed and constructed by JAERI as part of the collaboration, replaced the "High average power beam dump" shown as part of the MTX Microwave Diagnostics, next page. 


\section{MTX MICROWAVE DIAGNOSTICS}

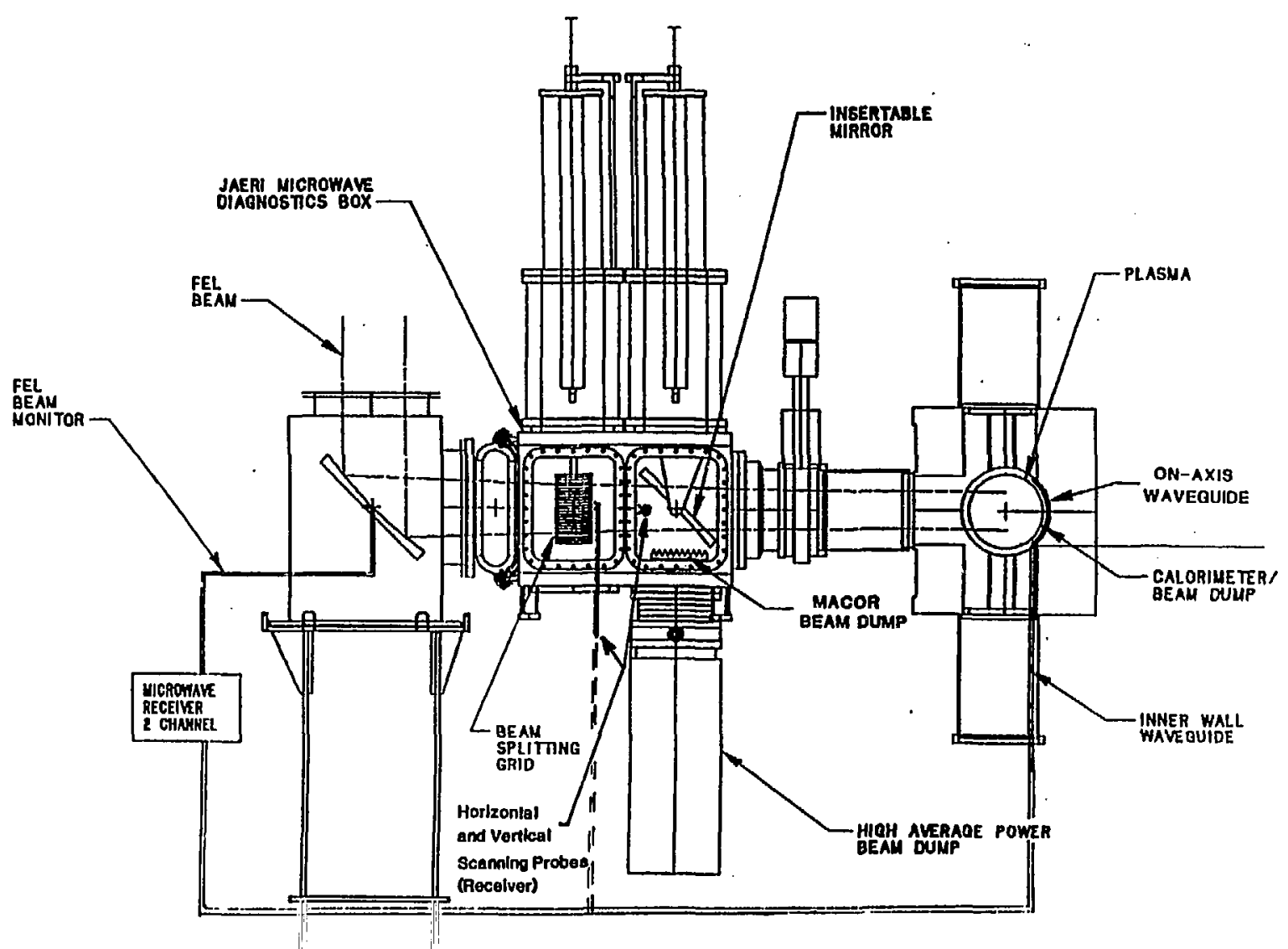




\title{
A.2.3 Microwave Calorimeter (S. Meassick* and M. E. Fenstermacher)
}

\begin{abstract}
A.2.3.1 Introduction. This report describes the design, ${ }^{1}$ construction and operation of the microwave calorimeter installed in MTX during August 1989. The calorimeter is designed to measure the spatial profile of $140 \mathrm{GHz}$ microwaves impinging upon it. The microwaves are generated in a FEL and propagated to the MTTX tokamak where they are injected radially inward through the plasma. The calorimeter is installed on the inside wall of the tokamak in order to measure the microwave energy transmitted through the plasma. From a knowledge of the encrgy incident upon the plasma and the energy remaining after propagation through the plasma, the energy a'ssorbed by the plasma can be determined. The active area of the calorimeter is an arc of $38 \mathrm{~cm}$ in the poloidal direction and a width of $8.4 \mathrm{~cm}$ in the toroidal direction. The calorimeter is instrumented with 57 sensors, 48 of which are digitized at a rate of $26 \mathrm{~Hz}$. The calorimeter operates on the principal of converting the microwave energy incident upon it into heat and measuring the temperature rise of the absorbing material. The absolute sensitivity of the calorimeter is approximately $0.01{ }^{\circ} \mathrm{C}$, corresponding to an energy flux of $0.005 \mathrm{~J} / \mathrm{cm}^{2}$. This sensitivity is required in order to measure the microwave energy expected to reach the calorimeter in MTX, which is less then $0.08 \mathrm{~J} / \mathrm{cm}^{2}$.

This report is organized as follows: Section 1 describes the microwave and thermal properties of the materials exposed to the microwaves. Section 2 describes the electrical characteristics of the temperature transducers and the associated electronics needed to make measurements of the incident energy flux. Section 3 describes the data acquisition software and the operation of the calorimeter as a diagnostic.
\end{abstract}

A.2.3.1 Physical Principals. MICROWAVE PROPERTIES. The calorimeter installed on MTX measures the microwave energy incident upon it by first converting the microwaves into heat and measuring the resultant temperature rise of the absorber. Since the heat capacity of the absorber is known the amount on energy incident upon the calorimeter can then be determined. The microwave absorber must satisfy a number of conditions to be applicable for use inside of the MTX tokamak. The absorber must have sufficient absorptivity of the microwaves in order to be able to absorb all of the microwaves incident upon it. In addition the material properties must be such that the absorber will have a large enough temperature rise to be measured accurately. Finally the absorber must be compatible with the ultra-high vacuum conditions encountered inside of the tokamak and must be able to withstand the temperature extremes encountered within the tokamak $\left(-100^{\circ} \mathrm{C}\right.$ to $\left.+125^{\circ} \mathrm{C}\right)$.

The material chosen to be the microwave absorber is silicon carbide (SiC) made by Standard Oil (Hexoloy SA Silicon Carbide). The material properties of 
silicon carbide are listed in Fig. A.23-1. This is a high density (low void fraction) sintered silicon carbide with an attenuation coefficient of $\alpha=11.1 \mathrm{~Np} / \mathrm{cm}$ and a dielectric constant of approximately 3.5. The dielectric constant and attenuation coefficient were determined by irradiating a $0.05 \mathrm{~cm}$ thick slab of $\mathrm{SiC}$ with microwave radiation and measuring the amount of radiation reflected and transmitted (Figs. A.2.3-2 and 3). It can be seen that there is a variation of the amount of radiation absorbed or reflected as a function of frequency. This is caused by interference due to radiation being reflected off the front and rear surfaces of the slab. The dielectric constant can be calculated by noting that the interference pattern is proportional to $\cos (\theta)$ where $\theta=2 \mathrm{~d} \omega n / c, n$ is the dielectric constant of the material, $d$ is the thickness of the material and $c$ is the speed of light. We thus have maxima when $\theta=2 \pi \mathrm{m}$ where $m$ is an integer representing the maxima. In order to determine the dielectric constant two maxima are compared yielding:

$$
n=c\left(m_{1}-m_{2}\right) /\left(2 d\left(f_{1}-f_{2}\right)\right)
$$

Figure A.2.3-4 shows the dielectric constant as a function of frequency. At $140 \mathrm{GHz}$ the dielectric constant for $\mathrm{SiC}$ is approximately 3.5. Silicon carbide, being a ceramic material is very vacuum compatible, with outgassing rates of less than $1 \times 10^{-9}$ Torr-L/sec/cm if properly baked out. Figure A.2.3-5 shows the measured outgassing properties as a function of time. With a high dielectric constant of 3.5, the silicon carbide will reflect a substantial fraction of the microwaves incident upon it. For normal incidence the reflection is given by:

$$
R=\left(\left(n_{2}-n_{1}\right) /\left(n_{2}+n_{1}\right)\right)^{2}
$$

where $n_{2}=3.5$ and $n_{1}=1.0$ are the dielectric constants of the silicon carbide and of a vacuum respectively. Thus we can expect $31 \%$ of the microwaves incident upon the silicon carbide to be reflected. This is close to the $33 \%$ reflection measured by irradiating a slab of silicon carbide with $140 \mathrm{GHz}$ microwaves. Figure A.2.3-6 shows the fractional absorption of $140 \mathrm{GHz}$ microwaves for normal incidence. The fluctuations in the absorption are caused by internal reflections of the microwaves giving rise to interference patterns. In order to absorb most of the microwaves on a single pass and not be to sensitive to small variations in thickness of the absorber a thickness of at least $0.25 \mathrm{~cm}$ of silicon carbide is required. The fractional power present at a distance $x$ from the front surface of the silicon carbide is given by $e^{-\alpha x}$. Thus for $0.25 \mathrm{~cm}$ of silicon carbide $94 \%$ of the incident microwave power is absorbed in one pass.

It was decided to add a layer of copper to the rear surface of the silicon carbide tiles in order to reflect microwaves not absorbed during their pass through the absorber. This was done in order to shield the temperature transducers from direct heating of the microwaves and the substantial electric fields of the microwaves radiation. The incident microwaves have electric fields of $180 \mathrm{kV} / \mathrm{cm}$, after passing through the silicon carbide the electric field is 
attenuated to a level of approximately $6 \mathrm{kV} / \mathrm{cm}$. In order to attenuate this to less than $1 \mathrm{~V} / \mathrm{cm}$ at least $11 \mathrm{skin}$ depths of attenuation are required. For a conductor, in this case copper, the skin depth is given by:

$$
\delta=\left(2 /\left(\mu_{0} g \omega\right)^{0.5}\right.
$$

where $g$ is the conductivity of copper and $\mu_{0}$ is the permittivity of free space. At $-60^{\circ} \mathrm{C}$ copper has a conductivity of $1.41 \times 10^{8} \mathrm{mho} / \mathrm{m}$ giving a skin depth of 1130 $\AA$, thus requiring at least $12400 \AA$ for the required attenuation. The copper layer chosen was $20000 \AA$ thick. The copper layer was grounded, forming a ground plane shielding the temperature transducers from noise emanating from the plasma.

In order to reduce the amount of microwaves reflected from the absorber a dielectric stack was added to the front surface. By using alternating layers of high and low dielectric constant materials interference can be used to minimize reflectance from the stack. The most suitable low dielectric constant material is a vacuum while the high dielectric constant material selected was Alumina $\left(\mathrm{Al}_{2} \mathrm{O}_{3}\right)$ of $99.9 \%$ purity. Alumina has a very low absorption of $140 \mathrm{GHz}$ microwaves $(\alpha=.05 \mathrm{~Np} / \mathrm{cm})$. Again, being a ceramic material, it has very good thermal and vacuum properties. Figure A.2.3-7 shows the dielectric stack used to minimize the reflectance of microwaves. The microwaves incident from the plasma must first pass through a tile of Alumina, a vacuum layer and then into the silicon carbide to be absorbed. Figure A.2.3-8 shows the reflectance of the dielectric stack for various thicknesses of the vacuum layer and Alumina tile. It can be seen that there is a minimum in reflectances for a vacuum layer of $0.9-1.0 \mathrm{~mm}$ and a tile thickness of approximately $1.0 \mathrm{~mm}$. The thickness picked for the Alumina are $0.985 \mathrm{~mm}$ and for the vacuum layer $.960 \mathrm{~mm}$. Figure A.2.3-9 shows the reflectance of microwaves as a function of their angle of incidence. It can be seen that the reflectance for both the $P$ and $S$ polarizations is small $(<10 \%)$ for angles of incidence less then 20 degrees from normal. Also shown is the reflectance resulting from a SiC tile with no dielectric stack. It can be seen that the dielectric stack reduces the reflectance substantially for small angles from normal incidence. The addition of the dielectric stack substantially increases the amount of microwave energy absorbed in the silicon carbide tile. Figure A.2.3-10 shows that over $90 \%$ of the incident microwave energy is absorbed in the silicon carbide tile when the dielectric stack is present.

The dielectric stack serves another function besides minimizing reflectance of the microwaves. It also shields the silicon carbide tiles from plasma bombardment and from radiation emanating from the plasma. The energy load on the walls from these two factors is much larger then that expected from the microwaves. Assuming an ohmic heating power of $500 \mathrm{~kW}$ for $0.25 \mathrm{sec}$ spread out over the walls of the tokamak yields an energy load of $3 \mathrm{~J} / \mathrm{cm}^{2}$, much larger then the incident microwave energy. 
THERMAL PROPERTIES. The thermal properties of the silicon carbide absorber must be such that the time for heat to diffuse from the front of the tile, where most of the energy will be deposited, to the thermistors must be small compared with the time for temperature irregularities to equilibriate between thermistors. This time must again be small compared with the time it takes for the heat to diffuse out of the tile into the supporting structure.

In order to calculate the thermal response of the tile we start with the heat transfer equation:

$$
\begin{aligned}
& \nabla^{2} \mathrm{~T}=\left(1 / \alpha^{2}\right) \partial \mathrm{T} / \partial \mathrm{t} \\
& \text { where } \\
& \alpha^{2}=k / s p
\end{aligned}
$$

and $T(x, t)$ is the temperature as a function of position and time, $k$ is the thermal conductivity of the silicon carbide, $s$ is the specific heat and $\rho$ is the density.

Solving the heat transfer equation in slab geometry for the time response gives a time constant of

$$
T(x, t)=\Sigma T_{n}(x) \exp \left(-t / \tau_{n}\right)
$$

where

$$
\tau_{n}=\operatorname{spL} L^{2} /\left(\mathrm{kn}^{2} \pi^{2}\right)
$$

In the equation for the time constant $L$ is the length if the slab, and $n$ is the mode number in question. The time for the heat to diffuse through the $0.25 \mathrm{~cm}$ thick tile is $10.5 \mathrm{~ms}$ for the lowest, $n=1$, mode. In addition it takes approximately $20 \mathrm{~ms}$ for the temperature in the thermistor to equilibriate. Thus the total time constant for equilibriation through the tile is $30 \mathrm{~ms}$. The time for the temperature to equilibriate between two thermistors that are spaced $1 \mathrm{~cm}$ along the $9 \mathrm{~cm}$ long tile $(\mathrm{L}=9 \mathrm{~cm}, \mathrm{n}=9)$ is $170 \mathrm{~ms}$. The time for the heat to diffuse out of the tile is given by the lowest mode $(n=1)$ for the tile and is $13.6 \mathrm{sec}$. Thus the time for heat to diffuse through the tile is much shorter the time for the temperature to equilibriate between thermistors which is again much shorter then the time it takes for the heat to diffuse into the surrounding mounting structure. In addition the time constant for the lowest order mode of the tile is much shorter the time between shcts, allowing the temperature to equilibrate in the tile and allowing all of the energy to diffuse out of the tile.

For a slab the energy flux can be related to the temperature rise by the relation:

$$
\Delta E=k \rho \times \Delta T
$$


where $\Delta E$ is the energy flux into the tile, $k$ is the specific heat of silicon carbide, $p$ is the density, $x$ is the thickness of the tile and $\Delta T$ is the temperature rise of the tile.

The temperature rise of the silicon carbide tiles is measured with negative temperature coefficient thermistors. The thermistors used must withstand the ultra-high vacuum conditions present in MTX and must accurately measure the temperature with a minimum perturbation of the thermal properties of the tiles. The thermistors chosen were $0.041 \mathrm{~cm}$ diameter ruggedized thermistors. These thermistors are glass coated, hermetically sealed and stabilized. Their maximum allowable temperature is $300^{\circ} \mathrm{C}$, sufficient to allow for baking to reduce their outgassing. The time response of these thermistors to an abrupt change in temperature is $16 \mathrm{~ms}$ and is comparable to the time for energy to diffuse through the silicon carbide tile.

The thermistors are bonded to the silicon carbide tiles with a ceramic cement, consisting largely of powdered $\mathrm{Al}_{2} \mathrm{O}_{3}$ with an inorganic binder. Again the cement was required to be ultra-high vacuum compatible, but since only minute quantities were required to attach the thermistors to the silicon carbide tile (a drop $0.051 \mathrm{~cm}$ diameter) this was not as urgent a requirement. The only significant outgassing product, besides $\mathrm{H}_{2} \mathrm{O}$ which can be baked out, was $\mathrm{SO}_{2}$. The outgassing rate of $\mathrm{SO}_{2}$ as a function of temperature is shown in Fig. A.3.2-11. Below $500^{\circ} \mathrm{C}$ the outgassing rate of the ceramic cement is less then $1 \times 10^{6}$ $\mathrm{cm}^{3} / \mathrm{min}$ at STP per gram of cement. For beads of cement $0.051 \mathrm{~cm}$ in diameter, this corresponds to an outgassing rate of $3.32 \times 10^{10} \mathrm{Torr} 1 / \mathrm{sec}^{-\mathrm{cm}^{2}}$. It was decided to add a $15000 \AA$ layer of $\mathrm{Al}_{2} \mathrm{O}_{3}$ between the grounded copper backplane and the thermistors in order to prevent any shorting of the thermistor leads.

The tiles are mounted in a stainless steel (type 304) frame, a cross section of which is shown in Fig. A.2.3-7. The frame consists of 3 hinged sections in order to facilitate insertion into the machine through a port. All parts that are in contact with the plasma are made out of arc cast moly in order to minimize impurity influx into the plasma. There is a copper braid mounted between the frame and the wall of the tokamak in order to aid in the heat transfer from the frame into the wall of the tokamak. The frame has a heater mounted to it in order to keep the tile temperature at a preset value. The location of the thermistors on the calorimeter is shown in Fig. A.2.3-12.

A.2.3.3. Electrical Characteristics and Electronics. The thermistors used as temperature sensors have a resistance that varies nearly exponentially with temperature. The temperature of the thermistor as a function of its resistance is given by:

$$
T=a_{0}+a_{1} \ln (R)+a_{2}(\ln (R))^{3}
$$

where $\mathbf{R}$ is the resistance of the thermistor, $\mathrm{a}_{0}, \mathrm{a}_{1}$ and $\mathrm{a}_{2}$ are calibration constants of the thermistor and $\mathrm{T}$ is the temperature of the thermistor. These constants are 
determined by measuring the resistance of the thermistors at three known temperatures, in this case $40^{\circ} \mathrm{C},-60^{\circ} \mathrm{C}$ and $-80^{\circ} \mathrm{C}$. The calibration constants were supplied by the ma: - ffacturer of the thermistors. The thermistors chosen have a nominal resistance of $1300 \Omega$ at $25^{\circ} \mathrm{C}$. At the operating temperature of approximately $-40^{\circ} \mathrm{C}$ the resistance of the thermistor will be approximately 20 $\mathbf{k} \Omega$.

The analog portion of data acquisition is shown in Fig. A.2.3-13. The thermistor is part of a resistive voltage divider circuit powered by separate $1.6 \mathrm{~V}$ mercury oxide batteries, two for each channel. The separate batteries for each channel are to insure maximum isolation from crosstalk between channels. The output of the voltage divider is fed into a unity gain buffer amplifier in order to prevent loading of the voltage divider. The signal from the buffer amplifier is fed into an amplifier with a gain of $10^{\text {in }}$ order to match the signal to the analog to digital converter. The amplifier has two single pole low pass filters, one set at $12.5 \mathrm{~Hz}$ and the other at $50 \mathrm{~Hz}$. These filters were inserted to eliminate aliasing in the analog to digital converter. The output voltage of the analog circuitry below the cutoff of the filters is given by:

$$
V=G\left(R_{1} V_{2}-R V_{1}\right) /\left(R+R_{1}\right)
$$

All of the analog signal conditioning circuitry is powered by a common battery power supply. The resistances of the resistors must be such that the drain on the batteries be low enough that they have a lifetime of at least 6 months. In addition the amount of power dissipated in the thermistor must be small enough to induce negligible self heating. The self heating of a thermistor in a voltage divider circuit is given by:

$$
\Delta T=V^{2} R D /\left(R_{1}+R\right)^{2}
$$

where $\Delta T$ is the self heating of the thermistor, $V$ is the voltage across the bridge, $R$ is the resistance of the thermistor, $R_{1}$ is the resistance of the reference leg of the voltage divider and $\mathrm{D}$ is the dissipation constant of the thermistor $\left(1670.0^{\circ} \mathrm{C} / \mathrm{W}\right)$. The resistances of the thermistor and the reference leg of the voltage divider should be equal in order to maximize the output of the voltage divider to a change in temperature. Thus the resistance of the reference leg was chosen to be $20 \mathrm{~K} \Omega$ Figure A.2.3-14 shows that the self heating of the thermistors will be less then $0.03^{\circ} \mathrm{C}$ for all temperatures at which the calorimeter is expected to operate. This is not negligible compared to the expected temperature rises dut to FEL pulses, but will not interfere with measurements since the self heating is essentially the same for the temperatures expected before and after the absorption of the FEL pulse. Since the energy absorbed is proportional to the change in temperature the self heating will subtract out.

The signals from the 48 analog data channels are fed into a battery powered digital card where the data is digitized and sent to a HP desktop computer in the control room. The 48 channels of analog data are time multiplexed into a single 
12 bit analog to digital converter. The dynamic range of the converter is -5.0 to $+5.0 \mathrm{~V}$, allowing the thermistors to operate between $-25^{\circ} \mathrm{C}$ and $-50^{\circ} \mathrm{C}$. Each channel is sampled at a rate of $26.18 \mathrm{~Hz}$. The output of the analog to digital converter is broken into a pair of 8 bit bytes to be serialized by a UART and sent to a HP series 200 computer via an optic fiber line at a rate of $28.8 \mathrm{~K}$ baud. The 4 higher order bits are transmitted first, followed by the 8 lowest order bits. Before the data is transmitted to the HP computer it is framed, each frame consisting of 2 start characters, 96 data bytes and 2 stop characters giving 100 bytes transmitted per frame. A data frame has the form:

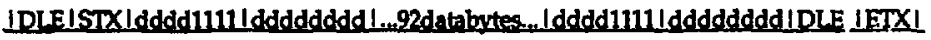

where DLE,STX are the start characters and DLE,ETX are the stop characters .

Data acquisition is triggered at $\mathbf{- 5}$ seconds via a pulse from the sequencer that is transmitted over an optic fiber line. A total of 256 frames are transmitted covering the time period from -5 to +5 seconds .

All of the electronics associated with analog signal conditioning and the digital card float at machine ground. Electrical isolation from the HP desktop computer is assured through use of an optic fiber link. Isolation from ground is assured with the use of isolation transformers for the battery charging circuitry and the use of breaker relays that turn off the battery chargers from 50 seconds before the shot to 15 seconds after the shot.

The heater mounied on the calorimeter frame has a power capacity of $400 \mathrm{~W}$ at $120 \mathrm{~V}$. The heater is controlled by an on/off controller with an adjustable setpoint. The temperature of the frame is monitored with 3 RTD temperature sensors, one on each section of the calorimeter frame. The voltage supplied to the heater is continuously adjustable with a variable transformer. As with the battery charging supplies, the heater is turned off and electrically isolated from 50 seconds before the shot until 15 seconds after the shot. The tile tempera'rure must be kept above $-100^{\circ} \mathrm{C}$ in order to keep thermistor resistance below $1 \mathrm{MQ}$ where noise pickup will become a problem. For normal operation with the calorimeter operating at $-40^{\circ} \mathrm{C}$, approximately 30 volts is supplied to the heater with a duty cycle of approximately $20 \%$. Thus the heating power is $25 \mathrm{~W}$ with an average power of approximately $5 \mathrm{~W}$.

A.2.3.4. Software. The data analysis software consists of two major sections, running on separate computers. The first part of the software runs on a HP series 200 computer. The HP computer is responsible for acquiring the data from the diagnostic, storing it on the VAX and on the SRM computer for insertion into the database and archival and doing a preliminary data analysis. The second part of the software runs under IDL on the VAX and calculates the spatial profiles of the energy incident upon the calorimeter and calculates the total energy incident on the calorimeter. 
DATA ACQUISITION SOFTWARE ON HP SERIES 200 COMPUTER. The software running on the HP computer acquires the data as it is being sent from the digital card at a data rate of $28.8 \mathrm{~K}$ baud. After acquisition, the data is unpacked so that the 12 bits of data for each data word are stored in a 16 bit integer. Thus the data is converted from the form:

\section{dddd1111 I dddddddd}

to

\section{o000dddd $\perp$ dddddddd}

The data is then checked to see if any of the channels saturated the digitizer, and if so notifies the operator of the HP computer. The raw data is stored cn the SRM computer for archiving in case the other data records are lost. The software calculates the time history of the temperature for each of the 48 channels. In order to accomplish this, the data word must be converted to a voltage from which the resistance of the thermistor can be calculated. From a knowledge of the thermistor resistance, the temperature can be calculated. In order to calculate the temperature accurately the battery voltages for each channel, the resistance of the reference resistor of the voltage divider, the gain of the analog board and the calibration constants for each thermistor are included for each channel. Once the temperature for each channel has been calculated, it is transmitted to the VAX for insertion into the MDS database system. Three signals are inserted into the database system. The first signal, CALR_PARAMS, contains 5 numbers: the first frame stored, the number of frames stored, the number of words in a frame and the voits per count of the digitizer. The second signal to be stored, CALR_TIME, contains the times of the data. It consists of a one dimensional array with a length of the number of frames times the number of words. The third signal stored in MDS, CALR_TEMP, contains the temperature, in degrees Celsius, of the thermistors at times CALR_TIME. In addition to sending the data to the VAX and to the SRM the data may also be stored on the loral disk, for an independent operation of the system. Additionally the temperature of up to 4 channels can be plotted in real time and printed locally.

DATA ANALYSIS SOFTWARE ON THE VAX COMPUTER. The main data analysis program on the VAX coinputer is an IDL program called CALOR_ENERG $Y$ that takes the data inserted into the MDS database system by the HP series 200 computer and calculates the spatial profile of the energy absorbed. In addition the total energy absorbed by the calorimeter can be calculated. The program caiculates the temperature rise of each channel by taking the average temperature before the shot and subtracting from the average temperature after the shot. The times before and after the shot are user specified. A baseline shot can be subtracted from the data if required. For both the data and baselines more then one shot may be specified to average over. The baseline shot(s) should be of the same type as the data shot(s), except it should not have 
the FEL data. The temperature rise is then converted to an energy flux into the tile. The program allows the user to specify data channels that should be excluded from the analysis. After the temperature rise is calculated for all channels, a surface spline is performed on the data in order to generate a constant grid. This data can then be contour or surface plotted. The program also calculates the total energy absorbed by the calorimeter by performing a surface integration on the splined data. Figure A.2.3-15 shows a contour plot of a representative plasma shot with a nonplasma shot being used as a baseline. Thus the contours show heating by the plasma discharge. The contour plot shows the energy gain of $0.15 \mathrm{~J} / \mathrm{cm}^{2}$ using 0.1 second averazes 2 seconds before the shot and 2 seconds after the shot. The energy gain is highest at the edge of the calorimeter, indicating that the ene $;$ is diffusing in from the frame. Figure A.2.3-16 shows the same data displayed as a surface plot. Typical contour and surface plots from FEL shots without plasma ("vacuum") and with plasma are shown in Fig. A.3.2-17a,b and A.3.2-18a,b respectively.

Two other programs are available in the same directory. The first, PLOT_CHANNEL, will plot the time history of the temperature of a user specitied channel. The other program PLOT_CHANNEL_BL will plot the time history of the temperature of a user specified channel after a baseline shot has been subtracted. Figure A.3.2-19 shows the temperature rise of channel 1 of a plasma discharge shot, while Fig. A.3.2-20 shows the temperature rise of a none plasma shot. It can be seen that the nonplasma shot has a bump in the temperature while the magnets are turned on.'This bump is aiso evident in the plasma i. Figure A.3.2-21 shows the difference of the two signals, showing only the plasma heating.

- Present address: Department of Ergineering, Highlands University, Las Vegas, NM 87701

1. M. Marinak, "Design of a Microwave Calorimeter for the Microwave Tokamak Experiment", UCID-21808 (October 7, 1988). 
Fig. A. 2.3-1

Figure A.2.3-1 Hexaloy SA grade SiC

PRAMETER

Composition

Density

Hardness -Kinoop

Flexural Strength 3 pt.

Fexural Strength 4 pt.

Compressive Strength

Modulus of Elasticity

Weibull Modulus (2 param)

Poisson Radio

Fracture toughness 8 RT double torsion \& SENB

Coefficient of thermal Expansion. RT-700 ${ }^{\circ} \mathrm{C}$

Maximum Service Temperature

Mean Specific heat RT

Thermal Conductivity @ RT

Thermal Conductivity $200^{\circ} \mathrm{C}$ Thermal Conductivity $400^{\circ} \mathrm{C}$

Permeability $8 \mathrm{RT}-1000^{\circ} \mathrm{C}$

Emissivity

Attenuation Coefficient $2140 \mathrm{GHz}$ Dielectric Constant @ $140 \mathrm{GHz}$
UNTTS

$\mathrm{kg} / \mathrm{m}^{3}$

$\mathrm{kg} / \mathrm{mm}^{2}$

$\mathrm{MPa}$

$\mathrm{MPa}$

$\mathrm{MPa}$

GPa

TYPICAL VAUE

$\mathrm{SiC}$

3100

2800

550

460

3900

410

10

0.14

MPa-ml/2

4.60

$10^{6} \mathrm{~mm} / \mathrm{mm}^{\circ} \mathrm{K}$

${ }^{\circ} \mathrm{C}$

4.02

1650

$\mathrm{J} / \mathrm{gm}^{\circ} \mathrm{K}$

$\mathrm{W} / \mathrm{m}^{\circ} \mathrm{K}$

$\mathrm{W} / \mathrm{m}^{\circ} \mathrm{K}$

$\mathrm{W} / \mathrm{m}^{\circ} \mathrm{K}$

Impervious to gasses

0.67

125.6

102.6

77.5

< $31 \mathrm{MPa}$

$\mathrm{Np} / \mathrm{cm}$

11.1

3.5 
Fig. A.2.3-2

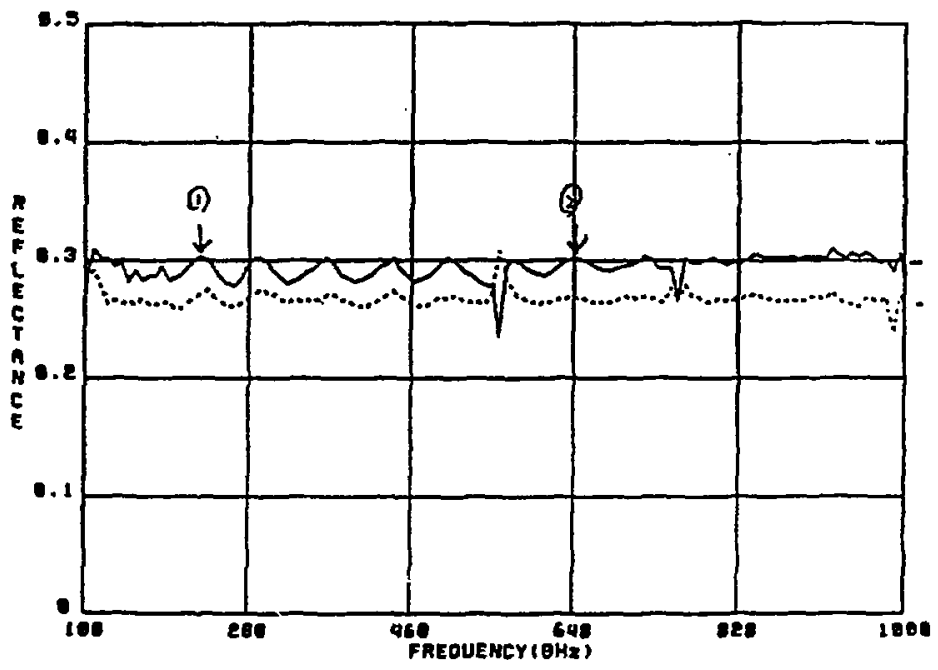


Fig. A.2.3-3

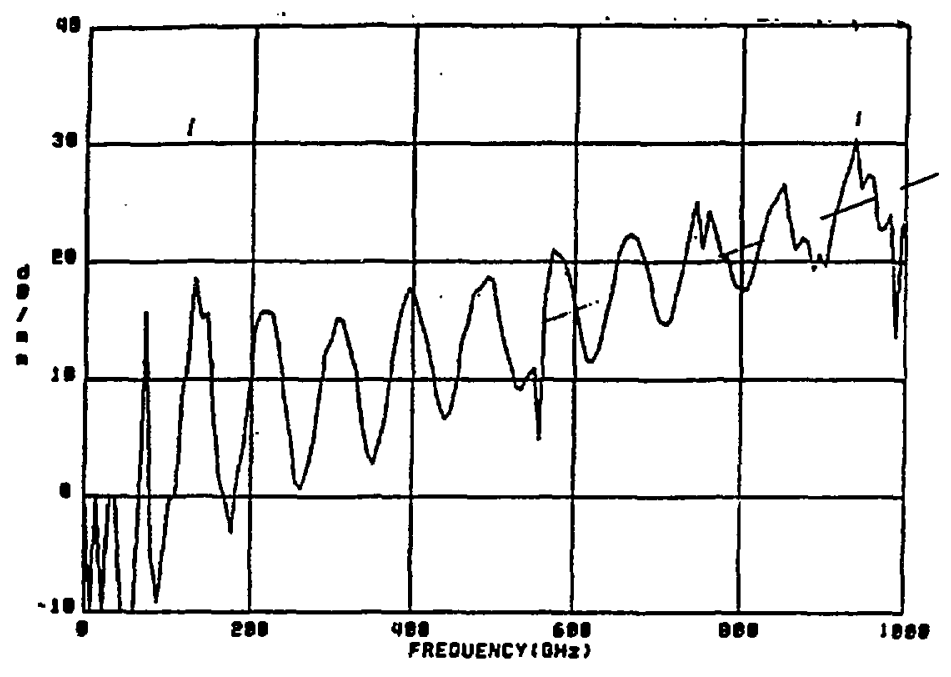

A. 2-13 
Fig. A. 2.3-4

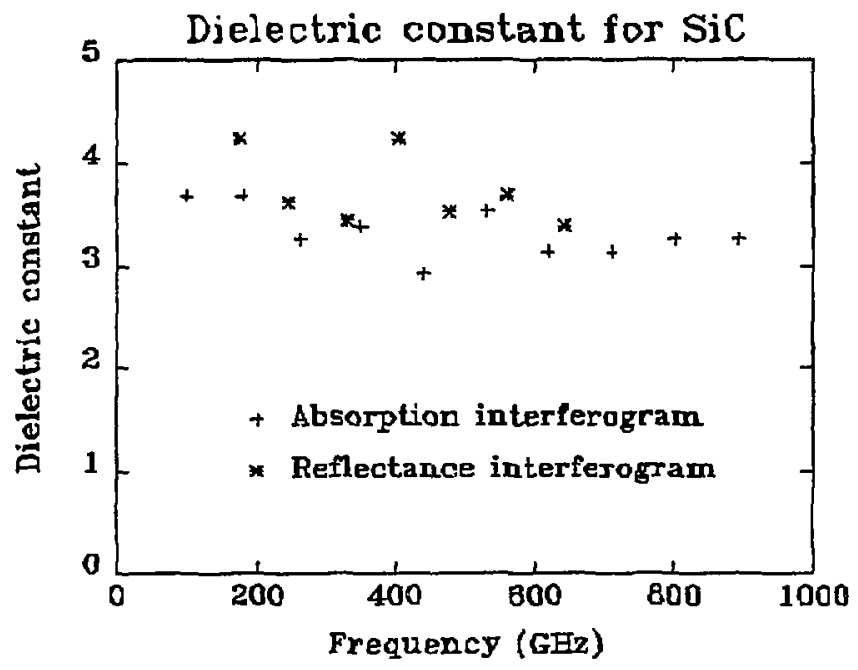

A. 2-14 
Fig. A.2.3-5

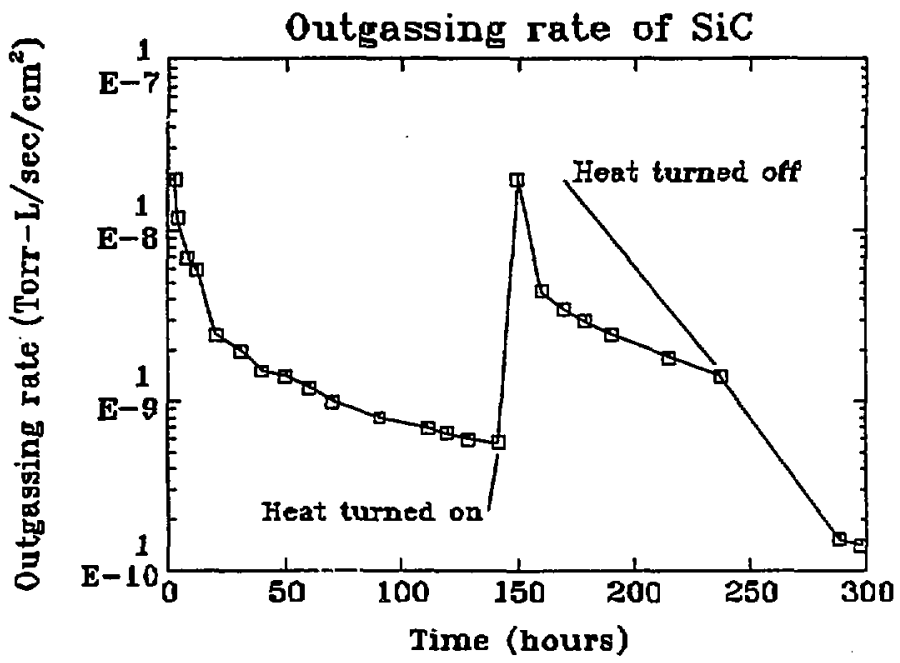

A . 2-15 
Fig. A.2.3-6

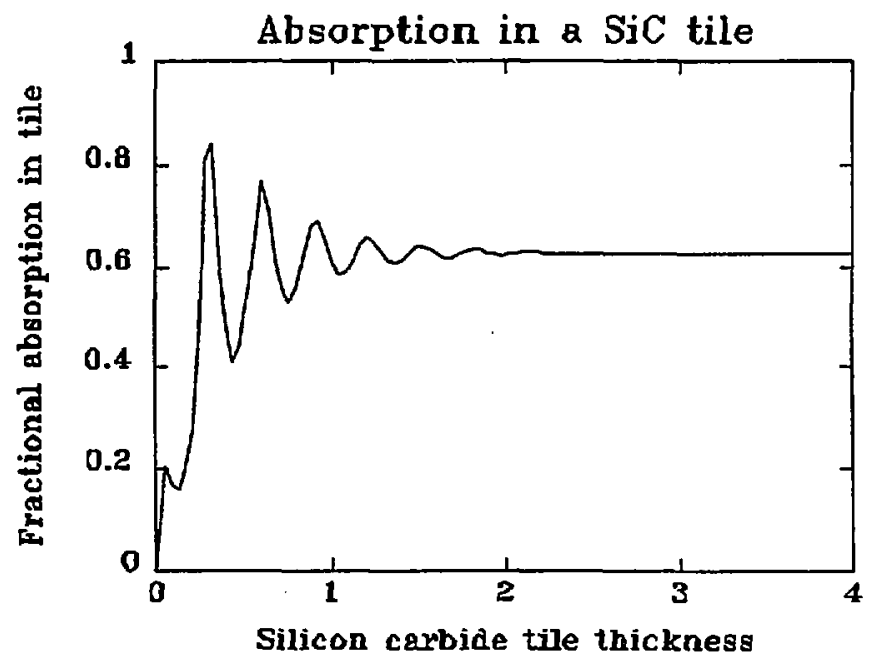

A. $2-16$ 
Fig. A. 2.3-7

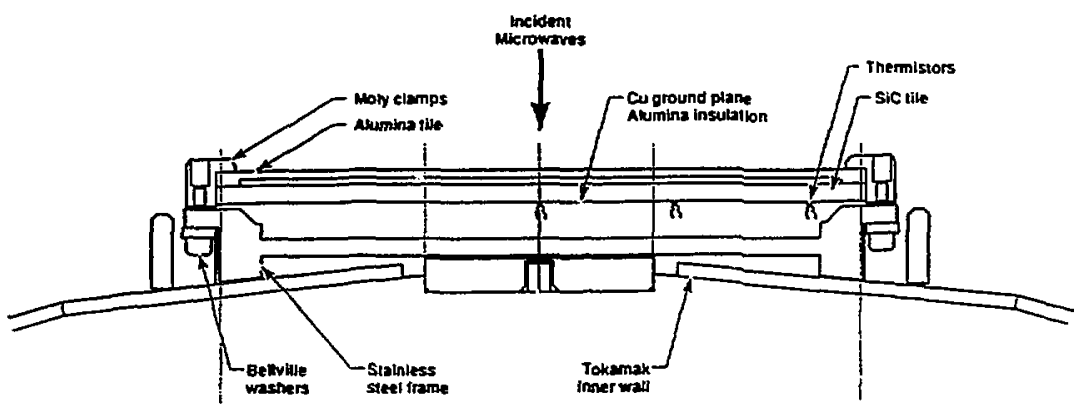


Fig. A. 2.3-8

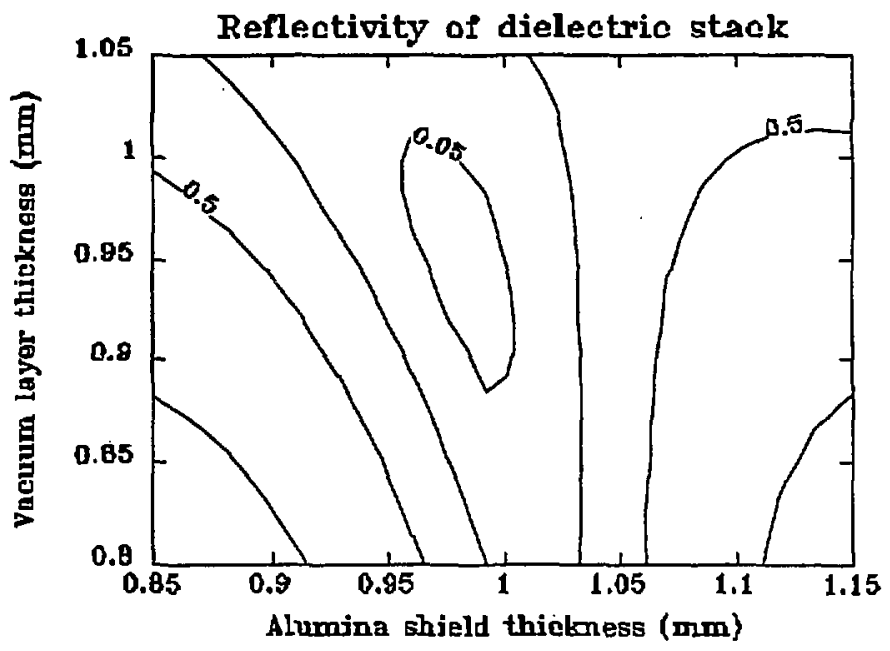




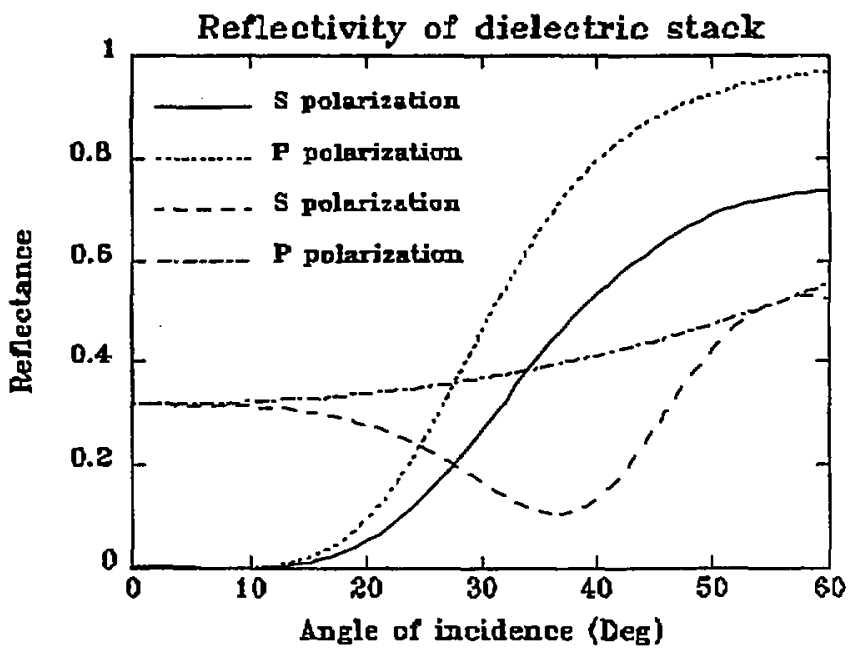

A. 2-19 
Fig. A. 2.3-10

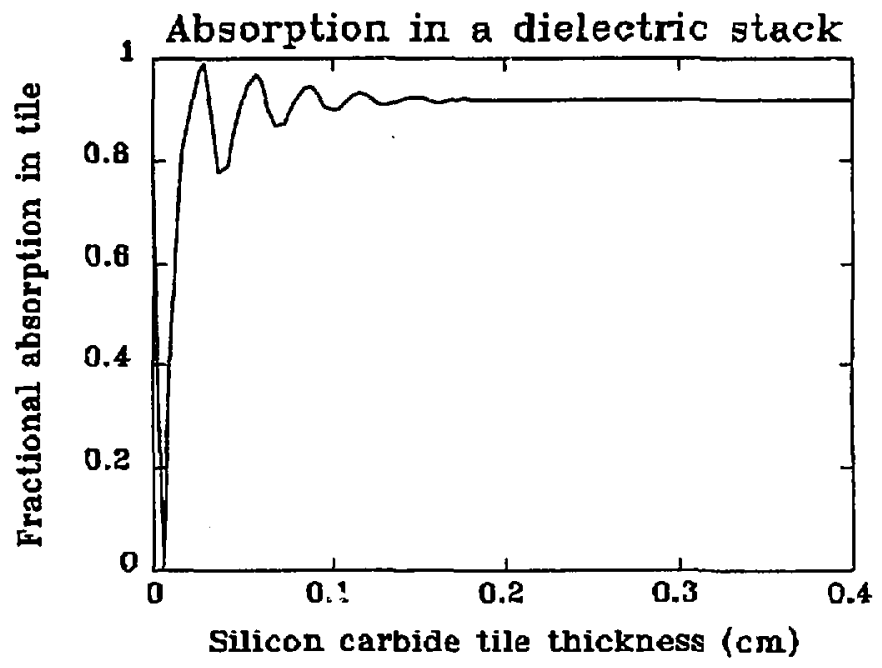


Fig. A.2.3-11

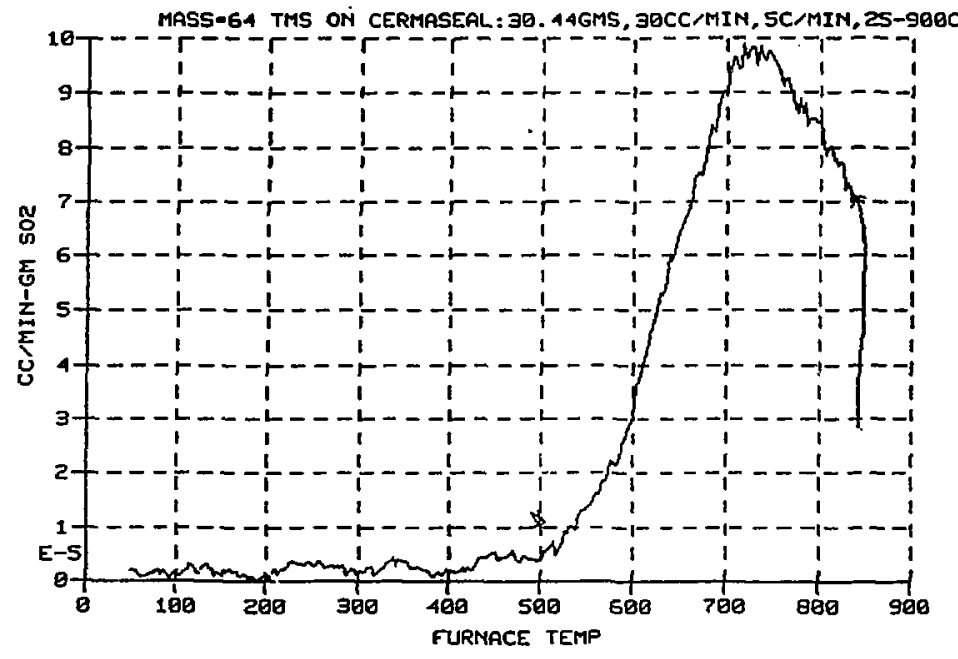


Fig. A. 2.3-12

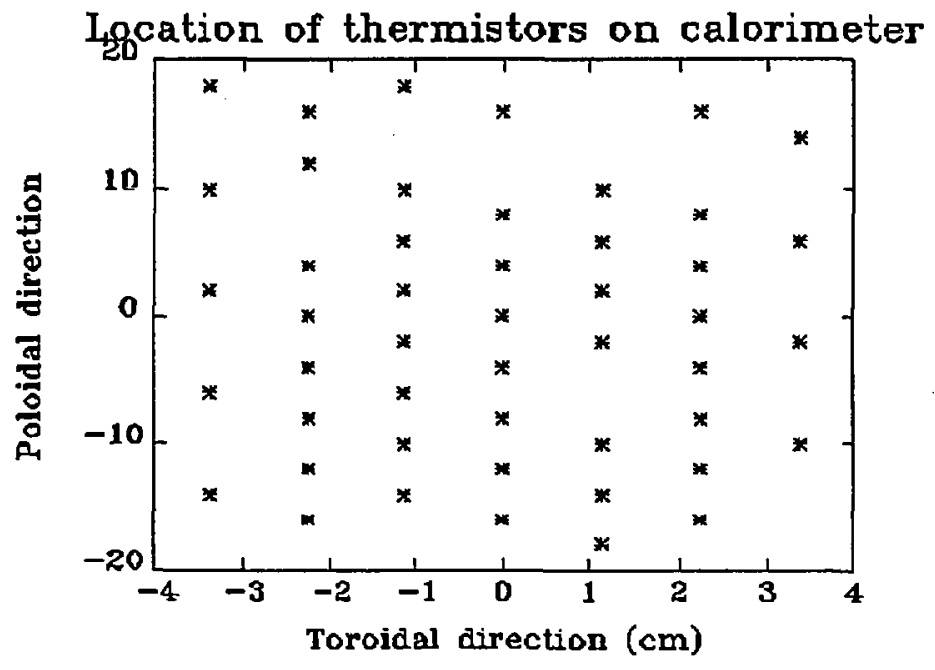

A. 2-22 


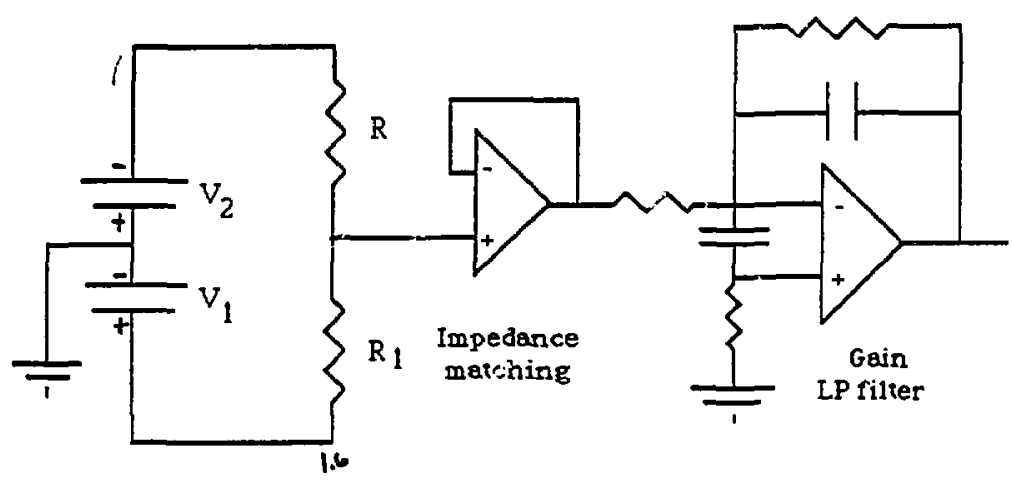


Fig. A. 2. 3-14

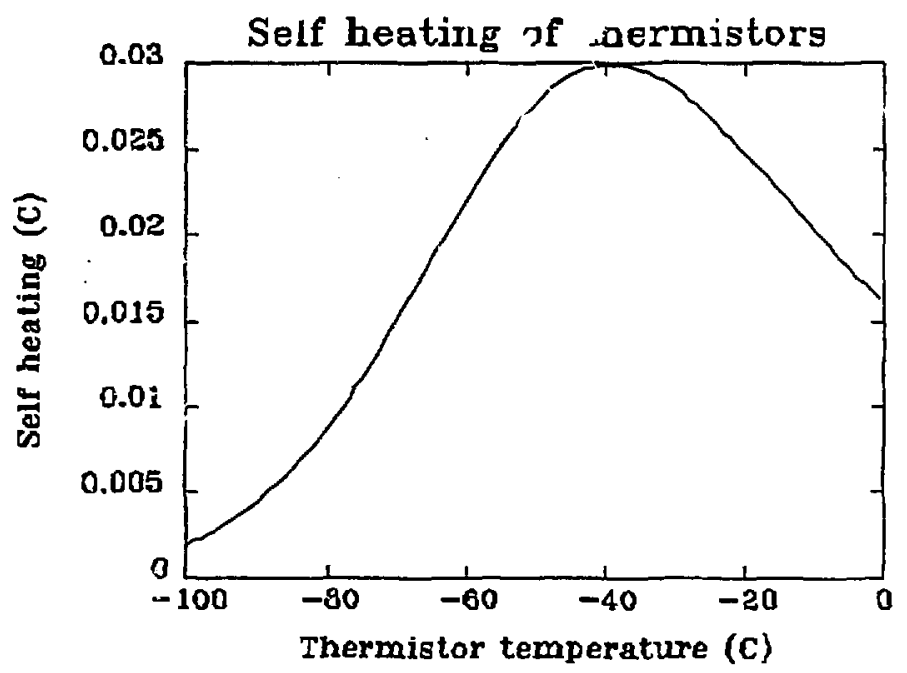




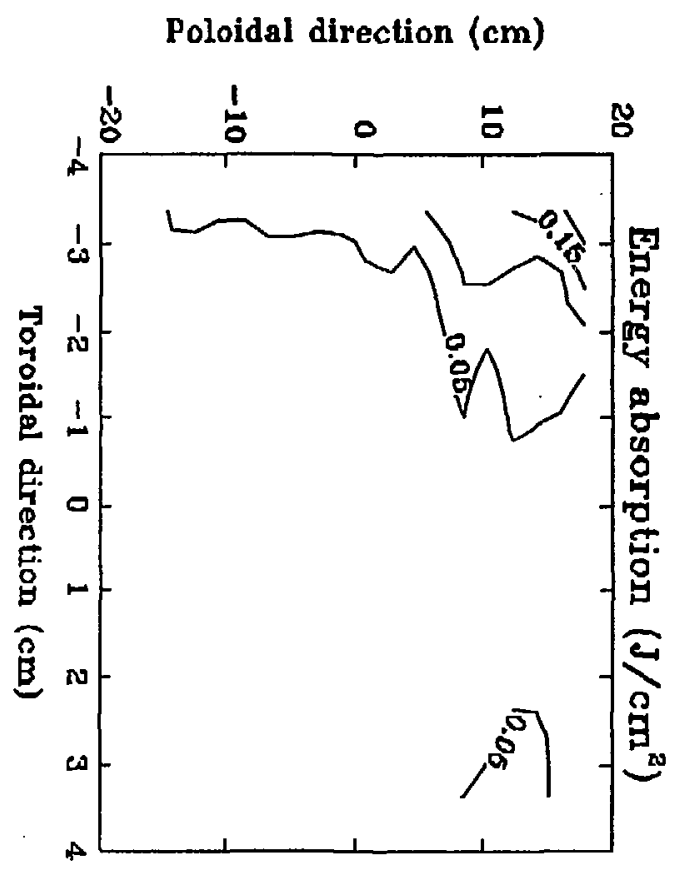


Fig. A. 2.3-16

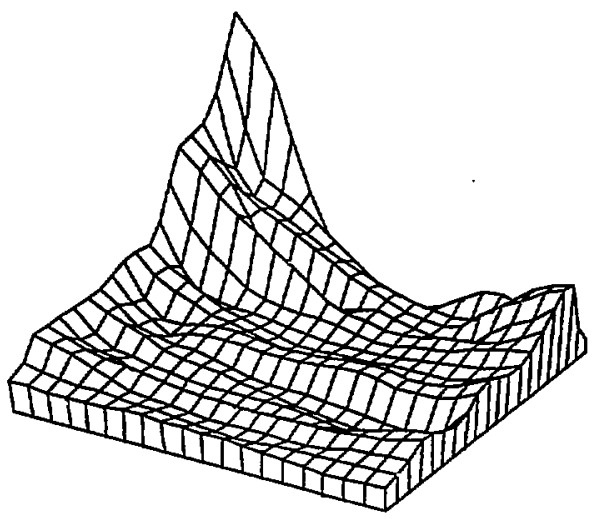




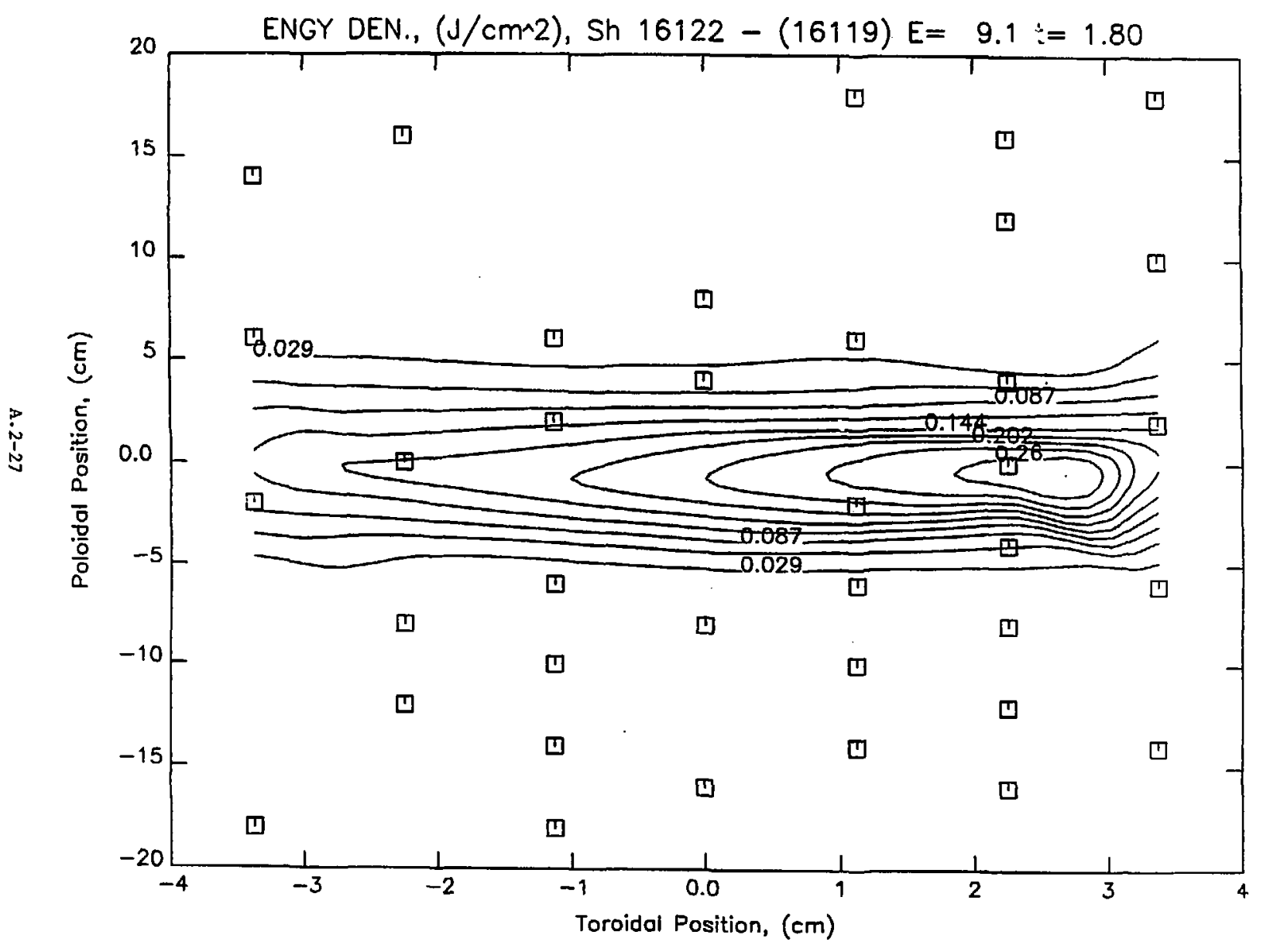


Fig. A. 2.3-17b

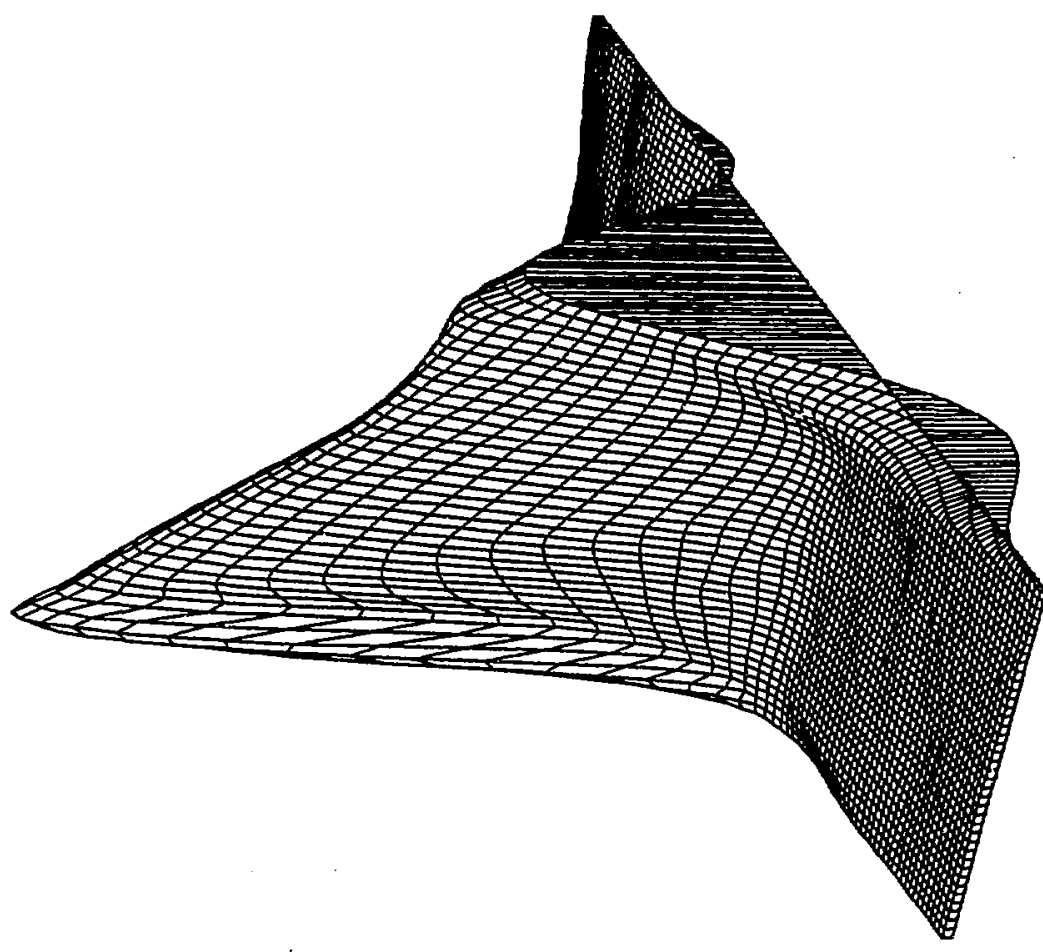




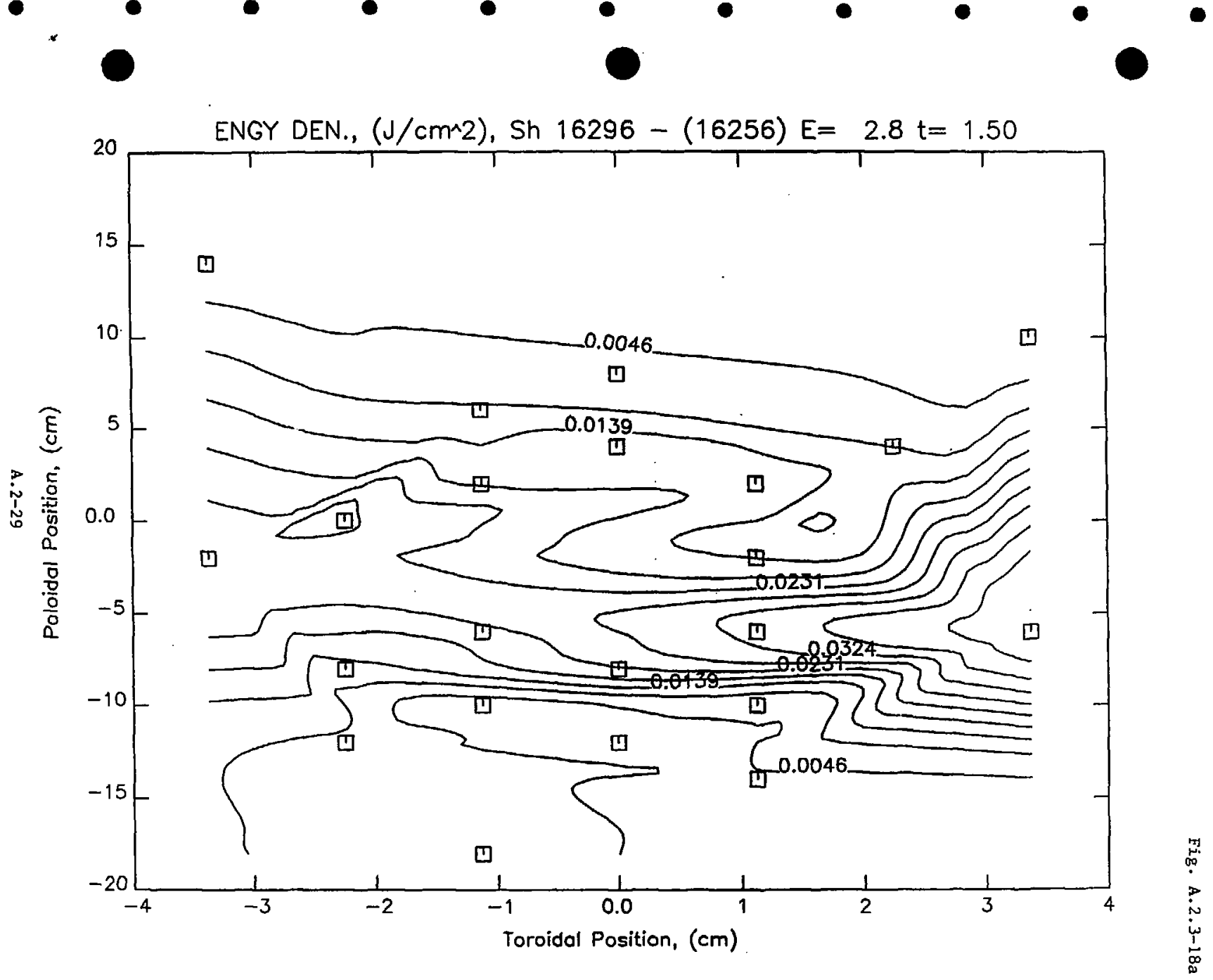


Fig. A. 2.3-18b

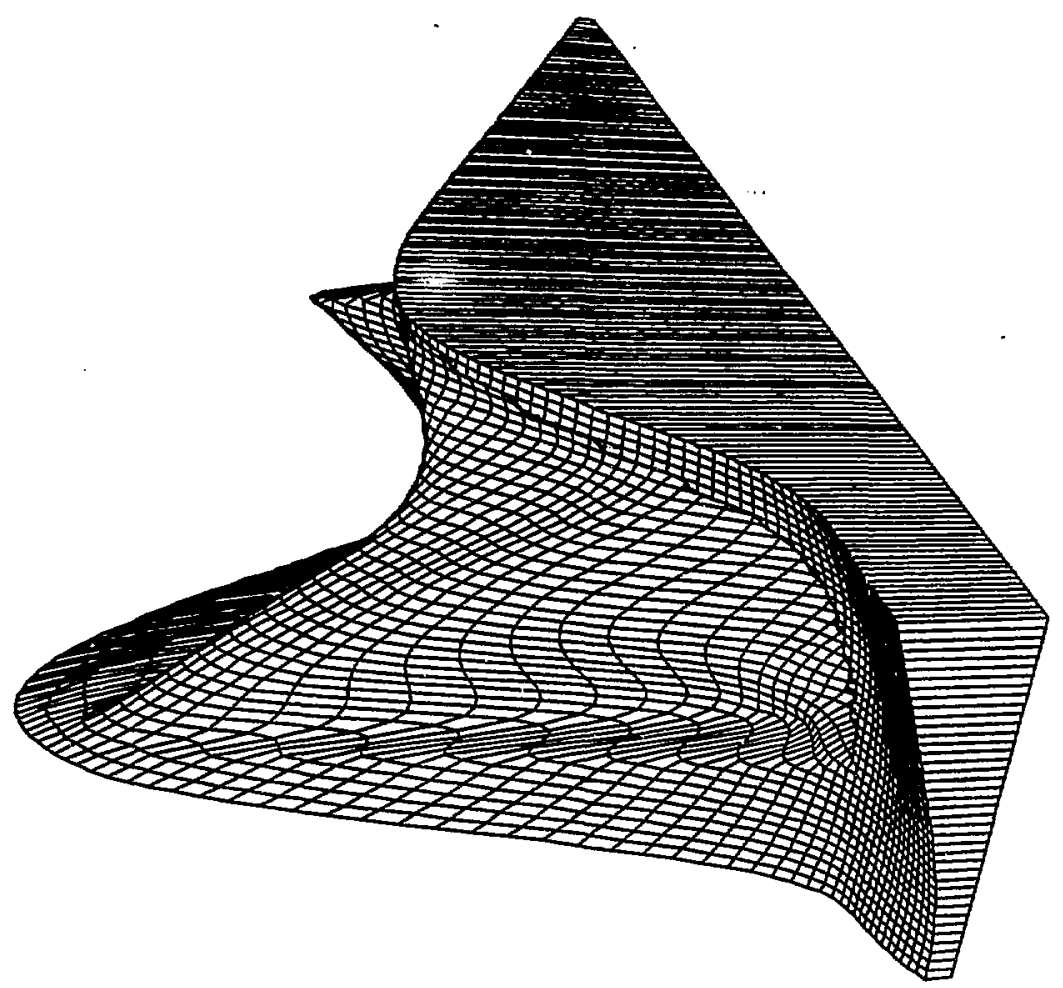


Fig. A. 2.3-19

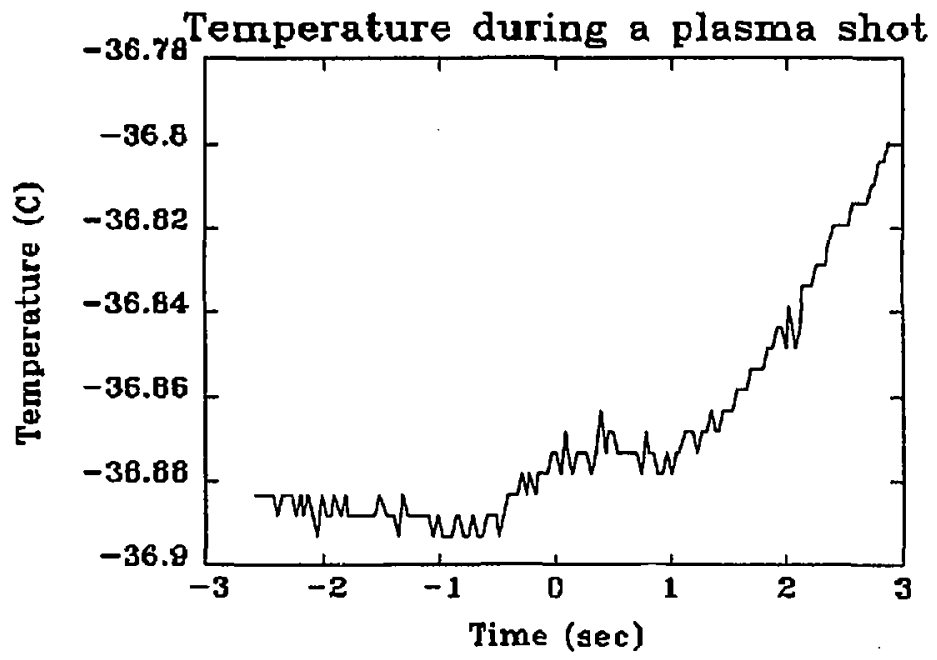


Fig. A. 2. 3-20

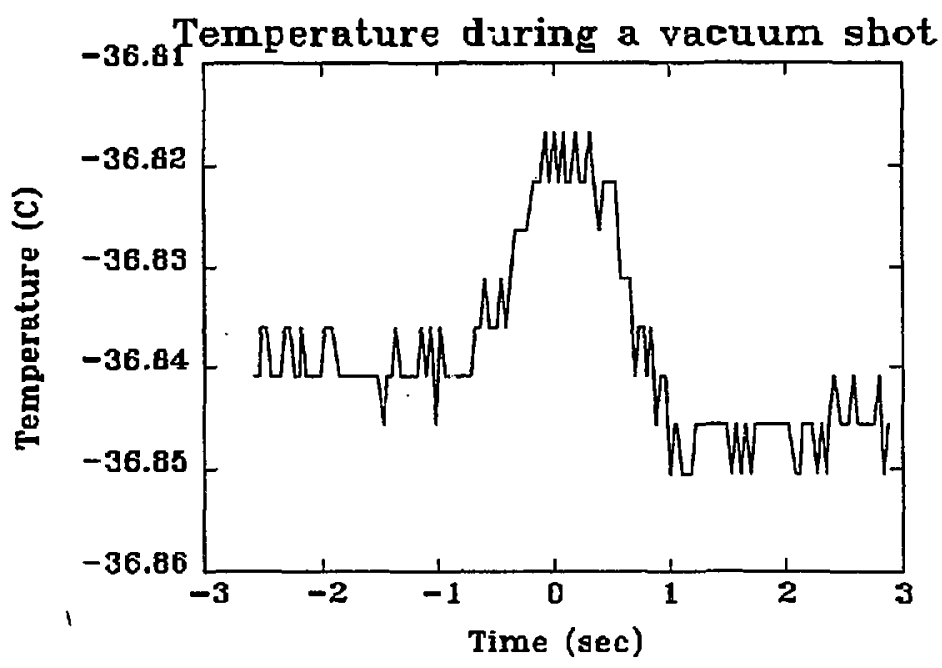




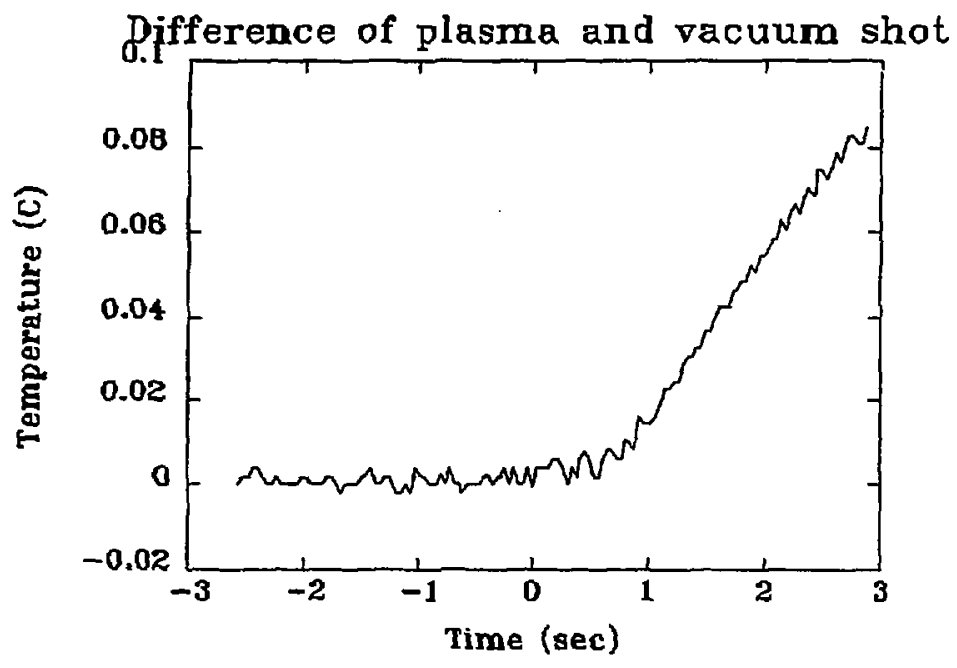




\section{A.2.4 LAPPS (J, H. Foote, T. Oda, K. Odajima, T. Ogawa, T. Ohgo)}

A.2.4.1 Technique. The LAPPS (Laser-Aided Particle Probe Spectroscopy) diagnostic system was designed and constructed to measure the electric field of the FEL when injected into the MTX plasma chamber. The apparatus allowed a crossed-beam measurement, with 2 -cm spatial resolution in the plasma, and included the FEL beam, a neutral-helium beam, and a dye-laser beam.

This approach combines laser-induced-fluorescence spectroscopy with a neutral-helium beam. The dye-laser beam pumps metastable atoms in the helium beam to a higher energy level. The Stark effect arising from the high FEL electric field produces energy-level mixing, causing forbidden transitions to be strongly induced. The intensity of emitted light resulting from a forbidden transition is a measure of the microwave electric field, being proportional to the square of the electric field. An elaborate light collecting and detecting system was necessary to measure the small signal levels expected and to exclude the large background light signals.

A.2.4.2 Equipment. Figure A.2.4-1 shows the relative locations of the LAPPS equipment with respect to the MTX vacuum chamber. We see in this figure the dye-laser beam transport and alignment system, the neutral-helium-beam source, the location of the detection optics, and the incident FEL beam. The meter scale indicates approximate sizes and distances involved.

In Fig. A.2.4-2, we see a more detailed view at the location of the MTX plasma chamber, which shows the FEL beam and the various LAPPS components (laser beam, neutral-helium beam, and the first stage of the lightdetection optics). The aperture at the top defines the viewing area at the center of the vacuum chamber.

To produce our laser beam, we used a Lambda Physik FL3002 dye laser pumped by a Lambda Physik EMG201 MSC excimer laser. The dye solution was QUI mixed in Dioxane. Table 1 lists some measured parameters of the laser beam. We obtained energy measurements of the laser beam using a Gentec, Inc. joulemeter with a pyroelectric ceramic head. The wavelengthscale meter on the dye laser was calibrated daily with a $\mathrm{He}(\mathrm{Li})$ hollow-cathodedischarge tube using the optogalvanic effect (usually for the $2^{3} S$ to $3^{3} P$ transition, with a 388.865-nm wavelength in air).

A series of seven mirrors transported the laser beam from the laser room to the MTX plasma chamber through air (see Fig. A.2.4-1). The position and rotation angle of each mirror were adjustable but, after initial alignment, usually only one mirror had to be fine-adjusted to keep the laser beam aimed at the center of the plasma chamber. Relative motion of the laser beam with respect to the MTX machine during a MTX magnetic-field shot appeared 
negligible. For eye protection from stray laser light, opaque tubing was installed all along the laser path.

To monitor each laser-beam pulse, we installed a Hannamatsu biplanar vacuum phototube near the location where the laser beam entered the MTX vacuum system. It was positioned to the side of the laser beam and was aimed perpendicularly to it at a beam-splitting mirror that reflected a small fraction of the laser light. Neutral-density filters were placed before the phototube to insure that there would not be non-linear saturation effects in the recorded signal.

The neutral helium beam was a major component of the LAPPS diagnostic system. Its apparatus consisted of an ion source and neutralizer, a vacuum-pumping system, Faraday Cup and thermocouple arrays for measuring the beam spatial profile and intensity, and auxiliary equipment. Table 2 gives the basic specifications.

Installed above the viewing-system defining aperture at the top of Fig. A.2.4-2 was the second stage of the viewing optics, shown in Fig. A.2.4-3. At the top of this schematic drawing, we plot the transmission characteristics of the dichroic mirror and the two interference filters used. The PMT1 detector was a 12-stage Hamamatsu R1333 (aiso used for the MTX Thomson scattering diagnostic; $-4 \%$ quantum efficiency at $703 \mathrm{~nm}$ ), while PMT2 was a Thorn EMI 9685R ( $-13 \%$ quantum efficiency at $585 \mathrm{~nm}$ ).

The signals from the two photomultiplier tubes, the laser monitor, and the FEL (when available) were recorded in two Tektronix 2440 highbandwidth digitizing oscilloscopes. From there, we transferred the data to the MTX data base for easy access.

A.2.4.3 Cascade-fluorescence measurements. As a checkout of the LAPPS diagnostic system after installation on MTX, we measured the cascadefluorescence spectroscopic line shown in Fig. A.2.4-4(a). To accomplish this, all systems (neutral-helium beam, laser beam, and viewing optics) had to be operated together successfully with synchronized timing and with the two beams injected into the MTX plasma region (no FEL or plasma present). The viewing optics used to measure the spectral line were the same as those used for the $\mathrm{VXB}$ and FEL electric-field measurements (discussed in Sec. 5.3).

These measurements involved first forming metastable triplet helium atoms [235 level in Fig. A.2.4-4(a)] in the deuterium-gas reutralizer. Then, through an allowed transition, the metastable atoms were excited to a higher energy level ( $\left.3^{3} \mathrm{P}\right)$ with the laser beam by matching the laser wavelength to the transition energy. When matching the wavelength, we tonk into account the Doppler shift caused by the laser and helium beams intersecting at $90^{0}-7.3^{\circ}=82.70$ rather than at $90^{\circ}$ (see Fig. A.2.4-2). Although the energy difference between the $2^{3} \mathrm{~S}$ and $3^{3} \mathrm{P}$ levels corresponds to a wavelength of 
$388.865 \mathrm{~nm}$ in air, we had to adjust the laser wavelength to be shorter by 0.234 $\mathrm{nm}$ to allow for the Doppler shift.

With the viewing syste'n shown in Figs. A.2.4-2 and A.2.4-3, we measured with PMT1 the 706.5-nm light emitted in the $3^{3} \mathrm{~S}$ to $2^{3} \mathrm{P}$ transition of a double-cascade-transition sequence as the atoms in the $3^{3} \mathrm{P}$ excited state decayed through spontaneous emission [see Fig. A.2.4-4(a)]. We could not measure the 388.9-nm resonant-fluorescence light because of the large amount of light at that wavelength scattered when the incoming laser beam hit the inside wall of the MTX vacuum chamber. But, this scattered light was mostly blocked from being detected with PMT1 by the dichroic mirror and interference filters shown in Fig. A.2.4-3. We selected these optical components so that wavelengths only near $703 \mathrm{~nm}$, far from $388.9 \mathrm{~nm}$, could pass. The bandwidth of the interference filter before PMT1 was centered at $703 \mathrm{~nm}$ rather than at $706.5 \mathrm{~nm}$ to allow for the approxirnately $3.0-\mathrm{nm}$ Doppler shift toward shorter wavelengths of the emitted light because of the forward motion of the excited helium atoms.

Figure A.2.4-4(b) shows sample recorded signals. The upper oscilloscope trace is the 706.5-nm cascade-fluorescence signal from PMT1. The lower trace shows the signai from the laser monitor. These signals are averages over eight laser puises. The PMT1 signal is spread out over a longer time than is the laser-moniter signal because of the lifetime of the $3^{3} \mathrm{P}$ state. The relative timing shown for the two signals in Fig. A.2.4-4(b) agrees with expectations after allowing for cable lengths, electronic time constants, and times of flight.

In Fig. A.2.45, we plot the measured PMT1 signal vs the wavelength of the laser beam. We have calibrated the wavelength scale using the 388.865$\mathrm{nm}$ wavelength in air corresponding to the $2^{3} \mathrm{~S}$ to $3^{3} \mathrm{P}$ transition. Each data point is averaged over eight laser pulses. Besides the cascade-fluorescence signal, PMT1 also detected background-light signals arising from scattered laser light and from helium-beam emissions. The scattered laser light probably explains the apparent background level near $200 \mathrm{mV}$-ns under the measured peak (the helium-beam light was subtracted before plotting the data in Fig. A.2.4-5). The 0.46-A FWHM of the peak is about the width expected for the range of Doppler shifts possible because of the spread of angles between the laser and helium beams.

The vertical line at the left shows the calculated laser-wavelength position of the cascade-fluorescence peak signal, taking into account the Doppler shift. The agreement between the calculated position of the peak and the experimentally measured position is good. These cascade-fluorescence results show that the LAPPS diagnostic system was functioning well and as planned. 
A.2.4.4 Further sources of LAPPS information. The LAPPS diagnostic has been extensively reported upon. During the planning stage, an article was published in the Reciew of Scientific Instruments 1 We produced a comprehensive status report during the design period for LAPPS. ${ }^{2}$ Reports have been presented at several conferences. ${ }^{3-10}$ 
Table A.2.4-1 Measured parameters of the LAPPS laser bean.

\begin{tabular}{ll}
\hline Parameter & Value \\
\hline $\begin{array}{l}\text { Energy at dye-laser exit, } 389 \mathrm{~nm}(\mathrm{~m}) \\
(-85 \% \text { of this energy measured at } 381 \mathrm{~nm})\end{array}$ & $\approx 30$ \\
Fraction of energy reaching MTX & $=0.50$ \\
Pulse fs:ll width at 1/e level (ns) & $25-30$ \\
Wavelength FWHM (A) & 0.07 \\
Repetition rate (Hz) & $0-80$ \\
$\begin{array}{l}\text { Polarization at plasma (vertical/horizontal } \\
\text { intensity components) } \\
\text { Beam size at plasma (height/width in num) }\end{array}$ & $4.8 / 1$ \\
\hline
\end{tabular}

Table A.2.4-2. Specifications of the LAPPS helium beam.

\begin{tabular}{ll}
\hline Parameter & Value \\
\hline Ion beam current (A) & 0.8 \\
Beam energy (keV) & $45-50$ \\
Pulse length (ms) & 5 \\
Repetition time (s) & 30 \\
Ion species & Helium \\
Neutralizer gas & Deuterium \\
$\begin{array}{l}\text { Atomic helium } 2^{35} \text { triplet density } \\
\left.\text { at } 45 \text { keV (cm }{ }^{-3}\right)\end{array}$ & $3 \times 10^{6}$ \\
Divergence half angle at 1/e level (deg) & 0.7 \\
\hline
\end{tabular}




\section{FIGURE CAPTIONS}

Fig. A.2.4-1. Schematic drawing showing the relative locations of the LAPPS dye-laser beam transport and alignment system, the neutral-helium-beam source, the detection optios, and the incident FEL beam.

Fig. A.2.42. Schematic drawing showing a detailed view at the MTX plasma chamber, including the various LAPPS components (laser beam, neutralhelium beim, and the first stage of the light-detection optics) and the FEL beam.

Fig. A.2.4. Second stage of the viewing optics, installed above the aperture shown in Fig. A.2.4-2. This schematic drawing also shows the transmission characteristics of the dichroic mirror (DM) and the two interference filters (IF] and IF2). PMT1 and PMT2 are photomultiplier tubes.

Fig. A.2.4. (a) Energy-level and transition diagram pertaining to our cascade-fluorescence measurements. (b) Upper oscilloscope trace is the signal from PMTI (see Fig. A.2.4-3) showing the 706.5-nm cascade-fluorescence signal. The lower trace shows the signal from the laser monitor. These signals are averages over eight laser pulses. Time runs from left to right at 50 ns/division.

Fig. A.24-5. Measured cascade-fluorescence signal vs wavelength of laser beam. The vertical line at the left shows the calculated position of the peak signal. 
1 T. Oda, K. Odajima, K. Mizuno, K. Ohasa, M. Shiho, K. Takiyama, J. H. Foote, and D. G. Nilson, Rev. Sci. Instrum. 61, 2964 (1990).

2 K. Odajima, K. Ohasa, M. Shiho, T. Oda, K. Takiyama, K. Mizuno, J. H. Foote, D. G. Nilson, S. L. Allen, and T. A. Casper, "MTX MicrowaveElectric-Field Diagnostic," Japan Atomic Energy Research Institute, Tokai, Japan, JAERI-M 90-088 (1990).

3 T. Oda, K. Odajima, K. Takiyama, J. H. Foote, K. Mizuno, T. Ogawa, K. Sato, K. Ohasa, K. Hoshino, H. Maeda, and D. G. Nilson, 'Development of a Diagnostic System for High Power Microwave (FEL) Electric Fields in the Microwave Tokamak Experiment," in Proceedings of the 1992 International Conference on Plasma Physics, edited by W. Freysinger, K. Lackner, $\mathbf{R}$. Schritter, and W. Lindinger (Innsbruck, Austria, June 29 - July 3, 1992), Part II, pp. 1191-1194.

4. T. Oda, K. Odajima, J. Foote, K. Mizuno, K. Takiyama, T. Ogawa, K. Ohasa, K. Hoshino, K Sato, and D. Nilson, "Spectroscopic Diagnostics of Tokamak Plasma Heating by High Power Microwave," in Proceedings of the 5th International Symposium on Laser-Aided Plasma Diagnostics, edited by $\mathbf{H}$. F. Dobele (Physikzentrum, Bad-Honnef, Germany, August 19-23, 1991), pp. 168-173.

5. J. H. Foote, T. Oda, K. Odaj̈ma, T. Ogawa, K. Ohasa, T. Ohgo, K. Takiyama, and D. G. Nilson, "Measurements on MTX with the FEL Microwave Electric Field Diagnostic (LAPPS)," Bull. Amer. Phys. Soc. 37, 1423 (1992).

6. K. Odajima, J. H. Foote, T. Oda, K. Takiyama, T. Ogawa, K. Sato, D. G. Nilson, K. Ohasa, K. Hoshino, and K. Mizuno, "FEL Microwave Electric Field Diagnostic for MTX (LAPPS)," Bull. Amer. Phys. Soc. 36, 2314 (1991).

7. J. H. Foote, T. Oda, K. Odajima, K. Mizuno, K. Ohasa, M. Shiho, K. Takiyama, and D. G. Nilson, Measuring High FEL Electric Fields in MTX Using the Stark Effect," Bull. Amer. Phys. Soc. 35, 1950 (1990).

8. K. Mizuno, T. Oda, J. Foote, K. Odajima, K. Ohasa, M. Shiho, K. Takiyama, and D. Nilson, "Development of New Electric Field Diagnostic (LAPPS) in Magnetic Fusion Device," Bull. Amer. Phys. Soc. 35, 2140 (1990).

9. K. Mizuno, J. Foote, T. Oda, K. Odajima, M. Shiho, K Oasa, D. Nilson, K. Takiyama, and Y. Matsucia, "Design of Microwave Electric Field Diagnostics (LAPPS) in MTX Tokamak Plasma," 17th IEEE International Conference on Plasma Science, Oakland, California, May (1990). 
10. T. Oda, K Mizuno, E. B. Hooper, Y. Matsuda, K. Takiyama, K. Kawasaki, K. Odajima, and K. Ohasa, "Electric Field Measurements in Fusion Plasmas," in Proceedings of the 4th International Symposium on Laser-Aided Plasma Diagnostics, edited by M. Akazaki (Fukuoka, Japan, Nov. 20-23, 1989), pp. 165-170. 


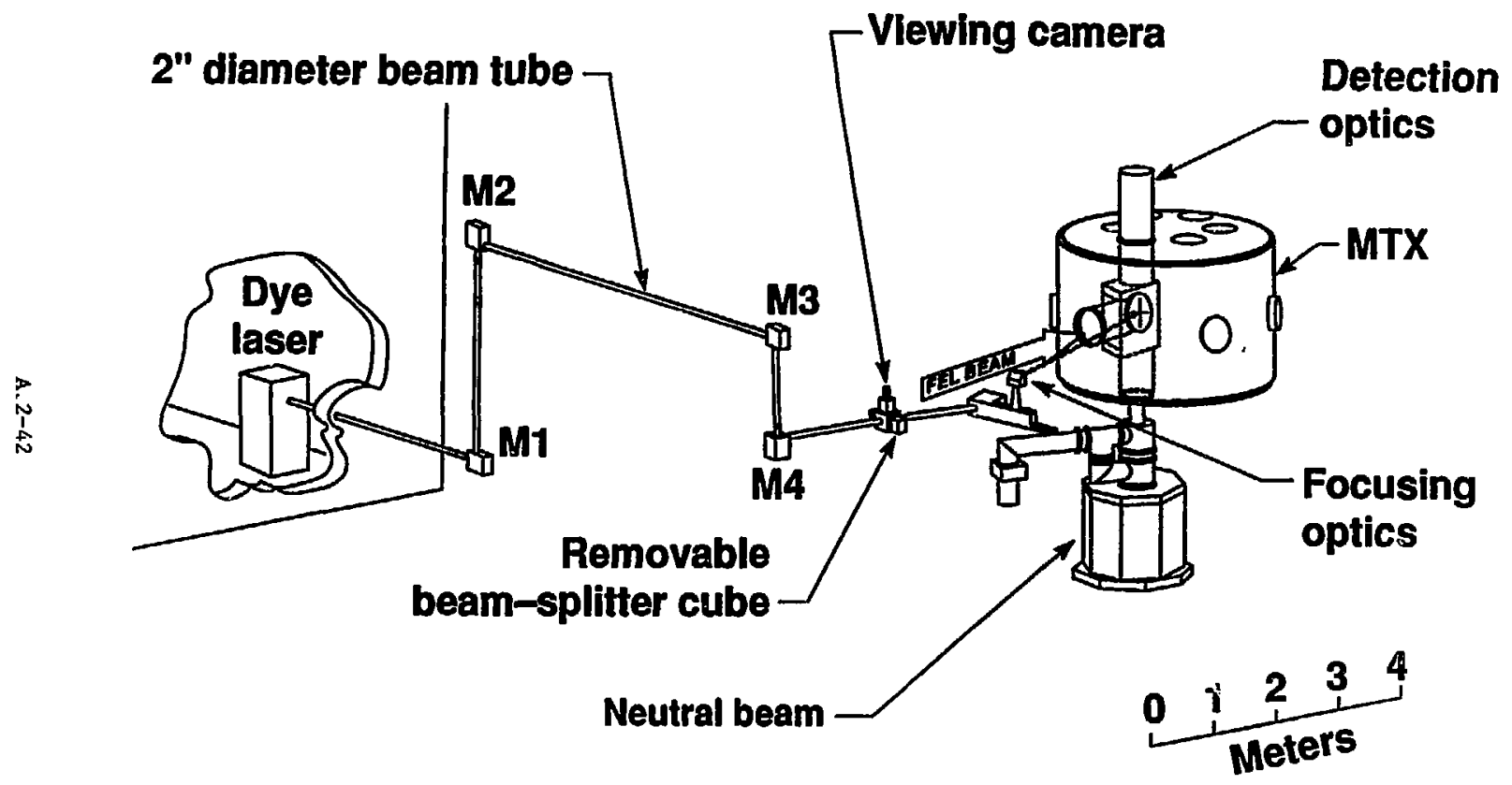

20-A-1292-0063B

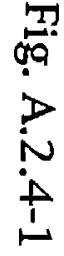


Fig. A.2.4-2

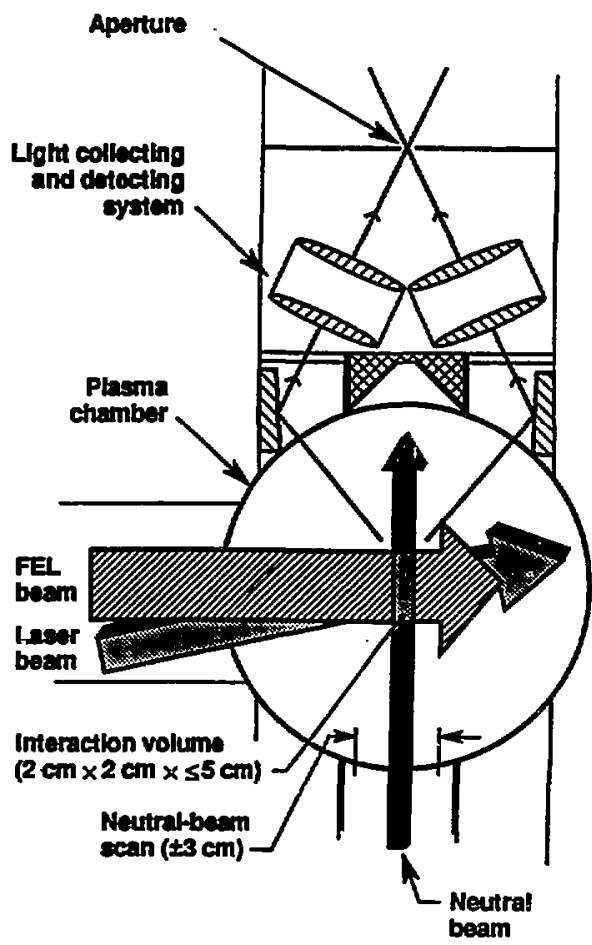


Fig. A.2.4-3
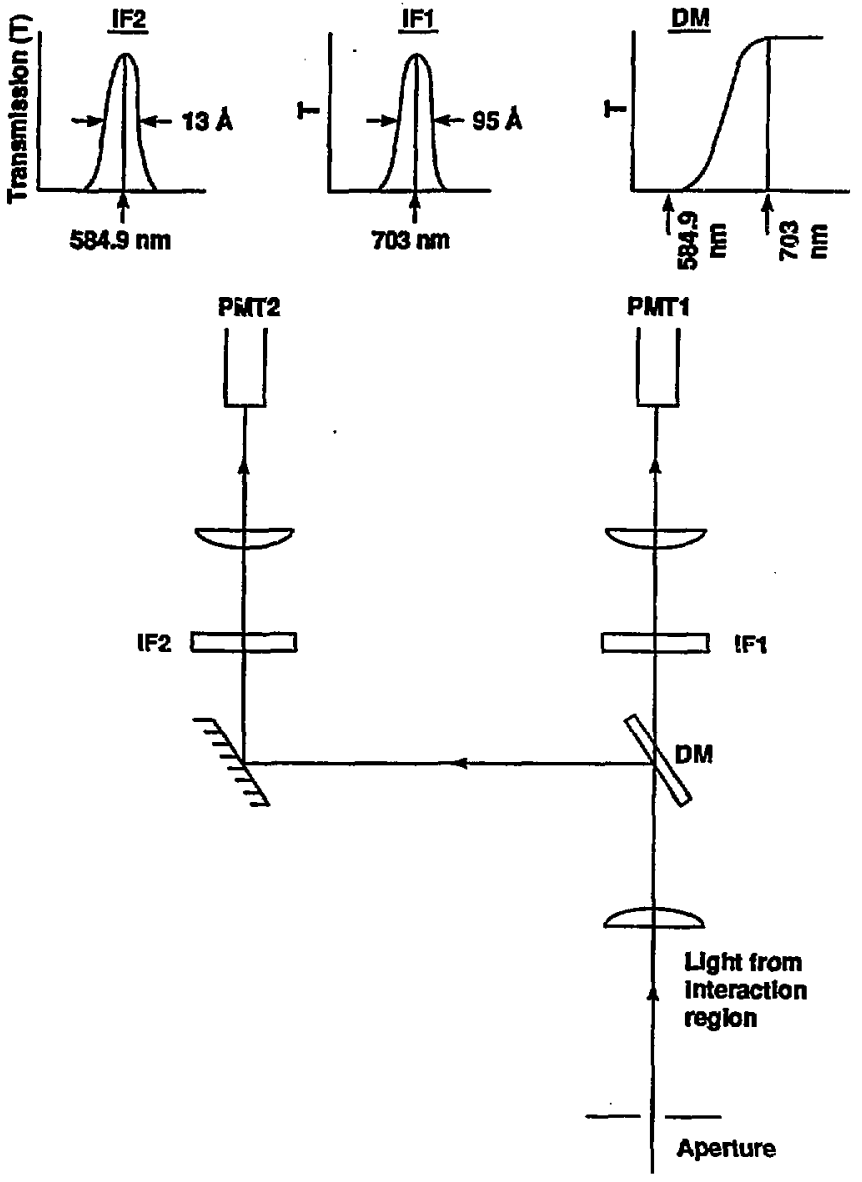
(a)

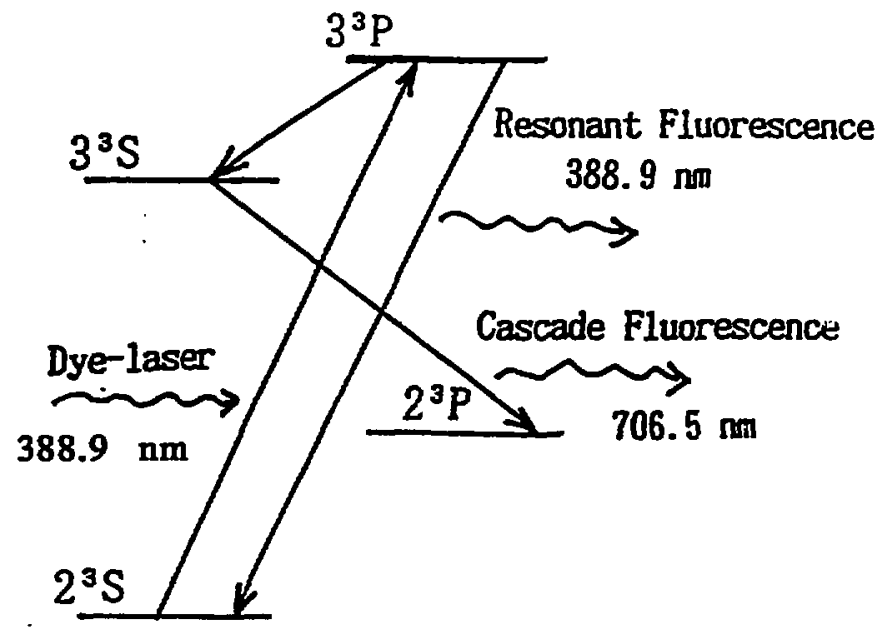

(b)

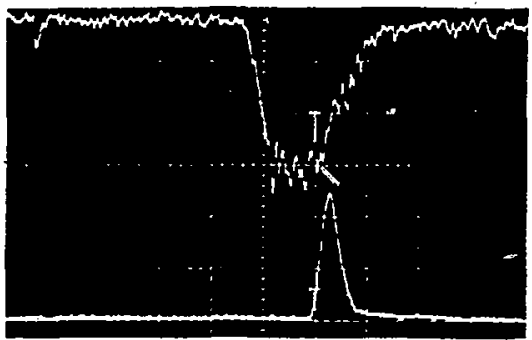




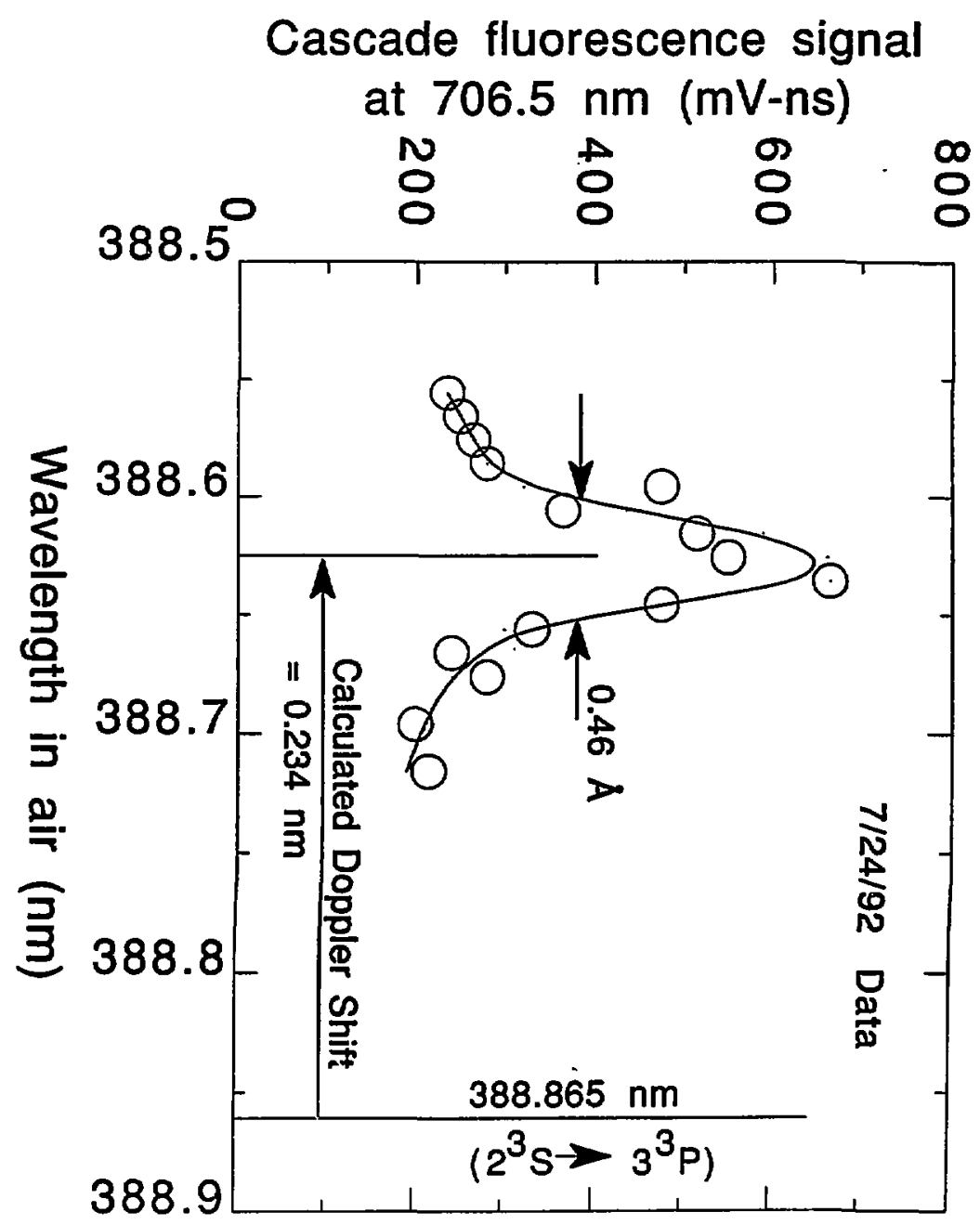




\section{A.3 Diagnostics: plasma}

\section{A.3.1 Thomson scattering (J. H. Foote)}

The Thomson scattering diagnostic system (TSS) on MTX routinely monitored electron temperature and density. Typical measured values and their estimated uncertainties at the plasma center under clean conditions were $900 \pm 70 \mathrm{eV}$ and $1-2 \times 10^{14}( \pm 30 \%) \mathrm{cm}^{-3}$. The TSS apparatus was compact, with all elements mounted on one sturdy, two-level optics table. Because of this, we maintained with minimum effort the alignment of both the rubylaser inpur optics and the scattered-light collecting optics. Undesired background signals, e.g., plasma light as well as ruby-laser light scattered off obstacles and walls, were generally small compared with the Thomson scattered signals we normally detected. In the MTX $T_{e}$ region, the TSS data are definitely fitted better when relativistic effects are included in the equations. The TSS apparatus, data, and method of data analysis are described more completely in a Review of Scientific Instruments article."

Figure A.3.1-1 shows the layout of the TSS apparatus. The laser beam dump consisted of layers of blue-green glass, with one section at the Brewster's angle for maximum absorption of the polarized laser light. The MTX vacuum-chamber configuration allowed only a minimal viewingsystem dump for reducing light reflected into the viewing line. For this purpose, a small blackened curved metallic section was installed on the inside wall of the vacuum chamber. All sections of the laser and viewing paths were in air except inside the spatial filter in the laser beam and inside the MTX vacuum chamber.

Typical reduced-data results are shown in Fig. A.3.1-2. The wavelength shift of the horizontal scale is with respect to the ruby-laser wavelength at 694 nm. Wavelength channel 3 is not useful because of the large scattered laser signal there. The curve is a least-squares fit to a Gaussian with a relativistic correction, with a peak displaced in wavelength from the ruby wavelength to best fit the data and yielding $T_{e}=1206 \pm 68 \mathrm{eV}$.

Besides determining the temperature of the Maxwellian electron distribution, the Thomson scattering system was designed to detect electron heating from GW-level free-electron laser pulses by measuring large wavelength shifts of the scattered laser photons. To make these expanded measurements, we rotated the diffraction grating in the spectrometer to allow wavelengths further than usual from the ruby-laser wavelength to fall on the light pipes leading to the photomultiplier tubes. The results obtained are discussed in Sec 5.4 of this report.

1. J. H. Foote, J. D. Barter, N. R. Sewall, J. J. Jolly, and L. F. Schlander, Rev. Sci. Instrum. 61, 2861 (1990). 


\section{FIGURE CAPTIONS}

Fig. A.3.1-1. Schematic drawing showing the arrangement of the Thomson scattering equipment on MTX.

Fig. A.3.1-2. TSS results (with standard-deviation statistical counting uncertainties) obtained after adjusting the raw data for the gain of each wavelength channel. 


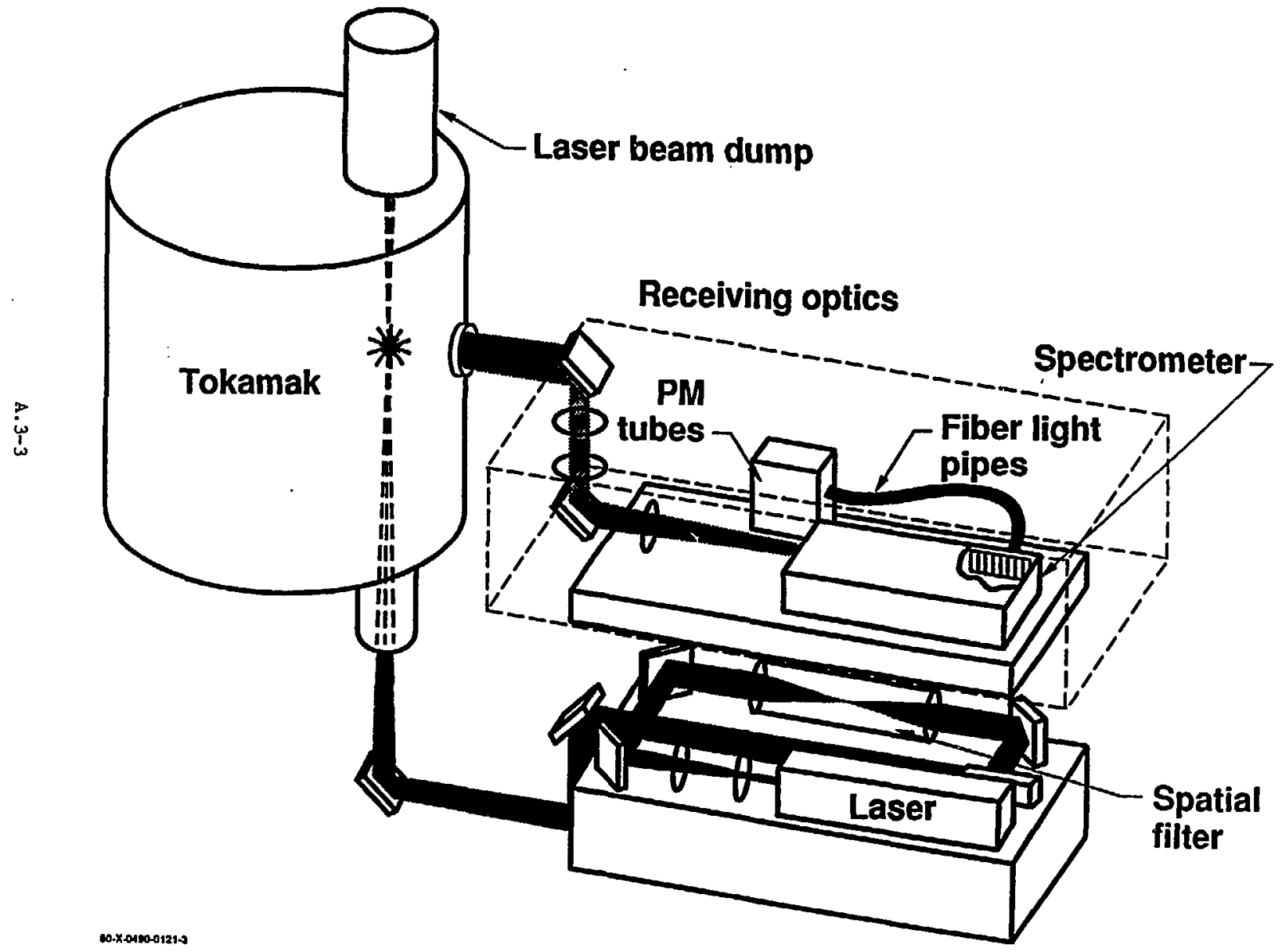


Fig. A.3.1-2

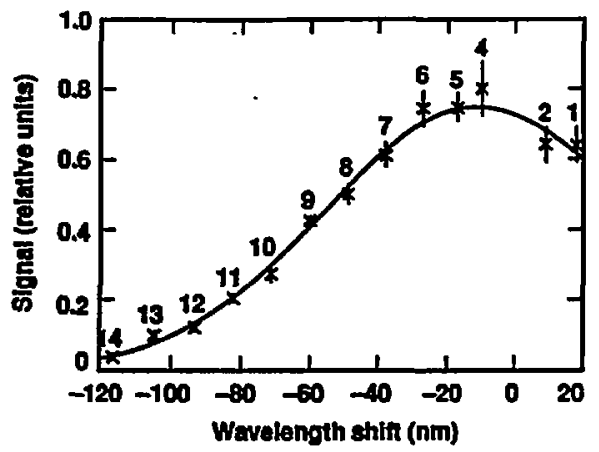

A. 3-4 


\section{A.3.2 Neutrons (T. Ogawa)}

\section{A.3.2.1. Introduction}

Thermonuclear fusion of deuterium is the process of interest here. The reaction $D(D, n){ }^{3}$ He produces a neutron with an energy of $2.45 \mathrm{MeV}$. The reaction rate is a very strong function of $t: 2$ ion temperature. Therefore neutron $x$.easurements have been used to diagnose the ion temperature of deuterium plasmas. We are also interested in the ion behavior in $\mathrm{ECH}$ experiments using intense FEL-produced microwaves. High-speed measurements are important to study the intense microwave absorption process. Wide dynamic range of neutron detection is required for total neutron I ate measurement to evaluate the ion temperature. We have designed i neutron diagnostic for MTX combining two different types of measurenent systems because there is no detector with the ability to provide both high-speed measurements and ion temperature measurements. Table 1 gives $c$ aracteristics of these measurements. One is a conventional neutron production rate measurement system using proportional counters and fission counters to estimate the ion temperatures with moderate time resolution. The system for the high-speed neutron measurement uses a plastic sc .tillator.

\section{A.3.2.2 Nitron production rate measurements}

Neutron production rate measurement systems must have a wide dynamic range for neutron flux detection. Our diagnostic measures the flux of neutrons without any energy discrimination using a polyethylene moderatc ${ }^{\prime}$ which thermalizes fast neutrons to get high detection sensitivity. The assen ibly is capable of detecing neutron flux over a range of five orders of magnitude in pulse count mode.

\section{Neasurement system}

Five neutron detectors of varying sensitivity are used in order to cover the entire range of neutron source strength with good counting statistics. The detectors are listed in Table 2 starting with the most sensitive. The detector $s /$ stem consists of two ${ }^{3} \mathrm{He}$ proportional counters, a $\mathbf{B F}_{3}$ proportional counter, a nd two $235 \mathrm{U}$ fission counters. A set of these detectors is installed in the cer er of a neutron moderator illustrated in. Fig.1. The $1 \mathrm{~mm}$ layer of cadmium strongly absorbs slow neutrons. Higher energy neutrons surviving the cadmium layer pass into a $10 \mathrm{~cm}$ layer of polyethylene on the way to the detectors. In this layer fast neutrons are converted into thermal neutrons. The $5 \mathrm{~cm}$ of lead reduces any hard $\mathrm{x}$-ray roise effect on the 
neutron signal.

Two detector assemblies in neutron moderators are placed beside MTX side ports $(114 \mathrm{~cm}$ from the plasma axis and $86 \mathrm{~cm}$ below the plasma midplane). Figures 2 and 3 show the location of these neutron detectors around the MTX device. The one detector assentbly is in front of port B and the other is port F. There are two fixed limiters inside the MTX vacuum vessel localed at port B and port E. Since one detector assembly (port B) is ciose to a limiter and one detector assembly (port F) is not, the photoneutron rate emitted from the limiters can be estimated from the difference between the counting rate of these two detector assemblies.

An electronic circuit for one detector assembly is illustrated in Fig.4. The electronics are selected by the requurement of high speed and high count rates capability. TC170/171 preamplifier is a fast-rise time charge-sensitive preamplifier. The timing output is differentiated with a $100 \mathrm{~ns}$ time constant. ORTEC 579 is a fast filter amplifier with 5 ns rise time and $250 \mathrm{X}$ gain. The $4608 \mathrm{C}$ is a eight channel high speed discriminator which has 150 MHz count rate capability. And it has three negative outputs per channels. The T8590 scaler has also eight channel inputs. Double scalers for each detector output signal are used for different sampling rate measurements.

Cable shielding and grounding configuration are important to eliminate electric noises from equipment around the MTX tokamak such as the liquid nitrogen pump and the bellows heater. The detector enclosure which is a neutron moderator is isolated from the support stand by insulated plates. Detectors and preamplifiers in the moderator are also isolated from the enclosure with wrapped kapton sheet. All cables between the enclosure and the diagnostic rack are covered with shielding tubes which connect the enclosure to the rack ground. Figure 5 shows the status of the grounding configuration. These connections make the detector enclosure and the rack appear as a single RF shield with a common groind connection.

\section{Calibration}

The neutron detectors were calibrated with a ${ }^{252} \mathrm{C}_{f}$ neutron source ( $\mathrm{S} / \mathrm{N}$ 401304) inserted in the MTX vacuum vessel on June 21,1990 . The source has a half-life of 2.65 years and its strength was $1 \mathrm{mCi}$ on November 30 , 1989. The source strength on the day of the calibration should have been 3.71 $\times 10^{6}$ neutrons $/ \mathrm{sec}(\mathrm{n} / \mathrm{s})$. The neutron source was located on the plasma axis at ports $B, D$, and F. Both ports $D$ and $F$ are $120^{\circ}$ away from port B. Figure 6 shows the calibration data of $B-1$ detector. In this figure, the toroidal angle $\theta$ is the angle along the plasma axis between port $B$ and source position. It is impossible to get a fitting curve for a calculation of detection efficiency from these three data points. We evaluate the efficiency based on a simple model 
calculation.

The source emits neutrons uniformly in every direction. The neutron flux should decrease in proportion to the square of the distance from the source, if the effects of scattering and absorption are not considered. Figure 7 shows a schematic diagram of the geometry showing the source position and the detector position. The distance $\ell$ is written as

$$
\ell^{2}=h^{2}+R^{2}+(R+d)^{2}+2 R(R+d) \cos \theta,
$$

where $\theta$ is a toroidal angle between the detector port and the neutron source port. The length $R$ is the major radius; $h$ and $d$ are the horizontal and vertical lengths between the plasma axis and the detector. The dotted lines in Fig.6 ar $\geq 1 / \ell^{2}$ curves normalized at the toroidal angle $\theta=0^{\circ}$ and $\theta=120^{\circ}$. These twi different curves mean that the neutron flux decreases not only by distance but also by some other factors. The neutrons from the vacuum vessel penetrate thick copper plates and a liquid nitrogen wall. They should be reduced there. Around $\theta=0^{\circ}$, neutrons come through almost directly without a:y reduction. Increasing $\theta$, neutrons are reduced by copper plates and liguid nitrogen. Therefore we estimate the average calibration factor from these two $1 / \ell^{2}$ curves under the assumption that the efficiency curve jumps down from one to the other at the toroidal angle $\theta=\theta_{c}$. Table 3 gives the calcuiatad efficiency for various $\theta_{c}$. The uncertainty in the calibration is a factor of two due to the assumption of the range of $\theta_{c}$ between $5^{\circ}$ and $30^{\circ}$.

Figure 8 shows the relative efficiency of the A-1 detector and the A-2 detector. The plots show detector count rate in a standard ohmic plasma. A straight line shows the relative efficiency obtained from the in situ calibration is consistent with the plasma data in the region of $5 \times 10^{3}-5 \times 10^{4}$ counts/sec (cps) for the A-1 detector count rate.

We can see the saturation count rate of the A-1 detector is $50 \mathrm{kcps}$ in Fig.8. Then wa choose these two detector ranges as follows

$$
\begin{array}{ll}
N=c_{1} \cdot N_{1} & \text { if } N_{1}<2 \times 10^{4} \mathrm{cps}, \\
N=\frac{1}{2}\left(c_{1} \cdot N_{1}+c_{2} \cdot N_{2}\right) & \text { if } 2 \times 10^{4} \leq N_{1} \leq 5 \times 10^{4} \mathrm{cps}, \\
N=c_{2} \cdot N_{2} & \text { if } N_{1}>5 \times 10^{4} \mathrm{cps},
\end{array}
$$

where $N_{1}$ and $N_{2}$ are the count rates of detector A-1 and A-2, respectively. The calibration factors are 


$$
\begin{aligned}
& c_{1}=3.3 \times 10^{5}, \\
& c_{2}=11.6 \times 10^{5} .
\end{aligned}
$$

The two detector assemblies show the toroidal symmetry of the neutron count rate in standard ohmic plasma discharges (the electron density $n_{e}>1 \times 10^{20} \mathrm{~m}^{-3}$ ). This means that photoneutrons are negligible compared with the thermonuclear fusion neutrons in ohmic plasmas of this density.

\section{(3) Calculation of ion temperature}

The thermonuclear neutron rate $\mathbf{N}$ from a Maxwellian plasma can be written as a function of the ion temperature and the deuteron density as the following equation:

$$
N=4 \pi R \int_{0}^{a} \frac{1}{2} n_{D}(r)^{2}<\sigma v>r d r,
$$

where $\langle\sigma v\rangle$ is the reaction rate averaged over the ion Maxwellian distribution function, and

$$
\begin{aligned}
& <\sigma v>=\frac{\alpha_{1}}{T_{i}(r)^{3 / 3}}\left[1+\alpha_{2} T_{i}(r)^{\beta} \exp \left(-\frac{\alpha_{3}}{T_{i}(r)^{1 / 3}}\right)\right] \\
& \alpha_{1}=2.72 \times 10^{-14}, \\
& \alpha_{2}=5.39, \\
& \alpha_{3}=19.8, \\
& \beta=0.917, \\
& a=0.165 \mathrm{~m} \text { (minor radius), } \\
& R=0.64 \mathrm{~m} \text { (major radius), } \\
& \mathrm{n}_{\mathrm{D}}(r) \text { is the deuterium density in } \mathrm{m}^{-3}, \\
& T_{i}(r) \text { is the ion temperature in } \mathrm{keV} .
\end{aligned}
$$

Figure 9 shows the calculated total neutron rate as a function of the central ion temperature. In this calculation, the deuteron density profile ar: 1 the ion temperature profile were taken to be

$$
\begin{aligned}
& n_{D}(r)=n_{D}\left[1-\left(\frac{r}{a}\right)^{2}\right], \\
& T_{i}(r)=T_{i}\left[1-\left(\frac{r}{a}\right)^{2}\right]^{2},
\end{aligned}
$$


$\mathrm{n}_{\mathrm{D}}$ is the central peak deuteriun density,

$T_{i}$ is the central peak ion temperature.

The neutron rate in Eq.(3) is a function of $n_{D}{ }^{2}$, so we introduce a normalized neutron rate $\mathrm{N}_{\mathrm{nrm}}$ as

$$
N_{n m m}=\frac{N}{n_{D}^{2}}
$$

$\mathbf{N}_{\text {nrm }}$ is a function of only the ion temperature. Equation (3) can be approximated by a convenient form

$$
N_{n m m}=p \cdot T_{i}^{q}
$$

where coefficients $p$ and $q$ vary with the range of $N_{n r m}$. Table 4 gives the parameters in the range of $N_{n r m}=1 \times 10^{7} \quad 1 \times 10^{12} \mathrm{n} / \mathrm{s}$ at $n_{D}=1 \times 10^{20} \mathrm{~m}^{-3}$. The difference between Eq.(3) and Eq.(6) in within $10 \mathrm{eV}$ for $T_{i}<1 \mathrm{keV}$, and within $20 \mathrm{eV}$ for $T_{i}>1 \mathrm{keV}$.

From Eq. (6), the ion temperature can be written

$$
T_{i}=\left(\frac{N_{r m}}{p}\right)^{\frac{1}{q}} \text {. }
$$

Parameters $1 / \mathrm{p}$ and $1 / \mathrm{q}$ are also shown in Table 4. Here we assume that the deuterium density is the same as the electron density and that the profile is given by Eq.(3). Therefore the central deuteron density $n_{D}$ is 1.5 times the line averaged electron density from the FIR central chord:

$$
n_{D}=1.5 \times \overline{n_{e}}:
$$

We can get the neutron production rate $\mathbf{N}$ from the neutron measurement given in section (2). The ion temperature is calculated for each plasma shot by combining the FIR data through the VAX compiter.

(4) Neutron production rate and ion temperature

The time evolution for typical MTX plasma parameters and the total neutron production rate are shown in Fig.10 and Fig.11. In Fig.11 the open circles shows the neutron measurement data and the closed circles show the calculated data from the neoclassical transport study. The calibration factor is calculated from $\theta_{c}=15^{\circ} \pm 5^{\circ}$. The uncertainty of the neutron data comes from the calibration factor. 
Figure 12 shows the trend of central electron and ion temperature with central peak electron density at the toroidal magnetic field $B_{t}=8 \mathrm{~T}$ and the plasma current $I_{p}=250-400 \mathrm{kA}$. The central electron temperatures and densities were obtained from the Thomson scattering system. The ion temperatures obtained from the neutron production rate are found to be smaller than the electron temperature by about $200 \mathrm{eV}$. The electron temperature decreases as the electron density increases, but the ion temperatures are almost constant above $n_{e}>2 \times 10^{20} \mathrm{~m}^{-3}$.

Here we discuss an uncertainty of the ion temperature deduced from neutron emission. The error comes from an uncertainty of the temperature profile, deuteron density, and the measured total neutron rate. In Eq. (7), the uncertainty of the temperature profile belongs to the factors $p$ and $q$. And the normalized neutron rate $\mathrm{N}_{\mathrm{nrm}}$ includes the uncertainty of the deuteron density and neutron rate shown as below:

$$
N_{n m m}=\frac{c_{j} \cdot N_{j}}{n_{D}^{2}},
$$

where $c_{j}$ and $N_{j}$ are the calibration factor and count rate of the $j$ th detector, respectively.

Figure 13 shows the variation of the central ion temperature calculated from neutron rates with profile dependence of the temperature and the density. In this figure $i_{T}$ and $i_{n}$ are the temperature and the density profile parameters:

$$
\begin{aligned}
& T_{i}(r)=T_{i} \cdot\left[1-\left(\frac{r}{a}\right)^{2}\right]^{i_{r}}, \\
& n_{D}(r)=n_{D} \cdot\left[1-\left(\frac{r}{a}\right)^{2}\right]^{i_{r}},
\end{aligned}
$$

$a$ is the plasma radius.

The central deuteron density is $n_{D}=1 \times 10^{20} \mathrm{~m}^{-3}$. The difference of the central ion temperature between $i_{T}=1$ and $i_{T}=2$ is within onily $75 \mathrm{eV}$ near the central ion temperature $T_{i}=1 \mathrm{keV}$.

The uncertainty in the deuteron density is rather complicated. If we know the central deuterium density, the error from the profile is very small, within $25 \mathrm{eV}$ as shown in Fig. 13. However, the error should be large when the deuterium density is deduced from the line averaged electron density measurement. The deuterium density is usually not equal to the electron density because of impurity contamination in the plasma. Here we assume the ions in the plasma consist of deuteron, hydrogen and eighth ionized oxygen. Then the ratio of deuteron density and electron density is 


$$
\xi=\frac{n_{n}}{n_{e}}=\frac{8-Z_{e f}}{7 \cdot(1+\zeta)},
$$

where $\zeta$ is the ratio of the hydrogen and deuterium, and $Z_{e f f}$ is the effective charge, these parameters defined by

$$
\begin{aligned}
& \zeta=\frac{n_{k}}{n_{b}}, \\
& Z_{\alpha}=\frac{\sum_{a} n_{a} \cdot Z_{\alpha}^{2}}{\sum_{\alpha} n_{a} \cdot Z_{\alpha}},
\end{aligned}
$$

$n_{\alpha}$ is the density of particle $\alpha$ and

$Z_{\alpha}$ is the charge of particle $\alpha$.

Figure 14 shows how the ratio of the deuteron to the electron density depends on the variables $\zeta$ and $Z_{\text {eff }}$. The ratio is 0.9 at $Z_{\text {eff }}=1.7$, if the contamination of hydrogen can be neglected. Under an assumption of the uniformity of $Z_{\text {eff }}, \zeta$, and $\xi$ in space, the deuteron line averaged density is

$$
\overline{n_{D}}=\xi \cdot \overline{n_{c}} \cdot \text {, }
$$

and the central peak deuteron density is

$$
n_{D}=k_{n} \cdot \overline{n_{D}},
$$

where $k_{n}$ is the coefficient of the density profile and is

$$
\begin{aligned}
k_{n} & =\frac{3}{2} & & \text { if } i_{n}=1, \\
& =\frac{15}{8} & & \text { if } i_{n}=2 .
\end{aligned}
$$

The error in the central deuteron density is due to the uncertainty of the density ratio $\xi$, the density profile coefficient $k_{n}$ and the measured electron density. The range of the density ratio is $\xi=\hat{s} .7$ to $\xi=0.9$ corresponding to $Z_{\text {eff }}=1.5 \sim 3.0$. And the range of the density profile coefficient is $k_{n}=1.5$ to $k_{n}$ $=1.9$ corresponding to $i_{n}=1 \sim 2$. The uncertainty in the central deuteron density from the alectron density measurement is 1.6 from these calculations, if the uncertainty of the measure $d$ electron density can be neglected.

The error in the measured total neutron rate is due to the uncertainty in the calibration factor and the statistics of the detector count rate. The calibration factor has the uncertainty of roughly a factor of two already discussed in Sec.(2). The error due to the counting statistics is only $10 \%$, 
because the typical count rate is 100 counts with $5 \mathrm{~ms}$ sampling time. That is negligible compare with the calibration factor. The normalized neutron rate is represented by

$$
N_{n \mathrm{~m}}=\frac{c_{j}}{\left(k_{n} \cdot \xi\right)^{2}} \cdot \frac{N_{j}}{\bar{n}_{e}^{2}},
$$

where the range of the denominator of the coefficient in Eq. (16) is $1.1 \sim 2.9$. The range of coefficient $c_{j}$ is $2.4 \times 10^{5} \sim 4.5 \times 10^{5}$ from Table 3 . Then the range of the coefficient of normalized neutron rate is $8.2 \times 10^{4} \sim 4.1 \times 10^{5}$, which corresponds to the range of the ion temperature $1.0 \sim 1.3 \mathrm{keV}$.

We conclude the error range of the ion temperature is $\pm 0.15 \mathrm{keV}$ around $T_{i}=1 \mathrm{keV}$. That becomes $\pm 0.10 \mathrm{keV}$, which comes from the uncertainty of the calibration factor, when we know the density profile and $\mathbf{Z}_{\text {eff }}$ -

\section{A.3.2.3 High-speed neutron measurement}

A high temporal resolution is required to observe the ion response to a microwave pulse from the FEL. A plastic scintillator (NE102A) with a nanosecond response is used for neutron detection.

\section{Measurement system}

The uncollimated scintillator, which is $5 \mathrm{~cm}$ in diameter and $10 \mathrm{~cm}$ in length, is inserted into the MTX top port and placed $48 \mathrm{~cm}$ above the horizontal plane of the plasma center. The original system used a $20 \mathrm{~m}$ fiber bundle to transmit the photon signal to the photomultiplier tube, as shown in Fig.15. The photomultiplier tube (R329-02) has a fast time response, typically with $2.5 \mathrm{~ns}$ rise time; the most sensitive wavelength is $420 \mathrm{~nm}$, which is optimum for the plastic scintillator. Figure 16 shows the electronics system, which can operate in both pulse-count mode and current mode. The amplifier has fast frequency response, $16 \mathrm{MHz}$ in pulse output and 160 $\mathrm{kHz}$ in direct current output. The pulse outputs connect to the CAMAC eight-channel scaler, which also is connected to the proportional counters and fission counters. The NE102A plastic scintillator has a 2.4 ns decay constant time, and the output pulse through the amplifier typically has a $7 \mathrm{~ns}$ rise time and $15 \mathrm{~ns}$ FWHM. The time resolution of this system is about 1 $\mathrm{kHz}$, limited by counting statistics. The transmission of the fiber bundle is not optimum for the photons from the plastic scintillator. The calculated total transmission efficiency of the fiber bundle is only $38 \%$ at $420 \mathrm{~nm}$ wavelength. Therefore, we redesigned this system using a long acrylic light guide instead of a fiber bundle to transmit the photon signal to a 
photomultiplier. Figure 17 shows the modified system. The photomultiplier tube is at $1.25 \mathrm{~m}$ above the top port, where the stray magnetic field is weak enough to be able to eliminate with a $10 \mathrm{~mm}$ thick magnetic shield. In this system, we have better counting statistics and the temporal resolution is up to $2 \mathrm{kHz}$.

\section{Response for deuterium pellet}

Figure 18 shows the time evolution of the plasma current, the lineaveraged electron density, the neutron emission, and the hard $x$-ray in a pellet injection experiment. The neutron signal comes from the plastic scintillator with $1 \mathrm{~ms}$ of sampling time in the pulse-count mode. The neutron signal has a large spike in the start phase of the discharge. The hard $x$-ray signal shows that this spike comes from intense $x$-ray emission. One deuterium pellet is injected into the plasma at $260 \mathrm{~ms}$ in this discharge. The neutron emission drops initlally at the pellet injection because of a decrease in the temperature. A soft $\mathrm{X}$-ray signal shows the abrupt drop of the electron temperature caused by cold particles from the evaporating pellet. The neutron rate then increases as the temperature recovers and becomes five times higher than the original value about $40 \mathrm{~ms}$ after the pellet injection in this discharge.

\section{A.3.2.4 Conclusion}

Two types of neutron measurement systems were installed and operated on the MTX device. The proportional counter system was absolutely calibrated using a ${ }^{252} \mathrm{Cf}$ source. The calibration swas carried out under the unsuitable configuration of the MTX tokamak. The uncertainty of the neutron rate is roughly a factor of two. However, the uncertainty of the ion temperature deduced from the neutron measurement is within \pm 0.15 $\mathrm{keV}$, because of the strong ion temperature dependence of the neutron rate. The total neutron production and the ion temperature were evaluated from the neutron measurements and compared with the calculation from the neoclassical transport study. The plastic scintillator system for high-speed measurements made fast time response measurements on pellet injected plasmas. Unfortunately, we did not see any response to the $1 \mathrm{GW}$ level single FEL pulse during physics studies of the MTX plasma. The temporal resolution of $2 \mathrm{kHz}$ wns not fast enough to respond to and detect the very short FEL pulse. It will be possible to get better counting statistics with faster sampling time in pulse-count mode by collection of data from more than one discharge of the same type. 


\section{Figure Captions}

Fig. 1 Schematic diagram of the neutron moderator consisting of $100 \mathrm{~mm}$ thick polyethylene, $50 \mathrm{~mm}$ thick lead, $1 \mathrm{~mm}$ thick cadmium, $2 \mathrm{~mm}$ thick permalloy and stainless steel. Neutron detectors and preamplifiers are set together inside the neutron moderator.

Fig. 2 Schematic diagram of the MTX tokamak plan view, showing the layout of the neutron detector assemblies and the scintillator.

Fig. 3 Elevation view of MTX tokamak, showing the layout of the neutron detectors.

Fig. 4 Electronic system of the neutron production rate measurement. The electronics are selected by the requirement of high speed and high count rates capability.

Fig. 5 Schematic diagram of the grounding for the neutron diagnostic. All cables between the enclosure and the diagnostic rack are covered with shielding tubes which sonnect the enclosure to the rack ground.

Fig. 6 The detection efficiency of the B-1 detector to the ${ }^{252} C_{f}$ source neutrons. The toroidal angle $\theta$ is the angle between port $B$ and the source position. The two dotted lines are $1 / 1^{2}$ curves normalized to the data points at the toroidal angle $\theta=0^{\circ}$ and $\theta=120^{\circ}$.

Fig. 7 Schematic diagram of the geometry sho ing the source position and the detector position

Fig. 8 The relative efficiency of the A-1 detector and the A-2 detector. The plots show detector count rate in standard ohmic plasma. A straight line shows the relative efficiency obtained from the in situ calibration.

Fig. 9 Calculated neutron production rate versus the central peak ion temperature. Temperature and density profiles of the form $T \propto\left[1-(r / a)^{2}\right]^{2}$ and $n \propto\left[1-(r / a)^{2}\right]$ are assumed. The central deuterium densities are $\mathrm{n}_{\mathrm{D}}=1 \times 10^{20} \mathrm{~m}^{-3}$ and $\mathrm{n}_{\mathrm{D}}=5 \times 10^{20} \mathrm{~m}^{-3}$.

Fig.10 Time evolution of typical MTX plasma parameters.

Fig.11 Time evolution of total neutron production rate. The open circles shows the neutron measurement data and the closed circles show the calculated data from the neoclassical transport study. The calibration factor is calculated for $\theta_{c}=15^{\circ} \pm 5^{\circ}$. The uncertainty of the neution data comes 
from the calibration factor.

Fig.12 The ion temperatures deduced from the neutron measurement are shown with the electron temperatures as a function of the central electron densities at $B_{1}=8 \mathrm{~T}, I_{P}=250 \sim 400 \mathrm{kA}$.

Fig.13 The central ion temperature calculated from neutron rates with profile dependence of the ion temperature and deuteron density.

Fig.14 The ratio of the deuteron to the electron density versus the ratio of the hydrogen to the deuteron density, with the dependence of $Z_{\text {eff }}$.

Fig.15 Schematic diagram of the scintillator system. The scintillator is $5 \mathrm{~cm}$ in diameter and $10 \mathrm{~cm}$ in length with a conical acrylic light guide and a polymer-dad silica bundle $(20 \mathrm{~m})$.

Fig.16 Electronic system of the high-speed neutron measurement. It operates in both pulse-count mode and current mode. The amplifier has fast frequency response, $16 \mathrm{MHz}$ in pulse output and $160 \mathrm{kHz}$ in direct current output.

Fig.17 Schematic diagram of the modified scintillator system. A long acrylic light guide is used instead of a fiber bundle to transmit the photon signal to a photomultiplier.

Fig.18 Time evolution of the plasma current, the line averaged electron density, the neutron signal from the NE102A plastic scintillator, and hard $x$ rays in a pellet injection experiment at $B_{l}=8 T$. A deuterium pellet is injected into the plasma at $260 \mathrm{~ms}$. The sampling time of the plastic scintillator is $1 \mathrm{~ms}$ in pulse-count mode. 
Table 1 Comparison of neutron diagnostics for MTX.

\begin{tabular}{|c|c|c|c|c|}
\hline Purpose & Detector & Electronics & Resolution & Merit \\
\hline $\begin{array}{l}\text { Neutron rate } \\
\text { and ion } \\
\text { temperature }\end{array}$ & $\begin{array}{l}\text { Proportional } \\
\text { counter } \\
\text { array }\end{array}$ & Pulse count & $5 \mathrm{~ms} \sim 10 \mathrm{~ms}$ & $\begin{array}{l}\text { Wide dynamic range } \\
\text { High sensitivity } \\
\text { X-ray shield }\end{array}$ \\
\hline $\begin{array}{c}\text { Ion behavior } \\
\text { to }\end{array}$ & $\begin{array}{c}\text { Plastic } \\
\text { scintillator }\end{array}$ & $\begin{array}{l}\text { Pulse count } \\
\text { or }\end{array}$ & $0.5 \mathrm{~ms} \sim 1 \mathrm{~ms}$ & $\begin{array}{l}\text { Fast temporal } \\
\text { resolution }\end{array}$ \\
\hline FEL pulse & & Current mode & & \\
\hline
\end{tabular}


Table 2 Neutron detectors

\begin{tabular}{ccccccc}
\hline $\begin{array}{l}\text { Detector } \\
\text { No. }\end{array}$ & $\begin{array}{l}\text { Model } \\
\text { No. }\end{array}$ & $\begin{array}{l}\text { Neutron } \\
\text { Bensitive } \\
\text { material }\end{array}$ & $\begin{array}{l}\text { Fill pressure } \\
\text { or } \\
\text { total quantity }\end{array}$ & $\begin{array}{l}\text { Active } \\
\text { length } \\
\text { (cm) }\end{array}$ & $\begin{array}{l}\text { Diameter } \\
\text { (cm) }\end{array}$ & $\begin{array}{l}\text { Thermal neutron } \\
\text { Bensitivity } \\
\text { (cps/nv) }\end{array}$ \\
\hline 1 & RS-P4-1609-201 & ${ }^{3} \mathrm{H}_{\mathrm{e}}$ & $5 \mathrm{~atm}$ & 229 & 5.1 & 80.9 \\
2 & RS-P4-0809-203 & ${ }^{3} \mathrm{H}_{\mathrm{e}}$ & $2 \mathrm{~atm}$ & 22.9 & 2.5 & 19.6 \\
3 & RS-P1-0809-205 & $\mathrm{BF}_{3}$ & $40 \mathrm{cmH} \mathrm{g}_{\mathrm{B}}$ & 21.1 & 25 & 4.1 \\
4 & RS-P6-1608-110 & $235 \mathrm{U}$ & $1.7 \mathrm{grm}$ & 18.1 & 5.1 & 0.74 \\
5 & RS-P6-1608-125 & $235 \mathrm{U}$ & $1.7 \mathrm{grm}$ & 18.1 & 5.1 & 0.23 \\
\hline \hline
\end{tabular}


Table 3 Detection Efficiency

\begin{tabular}{cc}
$\theta_{c}$ & Efficiency (counts/neutron) \\
\hline $0^{\circ}$ & $1.76 \times 10^{-6}$ \\
$5^{\circ}$ & $2.19 \times 10^{6}$ \\
$10^{\circ}$ & $2.60 \times 10^{-6}$ \\
$15^{\circ}$ & $3.01 \times 10^{6}$ \\
$20^{\circ}$ & $3.42 \times 10^{6}$ \\
$25^{\circ}$ & $3.81 \times 10^{-6}$ \\
$30^{\circ}$ & $4.19 \times 10^{6}$ \\
\hline \hline
\end{tabular}


Table 4 Coefficients of neutron rate calculation

\begin{tabular}{ccccc}
\hline Range & $p$ & $q$ & $1 / p$ & $1 / q$ \\
\hline $10^{7}<N_{n} \leq 10^{8}$ & $1.91 \times 10^{10}$ & 8.47 & $6.23 \times 10^{-11}$ & 0.118 \\
$10^{8}<N_{n} \leq 10^{9}$ & $9.89 \times 10^{9}$ & 7.41 & $1.01 \times 10^{-10}$ & 0.135 \\
$10^{9}<N_{n} \leq 10^{10}$ & $7.38 \times 10^{9}$ & 6.47 & $1.35 \times 10^{-10}$ & 0.155 \\
$10^{10}<N_{n} \leq 10^{11}$ & $7.65 \times 10^{9}$ & 5.73 & $1.31 \times 10^{-10}$ & 0.174 \\
$10^{11}<N_{n} \leq 10^{12}$ & $1.10 \times 10^{10}$ & 4.92 & $9.10 \times 10^{-11}$ & 0.203 \\
\hline
\end{tabular}




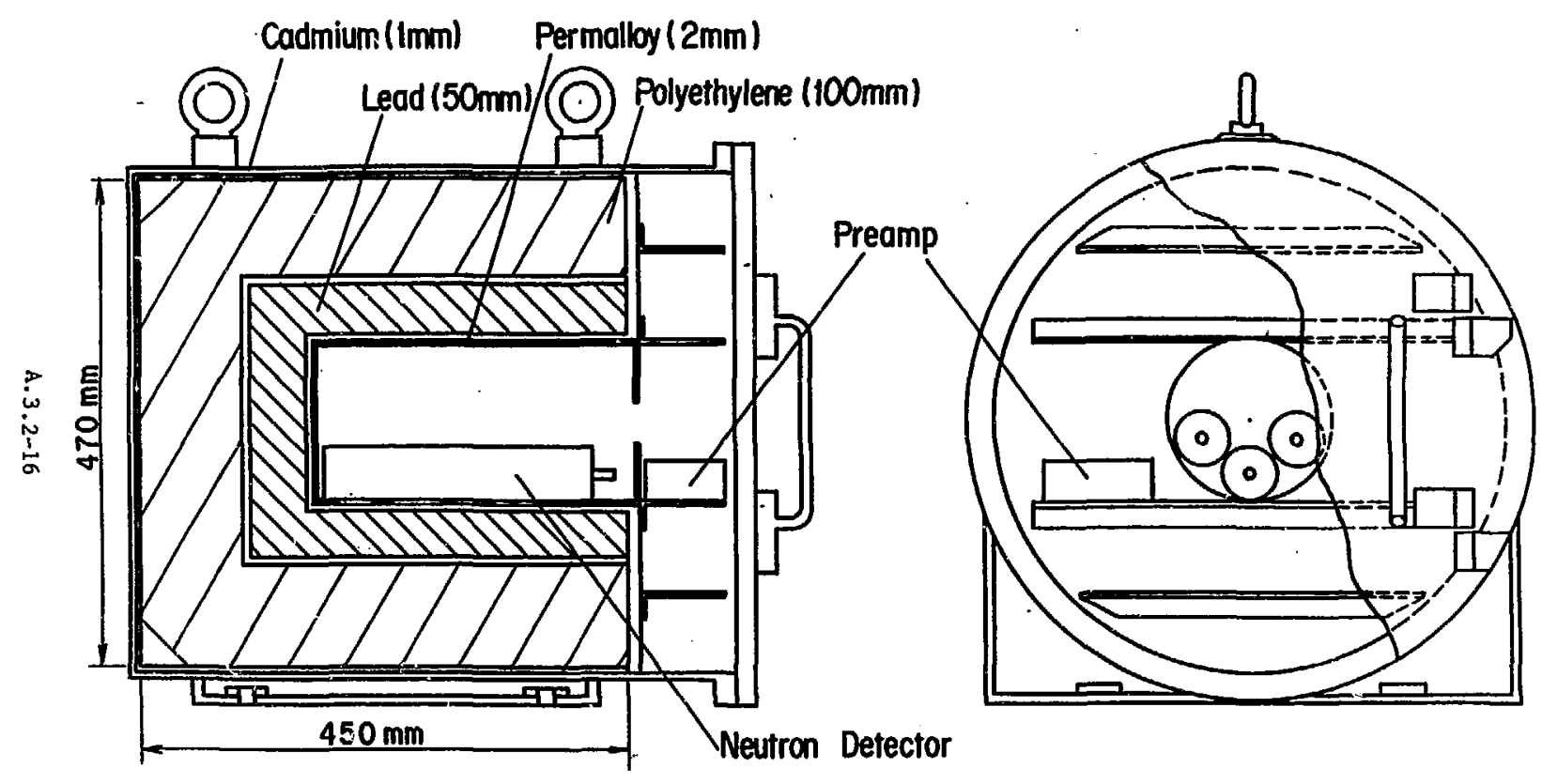


Fig. A.3.2-2

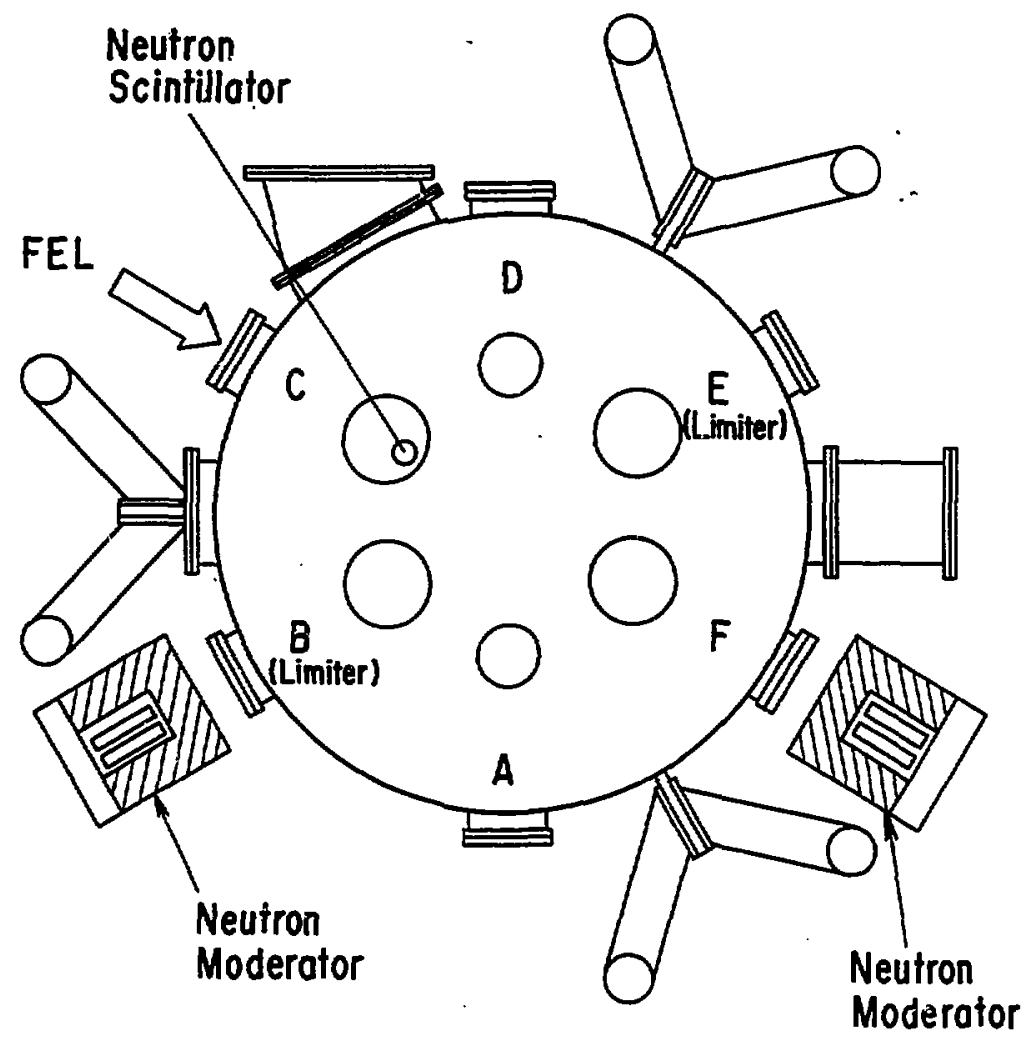




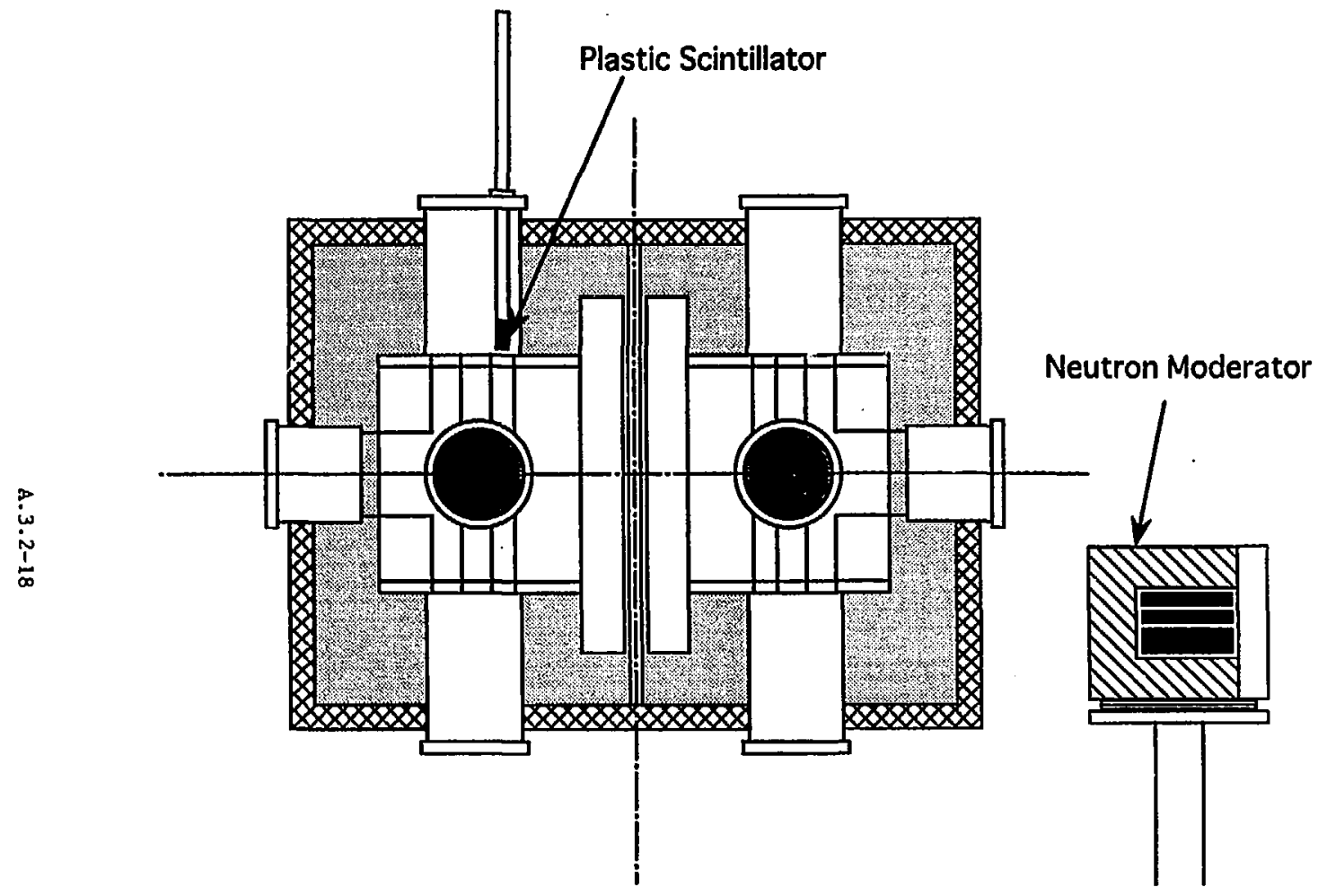




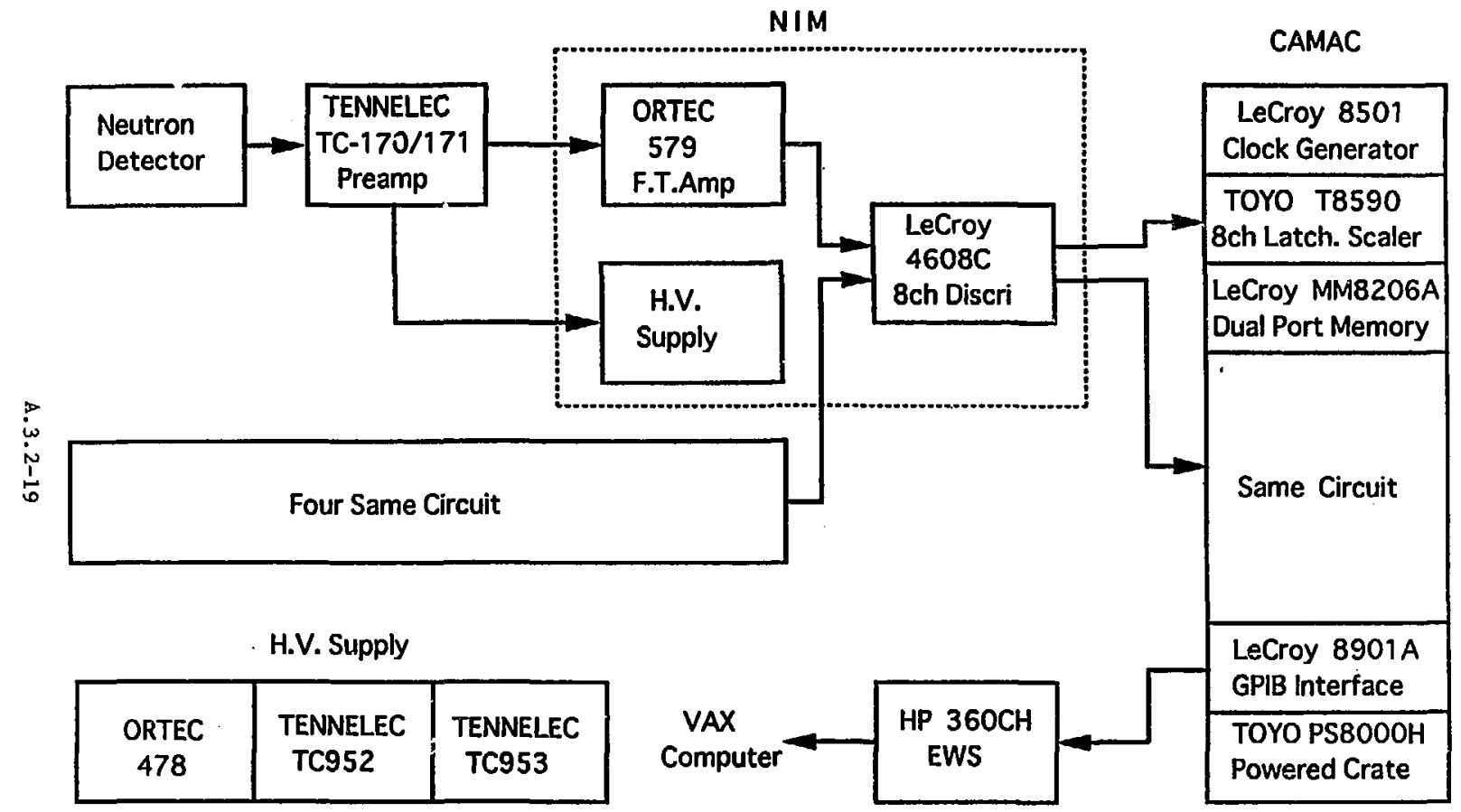




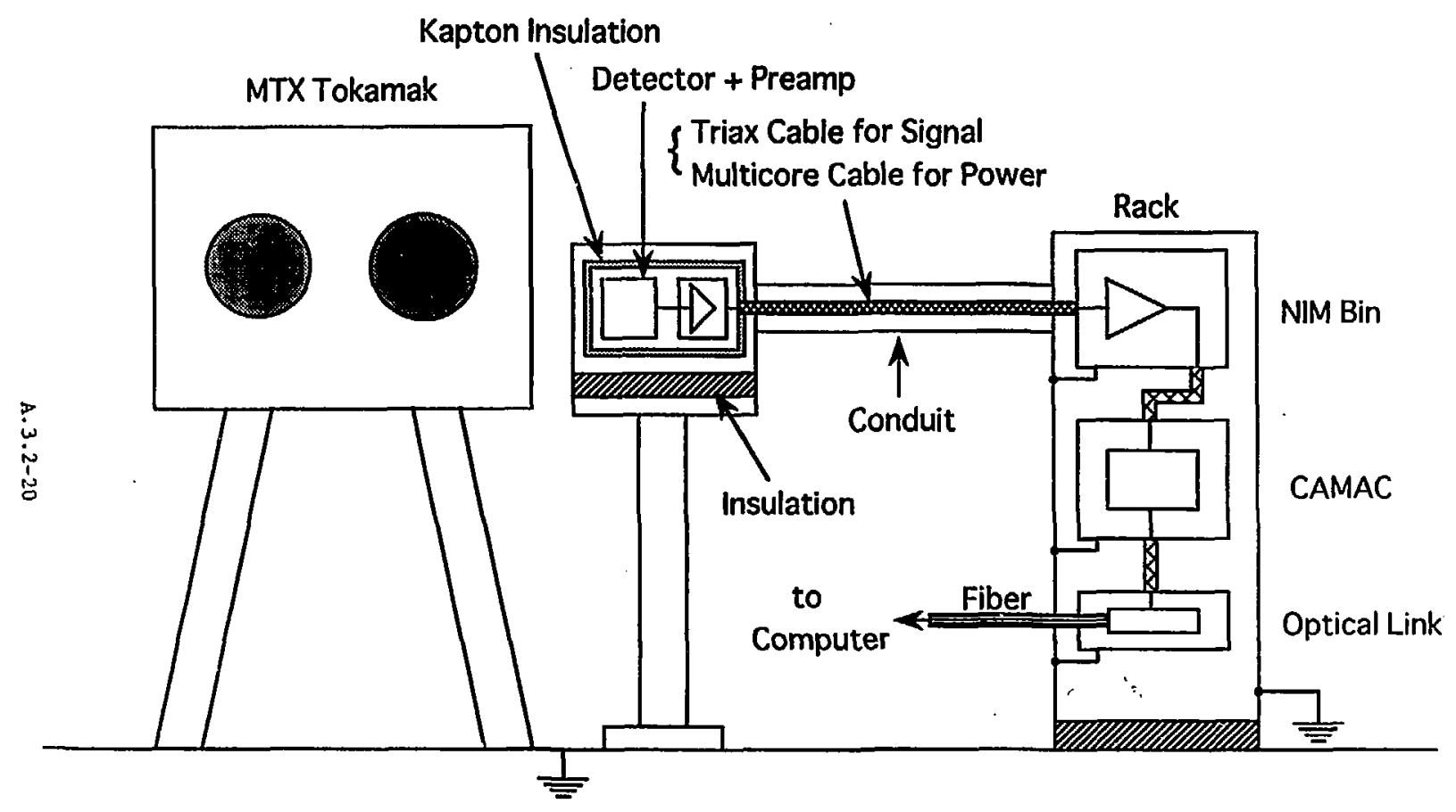


Fig. A.3.2-6

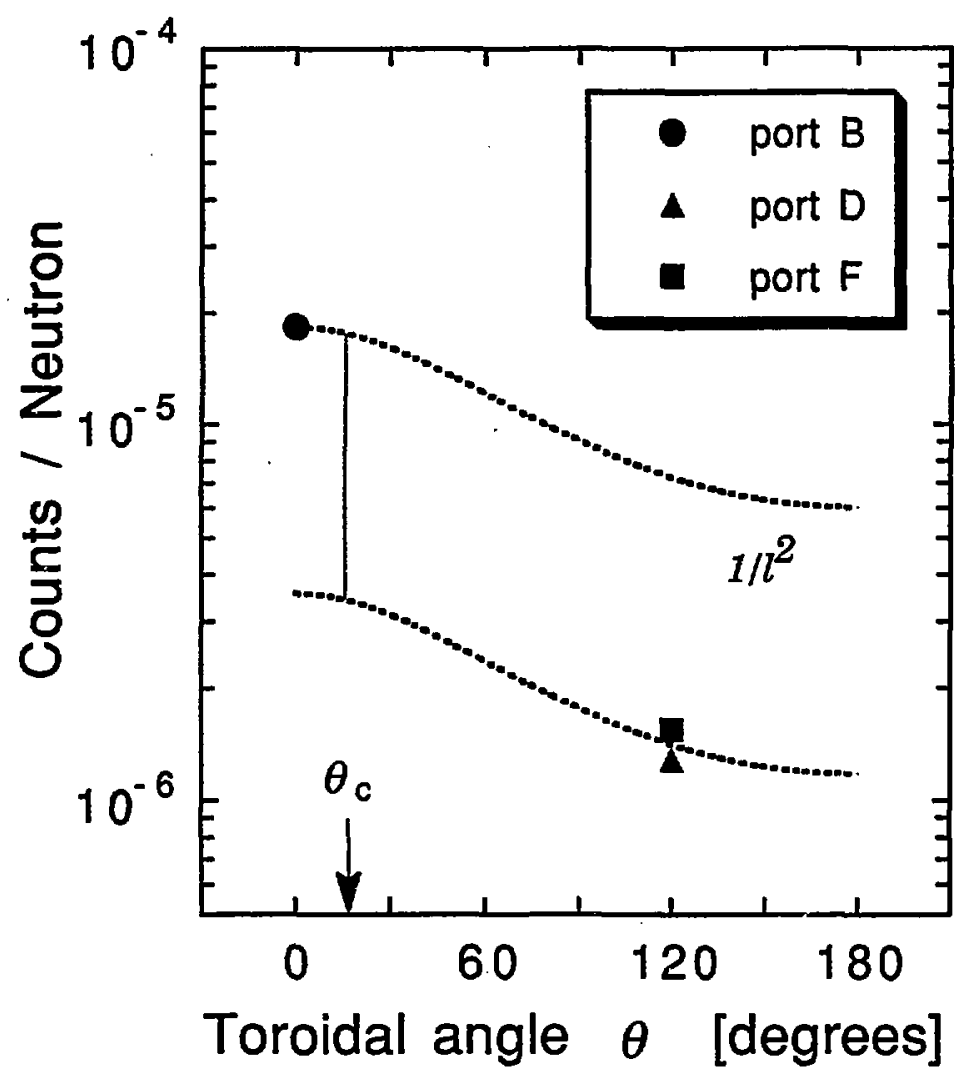

A. 3.2-21 


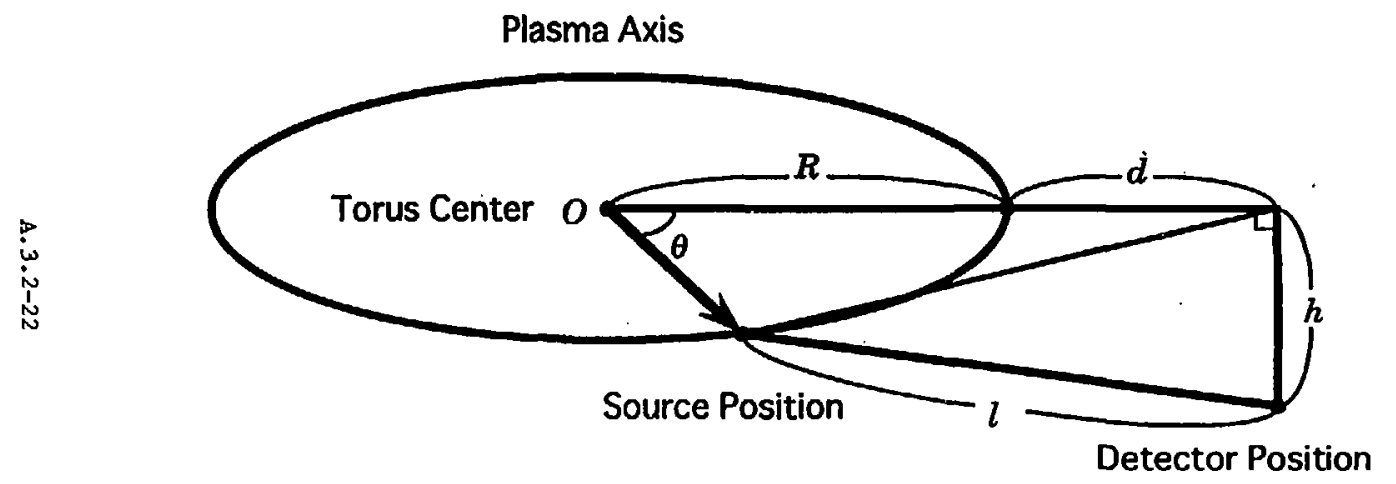

羿

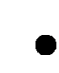


Fig. A. 3. 2-8

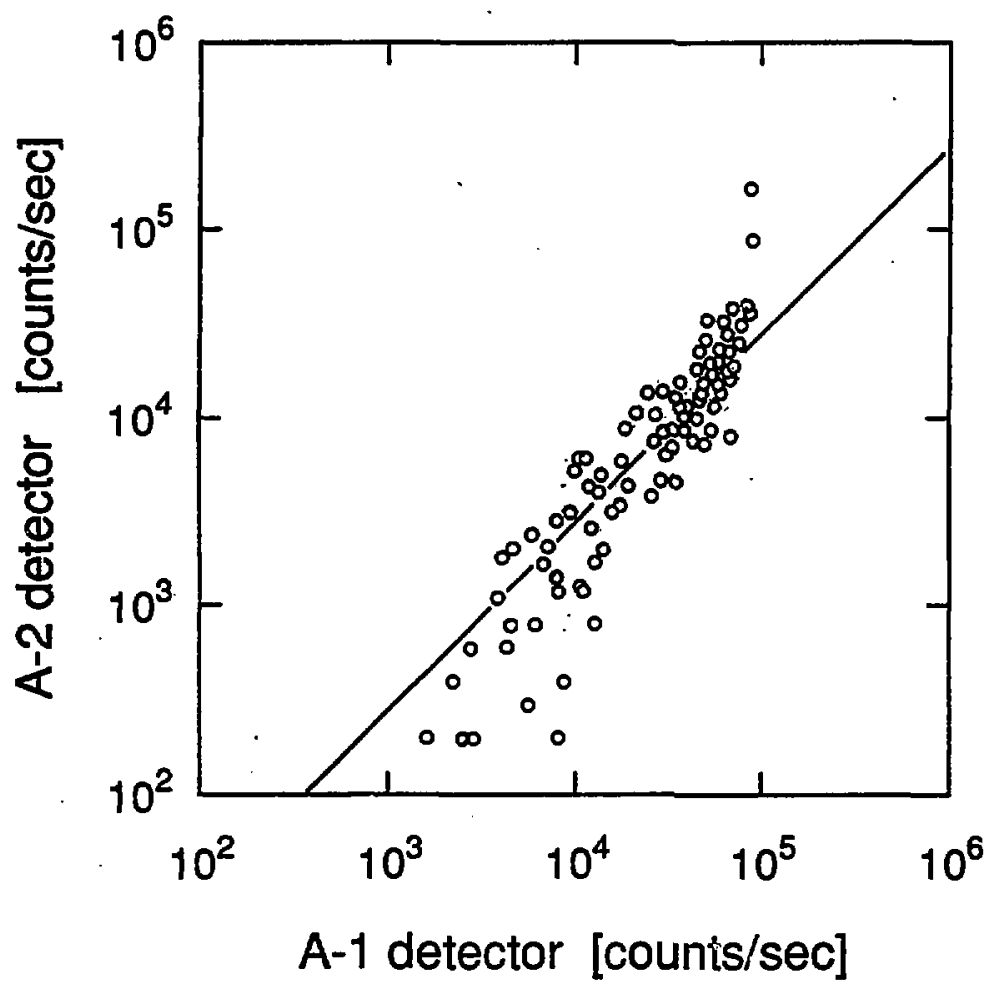

A. 3. 2-23 
Fig. A.3.2-9

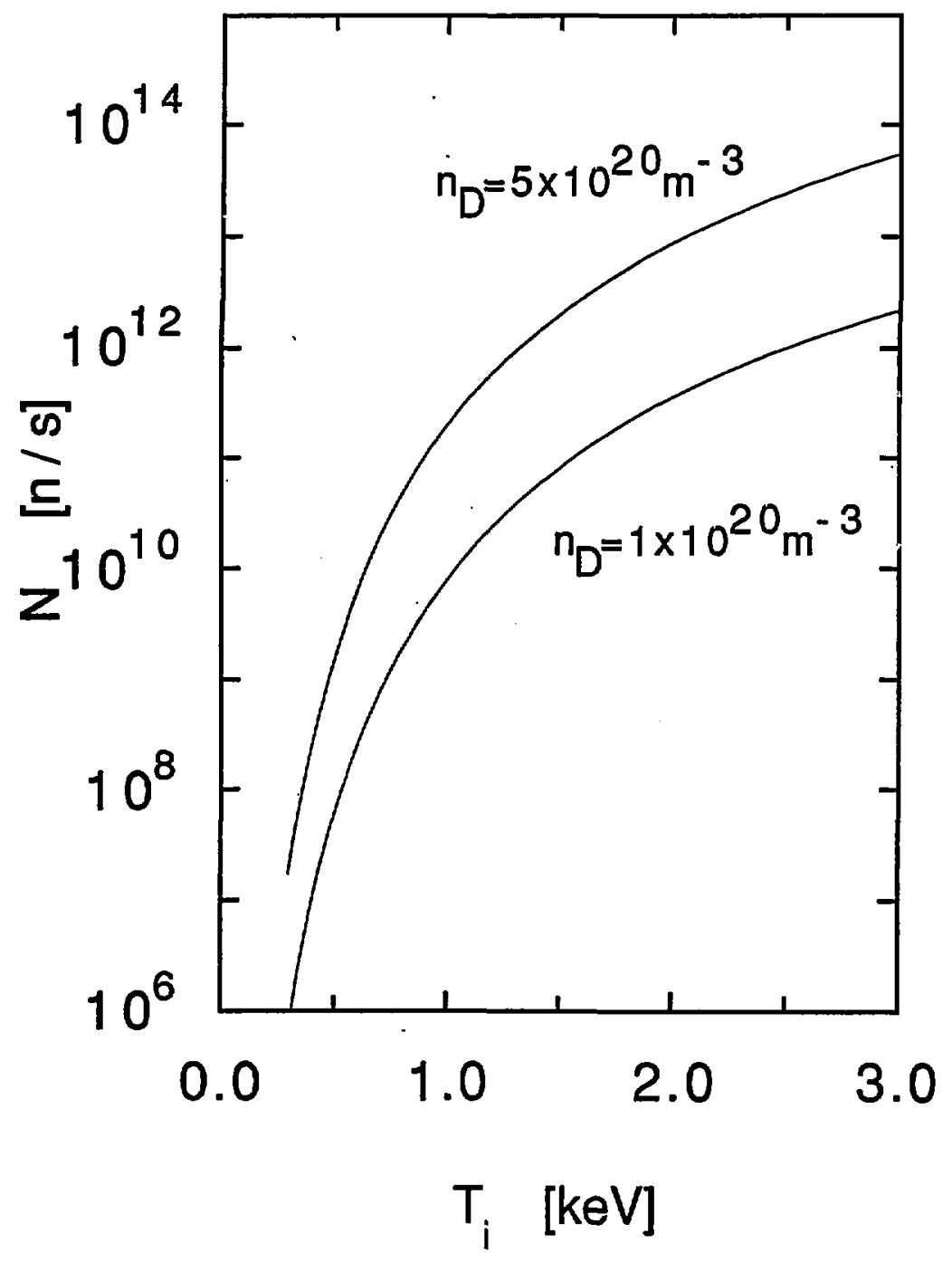

A. 3. 2-24 
Fig. A. 3.2-10
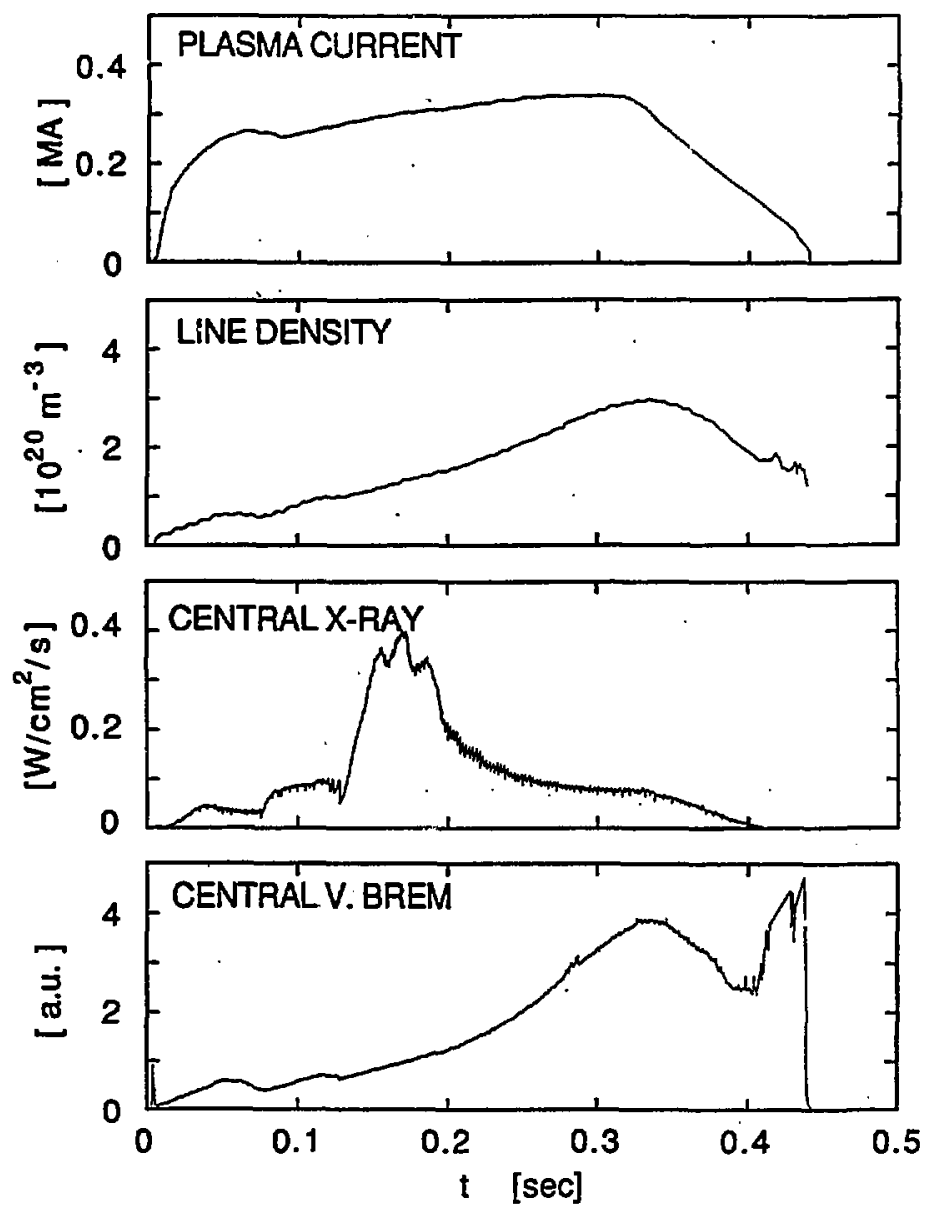


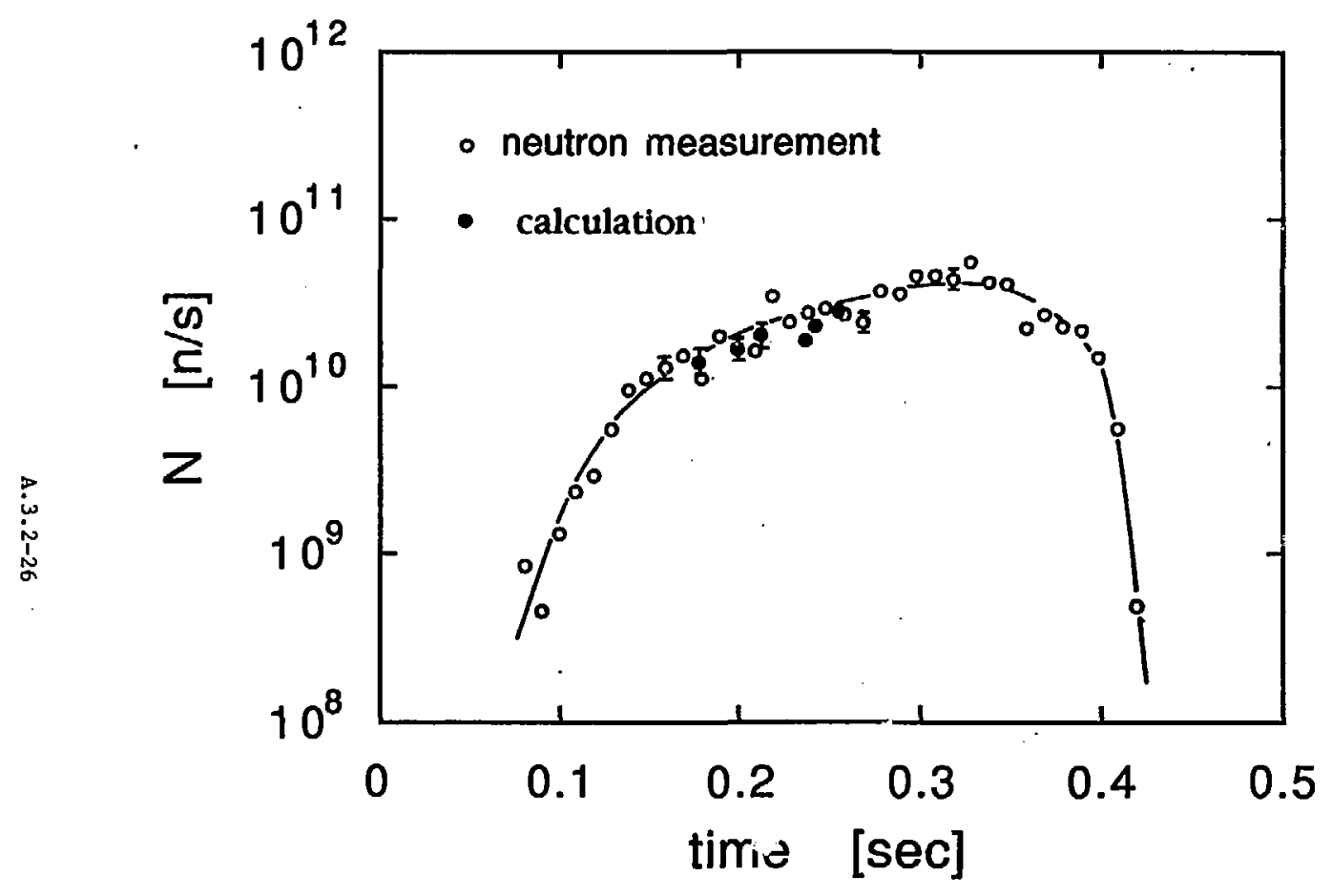




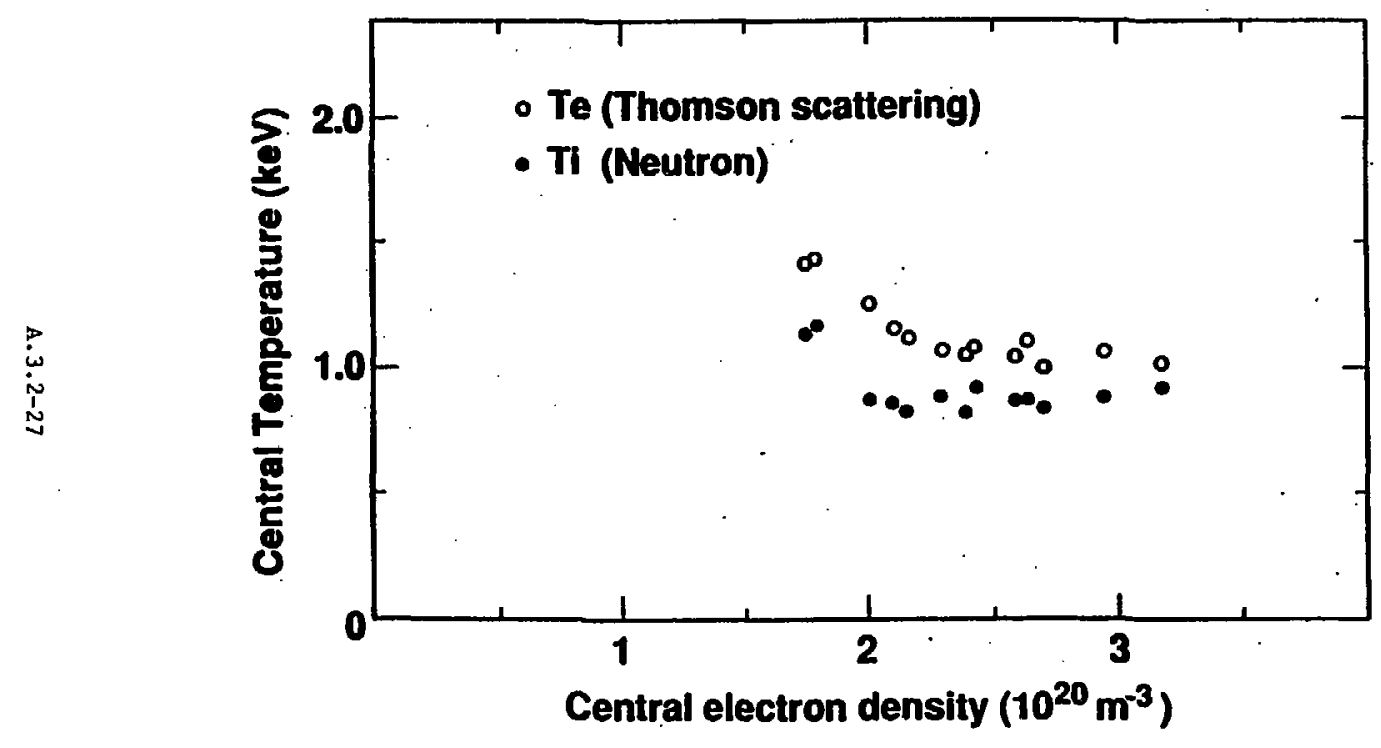


Fig. A. 3.2-13

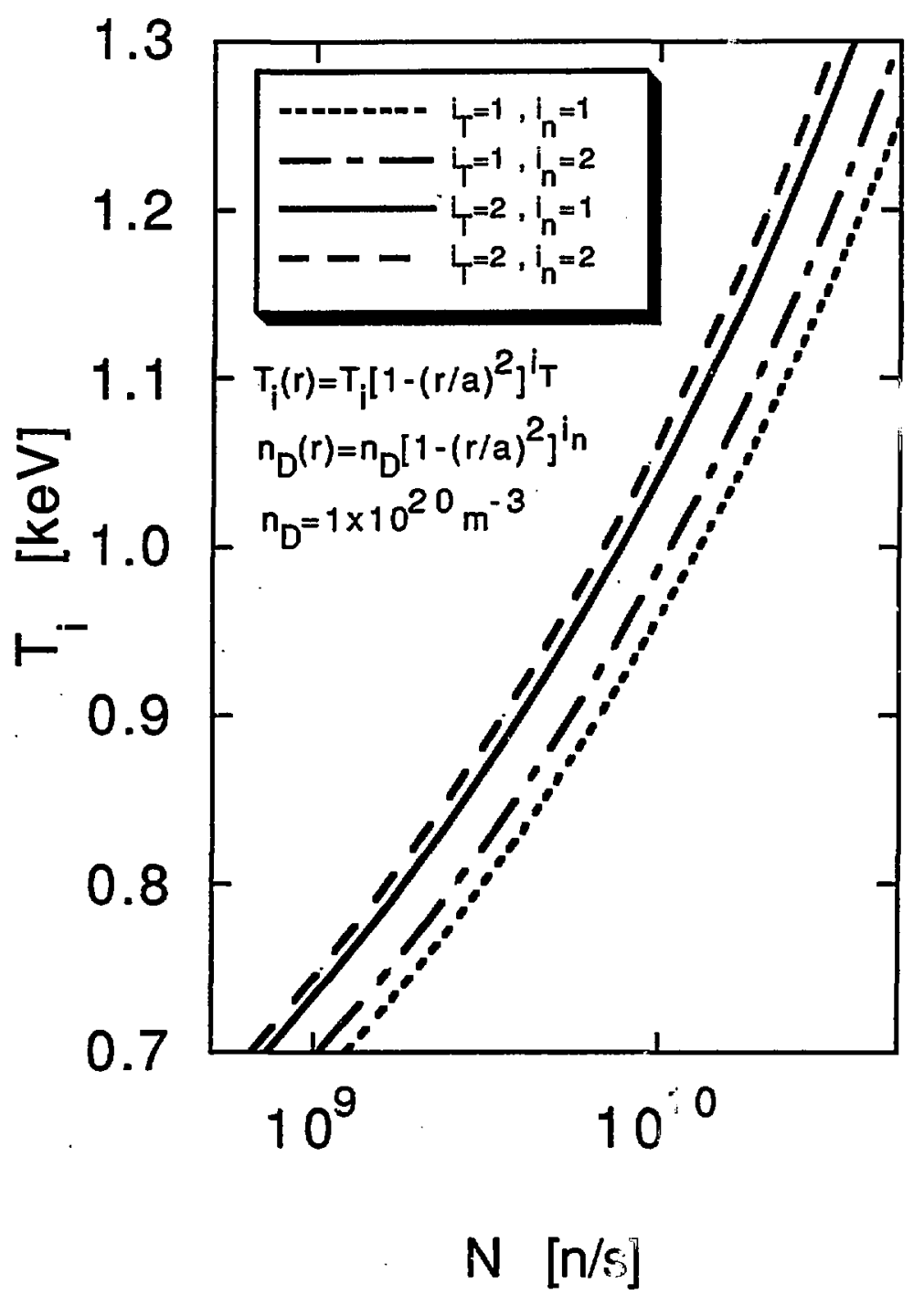




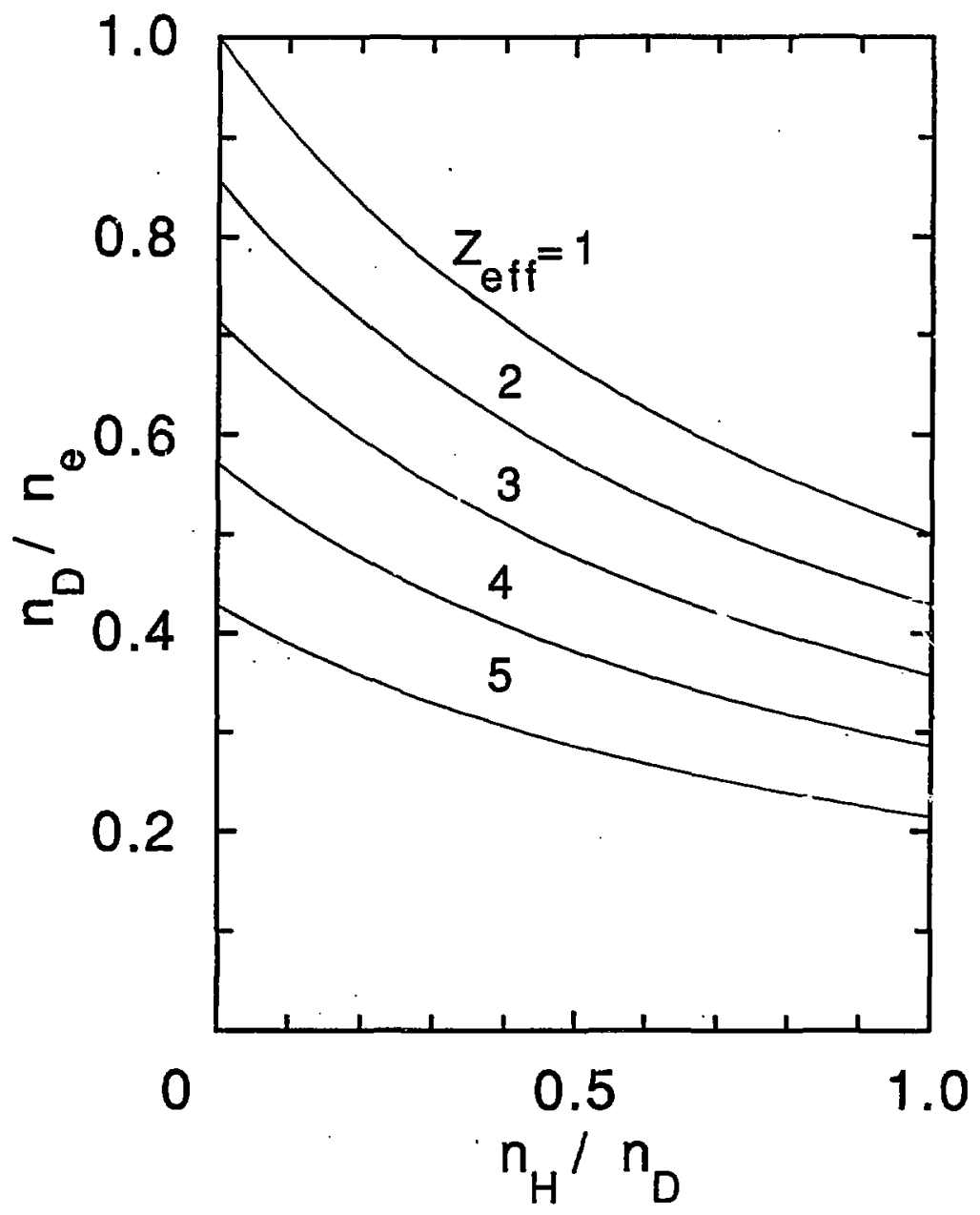




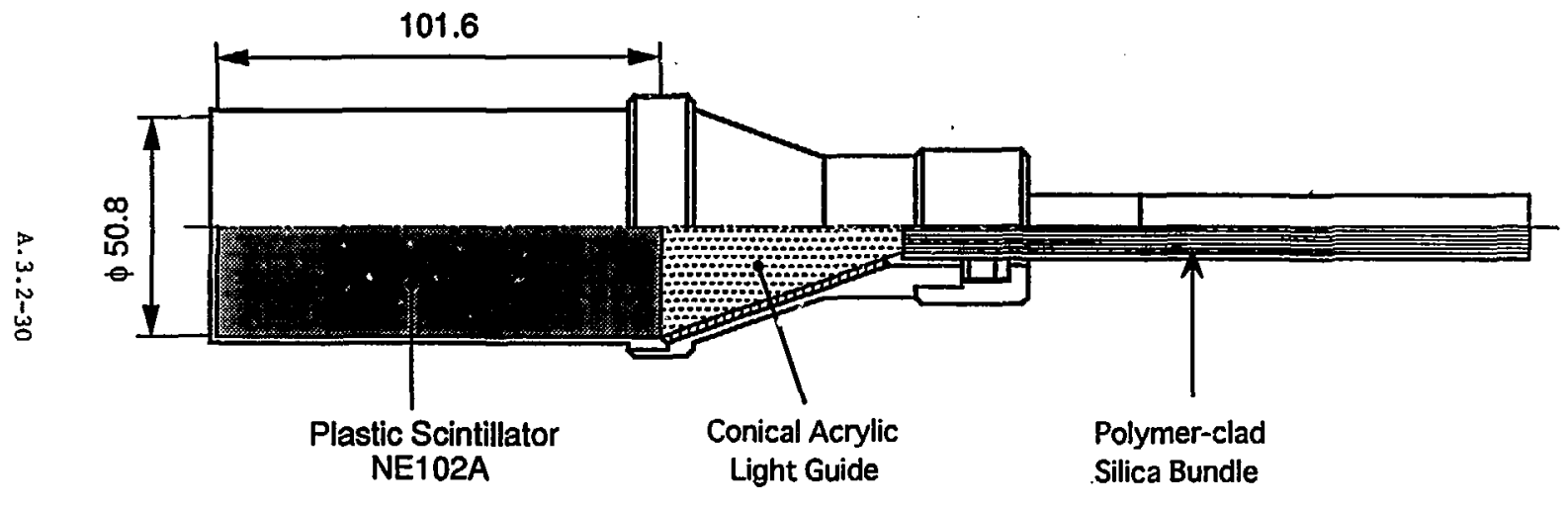


.
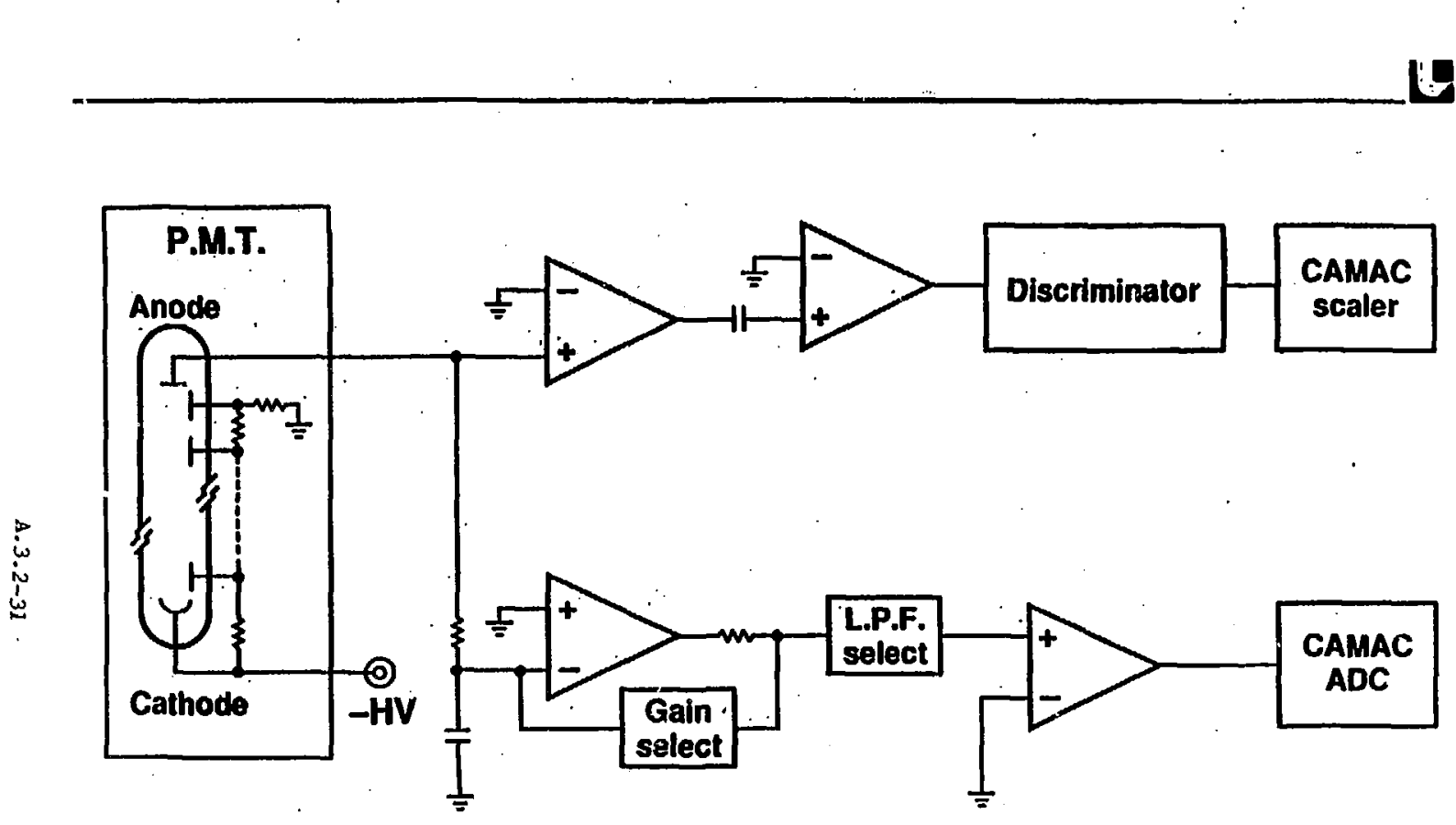


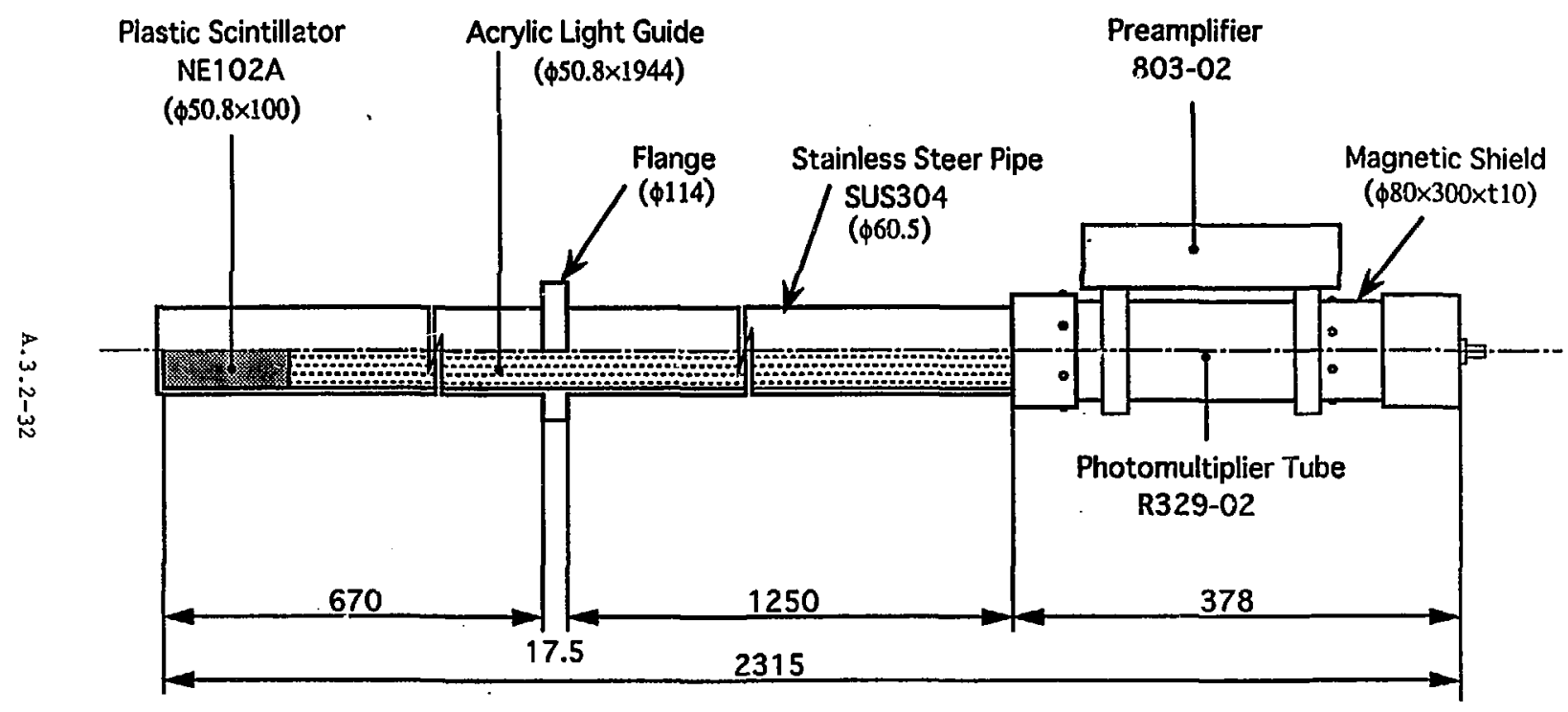


Eig. A. 3.2-18

O
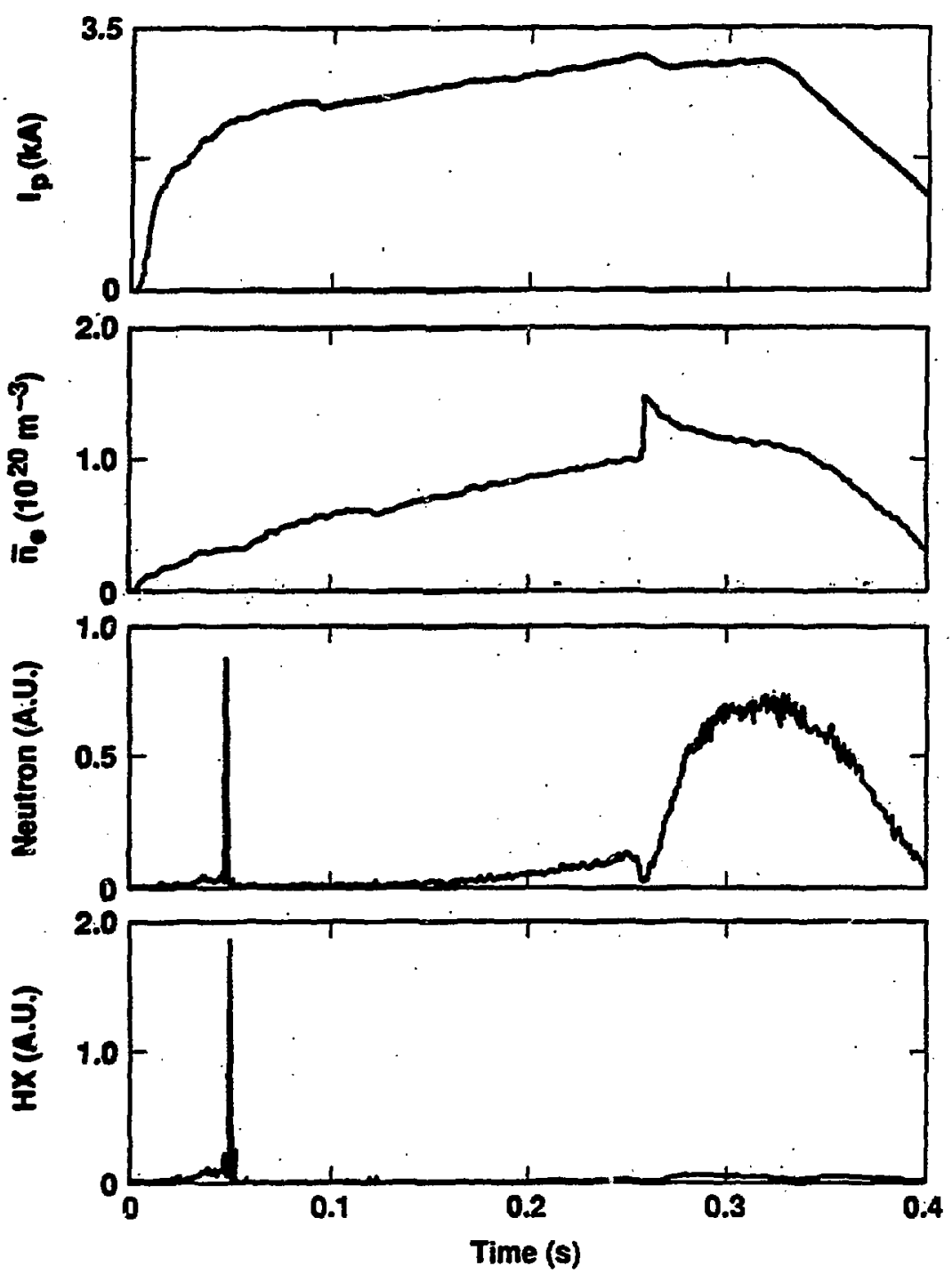

20-A-0500-0137

A. 3.2-33 


\section{A.3.3 JAERI Soft X-ray (K. Oasa)}

The following pages were part of a presentation at the 1991 Annual Meeting of the Division of Plasma Physics of the American Physical Society held in Tampa, Florida, during November 4 through 8 . The reference is: Bulletin of the American Physical Society 36, 2313 (October 1991).

Included in the following figures is an example of soft $x$-ray response for one MTX shot. 
Performance Test Result of MTX Upgrade High Speed Soft X-ray Measurement System on MTX ${ }^{\dagger} \mathrm{K}$. Oasa*, T. Ogawa*, K. Odajima*, M. Shiho*, H. Maeda*, MTX Group** Performance of an upgraded high speed soft X-ray measurement system has been tested during MTX gyrotron ECH experiments. The upgraded system has a $2 \times 10 \mathrm{PIN}$ photo diode array with a pre-amplifier installed on its backside, directly connected to it to maximize the time response of the system at reasonable signal intensity. The frequency response is designed to be more than $10 \mathrm{MHz}$ and has been checked by a fast rising visible wavelength light source. Each diode pair looking at the same radial position is designed to use various thickness Be filters to provide soft X-ray energy resolution in addition to the spatial resolution.

The system has been installed on the MTX port directly viewing the plasma-FEL interaction region. Its response to soft $\mathrm{X}$-ray emission from MTX gyrotron ECH plasmas has been checked except for time response to soft $\mathrm{X}$-ray signals. The observed fastest time variation of the MTX soft X-ray emission is about 20 micro-seconds and is too slow to check the detectors response. Test results will be shown.

*Japan Atomic Energy Research Institute, Tokai, Japan
**Lawrence Livermore National I-aboratory, Livermore, CA
tWork performed under the DOE-JAERI collaborative program in MTX
project and by LLNL for USDOE under Contract No. W-7405-ENG-48. 
spacial resolution (10 channels)

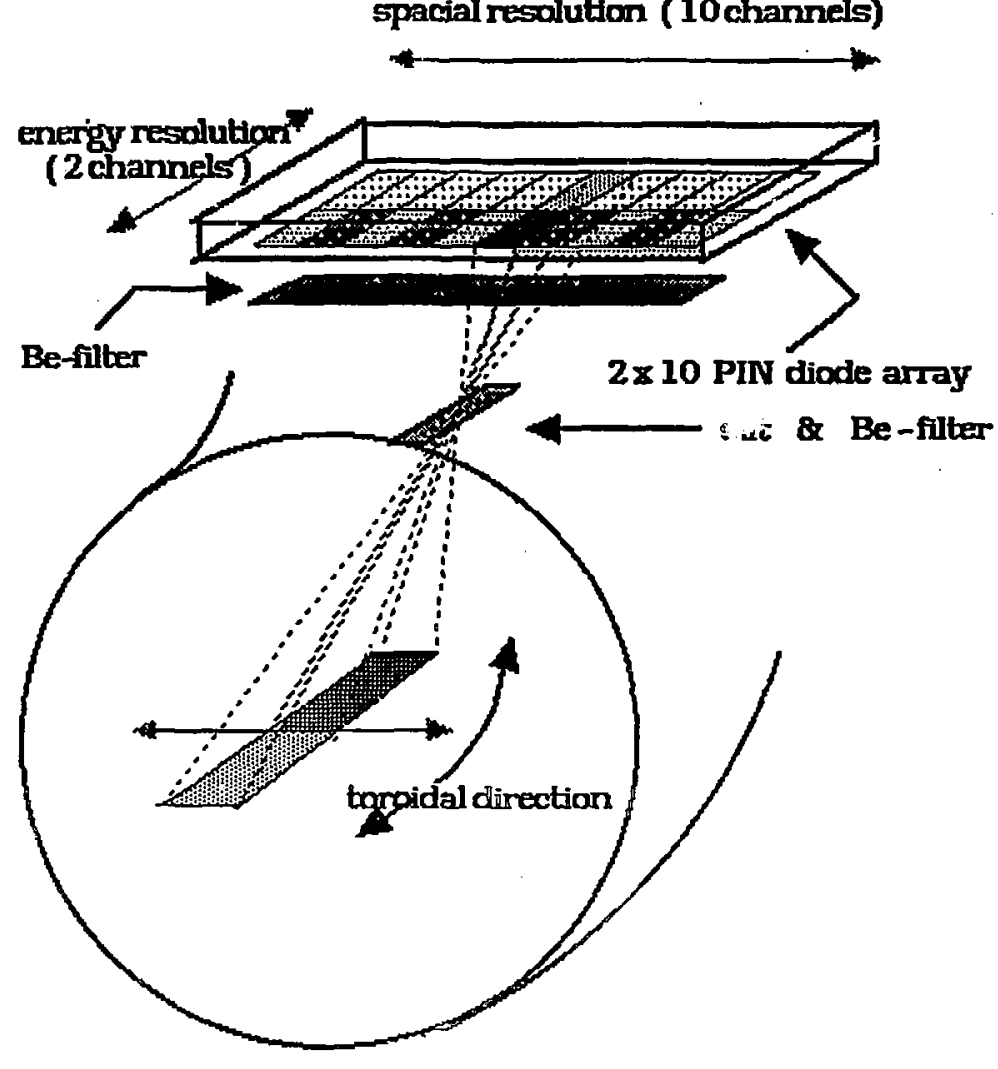


Preamplifier is connected to PIN diode directly.

$\Rightarrow$ reduction on stray capacitance.

$=\Longrightarrow$ improvement of response time.

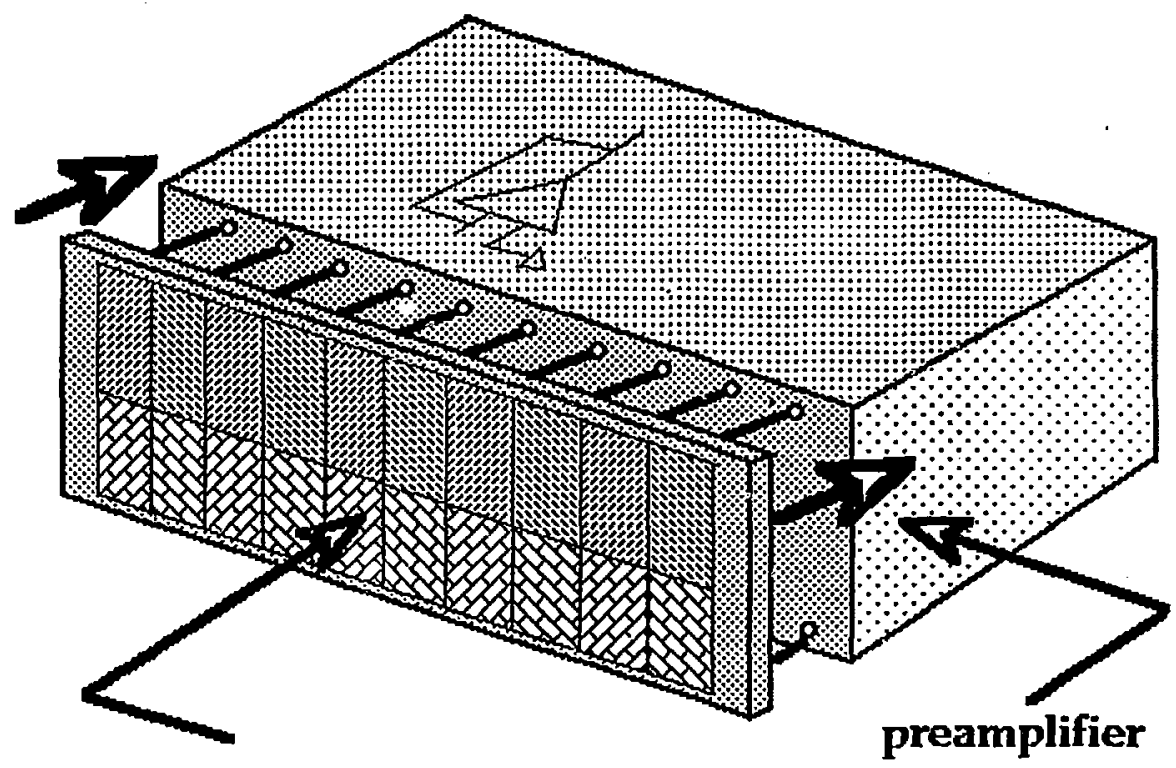

$2 \times 10$ PIN diode array 


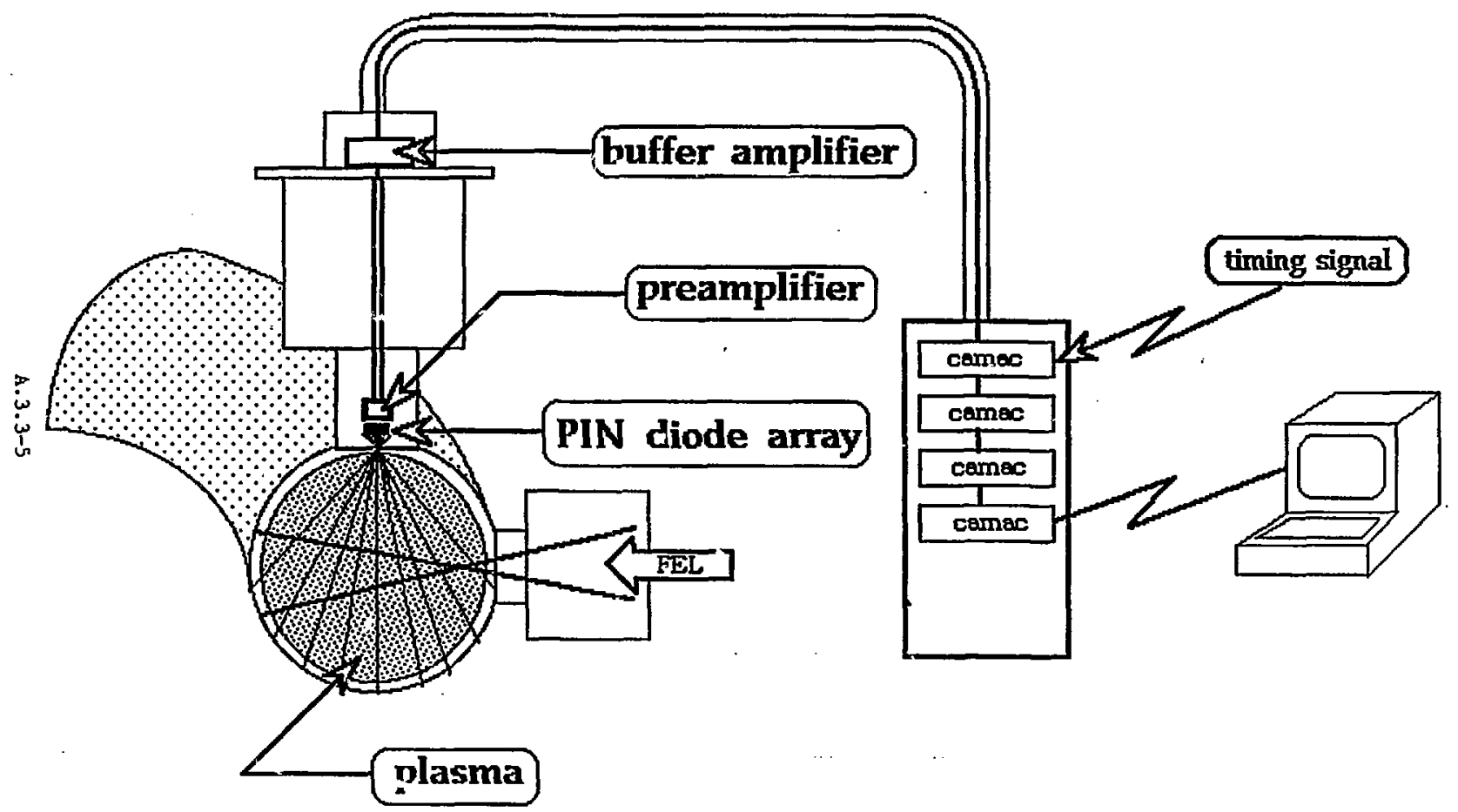




\section{PERFORMANCE TEST RESULTS}

\section{TIME RESPONSE}

observe $m=1$ oscillation associated with sawteeth oscillation.

response time to MTX soft X-ray emission is checked up to 5 micro second.

SPACIAL RESOLUTION

$2 \mathrm{~cm}$ spatial resolution is obtained. 

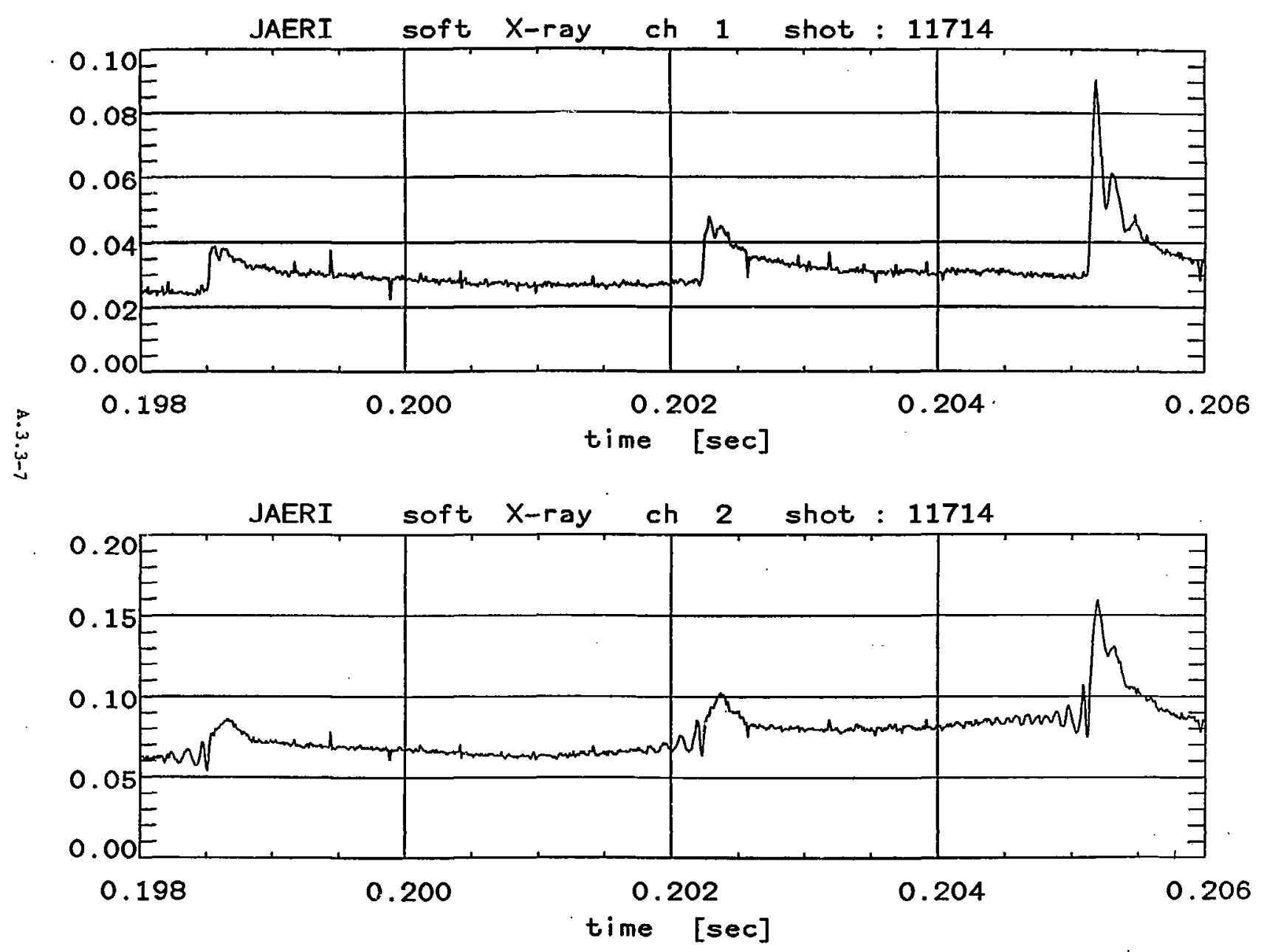

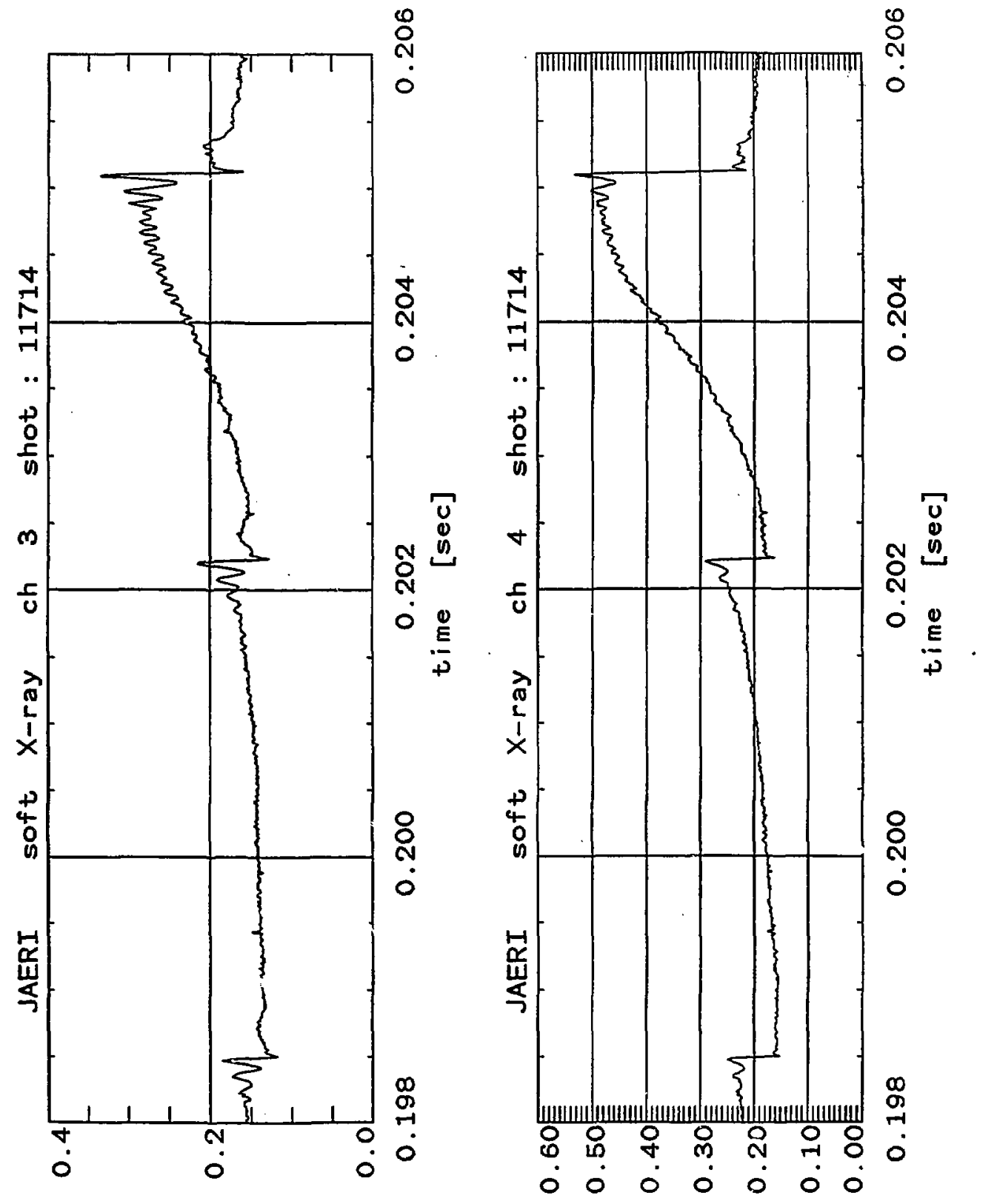

A. 3. 3-8 


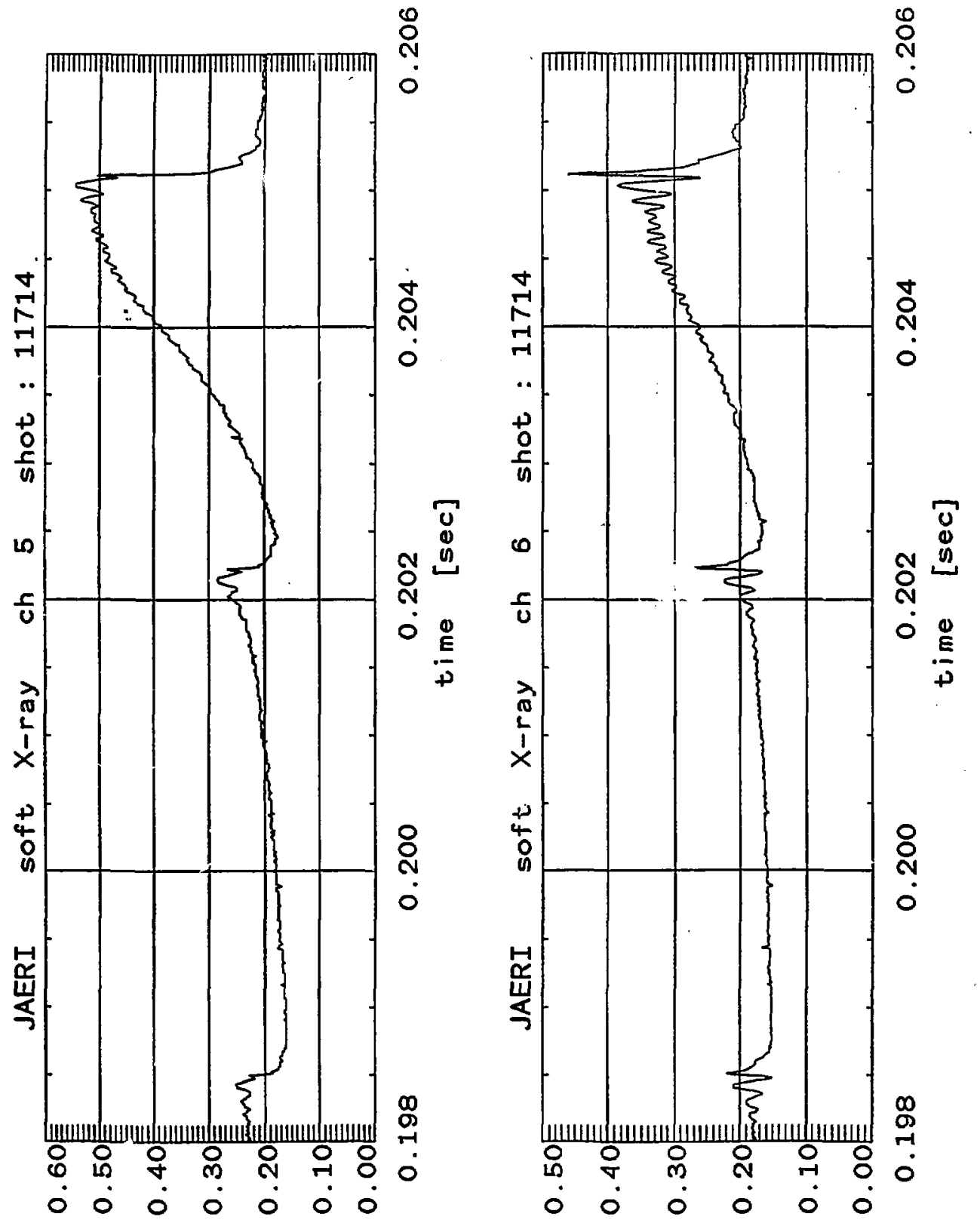




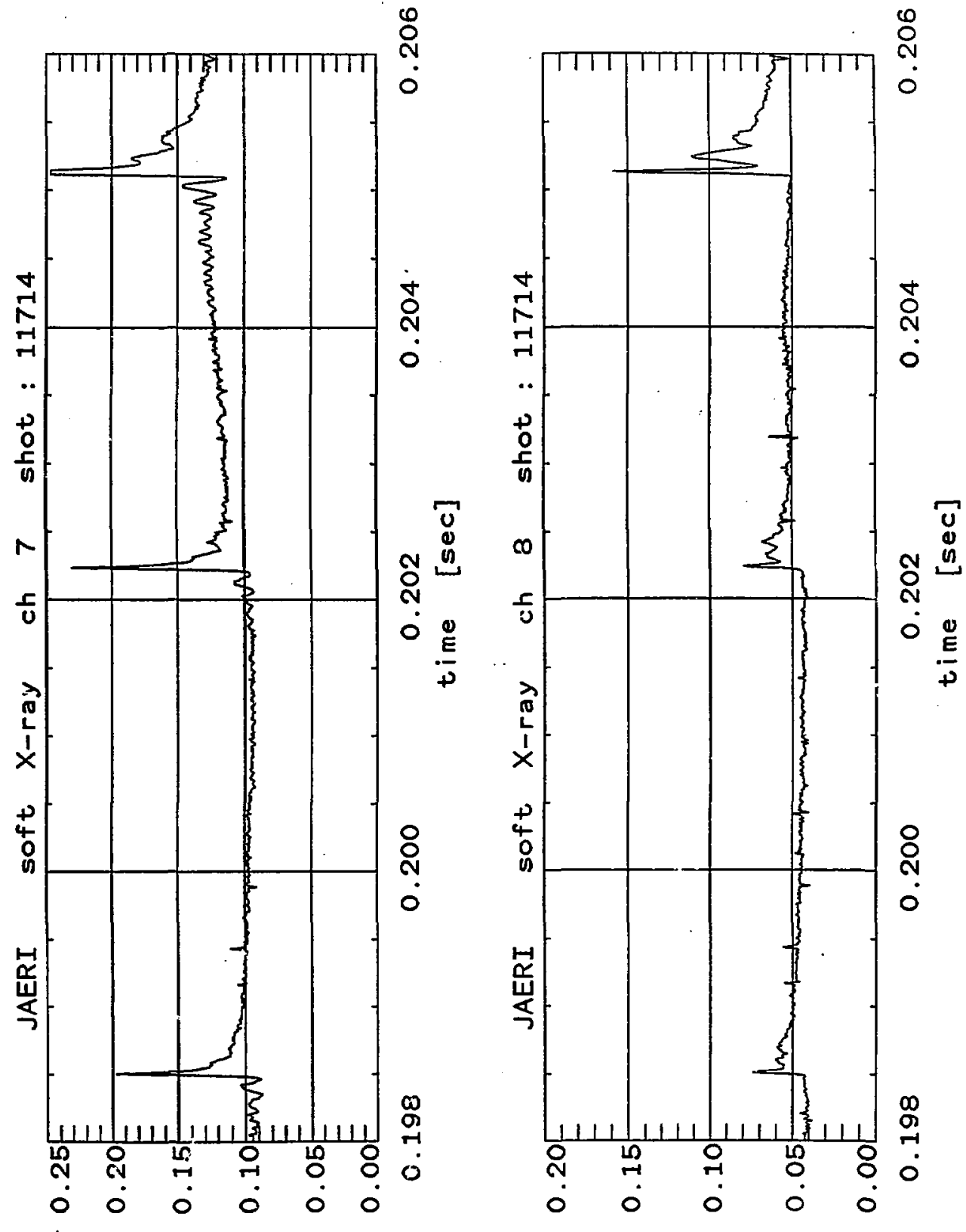

A. 3. 3-10 


\section{A.3.4 ECE Diagnostics (C. J. Lasnier)}

A.3.4.1 Viewing optics and wave guides. The Michelson Interferometer shared a wave guide wi h the ECE grating Polychromator, both viewing the plasma through the side E-port with no viewing dump in the tokamak. The line of sigit to the plasma was nearly horizontal, with a slight upward angle due to the wedged window at the port. $A$ lens at the port combined with another lens to form a Gaussian telescope, imaging ECE onto the opening of 2.75" ID plexiglas tubing acting as a dielectric wave guide. A rotatable mirror between the lenses was turned to view either the plasma or a calibration dump, with a wedged window and lens identical to those at the tokamak port (see Fig. A.3.4.1-1). The lenses, rotatable mirror, and the wave guide were all under vacuum induced by a mechanical pump.

A series of turning mirror blocks with vacuum seals transported the ECE radiation out of the vault through an opening under the North wall, to a polarizing beam-splitter in the hallway. This apparatus rotated the polarization of the incoming radiation by a selected amount, using a rotating rooftop mirror assembly. A wire-grid analyzer split the two E-field polarizations, sending one straight through to the Michelson, and one reflected to the Polychromator (Fig. A.3.4.1-2). Because of the extra mirrors in the subsequent transport to the polychromator, both instruments viewed vertically-polarized E-field at the Tokamak.

The heterody'ne receiver used two fundamental-mode $280-\mathrm{GHz}$ horn-mirror assemblies on the inner and outer walls of the tokamak at the B-port (Fig. A.3.4.13). The horns' viewing paths were nearly horizontal. The inner horn wave guide came out the top B-port, and the outer horn wave guide used the side B-port. The radiation was launched by stub WR-28 wave guides, and for used by a single lens at each port, into dielectric wave guide. The wave guides converged at a moveable mirror, which allowed the receiver to view either the inside or outside port. At the receiver, a lens matched the beam into the receiver.

The dielectric wave guide system for the heteroayne receiver suffered low throughput attributed to coupling losses at the input and output, and possibly not optimal alignment. Repeated alignments brought improvements, tut not to a satisfactory level. Th:e outside wave guide was not transmiting signal at the time the FEL data was taken bv the receiver. The inside wave guide worked well enough to provide evide ce of heating of a non-thermal electron distribution by the FEL (see section 5.2).

\section{A.3.4.2 Rapid-Scanning Michelson Interferometer. The Michelson} Interferometer (manufactured by SPECAC, loaned by General Atomics) had a stroke ler. gth of $1.6 \mathrm{~cm}$, and provided a clock pulse every $40 \mu \mathrm{m}$ of mirror motion (Fig. A 3.4.2-1). The mirror drive was by means of a voice-coil Ling vibrator. An InSb detector used : itially was loaned by GA, and had a liquid helin of $\sim$ I lay. This $w$ s replaced early on by an Infrared Labs cryostat and detector having a hold time of 6 days. 
Fig. A3.4.1-1

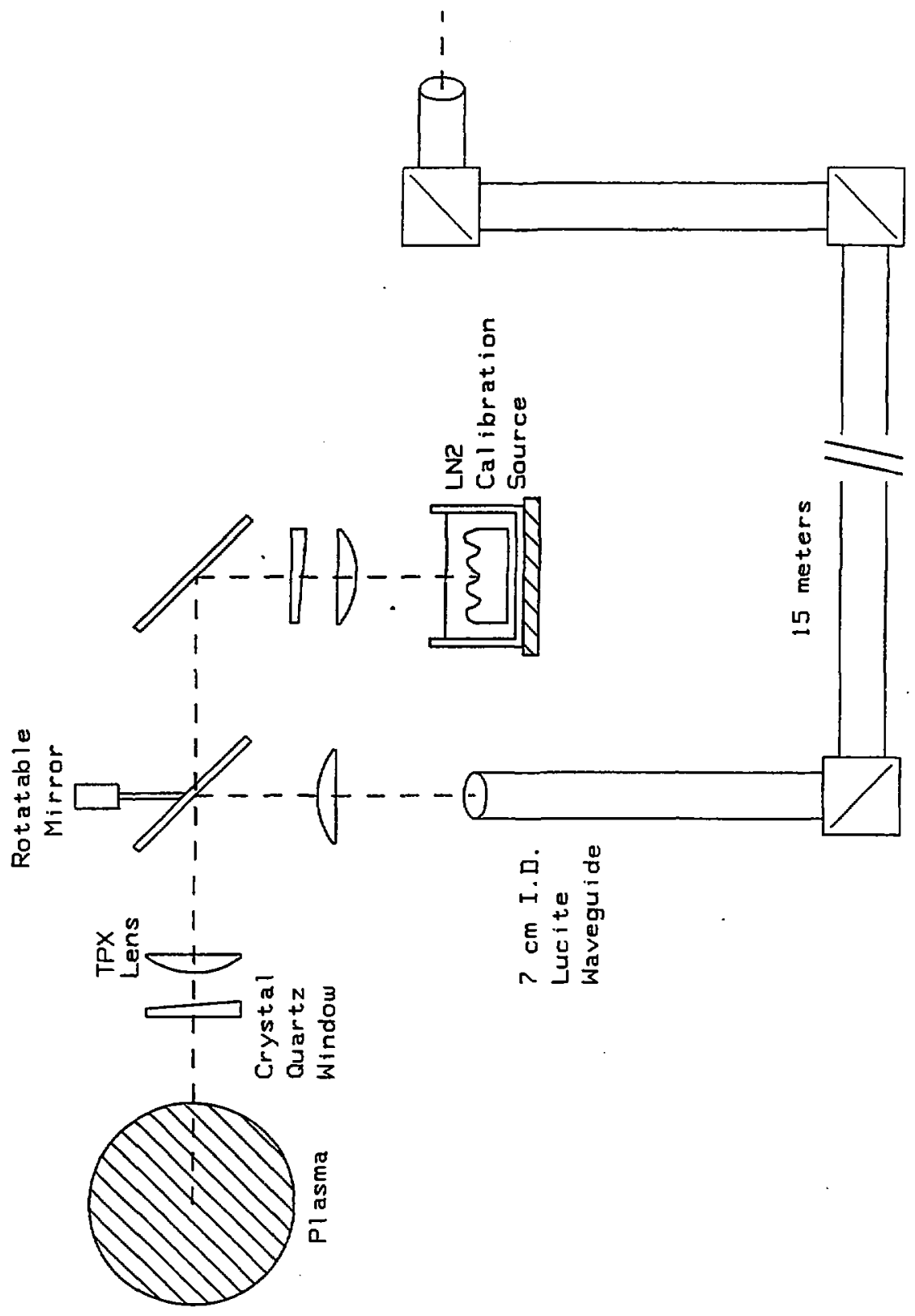

Fig. A3.4.1-1. Detail of ECE optics at the tokamak. 
BEAM-SPLITTER AND WAVEGUIDE FOR

UNIVERSITY OF MARYLAND GRATING POLYCHROMATOR

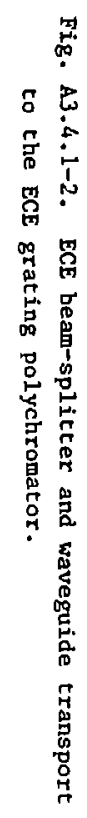

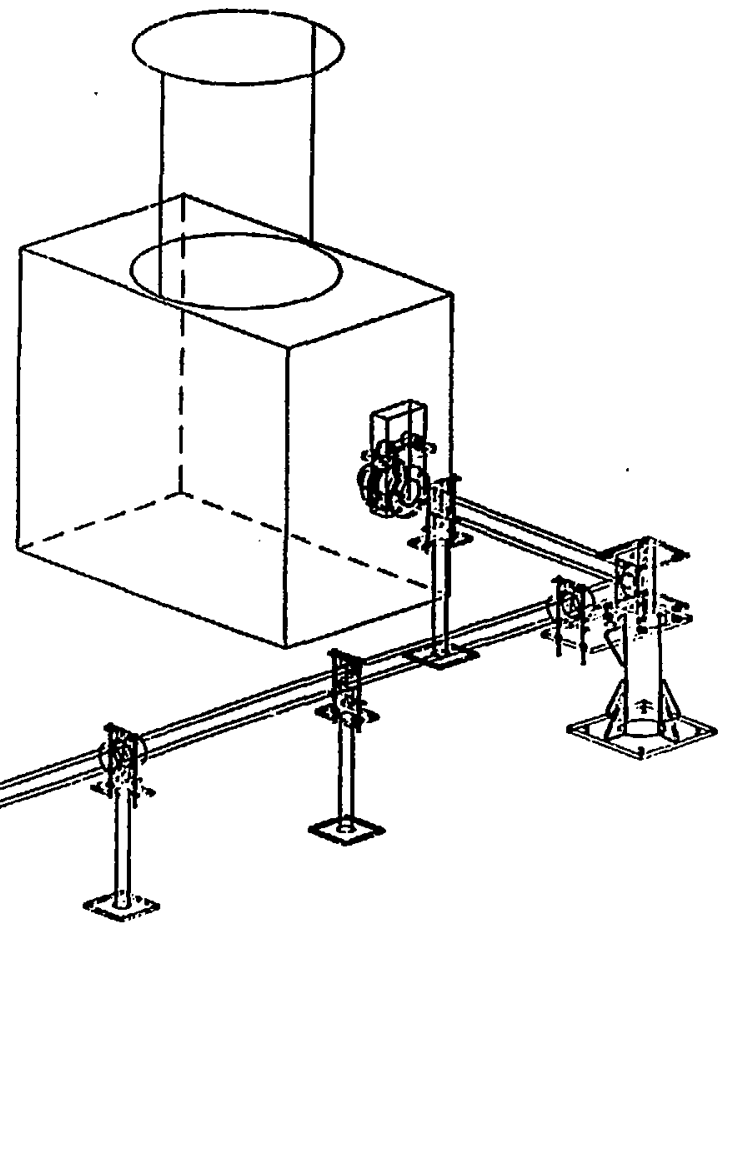




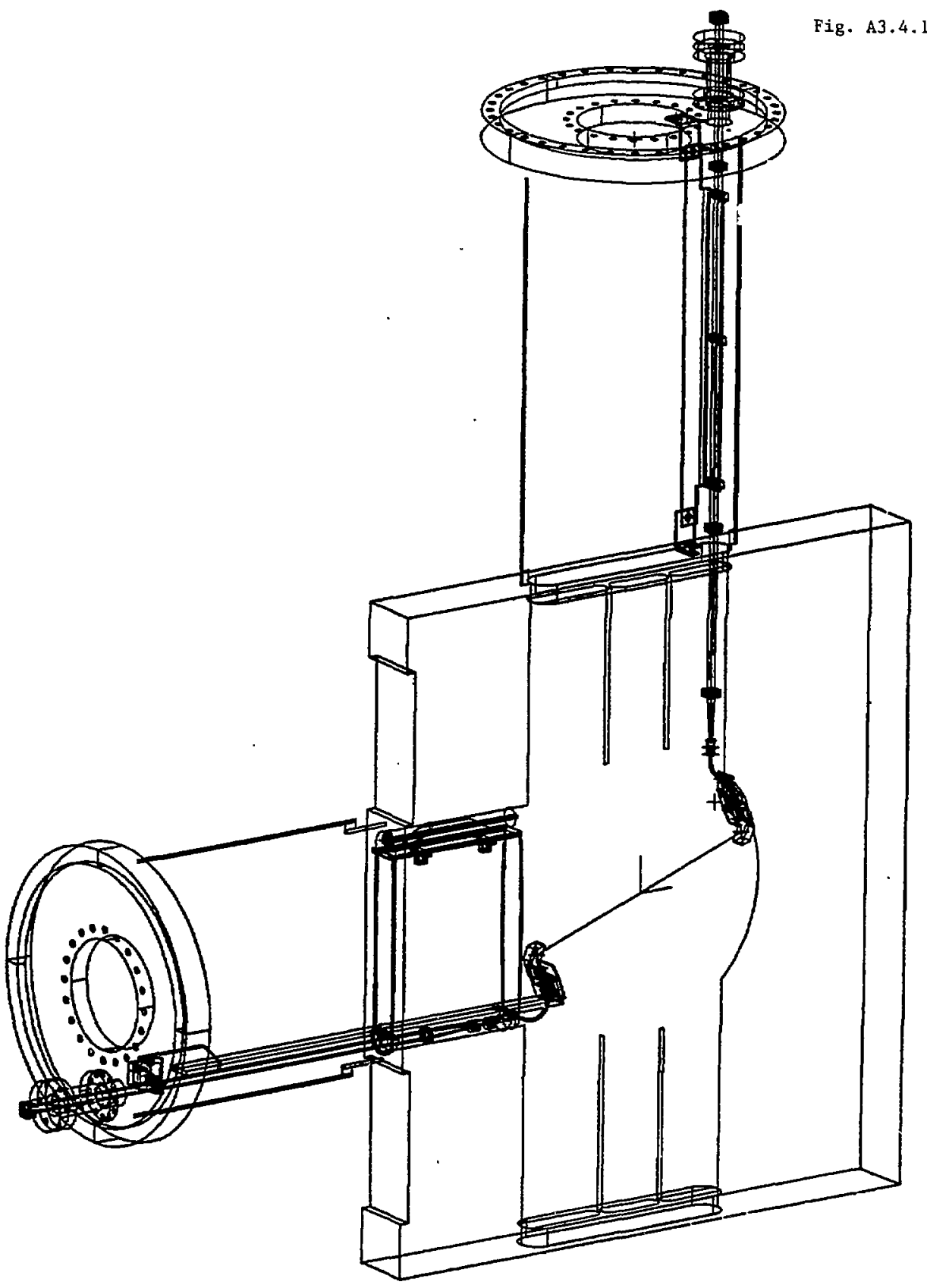

Fig. A3.4.1-3. Heterodyne ECE inboard and outboard horns. 


\section{ECE TABLE LAYOUT}

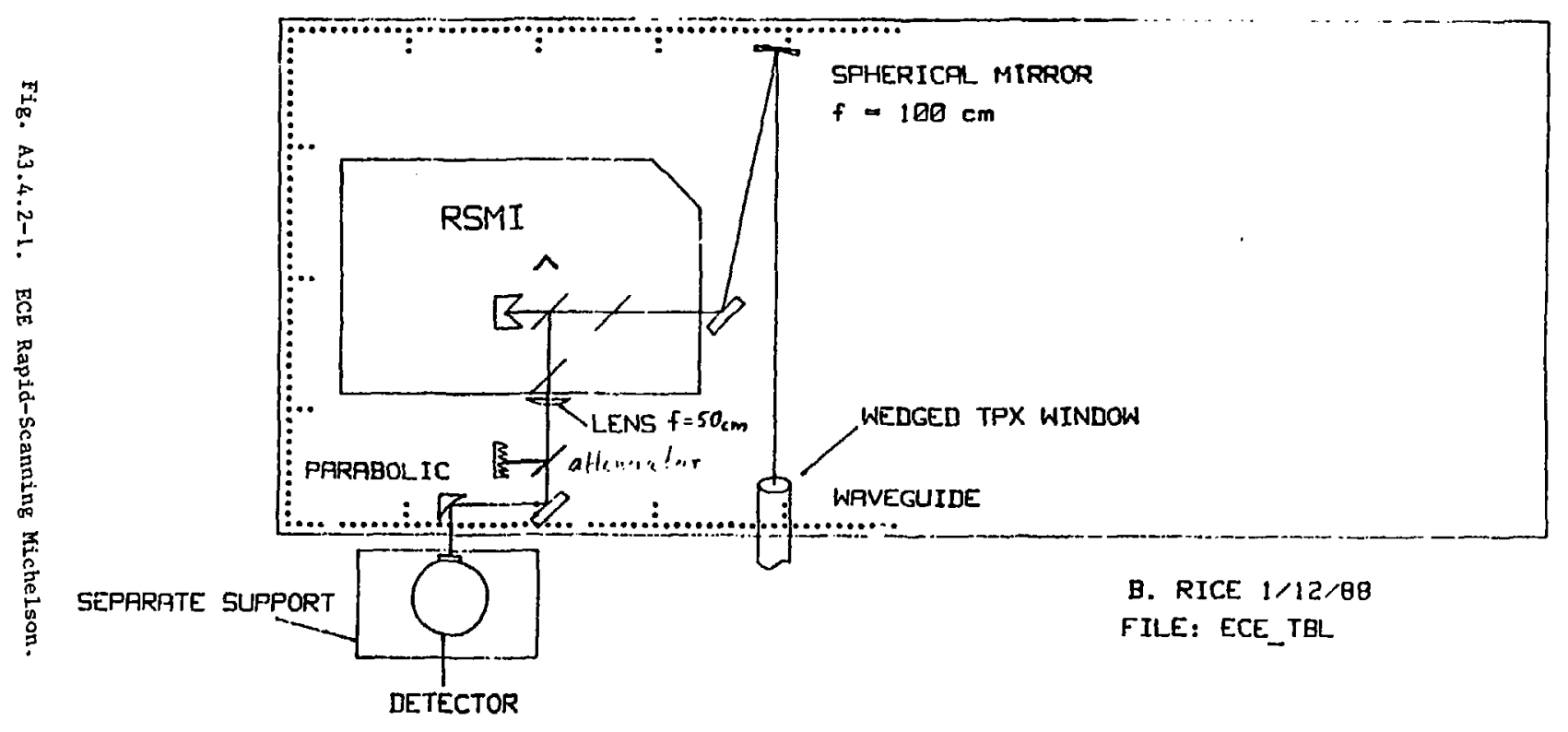


The detector signal was stored in one channel of a transient recorder, and another channel recorded the sweep direction signal. This recorder used the Michelson mirror position pulses as the clock signal. A second recorder also recorded the sweep signal, but used an MTX clock, so that the sweeps could be assigned a begin and end time in the MTX shot cycle.

The data was acquired and processed in a Hewlett-Packard Basic computer, with an Ariel board for the Fast Fourier Transforms. The processing algorithm was translated from the FORTRAN code used on DIII-D by the University of Maryland. the processed data was passed over the network to the VAX cluster for storage and analysis.

Calibration was done by repeated acquisition on signals using the transient recorders, while viewing a $L N$-temperature Eccosorb dump at the tokamak. This was done rather than using a signal averager, because the Michelson sweep length varied slightly during the scan, producing different numbers of clock pulses. This was later overcome using LED chopper-wheel sensors to produce a gate signal, which turned off the clock pulses outside the set scan length.

Absolute calibration agreemerit with Thomson scattering was elusive, so the temperature profile was normalized to the Thomson Scattering value.

A.3.4.3 ECE Grating Polychromator. The polychromator was designed and built by the University of Maryland under contract to LLNL (Fig. A.3.4.3-1). It was a 9-channel device, with slots in the cryostat for 10 additional detectors. The 9 installed InSb detectors were provided by QMC in an Oxford Instruments MD1840 cryostat. The detectors viewed through windows in the bottom of the cryostat. Three gratings were provided, of which Grating \#2 was the most used.

The liquid helium hold time of the cryostat varied from greater than 40 days to less than 1 day, which was attributed to poor filling technique. normally, it was topped off with liquid helium once per month. An automatic fill system kept liquid nitrogen in the cryostat. This was fed from a 160-liter storage dewar.

The body of the polychromator was kept under vacuum by a mechanical pump. The lowest pressure reached was $125 \mathrm{mT}$.

A separate vacuum chamber provided by LLNL for input optics contained a TPX focusing lens and a turning mirror, which could be replaced by one of three filter gratings. The input optics and gratings were provided by LLNL.

The detector bias circuits and pre-amplifiers were designed and built at the University of Maryland. The post-amplifiers and filters were provided by LLNL. The inside of the polychromator and input box were covered with Eccosorb at the end of the last run in attempt to reduce stray microwave pick-up from the FEL. This was only partially successful. 
Fig. A3.4.3-1. ECE Grating Polychromator.

Fig. A3 $4 \cdot 3-1$

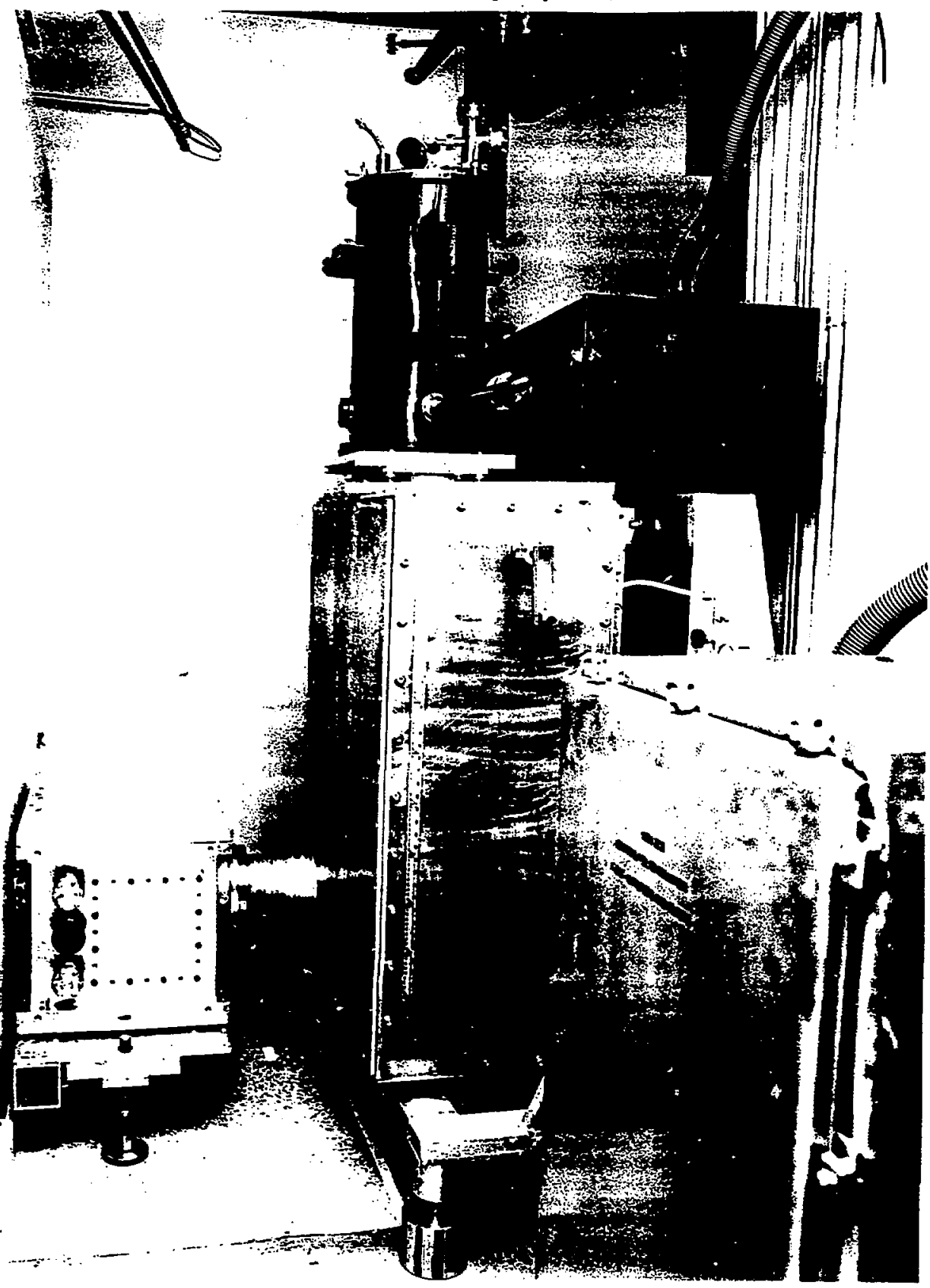




\section{A.3.5 Diamagnetic Loop (M. M. Marinak)}

A diamagnetic loop mounted at the center of an Alcator $\mathrm{C}$ bellows segment is used to measure the plasma diamagnetic flux, which is related to the perpendicular plasma thermal energy. Since the entire diamagnetic flux in Alcator $C$ is typically smaller than the contribution from the toroidal field by more than a factor of 1000 , unwanted contributions to the DML flux must be canceled to a very high degree of accuracy. The toroidal flux through the diamagnetic loop is canceled to first order through summation with the signal from a quadrupole Rogowski coil arr y which measures the total current in the toroidal field magnet. A summing circuit is employed which utilizes passive RC components to compensate for TF-induced eddy current contributions to the DML flux. The remaining signal is the sum of contributions from a number of sources in addition to the plasma diamagnetic flux: .

1. redistribution of current across the width of the toroidal field Bitter coils,

2. residual contributions from eddy currents in the bellows, flanges and toroidal field electrical bus caused by changes in the toroidal field current,

3. contributions from the direct coupling to the vertical field and ohmic heating coils as well as eddy currents generated by these coils, and

4. coupling to the poloidal field flux of the plasma. This contribution is estimated to be rather small for a properly centered plasma.

The linear transfer functions, which relate signals associated with sources of the above effecis to their resulting flux contribution to the DML output signal, were determined using multidimensional spectral analysis. Data from a number of magnet-only shots was analyzed to determine the components of the cross power spectral density matrix in eq. 1 and then solved for the desired transfer functions $\mathrm{H}$ in eq. 2.

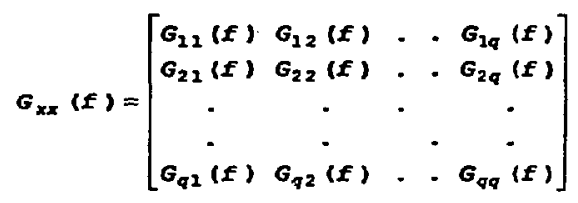

$B^{t}(f)=G_{x x}^{-1}(f) G_{x y}^{t}(f)$

(2)

With this method all of the desired transfer functions were determined 
with the necessary high degree of statistical accuracy, with the exception of the low frequency portion of the toroidal field compensation transfer function (below $5 \mathrm{~Hz}$ ). This low frequency portion of this transfer function reflects mostly the influence of the current redistribution across the width of the Bitter coils. The time constant for the fundamental mode of this transient is measured at some 2 seconds, in reasonable agreement with the calculated estimate. Because the cryogenic copper T.F. magnets undergo joule heating throughout the shot this transient progresses non-linearly throughout its duration. On MTX it is impractical to gather enough information to determine accurately a linear fit to this portion of the transfer function with the bandwidth ( $<1 \mathrm{~Hz}$ ) necessary to resolve features using the spectral method. Instead the low frequency portion of this transfer function is modeled with an expression of the form

$$
h(\omega)=\sum_{1} \frac{a_{1} j \omega \tau_{1}}{1+j \omega r_{1}}
$$

a summation of terms which simulate contributions from the current redistribution transient eigenmodes, with parameters determined by a least squares fit. The time constants $\tau_{l}$ in the fit expression are scaled together to compensate for changes in toroidal field magnet resistivity due to magnet temperature variations. As shown in Fig. 1 the required scaling ratio correlates well with the measured averaged TF coil resistance. The parameter fit is valid for small deviations about the reference case, such as those encountered during normal tokamak operation. The remaining linear transfer functions are of course valid for any current wave forms driven through the magnet system. These transfer functions succeed in canceling the contributions from items 1-3 above to a very high degree of accuracy. Remaining components at harmonics of $60 \mathrm{~Hz}$, due to noise from the power supplies, and at $37 \mathrm{~Hz}$ due to mechanical vibration of the bellows are removed with band stop filters.

In Fig. 2 the behavior of the total plasma thermal energy measured by the DML is shown during a typical shot 11925. As shown in table I the plasma thermal energy measured with the DML usually agrees with that calculated with the ONETWO transport code for a $-10 \mathrm{~kJ}$ plasma to well within $10 \%$. Fig. 3 compares the evolution of the toroidal beta measured during a current ramp in shot $\mathbf{1 2 4 0 6}$ with the value calculated with ONETWO.

Table I

\begin{tabular}{|l|l|l|l|}
\hline shot number & time (msec) & DML energy (J) & $\begin{array}{l}\text { ONETWO energy } \\
\text { (D) }\end{array}$ \\
\hline 13774 & 300 & 9500 & 8930 \\
\hline 13256 & 310 & 11550 & 11200 \\
\hline 13495 & 260 & 7500 & 7900 \\
\hline
\end{tabular}




\begin{tabular}{|l|l|l|l|}
\hline 13552 & 280 & 11450 & 11100 \\
\hline 13524 & 240 & 5800 & 5450 \\
\hline 14036 & 315 & 13200 & 12200 \\
\hline 13938 & 240 & 14000 & 13950 \\
\hline 13257 & 280 & 11450 & 11050 \\
\hline 14095 & 240 & 10650 & 9980 \\
\hline 13550 & 320 & 8700 & 8300 \\
\hline
\end{tabular}

Because the data analysis method employed places minimal reliance . upon filtering a time resolution of I msec is attainable when certain band stop filters are removed. This is limited by the time for the fields to penetrate the tokamak vessel wall. An example is shown in Fig. 4 where shot 12162 disrupts at 313 msec. A series of partial disruptions is visible on both the DML stored energy and channel 3 of the ECE polychrometer during shot 11993 shown in Fig. 5.

Figure 6 shows a rise in the plasma thermal energy during gyrotron heating in shot 11724. By scanning the toroidal field strength the radial location of the resonance position was varied across a number of shots with similar plasma conditions. The rise in stored energy during gyrotron heating showed little sensitivity to the radius of the microwave resonance zone for resonance positions within $\mathrm{r} / \mathrm{a}<0.5$. 
TIME CONSTANT MULTIPLIER VS. T.F. RESISTANCE

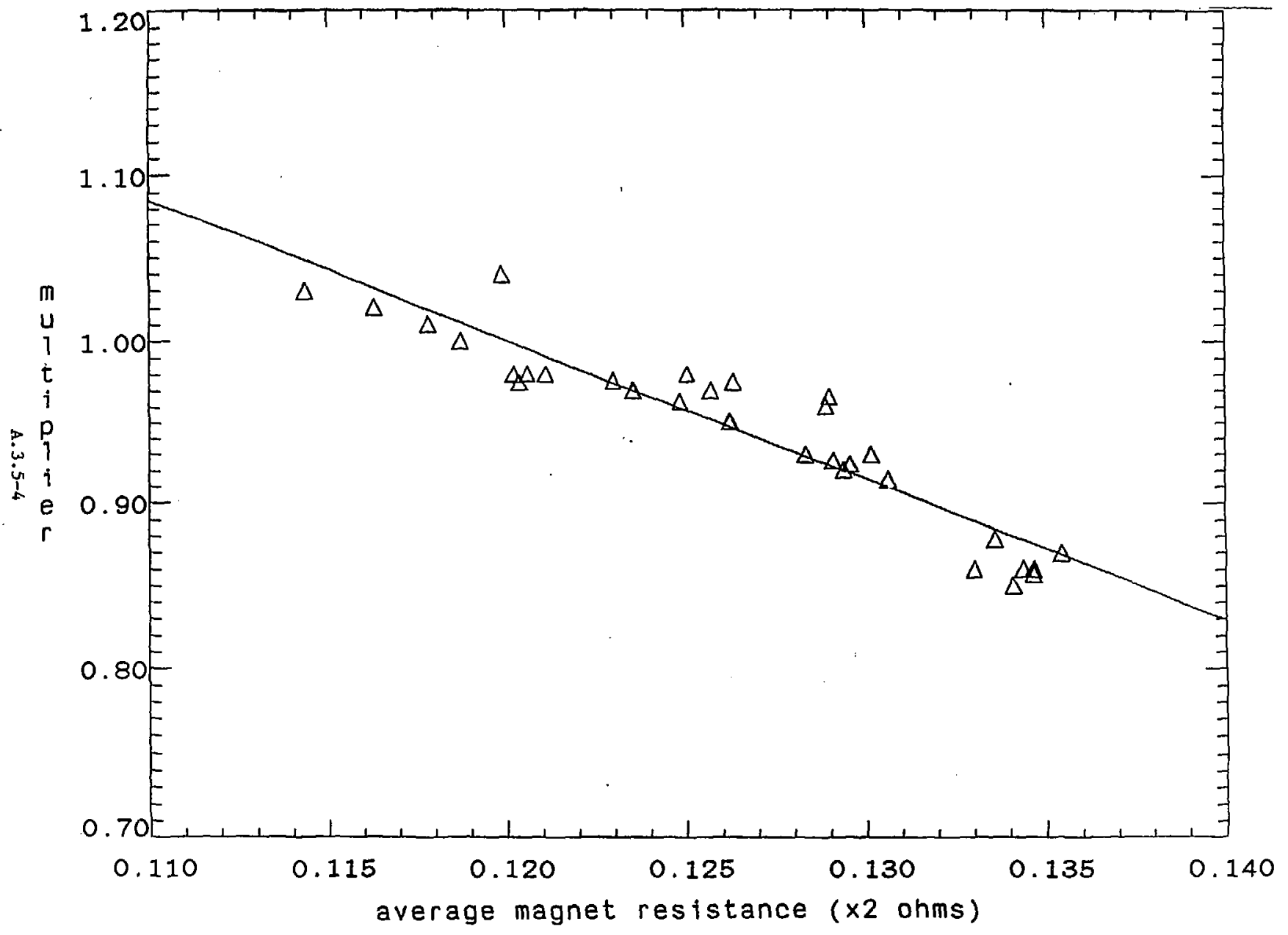

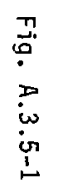


Fig. A.3.5-2

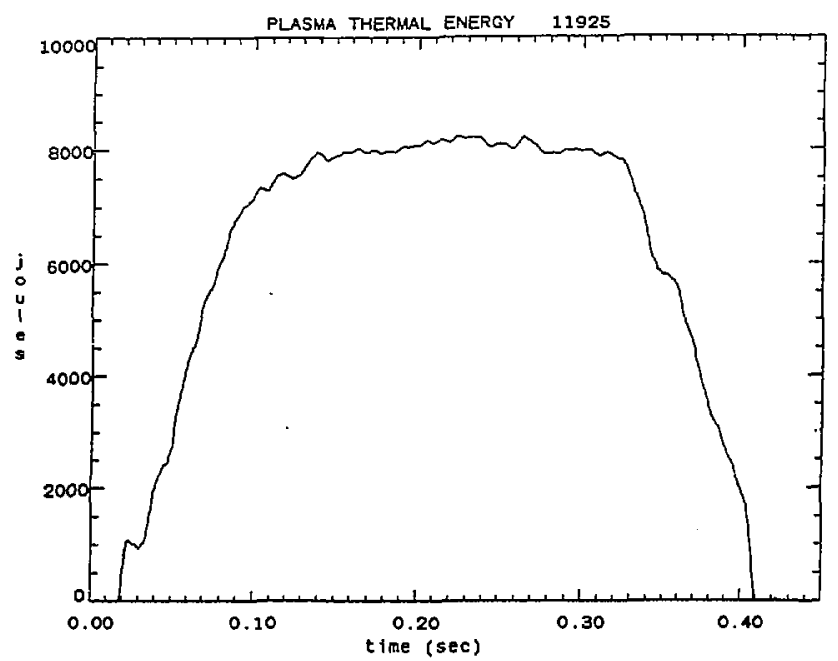

Fig. A.3.5-3

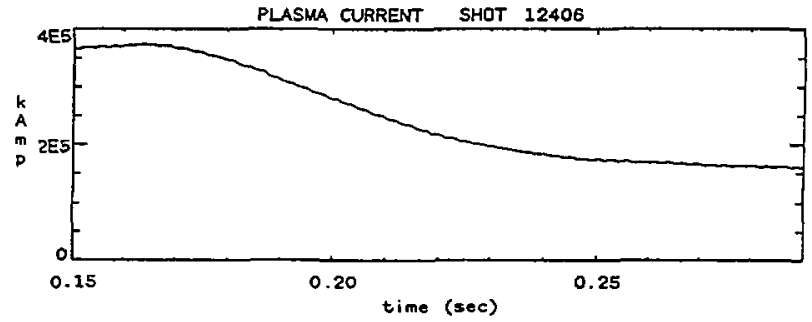

TOROIDAL BETA 5HOT 12406

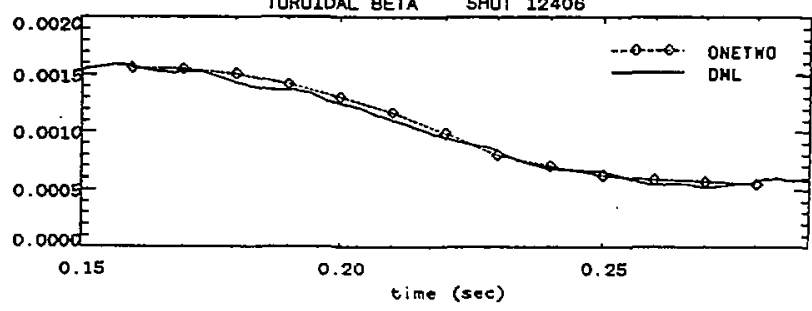

A. 3.5-5 
Fig. A.3.5-4

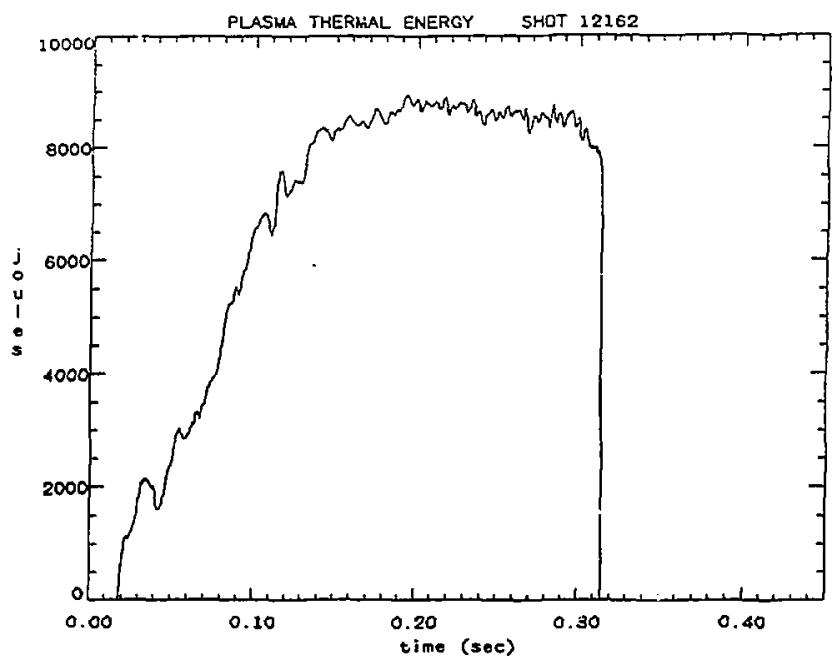

Fig. A.3.5-5
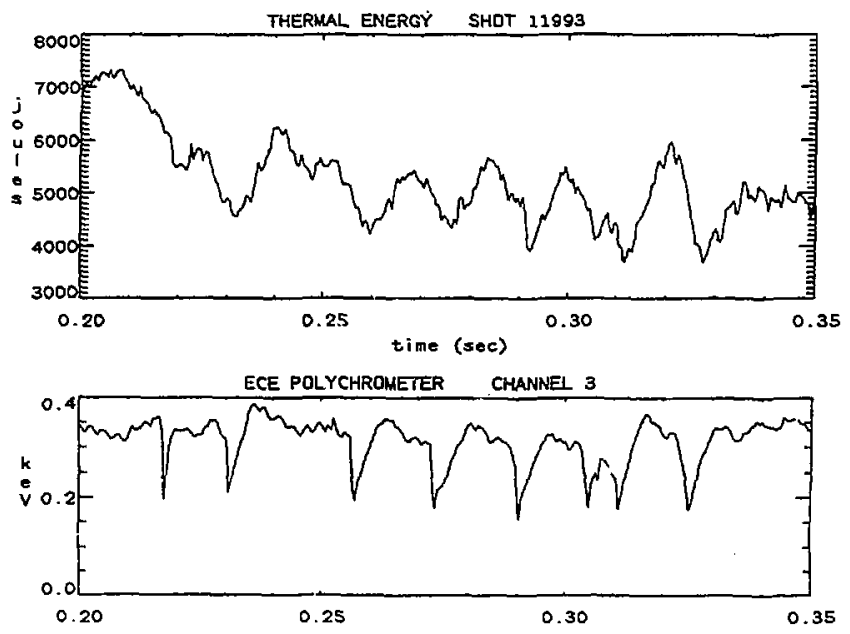
PLASMA THERMAL ENERGY 11724

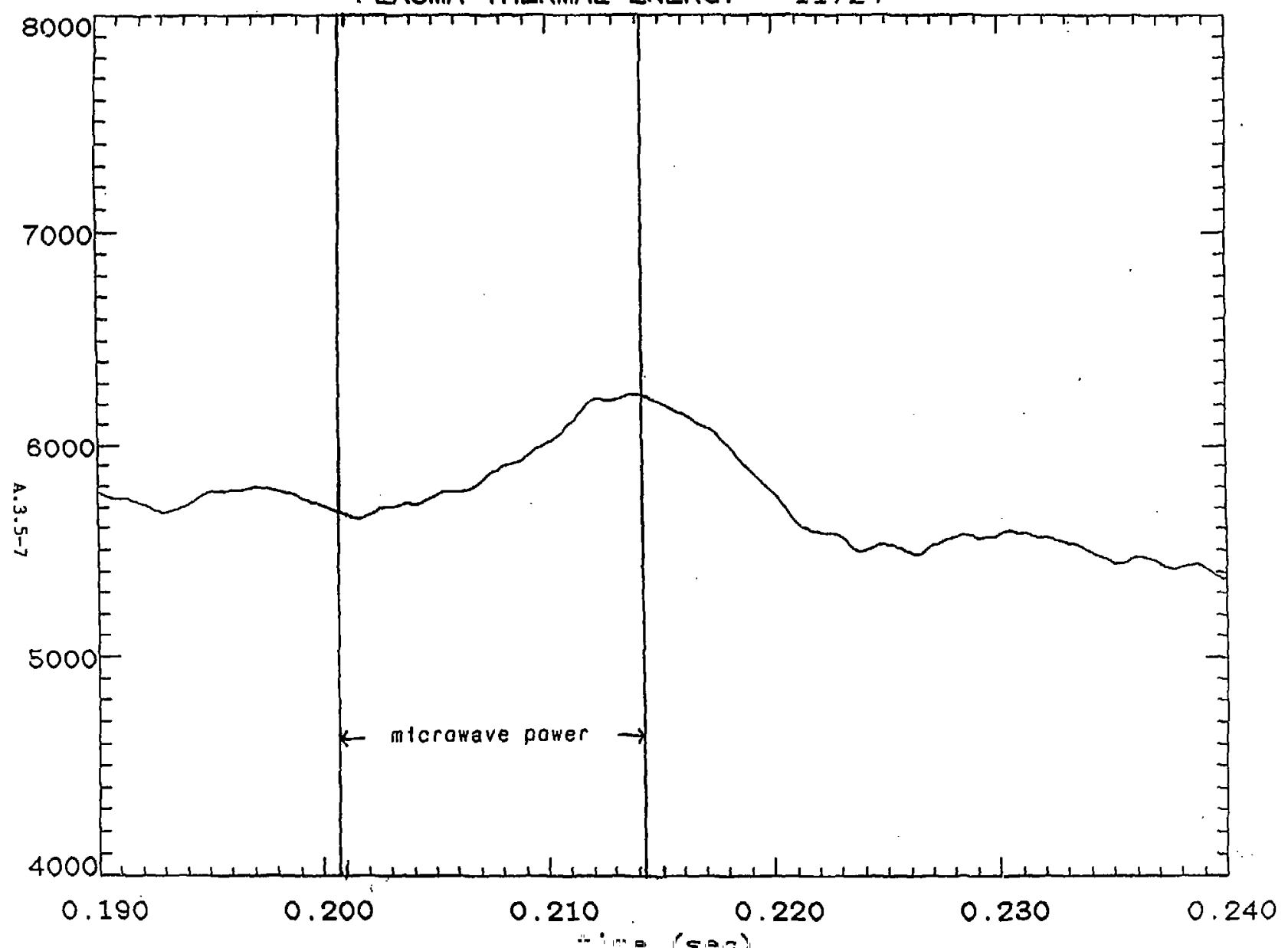




\section{A.4 MTX Data Acquisition System (T. A. Casper)}

The approach to the MTX data acquisition and analysis system is rather unique within the fusion community in that it employs a local area network (ethemet) of helerogeneous computers in a fully distributed environicent. Multiple vendors (HP; Sun, Solbourne, and DEC) and multiple operating systems (HPLX, SunOS, VMS, and HP-BASIC) are connected with an interprocess communications system (IPCS) in a cooperative computing environment. Software-driven synchronization of event times that follow a state transition model is implemented with message passing using IPCS as the underlyng mechanism. A single master timing process makes the connection between software and the MTX hardware timing system. This IPCS-based concept for synchronization was extended to the MAESTRO FEL controls interface and provided both the final solution to the real-time control of the FEL and its data-connection with the tokamak.

Most data are acquired from CAMAC modules although some special purpose systems are integrated as needed, for example, gigasample/sec transient recorders for FEL pulses, the inside wall tokamak calorimeter, and a SPRED spectrometer. We use LLNL-developed software for our UNDX-based data acquisition. The UNDX systems have an integrated data analysis package for intershot and interactive processing (based on a locally modified version of LLNL's VIEW image processing package). For data acquisition on our VAX cluster we use MIT's MDS software which was used for the previous AlcatorC operation. Typically, the IDL software (Research Systems, Inc., Boulder Co.) is used for analysis on the VAX cluster.

The distributed architecture provides performance and flexibility. The data are distributed over multiple files with data location information stored in a data base for archival and retrieval. The UNIX systems are autonomous but connected over a LAN and synchronized with themselves and the VAX cluster via IPCS. Processes on the various nodes perform intershot analysis with information passed transparently between the systems. For example, processed results from analysis on the UNIX machines can be written to the MDS data system. Our software and commercial products like IDL typically function with an IPCS interface to provide for real-time operations without operator intervention.

Technical aspects of the MTX data system have been described at technical meetings and, therefore, reports describing its development and operation already exist. We reproduce three of these reports in the following sections to make this final report a self-contained document:

- A.4.1 MTX Data Acquisition and Analysis Computer Network, UCRL-JC103311; also, in a somewhat reduced form but with the same title, as D.N.Butnes, et. al. Rev. Sci. Instr. 61, 3277 (1990). This section describes the overall computer system and our operational experience in most generality. 
- A.4.2 Integrated, Multivendor Distributed Data-Acquisition System by D.N. Butner, et. al., in Rev. Sci. Instr. 59, 1786 (1988). This publication provides a description of our interprocess communication system.

- A.4.3 Diagnostic Data Management on MTX by D. N. Butner et. al., in Proceedings of the 14th Symposium of Fusion Engineering, October 1992, IEEE 91CH3035-3(1992), Vol.2, p.847. This publication discusses the file/information flow within the MTX data system.

While there is some repetition of information in these articles, we felt it was best to keep them as complete, self-contained sections without rewriting. The final section, A.4.4, discusses the MAESTRO system which has not be published as a separate article to date. 
A.4.1

MTX Data Acquisition and Analysis Computer Network*,

D.N. Butner, T.A. Casper, M.D. Brown, M. Drlik, W.H. Meyer, and J.M. Moller

Lawrence Livermore National Laboratory, University of Califorria,

Livermore, CA 94550

\section{abstract}

For the MTX experiment, we use a network of computers for plasma diagnostic data acquisttios and analysis. This multivendor network employs VMS, ONIX, and BASIC based computers connected in a local area Ethernet network. Some of the data is acquired directly into a VAX/VMS computer cluster over a flber-optic serlaj. CAMAC highway. Several HPUnix workstations and AP-BASIC instrument control computers acquire and analyze data for the more data intensive or specialized diagnostics. The vax/iMS system is used for global analysis of the data and serves as the central data archiving and retrieval manager. Shot synchronization and control of data flow are implemented by task-to-task message passing using our interprocess communication system. The system has been in operation during our initial MTX tokamak and FEL experiments; it has operated reliably with data rates typically in the range of 5 megabytes/shot without limiting the experimental shot rate.

*Work performed under the auspices of the U.S. Department of Energy by the Iawrence Irivermore National Laboratory under contract number W-7405-ENG-48. 


\section{A General Purpose Data Acquisition System}

We have developed a shot based, general purpose data acquisition and analysis system for experiments at LLNL. The major functions this system provides include collection of experimental da:a using a heterogeneous network of computers, distributed intezshot processing over the network, automated, centralized arctiving of all data, and easy access to data for users and programs. This data acquisition system is guite flexitle with hardware and software configurations easily reazzarged and distributed among the various computers as required.

The keys to our system's generality are its ihot coordination scheme, its ability to communicate transparently among diverse computers and processes, and adherence to standardization where possible. The shot coordination scheme relies on the existence of software subsystems and a shot model that specifies a logical progression of states during acquisition and processing. The shot model is implemented by a supervisory program with diagnostic subsystem programs executing concurrently as a state machine. We achieved transparent communication by developing a portable Interprocess Communication System (IPCS) software package for passing messages and data between tasks that reside anywhere in a network of computers. 1

The system's flexibility includes its ability to run on a heterogeneous network of computers. This flexibility provides for easy expansion and was achleved through the use of modular programing techniques, IPCs, and portable computer languages. Use of IPCS allows the external interface of a program to remain the same even when internal functional changes are made. Most of our programs are written in the $C$ language, which allows us portability between VMS and UNIX computer systems. While our existing UNIX workstation inventory consists only of HPUX systems, much of our software has been run on SUN workstations and is readily portable to most mix based platforms.

One advantage of expandability is the ability to add new computers, diagnostics, and processing and to support multiple experiments. Reliance on commercial networking and the IPCS communtcation standard simplifies the integration of these new subsystems. Since we can move programs transparently around the network, our ability to add new computers to the network provides immediate use of the additional computational power. In addition, the system's flexibility and expandability allow us to distribute the intershot processing among the various computers in our network.

An important feature of the system is "transparent data access". which means that a program running anywhere in the computer network can access data stored anywhere in the network without knowing where the data are. To support 
transparent data access, we have a centralized data base that contains the current location information for all data whether on-line or off-line. We use a commercial data base manager to maintain the data location information. We have also integrated a commercial archiving system into the data transparency scheme. Transparent data access not only refers to the ability to find data anywhere, but also to the fact that subroutine calls to access the data and their arguments are invariant, regardless of location.

This general solution to data acquisition and analysis

has been applied to the Microwave Tokamak Experiment (MTX) ${ }^{2}$. It has also recently begun to support operations on the Ring Accelerator Experiment (RACE) and the High Eleld Test Eacility (HFTF) at LINL. We will now concentrate on details specific to the MTX implementation.

\section{Requisition syster Configuration}

The MTX Data Acquisition System us=s Digital Equipment Corporation (DEC) VAX computers located in our User Service Center (USC) running the VMS operating system, HewlettPackard UNIX (HPUX) workstations and HP-BASIC control computers, as indicated in Figure 1. Use of. these computers takes advantage of the much of the hardware investment made during the Tandem Mirror Experiment (TMX) program at LLNL 3 . The USC VAX cluster consists of three computers with redundant HSC hardware and approximately 6 gigabytes of disk storage. SMAUG, an 8200 , is the primary VAX data acquisition computer and is connected by a serial fiber optical highway to the MTX CAMAC hardware in the experimental area, approximately a quarter of a mile from the USC. IIL, an 8600 , is used for automated intershot processing and plotting and interactive analysis. In addition, LLI must support the standard USC tasks required for offlce operations and NERSC connection. ILV, an $11 / 780$, is used to maintain experiment status displays and to meet the needs of the administrative staff. Except for restrictions based on hardware connections

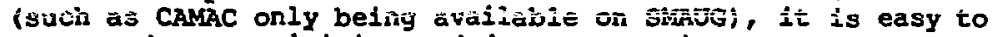
run our data acquisition and data processing programs on any of the three computers.

The HP computers, located both in the office building and in the experimental area, are connected to the USC via a fiber-optic local area Ethernet (LAN). Nine HPUX workstations are presently connected directly to the LAN and communicate with the VAX computers via the Internet TCP/IP network protocol. These autonomcus workstations typically have 8mytes of memory and 200mbytes local disc storage for the operating system and data. Processes running on these computers can communicate directly with processes running on the VAX computers via IPCS. Data acquired into files on the UNIX computers are easily moved to the VAX computers using -FTP or IPCS messages. 
We presently have seven HP-BASIC controllers in the acquisition system. Due to their lack of networking capabilities, these computers cannot be connected directly to the network. Instead, they are connected to a $400 \mathrm{Mbyte}$ shared disk system called the Shared Rescurce Manager (SRM) for data and message storage. A dedicated HPUx computer, DALE, acts as a communication gateway between the HP-BASIC computers and the other computers using files created on the SRM.

The DEC terminal servers located in the office building and in the experimental area allow users in either place to connect to the VAX computers. The TELNET function provides a direct connection between UNIX and VMS computers with FTP providing file transfers.

In the development of the MTX system, we integrated software from various sources: commercialiy developed software, software developed at national laboratories, and software developed at universities. We have also relied heavily on standardization and modularity for flexibility.

We use the $\mathrm{MDS}^{4}$ data acquisition software developed at MIT for our VAX/Vis acquisition subsystem. It acquires data from CAMAC modules using the ORNL drivers and stores the data in files. MDS allows a diagnostician to specify the types of modules used in a system, the pre-shot and post-shot commands for each module, and the files into which the data from each acquired signal are stored. MDS utilizes a signal naming convention that has been adopted as our defacto standard.

Our HP computers allow for more complex interactive control of diagnostics than does MDS. Acquisition in the HPDX workstations utilizes VIEW5, an LLNL-developed image processing software package, for analysis and database tools with our own IEEE -488 based CAMAC acquisition software. It utilizes a command based interface with English language syntax to provide acquisition and processing procedures. HPBASIC acguisition was implemented by standardizing, upgrading and extending (to accommodate IPCS messages) our earlier versions of $\mathrm{HP}-\mathrm{BASIC}$ acquisition software.

Each of these acquisition subsystems, MDS, HPUX, and HPBASIC, are fully integrated into the MIX data system via IPCS. Most intershot processing is completed with elther Interactive Data Language (IDI, a commercial product) on the VAX or with VIEW or the HPUX. Special purpose software is required for native analysis on the HP-BASIC computers. However, a significant portion of the data produced on these computers is stored in MDS data files as part of the intershot processing. The data is then available for further analysis on the vaX cluster.

\section{System Operation}

We show a diagram of the data acquisition software in Figure 2. The bubbles represent processes or sets of processes running on various computers. Many of the 
processes may run unmodified on any of several computers; many processes run with no source code changes on either VAX computers or HPUX computers. Most of the interprocess connections shown in $E$ igure 2 are implemented using IPCS. Control server programs provide access to commercial codes or to processes that do not know about IPCS. For example, MDCONTROL communicates with MDS using MDS events (VMS locks). MDSCONTROL thus provides an IPCS interface to MDS for communication with processes running on other computers. HPCONTROI provides a similar function for the HP-BASIC computers and provides IPCs message and data file transfer via the SRM.

The supervisory program, SHOTSYNC, which implements the shot model state machine, provides the shot coordination interface between the varjous diagnostic subsystem control programs. HPCONTROL On DALE, MDSCONTROL on the VAX computers, and the acquisition processes on the HPUX computers translate SHOTSYNC's state transitions into actions for their respective subsystems. A supervisory data base program, DESERVER, coordinates the collection of data location and shot configuration information.

SHOTSYNC accepts commands from the experiment leader via the SHOTLEADER user interface program. The shot supervisory program complies a list of diagnostic subsystems, supervisory dat bases, and various status-monitoring programs and coordinates their activities according to the state diagram. Much of this information is displayed by the SHOTLEADER program display as is shown in Figure 3. SHOTSYNC has no prior knowledge of what subsystems will be part of a shot. Instead, we use a dynamic check-in technique by which programs to be coordinated notify SHOTSYic that they need to receive shot coordination messages. SHOrSYNC maintains a list of checked-in diagnostic subsystems, and each subsystem remains active until it dies or is checked out by the experiment leader or diagnostician. The diagnostic subsystem status information is visible in the display shown in $F$ igure 3. For each shot, SHOTSYNC supplies the list of participants and other shot information as a package to the data base server at the times required by the state diagram. Thus we automatically record the actual shot configuration. Database utilities are available for subsequent interrogation to determine the experiment configuration and data location and availability for a given shot. A diagnostic subsystem or any other process that wants to synchronize its operations with the shot cycle must send a check-in message to SHOTSYNC and must respond as appropriate to shorsync's shot coordination messages.

The MASTERTIMER program provides the interface between SHOTSYic and the MTX timing system; it senses the times at which system hardware events occur. It is the single interface between the data acquisition system and the hardware timing system. The SHOTLEADER status display, Figure 3 , shows the existing state of acquisition and 
provides the necessary interface software for control as the data system proceeds through its shot model.

\section{Data Flow}

The data subsystems (MDS, HPUX, and HP-BASIC), upon completion of data aculisition into their local data bases, notify DBSERVER of the files generated. DBSERVER provides the IPCS interface to an ACCENT-R database on the VAX containing all the shot status information. A generic store/retrieve programming interface provides the transparent data access. Data are referred to by logical nine and there is a standard subroutine call interface. Nonlocal data are retrieved by interprocess communications with a database server, DATA DAENON. At present DATA DAEMON knows only how to store into and retrieve data from $\overline{\mathrm{MDS}}$ data bases. However, since DATA DAEMON is an IPCS-using task, it can be reached from any of the networked computers. Presently, VIEW processes (on HPUX systems) utilize this method for efficient exchange of data with MDS in support of automated intershot processing tasks comblning VMS and UNIX acquired data. For intershot processing in VMS using IDL procedures, UNIX data is typicaliy first written to MDS with the help of DATA DAEMON. Future versions will handle other data. subsystems and will perform more complex searches, thus eliminating such unnecessary intermediate steps as the writing of data to MDS above.

Currently, some highly processed data from the hP computers is stored in MDS files shortly after a shot occurs. The raw data from the HP computers is stored locally during operations. Later (i.e. overnight) it is compressed and moved to files on the VAX cluster via FTP for automatic archival. All raw data and some processed data from both the VAX computers and the HP computers are archived using a commercial archive product that provides for retrieval of data by filename.

Due to the parallel architecture of this system, the overall shot cycle time is determined by the slowest element. Presently, the dedicated VAx8200 running MDS requires approximately 1.5 minutes to acquire about 3 Mbytes of data per shot. An additional 2 Mbytes of processed data are received from workstations asynchronously. Since unIX and BASIC workstations have (so far) remained dedicated to specific instruments, the actual cycle time utilized for acquisition is minimal with the majority of the intershot time used for analysis. For example, the FIR interferometry system requires about 15 seconds for acquisition of data from 15 channels at 8 kwords/channel. The line averaged density is calculated, displayed locally, and a copy sent to MDS within 30 seconds of the shot. Local data storage on disc and hard copy plots (used for data verification and searches) requires an additional 2 minutes. At the end of this 2.5 ininute period, a "ready" signal is sent to the supervisory program thus allowing for another shot to start if all other 
systems are ready. This status information is displayed locally, Figure 3, from which any needed performance improvements are readily identified. About 2 minutes adcitional time is used for (interruptible) profile calculation and display. Any time-critical, uninterruptible work has been maintained at somewhat less than the cycle time determined by the cool-down time of the high field coils in MIX (> 3.5 minutes for tokamak operation at field strengths > 5T) Any additional processing load is scheduled on the vax 8600 and is allowed to lag behind the current shot.

\section{v. Summary and Future Fork}

Presently, our data volume is split about half from us and half from HP, most of whlch is HPUX. Many of the acquisition and processing tasks can run on either system with only special "commerclal" applications confined to a particular computer. The modularity allows for smooth migration of selected portions of the system to other computers as might be desired to take advantage of the rapidly evolving workstation industry. The application of distributed data acquisition and analysis has proved quite useful for off-line diagnostic development and for integration of visitor's diagnostics into our acquisition systen. The lack of single point catastzophic failures has provided a fairly robust system which can be operated (at reduced capacity) in the event of partial system failure. The reliasce on commercial products, local area networking, and IPCS has given us a flexible and reliable system maintained by only a few people. We anticipate the possibility of evolution towards a full unIX-based implementation in the future; this would, however, require replacement of several of our commercial products and of MDS with their UNIX equivalents.

He have designe: extensions to the present system that will provide fully transparent access to all data by signal name (for compatibility with MDS), whether the data exists in a file on any computer or on an archive tape. The Implementation depends heavily on the creation of data base data sets that define signal names and their related files and on data base server tasks that provide such information to any process running anywhere in the network. We designed our present system with these extensions in mind, thus providing for an evolutionary implementation. We are now concentrating on implementing additional utilities to facilitate the transparent data transfer, to provide more analysis tools, and to re-implement our journal file, a reduced resolution on-line database for multiple shot statistical analysis. 


\section{References}

1. G.G. Preckshot and D.N. Butner, IEEE-Trans. Nucl. Sci. NS34, 858 (1987).

2. K.I. Thomassen, presented at the $14 \mathrm{th}$ Conference on Plasma Physics, St. Andrews, Scotland, 1-3 July 1987.

3. T.A. Casper, H. Bell, M. Brown, M. Gorvad, S. Jenkins, W. Meyer, J. Moller, and D. Ferkins, Rev. Sci. Instrum. 57, 1880 (1986).

4. T.W. Fredian and J.A. Stillerman, Rev. Sci. Instrum. 57, $1907(1986)$.

5. J.M. Brase, V.J. Miller, and M.A. Wieting, LLNL UCID21368, March, 1988 . 


\section{Figure Captions}

Figure 1. Hardware configuration for the MTX data acguisition and analysis network.

Figure 2. Flow diagram of MTX data acquisition software; circles

represent tasks, arrows are communication/data messages, parallel lines indicate data files, and rectangles represent hardware (except possibly for the shot leader).

Figure 3. Typical state of the SHOTLEADER interface display: allowed interface functions appear in the left box while the right box shows the diagnostic conflguration status (systems "checked in") ard the present shot status for the MTX project. 


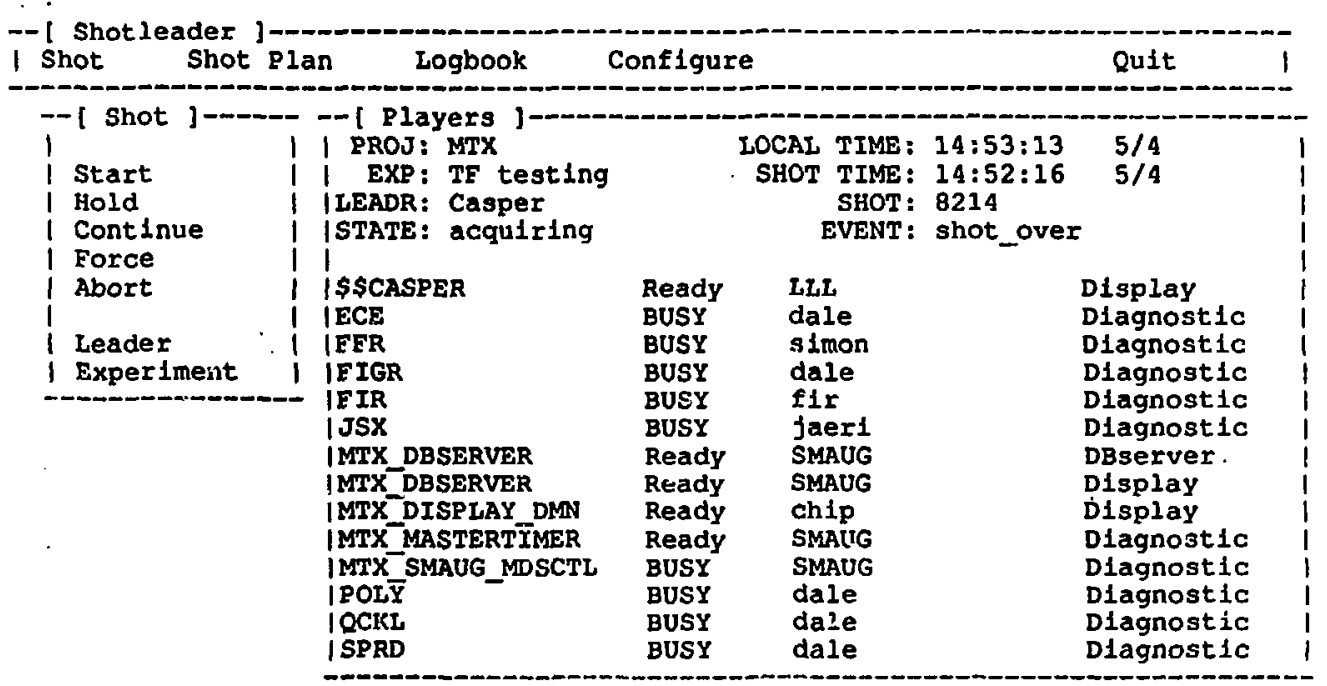




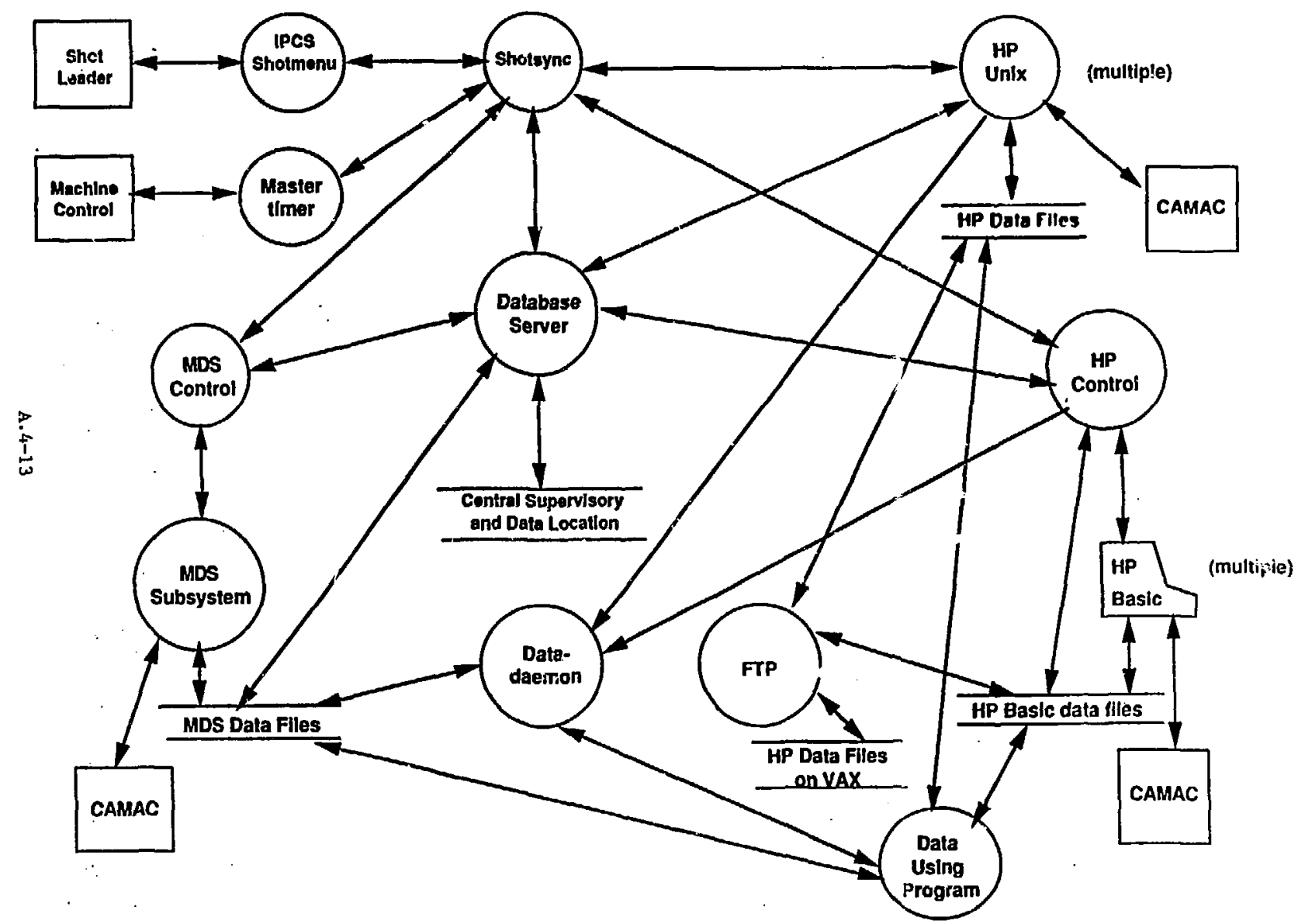




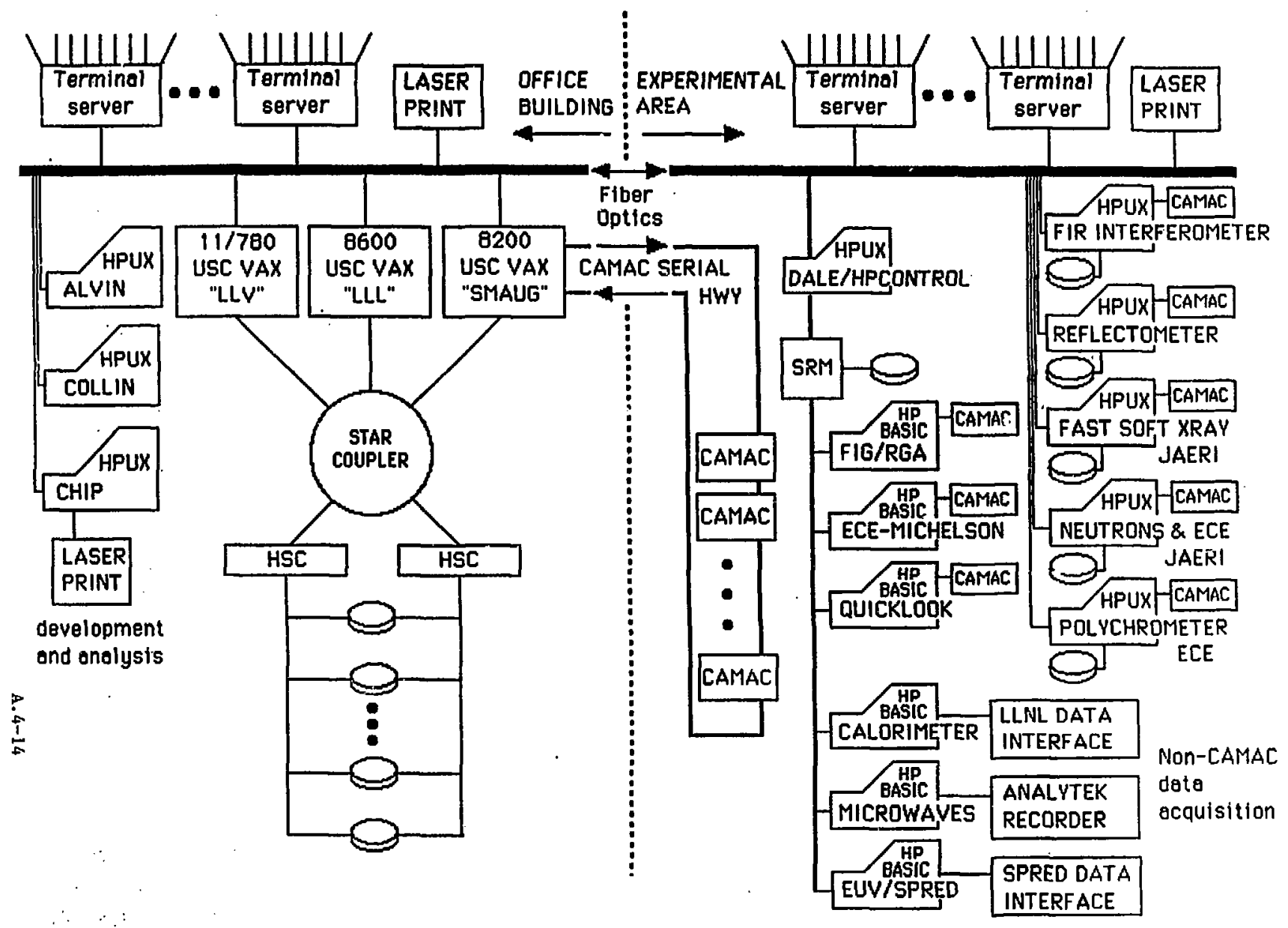




\title{
Integrated, multivendor distributed data-acquisition system
}

\author{
David N. Butner, Marena Drlik, WilliamH. Meyer, JeffreyM. Moller, and \\ George G. Preckshot \\ Lowrence Livermore National Laboraton, University of Caljfornia, Livermore, Colifornia 94550 \\ (Presented on 16 March 198;

\begin{abstract}
A distributed data-acquisition system that used various computer hardware and softwate is being developed to support magnetic fusion exp eriments at Lawrence Livermore National Laboratory (LLNL). The experimental sequence of operations is controlled by a su pervisory program, which coordinates software running on Digital Equipment Corporation (DEC) VAX computers, Hewlett-Packard (HP) UNIX-based workstations, and HP BASIC desktop computers. An interprocess communication system (IPCS) allows programs to communicate with one another in a standard manner regardless of program location in the network or of operating system and hardware cifferences. We discuss the design and implementation of this data-acquisition system with particular emphasis on the coordination model and the IPCS.
\end{abstract}

\section{MTX DATA-ACOUISITION SCENARIO}

We are developing a computer system to acquire data from the Microwave Tokumak Experiment (MTX).' This magnetic fusion experiment uses an MIT-developed dataacquisition software package that runs on DEC VAX com. puters and LLNL-developed data-acquisition software packages that run on HP series 300 UNIX computers and HP series 200 Basic desktop cornputers. Our problem is to develop an integrated data-acquisition system that uses this variety of software and hardware, that supports MTX and future experiments, and that allows us to add higher-performance computer equipment as it becomes available.

In magnetic fusion experiments, a plasma is generated in the tokamak for a period of a few seconds every few minutes; each plasma episode is called a shot. Our data-acquisition system is oriented toward such a shot scenario, but many of its features can be used for or adapted to a continuous process or experiment.

The tokamak used in MTX was also used for the Alcator experiment is MIT. The tokamak and various support equipment, such as power supplies and diagnostic equipment, were moved to LLNL in 1987. The data-acquisition systern developed at MIT, called MDS, ${ }^{2}$ acquires data from CAMAC modules and stores the data in files. MDS allows a diagnostician to specify the types of modules used in a system, the pre-shot and post-shot commands for each module, and the files into which the data from each acquired signal are stored. MDS also allows the diagnostician to specify that certain signals be automatically displayed after the shot. MDS is written in FORTRAN to tun on VAX computers; it relies on VMS services, so moving it to another type of computer would be impractical.

A previous magnetic fusion experiment at $\mathrm{LLNL}^{3}$ used HIP computers running UNIX and BAsIC for diagnostic data acquisition. MTX diagnosticians want to use some of this equipment because we have a large investment in previously developed soft ware for these computers. The HP computers also allow for more complex interactive control of diagnostics than does MDS.

In addition to acquiring experimental data and storing it in files, an ịntegrated data-acquisition system must keep track of where the data for each experiment and each diagnostic are stored so data can be retrieved in a reasonable manner. Our system includes a data location data base for this purpose. The details of tracking and retrieving the data in a standard manner are discussed elsewhere."

This article discusses two important features of the MTX data-acquisition system: the shor model and the IPCS. The shot model is the key to coordinating a variety of dataacquisition subsystems, and the IPCS provides a straightforward method so tasks on various computers can communicate with each other.

\section{COORDINATING DATA ACQUISITION-THE SHOT MODEL}

We created a method to coordinate data acquisition, the shot model, that relies on modular software subsystems and

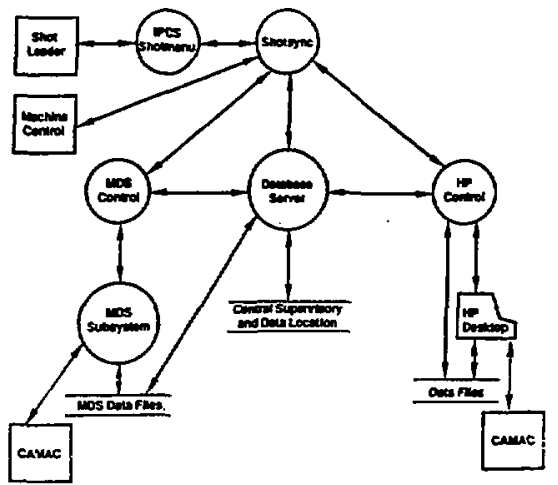

F16. 1. The shot supervisory program, SHOTSYNC, communicates with the diagnostic control programs MOSCONTROL and HPCONTROL MDSCONTROL and HPCONTROL communicate with DBSERVER, a data base program, and each coordinates its respective subsystem. 
a state machine. In this shot model, we separated the diag nostic subsystem programs and the data location data base programs into modules (Fig. 1). The modular spproach provides flexibility in designing the data-acquisition configuration for an experiment. Diagnostic subsystems can be modified, or new subsystems requiring different hardware/ software interfaces can be added without affecting the basic shot model. The modular approach also divorces subsystemdependent communication problems from the shot supervisory program and places this burden on the diagnostic subsystem programs.

The shot model is implemenled by a shot supervisory program and diagnostic subsister. programs executing concurrently as a state machine The rules that govern the sequence of events during a shol is: the considered as a state diagram (Fig. 2). The sho stipervisory program, SHOTSYNC is the coordinais inieflace between the shot leader and the diagnostte sutsistem control programs. HPCONTROL and MDSCONTROL, the diagnostic subsystem control programs (FIf 1). translate SHOTSYNC's state transitions into actions for ther respective subsystems. The supervisory data base program. DBSERVER, coordinates the collection of data location and shot configuration information.

SHOTSYNC accepts commands from a shot leader via a user-interface program. The shot supervisory program also compiles a list of diagnostic subsystems, supervisory data bases, and various status-monitoring programs and coordinates their activities according to the state-diagram. SHOTSYNC has no prior knowledge of what subsystems will be part of a shot. Instead, we use a "dynamic check-in" technique by which programs to be coordinated notify SHOTSYNC, via IPCS, that they need to receive shot coordination messages. SHOTSYNC maintains a list of

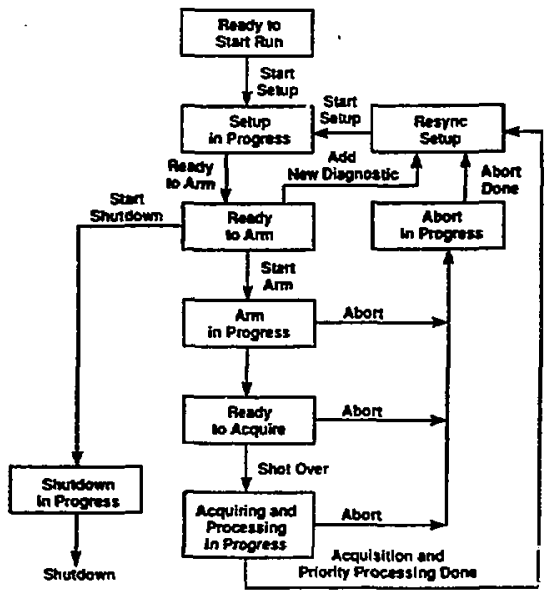

Fig. 2. The shot model state diagram. checked-in diagnostic subsystems, and each subsystem stays on the list until it dies or checks out. For each shot, SHOTSYNC supplies the list of participants and other shot information as a package to the data base server at the times required by the state diagram. Thus we automatically record the actual shot configuration. SHOTSYNC also sends shot cycle information to a logging device in the conerol room and supplies status information to user-interface programs. A diagnostic subsystem that wants to participate in a shot must send a check-in message to SHOTSYNC and must respond as appropriate to SHOTSYNC's shot coordination messages.

Currentl; we have two kinds of diagnostic subsystems: MDS acquiring from CAMAC modules, and HP BASIC computers and UNLX-based workstations acquiring from LLNL-developed diagnostics.

Multiple MDS subsystems can acquire data. Each subsystem is coordinated by an MDSCONTROL program, which communicales with SHOTSYNC using IPCS and with the MDS subsystem using MDS events. Each MDS can reside on a different VAX computer, which allows us to increase system capability by adding more computers and running in parallel.

HPCONTROL nuns on a UNIX-based workstation, talks via IPCS to SHOTSYNC, and uses the HP proprietary SRM protocol to communicate with the BASIC desktop computers. A major difference between HPCONTROL and MDSCONTROL is that HPCONTROL acts as an IPCS sponsor for many HP desktop diagnostics. All HP desktop IPCS communication is funneled through HPCONTROL.

\section{THE ROLE OF IPCS}

IPCS is an easy-to-use, portable software system for passing messages between tasks that reside anywhere in a network of computers. 3 By easy-to-use, we mean that programmers using IPCS need not know anything about the networks used by IPCS, and they have a few straightforward procedures to call to use IPCS. By portable, we mean that IPCS is written so it can be easily moved to another type of computer or operating system; portability is achieved by using a portable language and by judiciously using operating system services.

To send a message via IPCS, a programmer specifies the name of the receiving task, an optional set of class variables, and an optional variable-size data block (Fig. 3). A task that receives a message may use the message identification to return the message, optionally changing the class variables.

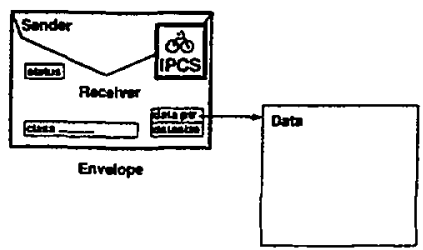

FiG. 3. Concepturi model of an IPCS message. 
The receiving task may also use the message identification to send a reply message, optionally including class variables and a data block. The task that retums or replies does not specify the riame of the task that is to receive the message; IPCS routes the message to the task that sent the original message.

To implement the logical structure of programs shown in Fig. 1, two features of IPCS are particularly useful: "addressing by task name" and the class variables. To send a message to another task, only the name of the task is specified, not the name of the computer on which the task runs. The class variables are eight 16-bit fields associated with every IPCS meseage, they are used to classify messages into a bierarchy of message types.

The method by which tesies communicate with SHOTSYNC is an example of the addressing-by-iask-name feature of IPCS. When any propram in the system wants to communicate with the shot syochronization task, it sends a mexsage using only "SFOTSYNC" as the name of the mesange receiver. It does not matter where theSHOTSYNC task is running in the network of computers; IPCS finds the SHOTSYNC task and delivers the message. This feature allows us to run a given tak on themost appropriate computer in the network. Without modification, a usk image file may be moved among computers running the same operating system on the same hardware. If a task dos not use a special feature of a computer or operating system, the source code is only recompiled and relinked to run on a different computer or operating system. With this portability of application code, we can easily change the processor load of various computers, operate with one or more computers unsvailable, or add more computers to the network.

The use of specific names for each task in the system is optional. Usually, only tasks that provide services, such as SHOTSYNC and DBSERVER, are named. Tasks that do not provide services are not named; instead IPCS creates a unique name for such tasks when they start. The reply and return mescage capabilities of IPCS ensure that response messages will be passed back to the appropriate task.

Class variables are used to implement a hierarchical structure of message types. The first class variable, called the major class, specifies a category of messages. In some cases, the major class designates a message to be handled by a certain task, such as DBSERVER. In other cases, the major class designates one of several types of messages handled by one task; for example, SHOTSYNC handles messages whose major classes are shot-registry and shot-command, among others. The second class variable, called the minor class, is used to specify functions involved specificaliy with the major class. For example, two minor classes for the shot-registry major class are "check in" and "check out"; two minor classes for shot-command are "start shot" and "bold shot." Other class variables can be used to indicate further subfunc. tions. With the message-type hierarchy, we can independently define message types for the various parts of the software system. The programmer who writes the message-handling interface for the DBSERVER does not need to worry about these messages conflicting with those of the programmer who writes the message-handling interface for SHOTSYNC.

\section{POATABILITY OF IPCS}

The IPCS software is implemented in C. This language is available on most computers, and it provides the tools we need to write a utility that is similar to an operating system. Also, c permits us to transfer IPCS software to various workstations, most of which run UNIX.

The only indispensable operating system functions required to implement IPCS are common memory areas, locks (to provide exclusive access to data structures), and the ability to awaken another process. Most multiusking operating systems provide these functions in some form. The code that interfaces with the operating system has beep isolated to a few software modules to aid in portability.

The VMS version of IPCS currently supports two network protocois: DECNET, the standard DEC network; and TCP $/ P$ using Wollongong's software package. The UNIX version of IPCS supports the TCP/P protocol. The IPCS has also been installed on Sun workstations.

\section{ACKNOWLEDGMENT}

This work was performed under the auspices of the U.S. DOE by LLNL under Contract No. W-7405-ENG-48.

'K. I. Thonnseen, presented at the 14th Conference oo Plasmn Physics, St Andrews, Scotlind, 1-3 July 1987.

'T. W. Fredien and J. A. Stillermen, Rev. Sci. Instram 57, 1907 (1986).

'T. A. Casper, H. Bell, M. Brown, M. Gorvad, S. Jentine, W. Meyer. J. Moller, and D. Perkint, Rev. Sci. Instrum. S7, IB80 (t986).

'G. G. Preckshot, D. N. Butner, M. D. Brown, and W. H. Meyer (these procedings).

${ }^{5}$ G. G. Preckshot and D. N. Butner, IEEE Trans. Nucl SCi NS-34, 858 (1987). 
A. 4.3

DLAGNOSTICS DATA MANAGEMENT ON MTX

D. N. Butmer, M D. Brown, T. A. Cusper, W. H. Meyer. and J. M. Moller

Lawrence Livermore National Luboratory

P.O. Box 5511, L-636

Livermore, CA 94551

\section{Abstref}

The Microwerve Tokmik Experimedi (ArTX) is a magnetic fusion

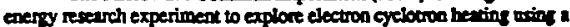
free electon leser operting in the microwave renge. The ditenosoc data from MTX is ecquired and procesed by a distributed, meitrvendor. computer netwot. Ench shot of the experimens produces den bies

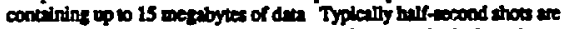
cikeo every 5 minites with 50 to 60 shots viven an a single dry. As meny s 60 full den shos heve been tiken on a yood day. Din files are creved

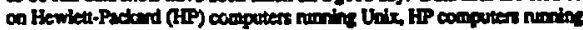

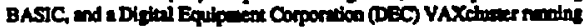

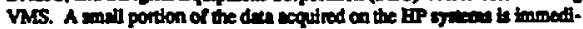

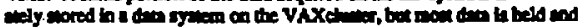

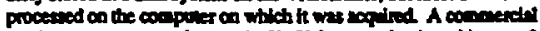

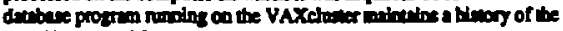
ditu files crested for each thot.

During the nifhe dan files on all conputers ere compresed to

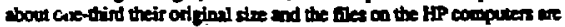

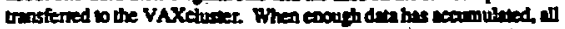
deta files the have not been previously actived axe uctived it $8 \mathrm{~mm}$ menedic tupe. Onoe the den ts on the VAXcluses, a single defined procedure cll may be uned wo obtin ding then was thien on any of the computers in the netroik.

Din that hes been achived to tape is minnined on ditt for a few

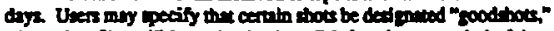
whoc dith fies win be mointiined co fist for a loper petiod of tive. If

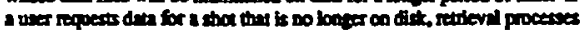
on the VAXcluses devermine which upes contin the dan, requet the

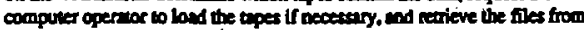
the tapes. The din is then ivilible for processing by programs numing on any computer in the network.

\section{Intodnetion}

The Microwave Tolonel Experimen (MIX) ts a mamedc fusion

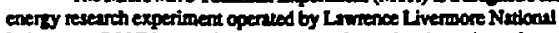
Labortory (UNL) wo exploce electon cyelowion bexing ving a free election hecr operating in the mitroweve fuct. The experiment his boen operating since June 19ik.

MTX is operited in a thoe mode Shas hrve a durtion of sbout soo milliseconds and occur at a rite of one per 5 minuter. During the shot diagnossic hadere sequires bout is neptones of den As the thot cermintites, the dath is transfered into files on a netwot of computer. Typieally, 50 wo 60 shots ere taken during one dyy, as many at 80 full dan shots hrve been taken on a good dy.

All of these data files moat be minned to meet wreari goals. Physicists must be able to eccess dren that wes then anybere in the networ. The files mua be moved from the compoter con which they were cretted to a conmon botion en thet they cen be more corventent)

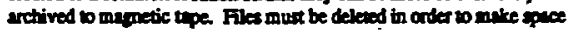
for new dwa, but dat for shots thet are parionlifly interening should rempin on disk far a while. There mus be en easy way for ditn ob be retrieved from magetic tape when a physicist wishes to andyze dat from old shots.

\section{Handwane}

The MTX Dan Acquisidon Systen uses Diginl Equipoen Corporetion (DEC) VAX computers locited in our Uner Service Cencer (USC) rening the VMS opering sysem, Hewlett-Pucled UNIX (tPUX) wotktutions and HP-BASIC contul computers, es lndicited is Fi. 1.

The USC VAXeluner condists of three compuese with retondex

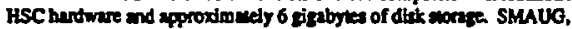

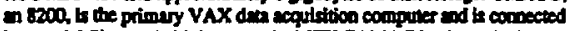
by a seril aber optic Wighay to the MTX CAMAC bendwan h the experimentil ues, eppoximaty a quiter of anile tom the USC. $L L$.

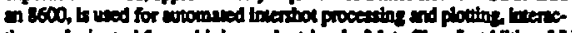

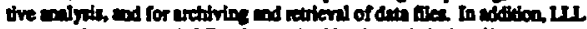

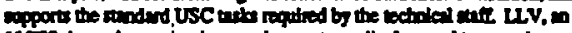

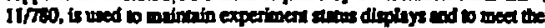

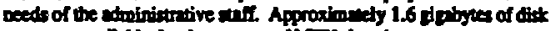
epece are arillable for the morate of MIX thot date

The HP UNDX computer, located both in the offec buitints and in the experimental area, are connected to the USC via a flber optic beal are Bhetna (LAN). Ten HPUX workthitions are preanily connected diredy to the LAN and communicute with the VAX compiness vit the

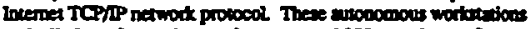

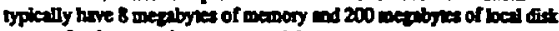
gure for the opering oratem ind date.

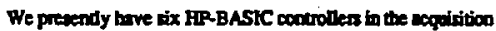

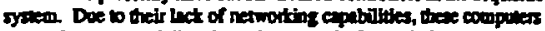

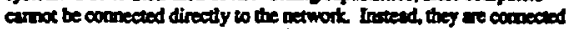

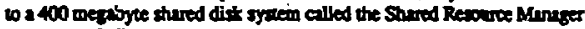
(SRMD. A dedleated FIPUX conpute, DAIE, ast as a communiention grteway between the HP.BASIC compureers and the other compules. using files created on the SRM.

The DSC terminal aervers located th the oftice builing and in the experimertal are allow users in either plece to conned bo the VAX conputers. The TENET function provides a direst contedion berween UNDX and VMS computers with FIP providing file tronsiers.

\section{Sofrome}

The cotware syom ts thown in Fiz. 2. The detils of the software system are discused in Rels. [1] ind [2]; only the appects related to dma manxement will be discussed berc. Most of the inverprocess consections shown in Fif. 2 are implemented uting our Interprocess

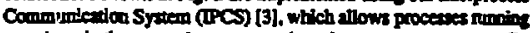
anymere th the aetwotk to communicate in a common mman. Contol server progrens provide sceess to commercial codes or lo procestes thet do not inow bout IPCS. MDSCONIROL thus provides an IPCS interfece wo the MIT-developed MDS dith scquistion pecher [4] nining on the VAX, end HFCONIROL provides a simile function for the HP BASIC comprters.

Sotware aspects of a shot tre coondinated by a single thot ryn-

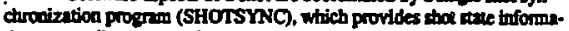
tion to any diagontic software that is runing when a shot cocurs. The diagnostic interfaces to SHOTSYNC are in MDSCONTROL.

\footnotetext{
"Wotk performed underthe muspices of the US. Deparment of Energy by the Lawrence Livermore Nationd Laboratory under conurea No. W-7405-ENG-48.
}

$$
\text { A. } 4-18
$$




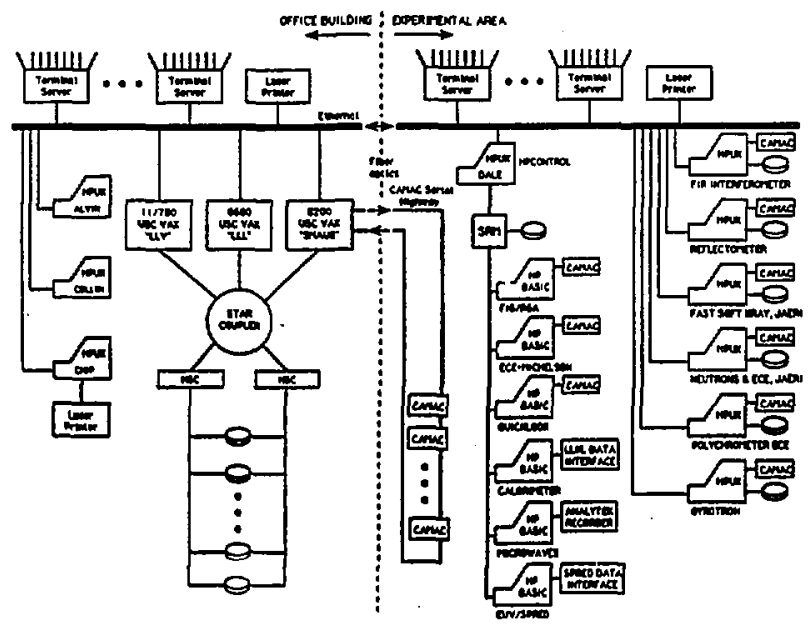

Figure 1. MTX dm exputition and dxa processing hardware

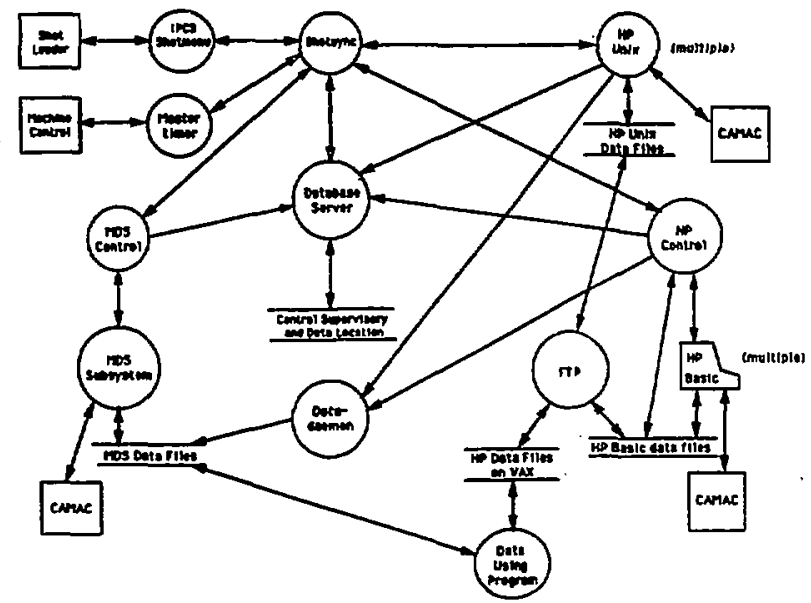

Figure 2 MTX dar acquisition and dus processing coftwar.

HPCONTROL, and molliple HP Unix symeme. SHOTSYNC and the

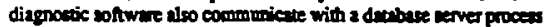
(DBSERVER), which maintirs informution conceming each thot in an

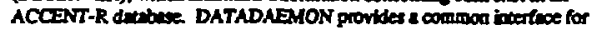

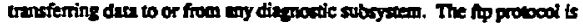
used 10 trinster files between conputers.

For every shot, SHOTSYIC ands a mexte to DBSERVER indicating which dispowic subyrems (MDSCONTROL, HPCONTROL. and multiple UNTX systems) participted in the shot. Exh diagnostic subsystem rends a message to DBSERVER indicating the files that if created for the thot

\section{Dan Mrnapement}

The din files for each of the three kinds of diagrastics (UNTX. SRM, and MDS) are manged somewhen difierenty, although all the files altimately resile cos the VAXchuser for the pupowes of archival und renieval By comention, the las five chareters of the min part of my filenime indicate the mumber of the shot for which the file was created.

The management of the data files for UNDX systems is shown in Fi2 3. For esch shot, wwo files are created in the dats direcrory on a dist connected to the UNLX computer, one file cortains the deta (the sit file). and the oher file contains information that describes the sizes and types of 


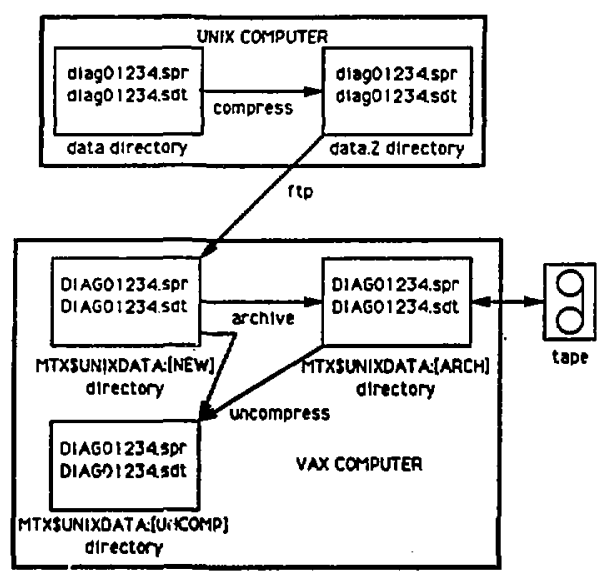

Figre 3. UNDX file management.

amps stored in the data the The format of thewe fives is determined using the LLNL-developed luage processins peckeg VIEW [S]. Piocessing and dispiay of the data is performed in real-time on ench UNIX computer. Since the files often contain slowly varying dath dencompression produces a signifient reduction in the sire of the file (in averaye of about 60\%). At night, all of ales tiken doring the day are compresed using the

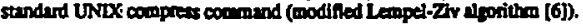

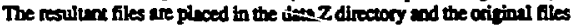
are deleted. Also during the night, the compresind niles are copied to the MTXSUNDXDATA:[NEW] directory on the VAXclonter using Ip.

Subequendy, the files are archived to magnetic tepe as discosed below.

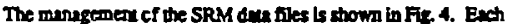
BASIC computer cremes in files on the SRM. Eech file coosins one or more signils for exch sionl, there is a texi decoription of the signal followed by the din. Procesing and fippiny of the duta is pertioned in renltime on ench BASIC compoter. At righ, each file the wis cresed on the SRM during the dyy ls.compresed ino a temporar fie and copled to the MTXSSRMDATA-DEEH direcony on the VAXelued exing fip. Sobcequently, the files are archived of minetic tope at ficosed below.

The menveneet of the MDS den files b thown in Fits. The

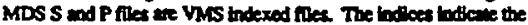

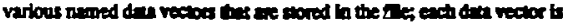

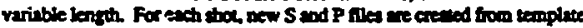

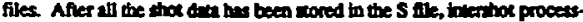

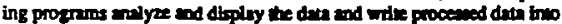
the P file In addition, den froc UNDX computes ts witen 10 the $P$ file

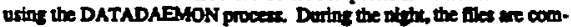

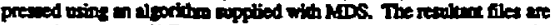
slored in the MTXSMDSDATA:[COMP] direcons and the original files are deleted Subequendy, the Eles are archived bo magnetic tope as discenssed below.

Eech night, after in of the UNDX and SRM comprased flles hrve been copied ot the VAXctures and atter the MDS files hre been compresed, irchive routines ron These arthive routines bee a commercil software package (V.X.MigDL) to schechule the vatious operetions and to maintain information abovt which files are on which upe and which tape is cumently being used for writing.

These routines fist delete any files which hwe, expired (the setting of expiration dates is described below). Next, these routines determine how much dist space is occupied by files in the

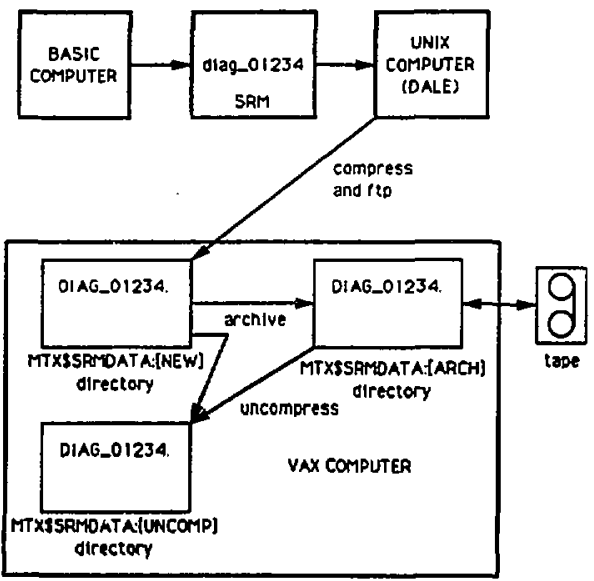

Figure 4. SRM file management.

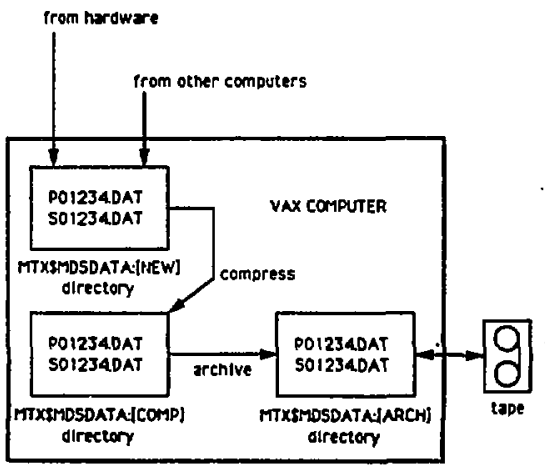

Figure S. MDS file management.

MTXSUNDXDATA:[NEW], MTX:SRMDATA:REW], and

MTXSMDSDATA:[COMP] diresorien If the fles cocopy more the 100 megubytes of dist spece and the correct lipe is mounied in the tupe dive. exch file in these directories is rensened in w the asocived (ARCF) direcory with the expintion date eet to a dne for th the fume (the epedol expiraion dy). Then, all files in the [ARCH] direcostes thet brve the

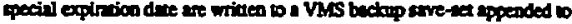

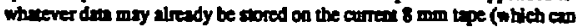
bold a will of abour 2000 mepabytes of din). Once til of the files brve

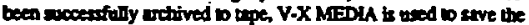
information boul tse bupe ind teve-set containiog the files, and the expirtion daves on all of the files are set wo be if for dyy from the date ot with the rective was perforwed. Alo, a copy of the archlve ape is made If the copy tupe is in the tape drive.

Users are allowed to change the expirvion dite for data files using 1 routine called GOODSHOT. GOODSHOr sets the expiratica defe of exh dist-residen file aseocinted with each specifled shot to a dre boot 1 moath for the dire a wich the GOODSHOT request is mide Optionally, GOODSHOT en be wed to request ther my files aseodiked with each specifled shot be retrieved from the archive ape. For thits cuse a set 
of processes uses information in the V-X MEDHA drabuse 20 detemine the upes and arve-sets containing the files ob te rovieved $A$ eat of VMS beclap comments is tenernied to retuleve the files in as colicient a mencer as possible. Two bope drives are reserved for we by MTX. 204 pigabyes of das is often aviliable on line Most ofter, the thot dati files are retrieved with no opernor ineervention. After the fles are retrieved, each file's expiration date is sel appropeiately.

\section{Amescing Din}

At any time the the dam files are residere on the VAXcluster, dare for a given signal on a tiven shot may be sccesed usire the iffrit nume and shor mumber. A commencial dignil procesthe code (IDL) is commonly used to procest the den contatned in these dith fllk. An ILNL wriuen IDL routine (GETDATAX), determines which file condins the requessed signul and searches the appropriate directorles for the file. If the file is not on dlat, an error is roumed to the calles, and the user may uve GOODSHOT to retrieve date tlias froo tupe. If the flle b cn disk, the dan is reurned to the IDL procesting code.

If the requeted duta is in w MDS file GETDATAX calls a

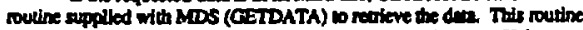
operises correctly whether the file has been compiened or bot If the requesed dat is in UNLX or seM fite, however, the tile on the VAX russ be uncompresed betore the dita can be read Therefore. GETDATAX his looks in the [UNCOMP] directory, if the nie is not there but is in the DEW] or (ARCH) direcery, en uncoupresed flle is

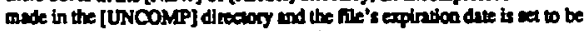
a few days from the time of uncomprassion.

The FDNDSHOT withy may be aned io determbe which dingostic subsyoums participated in a shot, whe files were creted and the current states of the files A siopic copy of the outut fion FINDSHOT is how below:

\section{EInatore 11907 \\ 11507}

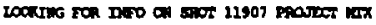

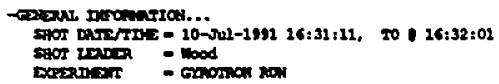

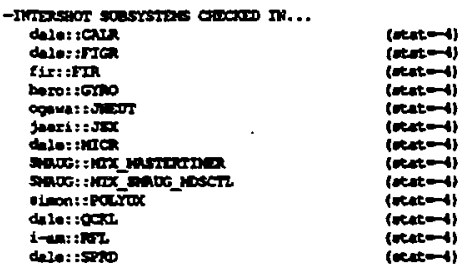

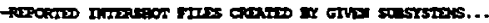

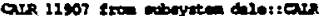

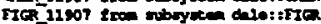

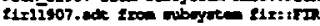

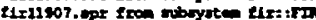

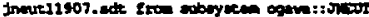

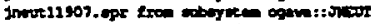

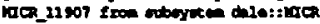

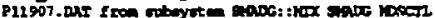

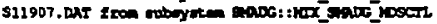

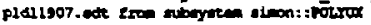

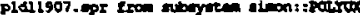

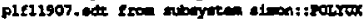

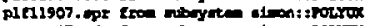

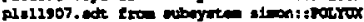

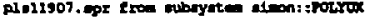

rf111907. edt fros wheysten 1-an: :Rr

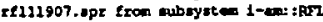
SPRD_11907 from aubyrice dale: SPPD

$\rightarrow$ Loak AT THE DIST FOR THIS SHOR...

Dinectory mTXS ONIXDATA: [ARCH]

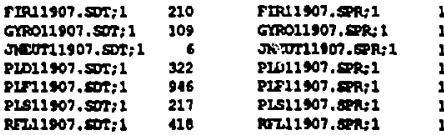

Diructory hasstorm: [NeY]

arn_11907.12 $12 \quad$ Fte 11907.11 nch-11907.12 21 SPO_11907.12

Diructory Mexsibsone! [Ared]

P11007.mat: $1195 \quad$ s11907.05;1 1221

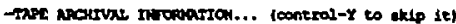

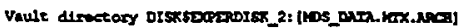

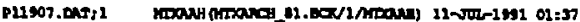

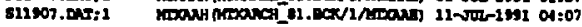

Totil of 2 riles

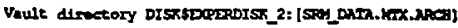

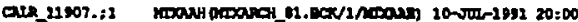

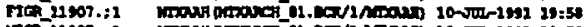

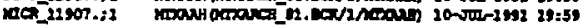

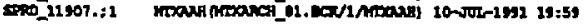

Torel of 4 41es

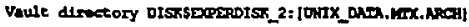

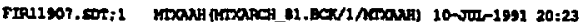

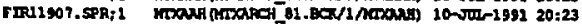

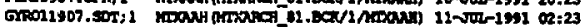

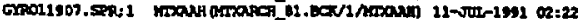

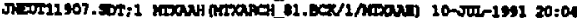

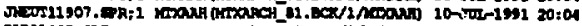

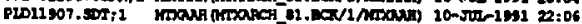

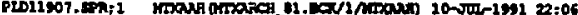

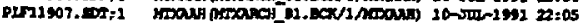

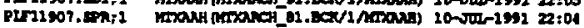

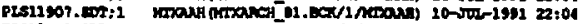

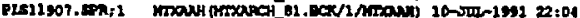

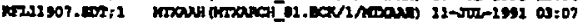

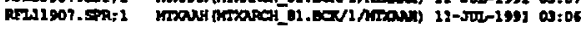

Total of 14 files

Grand coes of 3 dinectories, 20 files

\section{Softwre Genenility}

All of this softwari has been designed to be as independent of the experiment as possible. Many operations are controlled by texual configuration files. We are using much of the whwere unchinged in conjincdon with wo other experiments in the magnetic fusion ares at LLNL: RACE (the Ring Acceternot Experiment) and the Superconducting Magnet Developinent Group.

\section{Summary}

For each MTX shoh, several dala files we antomatically migrued through a nerwots of computers. All dath is avallable to the computer network for a few days afier a shot and may be scoesed th a comrion 
mamer no matter where the dats file was originally created. Physiciss may request tha data files for some shots remain on dist for a longer period of time and may essily requess thr data files for shots that are not on disk be retrieved from upe.

\section{Befermat:}

[1] D. N. Buner, M. Drlik, W. H. Meyer, J. M. Moller, and G. G. Preckshot, "Inuegrated, Multivendor Distribuled Datu-ecquisition System," Review of Scientific listuments, vol, 58, pp. 1786-1788, 1988.

[2] D. N. Butner, M. Dilik, M. D. Brown, W. H Meyer, and J. M. Motler. The MTX Dals Acquisition Sysrem." Pnacedings $13 \mathrm{ch}$ Symposium on Fusion Englneerine, Kroxville, TN, October 1989, Pp. $164-166$.
[3] G. G. Preckshot and D.N. Butner, "A Simple and Erficient Interprocess Commurieation System for Actully Using a Laboritory Computer Network," IEEE Transections on Nuclear Science, vol. NS-34, pp. 858-864.

[4] T. W. Fredian and J. A. Stilleman, "MDSMIT High-Speed Dak Acquisition and Analysis System." Review of Scientific Insmuments, vol. 57, pp. 1907-1909. 1986.

(S) J. M. Brase, V. J. Milke, and M. A. Wieting. Luwtence Livemore National Laboratory, Livemore, CA. UCTD-21368, March, 1988.

[6] T. A. Welch. "A Technique for High Performance Dau Compression," Computer, vol. 17, no. 6, pp. 8-19, June 1984. 


\section{A.4.4 Maestro (J. M. Moller)}

\section{A.4.4.1 Introduction}

Maestro, the Model And Expert System Tuning Resource for Operators, is a computer system designed to serve as an intelligent assistant to the accelerator operator.1,2,3,4In Maestro an object-oriented knowledge base containing detailed descriptions of the many ETA-II components, their physical properties, and their interrelationships, serves as the basis of a unified software environment encompassing accelerator control, diagnostics and modeling. The operator can manipulate these capabilities with a powerful graphical user interface.

This flexible environment supports diverse approaches to computerassisted accelerator tuning. Expert system methods attempt to "clone the operator" by incorporating into Maestro's procedures tuning strategies employed by expert accelerator operators. ${ }^{2}, 3$ Model-based tuning uses an online numerical linear induction accelerator model to calculate the electron beam trajectory given the magnetic field profiles and alignment information contained in the knowledge base.3,4 Manual tuning equips the operator with a variety of graphical tools for displaying and controlling accelerator parameters, and acquiring and interpreting diagnostic data.

\section{A.4.4.2 Maestro components}

Maestro runs under Unix on a Solbourne workstation. It is programmed in Allegro Common Lisp with the Allegro Common Windows graphical user interface. Non-Lisp procedures, such as the modeling, networking and signal processing codes, can be linked to Maestro through a "foreign function" interface.

Maestro's knowledge base is built using LLNL's FAR Frames and Rules system. FAR supports rule-based and object-oriented programming with a frame system based on class-subclass and class-member relationships. FAR supports slot inheritance, multi-valued slots, and active slots or daemons. Knowledge base class relationships are built and displayed using a graphical browser. FAR daemon functions are used to exchange dynamic control information with the online accelerator interface.

Spreadsheets based on FAR and Common Windows provide a good format for organizing the repetitive information describing the 60-cell ETAII. Dynamic information from the machine can be displayed along with static information. Axial profiles can be drawn on spreadsheet columns as color bar 
graphs. The spreadsheet cells are mouse-sensitive and can be linked to menus or action procedures.

A plot package based on FAR and Common Windows provides graphical display of diagnostic data. Graphical elements, including plotted points, are mouse-sensitive and can be linked to menus or action procedures.

Maestro's linear induction accelerator model, LIAM ${ }^{4}$, is a flexible simulation code that calculates beam centroid position and beam envelope from on-axis magnetic profiles. LIAM models the overlapping electromagnetic fields of multiple elements, including accelerating gaps, solenoids and steering dipoles. Beam energy can be derived by comparing model trajectories with diagnostic data from steering sweeps.

Maestro diagnostic data is stored using LLNL's VARS general purpose, self-describing, machine and language-independent data storage system. VARS stores named data items organized in a tree structure. VARS data structures can reside either in main memory or in data files.

LLNL's Inter Process Communication System, IFCS (Section A.4.1.1), connects Maestro to an earlier generation manual accelerator control system running on a network of Microvaxes. Maestro controls the accelerator by exchanging request messages with server processes on the Microvaxes. Separate IPCS servers support diagnostic scope trace acquisition, magnet power supply interface, and beambug highway control.

Vaxplay 5 is a diagnostic data analysis package developed by the Beam Program to analyze the digital data files, or dig files, output by an earlier generation of trace acquisition software on the Microvaxes. Maestro extends this development by translating its own diagnostic data into dig file format for analysis with Vaxplay. In addition, a $C$ language translation of Vaxplay functions, the signal processing library, allows some of the same data analysis to be performed inside Maestro in real time.

\section{A.4.4.3 Maestro control functions}

Connect to Control System. Maestro accelerator control has two modes of operation: connected and disconnected. When connected, Maestro control requests are passed to the control system via IPCS. A safety warning logo is displayed in the background window whenever Maestro is connected. When disconnected, Maestro control requests are written to dummy fields in the knowledge base for debugging.

There are two ways for Maestro to connect. Maestro can read both the target setting and the measured value of each control variable. Normally when Maestro reads a control variable the measured value is returned. But for some ETA-II subsystems the operators have determined that the target setting is more accurate than the measured value. Therefore a special 
connection mode is supported in which the target setting is returned.

Accelerator and Transport Magnet Control and Status. Magnet power supplies are controlled using spreadsheets in which each row corresponds to a magnet assembly, while columns correspond to horizontal and vertical steering coils and focus solenoids. Target currents are set by clicking the mouse on an appropriate spreadsheet cell.

Control spreadsheets display target currents, both numerically and as bar graphs. Status spreadsheets display both target and measured values along with power supply configuration information for detecting and identifying faulty power supply equipment. Configuration spreadsheets contain detailed information such as component name and type, location in the beamline, physical and electrical relationships to other components, electrical parameters, conversion factors from digital to engineering units, coil tilts and offsets, and power supply interfacing details.

Archioe of Past Magnet Settings. Magnet power supply target settings can be saved in VARS files. Settings are saved automatically before each data acquisition run, and can be saved manually with a main menu item. Past settings can be recalled and displayed as spreadsheets. The operator may choose to download selected portions of the spreadsheet onto the machine. Magnet settings spreadsheets can be subtracted from one another to show any differences.

Wiggler control. In the IMP wiggler (section 8.3) the magnetic field on a given pole is determined by a linear combination of the currents in tip and base coils powered by separate supplies. Each tip supply powers a magnet group of four poles, while each base supply powers a variable number of magnet groups, whose base currents are thereby held equal. In addition, the poles contain permanent magnets, divided axially into high field and low field sections to support tapering. The total field on each magnet group is subject to linearity limits, since driving the iron into saturation could destroy the precise balance between positive and negative poles, deflecting the beam. The iron also introduces the requirement to control hysteresis by making any current adjustments from the low field side, a process that can take several minutes.

The wiggler configuration spreadsheet has a $\mathrm{r} r ; \mathrm{w}$ for each magnet group and columns for each coefficient used in the field and linearity calculations, along with base supply configuration information. The wiggler cuntrol spreadsheet has tip and base currents, fields, linearity limits, and power supply current limits. The field profile and allowed limits are displayed on a bar graph, with red bars indicating field settings outside the limits.

The operator adjusts fields and base currents while Maestro computes the corresponding limits and tip currents. To avoid unnecessary hysteresis cycles. updated cell values are not immediately downloaded onto the supplies. But after the desired field profile has been achieved with all constraints satisfied 
then the operator can download all the power supply values onto the wiggler using a menu item. The microwave control spreadsheet controls various pieces of microwave diagnostic equipment such as variable attenuators and movable mirrors and probes. It also models the signal paths through the different waveguide segments and does the calibration bookkeeping of summing the measured attenuations.

\section{A.4.4.4 Maestro data acquisition}

All Maestro data is digitized by a bank of Tektronix 7912 oscilloscopes. Six of these scopes are connected to diagnostic probes, including inductive beam position sensors known as beambugs, through a beambug highway multiplexor. The beambug highway settings can be controlled by Maestro through a mechanism similar to the one used for magnet power supplies.

Before delivering trace data to Maestro, the server corrects it by subtracting a saved trace taken under similar conditions with the scope input grounded. Calibrate scopes grounds the scope inputs using the beambug highway and requests the acquisition server to acquire and save the resulting calibration traces.

Time-align scopes switches the beambug highway to a timing signal. The position of this signal on scope one serves as a reference. The selected scope is acquired repeated!y, along with scope one, and both signals are plotted. The operator can adjust the horizontal position knobs on the scopes until the two timing traces overlay.

Set scope attenuators displays a window with a colored button for each scope. The operator sets each button to match the color-coded attenuator being used for that scope. Since attenuators are installed manually, Maestro depends on the operator to keep the scope attenuators window up to date. Expert systeri rules remind the operator to check the scope attenuators whenever he changes to a new mode of data acquisition.

Energy Bugs are specia? beambugs configured for use in the beam energy analyzer. C-probe data is acquired from selected accelerator current and voltage probes. Generic Acquisition allows miscellaneous unmodelled signals to be acquired from the scopes. The results of these procedures are written to a dig file for analysis with Vaxplay.

Bugwalk acquisition is controlled by a spreadsheet with a row for each beambug. Bugwalk acquires data from selected beambugs at different axial locations, switching them into the scopes using the beambug highway. The results are written to a dig file for analysis with Vaxplay.

Afterwards Maestro analyzes the bugwalk data using the signal processing library and plots the beam current and corkscrew amplitude versus axial position. By clicking the mouse on a given data point in the plot, the raw data which produced that point can be examined in another window. 
VARS data for an extire day can usually be stored in memory. The data is written to a disk file automatically after each acquisition run, or manually with a main menu item. If a lor of data has been accumulated then the operator can close the VARS data file and start a new one. Past data files can be recalled and reviewed using a data browser.

\section{A.4.4.5 Corkscrew minimization}

Earlier work by the Beam Program identified beam corkscrew motion as a critical factor limiting the performance of ETA-II. Corkscrew motion is csised by a combination of beam energy sweep and beamline magnetic alignment errors. Alignment errors are now reduced during beamline construction using the SWAT stretched wire alignment technique. But residual alignment errors can be further reduced during accelerator operation with beam using correction coils.

Corkscrew minimization ${ }^{6}$ is a way of tuning the correction coils by measuring the corkscrew amplitude at a downsteam beambug for different values of the correction coil current. A minimum in the resulting curve represents the current setting for minimum corkscrew. This technique was developed using Vaxplay, but to handle ETA-II's 178 correction coils some automation was required.

Maestro corkscrew minimization is controlled by a spreadsheet with a row for each correction magnet set. Each magnet set has a horizontal and a vertical coil, which must be minimized separately. Two beambugs can be acquired simultaneously on the two channel beambug highway. Spreadsheet columns control which beambugs to use, and what values to use for the initial, final, and incremental current settings. Previous current settings are also shown to monitor convergence.

For each magnet set selected, Maestro steps through the selected current settings and acquires the beambug data. A one Hertz accelerator pulse rate allows just sufficient time between pulses to step the coil current to the next setting. After 15 to 20 pulses the data is analyzed using the signal processing library and the corkscrew amplitudes are plotted, along with a fitted curve. The operator may choose either to accept or override the fitted minimum. If the fitted minimum is satisfactory, then the operator can select fully automatic operation.

\section{A.4.4.6 Other Maestro functions}

The Knowledge Base Browser gives the operator a graphical display of the class-subclass and class-member relationships in the knowledge base.

Clicking the mouse on selected objects shows their attributes in more detail in another window. 
The Simuiation menu invokes an iconic Machine Interrogation and Control Interface, MICl. The operator clicks the mouse on a scale drawing of the beamline to select components, change settings, acquire simulation data, or browse historical data.

The IPCS menu is used to start, stop or restart the IPCS servers on the Microvaxes. Orderly shutdown disconnects from the accelerator control system and closes the VARS data files before stopping Maestro.

1. Darrel L Luger, Hai R. Brand, William J. Maurer, Fred Coffield, Frank Chambers and William Turner, Artificial Intelligence Techniques for Tuning Linear Induction A.ccelerators, Proceedings of the 1991 IEEE Particle Accelerator Conference, p. 3082.

2. D. L. L.ager, H. R. Brand, W. J. Maurer, F. Coffield, and F. Chambers, MAESTRO-A Model And Expert System Tuning Resource for Operators, Nuclear Instruments and Methods in Physics Research, vol. A293, 1990, pp 480-485.

3. D. L. Lager, H. R. Brand, W. J. Maurer, "An Expert System for Tuning Particle Beam Accelerators," in Advances in Artificial Intelligence Applications and theory, J. C. Bezdek, Ed., New Jersey: World Scientific, 1990, pp 53-65.

4. H. Brand, G. Caporaso, D. Lager, F. Coffield, and F. Chambers, LIAM - A Linear Induction Accelerator Model, Proceedings of the 1991 IEEE Particle Accelerator Conference, p. 3079.

5. F. W. Chambers, S. L. Allen, F. J. Deadrick, W. E. Nexsen, A. C. Paul, V. L. Renbarger and W. C. Turner, Diagnostics and Data Analysis for the ETAII Linear Induction Accelerator, Proceedings of the 1991 IEEE Particle Accelerator Conference, p. 3085.

6. S. L. Allen, H. R. Brand, F. W. Chambers, Y.-J. Chen, F. E. Coffield, F. J. Deadrick, L. V. Griffith, D. L. Lager, W. J. Maurer, W. E. Nexsen, A. C. Paul, S. Sampayan and W. C. Turner, Measurements of Reduced Corkscrew Motion on the ETA-II Linear Induction Accelerator, Proceedings of the 1991 IEEE Particle Accelerator Conference, p. 3094. 


\section{A.5 List of publications and reports}

\section{A.5.1 Publications}

W. M. Nevins, T. D. Rognilien, and B. I. Cohen, "Nonlinear Absorption of Intense Microwave Pulses," Phys. Rev. Letts. 59, 60 (1987).

M. Porkolab and B. I. Cohen, "Parametric Instabilities Associated with Intense Electron Cyclotron Heating in the MTX Tokamak," Nucl. Fusion 28, 239 (1988).

B. I. Cohen, R. H. Cohen, B. G. Logan, W. M. Nevins, G. R. Smith, A. V. Kluge, and A. H. Kritz, "Beat-Wave Current Drive with Intense Pulsed FreeElectron Lasers," Nucl. Fusion 28, 1519 (1988).

G. G. Preckshot, D. N. Butner, M. D. Brown, and W. H. Meyer, "Transparent Data Access in a Multi-Vendor, Distributed Data-Acquisition and Data Processing System," Rev. Sci. Instr. 59, 1774 (1988).

D. N. Butner, M. Drlik, W. H. Meyer, J. M. Moller, and G. G. Preckshot, "Integrated, Multivendor Distributed Data-Acquisition System," Rev. Sci. Instr. 59, 1786 (1988).

K. I. Thomassen, "Millimeter Wave Tokamak Heating and Current Drive with a High Power Free-Electron-Laser," 14th Annual Conference, IOP Plasma Physios Group, St. Andrews, Scotland, July 1-3, 1987, Plasma Physics and Controlled Fusion 30, 57 (1988).

J. A. Byers and R. H. Cohen, "A Microwave FEL Code Using Waveguide Modes," Ninth International Free Electron Laser Conference, Sept. 14-18, 1987, Nuclear Instruments and Methods in Physics Research A272, 595 (1988).

A. H. Kritz, G. R. Smith, W. M. Nevins, and R. H. Cohen, "Power Deposition and Current Drive by Intense Microwave Beams in Tokamaks," Phys. Fluids B 1, 142 (1989).

R. A. Jong et al., "TMP, A Free Electron Laser Amplifier for Plasma Heating in the Microwave Tokamak Experiment," Nuclear Instruments and Methods in Physics Research A285, 379 (1989).

K.I. Thomassen, "Electron Cyclotron Wave Sources and Applications for Fusion," J. Fusion Energy 9, I (1990).

iRR Stone, R. A. Jong, T. J. Orzechowski, E.T. Scharlemann, A. L. Throop, B. Kulke, K.I. Thomassen, and B.W. Stallard, "An FEL-Based Microwave System for Fusion," J. Fusion Energy 9, 77 (1990). 
J. H. Foote, J. D. Barter, N. R. Sewall, J. J. Jolly, and L. F. Schlander, "Thomson Scattering Diagnostic for the Microwave Tokamak Experiment," Rev. Sci. Instrum. 61, 2861 (1990).

T. Oda, K. Odajima, K. Mizuno, K. Ohasa, M. Shiho, K. Takiyama, J. H. Foote, and D. G. Nilson; "Stark-Effect Measurement of High Free-Electron Laser Electric Fields in MTX by Laser-Aided Particle-Probe Spectroscopy," Rev. Sci. Instrum. 61, 2964 (1990).

T. Ogawa (JAERD), K. Oasa (JAERD), K. Hoshino (JAERD), K. Odajima (JAERD), and H. Maeda (JAERD, "Neutron Diagnostic for MTX," Rev. Sci. Instr. 61, 3181 (1990).

T. Casper et al., "MTX Diagnostic and Timing System for Free-Electron Laser Heating Experiments," Rev. Sci. Instr. 61, 3274 (1990).

D. N. Butner et al., "MTX Data Acquisition and Analysis Computer Network," Rev. Sci. Instr. 61, 3277 (1990).

G. R. Smith, M. E. Fenstermacher, and E. B. Hooper, "Modelling of ElectronCyclotron Power Profiles on a Calorimeter in the Microwave Tokamak Experiment," Nucl. Fusion 30, 2505 (1990).

B. W. Stallard, "MAGICTRAC: A Novel Method for Conversion of Whispering Gallery Modes to a Free-Space Gaussian-like Beam," International Journal of Infrared and Millimeter Waves, London, UK, Vol. II, 1011 (1990).

S. M. Hibbs et al., "A Fuel Pellet Injector for the Microwave Tokamak Experiment," J. Vac. Sci. Tech. A9, No. 3 (1991).

B. I. Cohen, R. H. Cohen, W. M. Nevins, and T. D. Rognlien, "Theory of FreeElectron-Laser Heating and Current Drive in Magnetized Plasmas," Rev. Mod. Phys. 63, 949 (1991).

R. H. Cohen and T. D. Rognlien, "Gradient Effects on Intense, Pulsed Electron-Cyclotron-Wave Absorption and Current Drive," Phys. Fluids B 3, 3406 (1991).

B. W. Rice, "Fifteen Chord FIR Polarimetry System on MTX," Rev. Sci. Instrum. 63, 5002 (1992).

S. L. Allen et al., "Nonlinear Absorption of High Power FEL-Generated Microwaves at ECRH Frequencies in the MTX Tokamak," submitted to Phys. Rev. Lett. (1993). 
B. W. Rice and E. B. Hooper, "Poicidal Field Measurements During Sawteeth and Disruptions on MTX," submitted to Nucl. Fusion (1993). 


\section{A.5.2 Conference proceedings}

B. W. Stallard, G. R. Smith, R. A. James, K. I. Thomassen, A. H. Kritz, M. Makowski, T. Samec, and R. Yamamoto, "FCH in the Microwave Tokamak Experiment," 7th APS Topical Conference on Applications of RadioFrequency Power to Plasmas, Kissimmee, FL, May 4-6, 1987 (UCRL-96562), AIP Conference Proceedings 159, 21 (1987).

B. I. Cohen, "Particle Simulation of Intense Electron Cyclotron Heating and Beat-Wave Current Drive," Proceedings of the US-Japan Workshop on Plasma Modeling with MHD and Particle Simulation, Sept. 25-26, 1987, Napa, CA, C. K. Birdsall and T. Kamimura, editors, Electronics Research Laboratory, College of Engineering, University of California, Berkeley, CA 94720, p. 8 (UCRL-97359).

B. I. Cohen, R H. Cohen, G. Kerberl, B. G. Logan, Y. Matsuda, M. G. McCoy, W. M. Nevins, T. D. Rognlien, G. R. Smith, R. W. Harvey, A. H. Kritz (PPPL), P. Bonoli, and M. Porkolab, Microwave Heating and Current Drive in Tokamaks," 12th International Conference on Plasma Physics and Controlled Nuclear Fusion Research, Nice, France, Oct. 12-19, 1988, International Atomic Energy Agency, Vienna, Vol. 1, p. 759 (1989).

R. H. Cohen, B. I. Cohen, W. M. Nevins, T. D. Rngnlien, P. Bonoli, and M. Porkolab, "Current Drive by Intense Microwave Pulses," Cargese Workshop, Corsica, Italy, July 11-15, 1988. Conference proceedings published in Nonlinear Phenomena in Vlason Plasmas, F. Doveil, Ed., Editions de Physique, Orsay, p. 335 (1989), (UCRL-99583).

B. I. Cohen, R. H. Cohen, W. M. Nevins, and T. D. Rognlien, "Plasma Heating and Current Drive Using Intense, Pulsed Microwaves," presented at the Joint Varenna-Lausanne International School of Plasma Physics, Chexbres, Switzerland, Oct. 3-7, 1988, and published in Theory of Fusion Plasmas, CRPP, Association Euratom - Confederation Suisse, EPFL, p.597 (UCRL-99670).

A. Throop, R. Jong, S. Allen, et al., "140 GHz Microwave FEL Experiments Using ELF-II," Proc. of 11th Int. Conf. on Free Electron Lasers, Naples, FL, Aug. 28-Sept. 1, 1989 (UCRL-101946)

W. Turner, "Status of Microwave Free Electron Laser Applied to Tokamak Plasma Heating," presented at the 12th Intl. Conf. on Lasers and Applications, New Orleans, LA, Dec. 3-8, 1989.

J.A. Byers, R.H. Cohen, M.E. Fenstermacher, E.B. Hooper, S.Meassick, T.D. Rognilien, G.R. Smith, and B.W. Stallard, "Preparation for ECRH Propagation and Absorption Experiments in MTX," 8th Topical Conference on Radio 
Frequency Power in Plasmas, Irvine, CA, May 1-3, 1989, AIP Conference Proceedings 190, 36 (1989).

B.W. Stallard, J.A. Byers, E.B. Hooper, M. Makowski, S. Meassick, B. Rice, T.D. Rognlien, and J. Verboncour, "ECH on the MTX," 8th Topical Conference on Radio Frequency Power in Plasmas, Irvine, CA, May. 1-3, 1989, AIP Conference Proceedings 190, 84 (1989).

T. Oda, K. Mizuno, E. B. Hooper, Y. Matsuda, K. Takiyama, K. Kawasaki, K. Odajima, and K. Ohasa, "Electric Field Measurements in Fusion Plasmas," in Proceedings of the 4th International Symposium on Laser-Aided Plasma Diagnostics, edited by M. Akazaki (Fukuoka, Japan, Nov. 20-23, 1989), pp. 165170.

B. Stallard et al., "ECH by FEL and Gyrotron Sources on the MTX Tokamak," Proceedings of the 16th Symposium on Fusion Technology, London, UK, Sept. 3-7, 1990, North-Holland, p. 1050 (1991).

S. L. Allen et al., "Free Electron Laser Experiments in the Microwave Tokamak Experiment," Plasma Physics and Controlled Nuclear Fusion Research 1990, IAEA-CN-53/E-III-6, Vol. 1, International Atomic Energy Agency, Vienna, p. 783 (1991).

M. A. Makowski, B. W. Stallard and R. A. Jong, "A MAGICTRAC Design for the MTX Transport System," Proceedings of the 15th International Conference on Infrared and Millimeter Waves, Orlando, FL, Dec. 10-14, 1990, SPIE IRMM Vol. 1514, 538 (1990).

K. Thomassen, "Microwave Free-Electron Laser Applications for Eiectron Cyclotron Heating of Plasmas," SPIE Conference, Los Argeles, CA, Jan. 18-19, 1990, SPIE P Vol. 1227, 94 (1990).

M. E. Fenstermacher et al., "ECH Propagation and Absorption Exeriments at 140 GHz in MTX," Ninth Topical Conference on Radio Frequency Power in Plasmas, Charleston, SC, August 1991, AIP Conference Proceedings 244, 20 (1992).

K. I. Thomassen, S. L. Allen, T. A. Casper, M. E. Fenstermacher, J. H. Foote, E. B. Hooper, C. J. Lasnier, M. A. Makowski, W. H. Meyer, K. Oasa, G. D. Porter, B. Rice, K. Sakamoto, B. W. Stallard, and R. D. Wood, "High Power Millimeter Wave Heating in MTX," Proceedings of the 16th International Conference on Infrared and Millimeter Waves, Lausanne, Switzerland, August 1991.

T. Oda, K. Odajima, J. Foote, K. Mizuno, K. Takiyama, T. Ogawa, K. Ohasa, K. Hoshino, K. Sato, and D. Nilson, “Spectroscopic Diagnostics of Tokamak 
Plasma Heating by High Power Microwave," in Proceedings of the 5th International Symposium on Laser-Aided Plasma Diagnostics, edited by H. F. Dobele (Physikzentrum, Bad-Honnef, Germany, August 19-23, 1991), pp. 168173.

S. L. Allen and E. T. Scharlemann, "The ETA-II Linear Induction Accelerator and Imp Wiggler. A High-Average-Power Millimeter-Wave Free-ElectronLaser For Plasma Heating," Proceedings of the 9th International Conference on High-Power Particle Beams, Washington, DC, May 25-29, 1992, p. 247 (1992).

T. Oda, K. Odajima, K Takiyama, J. H. Foote, K. Mizuno, T. Ogawa, K. Sato, K. Ohasa, K. Hoshino, H. Maeda, and D. G.'Nilson, "Development of a Diagnostic System for High Power Microwave (FEL) Electric Fields in the Microwave Tokamak Experiment," in Proceedings of the 1992 International Conference on Plasma Physics, edited by W. Freysinger, K. Lackner, R. Schritter, and W. Lindinger (Innsbruck, Austria, June 29 - July 3, 1992), Part II, pp. 1191-1194.

S. L. Allen et al., "Electron Cyclotron Resonance Heating in the Microwave Tokamak Experiment," Plasma Physics and Controlled Nuclear Fusion Research 1992, International Atomic Energy Agency, Vienna (1993) (to be published).

B. W. Stallard et al., "Experiments on Nonlinear Absorption of ECH Waves in MTX and a Comparison with Theory," Tenth Topical Conference on Radio Frequency Power in Plasmas, Boston, MA, April 1993, AIP Conference Proceedings. 


\section{A.5.3 Symposia on Fusion Engineering papers}

Proceedings of the 12th Symposium on Fusion Engineering, Monterey, $C A$, October 12-16, 1987, IEEE 87CH2507-2 (1987):

B. Felker, W. Ferguson, J. Heefner, K. Krause, M. Makowski, and R. Yamamoto, "Microwave System for the Microwave Tokamak Experiment (MTX)," Vol. 1, p. 47.

S. M. Hibbs, H. H. Bell, F. M. Bowman, C. Hitchin, and M. Jackson, "Bringing the Mountain to Mohammed: The Relocation of Alcator C," Vol. 1, p. 204.

M. C. Jackson and R. C. Musslewhite, "Magnet Power System for the Microwave Tokamak Experiment (MTX)," Vol 1, p. 415.

B. Felker, "Shield Walls for the Microwave Tokamak Experiment (MTX) Facility," Vol. 1, p. 699.

K. I. Thomassen, B. I. Cohen, E. B. Hooper, D. D. Lang, and W. M. Nevins, "The Microwave Tokamak Experiment (MTX)," Vol. 2, p. 1168.

H. H. Bell, B. W. Rice, D. E. Petersen, and C. H. Herrera, "MTX Facility and Machine Grounding Plan," Vol. 2, p. 1465.

C. A. Brooksby, B. Rice, and W. A. Peebles, "Design of a Far Infrared Interferometer Diagnostic Support Structure," Vol. 2, p. 1478.

B. W. Rice, E. B. Hooper, and C. A. Brooksby, "MTX Plasma Diagnostics System," Vol. 2, p. 1524.

Proceedings of the 13th Symposium on Fusion Engineering, Knoxville, IN, October 2-6, 1989, IEEE 89CH2820-9, (1990):

S. W. Ferguson, R. Stever, A. Throop, B. Felker, and R. Franklin, "MTX/ELF II Microwave Power Measurements and Calibration for the 2-GW, 140-GHz, ELF II Free-Electron Laser," Vol. 1, p. 145.

D. N. Butner, M. Drlik, M. D. Brown, T. A. Casper, W. H. Meyer, and J. M. Moller, "The MTX Data Acquisition System," Vol. 1, p. 164.

D. E. Petersen, "Precision Timing and Interlocks Systems for FEL Heating Experiments on MTX," Vol. 1, p. 189.

N. R. Sewall and S. Meassick, "Floating Data Acquisition System for Microwave Calorimeter Measurements on MTX," Vol. 1, p. 679. 
D. D. Lang and H. H. Bell, "Microwave Tokamak Experiment: An Overview of the Construction and Checkout Phase," Vol. 2, p. 1064.

M. C. Jackson, "Microwave Tokamak Experiment (MTX) First Year of Operation and Future Plans," Vol. 2, p. 1068.

B. Felker and S. W. Ferguson, "Microwave Transport System for the MTX," Vol. 2, p. 1092.

M. C. Jackson, "Microwave Tokamak Experiment (MTX) Ohmic Heating System," Vol. 2, p. 1375.

Proceedings of the 14th Symposium on Fusion Engineering, San Diego, CA. Sept. 30 to Oct. 3, 1991. IEEE 91 CH 3035-3, (1992):

B. Felker and S. W. Ferguson, "The Intense Microwave Prototype (IMP) Free Electron Laser, 140 Gigahertz Microwave System for the Microwave Tokamak Experiment (MTX)," Vol. 1, p. 132.

S. W. Ferguson, B. Felker, M. Jackson, D. Petersen, N. Sewall, and R. Stever, "Installation and Operation of the $400 \mathrm{~kW} 140 \mathrm{GHz}$ Gyrotron on the MTX Experiment," Vol. 1, p. 138.

D. D. Lane, S. L. Allen, and H. H. Bell, "Overview of the Microwave Tokamak Experiment Operation and Developments," Vol. 1, p. 174.

D. E. Fetersen, "A Modular Timing System for Megawatt Gyrotrons," Vol. 2, p. 835.

M. C. Jackson, S. W. Ferguson, and D. E. Petersen, "The MTX Computer Control System for the 400 Kilowatt $140 \mathrm{GHz}$ Gyrotron," Vol. 2, p. 839.

D. N. Butner, M. D. Brown, T. A. Casper, W. H. Meyer, and J. M. Moller, "Diagnostics Data Management on MTX," Vol. 2, p. 847.

D. G. Nilson, F. J. Deadrick, S. M. Hibbs, D. E. Petersen, and S. E. Sampayan, "ETA-II Accelerator Upgrades," Vol. 2, p. 1179.

D. Nilson, T. Casper, J. Foote, C. Lasnier, P. Lopez, B. Rice, R. Stever, K. Hoshino, K. Odajima, T. Oda, and K. Mizuno, "Diagnostic Upgrades on MTX," Vol. 2, p. 1205. 


\section{A.5.4 IEEE Particle Accelerator Conferences}

Proceedings of the 1989 IEEE Particle Accelerator Conference, Chicago, IL, March 20-23, 1989:

W. Turner, J. Boyd, J. Clark, W. Nexsen, "Status of the ETA-II Linear Induction Accelerator-High Brightness Results," (UCRL-99579).

A. Throop, W., Turner, D. Atkinson, et al, "High Average Power Millimeter Wave FEL for Plasma Heating Using the ETA-II Accelerator," (UCRL-100225)

Proceedings of the 1991 IEEE Particle Accelerator Conference, San Francisco, CA, May 6-9, 1991, IEEE 91CH3038-7 (1991):

W. C. Turner, "Control of Energy Sweep and Transverse Beam Motion in Induction Linacs," Vol. 1, p. 581.

M. A. Makowski, "RF Transfer in the Coupled-Cavity Free-Electron Laser Two-Beam Accelerator," Vol. 4, p. 2548.

F. W. Chambers et al., 'Diagnostics and Data Analysis for the ETA-II Linear Induction Accelerator," Vol. 5, p. 3085.

S. L. Allen et al., "Measurement of Reduced Corkscrew Motion on the ETA-II Linear Induction Accelerator," Vol. 5, p. 3094.

W. E. Nexsen et al., "Reduction of Energy Sweep of the ETA-II Beam," Vol. 5, p. 3103.

A. C. Paul et al., "ETA-II Beam Brightness Measurement," Vol. 5, p. 3106.

Proceedings of the 1993 IEEE Particle Accelerator Conference, Washington, D.C. (1993):

S. L. Allen et al., "Generation of High Power $140 \mathrm{GHz}$ Microwaves with an FEL for the MTX Experiment."

C. J. Lasnier et al., "Burst Mode FEL with the ETA-III Induction Linac." 


\section{A.5.5 Lawrence Livermore National Laboratory reports}

K. I. Thomassen, "Free Electron Laser Experiments in Alcator-C," Lawrence Livermore National Laboratory, Livermore, CA, LLL-PROP-00202 (July 1986).

K. I. Thomassen, "Microwave Tokamak Experiment Administrative Plan," Lawrence Livermore National Laboratory, Livermore, CA, LLL-PROP-00202 Addendum 1 (July 1987).

E. B. Hooper, "Microwave Tokamak Experiment, Experimental Plan for FY 1989 to FY 1992," Lawrence Livermore National Laboratory, Livermore, CA, LLL-PROP-00202 Addendum 2 (May 1989).

K. I. Thomassen, "Microwave Tokamak Experiment Safety Analysis Doniment," Lawrence Livermore National Laboratory, Livermore, CA, LLLPROP.00202 Addendum 3 (Nov. 1989).

W. M. Nevins, E. B. Hooper, and I. B. Bernstein, "Use of Polarimetry to Measure the Current Profile in MTX," July 1987, (UCID-21123).

G. R. Smith, R. H. Cohen, and T. I. Mau, "Harmonic Overlap in ElectronCyclotron Current Drive at High $\mathrm{T}_{\mathbf{e}}$, "Lawrence Livermore National Laboratory, Livermore, CA, UCRL-96364 (1987).

H. H. Bell, "MTX Facility and Machine Grounding Plan," Lawrence Livermore National Laboratory, Livermore, CA, UCRL-96653, October 1987.

R. H. Cohen, "Mirror Theory Applied to Toroidal Systems," Lawrence Livermore National Laboratory, Livermore, CA, UCRL-96672, Rev. 1 (1987).

J. A. Byers and $\mathrm{R}$ H. Cohen, "A Microwave FEL Code Using Waveguide Modes," Lawrence Livermore National Laboratory, Livermore, CA, UCRL97348 (1987).

B. I. Cohen, "Particle Simulation of Intense Electron Cyclotron Heating and Beat-Wave Current Drive," Lawrence Livermore National Laboratory, Livermore, CA, UCRL-97359 (1987).

D. Prosnitz, "The Physics of Free Electron Lasers and Applications to Electron Cyclotron Heating," UCRL-98716 (1988).

J. A. Lorbeck, "Wire Grid Beam Splitter for Microwave Diagnostics on MTX," March 1989 (UCID-21644). 
E.B. Hooper, S. L. Allen, T.A. Casper, and K.I. Thomassen, 'MTX Diagnostic and Auxiliary Systems for Confinement, Transport, and Plasma Physics Studies," (UCID-21744).

M. Marinak, "Design of a Microwave Calorimeter for the Microwave Tokamak Experiment," Lawrence Livermore National Laboratory, Livermore, CA, UCID-21808 (1988).

M. Marinak (UCB) "An Extended Diffusive Model for Calculating Thermal Diffusivity from Single Monopole Tokamak Heat Pulse Propagation," Feb. 1990 (UCRL-ID-103119).

E. B. Hooper and M. A. Makowski, "Characterization of Disruptions in the Microwave Tokamak Experiment, MTX," Report of ITER R\&D Contribution, Task PH07, March 1990 (UCRL-ID-103473).

E. B. Hooper and S. L. Allen, "MTX - Overview and Status," UCRL-ID103486 (1990).

F. J. Deadrick and L. V. Griffith, "Progress in ETA-II Magnetic Alignment Using Stretched Wire and Low Energy Electron Beam Techniques," 1990 Linac Conference, Albuquerque, NM; also Lawrence Livermore National Laboratory, Livermore, CA, UCRL-103780.

M. E. Fenstermacher, "Comparison of Propagation and Absorption of FELGenerated Microwaves with Theory." DOE Milestone Report, Microwave Tokamak Experiment, Mar. 1990 (UCRL-ID-103808).

E. B. Hooper et al., "Physics Study of the Application of an FEL, to CIT," Final Report, FY 1990 (UCRL-ID-104103-Rev.1).

E. B. Hooper et al., "Anatomy of a Disruption in MTX," Report of ITER Ki\&D Contribution, Task PH07, Oct. 1990 (UCRL-ID-104867). 

A.5.6 Japan Atcinic Energy Research Institute reports
K. Odajima, K. Ohasa, M. Shiho, T. Oda, K. Takiyama, K. Mizuno, I. H. Foote, D. G. Nilson, S. L. Allen, and T. A. Casper, "MTX Microwave-Electric-Field Diagnostic," Japan Atomic Energy Research Institute, Tokai, Japan, JAERI-M 90-088 (1990).
C. J. Lasnier and K. Hoshino, "ECE Measurements During Extreme High- Power ECRH," Jaf an Atomic Energy Research Institute, Tokai, Japan, JAERI- memo 04-362 (1993). 